\section{Pacific Northwest}

National Laboratory

Operated by Battelle for the

U.S. Department of Energy

\title{
Factors Effecting the Fate and Transport of CL-20 in the Vadose Zone and Groundwater: \\ Final Report 2002 - 2004 SERDP Project CP/ER-1255
}

\author{
JE Szecsody \\ RE Riley \\ BJ Devary \\ DC Girvin \\ T Resch \\ JA Campbell
}

\author{
HL Fredrickson* \\ KT Thompson* \\ FH Crocker* \\ MM Qasim* \\ AP Gamerdinger** \\ L A Lemond**
}

June 2005

Prepared for the U.S. Department of Energy under Contract DE-AC06-76RL01830

*U.S. Army Engineer Research \& Development Center Environmental Laboratory at Waterways Experiment Station Vicksburg, Mississippi 39180-6199

**University of Arizona, Department of Soils Tucson, Arizona 85721 


\title{
DISCLAIMER
}

This report was prepared as an account of work sponsored by an agency of the United States Government. Neither the United States Government nor any agency thereof, nor Battelle Memorial Institute, nor any of their employees, makes any warranty, express or implied, or assumes any legal liability or responsibility for the accuracy, completeness, or usefulness of any information, apparatus, product, or process disclosed, or represents that its use would not infringe privately owned rights. Reference herein to any specific commercial product, process, or service by trade name, trademark, manufacturer, or otherwise does not necessarily constitute or imply its endorsement, recommendation, or favoring by the United States Government or any agency thereof, or Battelle Memorial Institute. The views and opinions of authors expressed herein do not necessarily state or reflect those of the United States Government or any agency thereof.

\author{
PACIFIC NORTHWEST NATIONAL LABORATORY \\ operated by \\ BATTELLE \\ for the \\ UNITED STATES DEPARTMENT OF ENERGY \\ under Contract DE-AC06-76RLO 1830
}

\section{Printed in the United States of America}

Available to DOE and DOE contractors from the

Office of Scientific and Technical Information, P.O. Box 62, Oak Ridge, TN 37831; prices available from $(615) 576-8401$.

Available to the public from the National Technical Information Service, U.S. Department of Commerce, 5285 Port Royal Rd., Springfield, VA 22161

This document was printed on recycled paper. 


\section{Factors Effecting the Fate and Transport of CL-20 in the Vadose Zone and Groundwater: Final Report 2002 - 2004 SERDP Project CP/ER-1255}

Jim E. Szecsody

Robert E. Riley

Brooks J. Devary

Don C. Girvin

Tom Resch

Jim A. Campbell

Herb L. Fredrickson*

Karen T. Thompson*

Fiona H. Crocker*

Mohammad M. Qasim*

Amy P Gamerdinger**

Luke A. Lemond**

June 2005

Pacific Northwest National Laboratory

Richland, Washington 99354

*U.S. Army Engineer Research \& Development Center

Environmental Laboratory at Waterways Experiment Station Vicksburg, Mississippi 39180-6199

**University of Arizona, Department of Soils

Tucson, Arizona 85721 


\section{Table of Contents}

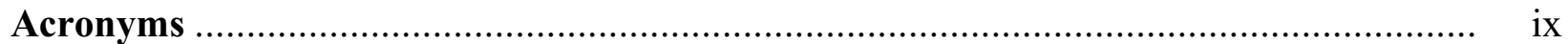

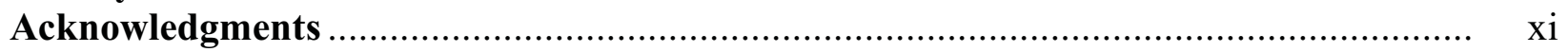

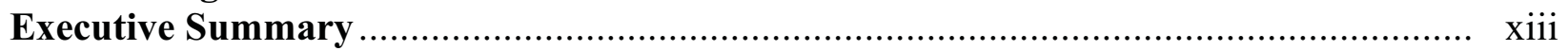

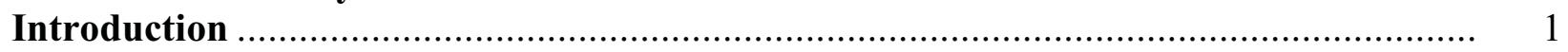

Background: Subsurface Fate of CL-20 ................................................................... 3

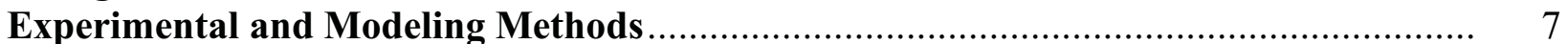

Task 1 Abiotic Fate of CL-20 in the Subsurface................................................... 7

Task 1.1 Sorption of CL-20 ...................................................................... 7

Task 1.2 Abiotic CL-20 Degradation and Other Reactions ..................................... 9

Task 1.3 CL-20 and Degradation Product Analysis .............................................. 11

Task 2 Microbial Degradation of CL-20 …………............................................... 13

Task 2.1 Anaerobic Degradation of CL-20................................................... 14

Task 2.2 Aerobic Degradation of CL-20 .................................................... 16

Task 3 Coupled Processes................................................................................ 18

Task 3.1 Coupled Sediment-Biotic Reactions with CL-20................................. 18

Task 3.2 Effect of Aging and CL-20/Sediment Reactions .................................... 19

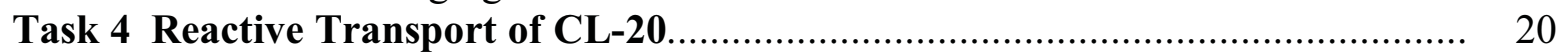

Task 4.1 Abiotic Transport of CL-20 in Saturated Porous Media ........................... 21

Task 4.2 Abiotic/Biotic Transport of CL-20 in Saturated Porous Media.................. 22

Task 4.3 Reactive Transport of CL-20 in Unsaturated Porous Media ..................... 23

Results

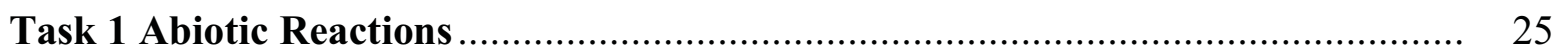

Task 1.1 Sorption of CL-20 to Sediments ............................................................ 25

Task 1.2 Abiotic CL-20 Degradation .............................................................. 30

Task 1.3 Analytical............................................................................. 44

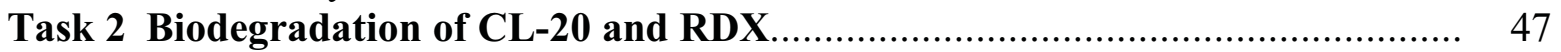

Task 2.1 Aerobic Biodegradation................................................................. 47

Task 2.2 Anaerobic Biodegradation ............................................................... 59

Task 3 Coupled Abiotic and Biotic Processes ....................................................... 63

Task 3.1 Coupled Abiotic/Biotic Processes ……….............................................. 63

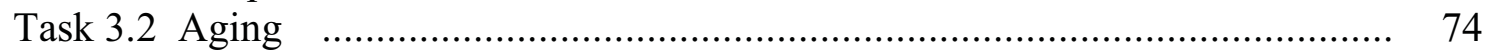

Task 4 Reactive Transport of CL-20........................................................... 80

Task 4.1/4.2 CL-20 Transport in Water-Saturated Systems ................................... 80

Task 4.3 CL-20 Transport in Low Water-Saturation Columns............................... 86

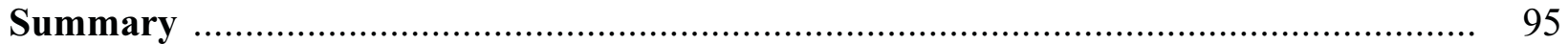

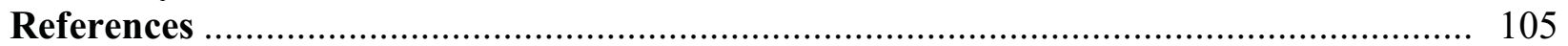

Attachment 1: Publications and Presentations ............................................................ 116

Appendix A - CL-20 Aqueous and Laboratory Minerals Degradation........................... 119

Appendix A1. CL-20 Reactions with Laboratory Materials ......................................... 119

Appendix A2. CL-20 Aqueous Degradation ........................................................ 120

Appendix B - CL-20 Degradation by Minerals ……................................................ 123

Appendix B1. CL-20 Degradation by Natural Minerals ............................................ 123

Appendix B2. CL-20 Degradation at Different Temperature ...................................... 125 
Appendix B3. CL-20 Degradation by Minerals with Adsorbed Fe(II) ......................... 127

Appendix B4. CL-20 Degradation by Minerals with Structural Fe(II) ......................... 129

Appendix B5. CL-20 Degradation by Minerals with Structural and Adsorbed Fe(II).... 131

Appendix C - CL-20 Sorption and Degradation in Oxic Sediments............................... 132

Appendix C1. CL-20 Sorption to Natural Sediments............................................... 132

Appendix C2. CL-20 Sorption Characterization ................................................ 134

Appendix C3. CL-20 Degradation in Oxic Sediments ............................................. 135

Appendix D - CL-20 Sorption and Degradation in Reduced Sediments ......................... 137

Appendix E - CL-20 Degradation Temperature Studies ............................................. 139

Appendix E1. Oxic Sediments and CL-20 Degradation................................................ 139

Appendix E2. CL-20/RDX Degradation in Reduced Sediment..................................... 143

Appendix F - CL-20 Coupled Degradation Studies ................................................. 146

Appendix G - CL-20 Mineralization Studies........................................................ 162

Appendix H - CL-20 Sorption and Degradation during 1-D Flow ............................... 165

Appendix H1. Oxic Norborne C Sediment ............................................................... 165

Appendix H2. Reduced Norborne C Sediment ......................................................... 170

Appendix H3. Oxic Westmoreland A1 Sediment ...................................................... 171

Appendix H4. Oxic Burbank AP Sediment ......................................................... 173

Appendix H5. Oxic Ocala C4 and Other Sediments ................................................ 175

Appendix I - CL-20/RDX Aging Studies with 1-D Flow ............................................ 178

Appendix I1. Aging Data for Westmoreland A1 Sediment .......................................... 178

Appendix I2. Aging Data for Sassafrass Sediment .................................................... 181

Appendix I3. Simulation Results of the Influence of Aging........................................ 184

Appendix I4. Breakthrough Curve Sensitivity Simulations ....................................... 186

Appendix I5. Tracer Breakthrough Simulations for the Westmoreland Sediment ......... 188

Appendix I6. Tracer Breakthrough Simulations for the Sassafrass Sediment................. 191

Appendix I7. RDX Breakthrough Simulations for the Westmoreland Sediment ............. 194

Appendix I8. RDX Breakthrough Simulations for the Sassafrass Sediment................... 197

Appendix I9. CL-20 Breakthrough Simulations for the Westmoreland Sediment .......... 200

Appendix I1. CL-20 Breakthrough Simulations for the Sassafrass Sediment................. 203

Appendix J - CL-20/RDX Transport during Unsaturated Flow ……........................ 206

Appendix J1. Unsaturated Flow Experiments in the Hanford MSC Sediment .............. 206

Appendix J2. Unsaturated Flow Experiments in the Hanford MSC Sediment .............. 217

Appendix J1. Unsaturated Flow Experiments in the Hanford MSC Sediment .............. 228 


\section{Table of Figures}

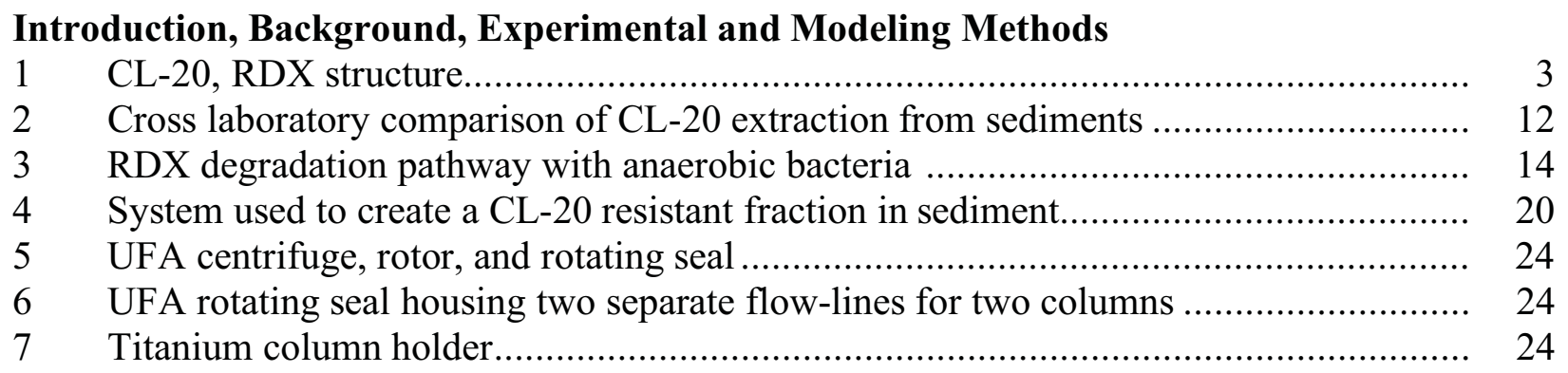

\section{Results: Task 1 - Abiotic Reactions}

1.1 CL-20 sorption rate on Norborne and Kenoma Bt1 sediment .................................... 26

1.2 Aqueous degradation rate of CL-20 by $24 \mathrm{~h}$ at different $\mathrm{pH}$.................................... 27

1.3 CL-20 and RDX sorption and organic matter, clay content, and iron content............... 28

1.4 CL-20 sorption isotherms and $\mathrm{pH}$ edge on Norborne $\mathrm{C}$ sediment .............................. 30

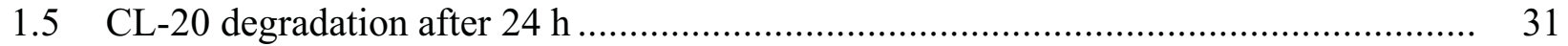

1.6 Degradation of CL-20 over time in contact with light and laboratory materials ........... 32

$1.7 \quad$ CL-20 degradation pathway in highly acidic conditions .......................................... 33

1.8 CL-20 degradation products showing carbon and nitrogen mass balance.................... 34

1.9 UV-Vis absorbance spectra of CL-20 degradation by alkaline hydrolysis.................... 35

1.10 CL-20 degradation rate in aqueous solution at elevated temperature........................... 36

1.11 CL-20 degradation at differing Eh in aqueous solution with reduced sulfur species...... 36

1.12 CL-20 degradation by adsorbed Fe(II) on differing iron oxides ................................ 37

1.13 CL-20 degradation by adsorbed Fe(II) on hectorite ................................................. 37

1.14 CL-20 degradation by kaolinite and hectorite......................................................... 38

1.15 CL-20 degradation by the oxic Cloudland AP sediment at different temperature.......... 39

1.16 CL-20 degradation rate in reduced Ft Lewis sediment and zero valent iron .................. 40

1.17 CL-20 degradation in hot aqueous solution and by alkaline hydrolysis....................... 41

1.18 CL-20/RDX degradation versus temperature.............................................................. 42

$1.19 \mathrm{CL}-20$ degradation at $4^{\circ} \mathrm{C}$ by reduced sediment and model simulations....................... 43

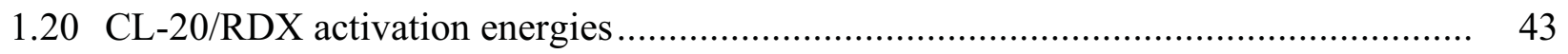

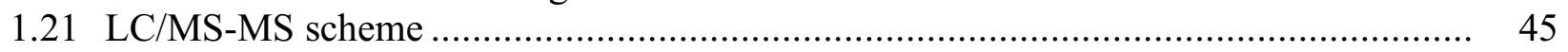

1.22 LC/MS identification of CL-20 with electrospray injection ....................................... 45

1.23 FTIR spectra of CL-20 and CL-20 reacted with OH................................................. 46

\section{Results: Task 2 - Biodegradation of CL-20 and RDX}

2.1 Degradation of CL-20 in China Lake soil microcosms under aerobic conditions .......... 47

2.2 Degradation of RDX in China Lake soil microcosms under aerobic conditions ............ 52

2.3 Cumulative mineralization of ${ }^{14} \mathrm{C}-\mathrm{CL}-20$ in China Lake soil microcosms................... 53

2.4 Change in CL-20 concentration in China Lake soil microcosms................................. 54

2.5 Growth of isolates on CL-20 and RDX ........................................................... 58

2.6 Cumulative mineralization and degradation of ${ }^{14} \mathrm{C}-\mathrm{CL}-20$ by Gordonia sp. KTC13 ..... 58

2.7 Degradation of CL-20 in China Lake soil microcosms under anaerobic conditions....... 59

2.8 Degradation of RDX in China Lake soil microcosms under anaerobic conditions ........ 60 
2.9 Degradation of CL-20 in China Lake soil microcosms under reducing conditions ........ 61

2.10 Cumulative mineralization of ${ }^{14} \mathrm{C}-\mathrm{CL}-20$ in China Lake soil microcosms.................. 62

\section{Results: Task 3 - Coupled Abiotic and Biotic Processes}

3.1 CL-20 mineralization in oxic sediment .......................................................... 63

3.2 CL-20 mineralization in an oxic environment without nutrients ............................. 64

3.3 CL-20 mineralization in anoxic sediment with glucose addition.............................. 64

3.4 CL-20 mineralization in reduced sediments with TSB addition ............................. 64

3.5 CL-20 mineralization in partially reduced sediments........................................ 65

3.6 Coupled CL-20 abiotic/biotic hypothesized in reducing conditions ......................... 66

3.7 CL-20 abiotic scoping experiments ............................................................... 66

3.8 Mossbauer of bioreduced FRC showing mainly green rust ................................. 67

3.9 CL-20 biodegradation with CN-32 without and with lactate and sterilized CN-32 ...... 67

3.10 CL-20 biodegradation with CN-32 with varying microbial population densities.......... 68

3.11 CL-20 abiotic degradation of oxic FRC sediment and bioreduced FRC sediment ........ 68

3.12 Coupled CL-20 abiotic/biotic degradation and prediction....................................... 68

3.13 CL-20 coupled degradation...................................................................... 69

3.14 Comparison of observed coupled degradation to predicted degradation..................... 70

3.15 Degradation of CL-20 with gibbsite only and in a coupled system .......................... 71

3.16 Degradation for coupled systems containing bioreduced FRC sediment ................... 73

3.17 CL-20 sorption and desorption in aged columns for the Westmoreland sediment ........ 75

3.18 CL-20 sorption and desorption in aged columns for the Sassafrass sediment.............. 76

3.19 CL-20 sorption and desorption in aged columns................................................. 77

3.20 Change in desorption $\mathrm{K}_{\mathrm{d}}$ /sorption $\mathrm{K}_{\mathrm{d}}$ for CL-20 and RDX ................................ 78

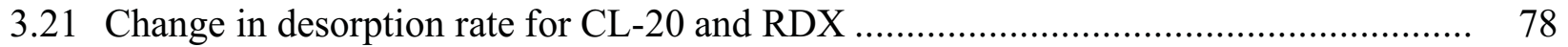

3.22 Change in degradation rate for CL-20 with aging time .......................................... 78

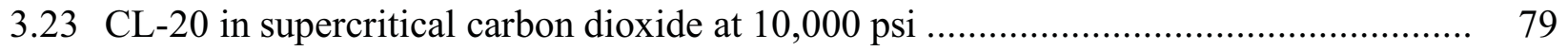

\section{Results: Task 4 - Reactive Transport of CL-20}

4.1 CL-20 reactive transport at five different velocities in Norborne sediment ................. 80

4.2 Reactive transport of CL-20 through oxic sediments ......................................... 81

4.3 CL-20 degradation in oxic and reduced Norborne sediment ................................ 82

4.4 CL-20 degradation products during 1-D transport in oxic sediments ....................... 84

4.5 Tracer transport in the Burbank sediment columns at differing water content............. 89

4.6 RDX and CL-20 transport in the Burbank sediment at differing water content ........... 90

4.7 Relationship of CL-20 and RDX sorption to water saturation during transport........... 91

4.8 Reactive transport of RDX and CL-20 through Burbank sediment ......................... 92

\section{Summary}

S1 Geochemical factors influencing CL-20 adsorption in sediments .......................... 95

S2 Influence of water saturation on CL-20 and RDX sorption................................. 96

S3 Potential for deep migration of CL-20 with different sediments and minerals............. 97

S4 CL-20 degradation rate as correlated with iron, temperature, $\mathrm{pH}$, and water saturation. 99 


\section{List of Tables}

Introduction, Background, Experimental and Modeling Methods

1 Organization of project tasks ....................................................................... 2

2 CL-20 and RDX properties....................................................................... 3

Results: Task 1 - Abiotic Reactions

1.1 CL-20 sorption and degradation and RDX sorption to sediments in 24 h.................... 26

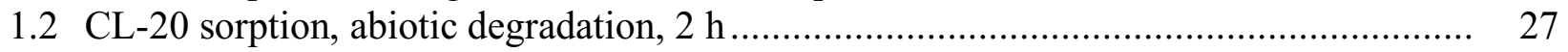

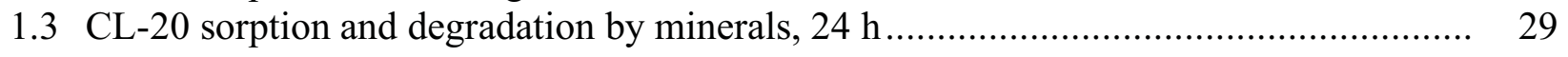

1.4 Small Molecular Weight Products of Abiotic Degradation ..................................... 34

1.5 Comparison of zero valent iron and reduced sediment CL-20 degradation rates ............ 40

1.6 CL-20 degradation factors ............................................................................. 41

1.7 Activation energies for degradation of CL-20 and RDX activation ........................... 44

1.8 Electrospray MS data of CL-20/reduced Norborne sediment reaction ........................ 46

Results: Task 2 - Biodegradation of CL-20 and RDX

2.1 Selected chemical and physical properties of soils

2.2 Coefficients from first-order fits for CL-20 and RDX aerobic degradation in China Lake soils....

2.3 Microbial biomass estimates in China Lake aerobic soil microcosms......................... 50

2.4 First-order rate constants for CL-20 and RDX aerobic degradation ............................ 51

2.5 Carbon balance of CL-20 mineralization in aerobic China Lake soil microcosms ......... 55

2.6 Coefficients from first-order model fits for CL-20 and RDX anaerobic degradation...... 56

2.7 Coefficients from first-order model fits under various electron acceptor conditions ....... 57

\section{Results: Task 3 - Coupled Abiotic and Biotic Processes}

3.1 Rates of coupled CL-20 abiotic/biotic degradation............................................ 71

3.2 CL-20 coupled degradation in gibbsite....................................................... 72

3.3 Coupled CL-20 degradation in FRC sediment after iron extractions ......................... 73

\section{Results: Task 4 - Reactive Transport of CL-20}

4.1 CL-20 sorption mass and degradation rates in 1-D columns .................................... 82

4.2 Selected $\mathrm{P} \backslash$ physical and chemical properties of soils ...................................... 86

4.3 Simulation results of low water saturation column experiments ............................ 88

\section{Summary}

S1 RDX and CL-20 sorption and degradation in oxic subsurface sediments .................... 97

S2 CL-20 degradation factors ................................................................. 98

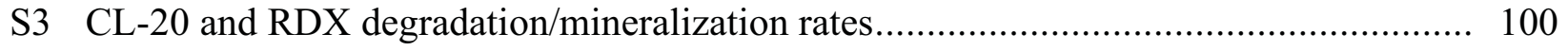

S4 Intrinsic rates of CL-20 and RDX biotransformation........................................... 101 


\section{Acronyms}

APCI atmospheric pressure chemical ionization

BRI Biotechnology Research Institute of the National Research Council, Canada

CERCLA Comprehensive Environmental Response, Compensation, and Liability Act

CL-20 2,4,6,8,10,12-Hexanitro-2,4,6,8,10,12-Hexaazaisowurzitane, CAS 135285-90-4

DCB dithionite-citrate-bicarbonate soil extraction for ferric oxides

DNX hexahydro-1,3-dinitroso-5-nitro-1,3,5-triazine, RDX degradation product

DOD U.S. Department of Defense

DOE U.S. Department of Energy

ECBC U.S. Army Edgewood Chemical Biological Center

EPA U.S. Environmental Protection Agency

ERDC U.S Army Research and Development Center

ESI electrospray ionization

ETFE plastic, polymer of tetrafluoroethylene and ethylene

FEP plastic, polymer of tetrafluoroethylene and hexafloropropylene

FRC Field Research Center at the U.S. DOE Oak Ridge Site

FTIR fourier transform infared spectroscopy

GC gas chromatography

HDPE high-density polyethylene

HMX 1,3,5,7-tetranitro-1,3,5,7-tetrazocane, high melting explosive, CAS 2691-41-0

HNIW hexanitrohexaazaisowurtzitane, CL-20

HPLC-UV high performance liquid chromatography with ultraviolet detection

LC/MS liquid chromatography/mass spectrometry

LDPE low-density polyethylene

MNX hexahydro-1-nitroso-3,5-dinitro-1,3,5-triazine, RDX degradation product

MSM mineral salt media used for microbial experiments

NCIMB National Collection of Industrial and Marine Bacteria

PFBA pentafluorobenzoic acid

PIPES 1,4-piperazinediethanesulfonic acid

PLFA phospholipids fatty acids

PNNL Pacific Northwest National Laboratory

RDX hexahydro-1,3,5-trinitro-1,3,5-triazine, royal demolition explosive, CAS 121-8-24

SERDP Strategic Environmental Research and Development Program

TCE trichloroethylene

TETRYL trinitrophenylmethylnitramine explosive, CAS 479-45-8

TNT trinitrotoluene, CAS 118-96-7

TNX hexahydro-1,3,5-trinitroso-1,3,5-triazine, RDX degradation product

UFA ultraflow centrifuge apparatus, column system within centrifuge

UV ultraviolet

WES U.S. ERDC, Waterways Experiment Station

ZVI zero valent iron 



\section{Acknowledgments}

We would like to acknowledge support from Dr. Robert Holst at the SERDP Office for funding and being involved in the collaboration of this and two other projects (CP-1254 and CP-1256) on CL-20 fate and toxicity. We would also like to acknowledge support from Mr. Bradley Smith, Executive Director for SERDP. Sharing preliminary data and hypotheses by Dr. Jalal Hawari (Biotechnology Research Institute, NRC) and Dr. Roman Kuperman (U.S. Army Edgewood Chemical Biological Center), principle investigators of these two other SERDP projects, was extremely useful for coordinating closely related tasks and to ensure fate/toxicity in various environments was being investigated. Significant early progress in breakdown products was made by information obtained in recently released technical documents at China Lake Naval Weapons Laboratory where CL-20 was originally developed. Access to these documents was made possible by Dr. Robin Nissin at China Lake. Molecular modeling of CL-20, RDX, and other energetics by Dr. Mo Qasim of the U.S Army Engineer Research and Development Center (ERDC) was instrumental in identification of the CL-20 degradation pathway. This hypothesized pathway was later confirmed by experimental evidence. This molecular modeling was partially funded by this project and partially by the COE Environmental Quality funding at the ERDC. We would also like to thank Dr. Scott Lusk and Dr. Scott Hamilton at ATK Thiokol for providing high purity CL-20 in various isotopic $\left({ }^{15} \mathrm{~N},{ }^{14} \mathrm{C}\right)$ forms for research on these three projects. Within this project, microbial work was mainly conducted at the U.S Army Engineer Research and Development Center (ERDC) by Dr. Fiona Crocker, Dr. Herb Fredrickson, and Karen Thompson. Geochemical, coupled process, and reactive transport work was mainly conducted at Pacific Northwest National Laboratory (PNNL) by Dr. Jim Szecsody, Brooks Devary, Dr. Don Girvin and Dr. Jim Campbell. Unsaturated column experimental work was conducted at PNNL by Luke Lemond and Dr. Amy Gamerdinger. 



\section{Executive Summary}

This SERDP-funded project was initiated to investigate the fate of CL-20 in the subsurface environment, with a focus on identification and quantification of geochemical and microbial reactions of CL-20. CL-20 can be released to the surface and subsurface terrestrial environment by: a) manufacturing processes, b) munition storage, and c) use with low order detonation or unexploded ordnance. The risk of far-field subsurface migration was assessed through laboratory experiments with a variety of sediments and subsurface materials to quantify processes that control CL-20 sorption-limited migration and degradation. Based on similarities in structure and functional groups, comparison of CL-20 to RDX sorption and degradation behavior provides insight into the migration potential. Sorption mass and degradation rates define the potential for deep migration of CL-20. Sorption was relatively small for CL-20 $\left(\mathrm{K}_{\mathrm{d}}=0.22\right.$ to $3.83 \mathrm{~cm}^{3} \mathrm{~g}^{-1}$; 15 subsurface sediments and 18 minerals) and RDX $\left(\mathrm{K}_{\mathrm{d}}=0.53\right.$ to $6.5 \mathrm{~cm}^{3} \mathrm{~g}^{-1}, 10$ subsurface sediments). CL-20 sorption in subsurface (low organic carbon) sediments is correlated with the mass of iron oxides in the sediment (not organic carbon or clay), whereas RDX sorption to subsurface sediments was correlated with the mass of clay (not organic carbon or mass of iron oxides). Based on the results of this study, CL-20 sorption can be estimated in surface (high organic carbon) and subsurface sediments from geochemical characterization information: a) fraction organic carbon, b) DCB-extractable Fe, c) percent clay, and d) water content. The $\mathrm{pH}$ of the sediment should be $<8.5$, as CL-20 degrades under alkaline conditions (described below). The empirical correlation of sediment properties to CL-20 sorption is:

$$
\mathrm{K}_{\mathrm{d}}\left(\mathrm{cm}^{3} \mathrm{~g}^{-1}, \mathrm{CL}-20\right)=\left[3.53(\% \mathrm{OC})^{1.14}+0.112 \mathrm{e}^{0.020 \mathrm{DCB}-\mathrm{Fe}}\right]\left[1.03 \mathrm{E}^{-3}(\% \mathrm{sat})^{1.51}\right]
$$

where $\% \mathrm{OC}$ is the percent organic carbon in the sediment, DCB-Fe is the DCB-extractable iron (Heron et al. 1994), and \% sat is the percent water saturation in the sediment. The empirical correlation of sediment properties to RDX sorption is:

$$
\mathrm{K}_{\mathrm{d}}\left(\mathrm{cm}^{3} \mathrm{~g}^{-1}, \mathrm{RDX}\right)=\left[1.25(\% \mathrm{OC})^{0.32}+0.133(\% \text { clay })^{0.705}\right]\left[2.28 \mathrm{E}^{-3}(\% \mathrm{sat})^{1.31}\right]
$$

where \% clay is the percent clay in the sediment. It is well known that RDX can migrate long distances from surface spills to groundwater and can move nearly unretarded in groundwater (Spalding and Fulton 1988). Because $\mathrm{K}_{\mathrm{d}} \mathrm{S}$ reported here are similar for CL-20 and RDX, CL-20 can be expected to move nearly unretarded through water-saturated sediments in groundwater, and has the potential to be a groundwater contamination problem similar to that of RDX. For sediments in the vadose zone, the water content has a significant effect on decreasing the sorption of both CL-20 and RDX, due to water traveling in only a portion of the sediment pores, so accessing less adsorption sites. For the shallow soil environment, surface soils $(<2 \mathrm{~m})$ in humid regions can have organic matter (fraction organic carbon or $\mathrm{f}_{\mathrm{oc}}$ ) as high as $40 \%$, whereas soils in arid regions have lower organic matter $(<5 \%)$. CL-20 sorption to surface soils (high $\mathrm{f}_{\mathrm{oc}}$ ) is mainly to the organic matter so can be generally predicted from the organic carbon content, although differing types of organic matter adsorbed CL-20 more strongly (Monteil et al. 2004). Therefore, in more humid regions, CL-20 will be retained in the soil zone where degradation (microbial, photodegradation) can occur. RDX is weakly correlated with fraction organic carbon relative to CL-20. 
While CL-20 subsurface transport is influenced only slightly by sorption, CL-20 degradation (abiotic, biotic) can have a major influence on the distribution of mass, and defines sediments with low degradation rates where there is great potential for subsurface migration. Abiotic and biotic degradation in oxic sediments is observed (hours to $1000 \mathrm{~s}$ of $\mathrm{h}$ ), which limits migration. CL-20 will degrade rapidly (minutes, hours) in alkaline waters ( $\mathrm{pH}>9.5$; hydrolysis), and in sediments with high adsorbed $\mathrm{Fe}^{\mathrm{II}}$ or with some smectite 2:1 clays. Most of sediments tested had very slow degradation (100 to $6400 \mathrm{~h}$ half-life), and so CL-20 would be persistent in the subsurface for years. At low water content, the CL-20 degradation rate decreased significantly. These results are consistent with low-water content batch experiments conducted by Jenkins et al. (2003) in which long-term studies showed CL-20 degradation half-lives ranging from 144 to 686 days. Long-term aging studies with CL-20 and RDX also showed change in the potential risk of deep migration. The smaller apparent Kd values for both CL-20 and RDX indicate that some mass remains on the sediment (i.e., lower migration risk) with long contact time. However, the same aging studies also showed that CL-20 persisted much longer in water saturated conditions with no flow, with degradation rates 1 to 2 orders of magnitude slower than determined in flow experiments (i.e., greater migration risk). The CL-20 abiotic degradation rate in oxic systems can be estimated from:

CL-20 abiotic degradation rate, oxic environment $\left(\mathrm{h}^{-1}\right)=$ $\left[1.85 \mathrm{E}^{-4}(\mathrm{DCB} \mathrm{Fe})^{1.72}+3.26 \mathrm{E}^{-12}\left(\mathrm{~T}^{\circ} \mathrm{C}\right)^{6.17}+2.85 \mathrm{E}^{-36}(\mathrm{pH})^{36}\right]\left[0.043 \mathrm{e}^{0.031 \% \mathrm{sat}}\right]$

where DCB Fe is the dithionite-citrate-bicarbonate extractable iron in the sediment, temperature is in Celsius, $\mathrm{pH}$ is the natural equilibrium $\mathrm{pH}$ of the sediment, and $\%$ sat is the percent water saturation. This estimate for CL-20 oxic abiotic degradation for natural subsurface systems shows that the rate is correlated with the DCB-extractable iron, water temperature (important if temp. $>35^{\circ} \mathrm{C}$ ) and $\mathrm{pH}$ (important if $\mathrm{pH}>8.5$ ). CL-20 is degraded rapidly in sediments under various reducing conditions:

CL-20 abiotic degradation rate, iron reducing conditions $\left(\mathrm{h}^{-1}\right)=$ $\left[5.7 \times 10^{-5} \pm 1.6 \times 10^{-5}\right]($ ferrous iron, $0.5 \mathrm{M} \mathrm{HCl}, 1 \mathrm{~h})$

where the ferrous iron is characterized by a 1 hour extraction with $0.5 \mathrm{M} \mathrm{HCl}$. The CL-20 abiotic degradation rate can be as rapid as 4 minutes (half-life) for a sediment with $100 \mu \mathrm{mol} / \mathrm{g}$ of ferrous iron. In comparison, the $\mathrm{RDX}$ degradation rate in the same iron reducing conditions is 50x more rapid:

RDX abiotic degradation rate, iron reducing conditions $\left(\mathrm{h}^{-1}\right)=$ $[0.011 \pm 0.004]$ (ferrous iron, $0.5 \mathrm{M} \mathrm{HCl}, 1 \mathrm{~h})$

CL-20 and RDX mineralization does not occur abiotically, although iron reducing conditions that promote rapid transformation to intermediates can then be biotically transformed to carbon dioxide. The rate of degradation and mineralization of CL-20 by bacterial isolates, isolates in sediment, and natural microbial microbial communities in sediments (oxic, anaerobic, and various reducing conditions) varied by four orders of magnitude, depending on microbial and geochemical factors. In contrast, the biotransformation rate (rate per microbe) varied by two orders of magnitude, so can be used to estimate the CL-20 or RDX biodegradation rate in 
sediment, given that the microbial population density is known. The microbial population density in soils ( 0 to $2 \mathrm{~m}$ depth) varies from $10^{6}$ to $10^{9} \mathrm{CFU} \mathrm{g}^{-1}$ (greater in humid soils), whereas subsurface sediments vary from $10^{3}$ to $10^{6} \mathrm{CFU} \mathrm{g}^{-1}$, so the actual observed CL-20 biodegradation rates variability appears to be predominantly due to the differences in microbial population. Estimation of the CL-20 biodegradation or mineralization rate given that the microbial population density is known:

\section{CL-20 biodegradation/mineralization rate $\left(\mathrm{h}^{-1}\right)=[$ intrinsic rate $][$ g sediment $]\left[\mathrm{CFU} \mathrm{g} \mathrm{g}^{-1}\right]$}

can be accomplished with a CL-20 intrinsic transformation rate of $8 \times 10^{-10} \mathrm{~h}^{-1} \mathrm{CFU}^{-1}$ (oxic, anaerobic environment, $\pm 10 \mathrm{x}$ ) or $1.3 \times 10^{-10} \mathrm{~h}^{-1} \mathrm{CFU}^{-1}$ (reducing environment) and mineralization rates of $8 \times 10^{-11} \mathrm{~h}^{-1} \mathrm{CFU}^{-1}$ (oxic, anaerobic, $\pm 10 \mathrm{x}$ ) or $1 \times 10^{-9} \mathrm{~h}^{-1} \mathrm{CFU}^{-1}$ (reducing environment). CL-20 biotransformation was fastest in reducing/coupled systems (i.e., combination of abiotic and biotic processes, half-life 15 to $50 \mathrm{~h}$ ), but was less than an order of magnitude faster than mineralization in oxic systems. Therefore, the redox environment (i.e., oxic or reducing) had only a slight influence on CL-20 biodegradation. In contrast, RDX biodegradation was three orders of magnitude more rapid in reducing systems relative to oxic systems.

CL-20 Degradation Mechanism and Pathway. Activation energies calculated from different abiotic CL-20 degradation systems indicate that there may be two different degradation pathways. Activation energies differed for the first step(s) of CL-20 transformation between: a) electron transfer reactions (CL-20/reduced sediment $=35 \mathrm{~kJ} \mathrm{~mol}^{-1} ; \mathrm{RDX} /$ reduced sediment $=$ $52 \mathrm{~kJ} \mathrm{~mol}^{-1}$ ), and b) non-electron transfer reactions (CL-20/hot $\mathrm{H}_{2} \mathrm{O}=133 \mathrm{~kJ} \mathrm{~mol}^{-1}$; CL-20/ glass $\left.+\mathrm{H}_{2} \mathrm{O}=146 \mathrm{~kJ} \mathrm{~mol}^{-1}\right)$. CL-20 degradation by hectorite $\left(43 \mathrm{~kJ} \mathrm{~mol}^{-1}\right)$ may involve electron transfer. CL-20 transformation by oxic sediment $\left(14 \mathrm{~kJ} \mathrm{~mol}^{-1}\right)$ is low and in the range of diffusion control (i.e., diffusion may be rate controlling rather than the chemical reaction). Molecular modeling predicted that the roof C-C bond of the CL-20 molecule should cleave (Qasim et al. 2004), based on calculations on the strained cage structure. Intermediates and end products several high molecular weight compounds, glyoxal, nitrate, nitrite, $\mathrm{N}_{2} \mathrm{O}, \mathrm{NH}_{3}$, formate, and $\mathrm{CO}_{2}$.

CL-20 biomineralization occurs in oxic, anaerobic, and reducing systems and was as high as 79\%. Most energetics, including RDX, are degraded/biomineralized more rapidly in reducing environments. Although natural or engineered abiotic electron transfer reactions (reduced sediment, zero valent iron) can rapidly transform energetics to intermediates, microbes are necessary for mineralization. Despite similarities in chemical structure, CL-20 and RDX soil biodegradation and mineralization differs. RDX-degrading aerobes (Rhodococcus 11Y, DN22) cannot use CL-20 as an N source. In addition, a CL-20 degrading aerobic isolate from China Lake sediment (Gordonia, $\mathrm{g}^{+}$) cannot use RDX as a sole $\mathrm{N}$ source. In oxic systems CL-20 degradation is 10x more rapid and mineralization 1000x more rapid relative to RDX. Behavior in reducing systems is significantly different as CL-20 degradation is 1000x slower and mineralization the same relative to RDX. The most rapid RDX mineralization rates occur in reduced sediments with sequential (series) abiotic reactions (first five transformation reactions) followed by final one or more biotic mineralization reactions. In contrast, CL-20 mineralization is most rapid in coupled reduced systems, with a coupled abiotic/biotic reaction. 
Implications to CL-20 Release in Surface and Subsurface Sediments. Results of this study show that CL-20 will exhibit differing behavior in the subsurface terrestrial environment:

1. CL-20 on the sediment surface will photodegrade and interact with plants/animals (described in other SERDP projects CU 1254, 1256). CL-20 will exhibit greater sorption in humid sediments to organic matter. Transport will be solubility limited (i.e., low CL-20 aqueous solubility).

2. CL-20 infiltration into soils $(<2 \mathrm{~m})$ from spills will be subject to sorption to soil organic matter (if present), and low to high biodegradation rates (weeks to years) depending on the microbial population (greater in humid environment).

3. CL-20 in the vadose zone ( $>2 \mathrm{~m}$ ) will be, in most cases, subject to low sorption and low degradation rates, so would persist in the subsurface environment and be at risk for deep migration. Low water content in arid regions will result in a decrease in both sorption and the degradation rate. Measured degradation rates in unsaturated sediments of years would result in significant subsurface migration distances.

4. CL-20 in groundwater will be subject to some sorption but likely very slow degradation rates. CL-20 sorption will be greater than RDX. Most CL-20 degradation will be abiotic (ferrous iron and other transition metals), because most deep subsurface systems have extremely low natural microbial populations. Degradation rates will range from weeks (iron reducing systems) to years.

Although CL-20 will move rapidly through most sediments in the terrestrial environment, subsurface remediation can be utilized for cleanup. Transformation of CL-20 to intermediates can be rapidly accomplished under: a) reducing conditions (CL-20 $4.1 \mathrm{~min}$. half-life, RDX 18 min. half-life), b) alkaline ( $\mathrm{pH}>10)$ conditions, and c) bioremediation with added nutrients. CL-20 degradation to intermediates may be insufficient to mitigate environmental impact, as the toxicity of many of these compounds is unknown. Biostimulation in oxic to reducing systems by carbon and nutrient addition can mineralize CL-20, with the most rapid rates occurring under reducing conditions. 


\section{Introduction}

Hexanitrohexaazaisowurtzitane (CL-20, HNIW) is a recently developed energetic material (Nielsen et al. 1998) that releases more energy on ignition and is more stable to accidental detonation than currently used energetic materials. It is expected to replace many of the energetic materials currently being used by the DoD. Hazardous wastes associated with the life cycle of DoD energetic materials currently constitute over $40 \%$ of hazardous waste produced by DoD operations. However, very little is known on the fate and transport of CL-20 in the environment (PPSON-02-01). The objective of this project is to provide data on the environmental fate and transport of CL-20 in the subsurface that can be directly inputted into contaminant transport models to assess and manage environmental risk form CL-20. The subsurface is one of the areas that have proved most problematic in the production and use (firing ranges) of energetic materials (SERDP 1993). More specifically, we identified the biotic and abiotic mechanisms that determine the fate of CL-20 in the subsurface environment, determine the rates of the significant reactions under conditions of weathering and aqueous transport, and provide this information in a format that can be inputted into existing groundwater/contaminant models. This report represents the final progress report for SERDP project CP-1255 entitled "Factors Effecting the Fate and Transport of CL-20 in the Vadose Zone and Groundwater" for the period March 2002 to May 2005. This laboratory study of CL-20 subsurface fate involves research at Pacific Northwest National Laboratory, the U.S. Army Engineer Research \& Development Center, Waterways Experiment Station, and the University of Arizona.

CL-20 release to different subsurface environments would result in a wide range of different geochemical and microbial interactions. To evaluate the fate of CL-20 in subsurface sediments, this study is focused on identification and quantification of geochemical and microbial reactions of CL-20 in sediments, the effects of weathering, and the influence of transport on these reactions in the subsurface environment (Table 1). The results can provide information and a method for predicting CL-20 fate for a range of subsurface conditions under which CL-20 may be released to the environment. 


\section{Table 1. Organization of project tasks}

Task 1. Abiotic Fate Identify CL-20-sediment geochemical reactions, effects of aging and quantify relative importance

batch sediment-water

fractions) and CL-20 specific mechanisms are important in different subsurface environments: task 1.1: CL-20 will be strongly retained by sediment organic matter and mineral surfaces, and

w will exhibit slow

sorption/desorption task 1.2: due to the similarities in functional groups, initial CL-20 degradation is likely to occur in reducing conditions on surfaces with transition metals ( $\mathrm{Fe}, \mathrm{Mn})$ task 1.3: analytical. initial and some final degradation products can be identified
Task 2. Microbial Rxns Task 3. Coupled Processes Task 4. Reactive Transport Identify CL-20

biodegradation pathway

in aerobic and anaerobic systems initially with RDX-degrading bactera batch microbe-water Evaluate synergistic/ antagonistic effects of reactive transport (abiotic, biotic degradation, aging) Demonstrate CL-20 and CL-20

batch CL-20, sedimentof flow effects on predictions

Biodegradation of CL-20

microbe-water, artificia

1-D sediment columns

(initial nitro- group removal) may be induced by RDXdegrading bacteria, although overall CL-20 (caged) vs RDX aged sediment (saturated, unsaturated), some with microbes biodegradation will be slower in the presence of sediments due to sorption (predicable) and additionally due to structure is different: task 2.1: anaerobic biodegradation. Bacteria that induce reduction of nitro-groups of RDX are likely to reduce nitromicrobial attachment (not predicable) task 3.2: aging. $\mathrm{Cl}-20$-sediment aging will slow the CL-20 desorption rate and availability for abiotic and groups of CL-20 biotic degradation task 4.1: abiotic saturated CL-20 transport will be highly dependent on the CL-20 sorption/desorption rate task 4.2: abiotic saturated CL-20 transport in reducing sediment will exhibit faster abiotic degradation than predicted from batch systems task 4.3: CL-20 abiotic transport subject in the vadose zone with sorption will be influenced by the differences in reactive surface access at differing biodegradation. Bacteria that oxidize RDX by complete nitro-group removal mav be reactive 


\section{Background: Subsurface Fate of CL-20}

The tasks in this project are designed to identify and quantify these processes and demonstrate coupled CL-20 reactive transport in saturated and unsaturated flow systems to develop prediction ability for the fate of CL-20 from potential spills. The current understanding of the environmental fate and reactivity of CL-20 in sediments and in groundwater is considerably greater due to three SERDP-funded projects (CP-1254, 1255, 1256). CL-20 is less toxic to plants tested relative to other energetics (and in some cases, stimulates growth), but is 1 to 4 orders of magnitude more toxic than other energetics. The subsurface fate from potential surface spills (ammunition storage, manufacturing spill) from two extremes of subsurface transport scenarios illustrates that different processes dominant in different environments. CL-20 infiltration in arid regions with a thick vadose zone would likely be dominated by slow aqueous transport (Task 4.3, Table 1), sorption (Task 1.1), and involve significant effects of aging (i.e., slow CL-20 release from sediments, Task 3.2). In addition, there may be minor effects from surface photodegradation (Task 1.2) and aerobic biodegradation (Task 2.2). In contrast, CL-20 subsurface transport in humid regions with a thin vadose zone will be dominated by fast saturated transport (Task 3.1, 3.2), sorption dependent on the flow rate (i.e., CL-20/sediment contact time, Task 1.1), and anaerobic abiotic and biotic degradation. Infiltration of CL-20 solubilized in nonaqueous solvents is not considered in this study.

CL-20 is a caged nitramine that shares some physical and chemical characteristics (Figure 1, Table 2) with hexahydro-1,3,5-trinitro-1,3,5-triazine (RDX). Because of these similarities, we hypothesize that many of the abiotic and biotic mechanisms that determine the environmental fate of RDX (Pennington et al., 1995) will also influence the fate of CL-20. As examples, sorption of RDX on sediments occurs reversibly (Singh et al. 1998a). Abiotic RDX degradation with $\mathrm{Fe}^{\mathrm{O}}$ occurs under reducing conditions (Singh 1998b, 1999) and in reduced sediment (Szecsody et al., 2004a). Guiot et al. (1999) also reported that biotransformation rate of RDX from contaminated soil was dependent on RDX concentration in soil even in the high ranges, suggesting that mass transfer limitations were important (Brannon et al., 1992). Like RDX, low water solubility $(<5 \mathrm{mg} / \mathrm{L})$ of CL-20 is likely to exhibit strong but slow sorption to specific sediment components (organic matter, clay and mineral surfaces). This could result in rapid transport of freshly introduced CL-20 (e.g., surface spills and low order detonations on firing ranges) through the subsurface. However, aged material would slowly desorb and limit the effectiveness of pump-and-treat approaches and the availability of CL-20 to microbial degradation. Clearly, more information on the physical sorptive behavior on environmental matrices is needed.

As a first approximation, it is also reasonable to

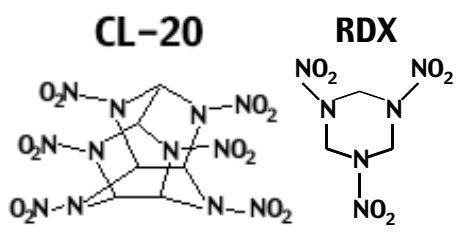

Figure 1. CL-20, RDX structure

Table 2. CL-20 and RDX properties

\begin{tabular}{|c|c|c|c|}
\hline \multicolumn{2}{|l|}{ Property } & RDX & CL-20 \\
\hline ENERGIES & ${ }^{\star} \mathrm{AM} 1$ & 104.88 & $\star \star \star \star 319.71$ \\
\hline$(\mathrm{Kcal} / \mathrm{mole})$ & *MNDO & 100.90 & $* * * 401.12$ \\
\hline \multicolumn{4}{|c|}{${ }^{*}$ Heat of formation } \\
\hline${ }^{* *}$ Steric en & **MM2 & $12.25,-1$ & $90^{* * * 139.37}$ \\
\hline \multicolumn{4}{|c|}{ *** CL-20 is a strained cage, higher steric energies } \\
\hline \multicolumn{2}{|c|}{ Volume (solvation, $A^{2}$ ) } & 88.13 & 134.48 \\
\hline \multicolumn{2}{|c|}{ Polarity (Debye) } & 11.20 & 3.61 \\
\hline \multirow[t]{2}{*}{ Structural } & $\mathrm{NO} 2$ groups & 3 & 6 \\
\hline & rings & $3(\mathrm{~N}-\mathrm{C})$ & 5 or $7(\mathrm{~N}-\mathrm{C})$ \\
\hline \multirow{3}{*}{$\begin{array}{l}\text { Bonds } \\
\text { (in ang.) }\end{array}$} & $\mathrm{C}-\mathrm{C}$ & none & 1.53 \\
\hline & $\mathrm{N}-\mathrm{N}$ & 1.40 & \\
\hline & $\mathrm{C}-\mathrm{N}$ & 1.49 & $1.44-1.57$ \\
\hline \multirow[t]{2}{*}{ Reactivity } & & at $\mathrm{C}-\mathrm{N}$ & $\begin{array}{c}\text { at } \mathrm{C}-\mathrm{C} \\
\text { etched } \mathrm{C}-\mathrm{N}\end{array}$ \\
\hline & reduction & nitro- & nitro- \\
\hline
\end{tabular}


assume that the biological mechanisms responsible for RDX transformation are also operable on CL-20. Biodegradation of RDX is widely reported (Waisner et al. 2001; Adrian and Lowder 1999; Binks et al. 1995; Freedmann and Sutherland 1998; McCormick et al. 1981; Ronen et al. 1998; Sheremata and Hawari 2000; Funk et al. 1993). The general consensus is that RDX was biodegraded cometabolically under aerobic as well as anaerobic conditions, but at a slower rate (Autenrieth et al. 1999; Hallgarth et al. 1999). Biodegradation under aerobic conditions resulted in production of no potentially carcinogenic byproducts (Knezovich and Daniels 1991). Presence of nitrate in the medium was strongly inhibitory to biodegradation of RDX (Ronen et al. 1998). In $40 \%$ to $44 \%(\mathrm{w} / \mathrm{v})$ soil slurries under anaerobic conditions, however, biodegradation could be achieved even in presence of very high concentrations of RDX (24,000 $\mathrm{mg} / \mathrm{kg}$ soil; Guiot et al. 1999). The degree of mineralization under anaerobic conditions exceeded 60\% (Waisner et al. 2000; Guiot et al. 1997, Young et al. 1997). A two-stage 'anaerobic-aerobic' bioremediation process (Ogden et al. 1994) involving a mixture of TNT, RDX, and HMX were effectively removed from soils (Greer et al. 1999), even at high concentrations of RDX $(100,000 \mathrm{mg} / \mathrm{kg})$.

A few studies have shown that CL-20 can be biodegraded in aerobic surface soils (Jenkins et al. 2003; Trott et al. 2003), by bacteria isolated from these soils (Bhushan et al. 2003a; Trott et al. 2003), and by functionally diverse bacterial enzymes (Bhushan et al. 2003a, 2003b). Similar to abiotic degradation processes, nitrite (1.7 to 2.3 moles), ammonium (0 to 1.3 moles), nitrous oxide (1.5 to 3.3 moles), formate (1.5 to 1.6 moles), and glyoxal (0 to 1 mole) were among the various end products formed per mole of CL-20 metabolized (Bhushan et al. 2003a, 2004a, 2004b). The enzymatic studies with purified salicylate 1-monooxygenase (Bhushan et al. 2004b) and nitroreductase (Bhushan et al. 2003a), and a membrane associated flavoenzyme (Bhushan et al. 2003a) have shown that the biotransformation of CL-20 is catalyzed by an oxygen-sensitive, one-electron transfer reaction that releases one nitrite ion from the molecule. Two one-electron transfer reactions were proposed to account for the formation of two nitrite ions. The loss of a single nitrite ion was proposed to produce an unstable intermediate that causes the weaker C-C attic bond to spontaneously break (Bhushan et al. 2003a, 2003b). Reduction of this molecule would release the second nitrite ion producing an unstable metabolite with a molecular weight of $345 \mathrm{Da}$ and empirical formula $\mathrm{C}_{6} \mathrm{H}_{6} \mathrm{~N}_{10} \mathrm{O}_{8}$. This intermediate has been proposed to be 1,4,5,8-tetranitro-1,3a,4,4a,5,7a,8,8a-octahydro-diimidazo[4,5- $b:$ 4', $^{\prime}$ ' e]pyrazine and it exists in two isomeric forms (Bhushan et al. 2003a). Abiotic hydrolysis of this compound eventually leads to the production of formic acid, glyoxal, nitrous oxide, and ammonia (Bhushan et al. 2003a, 2003b). Glyoxal has only been detected as a biological end product with the enzyme nitroreductase (Bhushan et al. 2003a). In these studies, ${ }^{14} \mathrm{C}-\mathrm{CL}-20$ mineralization was not investigated, due to the unavailability of ${ }^{14} \mathrm{C}$-labeled CL-20 at the time.

Due to the structural similarities between CL-20, RDX, and HMX, it is possible that biodegradation mechanisms will be similar. Both RDX and HMX were degraded under anaerobic conditions and the degradation pathways proposed include both biotic and abiotic steps, since some of the proposed intermediates are unstable in water (McCormick et al. 1981; Halasz et al. 2002; Hawari et al. 2000a; Hawari et al. 2001). McCormick et al. (1981) proposed an anaerobic biodegradation pathway whereby RDX undergoes sequential reduction of the nitro groups via nitroreductase enzymes to form the stable intermediates: MNX (hexahydro-1-nitroso-3,5dinitro-1,3,5-triazine), DNX (hexahydro-1,3-dinitroso-5-nitro-1,3,5-triazine), and TNX 
(hexahydro-1,3,5-trinitroso-1,3,5-triazine). According to the proposed pathway, additional enzymatic reduction steps and abiotic hydrolytic ring cleavage reactions subsequently yielded formaldehyde, methanol, hydrazine, and dimethyl hydrazine. Currently, there is no published evidence that supports this biotransformation mechanism for CL-20.

Recently, Hawari et al. (2000b) and Halasz et al. (2002) proposed an alternate pathway whereby the initial enzymatic attack on RDX caused the ring to break producing the unstable intermediates methylenedinitramine and bis(hydroxymethyl)nitramine. These intermediates then decomposed to nitrous oxide and formaldehyde. Methanol, formate, carbon dioxide, or methane was formed from the formaldehyde by various bacteria in anaerobic sludge (Hawari et al. 2000b; Halasz et al. 2002) or pure cultures (Zhao et al. 2002). Aerobic biodegradation of RDX was proposed to include denitration followed by ring cleavage yielding end products such as nitrite, ammonia, nitrous oxide, formaldehyde, carbon dioxide, and a dead-end product, 4-nitro-2,4diazabutanal (Bhushan et al. 2003b). Similarly, denitration and ring cleavage of RDX to produce nitrite, formate, and formaldehyde was postulated for aerobic degradation by Rhodococcus rhodochrous strain 11Y (Seth-Smith et al. 2002).

Due to the complex and strained molecular structure of CL-20, we hypothesized that CL-20 would be more susceptible to biological transformation reactions than RDX. In this study, we examined the biological and abiotic degradation of CL-20 and RDX, and the mineralization of uniformly labeled ${ }^{14} \mathrm{C}$-CL-20 in aerobic and anaerobic soil microcosms. Our objectives were to quantify and compare the rates of degradation and thus, determine the relative importance of biological versus abiotic degradation of CL-20 and RDX in these soils. This data will help to define differences in degradation mechanisms that affect the environmental fate and impact of these two explosives.

Because CL-20 transport through the unsaturated and saturated zones of the subsurface environment would be significantly attenuated by these geochemical and microbial processes, experimental investigations to define the processes are needed. Knowledge of individual processes can provide for an estimate of reactive transport behavior, but CL-20 behavior moving through the vadose or saturated zones will be additionally subjected to coupled process effects. Transport effects can also significantly influence the apparent manifestation of reactions during advective flow (Szecsody et al. 1998), particularly with multiple geochemical/microbial reactions. A sequence of scales of experiments can be used to initially quantify separate processes in small, highly controlled systems, then incorporate field-scale processes in largerscale coupled experiments. This experimental data describing CL-20 biogeochemical reactive transport is needed to provide an understanding of the relative importance of individual geochemical/microbial processes, coupled effects, and transport effects, so that CL-20 fate from potential spills can be assessed. 



\section{Experimental and Modeling Methods}

\section{Task 1. Abiotic Fate of CL-20 in the Subsurface}

The goal of this task is to develop an understanding of the nature and magnitude of abiotic mechanisms that influence CL-20 fate under varying subsurface conditions. Experiments will be performed in batch (i.e., no flow) sediment-water systems containing CL-20 and RDX (used as a baseline for degradation studies) under varying conditions (solute concentration, $\mathrm{pH}$, Eh, time, temperature). Reaction path modeling (i.e., numerical models that describe one or more reactions kinetically or at equilibrium) will be used to quantify reaction parameters with the experimental data. The expected results of this task are a conceptual and mathematical description of CL-20 sorption and degradation amount and rates. This information will be used to design coupled abiotic/biotic interactions (Task 3 ) and abiotic transport experiments (Task 4). The specific abiotic reaction mechanisms that will be addressed in this task are: sorption (Task 1.1), abiotic degradation (Task 1.2), and chemical analysis (Task 1.3).

Task 1.1. Sorption of CL-20

Hypothesis: CL-20 will be strongly retained by sediment organic matter (greatest affinity) and mineral surfaces (clays, $\mathrm{Fe}$-, Al-oxides), and exhibits slow sorption/desorption.

Sorption of CL-20 to various sediment components (i.e., organic matter, mineral surfaces) from aqueous systems involves both solvation energy and the solute/surface interaction energy (Sposito 1984). The solvent effect includes a decrease in water structure upon solute removal from solution (positive entropy) and an increase in hydrogen bonding between water molecules (negative enthalpy). For hydrophobic compounds, the solvent effect is the major driving force retaining compounds on surfaces (i.e., not specific solute-surface interactions), so hydrophobic compounds "partition" to the surface. The cage structure of CL-20 (Figure 1) has low water solubility ( $<5 \mathrm{mg} / \mathrm{L}$; Holtz et al. 1994). In comparison, RDX is a significantly smaller molecule (N-heterocyclic ring) with three nitro-groups and has a moderate water solubility $(42-55 \mathrm{mg} / \mathrm{L}$ at $25^{\circ} \mathrm{C}$, varies with ionic strength). CL-20 water solubility and Kow was not measured in this project, but by another SERDP/CL-20 project (CP-1256). The retention of CL-20 on sediments at equilibrium defines the distribution coefficient, $\mathrm{K}_{\mathrm{d}}$ :

$$
\mathrm{K}_{\mathrm{d}} \text { or } \mathrm{K}_{\mathrm{p}}=\mathrm{S}(\mu \mathrm{mol} / \mathrm{g}) / \mathrm{C}\left(\mu \mathrm{mol} / \mathrm{cm}^{3}\right)
$$

where $\mathrm{S}$ is the concentration of CL-20 sorbed and C is the concentration of CL-20 in the equilibrium solution. Although $\mathrm{K}_{\mathrm{d}}$ is not chemical mechanism specific, $\mathrm{K}_{\mathrm{d}}$ measurements are useful because it can describe the overall transport and attenuation of CL-20 reasonably well. With carbon referenced sorption:

$$
\mathrm{K}_{\mathrm{p}}=\mathrm{f}_{\mathrm{oc}} \mathrm{K}_{\mathrm{oc}}
$$

where $f_{o c}$ is the fraction carbon by weight and $\mathrm{K}_{\mathrm{oc}}$ is the partition coefficient and a hypothetical soil that is $100 \%$ organic carbon. Carbon referenced sorption is applicable to hydrophobic solutes at concentrations less than half their solubility, and deviation from this linear relationship 
are caused multiple sorption mechanisms and nonequilibrium behavior. For many compounds, $\mathrm{K}_{\mathrm{oc}}$ is related to the octanol-water partition coefficient $\left(\mathrm{K}_{\mathrm{ow}}\right)$ which a linear free energy relationship

$$
\log \mathrm{K}_{\mathrm{oc}}=\mathrm{x} \log \mathrm{K}_{\mathrm{ow}}+\mathrm{y}
$$

where $\mathrm{x}$ and $\mathrm{y}$ are empirical constants $(0.4,0.29$ for solvents; $0.524,0.618$ for pesticides). RDX has a fairly low $\log \mathrm{K}_{\text {ow }}(0.78)$, so partitions weakly to sediments. Field studies confirm this fact, as RDX is fairly mobile, with low $\mathrm{K}_{d}$ values of 1.0 to 5.0 (Singh 1999). In contrast, CL-20 should partition strongly to sediment organic matter (calculated $\log \mathrm{K}_{\mathrm{ow}}=3.2$ ). CL-20 sorption $\left(\mathrm{K}_{\mathrm{d}}\right)$ was measured on four to six well characterized sediments with a range of organic carbon content and a variety of sediment minerals at different CL-20 concentration (i.e., isotherms). Isotherms were conducted for 24 hours, with aqueous and solid phase CL-20 measurements. Solid phase measurement insures mass balance and enables calculation of $\mathrm{K}_{d}$ even with degradation. A small fraction of these sorption experiments will be over time to screen for degradation. Sorption will be confirmed by $\mathrm{K}_{\mathrm{d}}$ measurements on at least one sediment supplied by the other two SERDP CL-20 projects. Once it is determined, the time scale of sorption and that of mass loss, sorption, and desorption rate experiments will be conducted on a few sediments. Carbon-referenced sorption model applies when $\mathrm{f}_{\mathrm{oc}}>0.001$ ( $>0.1 \%$ carbon), below which sorption onto mineral surfaces is important. In this case, a weak dependence on the surface area of the soil, is observed:

$$
\log \mathrm{K}_{\mathrm{p}}=0.16 \log \mathrm{K}_{\mathrm{ow}}+\log \mathrm{Sa} / 200
$$

where $\mathrm{Sa}$ is the mineral surface area [25 solutes onto silica; McCarthy et al. 1981].

CL-20-sediment sorption affinity experiments were conducted to determine the mass of CL-20 that will partition to sediment surfaces and the sediment component (i.e., organic matter or specific mineral surfaces) that dominate sorption. A few well-characterized sediments representative of different possible sediment/CL-20 interactions were used: a) high- $\mathrm{f}_{\text {oc }}$, low clay sand (several, varied $\mathrm{f}_{\mathrm{oc}}$ ), b) a low- $\mathrm{f}_{\mathrm{oc}}$, high-clay sediment, and $\mathrm{c}$ ) a low- $\mathrm{f}_{\mathrm{oc}}$, low clay sand (iron and $\mathrm{Mn}$ oxide coated). Sorption to several major components can be predicted:

$$
\mathrm{K}_{\mathrm{d}}=\Sigma \mathrm{K}_{\mathrm{di} \rho \mathrm{I}}
$$

where the overall adsorption of the sediment $\left[\mathrm{K}_{\mathrm{d}}\right]$ is the sum of each component $\left(\mathrm{K}_{\mathrm{di}}\right)$ and the surface area contribution of that component $\left(\mathrm{p}_{\mathrm{i}}\right)$. This approach has been applied for metal adsorption (Ohnuki 1994).

Different types of sorption experiments will be conducted including: sorption isotherm, sorption $\mathrm{pH}$ edge, sorption rate, and sorption rate at different temperature. These experiments will all be conducted in batch vials sealed from the atmosphere (no headspace) in the dark (to minimize possible photoreactivity). Sorption isotherm experiments provide information about the reaction. For example, nonlinear sorption behavior at low concentration and a sorption maxima (Wise, 1003) indicates multiple, specific sorption sites. In contrast, an RDX sorption isotherm (Singh et al. 1999) with linear sorption indicates nonspecific sorption. It is 
hypothesized that CL-20 will exhibit linear sorption, but may undergo abiotic and biotic degradation reactions (other tasks). While CL-20 is not ionizable, a batch pH adsorption edge experiment will be conducted in order to determine if there are other reactions occurring at different $\mathrm{pH}$.

Time-course batch experiments were also conducted with CL-20. These experiments provide sorption and desorption rate data as well as rates of other reactions. Due to the size and complexity of the CL-20 molecule, it is expected that some slow approach to equilibrium will be observed, as observed with many organic solutes in sediments (Wu and Gschwend 1986; Coates and Elzerman 1986), with time scales as long as 4 weeks to reach equilibrium. The cause of the slow approach to equilibrium is physical (diffusion through immobile pore fluid) and chemical (slow reactions; Karickhoff 1984; Leehneer and Ahlrichs 1971). An example of a time course experiment (Figure 2, Appendix B) shows that sorption of this metal-chelate complex reached steady state by $0.5 \mathrm{~h}$ (at $\mathrm{pH} 4.5 ; 40 \mathrm{~h}$ at $\mathrm{pH} 6.5)$, but then dissociation was evident from 5 to $100 \mathrm{~h}$. This type of experiment is necessary in order to define the CL-20/sediment contact time used in sorption isotherm and $\mathrm{pH}$ edge experiments and the contact times that are useful for degradation experiments (Task 1.3). It is highly likely that CL-20 will only be observed in reducing conditions, so sorption will dominate the fate of CL-20 in the vadose zone (oxic, Figure 1), and only some groundwater systems may have the specific reducing conditions necessary to facilitate abiotic degradation. Analysis is described in Task 1.3.

Upscaling of any CL-20-sediment data to field sites is accomplished by the understanding of the underlying mechanisms and reactive surfaces that dominate the interactions. Along with experimental evidence for the relative adsorption strength of sediment organic matter, clays, (Fe, $\mathrm{Al}, \mathrm{Mn}$ )-oxides, a reaction model will be developed that can be used for prediction of fate in different sediments. The development of a reaction model is not a separate task, as mathematical simulations are an integral part of testing a conceptual description of a set of reaction and quantifying rates and affinities for different components (Szecsody et al. 1994). The one or more reactions used to quantify batch reactivity will be used in other tasks, as the code used is a reactive transport code with the flexibility to incorporate in equilibrium/kinetic forms of chemical reactions, physical effects (diffusion, density) as well as transport processes.

\section{Task 1.2. Abiotic CL-20 Degradation and Other CL-20/Sediment Reactions}

Hypothesis: Due to the similarities in functional groups between RDX and CL-20, initial abiotic CL-20 degradation is likely to occur in reducing conditions on surfaces with transition metals (Fe, Mn)

There is no evidence found as of 2002 for abiotic or biotic degradation of CL-20 under environmental conditions found in subsurface sediments, but this and the other two SERDPfunded projects have generated significant degradation data for a wide variety of surface and subsurface environments. Thermal degradation rates of three different polymorphs of CL-20 (Nedelko et al. 2000) are known, but likely unrelated to degradation under subsurface environmental conditions. Although a CL-20 precursor can undergo reduction, its structure is different from CL-20. However, CL-20 has functional group similarities to RDX and structural similarities to highly strained polycyclic (i.e., cage) molecules, so may share some similarities, in subsurface reactivity. The abiotic degradation of CL-20 was investigated after the initial 
sorption experiments (Task 1.1), which may provide some evidence of conditions under which CL-20 is unstable (i.e., pH, ionic strength, specific sediment components).

Batch sorption/degradation experiments were performed at $23^{\circ} \mathrm{C}$ to $25^{\circ} \mathrm{C}$ by adding an aqueous solution of CL-20 to sediments/minerals using solid/water ratios of 0.005 to $1.0 \mathrm{~g} \mathrm{~mL}^{-1}$ and contact times of 2 and $24 \mathrm{~h}$. Initial CL-20 concentrations were typically $3 \mathrm{mg} \mathrm{L}^{-1}$ (CL-20 water solubility in deionized water at $25^{\circ} \mathrm{C}$ is $3.6 \mathrm{mg} \mathrm{L}^{-1}$ or $6.9 \mu \mathrm{M}$; Monteil-Rivera et al. 2004). Unless otherwise noted, all batch experimental solutions contained $10^{-2} \mathrm{~mol} \mathrm{~L}^{-1} \mathrm{CaCl}_{2}$ as a background electrolyte, $10^{-3} \mathrm{~mol} \mathrm{~L}^{-1} \mathrm{HgCl}_{2}$ as a biocide to control possible microbial activity, and $10^{-2} \mathrm{~mol} \mathrm{~L}^{-1}$ PIPES (1,4-piperazinediethanesulfonic acid), a $\mathrm{pH}$ buffer, adjusted to $\mathrm{pH} 7$. PIPES is a common organic buffer, which experience has shown does not complex metals or react with sediments and/or CL-20. To determine the contact time required to obtain sorption equilibrium, a time series of batch sorption experiments was conducted under the conditions described above using $0.5 \mathrm{~g} \mathrm{~mL}^{-1}$ of three sediments. Sorption isotherm experiments and a single $\mathrm{pH}$ edge experiment were performed using $2 \mathrm{~h}$ contact times, with $0.5 \mathrm{~g} \mathrm{~mL}^{-1}$ of solid and solutions containing the same concentrations of electrolyte and biocide described above. For the sorption isotherm experiments, the CL-20 concentration ranged from 0.05 to $3.0 \mathrm{mg} \mathrm{L}^{-1}$. The $\mathrm{pH}$ edge experiment used no PIPES and the $\mathrm{pH}$ in individual samples was adjusted with $\mathrm{HCl}$. The influence of $\mathrm{pH}$ on the stability of CL-20 in homogeneous solution was examined for a period of $24 \mathrm{~h}$ at $25^{\circ} \mathrm{C}$. In these experiments, individual vials of distilled water were $\mathrm{pH}$-adjusted with $\mathrm{HCl}$ prior to the addition of CL-20. The initial concentration of CL-20 was $3 \mathrm{mg} \mathrm{L}^{-1}$ and individual samples covered a $\mathrm{pH}$ range of 4 to 12 . Teflon filters were used to separate the solid and solution phases.

Similar conditions and procedures were used for the batch RDX experiments, except that the initial RDX concentrations were $20 \mathrm{mg} \mathrm{L}^{-1}$ (solubility $45 \mathrm{mg} \mathrm{L}^{-1}$ ). At the end of all experiments, aqueous CL-20 or RDX was analyzed. For the $2 \mathrm{~h}$ and time-course experiments, sorbed CL-20 was extracted off sediment surfaces with methanol and analyzed. For the $24 \mathrm{~h}$ experiments, sorbed CL-20 or RDX was not measured but determined by the difference between the initial total and final aqueous sorbate concentrations. For one sediment (Norborne $\mathrm{C}$ ), $2 \mathrm{~h}$ batch experiments at several sediment/water ratios were performed under iron-reducing conditions at $\mathrm{pH} 7.0$ to examine the relationship between ferrous iron and CL-20 degradation. This Norborne sediment was chemically reduced in a packed column (described below) by passing 24 pore volumes of a $0.1 \mathrm{~mol} \mathrm{~L}^{-1}$ sodium dithionite, buffered with $0.4 \mathrm{~mol} \mathrm{~L}^{-1}$ potassium carbonate through the column over $120 \mathrm{~h}$. The column was then flushed with 50 pore volumes of a $10^{-2} \mathrm{~mol} \mathrm{~L}^{-1}$ solution of both $\mathrm{CaCl}_{2}$ and PIPES (adjusted to $\mathrm{pH} 7$ ) to remove the dithionite solution and $\mathrm{pH}$ equilibrate the sediment over 8 days. This treatment, which dissolved amorphous and some crystalline Fe oxides and resulted in primarily adsorbed ferrous iron (Szecsody et al. 2000a, b, 2004b), can also reduce structural iron in clays (Stucki et al. 1984). In an oxygen free glove box, the reduced sediment was then removed from the column, and portions were weighed and added to individual vials containing $3 \mathrm{mg} \mathrm{L}^{-1}$ of CL-20, in the same concentrations of electrolyte, buffer, and biocide noted above. The vials were then sealed and removed from the glove box. All other batch sorption-degradation, isotherms, and $\mathrm{pH}$ edge experiments were conducted in contact with air and designated as oxic experiments.

CL-20 did exhibit other reactions important in sediment systems (reactivity due to transition metals, photoreactivity). Some highly strained polycyclic molecules undergo oxidation reactions 
in the presence of transition metals, which result in different structural configurations (Gassman and Yamaguchi 1982). There is also evidence for increased electron transfer reaction rates for highly strained polycyclic molecules in the presence of transition metals (Nikolic et al., 1994). Transition metals are abundant on sediment surfaces, especially clays (adsorption). Some degradation experiments will be conducted varying common transition metals $\left(\mathrm{Fe}^{2+}, \mathrm{Mn}^{2+}\right)$ found in sediment-water systems. Some highly strained polycyclic molecules (i.e., 3-, 4-, 5- ring cage structures) are photoreactive (Zepp and Schlotzhauer 1981; Burton and Turley, 1995). This mechanism is of some importance for surface spills and will be investigated to a limited extent. These studies will also insure that any CL-20 degradation observed (in reducing sediments) is not partially caused by photodegradation. Prior to initiation of batch and 1-D column studies, experiments were conducted at $23^{\circ} \mathrm{C}$ to $25^{\circ} \mathrm{C}$ to assess the stability of aqueous CL-20 under two conditions: 1) exposure to sunlight or fluorescent light, and 2) in contact with laboratory materials used in batch and column experiments. Using an initial concentration of $3 \mathrm{mg} \mathrm{L}^{-1}$ of CL-20 in distilled water experiments were conducted using glass, high- and low-density polyethylene (HDPE and LDPE), FEP and PFA Teflon, Tefzel (ETFE), polycarbonate, polystyrene, polypropylene, $\mathrm{PEEK}^{\mathrm{TM}}$, delrin, tygon, and nylon materials. The loss of CL-20 from solutions adjusted to $\mathrm{pH} 7$ was followed over time under different lighting conditions. In addition, the affects of the $\mathrm{CaCl}_{2}$ electrolyte, the PIPES buffer, and the $\mathrm{HgCl}_{2}$ biocide on aqueous CL-20 were examined. The affects of these solution additives and the methanol or acetonitrile extractants on the HPLC analysis of aqueous CL-20 were also examined.

\section{Task 1.3. CL-20 and Degradation Product Analysis}

A number of analytical tools were screened for possible use in the identification and quantification of CL-20 and unknown breakdown products from abiotic and biotic degradation studies. We analyze CL-20 and breakdown products using high performance liquid chromatography with ultraviolet detection (HPLC-UV) and gas chromatography (GC) with either electron capture or mass spectrometry detection commonly used for trace levels of explosives. The EPA method 8330 (EPA 1997) for the analysis of explosives and metabolites in soils calls for the use of UV/Vis detection. However, a complex soil extract can often produce interfering peaks that can make absolute identification difficult.

High-performance liquid chromatography has been used to quantify CL-20 and precursor concentration (Bazaki et al. 1998; Nielson et al. 1989, 1994) at relatively high concentrations. FTIR spectroscopy has been used to identify different crystal forms of CL-20 (4 isomers; Kim et al. 1998). Introduction and recent improvements of ionization techniques such as electrospray have allowed the mass spectrometer to become more widely used in liquid chromatography. Previously, thermospray negative ion mode has shown utility in the detection of explosives containing nitro functional groups. Schilling (1996) also examined explosive components and metabolites using electrospray and APCI LC/MS. Schilling's results showed that compared to thermospray LC/MS, APCI, and ES were more sensitive than thermospray by at least an order of magnitude. RDX, nitroso-RDX metabolites, and other munitions in ground water have been analyzed using solid-phase extraction and isotope dilution liquid chromatography-atmospheric pressure chemical ionization (APCI) mass spectrometry (Cassada et al. 1999). The method detection limits indicate that nitramine and nitroaromatic compounds can be routinely determined in ground water samples using electrospray LC/MS with concentration techniques 
utilizing solid-phase extraction. Mobile phase additives to enhance the ESI intensities and spectra consistencies are typically used to stud nitrated explosives. Several of the explosives show nitrate adduct ions in the negative mode with ammonium nitrate as the mobile phase. The nitramines RDX and HMX showed the greatest enhancement in response of the explosives. The use of ammonium nitrate as the mobile phase made it possible to obtain consistent and interpretable LC/MS spectra at the nanogram level.

CL-20 itself was quantified in most batch and column experiments at Pacific Northwest National Laboratory by an HPLC method developed by the U.S. Army Waterways Experiment Station (Larson et al. 2001). With this HPLC system, compounds that are more polar or of lower molecular weight will elude sooner than CL-20. The CL-20 retention time in the HPLC is 8.0 minutes, with RDX at 4.2 minutes.

The specific conditions of the HPLC system include:

- Keystone NA C-18 column, $250 \mathrm{~mm} \times 4.6 \mathrm{~mm}$

- $55 \%$ methanol, $45 \%$ water (isocratic), degassed with continuous helium

- flow rate $0.8 \mathrm{~mL} / \mathrm{min}, 2200 \mathrm{psi}$, HP1050 series HPLC pump

- samples in 1.5 mL HDPE vials, 50 microliter injection volume

- ultraviolet detection at $230 \mathrm{~nm}$, HP1050 series multiple wavelength detector

Calibration of CL-20 from $0.05 \mathrm{mg} / \mathrm{L}$ to $3.5 \mathrm{mg} / \mathrm{L}$ (aqueous solubility) was linear. Degradation products were observed using the same HPLC method, although these are currently uncalibrated peaks, pending LC-MS identification. Two known degradation products that have been detected in trace concentrations include 2-oxaCL-20, and 2,12-dioxa-CL-20.

A cross-laboratory comparison of CL-20 extracted from sediments (Figure 2) by acetonitrile was conducted in 2003 between the three SERDP-funded projects (BRI, ECBC, and PNNL). Sediment
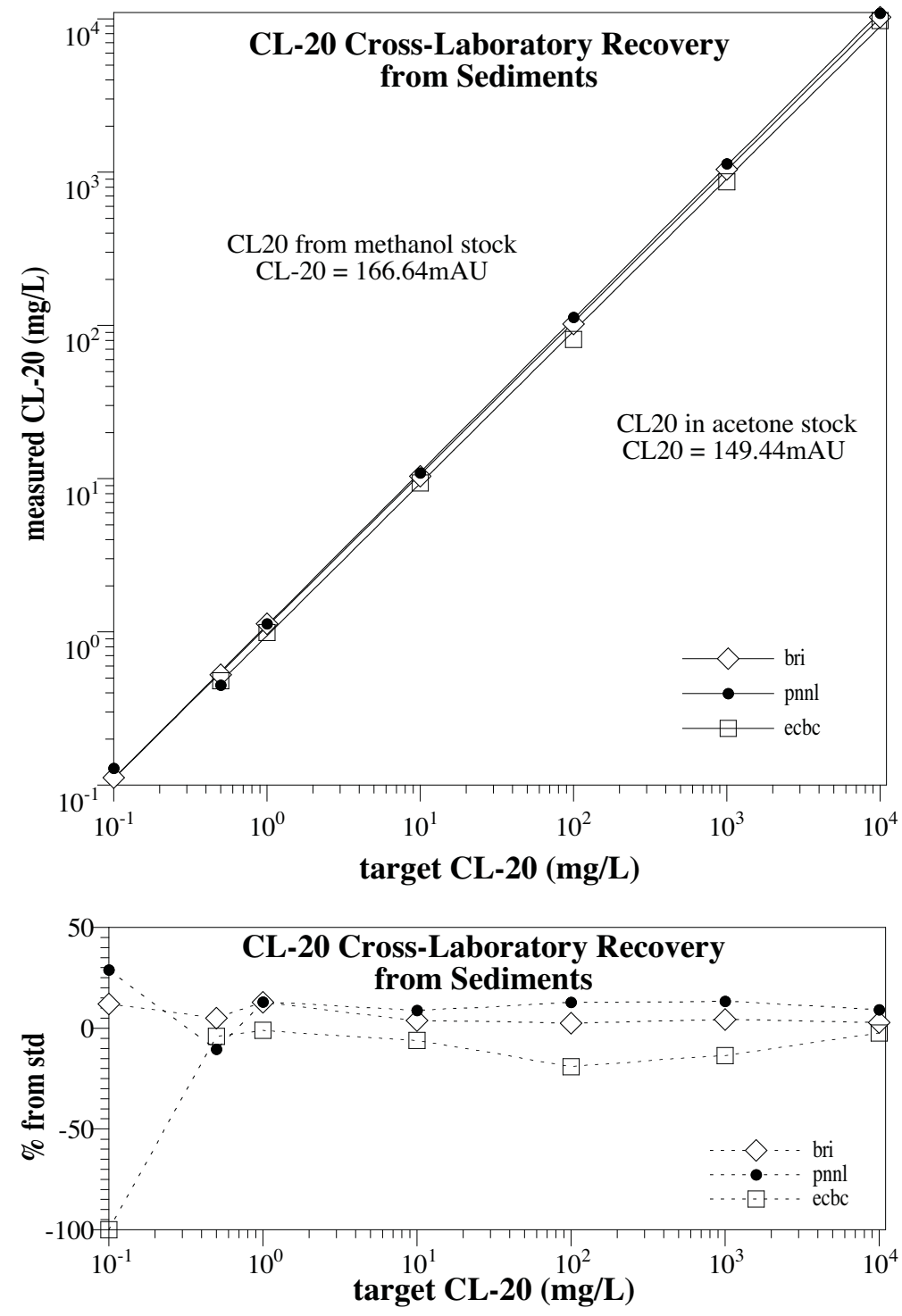

Figure 2. Cross-laboratory comparison of CL-20 extraction from sediments. 
samples were prepared by ECBC that contained CL-20 concentrations ranging from $0.1 \mathrm{mg} / \mathrm{g}$ to $10,000 \mathrm{mg} / \mathrm{g}$. Overall the cross comparison was good, although BRI and PNNL values were about $10 \%$ higher than the ECBC values.

A LC/MS-MS is currently being used for identification of CL-20 degradation unknowns. This liquid chromatograph system uses series mass spectrometry to positively identify the total molecular weight of individual compounds (first mass spectrometer) and identify the composition of each of these compounds by fragmentation in the second mass spectrometer. A method for the analysis of nonvolatile compounds in solution by mass spectrometry is electrospray ionization (ESI). Additional chemical techniques are being used to identify CL-20 degradation including absorption over the UV and IR range. As explained in the results section, absorption at specific wavelength is specific to certain bonds (i.e., C-C or C-H bond stretch), so a decrease in $\mathrm{N}-\mathrm{N}$ absorption (for example) is indicative of some nitro groups being removed from the CL-20 structure. The CL-20 cage structure contains C-N rings that are not aromatic. If any of the three $\mathrm{C}-\mathrm{C}$ bonds in the CL-20 cage breaks (one specific $\mathrm{C}-\mathrm{C}$ bond is predicted to break by molecular modeling), then a flat molecular structure results containing three rings (also not aromatic). However, with the additional removal of any $-\mathrm{NO}_{2}$ groups, aromatic rings can result (i.e., pi electrons), which can be detected by IR absorption.

\section{Task 2. Microbial Degradation of CL-20}

Hypothesis: Biodegradation of CL-20 will be initiated by similar groups of microorganisms that degrade RDX and will employ similar enzymatic mechanisms.

The goal of this task is to identify the microbiological degradation mechanisms of CL-20 (anaerobic in Task 2.1; aerobic in Task 2.2), identify the taxa of microorganisms that execute these mechanisms, and determine the physiological requirements and environmental distributions of CL-20 degrading microorganisms. In this way, we will identify CL-20 biodegradation products, determine optimal conditions (i.e., Eh, $\mathrm{pH}$, ionic strength conditions) for CL-20 biodegradation, and develop a means to estimate potential for in situ CL-20 degradation. RDX is catabolized via a co-oxidative process whereby energy derived form the oxidation of another compound is used to drive RDX biodegradation. Given the similarity in some structural features between RDX and CL-20 and the low water solubility of CL-20 $(<5 \mathrm{mg} / \mathrm{L})$, we expect the major environmental biological degradation mechanisms for CL-20 will be a co-oxidative mechanism.

Surface and subsurface soils used in this task were obtained from China Lake, CA (January 2003 and October 2003) and from active firing ranges at Ft. Bliss, TX (June 2002) and Ft. Polk, LA (July 2003). China Lake is a Naval Air Warfare Center Weapons Division site that is located about $250 \mathrm{~km}$ north of Los Angeles, CA. Surface soil at China Lake was collected from Site 8, an area that receives surface water run-off and is therefore an active fluvial deposit. This area may have been contaminated with explosive residues in the past, however, we did not detect any explosive residues in this soil via HPLC analysis (see below). Subsurface soil was collected from China Lake Site 7 at a depth of $4.5 \mathrm{~m}$ in January 2003. Ft. Bliss is located near El Paso, TX and soils in this area are arid, loamy-sands (5\% to 7\% clay). Soil was collected from the center of a detonation crater (soil A), next to a low-order (incomplete) detonation (soil B), and $1 \mathrm{~m}$ from a tank target (soil C). Explosive residues were not detected in soils A or C, while soil B 
was contaminated with 2,4,6-trinitrotoluene (TNT; $3649 \mathrm{mg} \mathrm{kg}^{-1}$ ) and 2-amino-4,6dinitrotoluene (2ADNT; $177 \mathrm{mg} \mathrm{kg}^{-1}$ ). Ft. Polk is located $100 \mathrm{~km}$ north of Lake Charles, LA. Soil collected at Ft. Polk was from a grenade range and did not contain any detectable explosive residues. All soils were stored at $4^{\circ} \mathrm{C}$ and homogenized at the obtained field water content before use. Surface soils were passed through a $2-\mathrm{mm}$ sieve to remove small pebbles and rocks. The China Lake surface soil collected in October 2003 had very low moisture content and so it was amended with sterile water to the same moisture content $(6 \% \mathrm{w} / \mathrm{w})$ as the soil collected in January 2003 and incubated for 2-3 days at room temperature to stimulate microbial activity in the soil.

\section{Task 2.1. Anaerobic Degradation of CL-20}

Hypothesis: Bacteria that induce nitro-group reduction of $R D X$ can likely reduce nitro-groups of CL-20.

The most likely biotic degradation pathway is via sequential reduction of the nitro groups to nitroso derivatives followed by ring cleavage. This pathway results in approximately 25 different compounds, some of which are only hypothesized and have not been detected. This RDX pathway has been repeatedly suggested from: a) the identification of the first three degradation products (MNX, DNX, and TNX; see Figure 3); b) quantification of final products, $\mathrm{CO}_{2}, \mathrm{~N}_{2}$, $\mathrm{N}_{2} \mathrm{O}$; and $\mathrm{c}$ ) mass balance of $\mathrm{N}$ and $\mathrm{C}$ by isotopic means $\left({ }^{14} \mathrm{C},{ }^{15} \mathrm{~N}\right)$. Even if some of the reaction intermediates are not known, carbon mass balance has shown up to $80 \%$ mineralization (i.e., $\mathrm{CO}_{2}$ ) of $\mathrm{RDX}$.

Continually mixed, batch time course microcosm

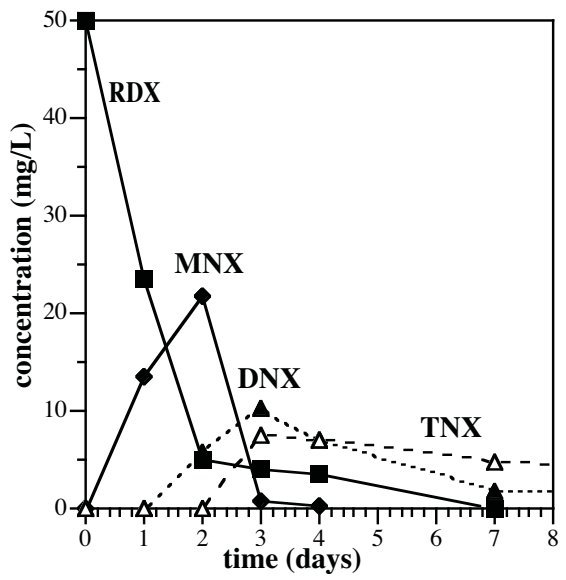

Figure 3. RDX degradation pathway with anaerobic bacteria (McCormick et al., 1981). experiments will be used to determine CL-20 disappearance, the flux of initial degradation products, and $\mathrm{C}, \mathrm{N}$ mass balance (by compound and isotopic means). A partial factorial experimental design will be used to evaluate the interactions between $\mathrm{pH}$, ionic strength, oxidationreduction potential, co-oxidative carbon sources, and types of bioaugmentation inocula. A wide range of well-characterized explosive-degrading microorganisms are maintained in the U.S. Army Engineer Research and Development Center's culture collection and will be used in these experiments. A mineral salts medium containing $2 \mathrm{mg} / \mathrm{L}$ each of $\mathrm{FeSO}_{4} \cdot 7 \mathrm{H}_{2} \mathrm{O}$, $\mathrm{MgSO} 4 \cdot 7 \mathrm{H}_{2} \mathrm{O} ; 5 \mathrm{mg} / \mathrm{L}$ each of $\mathrm{MnCl}_{2}, \mathrm{H}_{3} \mathrm{BO}_{3}, \mathrm{Na}_{2} \mathrm{SeO}_{3}, \mathrm{Na}_{2} \mathrm{MoO}_{4} \cdot 2 \mathrm{H}_{2} \mathrm{O}, \mathrm{CoCl}_{2} \cdot 6 \mathrm{H}_{2} \mathrm{O}$, $\mathrm{NiSO}_{4} \cdot 6 \mathrm{H}_{2} \mathrm{O}, \mathrm{CaSO}_{4} \cdot 5 \mathrm{H}_{2} \mathrm{O}$; and $\mathrm{ZnSO}_{4} \cdot 7 \mathrm{H}_{2} \mathrm{O}$, pH adjusted with phosphate buffer, and ionic strength adjusted with $\mathrm{NaCl}$. Microcosms will consist of Teflon-lined, screw-capped test tubes (120 x $15 \mathrm{~mm}$ ) placed on a revolving carousel in a Coy glove bag filled with a nitrogen and hydrogen mixture (98:2 vol./vol.). Tubes will be completely filled to eliminate headspace. At specified times, replicate tubes will be removed, $0.1 \square \mathrm{m}$ filtered, and analyzed for CL-20 remaining and degradation products (Task 1.3). Because CL-20 is likely to partition to organic matter in sediments, it is also likely to sorb to the microbial surface. It is important to distinguish between CL-20 removal by microbes that are due only to sorption from degradation (i.e., 
measurement of reaction products). Mineralization of isotopically labeled CL-20 to inorganic C and $\mathrm{N}$ compounds will be used to assess the extent of CL-20 binding to microbial cells and soil matrices.

Aerobic enrichment cultures were prepared using the surface soil in microcosms with a soil/ MSM ratio of $0.1 \mathrm{~g} / \mathrm{mL}$ amended with glucose $(5 \mathrm{mM})$, glycerol $(10 \mathrm{mM})$, succinate $(5 \mathrm{mM})$, and CL-20 ( $\sim 91 \mu \mathrm{M}$ or $40 \mathrm{mg} / \mathrm{L})$. The microcosms were incubated at $30^{\circ} \mathrm{C}$ with shaking. Once the CL-20 had been removed, $10 \%$ of the culture volume was transferred to fresh medium and the degradation of CL-20 was followed again. A total of 4 transfers were made before the enrichment was serially diluted and plated onto 5\% PTYG and MSM + CL-20 agar plates. Growth was poor on the latter plates and so single colonies growing on the 5\% PTYG plates were tested for their ability to grow on CL-20 as the sole nitrogen source. Anaerobic enrichments were prepared by adding $10 \mathrm{mg} / \mathrm{L}$ of CL-20 to the anaerobic microcosms without sodium sulfide after 62 days of incubation. The loss of CL-20 was then followed as above.

Two well-known, aerobic, RDX-degrading bacterial strains were tested for their ability to grow and utilize CL-20 as a nitrogen source (Kitts et al. 1994). Rhodococcus sp. strain DN22 was grown in the MRS medium of Coleman et al. (1998), while Rhodococcus rhodochrous strain $11 Y$ (Seth-Smith et al. 2002) was grown in the same nitrogen-free mineral salts medium used for the CL-20 enrichments. Each medium was amended with either RDX or CL-20 at $40 \mathrm{mg} / \mathrm{L}$. Bacterial growth was measured spectrophotometrically at $595 \mathrm{~nm}\left(A_{595}\right)$. RDX and CL-20 concentrations were measured by HPLC on filtered $(0.45 \mu \mathrm{m})$ cell extracts.

Sacrificed microcosms were extracted with an equal volume of methanol (CL-20) or acetonitrile $(\mathrm{RDX})$ followed by sonication for $18 \mathrm{~h}$ at $15^{\circ} \mathrm{C}$. The microcosms were then centrifuged to pellet the soils/sediments and the supernatants were filtered using PTFE $0.45 \mu \mathrm{m}$ filters and analyzed via HPLC. CL-20 is stable in methanol and methanol:water (1:1, v:v) mixtures, and unstable in acetonitrile and acetonitrile:water $(1: 1, \mathrm{v}: \mathrm{v})$ mixtures. Alternately, the aqueous phase $(100 \mu \mathrm{L})$ of microcosms was directly analyzed via HPLC, similar to the system described earlier.

${ }^{14} \mathrm{C}$-labeled CL-20 was used to investigate the rate and extent of CL-20 mineralization in the China Lake surface and subsurface soils. Microcosms were prepared in 125-mL serum bottles containing $5 \mathrm{~g}$ of soil, $50 \mathrm{~mL}$ of the mineral salts medium ( \pm glucose, $\left.1 \mathrm{~g} \mathrm{~L}^{-1}\right)$, and an inner vial $(10 \times 75 \mathrm{~mm})$ containing $1 \mathrm{ml}$ of $1 \mathrm{~N} \mathrm{KOH}$. The serum bottles were closed with Teflon ${ }^{\mathrm{TM}}$-coated butyl rubber stoppers and aluminum crimp seals. The mineral salts medium was amended with both unlabeled and ${ }^{14} \mathrm{C}$-labeled CL-20 to achieve an initial concentration of $3.5 \mathrm{mg} \mathrm{L}^{-1}$ and $1.44 \mathrm{nCi} \mathrm{mL}^{-1}$. The microcosms were incubated in the dark at $25 \pm 1{ }^{\circ} \mathrm{C}$ with constant shaking at $175 \mathrm{rpm}$. Control microcosms included soils amended with $\mathrm{HgCl}_{2}\left(300 \mathrm{mg} \mathrm{L}^{-1}\right)$ or only the sterile mineral salts medium. At each sampling time, the bottles were opened and the $\mathrm{KOH}$ removed for liquid scintillation counting. In addition, $0.6 \mathrm{~mL}$ of the aqueous phase was collected to determine the aqueous concentration of CL-20. The aqueous sample was mixed with an equal volume of acidified acetonitrile (Monteil-Rivera et al. 2004), filtered, and analyzed by HPLC. A fresh volume of $\mathrm{KOH}(1 \mathrm{~mL})$ was added to the inner vial and the microcosm closed. At the end of the incubation period, the soil was collected by centrifugation $(5,000 \times g$ for $10 \mathrm{~min})$ and the radioactivity in the aqueous phase (1-mL) and soil was measured. One g of soil was mixed with $5 \mathrm{ml}$ of acidified acetonitrile and then the soil was treated as described above. The filtered soil 
extract was diluted with an equal volume of water and then analyzed by HPLC and liquid scintillation counting. The acetonitrile-extracted soil was air-dried and the amount of soil-bound ${ }^{14} \mathrm{C}$-labeled CL-20 or ${ }^{14} \mathrm{C}$-labeled CL-20 degradation products remaining was determined by combustion to ${ }^{14} \mathrm{C}-\mathrm{CO}_{2}$ using a Model 307 Packard Sample Oxidizer (Packard Co., Meriden, CT).

\section{Task 2.2. Aerobic Degradation of CL-20}

Hypothesis: Bacteria that oxidize RDX by complete nitro-group removal may be reactive with CL-20.

In contrast to the mechanism proceeding via stepwise reduction of nitro groups (Figure 5) an aerobic bacterium, strain DN22 (Coleman et al. 1998) can directly remove an entire nitro-group from RDX resulting in an increase in nitrite in solution. Other reaction products include nitrate, glutamine, and ammonia. While there are some similarities between CL-20 and RDX structure (Figure 1; nitro-groups attached to nitrogen-heterocyclic rings), the cage structure of CL-20 will behave significantly differently than the planar RDX structure. We will use DN22, other aerobic RDX-degrading strains, and the microcosms described above (only aerobic) to assess their ability to aerobically degrade CL-20.

Aerobic soil microcosms consisted of $15 \mathrm{~mL}$ screw cap test tubes containing $1 \mathrm{~g}$ of soil plus $5 \mathrm{~mL}$ of a nitrogen-free mineral salts medium ( $\mathrm{pH}$ 7.0). The medium composition was similar to a medium designed to culture anaerobic bacteria described by Shelton and Tiedje (1984). The medium consisted of (per liter): $\mathrm{KH}_{2} \mathrm{PO}_{4}, 0.272 \mathrm{~g} ; \mathrm{K}_{2} \mathrm{HPO}_{4}, 0.348 \mathrm{~g} ; \mathrm{MgSO}_{4} .7 \mathrm{H}_{2} \mathrm{O}, 0.2 \mathrm{mg}$; $\mathrm{FeSO}_{4} .7 \mathrm{H}_{2} \mathrm{O}, 2 \mathrm{mg} ; \mathrm{CaCl}_{2} .2 \mathrm{H}_{2} \mathrm{O}, 0.03 \mathrm{mg} ; \mathrm{MnCl}_{2} .4 \mathrm{H}_{2} \mathrm{O}, 0.5 \mathrm{mg} ; \mathrm{H}_{3} \mathrm{BO}_{3}, 0.05 \mathrm{mg} ; \mathrm{ZnCl}_{2}$, $0.05 \mathrm{mg} ; \mathrm{CuCl}_{2}, 0.03 \mathrm{mg} ; \mathrm{Na}_{2} \mathrm{MoO}_{4} .2 \mathrm{H}_{2} \mathrm{O}, 0.01 \mathrm{mg} ; \mathrm{CoCl}_{2} .6 \mathrm{H}_{2} \mathrm{O}, 0.5 \mathrm{mg} ; \mathrm{NiCl}_{2} .6 \mathrm{H}_{2} \mathrm{O}, 0.05 \mathrm{mg}$; and $\mathrm{Na}_{2} \mathrm{SeO}_{3}, 0.5 \mathrm{mg}$. The $\mathrm{NH}_{4} \mathrm{Cl}$, resazurin, $\mathrm{NaHCO}_{3}, \mathrm{Na}_{2} \mathrm{~S}_{9} 9 \mathrm{H}_{2} 0$, and vitamins were omitted. Procedures to minimize oxygen saturation (i.e., boiling and degassing with $\mathrm{N}_{2}$ ) were omitted when the medium was used for aerobic incubations. Glucose $\left(1 \mathrm{~g} \mathrm{~L}^{-1}\right)$ was added to the indicated microcosms as an exogenous carbon source, and CL-20 (3 or $10 \mathrm{mg} \mathrm{L}^{-1}$ ) or RDX (3 or $10 \mathrm{mg} \mathrm{L}^{-1}$ ) was added as the nitrogen source. CL-20 and RDX were prepared as methanol or acetone stock solutions, respectively. Final concentrations of CL-20 or RDX at $3 \mathrm{mg} \mathrm{L}^{-1}$ were obtained by adding $0.3 \mathrm{ml}$ of a $10,000 \mathrm{mg} \mathrm{L}^{-1}$ stock to the bottom of a sterile flask, allowing the solvent to evaporate before adding $1 \mathrm{~L}$ of sterile medium, and then stirring the medium overnight in the dark to allow the explosives to dissolve. Final concentrations of $10 \mathrm{mg} \mathrm{L}^{-1}$ were obtained by directly adding $10 \mu \mathrm{L}$ of CL-20 or RDX stock solution $\left(5000 \mathrm{mg} \mathrm{L}^{-1}\right)$ to $5 \mathrm{~mL}$ of sterile medium in a test tube and then vortex mixing to disperse.

To quantify abiotic degradation rates associated with soil minerals and clays, biologically attenuated soil control microcosms were used. These microcosms contained sterile mineral salts medium and either soil that had been autoclaved three consecutive times $\left(1 \mathrm{~h}\right.$ at $\left.121^{\circ} \mathrm{C}\right)$ or soil that was amended with $\mathrm{HgCl}_{2}$ (final concentration, $300 \mathrm{mg} \mathrm{L}^{-1}$ ). Abiotic soil-free control microcosms contained only sterile mineral salts medium and these were included to quantify reactions caused by the glassware and medium components (Szecsody et al. 2004a). Microcosms were continually mixed on a tissue culture rotator (Glas-Col, Terre Haute, IN) at $30 \mathrm{rpm}$ and incubated in the dark at $25 \pm 1{ }^{\circ} \mathrm{C}$. At day 0 and day 21 , microbial biomass estimates in the China Lake soil microcosms incubated under aerobic conditions were obtained from the concentration of esterlinked phospholipid fatty acids (PLFA). PLFA analyses were performed according to the 
procedures of Balkwill et al. (1988) and White and Ringelberg (1998). Two to three replicate microcosms were analyzed at selected time intervals. The total amount of energetic remaining (aqueous plus soil-sorbed) was determined with an organic extraction consisting of the addition of five milliliters of methanol (CL-20) or acetonitrile (RDX) to each microcosm. Microcosms were placed into an ultrasonic bath (Branson, Danbury, CT) for $18 \mathrm{~h}$ at $15^{\circ} \mathrm{C}$. Following centrifugation at $2,800 \times \mathrm{g}$ for $10 \mathrm{~min}$ the supernatant was filtered through a $0.45-\mu \mathrm{m}$ PTFE filter and analyzed by HPLC.

Isolation of an Aerobic CL-20-Degrading Bacterium. Aerobic, CL-20 (40 mg L $\left.{ }^{-1}\right)$ enrichment cultures were prepared from the China Lake surface soil microcosms described above, except that glucose $\left(0.9 \mathrm{~g} \mathrm{~L}^{-1}\right)$, succinate $\left(1.35 \mathrm{gL}^{-1}\right)$, and glycerol $\left(0.92 \mathrm{~g} \mathrm{~L}^{-1}\right)$ were added as carbon sources and the incubation temperature was $30^{\circ} \mathrm{C}$. Once the CL-20 had been completely degraded, as determined via HPLC, $10 \%$ of the culture volume was transferred to fresh medium and the degradation of CL-20 was followed again. A total of four consecutive transfers were made before serial dilutions of the enrichment culture were spread onto 5\% PTYG agar (Balkwill et al., 1988) and MSM + CL-20 (10 $\left.\mathrm{mg} \mathrm{L}^{-1}\right)$ agar. Growth was poor on the latter agar medium and so colonies growing on the 5\% PTYG agar were tested for their ability to grow on CL-20 in liquid culture as the sole nitrogen source. A single bacterial strain, KTC13, was isolated with the ability to utilize CL-20 as a sole nitrogen source. The bacterial strain was identified using $16 \mathrm{~S}$ rRNA gene sequence analysis (Ausubel et al. 1989; Lane et al. 1985).

Growth of Pure Bacterial Cultures on CL-20 and RDX. The CL-20-degrading strain, KTC13, and two RDX-degrading bacterial strains, Rhodococcus sp. strain DN22 (Coleman et al. 1998) and Rhodococcus rhodocrous strain 11Y (Seth-Smith et al. 2002), were grown in liquid culture with CL-20 or RDX as the sole nitrogen source. Strain KTC13 and $R$. rhodocrous strain $11 \mathrm{Y}$ were grown in a mineral salts medium $(\mathrm{pH} 7.0)$ consisting of (per liter): $\mathrm{K}_{2} \mathrm{HPO}_{4}, 0.38 \mathrm{~g}$; $\mathrm{MgSO}_{4} .7 \mathrm{H}_{2} \mathrm{O}, 0.2 \mathrm{~g} ; \mathrm{FeCl}_{3} .6 \mathrm{H}_{2} \mathrm{O}, 0.05 \mathrm{~g}$; glucose, $0.9 \mathrm{~g}$; succinate, $1.35 \mathrm{~g}$; and glycerol, $0.92 \mathrm{~g}$. Rhodococcus sp. strain DN22 was grown in MRS medium (Coleman et al. 1998). CL-20 (40 $\mathrm{mg} \mathrm{L}^{-1}$ ) or RDX (40 mg L ${ }^{-1}$ ) was added as the sole nitrogen source. Cultures were incubated in the dark at $30^{\circ} \mathrm{C}$ and were agitated $(150 \mathrm{rpm})$. Growth was monitored spectrophotometrically at $595 \mathrm{~nm}\left(A_{595}\right)$ with a UV-1600A recording spectrophotometer (Shimadzu Corp., Kyoto, Japan). CL-20 and RDX concentrations were measured periodically by HPLC on filtered (0.45 $\mu \mathrm{m}$ PTFE filters) cell extracts. Rhodococcus sp. strain DN22 was kindly provided by Nicholas V. Coleman and $R$. rhodocrous strain 11 Y (NCIMB 40820) was obtained from the National Collection of Industrial and Marine Bacteria (NCIMB), Aberdeen, United Kingdom.

Degradation Kinetics. The concentrations of CL-20 and RDX were plotted as $C / C_{i}$ vs. time $(t)$, where $C$ is the concentration of energetic remaining at time, $t(\mathrm{~d})$ and $C_{i}$ is the initial concentration of energetic at time 0 . To determine $k$, the first-order degradation rate constant $\left(\mathrm{d}^{-1}\right)$, the degradation curves were fit according to first-order kinetics as follows:

$$
Y=Y_{\max } \times \exp (-k t)
$$


where $Y$ is the value of $C / C_{i}$ at a given time, $t, Y_{\max }$ is the maximum value of $C / C_{i}$ at time 0 . This general model was adapted to provide better fits to some of the data that exhibited lag phases and/or incomplete degradation as follows:

$$
Y=\text { If }\left\{t<t_{L}, \operatorname{Lag}, Y_{\min }+\left(\operatorname{Lag}-Y_{\min }\right) \times \exp \left[-k\left(t-t_{L}\right)\right]\right\}
$$

where $t_{L}$ is the time when the lag phase ends, Lag is the maximum value of $C / C_{i}$ during the lag phase, and $Y_{\min }$ is the minimum, measured value of $C / C_{i}$. Values for $k$ were calculated for each curve using GraphPad Prism version 4.00 for Windows, GraphPad Software, San Diego, California, USA. Values for $t_{L}, Y_{\min }, Y_{\max }$, and $L a g$ were either calculated or were set to initial values in order to achieve the best curve fit as determined by coefficients of fit values $\left(r^{2}\right)$ and how well the curve-fit reflected the actual data. If values for $t_{L}$ and $Y_{\min }$ were set to 0 , then equation (7) collapsed to equation (6) (first-order degradation kinetics without a lag phase and complete degradation). If only the value for $t_{L}$ was set to 0 , the equation modeled the situation with no lag phase and incomplete degradation. The degradation half-life (d) was then calculated from

$$
\mathrm{t}_{1 / 2}=\ln (2) / k
$$

The rate data were analyzed using the general linear models procedure (PROC GLM) of SAS (SAS Institute 2003).

\section{Task 3. Coupled Processes}

Two specific interactions between geochemical and microbially mediated processes will be assessed: a) the effect of geochemical reactions on microbial degradation of CL-20 (Task 3.1), and $b$ ) the effect of aging on geochemical reactions of CL-20 with sediments (Task 3.2). Hypotheses are listed in these separate tasks. Interactions between coupled processes are complex (e.g., synergistic or antagonistic) and often not predicable from measurements of the individual processes or reactions (Bryce and Elzerman 1994; Ma and Selim 1994). The dominant process(es) that occurs in the natural environment may be different from that in segregated systems. Reasons for this disparity include: a) reactant concentrations are different than in individual reaction systems, b) reaction conditions are different (i.e., surface versus in aqueous solution), and c) a reaction system is different. Testing the extent of a coupled-process effect will be accomplished using a multiple-reaction model and the separate reaction parameters to predict results in coupled process experiments.

\section{Task 3.1. Coupled Sediment-Biotic Reactions with CL-20}

Hypothesis: CL-20 coupled abiotic/biotic degradation in anaerobic systems will be more rapid than separate abiotic or biotic degradation due to an actual coupled reaction. CL-20 degradation in oxic systems in the presence of abiotic and microbes will not result in coupled reactions.

The above-described (Task 2.1) microcosms will be again employed in a partial factorial experimental design wherein different environmentally relevant matrices are assessed. The interactions between varying proportions are a) varying amount of clean silica sand (no organic matter, Fe- oxides, no clays), b) varying amount of a high-organic content sand used in sorption experiments (Task 1.1), and c) varying amount of $\mathrm{Fe}$ - and Mn-oxides and/or clays used in sorption experiments. The clean sand experiments are designed to have progressively greater 
attachment of microbes, but little sorption of CL-20 and products. A microbial sorption isotherm on the sand will be developed from measurements of the microbial population remaining in solution at different sand and microbe concentrations. It is likely that the attachment will be very strong. If progressively greater sorption of microbes is correlated with slower degradation, it may indicate less microbial reactivity of CL-20 in the presence of natural sediments. An example of this coupled effect is the biodegradation of quinoline in the presence of sediments, which was slowed by the binding of microbes onto sediment surfaces (Szecsody et al. 1993). Coupled abiotic/biotic degradation experiments with the other sediments containing organic matter, oxides, and clays will have increased sorption of CL-20. The coupled rate of sorption and biotic degradation should be predictable from individual experiments (CL-20 sorption onto surfaces, Task 1.1) if no additional effects are present. A sorption effect example is the biotic reduction of iron oxides (on sediments), which was slowed by the coating of $\mathrm{Fe}$ (II) produced on microbes (Liu et al. 2000). As the microbial degradation pathway is established (Task 2.1) and CL-20 sorption to mineral surfaces and the microbial surface is established, more complete or different coupled hypotheses may be determined.

\section{Task 3.2. Effect of Aging and CL-20/Sediment Reactions}

Hypotheses: CL-20-sediment aging will slow the CL-20 desorption rate and availability for abiotic and biotic degradation. Desorption and availability will also be influenced by the magnitude of the carbon content of the sediment subjected to the aging process.

Aging is the process by which a contaminant is slowly incorporated into sediment organic matter and microfractures (Pignatello and Xing 1996; Luthy et al. 1997), or metals being incorporated into Fe-oxides. Release of the contaminant involves this mechanism acting in reverse with the contaminant slowly diffusing out of amorphous and microcrystalline organic material to reach the bulk fluid (e.g., soil pore water) in very low concentration. Rather than being sequestered, the contaminant in this state is considered to be part of what has become to be known as a "resistant fraction." CERCLA remedial actions driven by Records of Decision require cleanup levels for soils and predictions of groundwater impacts as part of a site's closure process. Being able to quantify the amount of CL-20 present as a "resistant fraction" in soils of varying organic carbon content, under both saturated and unsaturated flow conditions, could provide information that would allow the setting of a more realistic regulatory standard for remediation of CL-20 contaminated soils.

Because aging imparts additional chemical and physical (i.e., diffusional) contaminantsediment rate limiting processes, it is hypothesized that a CL-20 resistant fraction will be generated and that the desorption rate will be slower as a result of aging. In addition, because microbes and abiotic reactive surfaces are typically near sediment surfaces and not in microfractures, it is expected that aging will result in less availability of the CL-20 for abiotic and biotic degradation reactions. Failure to account for the amount of CL-20 present as a "resistant fraction" in contaminated soils could overestimate the environmental risk of CL-20 (Alexander 2000).

We studied the potential effects of weathering of soils on the slow release and availability of CL-20 to the environment. Soils from sites of possible CL-20 contamination and with varying organic carbon content will be subjected to CL-20 weathering using circulating supercritical 
carbon dioxide (Figure 4). Because of favorable properties of supercritical carbon dioxide (e.g., high diffusivity, low viscosity [Lee and Markides 1990]), the chemical adsorption and equilibrium process can occur on a time scale (hours) amenable to laboratory study without the potential for introduction of chemical artifacts (carbon dioxide is considered benign). The system has been used successfully to observe the presence of a "resistant fraction" of phenanthrene in a porous silica and soil (Riley et al. 2000; Wang et al. 2000). Concentrations of CL-20 on sediments aged with supercritical carbon dioxide will be determined by solvent extraction and LC analysis and supercritical fluid in-line measurements.

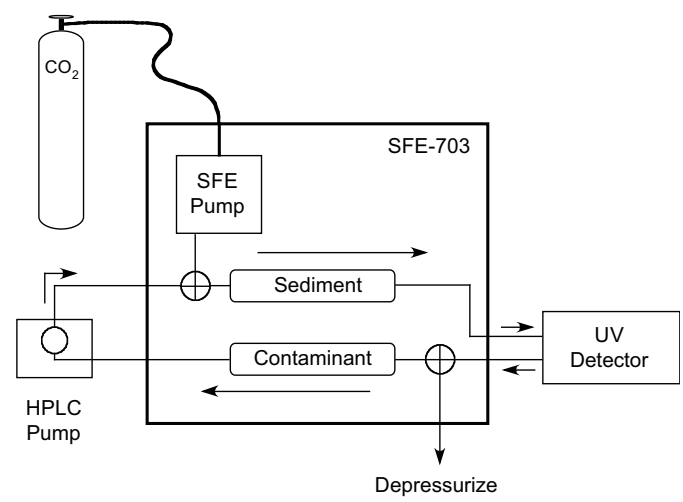

Figure 4. System used to create a CL-20 resistant fraction in sediment

Soils with varying organic carbon content and artificially aged with CL-20 will be subjected to assessment in laboratory batch and column leaching experiments, with LC or LC-MS CL-20 analysis (Task 1.3). At the termination of the experiments, amounts of CL-20 remaining on the soils will be determined and overall mass balances performed. The amount of CL-20 "resistant fraction" present for soils of different carbon content will be determined from the mass balance information and the aqueous leachate profiles of the column experiments. A corresponding set of experiments will be performed under unsaturated flow conditions, where aging may dominate (Figure 1). In a set of batch experiments, sediments subjected to short and long contact times with CL-20 will be assessed for abiotic and biotic degradation rates and the effect of aging.

Due to degradation of CL-20 in the liquid $\mathrm{CO}_{2}$, the potential effects of weathering of soils on the slow release and availability of CL-20 to the environment was studied conventionally with long contact time before desorption. A series of ten columns were loaded with CL-20 and RDX and flow stopped for $1 \mathrm{~h}, 100 \mathrm{~h}, 670 \mathrm{~h}$ (1 month), $2200 \mathrm{~h}$ ( 3 months), and $2200 \mathrm{~h}$ at $35^{\circ} \mathrm{C}$. Two different sediments were chosen that mainly exhibited little degradation, the Sassafrass sediment (used by ECBC and BRI), and the Westmoreland A1 sediment. Diffusion of CL-20 into microfractures will occur more rapidly at elevated temperature, so $2200 \mathrm{~h}$ at elevated temperature is equivalent to a longer period of time. A temperature greater than $35^{\circ} \mathrm{C}$ was not used because $\mathrm{CL}-20$ is unstable at neutral $\mathrm{pH}$ in water at a temperature $>35^{\circ} \mathrm{C}$. These aging experiments were initiated in year 2, and ended in year 3. Extensive simulation of experiments was conducted in year 3 to determine the influence of aging upon: a) CL-20 and RDX sorption mass $\left(\mathrm{K}_{\mathrm{d}}\right)$; b) CL20 and $\mathrm{RDX}$ desorption rate $\left(\mathrm{k}_{\mathrm{b}}\right)$, and c) CL-20 abiotic degradation rate.

\section{Task 4. Reactive Transport of CL-20}

In this task we quantified at a subset of abiotic and biotic reactions of CL-20 (Tasks 1 and 2) in flowing systems. Experiments conducted in 1-D flowing systems will provide an understanding of CL-20 reactive transport behavior by: a) demonstrating CL-20 transport subjected to significant reactions determined in batch experiments (sorption, abiotic degradation, biotic degradation), and to evaluate prediction of the behavior based on the batch knowledge. While 
the nature of the reaction network (i.e., reaction identity and stoichiometry) is identical in batch and column systems, batch-derived reaction parameters can fail to accurately predict transport because transport effects can significantly influence the apparent manifestation of nonlinear or coupled reactions during advective flow.

\section{Task 4.1. Abiotic Reactive Transport of CL-20 in Saturated Porous Media} Hypothesis: Prediction of abiotic saturated CL-20 transport from batch data will be highly dependent on the CL-20 sorption/desorption rate and abiotic degradation rate.

The initial step in applying batch CL-20/sediment reaction information to larger-scale field systems is to access reactivity during flow in homogeneous, water-saturated columns. Batch information can fail to accurately predict reactive transport behavior due to: a) aqueous reactant advection away from solid-phase reactants in columns, b) higher sediment-water ratios in columns, c) impact of reaction rates in flowing systems with limited contact time, d) increased physical rate limitations (i.e., diffusion) in porous media systems, e) particle-scale heterogeneities, or f) differing water content changing reactive surface site access. The first three (a through c) reasons should be predicted with batch data, assuming that there is complete knowledge of the reaction network and other differences between batch and flowing systems are correctly accounted for. Simple, linear reactions have been shown to accurately predict reactive transport in homogeneous porous media (Szecsody et al. 1992; Chen et al. 1992), but difficulties predicting transport with nonlinear (Langmuir, Freundlich) adsorption arise due to the reaction mechanism being more complex than described initially in batch systems (Spurlock et al. 1995; Burglsser et al. 1993; Huang et al. 1995). The latter three reasons (d through f) refer to differences in natural sediments that occurs between reacting a solute with unconsolidated sediment (batch) during flow through natural sediment. While 1-D column experiments are only idealized representations of field scale transport, they are useful in demonstrating the relative importance of these flow-related processes to the geochemical or microbial reactions. Our own RDX transport experiments in sediments (Ainsworth et al. 1993) showed little sorption (as predicted), so differences in clay and organic matter had only a minor effect on breakthrough.

We initially used small 1-D saturated column experiments to derive transport-system reaction parameters to compare with CL-20 batch studies (Task 1.1). 1-D column experiments under oxic conditions (sorption only) will be conducted varying the flow velocity between experiments in order to have a different residence time (i.e., CL-20/sediment contact time). This series of experiments tested the sorption mass and sorption rate applicability of the batch-derived parameters, as the breakthrough curve shape is highly dependent on the ratio of sorption rate to residence time. Column experiments consist of injecting a pulse of CL-20-laden solution (with tracer) into a saturated sediment column for sufficient time to allow for CL-20-sediment equilibrium (likely 100 s of pore volumes), then injecting a pulse of solute free solution into the column until most of the CL-20 mass is desorbed. It is expected that there will be some adjustment to the sorption rate determined in columns relative to batch systems. The $\mathrm{K}_{\mathrm{d}}$ or "linear" sorption approach (Task 1.1, Equation 1), can define a CL-20 retardation or velocity relative to groundwater flow for a reaction at equilibrium (i.e., no kinetic effect):

$$
\mathrm{R}_{\mathrm{f}}=1+\rho_{\mathrm{b}} \mathrm{K}_{\mathrm{d}} / \theta
$$


where $\rho_{b}$ is the dry bulk density $\left(\mathrm{g} \mathrm{cm}^{-3}\right)$, and $\theta$ is the porosity. While this $\mathrm{K}_{\mathrm{d}}$ concept is applicable for CL-20 subject to sorption only (no degradation), it additionally requires sorption equilibrium. It is highly likely that the CL-20 sorption/desorption rates will be slow (10s of hours). Column breakthrough of a slowly sorbing solute will show some breakthrough much sooner than expected (some CL-20 will not have time to sorb) and other mass eluting much later in time than expected (i.e., some CL-20 desorbing slowly). This "spreading" or "tailing" commonly associated with sorption kinetics can be described using a reactive transport model incorporating the sorption/desorption rate (Szecsody et al. 1998). Degradation reactions result in mass loss, so do not alter the apparent breakthrough velocity of CL-20, but will result in effluent mass being less than the injected mass. Because CL-20 sorption is expected to be large (and the rate slow), fast flow with small CL-20 injected pulses and elevated temperatures will enable column experiments to be accomplished, even with a large $\mathrm{K}_{\mathrm{d}}$, based on the relative reaction rate to the flow rate. The Damkohler I number $\left(\mathrm{N}_{\mathrm{d}}\right)$ is the ratio of solute residence time in a column to the reaction half life for reaction $\mathrm{i}$ :

$$
\mathrm{N}_{\mathrm{d}, \mathrm{i}}=\frac{\mathrm{L}}{\mathrm{v}} \frac{\rho_{\mathrm{b}} \mathrm{K}_{\mathrm{d}, \mathrm{i}} \mathrm{k}_{\mathrm{f}, \mathrm{i}}}{\theta}
$$

where $\mathrm{L} / \mathrm{v}$ defines the residence time in the column, and $\mathrm{K}_{\mathrm{d}, \mathrm{i}}$ is the sorption to each type of site.

A second limited set of 1-D column experiments will be under reducing conditions (Task 1.2) that promote CL-20 degradation. At these greatly increased sediment/water ratio (relative to batch systems), degradation rates will obviously be faster, but the rate per unit sediment mass will be similar. It is expected to observe a somewhat slower degradation rate per gram of sediment in columns, due to slower mass transfer. The abiotic degradation of TCE in reduced sediment in batch systems $3.3 \mathrm{~h}$ was also observed to be somewhat slower in 1-D columns $(5.6 \mathrm{~h}$ in 23 experiments; Szecsody et al. 2000b, 2004b). The direct applicability of the batch-derived CL-20/ sediment reaction parameters to predict CL-20 transport in columns will involve a comparison of predictions to 1-D column experimental results. Additional column experiments will be conducted based on sensitivity simulations. The CL-20/sediment sorption/degradation reaction system will likely involve multiple nonlinear reactions.

\section{Task 4.2. Abiotic/Biotic Reactive Transport of CL-20 in Saturated Porous Media} Hypothesis: Biotic saturated CL-20 transport in reducing sediment will exhibit slower biotic degradation than predicted from batch systems.

Biodegradation of CL-20 occurs more rapidly in reducing conditions, so applicability to the field scale is in saturated groundwater systems, which exhibit reducing conditions. A limited set of 1-D saturated column experiments with uniformly attached (RDX-degrading assumed to be CL-20 degrading) bacteria will be conducted to demonstrate CL-20 biodegradation can occur in field-scale conditions (CL-20). These experiments are similar to those described in Task 4.1, but with a uniform initial population of microbes mixed with the porous media for $24 \mathrm{~h}$ before flow is started (to allow for attachment; Szecsody et al. 1994). Part of this task is to determine the rate at which CL-20 biodegradation does occur in the packed porous media. It is likely to be significantly less than in batch systems, where ideal conditions of major and trace nutrients can be easily maintained. Bioreactivity in columns have been reported with slower rates than batch systems (Szecsody et al. 1994) or at a greater rate (Liu et al. 2000). An example of the impact of 
flow on microbial activity is the biodegradation of a metal-chelate complex showed decreased microbial activity during flow. Biodegradation and sorption were well characterized by batch experiments. A 1-D column experiment showed significantly (40x) less biodegradation in the flowing system than predicted from batch data, likely due to less trace nutrient delivery than in batch. This effect can be characterized; field-scale bioremediation systems typically use alternating pulses of nutrients/no nutrients to minimize the effect.

\section{Task 4.3. Reactive Transport of CL-20 in Unsaturated Porous Media}

Hypothesis: CL-20 abiotic transport subject in the vadose zone with sorption will be influenced by reactive surface access at differing water contents.

Transport behavior for near-surface spills is of high significance, so in this task, we quantified CL-20 sorption and abiotic degradation during unsaturated transport and evaluate mechanisms that cause CL-20 sorption/degradation to differ from predictions that are based on behavior in water-saturated systems. The impact of mobile-immobile water domains on sorption during transport in unsaturated sediments has only recently been investigated (Gamerdinger and Kaplan 2000; Gamerdinger et al. 2001a, 2001b). This research has shown that changes in the water flow regime in unsaturated sediments may affect the accessibility of mobile solutes to reactive surface sites, and rate-limited sorption reactions.

At low moisture contents, it is hypothesized that accessibility to reactive surface sites will change due to increasing immobile water. A corresponding change in sorption and transport will depend on the correlation between particle surface reactivity and particle size. As sediments are progressively de-saturated, dual porosity or mobile-immobile water regions develop, particularly in sandy sediments (Nielson and Biggar 1961; Gaudet et al. 1977). Previous research indicates that the resulting hydraulic heterogeneity is linked to chemical heterogeneity at the particle scale and the association of the unsaturated mobile flow regime with sand versus silt-sized particles is expected to vary with sediment texture (Kaplan et al. 1994; Gamerdinger et al. 1998).

Research in this task will use sediments that are being used in the batch sorption experiments (Task 1.1) at differing water content using centrifugation methods. A centrifuge method that allows for highly controlled, steady-state unsaturated flow at low water contents was selected for conducting transport experiments (Gamerdinger et al. 1998; Gamerdinger and Kaplan 2000). Breakthrough curves for the non-sorptive pentafluorobenzoic acid (PFBA) tracer will be used to quantify the ratio of mobile-immobile water at low water content ( $10 \%$ to $20 \%$ moisture saturation) and to characterize hydrodynamic conditions that determine CL-20 transport. The unsaturated column system includes two infusion pumps and an "unsaturated flow apparatus" (L8-UFA ${ }^{\circledR}$, Beckman Coulter, Inc., Fullerton, CA). A rotating seal is at the center of the rotor and is the conduit through which fluid is delivered from the pump to the columns, by two independent flow paths. Miscible displacement or unsaturated transport experiments are initiated when the columns reach a steady-state average water content ( $>10$ pore volumes). During operation of the UFA, centripetal acceleration forces water from the column, which is replaced by fluid delivery via the pump through a rotating seal (see Figures 5 and 6), with step inputs of solute-free and solute-laden fluid. Column effluent is collected below the sediment column (Figure 7) and analyzed for CL-20 (Task 1.3) to determine the flux-averaged effluent concentration. The observed data are described with a two-region transport model (Gamerdinger et al. 2001b). 


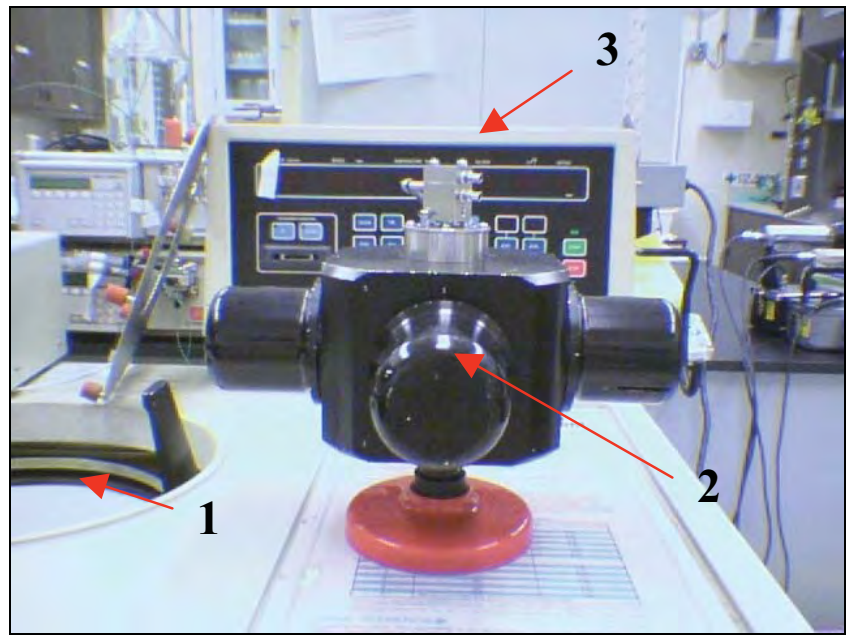

Figure 5. UFA centrifuge (1), rotor (2), and rotating seal (3)

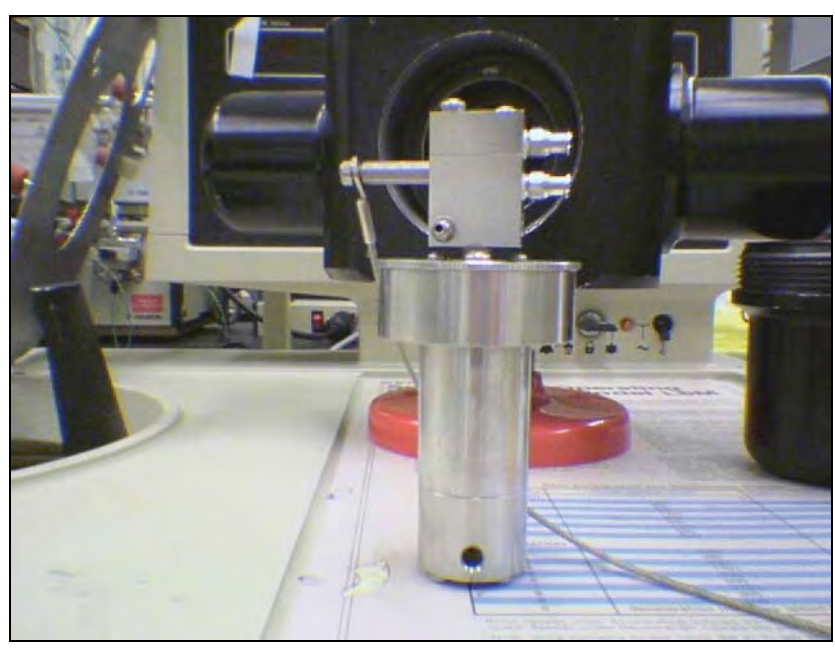

Figure 6. UFA ${ }^{\circledR}$ rotating seal housing two separate flow-lines for two columns

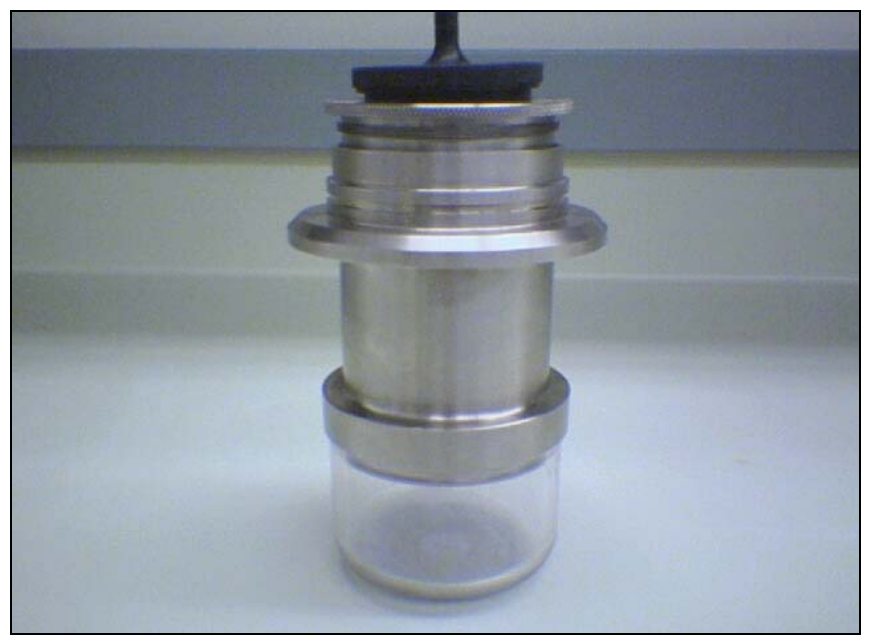

Figure 7. Titanium column holder 


\section{Results}

\section{Task 1. Abiotic Reactions}

\section{Task 1.1. Sorption of CL-20 to Sediments}

CL-20 can be released to the surface and subsurface terrestrial environment by: a) manufacturing processes, b) munition storage, and c) use with low order detonation or unexploded ordnance. CL-20 and RDX toxicity to specific organisms in terrestrial and aquatic ecosystems was determined. CL-20 to RDX sorption and degradation behavior in surface and subsurface sediments was quantified to provide insight into the migration potential.

CL-20 Behavior in Surface Soils. CL-20 sorbs to specific sediment components (organic matter, clays, oxides). Surface soils $(<2 \mathrm{~m})$ in humid regions can have organic matter (fraction organic carbon or $\mathrm{f}_{\mathrm{oc}}$ ) as high as $40 \%$, whereas soils in arid regions have lower organic matter $(<5 \%)$. As predicted, CL-20 sorption to surface soils is mainly to the organic matter so can be generally predicted from the organic carbon content:

$$
K_{d}, C L-20\left(f_{o c}>1 \%\right)=\left[3.53(\% \mathrm{OC})^{1.14}\right]
$$

although differing types of organic matter adsorbed CL-20 more strongly (Monteil-Rivera et al. 2004). Therefore, in more humid regions, CL-20 will be retained in the soil zone where degradation (microbial, photodegradation) can occur. A more extensive analysis of sorption to surface soils is found in a companion SERDP project (CU-1254, J. Hawari).

CL-20 Behavior in Subsurface Sediments (Vadose Zone, Groundwater). Both CL-20 sorption and degradation control subsurface migration risk. CL-20 sorption in the subsurface below the soil zone ( $<2 \mathrm{~m}$; vadose zone, groundwater aquifers; organic carbon $<0.5 \%$ ) is small for CL-20 $\left(\mathrm{K}_{\mathrm{d}}=0.22\right.$ to $\left.3.83 \mathrm{~cm}^{3} \mathrm{~g}^{-1}\right)$ and for $\mathrm{RDX}\left(\mathrm{K}_{\mathrm{d}}=0.53\right.$ to $\left.6.5 \mathrm{~cm}^{3} \mathrm{~g}^{-1}\right)$. While there will be some retention in sediments, realistically, both CL-20 and RDX can migrate deep in the subsurface. RDX is well documented as a groundwater contaminant, moving nearly unretarded. CL-20 sorption for low $\mathrm{f}_{\mathrm{oc}}$ sediments can be estimated from:

$$
K_{d}, C L-20\left(f_{o c}<1 \%\right)=\left[0.112 \mathrm{e}^{0.020 \mathrm{DCB}-\mathrm{Fe}}\right](\mu \mathrm{mol} / \mathrm{g})
$$

Abiotic and biotic degradation in oxic sediments is observed (hours to 1000s of h), which limits migration. CL-20 will degrade in alkaline waters ( $\mathrm{pH}>9.5$; hydrolysis), and with in sediments the degradation rate is related to adsorbed $\mathrm{Fe}^{\mathrm{II}}$ or 2:1 clays. Of 15 sediments (varied properties), 7 had very slow degradation (100 to $6400 \mathrm{~h}$ half-life), and so would be persistent in the subsurface for years.

CL-20 sorption to sediments and sediment minerals was small, ranging from 0.02 to $4.3 \mathrm{~cm}^{3} / \mathrm{g}$ (Table 1.1). CL-20 sorption to most sediment minerals, such as some clays (kaolinite, chlorite, muscovite), silica, albite, ferrihydrite, and aluminum oxide was relatively small $\left(0.07 \mathrm{~cm}^{3} / \mathrm{g}\right.$ for albite; $1.8 \mathrm{~cm}^{3} / \mathrm{g}$ for chlorite). To confirm that aqueous CL-20 mass loss from solution was sorption, methanol extractions of organic mass from mineral surfaces and sediments were conducted. These results showed that sorption was occurring on nearly all surfaces (i.e., 
CL-20 mass was extracted from the surface with methanol). In addition, most minerals and sediments reacted with CL-20, producing degradation products, which were also removed with the methanol extraction.

Table 1.1. CL-20 sorption and degradation and RDX sorption to sediments in $24 \mathrm{~h}$.

\begin{tabular}{|c|c|c|c|c|c|c|c|c|}
\hline Sediment & $\begin{array}{l}\text { CL-20 apparent } \\
\mathrm{K}_{\mathrm{a}}\left(\mathrm{cm}^{3} \mathrm{~g}^{-1}\right)\end{array}$ & $\begin{array}{c}\text { RDX } \\
K_{d}\left(\mathrm{~cm}^{3} / g\right)\end{array}$ & $\begin{array}{c}\mathrm{Fe}^{\mathrm{II}}{ }_{\text {tot }} \\
\left.(\mu \mathrm{mol} \mathrm{g})^{-1}\right)^{2}\end{array}$ & 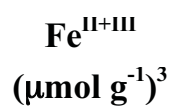 & $\begin{array}{l}\text { clay } \\
(\%)\end{array}$ & $\begin{array}{l}\text { foc } \\
(\%)\end{array}$ & $\begin{array}{c}\text { CEC } \\
\left(\text { meq } 100 g^{-1}\right)\end{array}$ & pH \\
\hline$\overline{\text { Burbank Ap }}$ & $3.4 \pm 0.86$ & $6.5 \pm 0.14$ & - & 110 & 4 & 0.5 & 5.5 & 7.00 \\
\hline Cloudland Ap & $4.8 \pm 1.8$ & $0.53 \pm 0.11$ & 25 & 90 & 11 & 1.19 & 2.49 & 5.30 \\
\hline Kenoma Bt1 & $1.2 \pm 0.37$ & $1.7 \pm 0.25$ & 45 & 163 & 44 & 1.43 & 26 & 5.60 \\
\hline Norborne C & $0.31 \pm 0.06$ & $0.68 \pm 0.06$ & - & 55 & 13 & 0.23 & 9.14 & 7.10 \\
\hline Ocala C4 & $>10$ & $3.0 \pm 1.4$ & 72 & 6.1 & 33 & 0.08 & 33.5 & 10.70 \\
\hline Westmoreland A1 & $6.1 \pm 1.1$ & $0.69 \pm 0.22$ & - & 172 & 14 & 2.03 & 6.91 & 4.90 \\
\hline
\end{tabular}

${ }^{1} 5 \mathrm{M} \mathrm{HCl}$ extraction

${ }^{2}$ dithionite-citrate-bicarbonate extraction

Time-series experiments were conducted with the Norborne and Kenoma sediments (Figure 1.1) to determine the contact time required to ensure that CL-20 sorption equilibrium was obtained in batch sorption-degradation studies described below. Both solution and sorbed CL-20 concentrations were measured in individual samples after 1 and 30 minutes, and 1.5, 18, and $48 \mathrm{~h}$ contact times. Sorption equilibrium was reached for both sediments within 4 minutes (sorption half-life $\sim 0.8 \mathrm{~min}$.). The 2 and $24 \mathrm{~h}$ contact times used in batch experiments (described below) were thus sufficient for sorption equilibrium to be obtained for these three sediments. Based on this result, it has been assumed that $2 \mathrm{~h}$ contact times were sufficient to
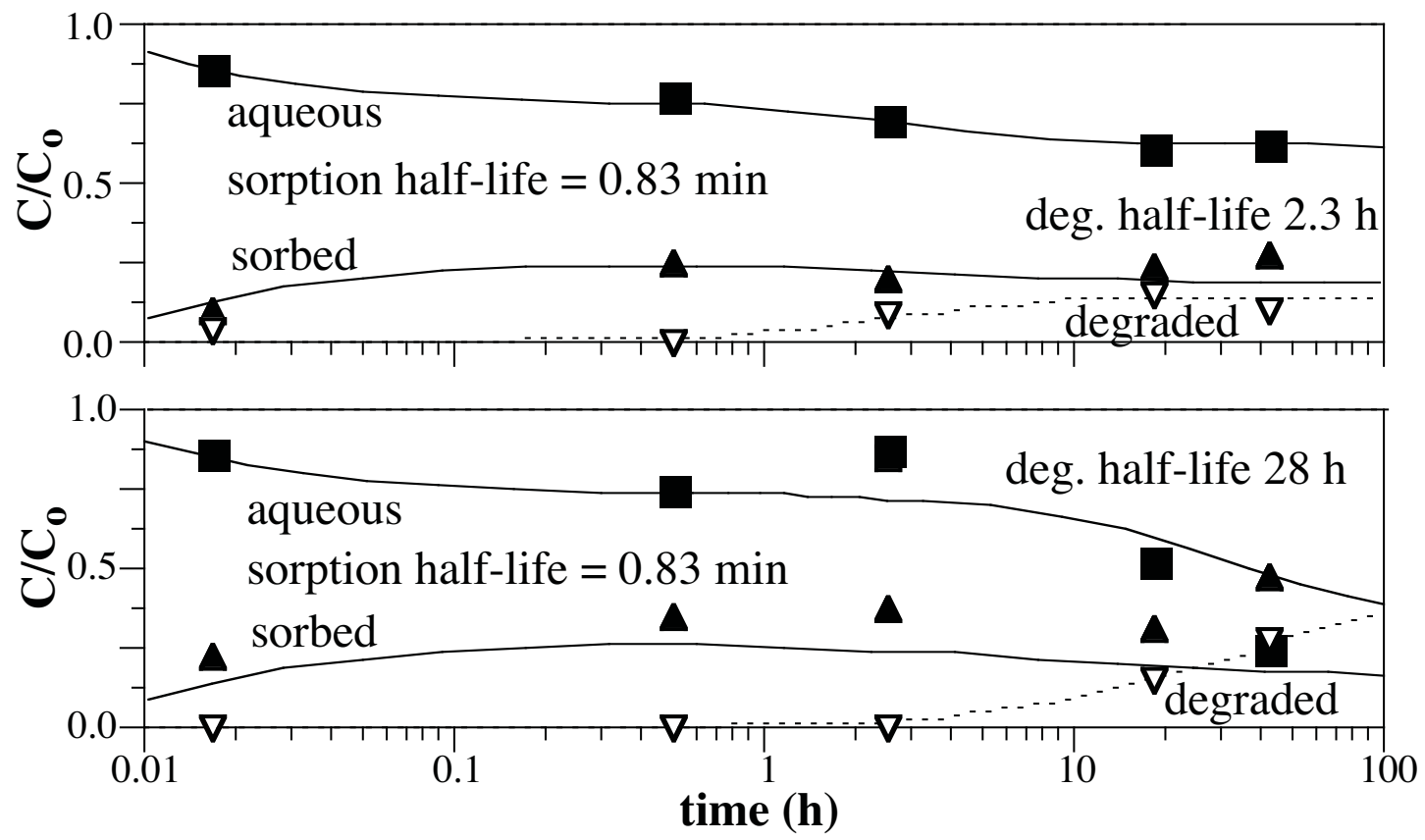

Figure 1.1. CL-20 sorption rate on: a) Norborne $\mathrm{C}$ sediment and b) Kenoma Bt1 sediment. The initial CL-20 concentration was $3.2 \mathrm{mg} \mathrm{L}^{-1}$ at $\mathrm{pH} 7.0$. Sorbed phase was quantified by methanol extraction of CL-20 from the sediment. 
ensure that sorption equilibrium was obtained for the other solids examined in this study and that the $\mathrm{K}_{\mathrm{d}}$ values presented here are indeed equilibrium values.

CL-20 is degraded by hydrolysis in homogeneous solution at a $\mathrm{pH}>8.5$ (Figure 1.2), although degradation in sediment-water systems was observed at all $\mathrm{pHs}$ examined. RDX and HMX alkaline hydrolysis is reported (Heilmann et al., 1996). To eliminate CL-20 hydrolysis, batch and column experiments were conducted in CL-20 solutions buffered to $\mathrm{pH}$ 7.0. The interactions via sorption and abiotic degradation of aqueous

CL-20 with sediments having a range of organic carbon, clay, and iron oxide content were examined in batch $24 \mathrm{~h}$ experiments (Table 1, Figure 1.3, open diamonds) and $2 \mathrm{~h}$ experiments (Table 1.2, Figure 1.3, solid diamonds). CL-20 degradation occurred in nearly all experiments, as indicated by the presence of degradation products in either the final solution or surface extraction phases or both.
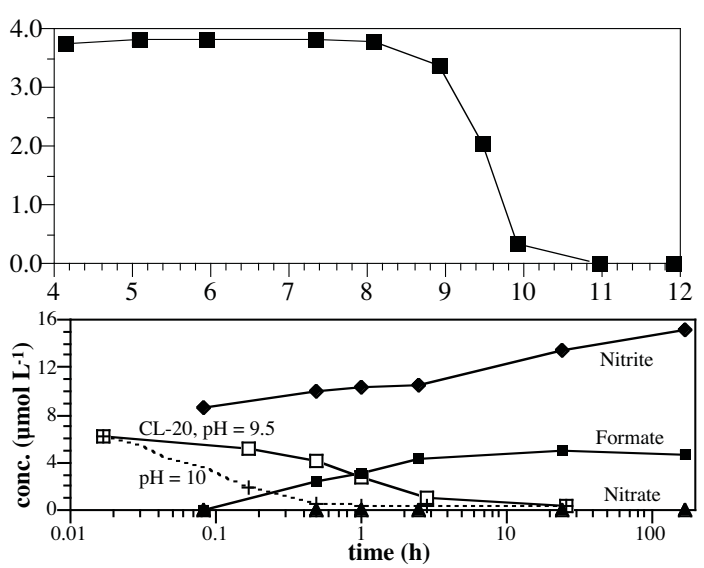

Figure 1.2. Aqueous degradation rate of CL-20: a) by $24 \mathrm{~h}$ at different $\mathrm{pH}$ and b) at $\mathrm{pH} 9.5$ (solid lines) and $\mathrm{pH} 10$ (dashed line) at $25^{\circ} \mathrm{C}$. The initial CL-20 concentration was $2.7 \mathrm{mg} \mathrm{L}-1$ (6.7 $\mu \mathrm{mol} \mathrm{L-1).}$ CL-20 removal from solution was described in terms of an apparent distribution coefficient $\left(\mathrm{K}_{\mathrm{a}}\right.$, Table 1.1) because the solid phase concentration was not measured, but determined from the difference between the total initial CL-20 $\left(\mathrm{C}_{\mathrm{i}}\right)$ and final solution $(\mathrm{C})$ concentration, as noted above. Thus, $\mathrm{K}_{\mathrm{a}}\left(=\left(\mathrm{C}_{\mathrm{i}}-\mathrm{C}\right) / \mathrm{C}\right)$ overestimated sorption because degradation of CL-20 occurred. Although CL-20 degradation occurred in all but one $2 \mathrm{~h}$ experiment, sorption could be quantified by the standard distribution coefficient $\left(\mathrm{K}_{\mathrm{d}}=\mathrm{S} / \mathrm{C}\right.$, Table 1.2) because final CL-20 solution (C)

and solid (S) concentrations were measured.

Based on the degradation half-lives and $\mathrm{K}_{\mathrm{d}} \mathrm{S}$ from $2 \mathrm{~h}$ experiments (Table 1.2), the final solution concentrations for the Cloudland and Westmoreland sediments in $24 \mathrm{~h}$ experiments would have been less than $4 \%$ and $1 \%$ of the total concentration, respectively, resulting in an overestimate of sorption by $\mathrm{K}_{\mathrm{a}}$. Comparison of $\mathrm{K}_{\mathrm{a}}$ and $\mathrm{K}_{\mathrm{d}}$ values for these sediments confirms this. Thus, the difference in Figure 1.3a between the apparent $\mathrm{K}_{\mathrm{a}}$ (open diamonds) and the $\mathrm{K}_{\mathrm{d}}$ (solid diamonds) for each sediment is primarily due to degradation because sorption was fast, relative to the degradation half-lives derived for these soils from the $2 \mathrm{~h}$ experiments (Table 1.2).

CL-20 sorption by these sediments after $2 \mathrm{~h}$ is small, with $\mathrm{K}_{\mathrm{d}}$ values ranging from 0.22 to 3.8
Table 1.2 CL-20 sorption, abiotic degradation, $2 \mathrm{~h}$.

\begin{tabular}{lcc}
$\begin{array}{l}\text { Sediment/ } \\
\text { Mineral }\end{array}$ & $\begin{array}{c}\mathbf{K}_{\mathbf{d}} \\
\left(\mathbf{c m}^{\mathbf{s}} \mathbf{g}^{-1} \mathbf{)}\right.\end{array}$ & $\begin{array}{c}\text { degradation } \\
\text { half-life } \\
\mathbf{( h )}\end{array}$ \\
\hline Norborne C & $0.52 \pm 0.28$ & $5.0 \pm 1.6$ \\
Kenoma Bt1 & $1.23 \pm 0.37$ & $>1000$ \\
Ocala C4 & -- & $<0.2$ \\
Westmoreland A1 & $3.43 \pm 0.34$ & $5.5 \pm 3.9$ \\
Cloudland AP & 1.98 & 3.5 \\
Burbank & $1.92 \pm 0.43$ & $13 \pm 13$ \\
China Lake & $0.25 \pm 0.08$ & $600 \pm 170$ \\
silica sand & 0.013 & 45 \\
Fe(OH) $)_{3}$-sand & $0.13 \pm 0.10$ & $6.1 \pm 3.5$ \\
magnetite & 0.93 & 12.6 \\
Al $\mathrm{O}_{3}$ & 0.62 & $n o n e$ \\
kaolinite & 0.32 & 17.8 \\
hectorite & 1.4 & $<0.2$ \\
montmorillonite & 1.5 & 0.44 \\
illite & 1.21 & 3.4 \\
biotite & 0.85 & 3.3 \\
\hline
\end{tabular}



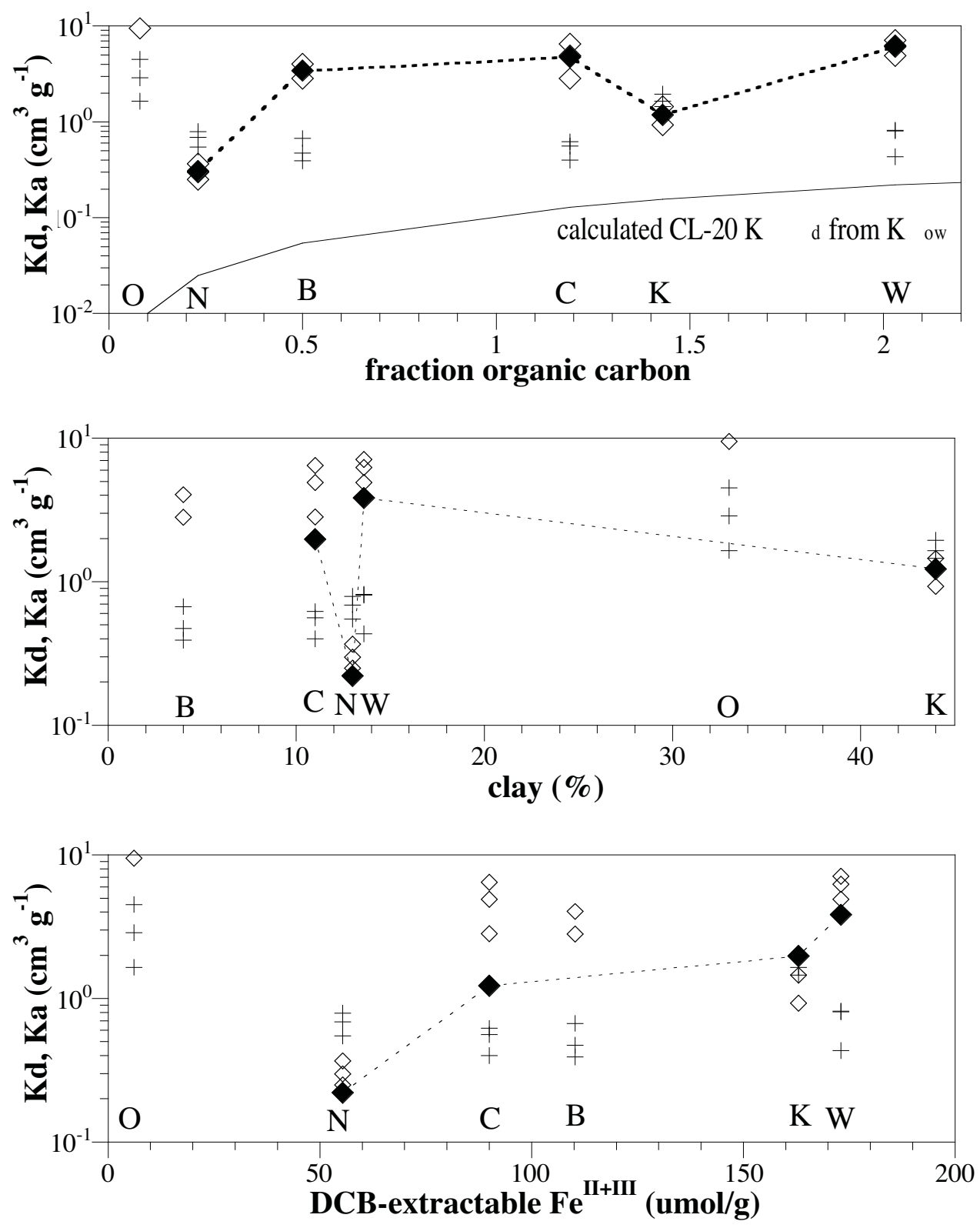

Figure 1.3. Correlation of CL-20 and RDX sorption (Kd) and CL-20 sorption and degradation $(\mathrm{Ka})$ on natural sediments with: a) fraction organic matter, b) clay content, and c) DCB-extractable iron content. Letters refer to soil name.

$\mathrm{cm}^{3} \mathrm{~g}^{-1}$. These $\mathrm{K}_{\mathrm{d}} \mathrm{s}$ were correlated $\left(\mathrm{R}^{2}=0.90\right)$ with $\mathrm{f}_{\mathrm{oc}}$ (Figure 1.3a, solid diamonds), but was about an order of magnitude greater than estimated from the CL-20 octanol-water partition coefficient, $\mathrm{K}_{\mathrm{ow}}$, and the carbon-reference model (Figure 1.3a, line). To a first approximation, the carbon-reference model (Karickhoff 1984) assumes that sorption of hydrophobic compounds with water solubilities $<10^{-3} \mathrm{M}$ is dominated by the organic carbon (humin-kerogen) in sediments and soils and that the sorption $\mathrm{K}_{\mathrm{d}}$ varies linearly with the $\mathrm{f}_{\mathrm{oc}}$ in soils or sediments. That is, $\mathrm{K}_{\mathrm{d}}=\mathrm{K}_{\mathrm{oc}} \mathrm{f}_{\mathrm{oc}}$, where $\mathrm{K}_{\mathrm{oc}}$ is the carbon-referenced partition coefficient and is constant for a wide range of soils or sediments for which $\mathrm{f}_{\mathrm{oc}}>0.001$. The empirical linear regression, $\log \mathrm{K}_{\mathrm{oc}}=$ 
$0.82 \log \mathrm{K}_{\mathrm{ow}}+0.14$, derived for a wide class of neutral organic compounds (Karickhoff 1979), was used here, along with the measured value of $\mathrm{K}_{\mathrm{ow}}\left(\log \mathrm{K}_{\mathrm{ow}}=1.92\right.$; Monteil-Rivera et al. 2004 ), to estimate a value of $\mathrm{K}_{\mathrm{oc}}=51.8$. The line in Figure 1.3a used this value. This underprediction of the $K_{d}$ vs. $f_{o c}$ indicates that most (>90\%) of the CL-20 sorption on these sediments is dominated by mineral surfaces and not by organic carbon. This is consistent with hypothesis (ii), namely that CL-20 sorption will not be controlled by sediment organic carbon. While CL20 sorption after $2 \mathrm{~h}$ was not related to the total clay fraction in sediments (Figure 1.3b), measured $K_{d}$ values for individual clays (Table 1.2) varied from $0.32 \mathrm{~cm}^{3} \mathrm{~g}^{-1}$ (kaolinite) to 0.85 $\mathrm{cm}^{3} \mathrm{~g}^{-1}$ (biotite) to $1.2 \mathrm{~cm}^{3} \mathrm{~g}^{-1}$ (illite), indicating a preference for specific clays. Sorption $\mathrm{K}_{\mathrm{d}} \mathrm{S}$ after $2 \mathrm{~h}$ showed a linear dependence on the mass of DCB-extractable $\mathrm{Fe}\left(\mathrm{R}^{2}=0.81\right.$, Figure 1.3c). Although this data is limited, this may suggest that DCB-extractable Fe (i.e., iron oxides) is a key CL-20 sorption phase. For these $2 \mathrm{~h}$ sediment experiments, no relationships were observed between $\mathrm{K}_{d}$ and extractable $\mathrm{Fe}^{\mathrm{II}}$, or between CL-20 degradation and either total clay, DCB-extractable Fe or extractable $\mathrm{Fe}^{\mathrm{II}}$.

For the $24 \mathrm{~h}$ experiments, CL-20 sorption (with degradation) was not related to sediment $\mathrm{f}_{\mathrm{oc}}$ (Figure 1.3a, open diamonds), total clay fraction (Figure 1.3b), or DCB-extractable Fe (Figure $1.3 \mathrm{c}$ ). The CL-20 abiotic degradation varied considerably among sediments, accounting for $<5 \%$ of the mass for the Kenoma sediment after $24 \mathrm{~h}$ and for $100 \%$ of the mass for the Ocala sediment after $2 \mathrm{~h}$. Although these results show significant degradation with some sediments, the general properties of total clay or iron content are insufficient for prediction of abiotic degradation. Therefore, additional CL-20 degradation experiments were conducted with individual clay and mineral phases (next section).

RDX was sorbed to the same sediments in separate $24 \mathrm{~h}$ experiments (Table 1.3, Figure 1.3). No RDX degradation was observed with these oxic soils. This is in agreement with the observations of Singh et al. (1998) for a Sharpsburg montmorillontic surface soil. RDX sorption by these sediments showed no dependence on sediment $f_{\text {oc }}$, sediment clay fraction, or DCB-extractable Fe (Figure 1.3). Sorption of explosives such as CL-20 and RDX is small as shown by the low $\mathrm{K}_{\mathrm{a}}$ and $\mathrm{K}_{\mathrm{d}}$ values in Tables 1.2 and 1.3 for these sediments and by $\mathrm{K}_{\mathrm{d}}$ values of 1.2 (RDX) and 5.8 (TETRYL) on montmorillonite (Haderlein et al. 1996) and 0.97 (RDX) on a Sharpsburg montmorillontic surface soil (Singh et al.1998).

Because CL-20 sorption appears to be dominated by mineral phases, sorption isotherms were measured to determine whether site limitations occur with increased surface loading of CL-20. Such site limitations would

Table 1.3 CL-20 sorption and degradation by minerals, $24 \mathrm{~h}$.

\begin{tabular}{|c|c|c|c|}
\hline Mineral & $\begin{array}{c}\text { CL-20 apparent } \\
K_{\mathrm{a}}\left(\mathrm{cm}^{3} \mathrm{~g}^{-1}\right)\end{array}$ & $\begin{array}{c}\text { Deg. } \\
\text { extent }\end{array}$ & 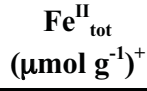 \\
\hline \multicolumn{4}{|l|}{$\overline{\text { Oxides }}$} \\
\hline Silica (accusand) & 0.013 & $\mathrm{~S}$ & 0 \\
\hline Sand (\#70, 1\%biotite) & 0.65 & M & 182 \\
\hline Goethite coated sand & 0.022 & $\mathrm{~N}$ & 0 \\
\hline Ferrihydrite coated sand & 0.002 & $\mathrm{~S}$ & 0 \\
\hline Magnetite & 1.6 & $\mathrm{~L}$ & 22,000 \\
\hline$\alpha-\mathrm{Fe}(\mathrm{OH})_{3}$ & 0.52 & $\mathrm{~S}$ & 0 \\
\hline $\mathrm{Al}_{2} \mathrm{O}_{3}$ & 0.62 & $\mathrm{~S}$ & 0 \\
\hline $\mathrm{MnO} 2$ & 1.5 & $\mathrm{~L}$ & 0 \\
\hline Albite & 0.07 & $\mathrm{~S}$ & 0 \\
\hline \multicolumn{4}{|l|}{ Phyllosilicates } \\
\hline 1:1 Kaolinite & 0.04 & $\mathrm{~S}$ & 0 \\
\hline 2:1 Vermiculite & 0.8 & $\mathrm{~S}$ & 0 \\
\hline \multicolumn{4}{|l|}{$2: 1$ Smectite clay group } \\
\hline Hectorite & $>10$ & $\mathrm{~L}$ & 0.2 \\
\hline Montmorillonite & 15 & $\mathrm{~L}$ & 2300 \\
\hline Nontronite & $>10$ & $\mathrm{~L}$ & 141,000 \\
\hline \multicolumn{4}{|l|}{ 2:1 Mica group } \\
\hline Illite & 6.9 & $\mathrm{~L}$ & 19,000 \\
\hline Muscovite & 0.43 & M & 0 \\
\hline Biotite & 1.5 & $\mathrm{~L}$ & 16,700 \\
\hline Chlorite & 1.8 & $\mathrm{~L}$ & 24,400 \\
\hline
\end{tabular}


be indicated by an isotherm whose slope decreased significantly with increasing CL20 concentrations. Sorption isotherms were measured for the Norborne sediment and an aluminum oxide using $2 \mathrm{~h}$ contact time and a sediment/water ratio of $0.5 \mathrm{~g}$ $\mathrm{mL}^{-1}$. The sorption isotherms were nonlinear with Freundlich isotherm exponents of 0.83 and 0.62 for the Norborne and the oxide, respectively (Figure 1.4a). This is consistent with adsorption of CL-20 to specific surface sites in agreement with hypothesis (ii), rather than purely hydrophobic organic compound linear partitioning to organic matter (Holmen and Gschwend 1997; Winget et al. 2000), or linear partitioning to mineral surfaces (McCarty et al. 1981). Over the CL-20 concentration range examined ( 0.05 to $\left.3.6 \mathrm{mg} \mathrm{L}^{-1}\right)$, surface site limitations were not observed.

Because CL-20 is not ionizable, no $\mathrm{pH}$ dependence was expected. However, a pH edge experiment was conducted for the Norborne sediment using $2 \mathrm{~h}$ contact time, a sediment/water ratio of $0.5 \mathrm{~g} \mathrm{~mL}^{-1}$ and a CL-20 concentration of $3 \mathrm{mg} \mathrm{L}^{-1}$. Relatively little $\mathrm{pH}$ dependence was observed, as expected (Figure 1.4b; Szecsody et al. 2004a).

\section{Task 1.2. Abiotic CL-20 Degradation}

Oxic batch experiments were conducted for $24 \mathrm{~h}$ with aqueous CL-20 and separate oxides and phyllosilicates to determine which phases could either react with CL-20 or catalyze its reaction with other species. In general, the 2:1 clays, the ferrous-iron containing minerals (e.g., magnetite and biotite), and $\mathrm{MnO}_{2}$ exhibited the greatest amount of abiotic CL-20 degradation (indicated by ' $\mathrm{L}$ ' in Table 1.3). The other minerals exhibited significantly less degradation (e.g., silica, $\left.\alpha-\mathrm{Fe}(\mathrm{OH})_{3}\right)$. The amount of CL-20 abiotic degradation observed for the clays varies significantly (Table 1.3), with only minor (S) degradation for kaolinite and nearly complete (L) degradation for montmorillonite (Table 1.3), at the solid/solution ratio of $0.5 \mathrm{~g} \mathrm{~cm}^{-3}$. Degradation was greatest for the smectite group of clays, with total degradation of the CL-20 occurring for hectorite and nontronite. The $2 \mathrm{~h}$ sorption-degradation experiments (Table 1.2) were in relatively good agreement with these $24 \mathrm{~h}$ results (Table 1.3). Qualitatively, the amount of abiotic CL-20 degradation was generally greater for those solids with higher ferrous iron content (Table 1.3) in agreement with hypothesis (iii), but minerals with little or no ferrous iron (i.e., hectorite and $\mathrm{MnO}_{2}$ ) could also degrade CL-20.

The moles of CL-20 lost from solution in contact with sediment and mineral phases for $24 \mathrm{~h}$ showed a distinct increase with the mass of solid present (Figure 1.5a). This is most pronounced 
for the 2:1 phyllosilicates (montmorillonite, hectorite, biotite, illite), $\mathrm{MnO}_{2}$, and magnetite. Kaolinite and nontronite exhibited the smallest loss and the slowest CL-20 degradation, which for kaolinite is consistent with Table 1.3. This suggests that the degradation capacity under oxic conditions is limited and increases with the quantity of sediment or mineral phase in $24 \mathrm{~h}$ batch experiments. Therefore, while the total clay content of sediments is not related to abiotic CL-20 degradation, as noted in the previous section, degradation is related to the mass of specific clays (Figure 1.5a).

The role that reactive ferrous iron may have in contributing to the degradation capacity was examined (hypothesis (iii)). For oxic conditions, no relationship was observed between CL-20 degradation and the $0.5 \mathrm{M} \mathrm{HCl}-$ extractable ferrous iron content of sediments (Figure 1.5b, open squares) or discrete mineral phases (Table 1.3 and Figure $1.5 \mathrm{~b}$, crosses). However, when the Norborne sediment was chemically reduced with sodium dithionite, CL-20 loss was clearly dependent upon the mass of sediment and thus the mass of extractable $\mathrm{Fe}^{\mathrm{II}}$ present (Figure 1.5, solid squares). This chemical reduction technique (Szecsody et al. 2000a,

a)

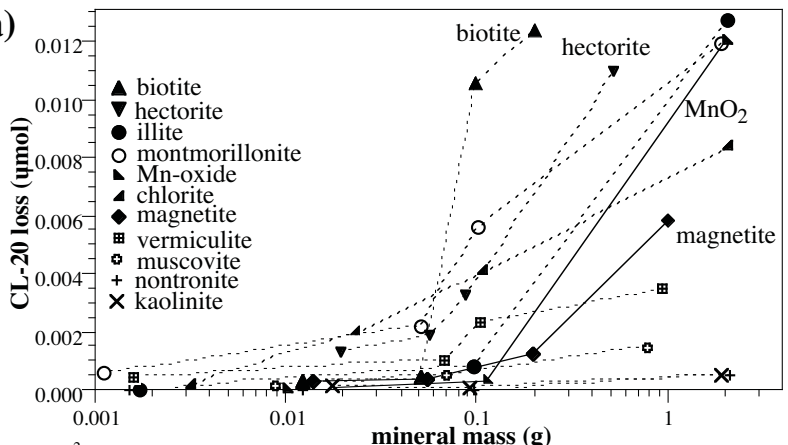

b)

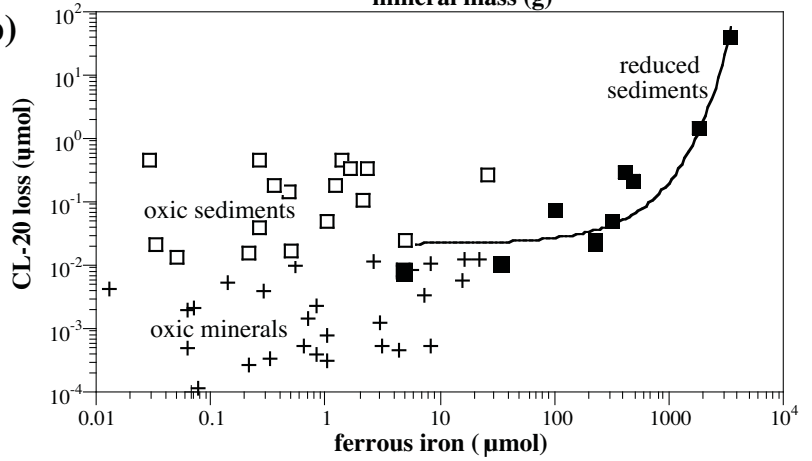

Figure 1.5. CL-20 degradation after $24 \mathrm{~h}$ as a function of: a) mineral mass and b) mass of $0.5 \mathrm{M}$ $\mathrm{HCl}$-extractable ferrous iron. Data points represent several solid to solution ratios for individual solids. 2004b, Vermeul et al., 2000) dissolves and reduces iron oxides, and can reduce some of the structural ferric iron in clays. Other explosives (RDX, TNT) are rapidly reduced abiotically with iron-reducing surfaces (Szecsody et al. 2001; Hofstetter et al. 1999; Klausen et al. 1995). Considering the oxic minerals and sediments and the reduced Norborne sediments together (Figure 1.5b), CL-20 degradation begins to show a strong exponential dependence on $\mathrm{Fe}^{\mathrm{II}}\left(\mathrm{R}^{2}=0.98\right)$ as the molar ratio of Fe ${ }^{\mathrm{II}}$ to CL-20 exceeds 1:100. This apparent threshold may simply reflect the decrease in Eh that accommodates the persistence of higher concentrations of reduced iron in this reduced sediment.

While degradation is greatly enhanced under reducing conditions, the main reactive phases promoting CL-20 abiotic degradation under oxic conditions were specific 2:1 phyllosilicates. Significant degradation occurred in the presence of these 2:1 clays and micas (Tables 1.2 and 1.3), even though no apparent dependence on $\mathrm{Fe}^{\mathrm{II}}$ was observed (Figure $1.5 \mathrm{~b}$, crosses). In addition, some oxic sediments promoted significant CL-20 degradation within $2 \mathrm{~h}$ (Cloudland and Westmoreland, Table 1.2). Slow but steady degradation occurred for some oxic minerals that contained no ferrous iron or clay (albite, goethite, aluminum oxide, ferrihydrite-coated sand, silica sand). Acid-cleaned silica sand exhibited some CL-20 degradation within $24 \mathrm{~h}$ and was consistent with the slow CL-20 degradation with glass (Figure 2). This slow degradation along with the fact that CL-20 was rapidly degraded by hectorite, which contained only trace amounts 
of ferrous iron, suggests that other reactive constituents may promote CL-20 degradation. Clearly, $\mathrm{Fe}^{\mathrm{II}}$ is not the only reactive species promoting abiotic CL-20 degradation, as suggested by hypothesis (iii).

\section{Interactions of CL-20 with Laboratory Materials, Light, and Aqueous Constituents.} When exposed to sunlight, CL-20 was found to be photoreactive, which is consistent with the findings of others (Hawari 2003). Eighty percent of the CL-20 was degraded by sunlight after $200 \mathrm{~h}$ in either glass or milky-colored HDPE vials (Figure 1.6a). In comparison, no mass loss was observed for milky- and brown-colored HDPE vials exposed to fluorescent light after $200 \mathrm{~h}$. Additional laboratory materials were tested under fluorescent light over a period of $400 \mathrm{~h}$ (Figure 1.6b). Between $70 \%$ and $100 \%$ of the CL-20 in contact with delrin, glass, and tygon was degraded or sorbed, while only $5 \%$ mass loss occurred with polycarbonate. Over the same time frame, CL-20 was nonreactive when pumped continuously through $\mathrm{PEEK}^{\mathrm{TM}}$ tubing and columns or when contained in the following materials: FEP and PFA Teflon, Tefzel (ETFE), HDPE, LDPE, polystyrene, and polypropylene. The use of Teflon filters with CL-20 did not result in any mass loss, although 100\% loss occurred with nylon filters. A methanol flush of the nylon filter recovered $80 \%$ of the CL- 20 , so the nylon was mainly sorbing CL-20, although there was some $(20 \%)$ degradation. Based on these results, all experiments were conducted using Teflon filters and HDPE, LDPE, polypropylene, PEEK $^{\mathrm{TM}}$ or other non-
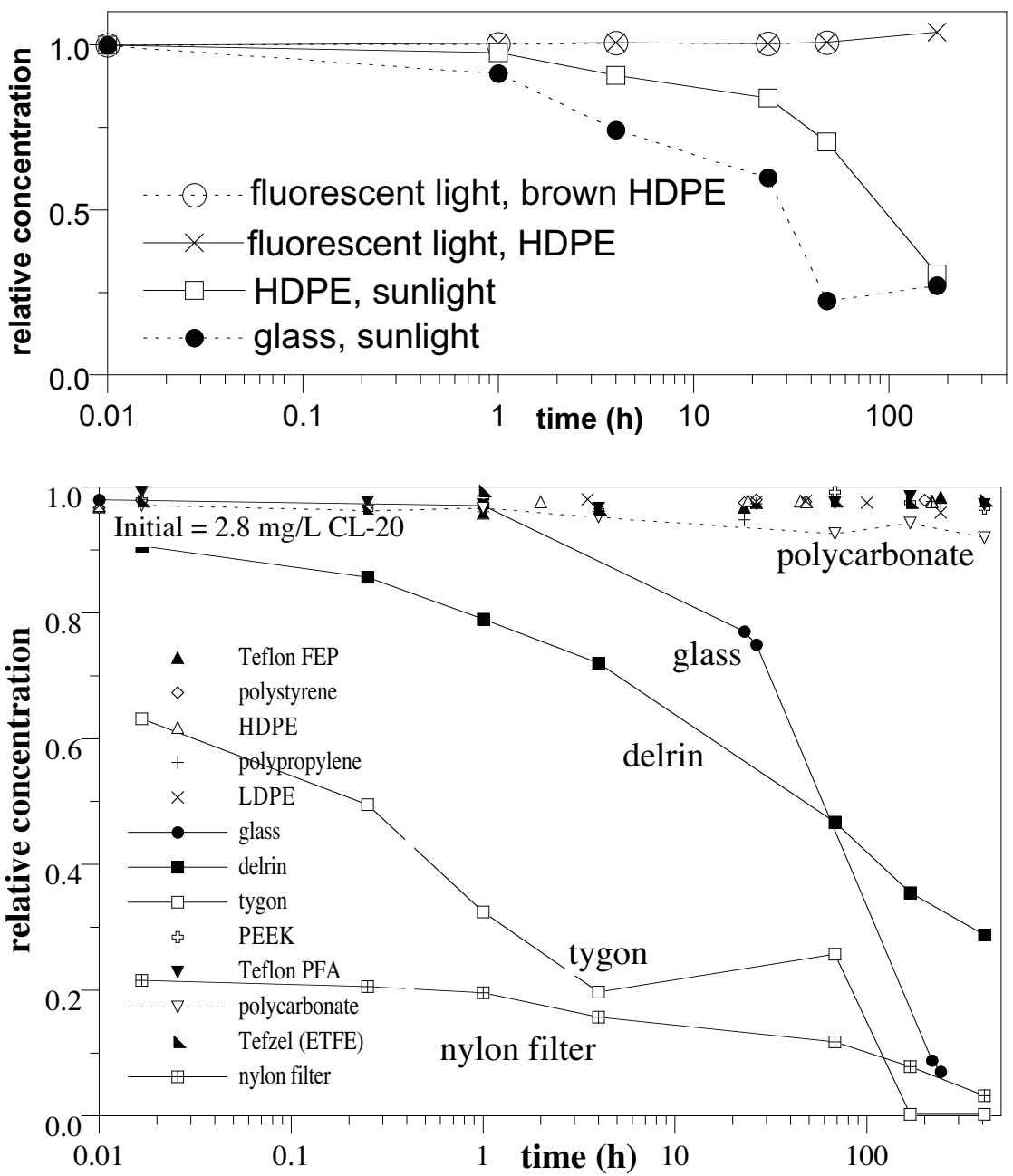

Figure 1.6 Degradation of Cl-20 over time in contact with light and laboratory materials.

reactive materials, with no exposure to sunlight.

The addition of $\mathrm{CaCl}_{2}$, PIPES and $\mathrm{HgCl}_{2}$ to CL-20 solutions, at concentrations specified above, did not influence CL-20 interactions with solids or its degradation. Neither the presence 
of these constituents nor the presence in solution of methanol, which was used to extract CL-20 and its degradation products from solids, produced any HPLC peaks or modified the signature CL-20 peak or degradation product peaks.

Therefore, these added constituents had no affect on CL-20 interactions or analysis.

\section{CL-20 Degradation Pathway.}

Molecular modeling predicted that the roof (attic) C-C bond of the CL-20 molecule should cleave (Qasim et al. 2004, 2005), based on calculations on the strained cage structure (Figure 1.7). Intermediates and end products were first identified by Hawari (2003, 2004) that include compounds 3 and 5 (Figure 1.7), glyoxal, nitrate, nitrite, $\mathrm{N}_{2} \mathrm{O}, \mathrm{NH}_{3}$, and formate. The current proposed CL-20 degradation pathway for hydrolysis and zero valent iron (J. Hawari) involves removal of two $\mathrm{NO} 2$ groups before the roof $\mathrm{C}-\mathrm{C}$ bond breaks (as shown in Figure 1.7). Additional molecular modeling in 2004 indicates that two intermediates may form (compounds 6 and 7 , Figure 1.7), which are not part of the hydrolysis/zvi pathway proposed by Jalal Hawari. The presence of an aromatic compound during degradation with reduced sediment and hydrolysis is consistent with these proposed compounds, although tentative LC/MS-MS identification (see Task 1.3) has not positively identified these compounds.

Carbon and nitrogen mass balance is useful in demonstrating that only part of the pathway has been identified, and further identification is needed (Table 1.4, Figure 1.8). The CL-20 molecule contains six carbon atoms and 12 nitrogen atoms. Carbon mass balance of intermediates that include formate, glyoxal, and glycolic acid (Figure 1.8a) in the $<100 \mathrm{~h}$ time frame account for $<50 \%$ of the $\mathrm{C}$ mass, although mass balance is actually better than shown since high molecular weight degradation products (Figure 1.7, compounds 3, 5, 6, 7) are not quantified. 
Beyond $100 \mathrm{~h}$, the carbon mass balance increases to a high of $79 \%$ (by $1600 \mathrm{~h}$ ), as the main product is carbon dioxide. All of these mineralization experiments involve microbes. The remaining carbon mass may be incorporated into the microbial biomass or form other compounds such as methane. It is clear that abiotic processes alone, while rapidly degrading CL-20 10x to $100 x$ faster than biotic processes, result in only minor $(<30 \%)$ of the CL-20 carbon mass becoming smaller molecular weight (identified) $\mathrm{C}$ products. Microbial degradation of the initial CL-20 or intermediates is apparently necessary to break some of the bonds.

Nitrogen mass balance is slightly better than carbon, with as high as $80 \%$ mass balance at $>100 \mathrm{~h}$ for reduced sediment and hydrolysis (Figure 1.2). As with carbon mass balance, low $\mathrm{N}$ balance at early times $(<100 \mathrm{~h})$ do not include high molecular weight degradation products, which contain considerable $\mathrm{N}$ mass. Low molecular weight $\mathrm{N}$ products include nitrate, nitrite and nitrous oxide. Further work here using a combination of the uniformly N-15-labeled CL-20 and ring only N15-labeled CL-20 molecules could help distinguish that early time scale $\mathrm{N}$ products may be from nitroso ring intermediates.

Ultraviolet analysis of CL-20 hydrolysis (Figure 1.9) suggests that aromatic compounds are forming (i.e., products noted in Figure 1.7). For the UV absorbance spectra, degraded results were compared to standards. Standards (OH-MeOH in purple; CL-20 in Methanol in yellow;
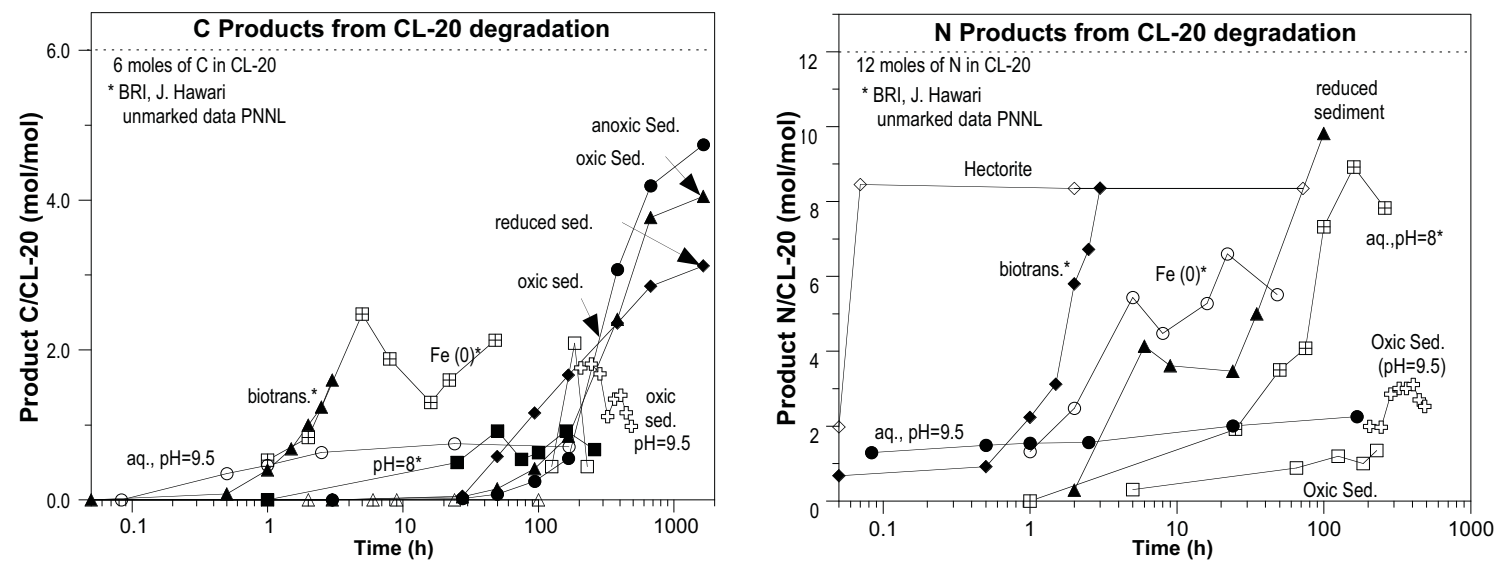

Figure 1.8. CL-20 degradation products showing: a) carbon and b) nitrogen mass balance 
Methanol only in light blue) contain no aromatic pi electrons, so there is no absorbance in the 300 to $400 \mathrm{~nm}$ range. When CL-20 was reacted in aqueous solution at $\mathrm{pH} 10$ (Figure 1.9, dark blue), there is major absorbance in the 300 to $400 \mathrm{~nm}$ range, indicating the presence of one or more aromatic compounds. This type of analysis is one piece of evidence suggestive of a degradation pathway, and will be conducted with CL-20 reacted with sediments.

In addition, preliminary FTIR analysis indicates a decrease in C-C bonding (Figure 1.23, Task 1.3), which also suggests that cage breakdown is occurring. These results are suggestive

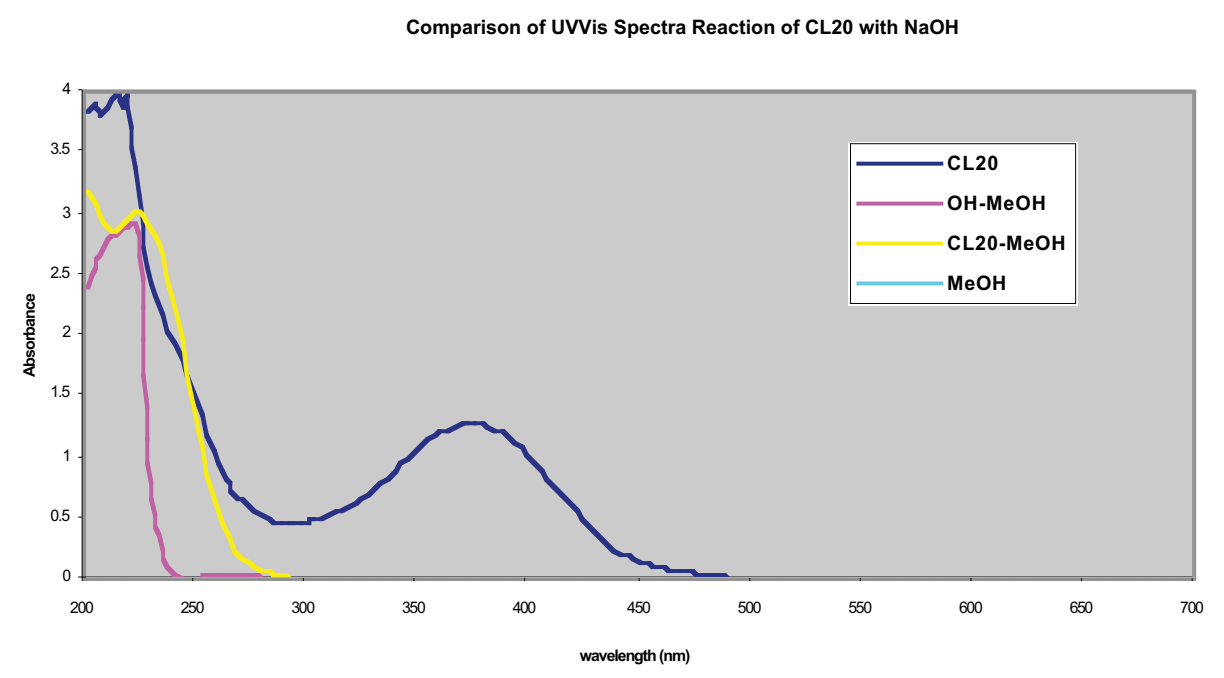

Figure 1.9. UV-Vis absorbance spectra of CL-20 degradation by alkaline hydrolysis

(but not proof) that CL-20 degradation (pathway in Figure 1.7) is occurring. The initial high molecular weight compounds that are formed are not volatile, so cannot be detected by GC-MS.

CL-20 Degradation Mechanisms. A summary of the types of sediment/water systems in which CL-20 degradation is observed is presented below.

CL-20 Degradation in Aqueous Solution. CL-20 degrades in contact with specific mineral (and man-made) surfaces, as well as under specific aqueous conditions. The existence of aqueous degradation is significant, as some compounds are only degraded in contact with solid surfaces that can delocalize electrons. CL-20 is well documented to degrade at a $\mathrm{pH}>8.5$ in aqueous solution (Figure 1.10). Degradation products include 3.5 moles of nitrite and 0.75 moles of formate (carbon) per mole of CL-20 degraded after $200 \mathrm{~h}$ (Figure 1.8).

CL-20 is also degraded in sunlight, but not in laboratory fluorescent light (Fig. 1.6a). CL-20 is unstable in aqueous solution at a temperature $>35^{\circ} \mathrm{C}$ at neutral $\mathrm{pH}$ (Figure 1.10), although is apparently stable to $90^{\circ} \mathrm{C}$ in highly acidic conditions (Nielson et al. 1989, 1994). 
PZZB: CL-20 Aqueous Stability vs. temp. $40^{\circ} \mathrm{C}$

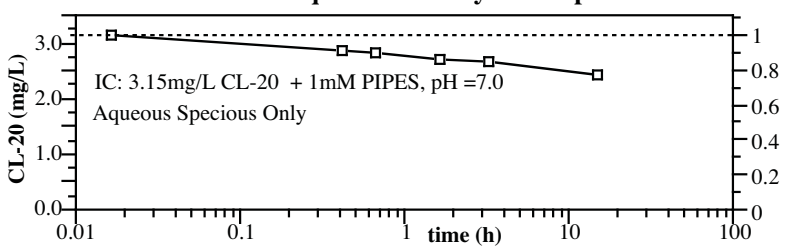

PZC-St. : CL-20 and RDX Aqueous Stability at $65{ }^{\circ} \mathrm{C}$

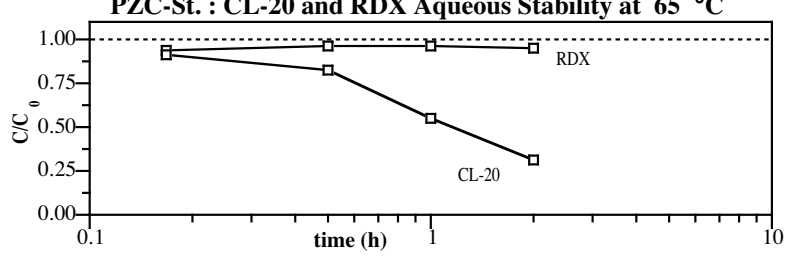

Figure 1.10. CL-20 degradation rate in aqueous solution at elevated temperature

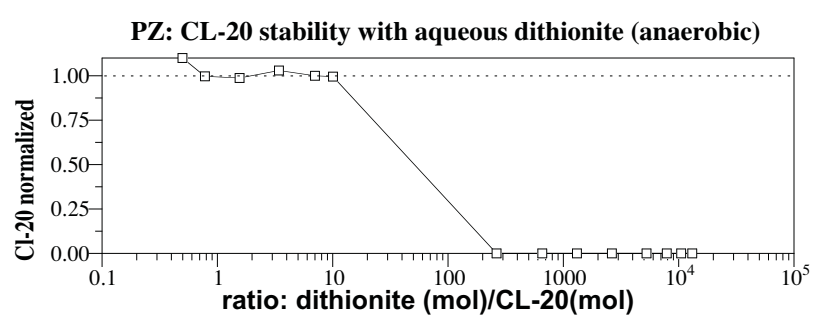

Figure 1.11. CL-20 degradation at differing Eh in aqueous solution with reduced sulfur species
For comparison, RDX was shown to be stable at elevated temperatures. These results indicate that water is key to at least one degradation pathway. These results also promote a hypothesis that CL-20 in sediments at low water content will likely degrade more slowly (see Results Section 3, coupled processes). Elevated temperature can be used as a remediation technology that has minimal damage to a subsurface environment. Of great interest in this study is the stability of CL-20 in differing redox environments. In aqueous solution at $\mathrm{pH}$ 7.00, CL-20 is stable in the absence of

oxygen and is stable under slightly reducing conditions (Figure 1.11) of an aqueous reduced sulfur species (sodium dithionite). However, if the Eh $<-0.0$, CL-20 is rapidly degraded in aqueous solution.

The aqueous degradation of CL-20 in the presence of reduced sulfur species (differing mixtures, so differing Eh, Figure 1.11) has implications for other aqueous and solid phase reduction systems. These results suggest that aqueous ferrous iron (Eh $=+0.6$ volts) is an insufficient reduced system to promote CL-20. This was confirmed experimentally (Appendix D, experiment PZL-A), where 143x more aqueous ferrous iron relative to CL-20 (pH 7.00) did not cause any degradation over 300 hours. However, ferrous iron adsorbed to ferric oxides (and clays) are more reducing (Eh -0.1 volts for ferrous iron on amorphous iron oxide), so CL-20 degradation is likely promoted on by ferrous iron adsorbed to some of the iron oxides, which is described in detail in the next section.

CL-20 Degradation by Adsorbed Fe(II). Ferrous iron was adsorbed to a series of surfaces which have differing ability to delocalize electrons, and thus may have a differing ability to degrade CL-20, if electron transfer is involved in CL-20 degradation. CL-20 was degraded by adsorbed Fe(II) on iron oxides, so behaved as predicted. With Fe(II) adsorbed on sand (Appendix E, experiment PZM-A), no degradation occurred, as predicted (the iron oxide surface is needed). When ferrous iron was adsorbed to a pure alpha $\mathrm{Fe}(\mathrm{OH})_{3}, \mathrm{CL}-20$ was not degraded (Figure 1.12a), but when ferrous iron was adsorbed to an amorphous iron oxide (2-line ferrihydrite; Figure 1.12b), CL-20 was degraded at a slow rate. The actual rate is not that significant in these batch experiments, as actual sediments can have orders of magnitude greater iron, so would degrade CL-20 at a proportionally greater rate. The mass loss in this experiment was confirmed to be degraded CL-20, because a methanol extraction was used to recover any adsorbed and degraded CL-20 mass from the surface. 
The final surface used for these adsorbed ferrous iron studies was hectorite, which is a 2:1 smectite clay that contains no structural ferrous or ferric iron. Hectorite by itself will degrade CL-20. The rate of CL-20 degradation by pure hectorite was about $0.00205 / \mathrm{h}$ (half life $337 \mathrm{~h}$ ) in the batch study with a clay/water ratio of $0.2 \mathrm{~g} / \mathrm{cm}^{3}$. Using a clay/water ratio of $0.005 \mathrm{~g} / \mathrm{cm}^{3}$ (i.e., 40x less, Figure 1.13), CL-20 was degraded at a rate of $6.76 / \mathrm{h}$ (half life $6.2 \mathrm{~min}$ ), or $3200 \mathrm{x}$ faster than the pure clay. At the equivalent clay/water ratio, it is estimated that the rate is $10^{5}$ times faster.

Therefore, adsorbed ferrous iron on iron oxides and most importantly on 2:1 smectite clays appears to promote rapid CL-20 degradation. This implies (but does not prove) that electron transfer is occurring, and also suggests that the ability of a surface to delocalize electrons significantly increases the rate of CL-20 degradation.

\section{CL-20 Degradation by Minerals} without Iron. A series of seven experiments were conducted with differing minerals that contain no structural iron or any adsorbed ferrous
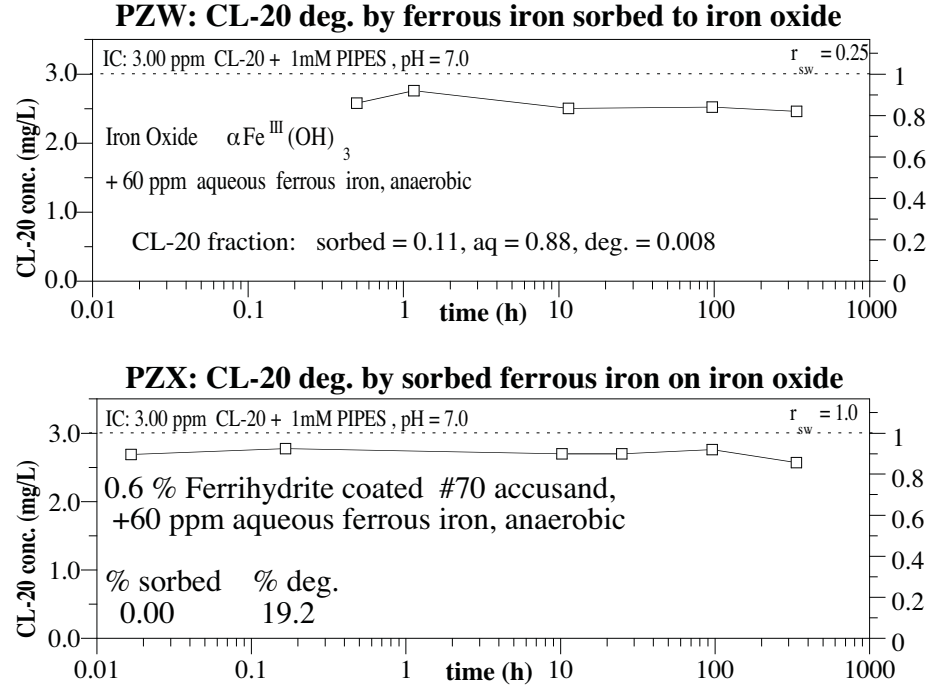

Figure 1.12. CL-20 degradation by adsorbed Fe(II) on differing iron oxides: a ) $\alpha \mathrm{Fe}(\mathrm{OH}) 3$ and b) semicrystalline ferrihydrite.

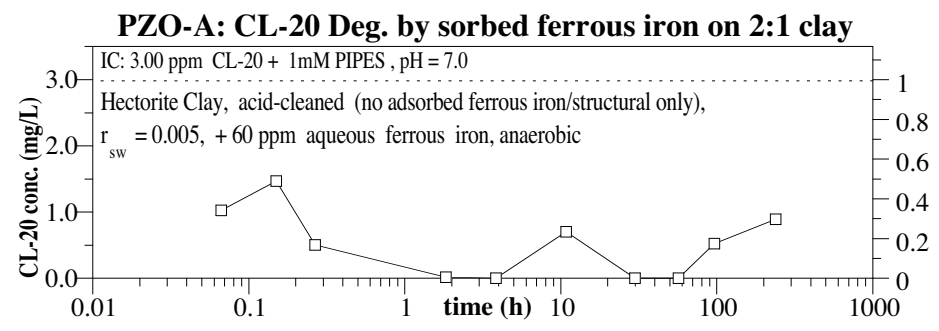

Figure 1.13. CL-20 degradation by adsorbed Fe(II) on hectorite. The degradation rate observed was $6.76 / \mathrm{h}$ (half-life of $6.2 \mathrm{~min}$ ). iron (Appendix B). All surfaces were acid cleaned $(0.5 \mathrm{M} \mathrm{HCl})$ to remove any adsorbed species (which includes ferrous iron and $\mathrm{Mn}^{2+}$ ). The purpose of these studies is to determine which surfaces will promote CL-20 degradation by some mechanism in the complete absence of ferrous iron, which primarily includes clays. As expected, sand and high surface area silica either do not degrade CL-20 or degrade at sufficiently slow rates $(<0.2 \%$ in $100 \mathrm{~h}$, Appendix B, experiments PZH-A, PZH-B).

Two additional minerals were investigated - a 1:1 clay (kaolinite) and a 2:1 smectite clay (hectorite). CL-20 was degraded by kaolinite, but only at extremely slow rates (2000 to $3600 \mathrm{~h}$ half-life, Figure 1.14a). In contrast, acid-cleaned hectorite with no adsorbed ferrous iron under exactly the same conditions (clay/water ratio, $\mathrm{pH}, \mathrm{CL}-20$ concentration) degraded CL-20 about 10 times faster (Figure 1.14b), with $330 \mathrm{~h}$ degradation half-life. In a separate degradation experiment with hectorite, ion chromatography was used to determine the concentrations of the 

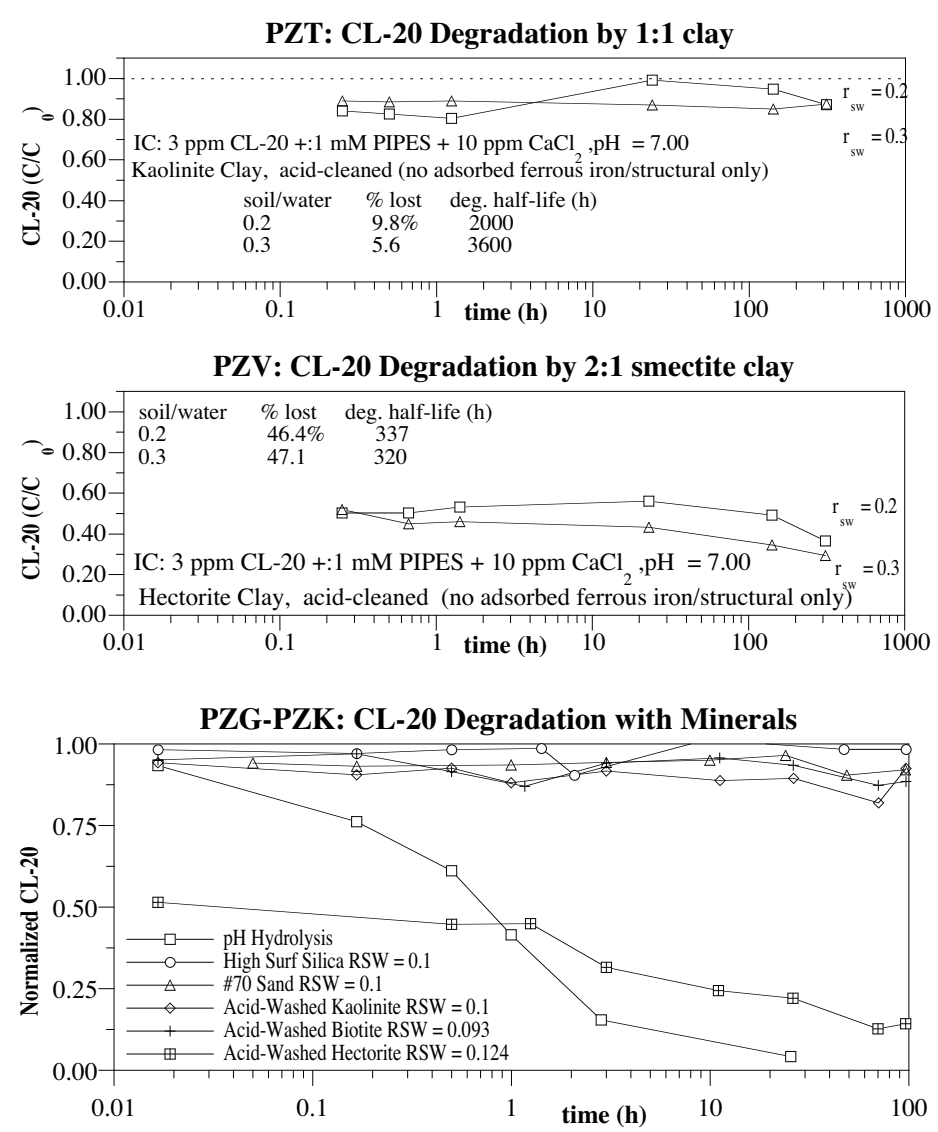

Figure 1.14. CL-20 degradation by: a) kaolinite and b) hectorite, both with methanol extraction indicating sorbed and degraded fractions, and c) different minerals low molecular weight degradation products (Appendix D, experiment PZK). Similar low molecular weight degradation products were produced (nitrite, nitrate, formate) as with adsorbed $\mathrm{Fe}(\mathrm{II})$ on iron oxides and with reduced sediments.

\section{CL-20 Degradation by Minerals} with Only Structural Iron. In order to determine whether structural iron can degrade CL-20, specific minerals were chosen that contain differing amounts of structural ferrous iron. These were biotite ( $3 \%$ iron), montmorillonite (3\% iron) and nontronite (22\% iron), in Figure 1.14c. Both montmorillonite and nontronite are 2:1 smectite clays, but with differing iron substitution in the structure. All minerals were cleaned with $0.5 \mathrm{~mol} / \mathrm{L}$ $\mathrm{HCl}$ to remove any adsorbed cations, then $\mathrm{pH}$ equilibrated for several days to $\mathrm{pH}$ 7.00. The montmorillonite and nontronite (which contain a mixture of ferrous and ferric iron) were then chemically reduced for $120 \mathrm{~h}$ with $0.09 \mathrm{~mol} / \mathrm{L}$ sodium dithionite. After three successive washings, these clays were again $\mathrm{pH}$ equilibrated for several days to $\mathrm{pH}$ 7.00.

The acid-cleaned biotite degraded CL-20 at a slow rate (1400 to $2100 \mathrm{~h}$ half-life; Figure 1.14c). Experiments conducted with biotite in 2002 showed much faster degradation rates, so may have been caused by adsorbed ferrous iron on the biotite surface. Montmorillonite at a clay/water ratio of $0.05 \mathrm{~g} / \mathrm{cm}^{3}$ showed little CL-20 degradation, although methanol extractions were not conducted. This may be indicative that adsorbed iron on the montmorillonite was promoting degradation. Nontronite at the same clay/water ratio showed rapid CL-20 degradation (Appendix F, experiment PZR-A), with a degradation half-life of $0.55 \mathrm{~h}$. The conclusion from this series of experiments is that structural iron is generally not as available for promoting CL-20 degradation for most minerals, although CL-20 degradation can be rapid for a 2:1 smectite clay (nontronite).

CL-20 Degradation by Minerals with Structural and Adsorbed Fe(II). Montmorillonite at an extremely small clay/water ratio $\left(0.00025 \mathrm{~g} / \mathrm{cm}^{3}\right)$, which was chemically reduced and additionally contained adsorbed ferrous iron, showed CL-20 degradation $(3.3 \%$ in $400 \mathrm{~h})$. In addition, the methanol extraction of the clay showed no adsorbed CL-20 (i.e., once on the surface, it was apparently degraded; Appendix G, experiment PZZ). At a sediment water ratio of $0.2 \mathrm{~g} / \mathrm{cm}^{3}$, a $10 \mathrm{~h}$ degradation half-life is estimated. This is significantly faster than the same 2:1 
smectite clay with no structural or adsorbed iron (hectorite; Figure $12 \mathrm{~b} ; 330 \mathrm{~h}$ half-life), but slower than hectorite with adsorbed iron (Figure 1.14c, 6.2 min half-life).

The overall conclusion of CL-20 degradation promoted by $2: 1$ smectite clays is that it does occur at relatively rapid rates, but ferrous iron adsorbed on clays or iron oxides promote CL-20 degradation much more rapidly.

CL-20 Degradation Rate in Sediments - Temperature Studies. The degradation rate of CL-20 in oxic and reduced sediments (Cloudland and Norborne sediments) is being determined at different temperature in order to calculate the activation energy of the degradation reaction. While it is well established that CL-20 degrades faster in reducing conditions, if the activation energy is the same in reduced and oxic sediments, it suggests that the degradation mechanism is the same. The RDX activation energy in reduced sediments will also be investigated.

Seven batch experiments are completed in this study (Appendix C), although additional experiments are planned at temperatures ranging from $4^{\circ} \mathrm{C}$ to $65^{\circ} \mathrm{C}$. Because CL-20 degrades in warm water $\left(>40^{\circ} \mathrm{C}\right)$, the sediment degradation rate in the elevated temperature experiments will be the CL-20 loss rate in excess of that in aqueous solution (Figure 1.15). Results demonstrate that, as expected the degradation rate increases with temperature. Additional experiments were conducted in order to make comparisons between sediments, compounds (CL-20 vs. RDX) and differing redox conditions (described in a later section on activation energy). Since CL-20 degrades in aqueous solution at elevated temperature, that influence needs to be separated from the degradation by sediments, so it is likely that only data $<45^{\circ} \mathrm{C}$ will be used to calculate the activation energy.

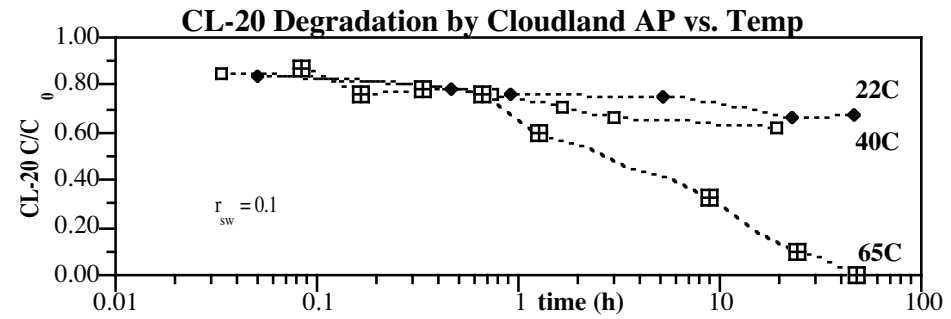

Figure 1.15. CL-20 degradation by the oxic Cloudland AP sediment at different temperature

CL-20 Degradation by Reduced Sediments and Zero Valent Iron. The reduced system of real interest is in natural sediments, which contains multiple mineral phases. In previous sections, pure mineral phases without and with adsorbed iron were examined in experiments in order to compare with results in natural sediments. Chemically (or biologically) reduced sediments have one or more ferrous iron phases present, which promotes CL-20 degradation at a rapid rate. At $\mathrm{pH} 10, \mathrm{CL}-20$ is degraded by alkaline hydrolysis rapidly (1.8 min half-life, Figure 1.2), and so reduced sediment at $\mathrm{pH} 10$ is degraded by both ferrous iron and alkaline hydrolysis. Three experiments at different sediment/water ratios (Appendix B, experiment PZP) showed very rapid CL-20 degradation $(<1 \%$ mass remaining after 1.0 minute, so half-life $<9$ seconds).

The rate of sediment reduction of CL-20 was also quantified at $\mathrm{pH} 8.0$ (three experiments) and at pH 6.0 (one experiment; all in Appendix F), where rates were sufficiently slow (i.e., minutes to hours) that they could be modeled. In a reduced natural sediment environment, the rate of CL-20 removal from the system was 4 times faster ( $62 \mathrm{~h}$ half-life) at $\mathrm{pH} 6.0$ than at 
$\mathrm{pH} 8.0$ (Figure 1.16a). In comparison, the rate of removal of CL-20 by zero valent iron at the same solid phase/water ratio (i.e., $0.1 \mathrm{~g} / \mathrm{cm}^{3}$ ) was $0.3 \mathrm{~h}$, or at least 200 times faster than the reduced sediment.

Actual comparison of reaction rates of surfaces requires conversion of the experiment-specific rates (expressed as first-order half-lives in this case) to intrinsic reaction rates by normalizing to: a) total surface area or b) moles of reactive surface phase. Normalizing to surface area is a viable technique if pure mineral phases are being used, as the only physical characteristic that differs is surface area. However, normalizing reaction rates by surface area for sediments is not entirely correct, as there are multiple surface phases (each which have surface area) and only some are redox reactive. For
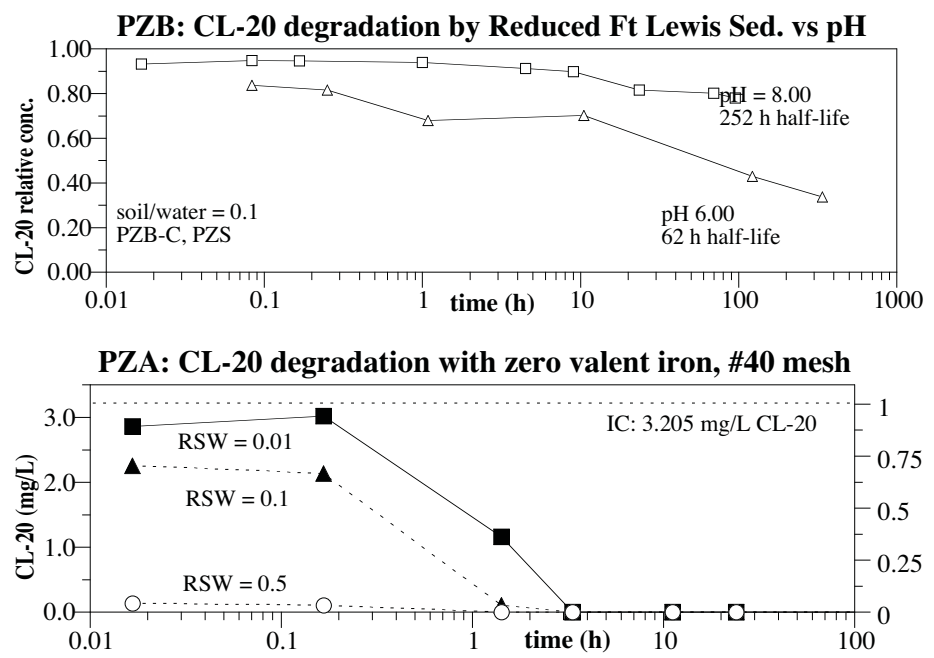

Figure 1.16. CL-20 degradation rate in a) reduced Ft. I ewis sediment and h) zern valent iron the purpose of remediation, CL-20 degradation rates for reduced sediments were calculated for field soil/water ratio $\left(5.0 \mathrm{~g} / \mathrm{cm}^{3}\right)$ to compare with zero valent iron at the same solid/water ratio (Table 1.5).

Table 1.5 Comparison of zero valent iron and reduced sediment CL-20 degradation rates.

\begin{tabular}{lcccc} 
surface & $\begin{array}{c}\text { first-order } \\
\text { half-life }(\mathbf{h})\end{array}$ & $\begin{array}{c}\text { soil/water } \\
(\mathbf{g} / \mathbf{m L})\end{array}$ & $\begin{array}{c}\text { estimated half-life }(\mathbf{h}) \\
\text { soil/water }=\mathbf{5 . 0}\end{array}$ & $\begin{array}{c}\text { half-life normalized } \\
\text { to } \mathbf{1 0 0 \%} \text { reactive phase }\end{array}$ \\
\hline $\begin{array}{l}\text { zero valent iron } \\
(\# 40 \text { mesh, Aldrich) }\end{array}$ & $0.75 \mathrm{~h}$ & 0.01 & 0.0015 & $0.1 \mathrm{~min}$ \\
$\begin{array}{c}\text { reduced Ft Lewis sediment } \\
(160 \text { umol/g ferrous iron) }\end{array}$ & $62-252 \mathrm{~h}$ & 0.1 & $1.2-5.0$ & $0.29 \mathrm{~min}$ \\
\hline
\end{tabular}

Results of the batch studies (Figure 1.16a) with sediments estimate a CL-20 field degradation rate of 1.2 to $5.0 \mathrm{~h}$, whereas an actual reduced sediment column degradation rate (lab experiment, Section 4) had a degradation rate of 2.2 minutes (32 times faster), and zero valent iron (half life $0.1 \mathrm{~min}$ ) was about 800 times faster. Because the reduced sediment contains only $0.4 \%$ ferrous iron by weight, normalizing to $100 \%$ reactive phase shows that the zero valent iron and adsorbed ferrous iron on the natural sediment have nearly the same reactivity. Therefore, knowing the mass of ferrous iron in the natural sediment may be used to predict the CL-20 degradation rate.

Summary: CL-20 Degradation by Minerals and Sediments. CL-20 exhibits very slow or no degradation in the following systems: a) minerals with no adsorbed or structural ferrous iron, b) minerals including 2:1 clays with structural ferrous iron, c) in an aqueous solution of ferrous iron, and d) silica or 1:1 clay with adsorbed ferrous iron. CL-20 exhibits rapid degradation (minutes to hours) in the following systems: a) hot aqueous solution, b) alkaline $(\mathrm{pH}>9)$ 
conditions, c) adsorbed ferrous iron on iron oxides (minutes), d) adsorbed ferrous iron on 2:1 smectite clays (minutes), and e) 2:1 smectite clay hectorite (no structural or adsorbed ferrous iron).

These findings indicate that CL-20 will rapidly degrade with an electron transfer (i.e., ferrous iron donor is needed), but also a surface is needed to promote rapid electron transfer (i.e., aqueous ferrous iron will not degrade, but ferrous iron adsorbed on iron oxides/clays will degrade CL-20). However, other systems will degrade CL-20 in minutes to hours that are not electron transfer reactions (i.e., hot aqueous solution, hectorite, various plastics, glass, Table 1.6), so there may a separate pathway that when CL-20 is adsorbed on the surface will promote the strained cage opening (possibly), which would only produce some high molecular weight intermediate. Both hot

Table 1.6. CL-20 degradation factors

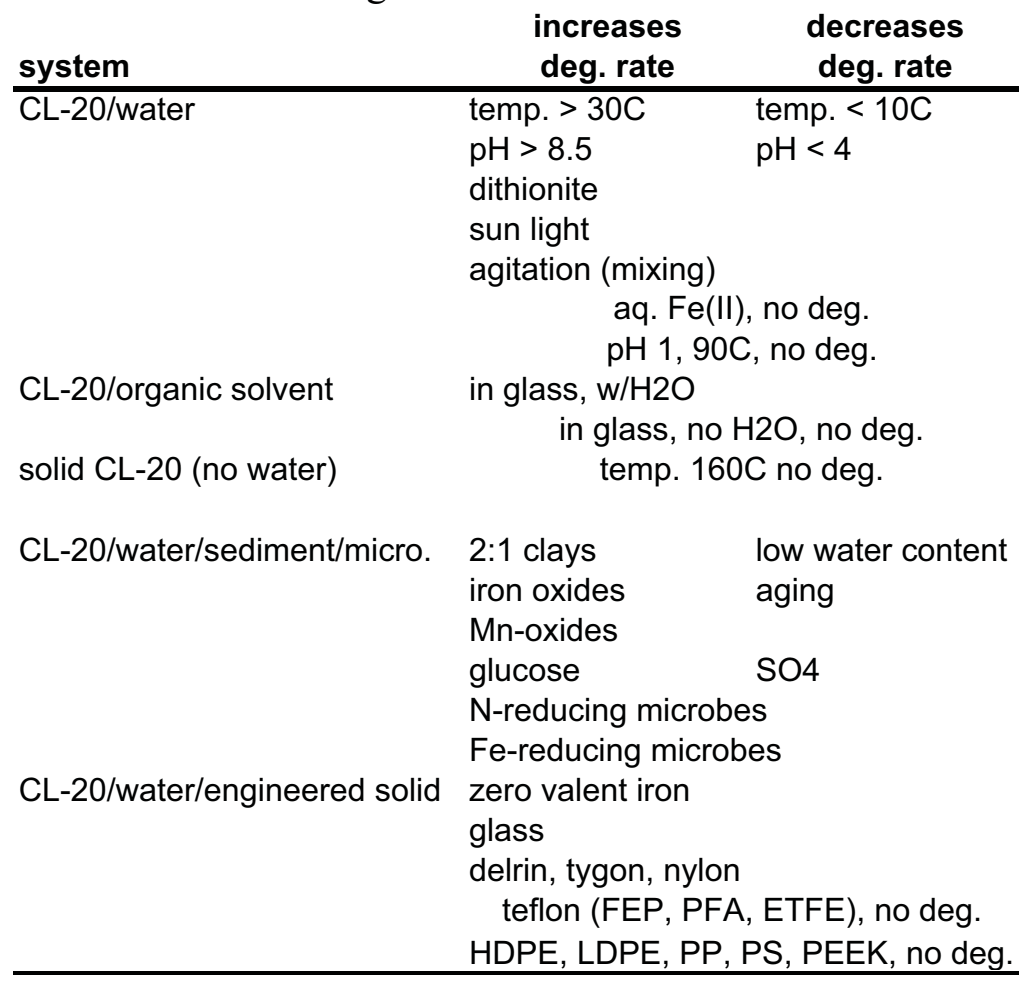

aqueous solution and alkaline hydrolysis for $1000 \mathrm{~h}$ did not produce $\mathrm{CO}_{2}$ (Figure 1.17). The apparent counts in the $\mathrm{CO}_{2}$ trap for the hot aqueous solution sample was not $\mathrm{CO}_{2}$ (i.e., an unidentified intermediate). Activation energies for various abiotic pathways were quantified to determine if more than one CL-20 degradation pathway exists (next section).

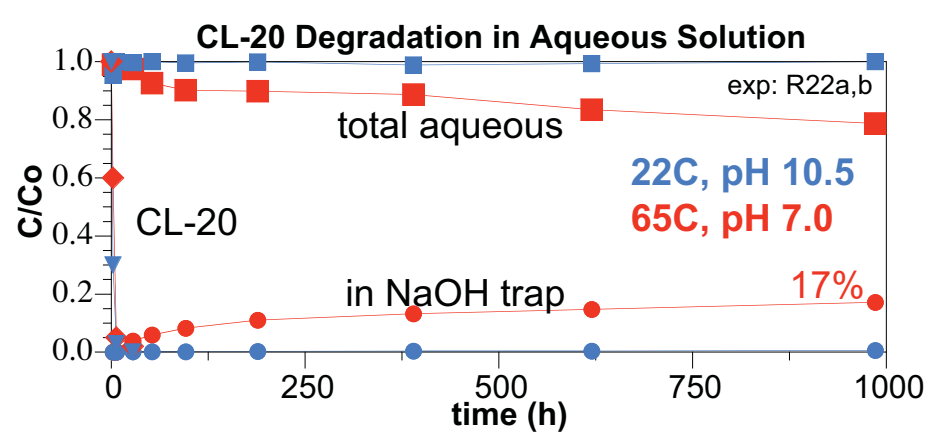

Figure 1.17. CL-20 degradation in hot aqueous solution (red) and by alkaline hydrolysis

CL-20 Abiotic Degradation and Activation Energies. The rate of CL-20 degradation in systems with electron donors and other systems without electron donors was quantified at different temperature to determine the activation energy. The electron donor systems include: 

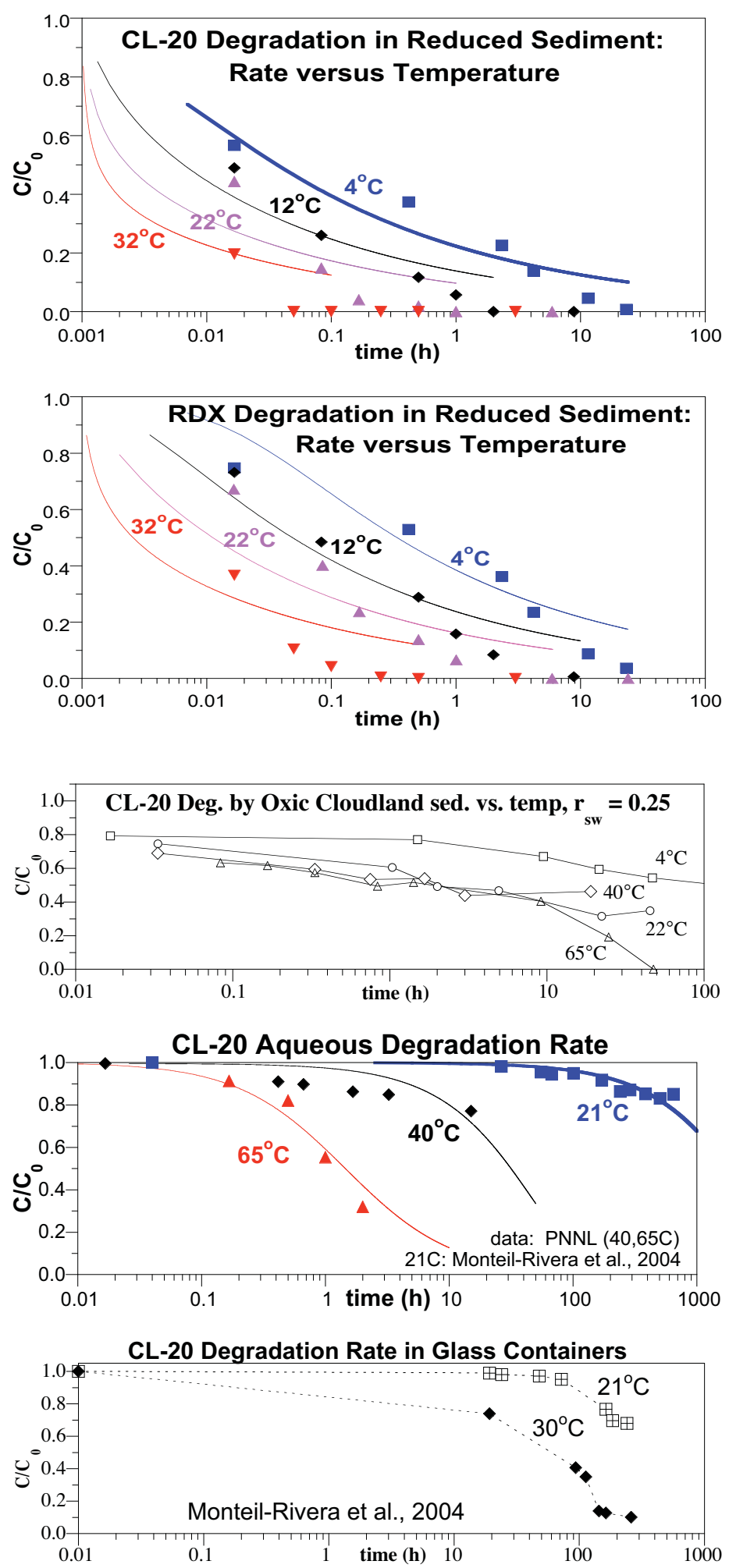

Figure 1.18. CL-20/RDX degradation versus temperature (data from this study or other referenced). a) aqueous dithionite and b) reduced sediment (both CL-20 and RDX). The systems with no electron donor included: a) hot aqueous solution, b) glass, c) oxic sediment, and d) hectorite (a 2:1 smectite clay with no structural or adsorbed ferrous iron).

The raw data (Figure 1.18 and Appendix E) was modeled to determine an actual rate. The hypothesized reaction pathway (Figure 1.19) indicates for electron transfer, the first loss of an $\mathrm{NO}_{2}$ group involves two electrons (or four for the loss of two $\mathrm{NO}_{2}$ groups); one mole of CL-20 needs four moles of electrons for these first two steps. A simple first-order reaction (i.e., CL-20 with a single electron transfer reaction) did not fit the experimental data (Figure 1.19). A fourth or fifth order reaction was needed to fit the slope of the experimental data, but this was four moles of CL-20 to one mole of electron donor. If the electron transfer reaction was the rate limiting step, then the reaction order would be 1 with respect to CL-20 and 4 with respect to the electron donor (such as ferrous iron). Clearly with a reaction order significantly different from this, something else (i.e., a catalyst) is controlling the reaction order, and not the electron transfer step.

Fitting all of the experimental data at different temperature, as shown in Figure 1.20, indicates that in all cases, CL-20 degradation was 
more rapid at elevated temperature. A plot of the absolute temperature versus the rate coefficient provides the activation energy. Two shown for reduced sediment and CL-20/RDX have nearly the same activation energy, indicating the first transformation reaction appears to have a similar rate-limiting process (Table 1.7).

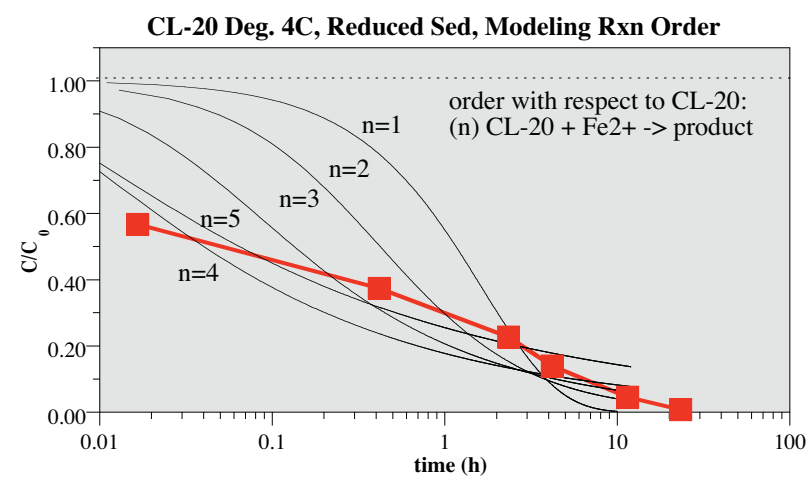

Figure 1.19. CL-20 degradation at $4{ }^{\circ} \mathrm{C}$ by reduced sediment and model simulations with differing reaction order
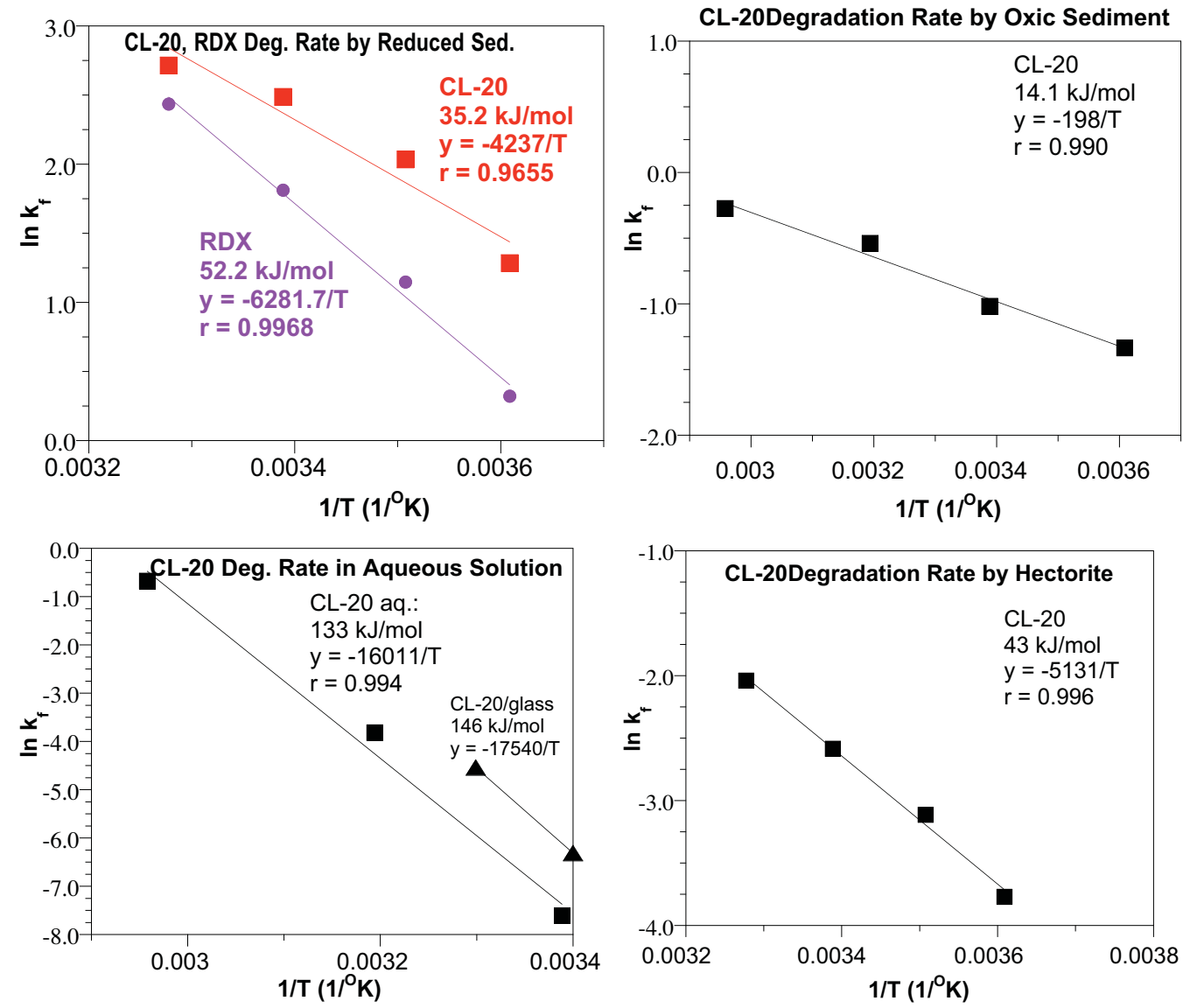

Figure 1.20. Activation energies of CL-20 and RDX degradation. 
Table 1.7. Activation energies for degradation of CL-20 and RDX activation

\begin{tabular}{llc} 
Compound & Degradation System & Energy $(\mathrm{kJ} / \mathrm{mol})$ \\
\hline CL-20 & hot aqueous solution & 133 \\
& glass, aqueous & 146 \\
& solution & \\
& hectorite & 42.6 \\
& oxic sediment & 14.1 \\
& reduced sediment & 35.2 \\
RDX & reduced sediment & 52.2
\end{tabular}

There are low activation energies for known electron transfer reactions (reduced sediment degrading CL-20 and RDX). However, several systems have very high activation energies (132 to $146 \mathrm{~kJ} / \mathrm{mol}$ ) including the hot aqueous solution and glass with an aqueous solution. The implication here is these systems degrade CL-20 by a different transformation step (possibly) that is similar within these systems. CL-20 degradation by hectorite and "oxic" sediment (Cloudland) may be electron transfer reactions, based on the similarity in the activation energies with known electron transfer systems (reduced sediment). For comparison, reduction of vinyl chloride by zero valent iron (homogeneous electron transfer reaction) has an activation energy of $46 \mathrm{~kJ} / \mathrm{mol}$ (Deng et al., 1999). While the pure hectorite (a 2:1 smectite clay) does not contain any adsorbed or structural ferrous iron, it does contain significant $\mathrm{Mg}$ and can have other transition metal substitution in the structure. The oxic Cloudland sediment contains $11 \%$ clay (Table 1.1), so may have similar clay reactions occurring. In conclusion, there appear to be multiple degradation pathways for the initial CL-20 transformation step.

\section{Task 1.3. Analytical}

An LC/MS-MS and other analytical tools were used in identifying high molecular weight CL-20 degradation products. The CL-20 aqueous concentration is being quantified by high performance liquid chromatography, as described in the experimental methods section. An array of different analytical techniques are being used to identify CL-20 degradation products, which includes:

- LC-MS/MS electrospray (measurement of high molecular weight compounds)

- GC/MS (low molecular weight, volatile compounds)

- derivatized GC/MS (combines low molecular weight nonvolatile compounds with organic silane compounds to render the volatile)

- direct insertion MS

- ion chromatography (low molecular weight anions)

- UV/VIS

- FTIR

The LC-MS/MS electrospray ionization method (first mass spectrometer) is used to quantify the total mass of the intact compound, and the second mass spectrometer is used to identify the composition of each peak identified on the first mass spectrometer by a more conventional 
fragmentation method (Figure 1.21). The technique needed to be developed first for CL-20 before degradation products could be analyzed. RDX, TNT, and CL-20 were analyzed using electrospray ionization mass spectrometry in the negative ion mode. For RDX, the base ion was (molecular weight or "M" $+\mathrm{Cl}$ ). . The negative ion electrospray mass spectrum of CL-20 produced ions equivalent to (M-H)' at $\mathrm{m} / \mathrm{z} 437$ and an adduct ion at $\mathrm{m} / \mathrm{z} 499$ (Figure 1.22). This ion has been only tentatively identified as $\left(\mathrm{M}+\mathrm{ONO}_{2}-\mathrm{H}\right)^{--}$. This adduct ion has also been observed in the negative ion electrospray mass spectrum of nitroglycerine (Shah and Campbell, 1997).

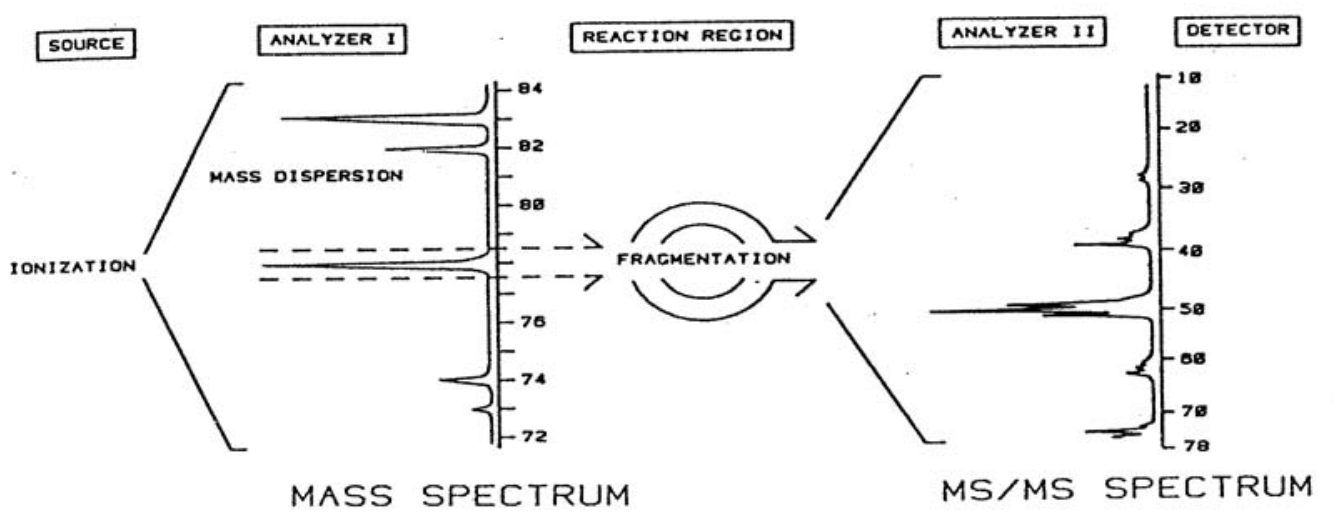

Figure 1.21. LC/MS-MS scheme

An MS/MS experiment can be described as selection of a precursor ion in MS1, directed through a collision cell or reaction region, with subsequent fragmentation detected of the precursor ion detected on MS2. This is shown schematically in Figure 1.21. In this particular case, the ionization is electrospray ionization. Ions of interest in both the 1) PZD samples and 2) base hydrolysis of CL-20 samples were analyzed using MS/MS. Table 1.8 shows the ions of interest selected MS/MS studies of the PZD samples and base hydrolysis samples and not present in the associated blanks. As an example, MS/MS studies of m/z 349 (base hydrolysis) produced fragment ions $\mathrm{m} / \mathrm{z} 330,317$, and 299. All of the MS/MS data has been collected and is presently being evaluated.

A portion of a base hydrolysis sample was lyophilized and a yellow solid was observed. The residue was then derivatized with $\mathrm{N}$-methyl-N-(t-butyldimethylsilyl)-trifluoroacetamide (MTBSTFA). This reagent reacts with hydroxyl and amine groups to form a-Si- $\left(\mathrm{CH}_{3}\right)_{2}(\mathrm{t}$-butyl) derivative. The derivatized residue was analyzed using electron ionization mass spectrometry. Three major components were observed with mass spectra indicating ions $\mathrm{m} / \mathrm{z} 291$ (odd mass 
contains odd number of nitrogens), $\mathrm{m} / \mathrm{z} 248$ (even number of nitrogens) and $\mathrm{m} / \mathrm{z} 306$. The last major component also contained a fragment ion $\mathrm{m} / \mathrm{z} 260$ (loss of 46, possibly loss of a nitro group).

Table 1.8. Electrospray MS data of CL-20/reduced Norborne sediment reaction

\begin{tabular}{lccc}
\multicolumn{1}{c}{ Sample } & $\begin{array}{c}\text { Contact Time } \\
(\mathrm{h})\end{array}$ & Positive Adduct Ions & Negative Adduct Ions \\
\hline PZD-10 & 0.3 & 159,191 & $367,383,399$ \\
PZD-11 & 3.0 & 115,145 & 150,323 \\
PZD-12 & 35.0 & 145,159 & -- \\
Base Hydrolysis & 30.0 & 333,349 & 293,309
\end{tabular}

FTIR is useful in identifying that the cage structure of the CL-20 molecule is breaking apart and some sort of compound containing aromatic rings is forming. UV-Vis spectra (Figure 1.9) clearly showed that CL-20 breakdown due to alkaline hydrolysis produced one or more compounds that contain aromatic groups. The FTIR spectra (Figure 1.23) more clearly show the formation of aromatic C-H bonds for degraded samples (Qasim et al., 2004, 2005).

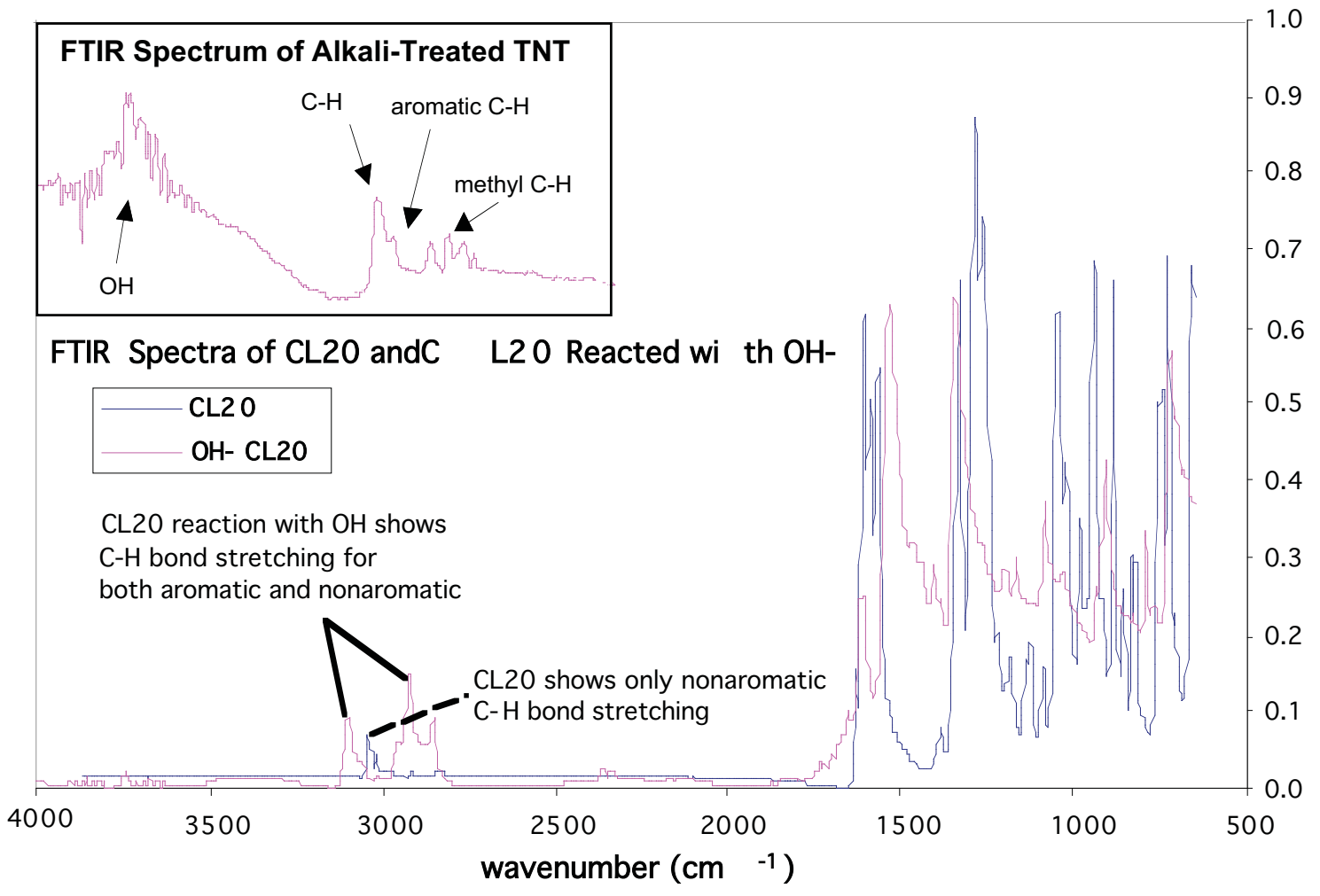

Figure 1.23. FTIR spectra of CL-20 and CL-20 reacted with OH 


\section{Task 2. Biodegradation of CL-20 and RDX}

\section{Task 2.1. Aerobic Biodegradation}

Biodegradation of CL-20 in China Lake Surface and Subsurface Soils. CL-20 (10 $\mathrm{mg} \mathrm{L}^{-1}$ ) was biodegraded in China Lake surface and subsurface soil microcosms incubated under aerobic conditions (Figure 2.1). In the surface soil, the biodegradation of CL-20 was significantly faster in soil amended with glucose than in soil without glucose (Figure 2.1A, Table 2.2). CL-20 was completely biodegraded in the glucose-amended soil after 13 days, while $60 \%$ to $65 \%$ of the CL-20 was degraded in the unamended soil after 21 days. Similarly, glucose enhanced the rate and extent of biodegradation in the subsurface soil (Figure 2.1B, Table 2.2). The rates of biodegradation were slower in the subsurface soil microcosms compared to the surface soil (Figure 2.1, Table 2.2). In the glucose-amended soil microcosms, the rate of biodegradation was 2.5 times slower in the subsurface soil (half-life $=4.2 \mathrm{~d}$ ) than in the surface soil (half-live $=1.7 \mathrm{~d}$ ). In the unamended soils, there was a two-fold difference in CL-20 biodegradation rates between the surface and subsurface China Lake soils (Table 2.2). Microbial biomass estimates of the surface and subsurface soils were similar prior to incubation at day 0 (Table 2.3). After 21 days in the presence of CL-20, an increase in biomass was observed in both surface and subsurface soil microcosms with or without glucose, suggesting that CL-20 was being used to support growth of the indigenous soil microorganisms. The microbial biomass was about 3 times higher in the surface soil microcosms $\left(1.48 \times 10^{8}\right.$ cells $\mathrm{g}^{-1}$ [wet weight]) than the subsurface soil microcosms $\left(5.31 \times 10^{7}\right.$ cells $^{-1}$ [wet weight] $)$ probably as a result of the faster biodegradation rate in the surface soil microcosms. The surface soil had slightly higher nitratenitrogen and clay contents than the subsurface soil (Table 2.1), which may have contributed to higher microbial activities in the surface soil.
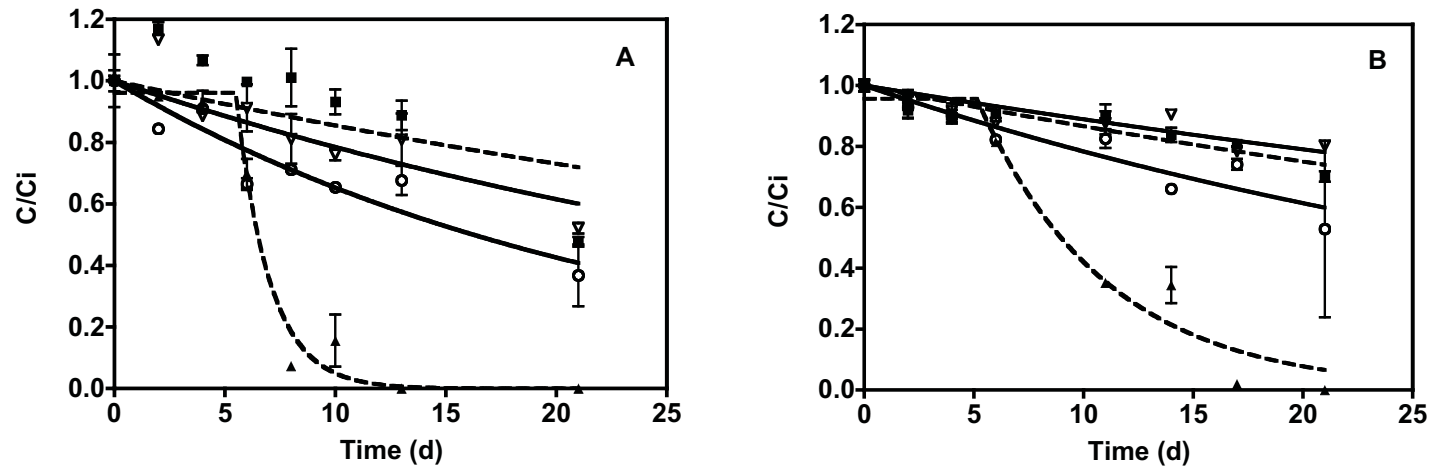

Figure 2.1. Degradation of hexanitrohexaazaisowurtzitane (CL-20) in China Lake soil microcosms incubated under aerobic conditions A) surface soil and B) subsurface soil. Symbols: Unamended soil: - - - ; Soil + glucose -- $\mathbf{\Delta - - ;}$ Soil + glucose $+\mathrm{HgCl} 2$ $-\nabla-$; Mineral salts medium + glucose -- $\mathbf{-}--$. 


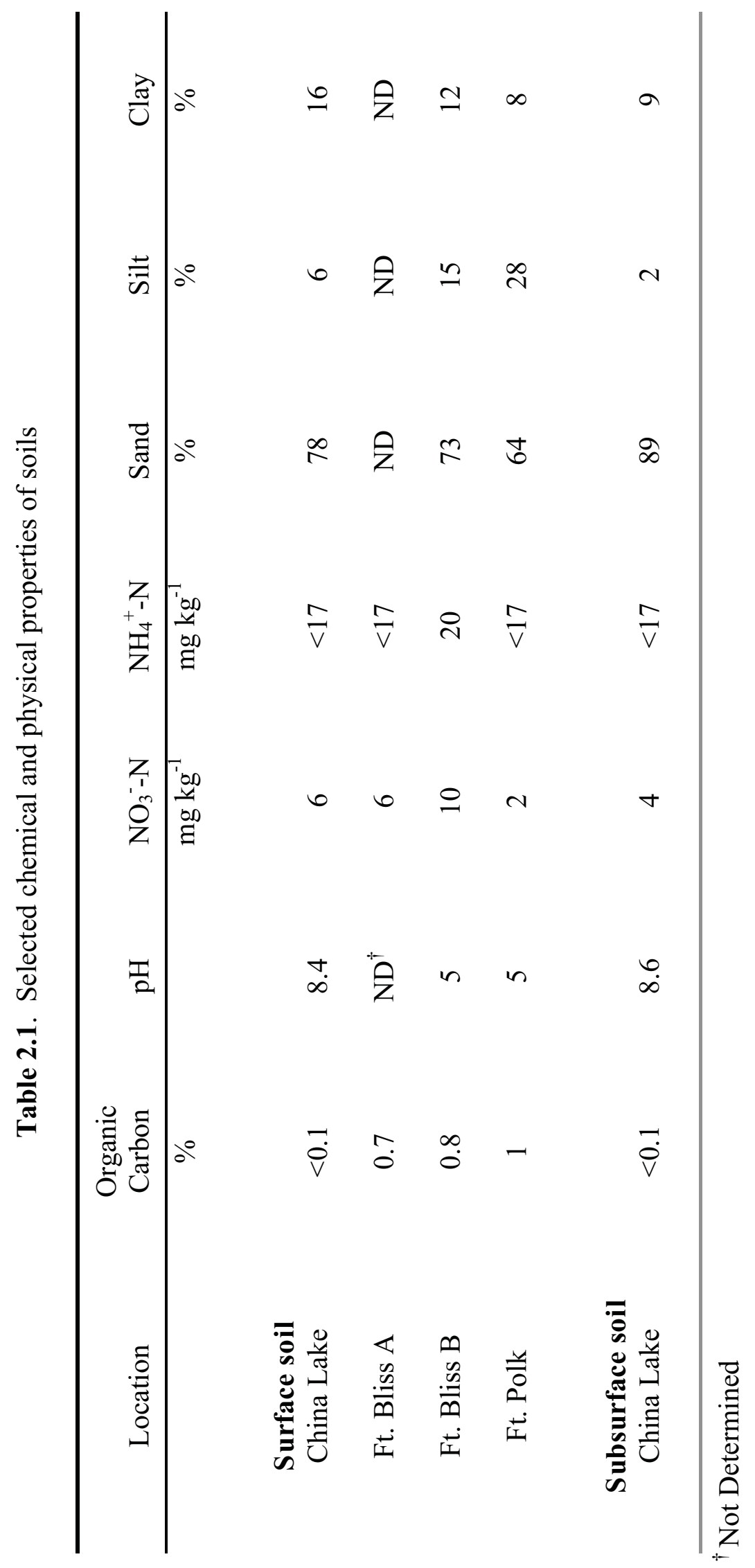




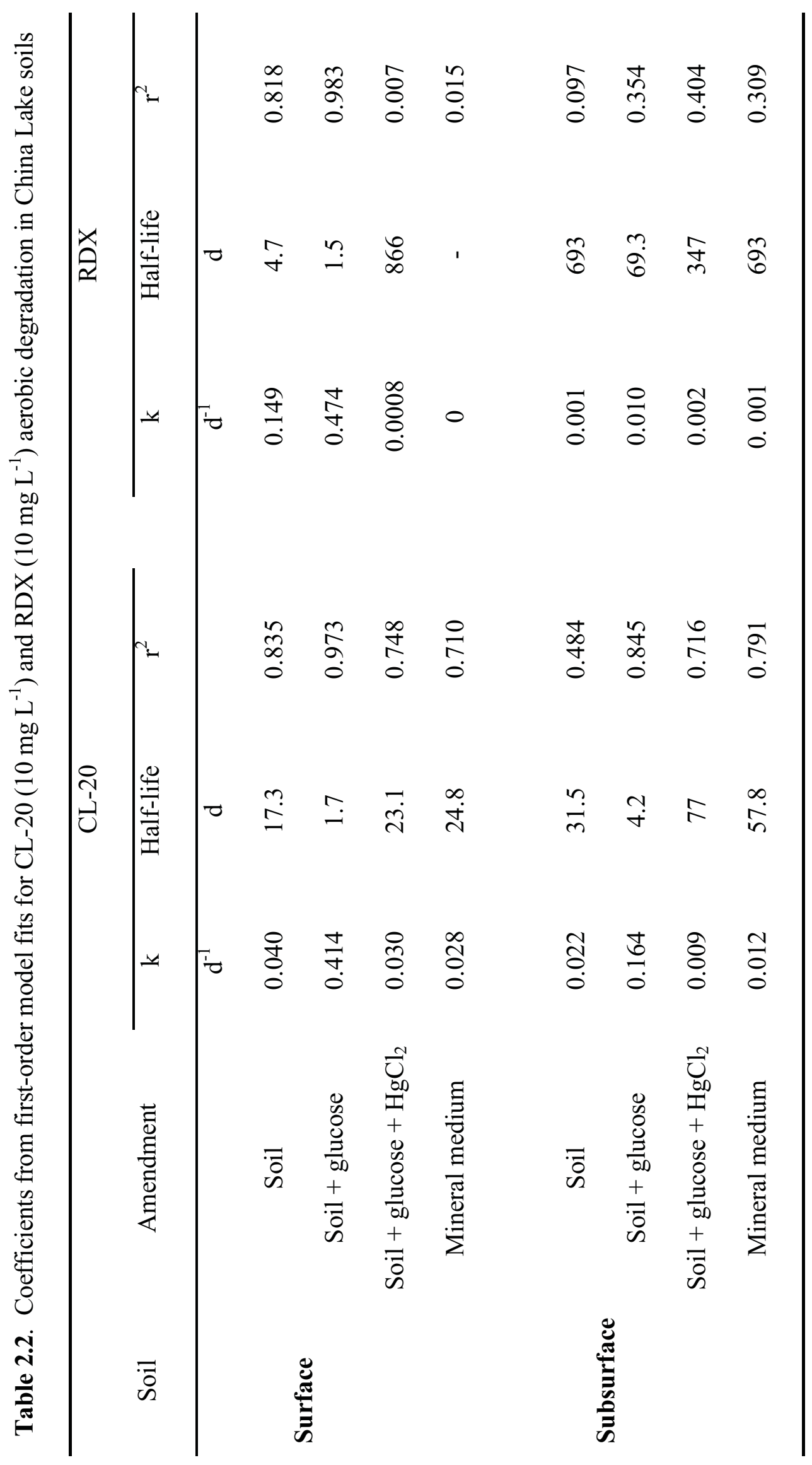




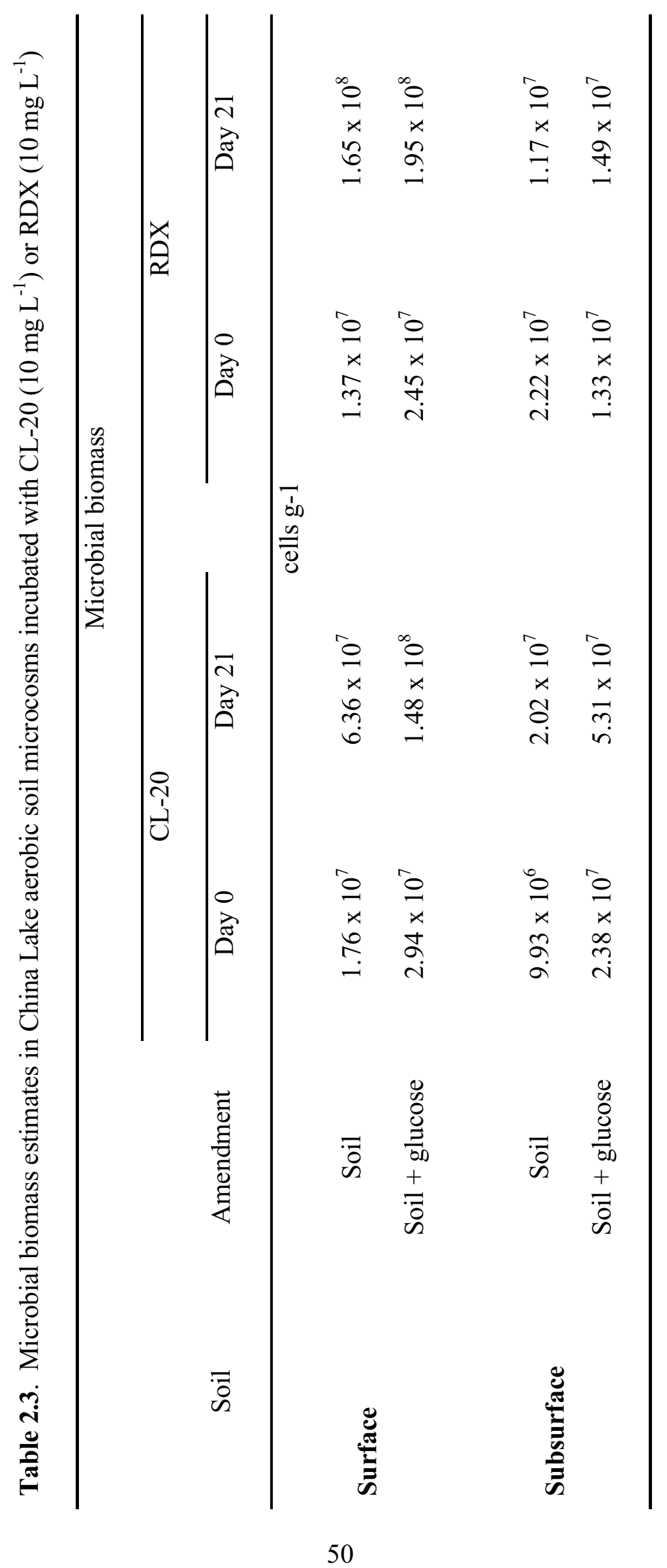




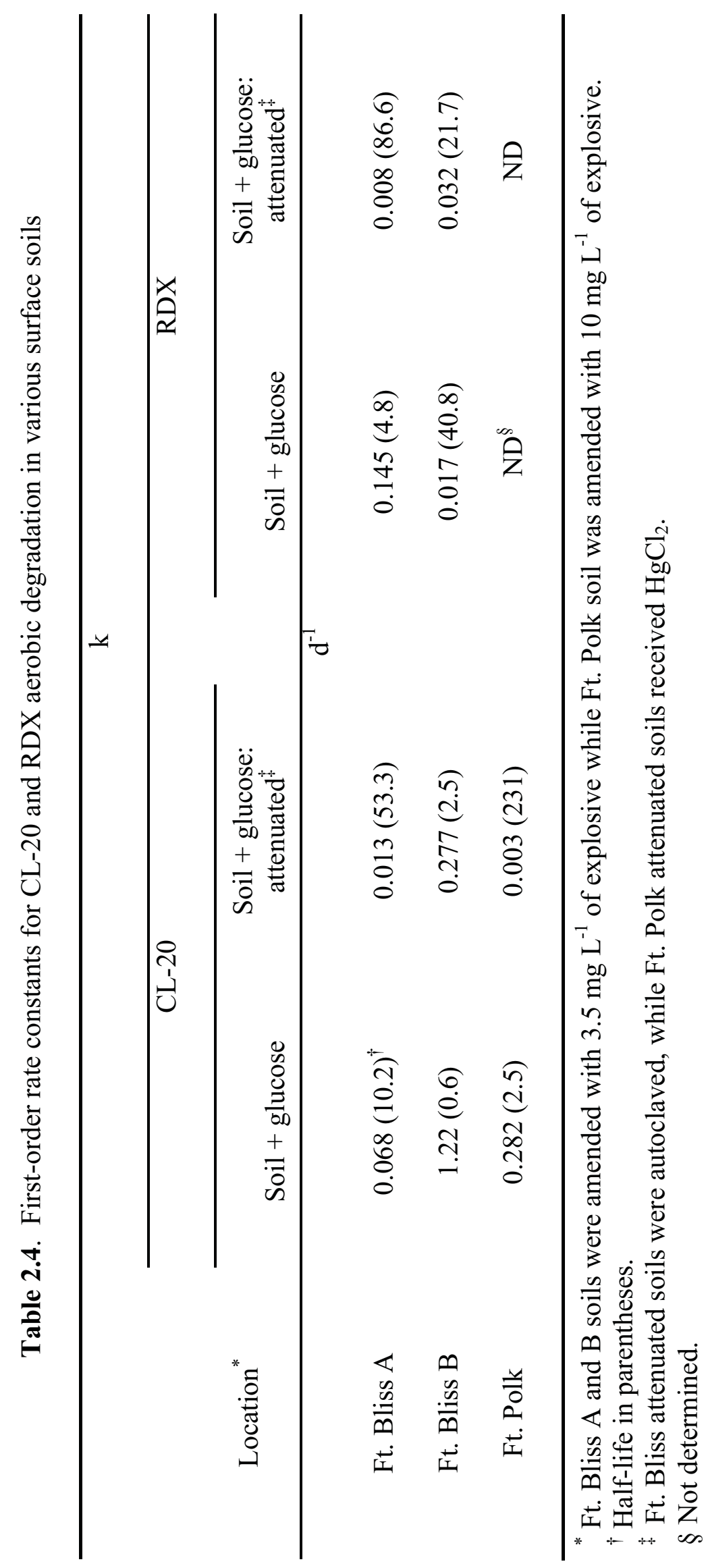


Biodegradation of RDX in China Lake Surface and Subsurface Soils. Under aerobic conditions, RDX was completely biodegraded after 8 or 13 days of incubation in the surface soil amended with or without glucose, respectively (Figure 2.2A). The rate of RDX biodegradation (half-life $=1.5 \mathrm{~d}$ ) was very similar to the rate of CL-20 biodegradation when the surface soil was amended with glucose (Table 2.2). The RDX biodegradation rate (half-life $=4.7 \mathrm{~d}$ ) was significantly faster than the CL-20 biodegradation rate (half-life $=17.3 \mathrm{~d}$ ) when glucose was not added to the surface soil microcosms (Table 2.2). After 21 days, biomass increases were nearly 10-fold in the surface soil amended with RDX and with or without glucose (Table 2.3). In the subsurface soil microcosms, RDX was biodegraded slowly with approximately $15 \%$ of the RDX transformed in 21 days in soil amended with glucose, while there was no apparent biodegradation in the subsurface soil that did not receive glucose (Figure 2.2B, Table 2.2). There were only minor changes in microbial biomass in these microcosms during the incubation period (Table 2.3).
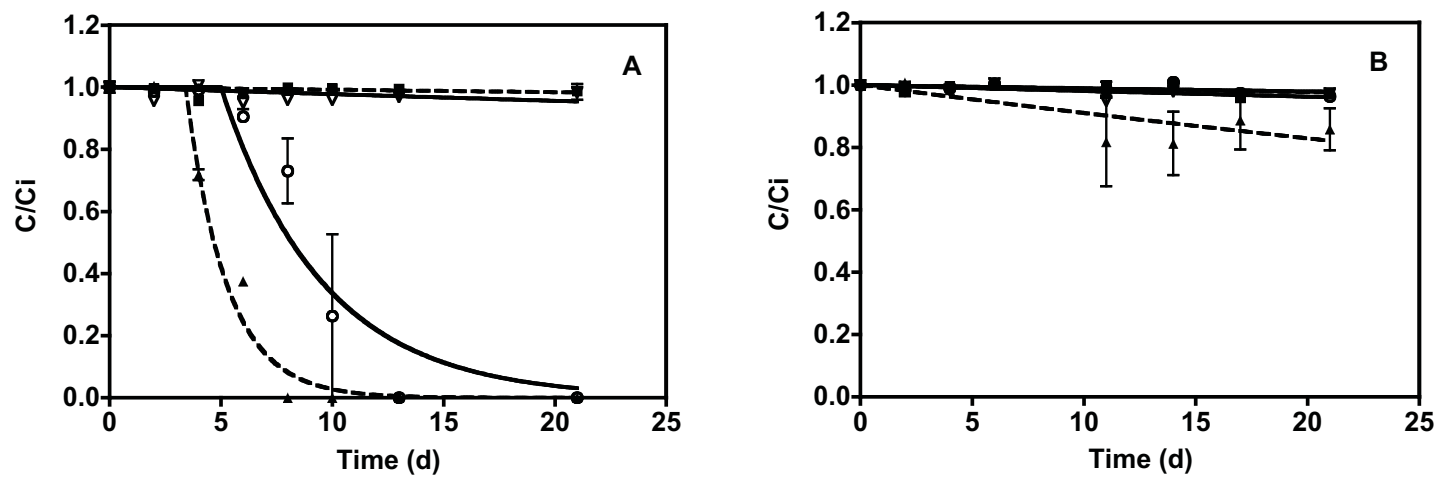

Figure 2.2. Degradation of hexahydro-1,3,5-trinitro-1,3,5-triazine (RDX) in China Lake soil microcosms incubated under aerobic conditions. A) surface soil; B) subsurface soil. Symbols: Unamended soil: $-\mathrm{O}-$; Soil + glucose -- $\mathbf{\Delta - - ;}$ Soil + glucose $+\mathrm{HgCl} 2$ - $\nabla$-; Mineral salts medium + glucose -- $\mathbf{-}--$.

Abiotic Degradation of CL-20 and RDX. CL-20 was prone to abiotic degradation in addition to biological degradation under aerobic conditions (Figure 2.1). This abiotic degradation occurred in the China Lake soil microcosms amended with $\mathrm{HgCl}_{2}$ and in microcosms containing only sterile mineral salts medium. The rates of abiotic CL-20 degradation were 0.009 to $0.030 \mathrm{~d}^{-1}$ (half-lives $=23.1$ to $77 \mathrm{~d}$ ) and were not significantly different between soils amended with $\mathrm{HgCl}_{2}$ and the sterile mineral salts microcosms (Table 2.2). Furthermore, these abiotic rates of CL-20 degradation were similar to the biological rate of CL-20 degradation in China Lake soil microcosms that did not receive glucose. In contrast, RDX was not degraded by abiotic mechanisms in aerobic surface or subsurface soils treated with $\mathrm{HgCl}_{2}$ or in microcosms only containing sterile mineral salts medium (Figure 2.2, Table 2.2).

Biodegradation of CL-20 and RDX in Various Surface Soils. For a broader perspective on the fate of CL-20 in soils, we compared CL-20 degradation in the China Lake surface soil with three firing range soils (Tables 2.2 and 2.4). The rates of CL-20 biodegradation in biologically active soil microcosms amended with glucose varied greatly between surface soils from China Lake, Ft. Polk, and Ft. Bliss $\left(0.068<k<1.222 \mathrm{~d}^{-1}\right)$. For each soil type, rates of CL-20 degradation were significantly faster in active soils than in biologically attenuated soils 
(Tables 2.2 and 2.4). The organic carbon content, $\mathrm{pH}, \mathrm{NO}_{3}$-nitrogen, or clay content of the soils (Table 2.1) could not explain the different CL-20 biodegradation rates in these soils. Similarly, rates of RDX biodegradation were very variable among the 4 surface soils amended with glucose $\left(0.017<k<0.474 \mathrm{~d}^{-1}\right)$. The rate of RDX biodegradation in these soils was not related to the rate of CL-20 biodegradation in the same soil (Tables 2.2 and 2.4). In active surface soil microcosms amended with glucose, the fastest CL-20 biodegradation rate occurred with the Ft. Bliss B soil followed in order by China Lake, Ft. Polk, and Ft. Bliss A soil. With respect to RDX, the order of biodegradation rates was as follows: China Lake $>$ Ft. Bliss A soil $>$ Ft. Bliss B soil.

Mineralization of ${ }^{\mathbf{1 4}}$ C-CL-20 in China Lake Soils. The aerobic mineralization of CL-20 in biologically active China Lake surface and subsurface soil microcosms was extensive with between 41.1 and $55.7 \%$ of the initial ${ }^{14} \mathrm{C}-\mathrm{CL}-20$ recovered as ${ }^{14} \mathrm{C}-\mathrm{CO}_{2}$ after 41 days of incubation (Figure 2.3, Table 2.5). The production of ${ }^{14} \mathrm{C}-\mathrm{CO}_{2}$ was slower than the biodegradation of CL-20, since ${ }^{14} \mathrm{C}-\mathrm{CO}_{2}$ did not appear until after a lag phase of between 2 to 7 days (Figure 2.3). Lag phases were not evident from the CL-20 disappearance data (Figure 2.4). In the subsurface soil amended with glucose, CL-20 was completely degraded in 4 days, but the maximum extent of ${ }^{14} \mathrm{C}-\mathrm{CO}_{2}$ evolved was not reached until after 14 days. Similarly, CL-20 was completely biodegraded in the surface soil microcosms treated with or without glucose in 18 days, but the extent of ${ }^{14} \mathrm{C}-\mathrm{CO}_{2}$ evolution only approached the maximum after 41 days. While biodegradation of CL-20 appeared to be similar between the glucose-amended surface soil and the unamended surface and subsurface soils, the distribution of ${ }^{14} \mathrm{C}$-label from ${ }^{14} \mathrm{C}-\mathrm{CL}-20$ was different and may simply reflect that mineralization had progressed further towards completion in the surface soil microcosms. In subsurface soil microcosms without glucose, a slightly lower amount of ${ }^{14} \mathrm{C}-\mathrm{CO}_{2}$ was evolved (41.1\% vs. $50.8 \%$ to $55.7 \%$ ), and larger amounts of the ${ }^{14} \mathrm{C}$-label were recovered from the aqueous phase $(27.1 \%$ vs. $9.7 \%$ to $16.4 \%)$ and soil $(24.2 \%$ vs. $10.5 \%$ to 12.1\%; Table 5).
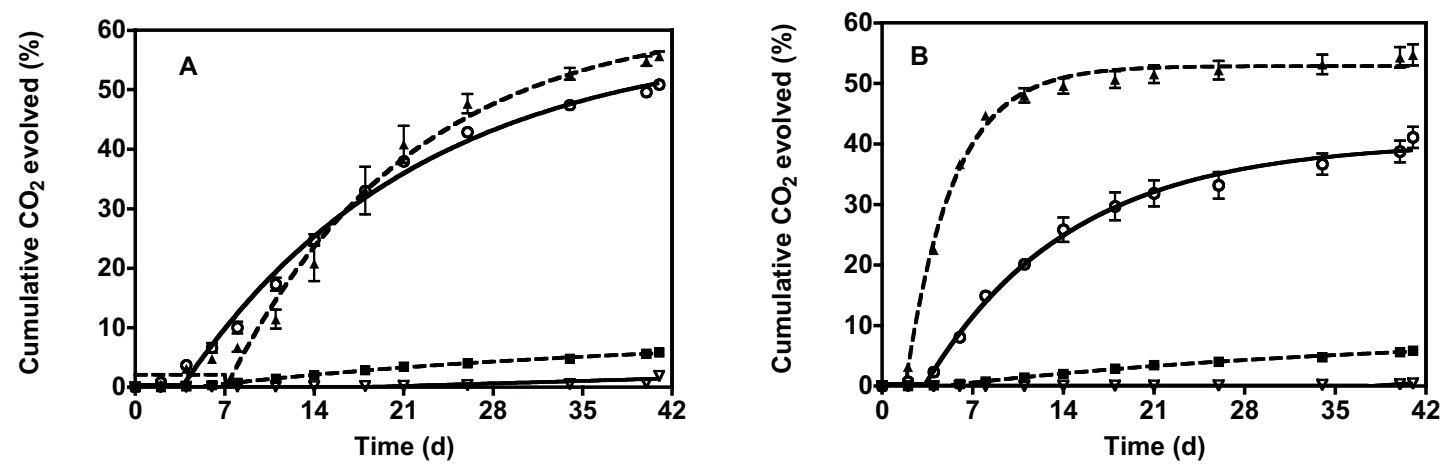

Figure 2.3. Cumulative mineralization of ${ }^{14} \mathrm{C}$-hexanitrohexaazaisowurtzitane $\left({ }^{14} \mathrm{C}-\mathrm{CL}-20\right)$ to ${ }^{14} \mathrm{C}-\mathrm{CO}_{2}$ in China Lake soil microcosms. A) surface soil; B) subsurface soil. Symbols: Unamended soil: $-\bigcirc-$; Soil + glucose $--\mathbf{A}--$; Soil + glucose $+\mathrm{HgCl} 2-\nabla-$; Mineral salts medium + glucose -- $\mathbf{-}--$.

Little mineralization of CL-20 was observed in the $\mathrm{HgCl}_{2}$-amended soil microcosms or soilfree microcosm (Figure 2.3). Between $0.46 \%$ to $1.3 \%$ of the ${ }^{14} \mathrm{C}$-label was evolved as ${ }^{14} \mathrm{C}-\mathrm{CO}_{2}$ in the $\mathrm{HgCl}_{2}$-amended soils, while $5.8 \%{ }^{14} \mathrm{C}-\mathrm{CO}_{2}$ was produced in the soil-free microcosm 
(Table 2.5). In contrast, the abiotic degradation of CL-20 in these control microcosms was extensive (54\% to $90 \%$ degraded) after 41 days of incubation (Figure 2.4). The higher agitation rate used with these microcosms was probably responsible for the increase in abiotic degradation compared to our earlier results (Figures 2.1 and 2.4 ). The majority of the ${ }^{14} \mathrm{C}$-label $(52.7 \%$ to $79.1 \%$ ) was found as unidentified water-soluble metabolites (Table 2.5).
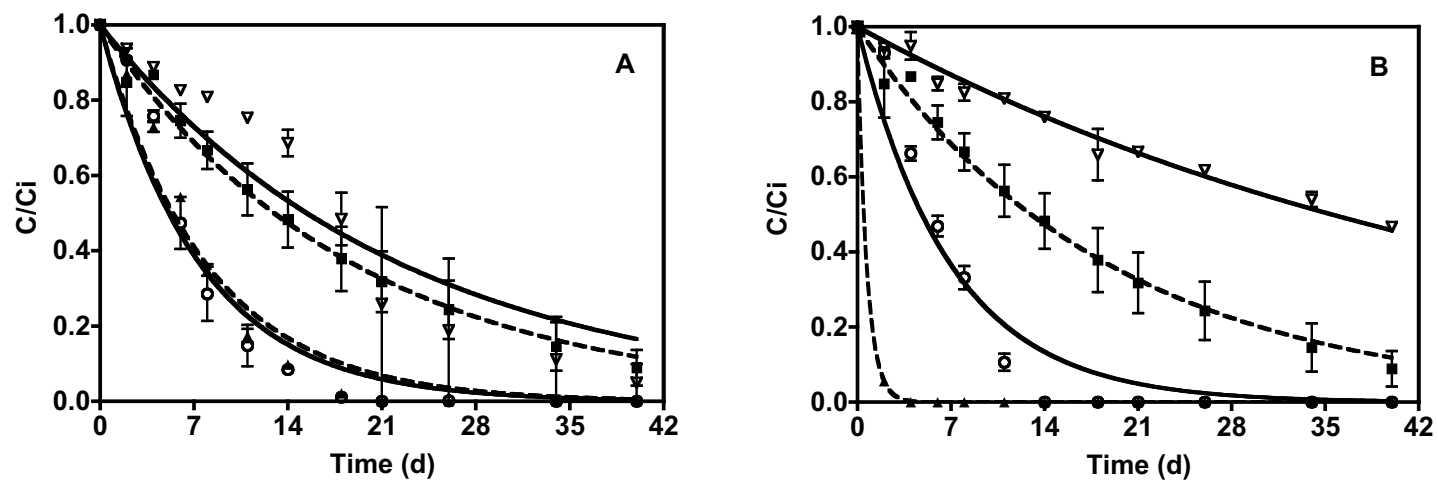

Figure 2.4. Change in CL-20 concentration in China Lake soil microcosms amended with

${ }^{14} \mathrm{C}$ hexanitrohexaazaisowurtzitane $\left({ }^{14} \mathrm{C}-\mathrm{CL}-20\right)$. A) surface soil; B) subsurface soil. Symbols: Unamended soil: $-\bigcirc-$; Soil + glucose -- $\mathbf{A}--$; Soil + glucose $+\mathrm{HgCl} 2$ - $\nabla$-; Mineral salts medium + glucose $-\mathbf{-} \mathbf{-}-$.

Growth of Pure Bacterial Cultures on CL-20 and RDX. A single CL-20-degrading bacterial strain, KTC13, was isolated from a China Lake surface soil enrichment culture that grew on CL-20 as a nitrogen source (Figure 2.5). Strain KTC13, was unable to grow on CL-20 as a sole carbon and nitrogen source or in a nitrogen-free mineral salts medium. Based on partial 16S rRNA gene sequencing strain KTC13 was identified as a Gordonia sp. Since two Rhodococcus strains were recently isolated with the ability to use RDX as a nitrogen source for growth under aerobic conditions (Coleman et al. 1998; Seth-Smith et al. 2002), these strains and Gordonia sp. strain KTC13 were tested for their ability to use CL-20 or RDX (Figure 2.5). RDX did not support the growth of Gordonia sp. strain KTC13, nor was it degraded by strain KTC13. In contrast, Rhodococcus spp. strain DN22 and strain 11Y grew on RDX with concomitant loss of RDX, but they did not grow with or degrade CL-20.

Mineralization of ${ }^{14}$ C-CL-20 by Gordonia sp. Strain KTC13. Gordonia sp. KTC13 aerobically mineralized ${ }^{14} \mathrm{C}-\mathrm{CL}-20$ to ${ }^{14} \mathrm{C}-\mathrm{CO}_{2}$ to a maximum extent of $20.8 \%$ of the initial ${ }^{14} \mathrm{C}-\mathrm{CL}-20$ recovered after 34 days of incubation (Figure $2.6 \mathrm{~A}$ ). The production of ${ }^{14} \mathrm{C}-\mathrm{CO}_{2} \mathrm{did}$ not appear until after a short lag phase of $2 \mathrm{~d}$, and then the production of ${ }^{14} \mathrm{C}-\mathrm{CO}_{2}$ continued for 3 weeks after the CL-20 was no longer detected in the culture media (Figure 2.6B). Mineralization of ${ }^{14} \mathrm{C}-\mathrm{CL}-20$ did not occur in the mineral salts medium microcosms or in microcosms containing strain KTC13 and the inhibitor gluteraldehyde (Figure 2.6A), although abiotic degradation of CL-20 did occur at a slow rate (Figure 2.6B). Between $84 \%$ and $86 \%$ of the ${ }^{14} \mathrm{C}$ label remained in the aqueous phase as unidentified metabolites in the mineral salts medium and gluteraldehyde treated cell cultures. About $60 \%$ of the ${ }^{14} \mathrm{C}$ label remained in the aqueous phase in the live cell cultures (data not shown). 


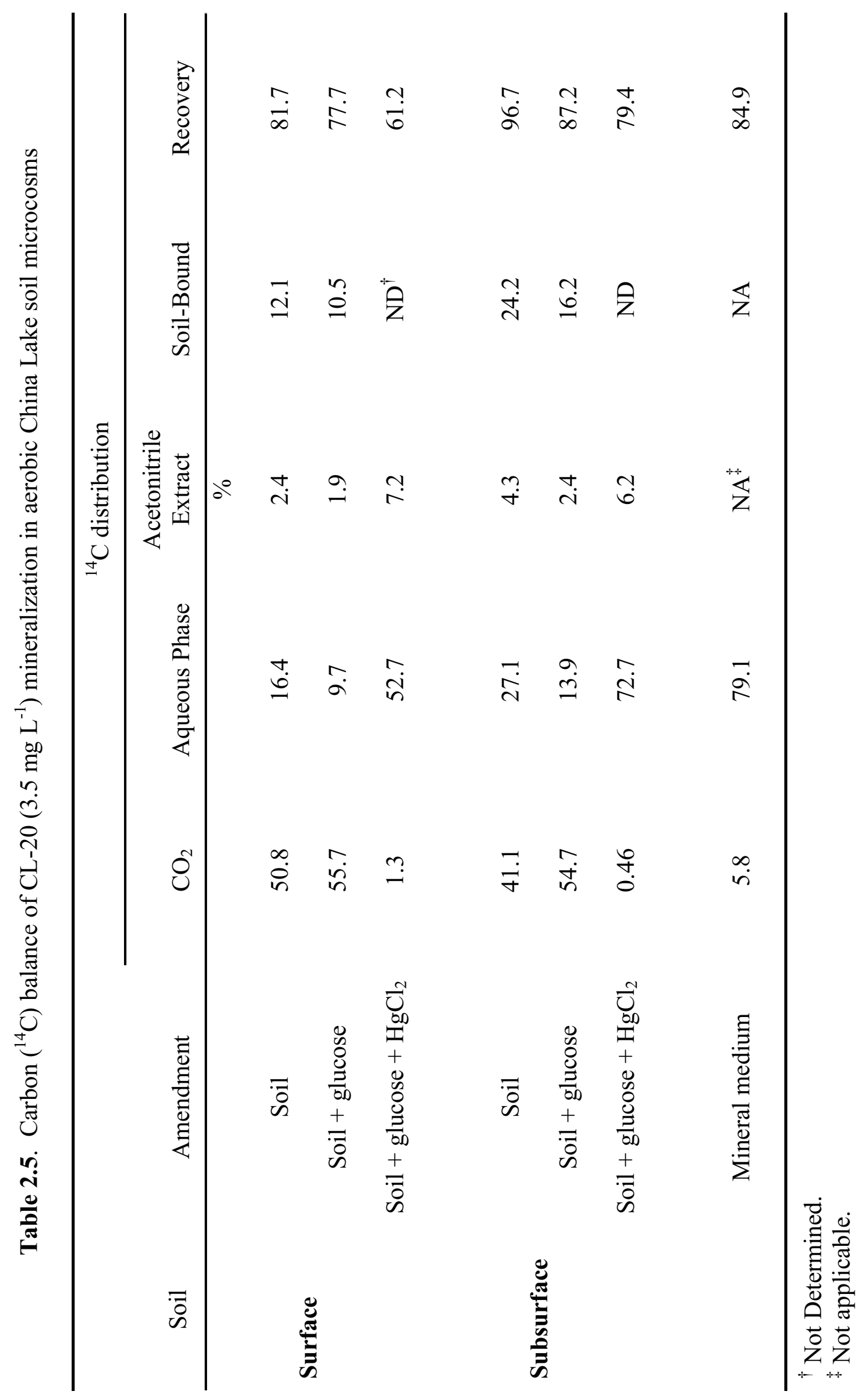




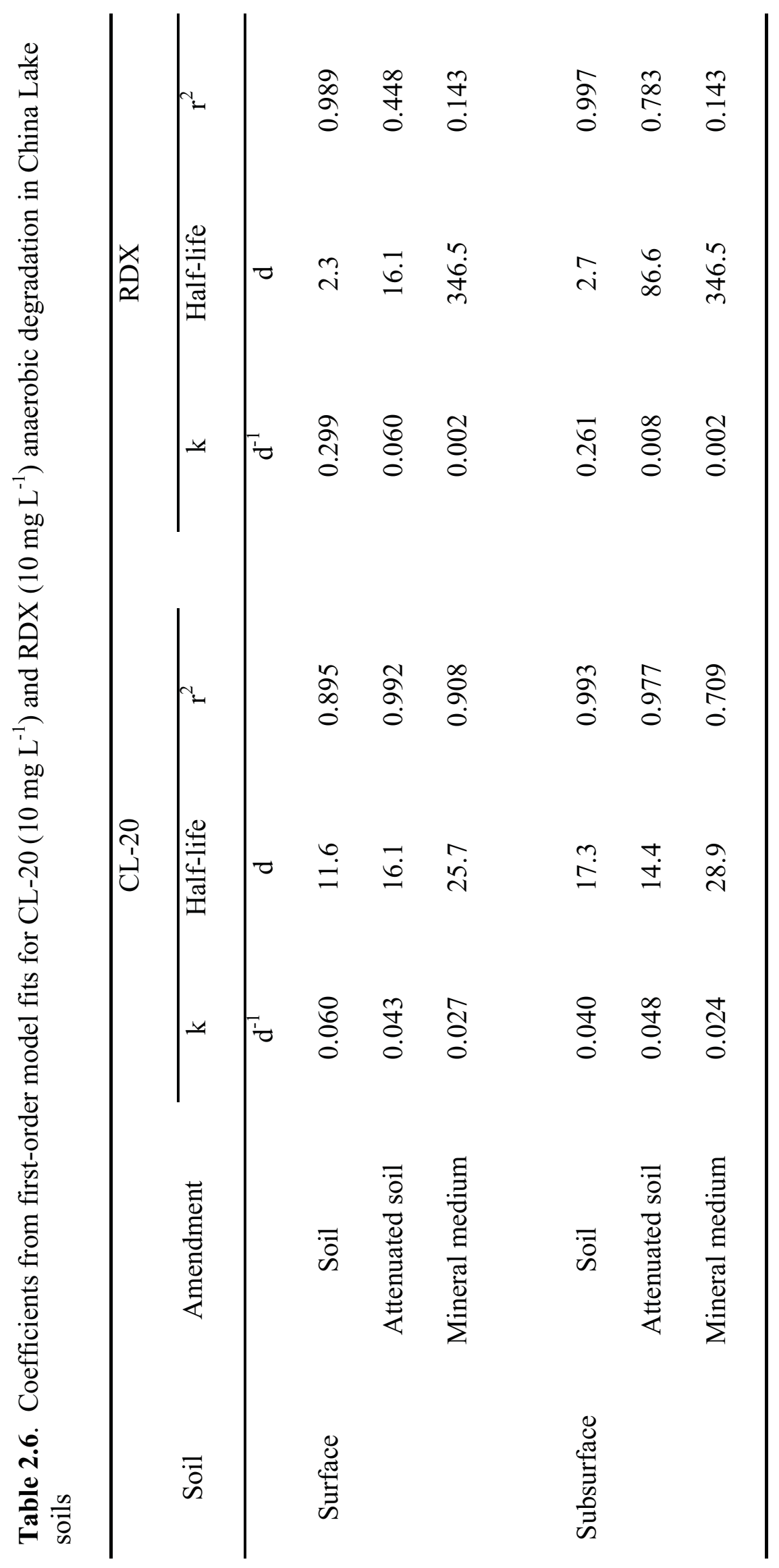




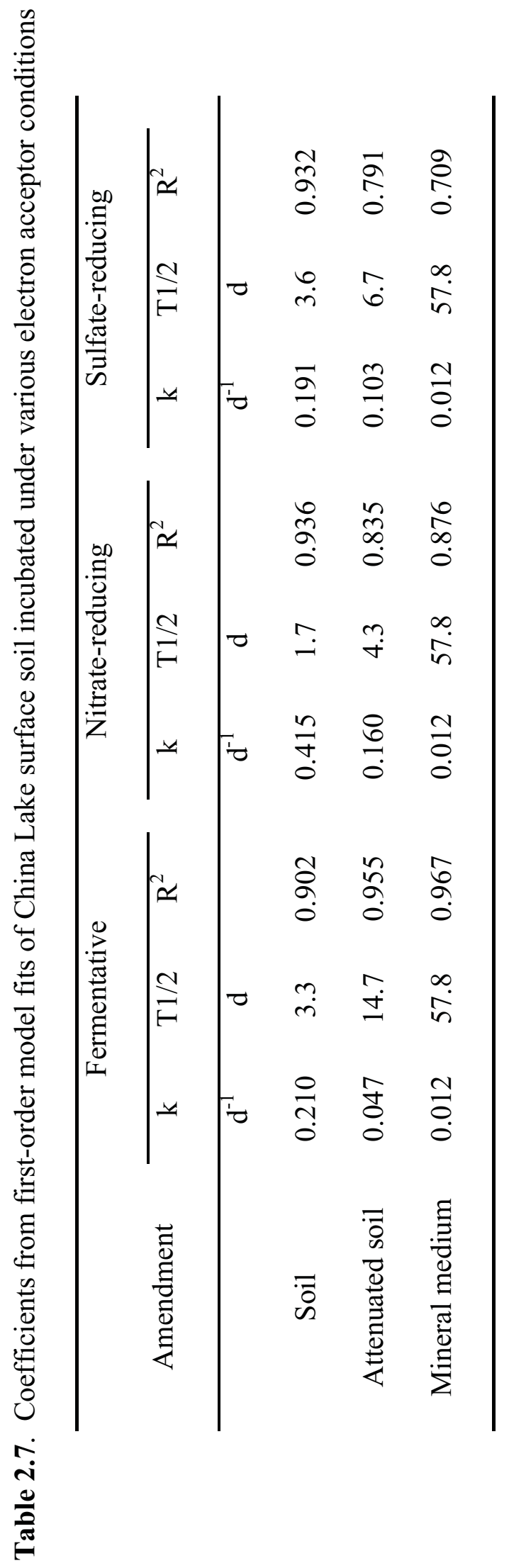



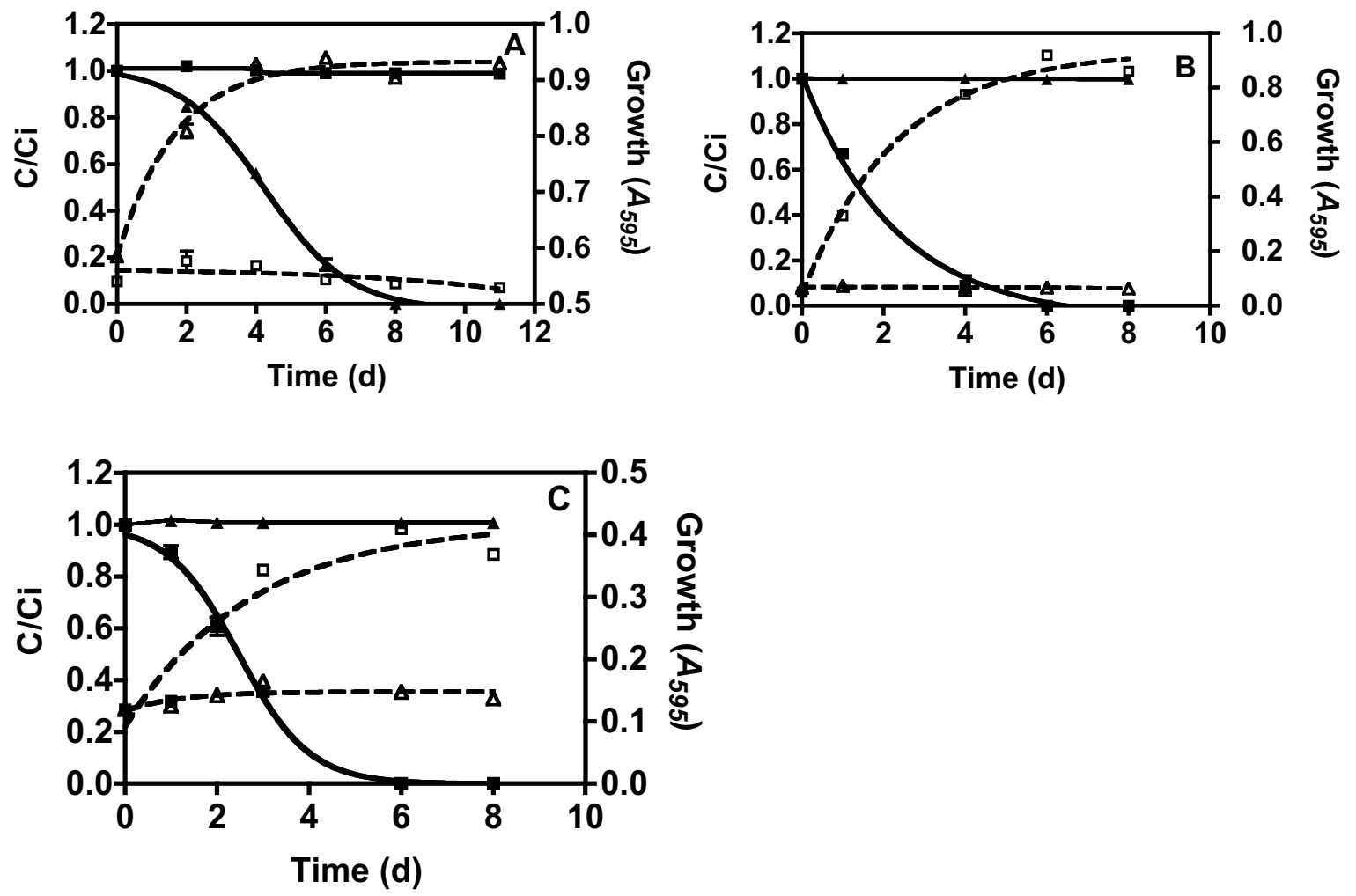

Figure 2.5. Growth of A) Gordonia sp. strain KTC13; B) Rhodococcus rhodocrous strain 11Y; and C) Rhodococcus sp. strain DN22 on hexanitrohexaazaisowurtzitane (CL-20) or hexahydro1,3,5-trinitro-1,3,5-triazine (RDX) as a sole nitrogen source. Symbols: Growth $\left(A_{595}\right)$ on CL-20: $--\triangle--$; Growth $\left(A_{595}\right)$ on RDX: $--\square--$; Concentration of CL-20: - $\mathbf{\Delta -}$; Concentration of RDX:- - -
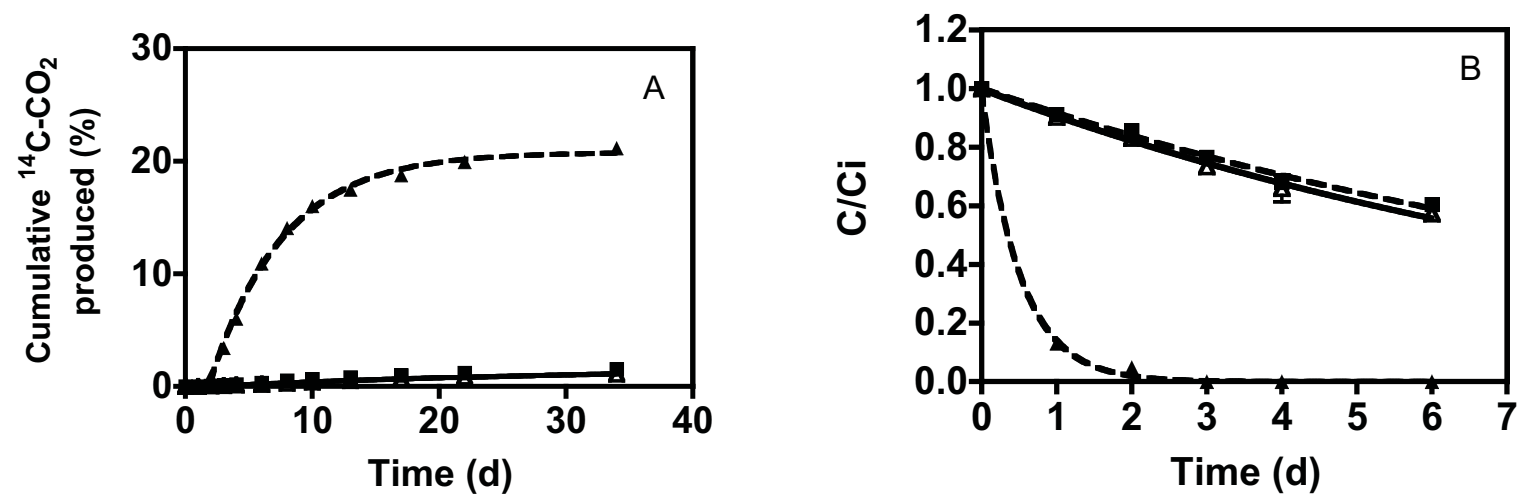

Figure 2.6. Cumulative mineralization and degradation of ${ }^{14} \mathrm{C}$-hexanitrohexaazaisowurtzitane $\left({ }^{14} \mathrm{C}-\mathrm{CL}-20\right)$ to ${ }^{14} \mathrm{C}-\mathrm{CO}_{2}$ by Gordonia sp. KTC13. A) Cumulative ${ }^{14} \mathrm{C}-\mathrm{CO}_{2}$ production; B) ${ }^{14}$ C-CL-20 degradation. Symbols: KTC13 live cells -- $\mathbf{\Delta - -}$; KTC13 cells + gluteraldehyde $-\nabla$-; Mineral salts medium -- $\mathbf{- -}$. 


\section{Task 2.2. Anaerobic Biodegradation}

Anaerobic CL-20 Biodegradation in China Lake Soils. Degradation of CL-20 (10 mg L $\left.{ }^{-1}\right)$ was observed under anaerobic conditions in biologically active surface and subsurface soils (Figure 2.7). In biologically active surface soil microcosms, greater than $90 \%$ of the CL-20 was degraded in 24 days (Figure 2.7A). In comparison, only $61 \%$ of the CL-20 was degraded within 24 days in the biologically active subsurface microcosms (Figure 2.7B). First-order degradation rates for CL-20 (Table 2.6) were faster in the biologically active surface soil microcosms (halflife $=11.6 \mathrm{~d}$ ) than the subsurface soil microcosms (half-life $=17.3 \mathrm{~d})$. A few metabolites were formed in these microcosms as observed via HPLC. However, the metabolites were sporadically detected in very minor amounts and remain unidentified.
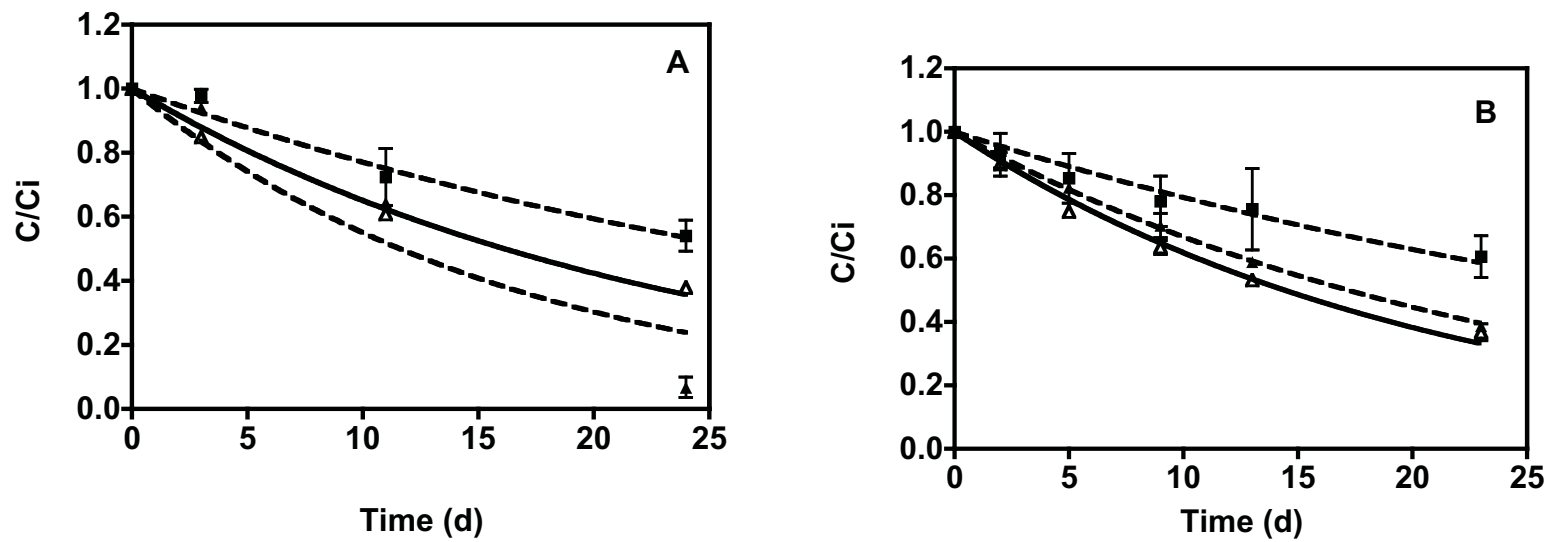

Figure 2.7. Degradation of hexanitrohexaazaisowurtzitane (CL-20) in China Lake soil microcosms incubated under anaerobic conditions. A) surface soil; B) subsurface soil. Symbols: Soil -- $\mathbf{\Delta - -}$; Attenuated soil $-\nabla-$; Mineral salts medium -- $\mathbf{-}--$.

Anaerobic Biodegradation of RDX in China Lake Soils. Anaerobic RDX (10 $\left.\mathrm{mg} \mathrm{L}^{-1}\right)$ degradation in the biologically active surface and subsurface microcosms began after a lag phase of 2 and 12 days, respectively (Figure 2.8). In the surface soil microcosms, the RDX was completely biodegraded after 13 days, while greater than $90 \%$ was biodegraded by the subsurface soil in 23 days. The nitroso metabolites MNX, DNX, and TNX were produced in both the biologically active surface and subsurface microcosms (Appendix K). MNX and DNX were transiently produced and eventually converted to TNX in the surface soil microcosms. MNX, DNX, and TNX appeared in the subsurface microcosms between days 13 and 21 in agreement with the start of RDX biodegradation. First order rates of RDX degradation were similar between the biologically active surface (half-life $=2.3 \mathrm{~d}$ ) and subsurface soils (half-life $=2.7 \mathrm{~d}$ ) once degradation began (Table 2.6). The rates of RDX biodegradation were significantly faster than the anaerobic rates of CL-20 biodegradation in these same soils (Table 2.6).

Abiotic Degradation of CL-20 and RDX under Anaerobic Conditions. Abiotic degradation of CL-20 (10 $\left.\mathrm{mg} \mathrm{L}^{-1}\right)$ was observed under anaerobic conditions in the biologically attenuated soils and mineral salts medium (no soil) (Figure 2.7). About 62\% and 64\% of the CL-20 was degraded in 24 days in the attenuated surface and subsurface soil microcosms, respectively. In the mineral salts medium microcosms between $39 \%$ and $46 \%$ of the CL-20 was degraded. 
Anaerobic rates of CL-20 degradation were slower in the mineral salts medium microcosms (half-lives $=25.7$ to $28.9 \mathrm{~d}$ ) than in the attenuated soil microcosms (half-lives $=14.4$ to $16.1 \mathrm{~d}$; Table 2.6). The latter rates were equal to or slightly slower than the rates of CL-20 degradation in the active soil microcosms. In the subsurface soil, the anaerobic degradation of CL-20 in the attenuated soil microcosms was very similar to the anaerobic degradation of CL-20 degradation in the biologically active soil microcosms.

Abiotic degradation of RDX $\left(10 \mathrm{mg} \mathrm{L}^{-1}\right)$ in anaerobic mineral salts medium soil was not observed (Figure 2.8). Abiotic degradation of RDX in attenuated surface soil microcosms was not observed for 13 days (Figure 2.8A). The loss of RDX between days 13 and 23 was probably due to microbial growth in one of the attenuated microcosms. In the subsurface attenuated soil microcosms a slow rate of RDX loss was observed after a lag period of 10 days (Figure 2.8B).
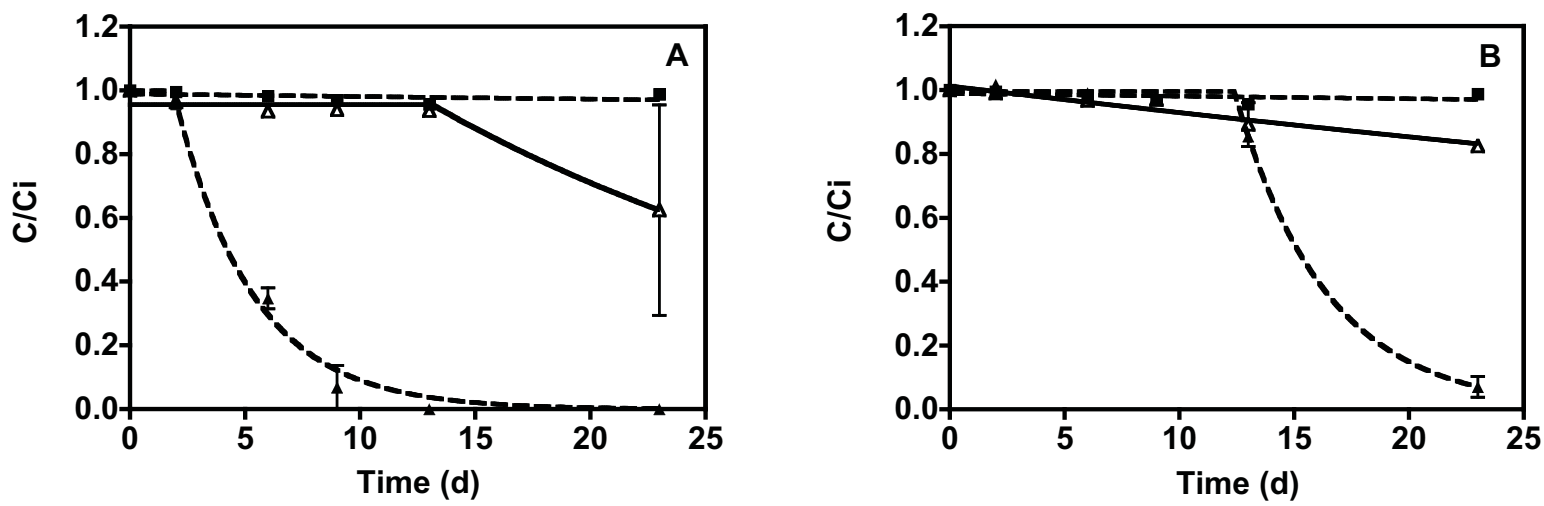

Figure 2.8. Degradation of hexahydro-1,3,5-trinitro-1,3,5-triazine (RDX) in China Lake soil microcosms incubated under anaerobic conditions. A) surface soil; B) subsurface soil. Symbols: Soil -- $\mathbf{\Delta - -}$; Attenuated soil $-\boldsymbol{\nabla}-$; Mineral salts medium -- --- .

Anaerobic CL-20 Degradation in Different Terminal Electron Acceptor Conditions. CL-20 (10 $\left.\mathrm{mg} \mathrm{L}^{-1}\right)$ was degraded under fermentative, nitrate-reducing, and sulfate-reducing conditions by the China Lake surface soil (Figure 2.9). Under fermentative conditions, CL-20 was completely degraded in the active soil microcosms by $21 \mathrm{~d}$ (Figure 2.9A). Under nitratereducing conditions and sulfate-reducing conditions, CL-20 was completely degraded within $10 \mathrm{~d}$ in the biologically active soil microcosms (Figure 2.9B and 2.9C). The soil in these microcosms turned black by day 14 and a strong sulfide smell was detected when the microcosms were opened. In active soil microcosms, biological CL-20 degradation was significantly faster under nitrate-reducing conditions (half-life $=1.7 \mathrm{~d}$ ) compared to the rates under fermentative (half-life $=3.3 \mathrm{~d}$ ) or sulfate-reducing conditions (half-life $=3.6 \mathrm{~d}$; Table 2.7). Various metabolite peaks that were unique to each anaerobic condition were detected via HPLC, but the identity of these peaks is unknown at this time.

Abiotic degradation of CL-20 was observed in the biologically attenuated surface soil microcosms and the mineral salts medium microcosms incubated under each anaerobic condition (Figure 2.9). CL-20 was incompletely degraded in the attenuated soil microcosms (73\%) and 
mineral salts medium (26\%) microcosms, respectively with no added electron acceptor (Figure 2.9A). Under nitrate-reducing conditions, CL-20 was completely degraded within $21 \mathrm{~d}$ in the biologically attenuated soil microcosms, while only $25 \%$ of the CL-20 was degraded in the nitrate mineral salts medium microcosms after $21 \mathrm{~d}$ of incubation (Figure 2.9B). Under sulfatereducing conditions, the amount of CL-20 degraded after $21 \mathrm{~d}$ of incubation was $79 \%$ and $22 \%$ in the attenuated soil and sulfate mineral salts medium microcosms, respectively (Figure 2.9C). The rate of CL-20 degradation in the biologically attenuated soil microcosms was significantly faster under nitrate-reducing conditions (half-life $=4.3 \mathrm{~d}$ ) than fermentative (half-life $=14.7 \mathrm{~d}$ ) or sulfate-reducing (half-life $=6.7 \mathrm{~d}$ ) conditions (Table 2.7). Abiotic degradation of CL-20 in the mineral salts medium microcosms without soil was slow under all anaerobic conditions (halflives $=57.8 \mathrm{~d}$; Table 2.7). For each anaerobic condition, the rates of CL-20 degradation in the attenuated soil microcosms and mineral salts medium microcosms were significantly different than the rates of CL-20 degradation in the active soil microcosms.
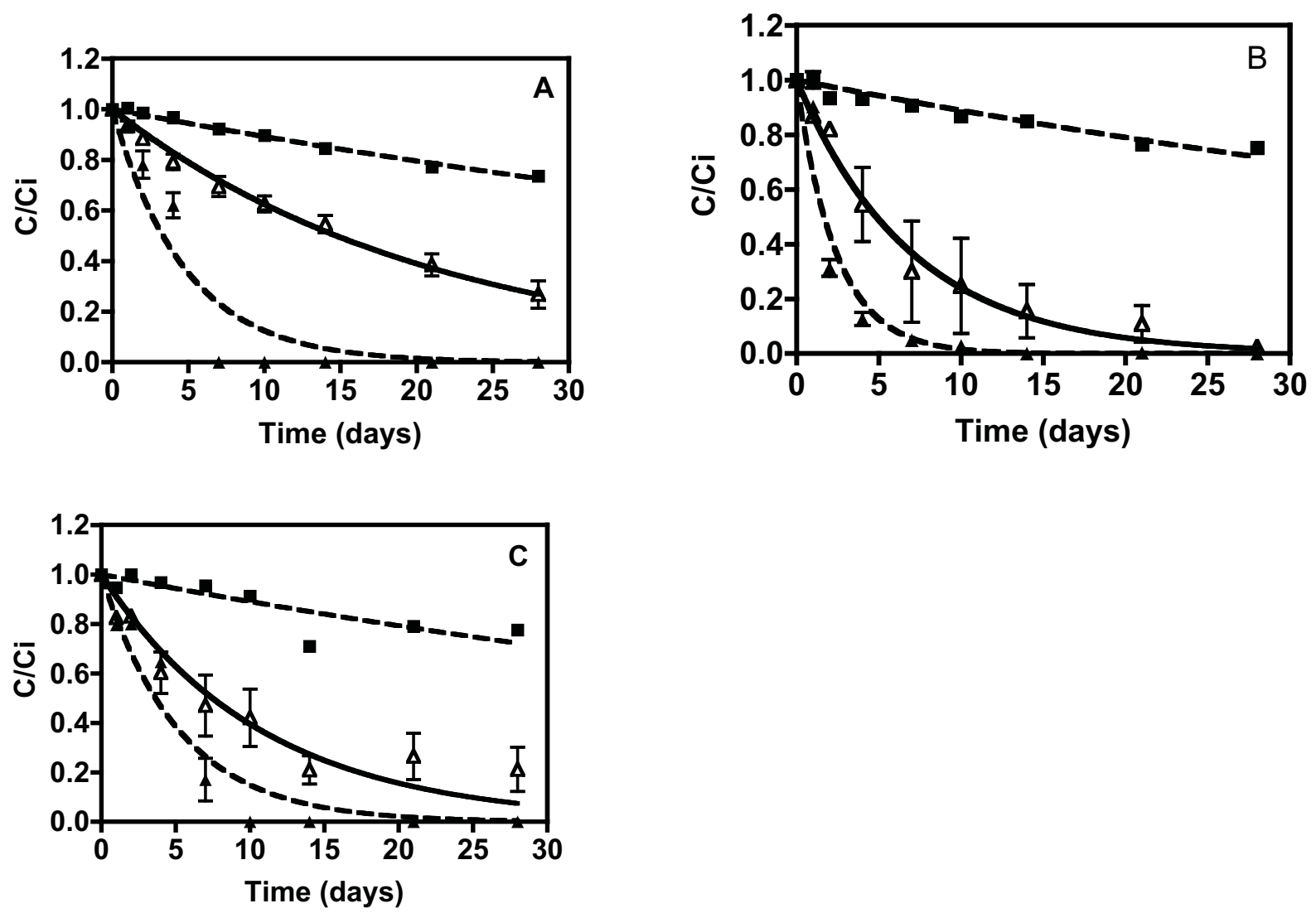

Figure 2.9. Degradation of hexanitrohexaazaisowurtzitane (CL-20) in China Lake soil microcosms incubated under various anaerobic conditions. A) Fermentative; B) Nitrate-reducing; C) Sulfatereducing. Symbols: Soil $--\mathbf{A}--$; Attenuated soil $-\nabla-$; Mineral salts medium $--\boldsymbol{\square}--$.

${ }^{14}$ C-CL-20 Anaerobic Mineralization. The mineralization of ${ }^{14} \mathrm{C}-\mathrm{CL}-20\left(3.5 \mathrm{mg} \mathrm{L}^{-1}\right)$ was also followed under fermentative, nitrate-reducing, and sulfate-reducing conditions (Figure 2.10). Similar to the mineralization of ${ }^{14} \mathrm{C}-\mathrm{CL}-20$ under aerobic conditions (Figure 2.3), the production of ${ }^{14} \mathrm{C}_{-} \mathrm{CO}_{2}$ under anaerobic conditions was slower than the anaerobic degradation of 

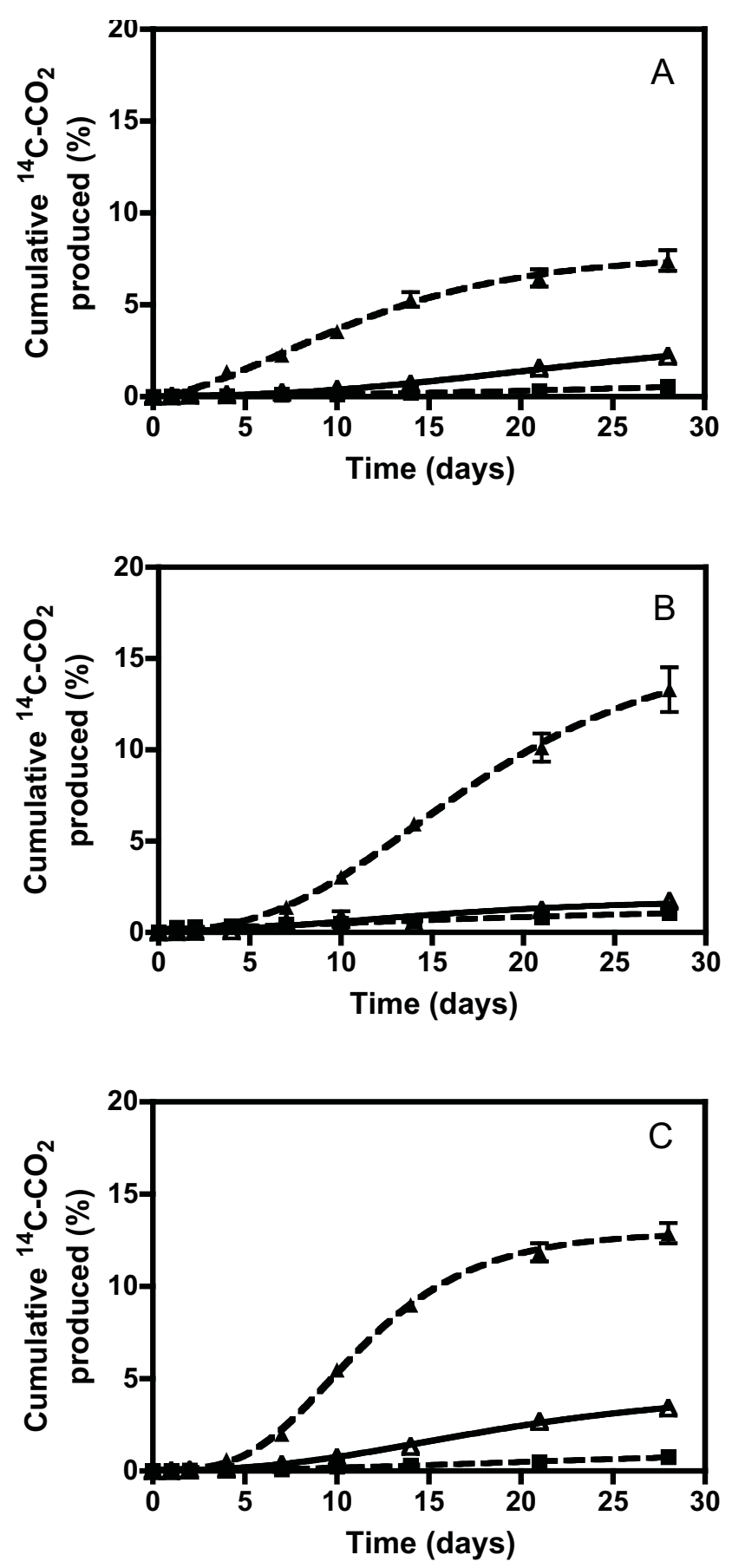

Figure 2.10. Cumulative mineralization of

${ }^{14} \mathrm{C}$-hexanitrohexaazaisowurtzitane $\left({ }^{14} \mathrm{C}-\mathrm{CL}-20\right)$ to

${ }^{14} \mathrm{C}-\mathrm{CO}_{2}$ in China Lake soil microcosms incubated under various anaerobic conditions. A) Fermentative; B) Nitrate-reducing; C) Sulfate-reducing. Symbols: Soil -- $\mathbf{\Delta - - ;}$ Attenuated soil $-\nabla-$; Mineral salts medium -- $\mathbf{- -}$.
CL-20 (Figure 2.9). In contrast, the amount of ${ }^{14} \mathrm{C}-\mathrm{CO}_{2}$ produced was lower under anaerobic conditions than under aerobic conditions and the rate and extent of ${ }^{14} \mathrm{C}_{-} \mathrm{CO}_{2}$ produced differed under each of the anaerobic conditions. Under fermentative conditions, mineralization of ${ }^{14} \mathrm{C}-\mathrm{CL}-20$ to ${ }^{14} \mathrm{C}-\mathrm{CO}_{2}$ slowly increased to $7.4 \%$ of the initial ${ }^{14} \mathrm{C}$-label added. The rates of ${ }^{14} \mathrm{C}-\mathrm{CO}_{2}$ evolution under nitratereducing and sulfate-reducing conditions were faster than under fermentative conditions with $13.3 \%$ and $12.9 \%$ of the ${ }^{14} \mathrm{C}-\mathrm{CL}-20$ mineralized to ${ }^{14} \mathrm{C}-\mathrm{CO}_{2}$ in $28 \mathrm{~d}$. The evolution of ${ }^{14} \mathrm{C}_{-} \mathrm{CO}_{2}$ had not yet reached the maximum amount in the nitrate-reducing soil microcosms by $28 \mathrm{~d}$. The abiotic mineralization of ${ }^{14} \mathrm{C}-\mathrm{CL}-20$ in the attenuated soil microcosms was less than $3.5 \%$ and less than $1.1 \%$ in the mineral salts medium for the anaerobic conditions examined in this study (Figure 2.10). In these microcosms, the majority of the ${ }^{14} \mathrm{C}$-label (69 to $82 \%$ ) remained in the aqueous phase as ${ }^{14} \mathrm{C}-\mathrm{CL}-20$ and unidentified CL-20 metabolites. 


\section{Task 3. Coupled Abiotic and Biotic Processes}

\section{Task 3.1 Coupled Abiotic/Biotic Processes}

Both abiotic and biotic processes have been shown to result in the degradation of aqueous CL-20. As operational definitions, let us define the abiotic or interfacial process as occurring at the water-sediment interface where the interaction of sorbed CL-20 with either surface functional groups and/or Fe(II) in a reducing environment. This results in the initial fragmentation of CL-20's strained cage and the subsequent removal of nitrite groups from the resulting structure. Similarly, biotic degradation is defined here as surface degradation of sorbed CL-20 occurring on bacterial cell walls and /or direct bacterial utilization of CL-20 as a carbon and/or nitrogen source. Biotic degradation could result in the complete mineralization of CL-20 whereas abiotic degradation most likely will not. The objective of this task is to separately examine biotic degradation, then abiotic degradation to derive rate expressions and rate constants for these separate processes and finally to use this information to model (or attempt to model) the competitive processes of degradation observed in a coupled biotic-abiotic system. This objective will be achieved using the a well characterized dissimlatory metal reducing bacteria (Shewanella putefaciens, $\mathrm{CN}-32$ ) and a well characterized sediment that has been bioreduced by $\mathrm{S}$. putefaciens thus reducing a significant fraction of the structural $\mathrm{Fe}(\mathrm{III})$ and $\mathrm{Fe}(\mathrm{III})$ oxides to $\mathrm{Fe}(\mathrm{II})$. Separate experiments will be conducted with S. putefaciens only, then with only bioreduced sediment with no viable cells remaining. Finally the coupled system will consist of previously bioreduced sediment plus a fresh population of $\mathrm{S}$. putefaciens with a cell count chosen to yield comparable rates of biotic and abiotic degradation.

CL-20 Oxic Mineralization. A set of experiments demonstrated the importance of abiotic and biotic processes to CL-20 mineralization. In oxic sediment with no bactericide, abiotic and biodegradation processes can occur. CL-20 oxic mineralization occurred in a system with the addition of only trace nutrients (no C or N, Figure 3.1) with a half-life of $230 \mathrm{~h}$.

What is surprising is that with the addition of all necessary nutrients, microbes did not mineralize CL-20 at all (Figure 3.1), possibly because it was not needed for survival and growth (i.e., too much addition of nutrients). Further experiments were conducted in which only a carbon source (glucose) was added to oxic sediment (Figure 3.2). Up to 63\% mineralization has been observed (Figure 4a) by $670 \mathrm{~h}$, but would the same pathway as nitroreductase catalyzed biotransformation (Bhushan et al. 2004) occur, which would mean that abiotic reactions are not necessary.

\section{CL-20 Mineralization in Anoxic} Systems. To test whether CL-20 biodegradation processes more rapidly

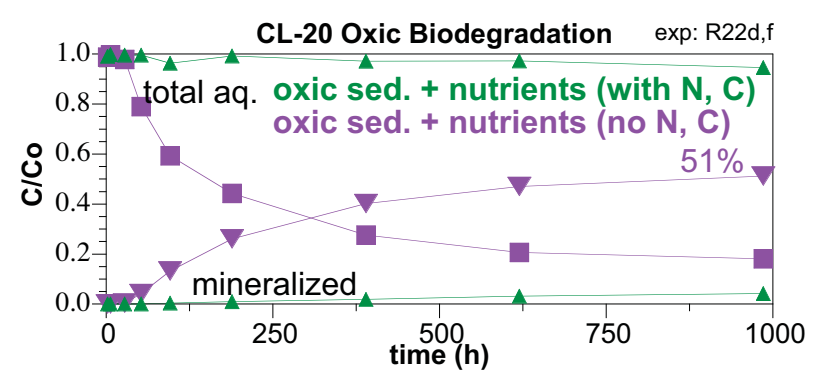

Figure 3.1. CL-20 mineralization in oxic sediment with the addition of trace nutrients (purple) and with the addition of tryptic soy broth (TSB), which contains $\mathrm{C}, \mathrm{N}$, and other necessary nutrients 


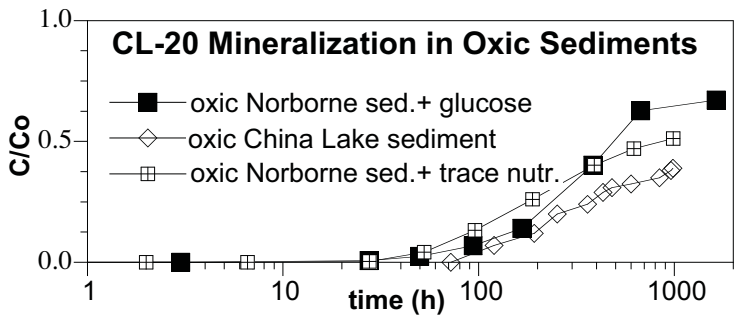

Figure 3.2. CL-20 mineralization in an oxic environment without nutrients, trace metals only, and glucose

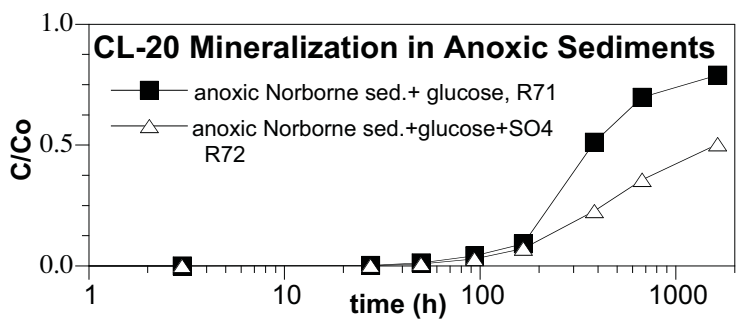

Figure 3.3. CL-20 mineralization in anoxic sediment with glucose addition and anoxic sediment with glucose and $\mathrm{SO}_{4}$

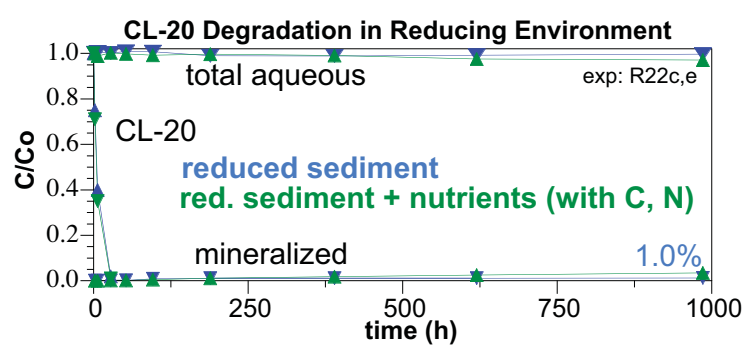

Figure 3.4. CL-20 mineralization in reduced sediments (blue) with TSB addition (green) utilize oxygen or something else as an electron acceptor, similar experiments were conducted in sediment/water systems with helium headspace (anoxic, not reducing).

In anoxic sediment with only $\mathrm{C}$ addition (glucose), the CL-20 mineralization rate was more rapid than in oxic sediment. Up to $70 \%$ mineralization has been observed (Figure 3.3) by $670 \mathrm{~h}$. It is hypothesized that this is the same pathway as with oxic sediments (i.e.,

$\mathrm{N}$-reducing bacteria), and the slightly more rapid rate because of removal of the oxygen electron acceptor; nitrate is being used as the electron acceptor. The specific proportion of glucose added (Figures 3.2 and 3.3) was with a $\mathrm{C} / \mathrm{N}$ ratio of $20 / 1$ (considering all $12 \mathrm{~N}$ of CL-20). In an attempt to stimulate sulfate reducers, an additional anoxic experiment was conducted in which glucose and sulfate were added (Figure 3.3). This actually had a slower CL-20 mineralization rate relative to oxic and anoxic sediment experiments with the addition of only glucose.

\section{CL-20 Mineralization in Reduced}

Sediments. RDX, HMX, and TNT are more rapidly degraded in reducing environments and RDX undergoes the most rapid mineralization in reducing environments. Although CL-20 does not behave similarly to RDX in terms of microbial reactions, it is hypothesized that reducing systems will show more rapid mineralization compared with oxic systems. Two

preliminary experiments (Figure 3.4) actually show essentially no CL-20 mineralization in reduced sediments with a) no additions, and $\mathrm{b}$ ) with $\mathrm{C}, \mathrm{N}$ addition.

An additional set of three mineralization experiments were conducted in partially reduced sediment (Figure 3.5) and fully reduced sediments. A companion SERDP project (1376) has demonstrated that the concentration of dithionite is directly proportional to the microbial population survival (i.e., higher dithionite concentrations kills more microbes), although even high dithionite concentrations leave sufficient $(10 \%)$ microbial populations to easily promote mineralization of acetate, RDX, TNT, and CL-20. Up to $48 \%$ mineralization is observed (Figure 3.5) by $670 \mathrm{~h}$, but the initial mineralization was more rapid than oxic systems. It is unclear if the products are the same as zero valent iron (i.e., initial nitrite, but final nitrous oxide and ammonia). Ferrous iron sorbed on iron oxide and clay systems appear to act as an electron 
donor and catalyst that causes very rapid CL-20 transformation, but it is unclear if these intermediates are more rapidly mineralized. The data (Figure 3.5) suggests not, although dithionite treatment of sediments does kill a fraction (not all) of the microbial population. Some experiments will involve ferrous iron addition to sediment, to not kill microbes.

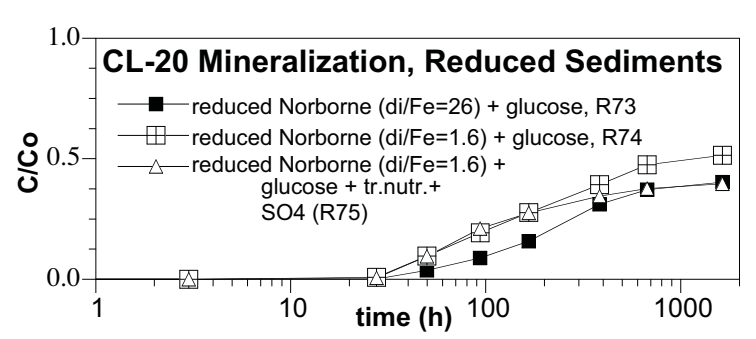

Figure 3.5. CL-20 mineralization in

In this study, more reduced sediment promoted a more rapid CL-20 mineralization rate. The total amount of mineralization was a) partially reduced sediment with glucose, b) fully reduced sediment with glucose, and

c) fully reduced sediment with glucose, $\mathrm{SO}_{4}$. greatest for the oxic system, suggesting degradation pathways of intermediates (microbially) is more rapid in oxic systems. These results may simply infer initial abiotic CL-20 transformation can promote more rapid CL-20 mineralization for some time, but microbes are rate limiting for CL-20 and intermediate transformation reactions. The addition of sulfate in reduced sediments appeared to slightly increase the rate of CL-20 mineralization.

Coupled Process Experiments. Both abiotic and biotic processes have been shown to result in the degradation of aqueous CL-20. Abiotic or interfacial process occur at the water-sediment interface where the interaction of sorbed CL-20 with either surface functional groups and/or $\mathrm{Fe}(\mathrm{II})$ in a reducing environment. This results in the initial fragmentation of CL-20's strained cage and the subsequent removal of nitrite groups from the resulting structure. Similarly, biotic degradation is defined here as surface degradation of sorbed CL-20 occurring on bacterial cell walls and/or direct bacterial utilization of CL-20 as a carbon and/or nitrogen source. Biotic degradation could result in the complete mineralization of CL-20 whereas abiotic degradation most likely will not. The objective of this task is to separately examine biotic degradation, then abiotic degradation to derive rate expressions and rate constants for these separate processes and finally to use this information to model (or attempt to model) the competitive processes of degradation observed in a coupled biotic-abiotic system. This objective will be achieved using the a well characterized dissimlatory metal reducing bacteria (Shewanella putefaciens, CN-32 ) and a well characterized sediment that has been bioreduced by $S$. putefaciens thus reducing a significant fraction of the structural Fe(III) and Fe(III)oxides to Fe(II). Separate experiments were conducted with $S$. putefaciens only, then with only bioreduced sediment with no viable cells remaining. Finally the coupled system will consist of previously bioreduced sediment plus a fresh population of $S$. putefaciens with a cell count chosen to yield comparable rates of biotic and abiotic degradation.

It is hypothesized that in oxic sediment/water systems, abiotic and biotic CL-20 degradation processes occur separately and are additive (i.e., no coupled abiotic/biotic reactions). Oxic abiotic/biotic systems were, therefore, not investigated. In contrast, in reducing sediment/water systems it is hypothesized that while separate abiotic and biotic CL-20 degradation reactions do occur, additional coupled abiotic/biotic reactions can occur (Figure 3.6) - the results of which could not be readily predicted from separate abiotic and biotic experiments. CL-20 degradation in coupled abiotic/biotic systems was extensively investigated in this task. 


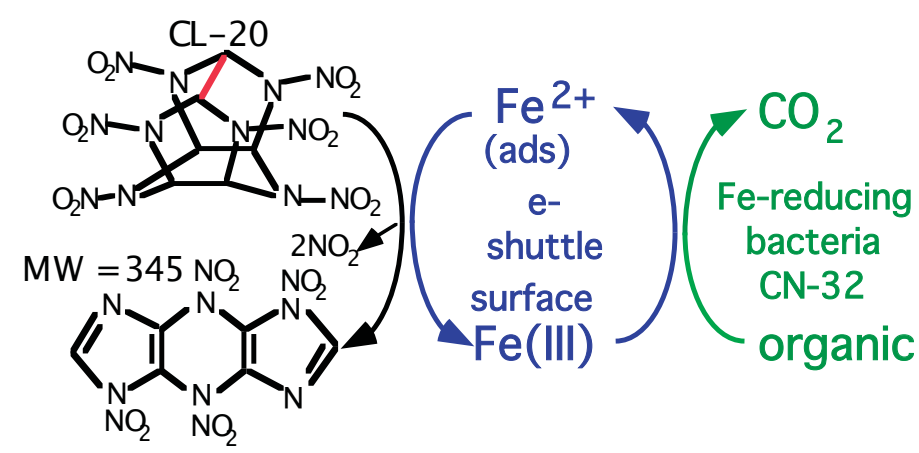

Figure 3.6. Hypothesized coupled CL-20 abiotic/biotic reactions in reducing conditions
Initial scoping experiments were used to define conditions for abiotic and biotic rate experiments. In addition, the biotic rate experiments have been completed and analyzed and the abiotic rate experiments are in progress. The abiotic scoping experiments (Figure 3.7) used $1 \mathrm{~g}$ of bioreduced sediment containing $100 \mu \mathrm{mol}$ of $\mathrm{Fe}(\mathrm{II})$ and $0.46 \mu \mathrm{mol}$ of CL-20 for a $\mathrm{Fe}(\mathrm{II})$ :CL-20 ratio of 217:1. The CL-20 half-life for this ratio was 61 to $115 \mathrm{~h}$ for the two

replicates (lower two curves Figure 3.6). Based on these results we have completed the 60-day bioreduction by $S$. putefaciens of 1, 3, and $10 \mathrm{~g}$ of sediment to yield 100, 214 and $880 \mu \mathrm{mol}$ of $\mathrm{Fe}(\mathrm{II})$, respectively, in these sediments. Experiments to determine abiotic rates using 1, 3, and $10 \mathrm{~g}$ of bioreduced sediment and $0.46 \mu \mathrm{mol}$ of CL-20 (2 ppm) are currently underway. The $\mathrm{Fe}(\mathrm{II}): \mathrm{CL}-20$ ratios of 217:1, 465:1, and 1913:1 in these experiments will provide a range of rates that will overlap those that have been found in the completed biotic degradation experiments (see below).

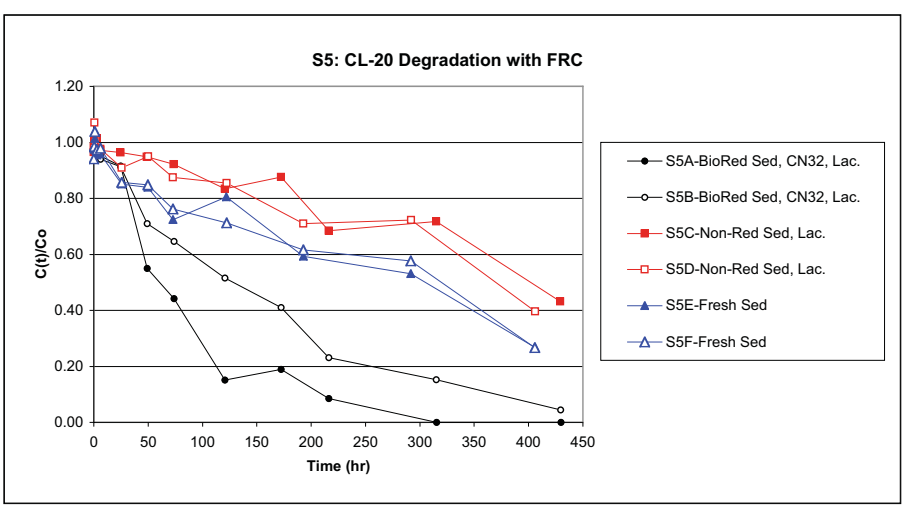

Figure 3.7. CL-20 abiotic scoping experiments
Mossbauer spectra of the unreduced and bioreduced sediment (60 day bioreduction) has shown that the dominant ferrous iron phase produced by the microbes is green rust (Figure 3.8; Kukkadapu et al., 2005)). A $0.5 \mathrm{M} \mathrm{HCl}$ extraction of the sediment was used to determine the total mass of ferrous iron was $92 \mu \mathrm{mol} / \mathrm{g}$ (this study). In separate bottles 1,3 , and $10 \mathrm{~g}$ of FRC sediment were bioreduced for 60 days by $\mathrm{CN}$ 32 (at $\left.9 \times 10^{7} \mathrm{CFU} / \mathrm{ml}\right)$ in the presence of $30 \mathrm{mM}$ pipes buffer ( $\mathrm{pH} 7)$ and

$10 \mathrm{mM}$ lactate as an electron acceptor, and then pasteurized to eliminate biological activity. The most reactive biogenic Fe(II) produced by CN-32 was extracting for $1 \mathrm{hr}$ with $0.5 \mathrm{M} \mathrm{HCl}$ and analyzed by the ferrozine method $(92 \mu \mathrm{mol} / \mathrm{g})$.

A single strain of an iron-reducing bacteria was used in these coupled experiments (Shewanella putefaciens, strain CN-32). The biotic scoping experiment (Figure 3.9), containing $3.3 \mu \mathrm{mol}(1.8 \mathrm{ppm})$ of CL-20 and $4.2 \times 10^{8}$ colony forming units per $\mathrm{ml}(\mathrm{CFU} / \mathrm{mL})$ of CN-32, yielded a CL-20 half life of $10 \mathrm{~h}$. 
Based on this result the biotic rate experiments (Figure 3.10) were conducted using the same CL-20 concentration and $4.77 \times 10^{7}, 3.3 \times 10^{8}$ and $7.4 \times 10^{8} \mathrm{CFU} / \mathrm{mL}$ of $\mathrm{CN}-32$ yielding half lives of 46, 10 and $4.2 \mathrm{~h}$, respectively. The coupled experiments will thus utilize a population of CN-32 of approximately $3.5 \times 10^{7} \mathrm{CFU} / \mathrm{mL}$ and $10 \mathrm{~g}$ of bioreduced sediment.

The intrinsic rate of microbial biodegradation of CL-20 was $2.5 \pm$ $0.6 \mathrm{E}^{-10} \mathrm{CFU}^{-1} \mathrm{~h}^{-1}$ (i.e., rate per microbe), and this intrinsic rate was very constant over the range of microbial cell densities used (Figure 3.10). One might expect a slower intrinsic biodegradation rate an extremely high cell counts, as the efficiency of cells coming in contact with CL-20 is less, although the cell numbers were not that great in these studies.

A similar set of CL-20 degradation rate experiments were conducted with the bioreduced sediment over a range of sediment/water ratios (Figure 3.11). These results were very similar to the microbial results, in that the intrinsic rate of CL-20 degradation (5.1 \pm $1.4 \mathrm{E}^{-5} \mu \mathrm{mol}^{-1} \mathrm{~h}^{-1}$ (i.e., rate per micromol of ferrous iron) was very constant over the range of conditions. In these experiments, the ratio of ferrous iron/CL-20 (mole/mole) was 200:1, 400:1, and 1600:1.

\section{Coupled CL-20 Abiotic/Biotic}

Degradation. With the separate abiotic and biotic CL-20 degradation data, the coupled system hypothesis could then be tested by a system containing both bioreduced FRC (U.S. DOE NABIR Field Research Center at Oak Ridge National Laboratories) sediment and the CN-32 microbes. In the first coupled experiment, results of biotic experiments shown (Figure 3.10) and abiotic rate experiments (Figure 3.11) were used to determine a soil/water/microbe ratio where the separate rates of abiotic and biotic degradation were roughly the same (i.e., so one process was not 


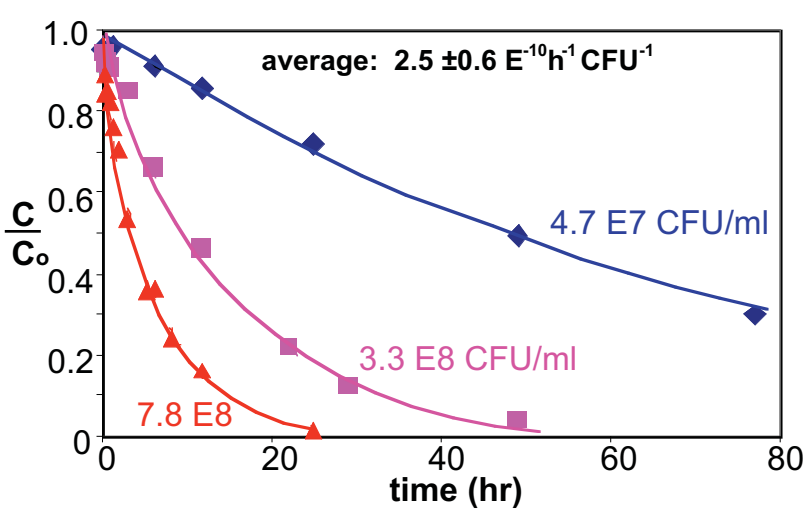

Figure 3.10. CL-20 biodegradation with CN-32 with varying microbial population densities

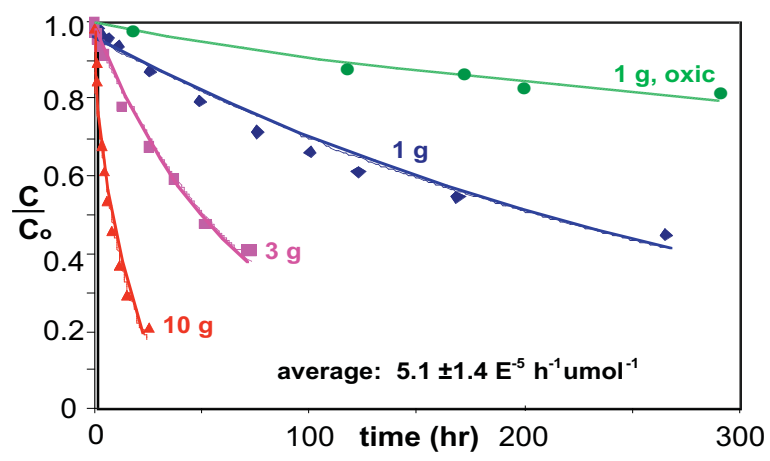

Figure 3.11. CL-20 abiotic degradation of oxic FRC sediment and bioreduced FRC sediment

\section{CL20 Deg. in Coupled System}

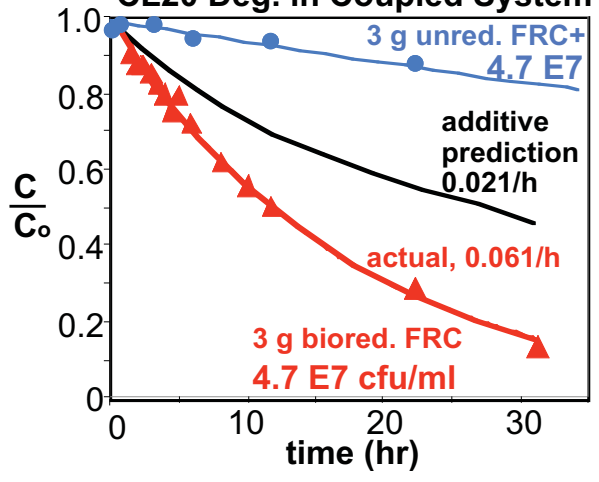

Figure 3.12. Coupled CL-20 abiotic/biotic degradation (red) and prediction from separate abiotic and biotic experiments (black). A microbial hypothesis was tested (blue data), which indicated a coupled biogeochemical reaction was occurring. dominant), in order to determine if there was an actual coupled biogeochemical reaction occurring or the separate processes occurring in the same experimental system were simply additive.

Results of the first coupled reaction system (Figure 3.12) indicated that CL-20 was being degraded at a rate about $3 \mathrm{x}$ faster than could be quantified by the separate abiotic and biotic degradation reactions. Because these reaction rates have been quantified at different cell and sediment/ water concentrations (with duplicates), there is very good reproducibility of the data. Therefore, the $3 \mathrm{x}$ increase in CL-20 degradation rate is significant, although by itself does not prove a specific hypothesis is correct. The implications of this data are that coupled reactions are significant if microbes are present in a reducing environment at $10^{7} \mathrm{CFU} / \mathrm{g}$ or greater.

There are three hypotheses that could explain the observations in the coupled experiment:

Hypothesis 1: An actual coupled biogeochemical reaction is occurring (shown in Figure 3.6), where ferrous iron (in the green rust or clay) is rapidly degrading the CL-20, and producing a labile Fe(III) phase, which the microbes are rapidly re-reducing.

Hypothesis 2: CL-20 degradation is predominantly microbially mediated. The mineral surface is not involved in electron transfer and is only breaking the CL-20 cage by non-electron transfer reactions (as is observed for many minerals, at a slow rate).

Hypothesis 3: Ferrous iron is desorbing from the sediment and adsorbing to the microbial surface $(\mathrm{CN}-32)$, so the 
reaction is actually occurring on the microbial surface and the mineral phase is simply acting as a ferrous iron source. This sequential abiotic, biotic reaction sequence is not sustainable.

Hypothesis 2 was tested in several ways. First, the same (unreduced) sediment was used with the same concentration of microbes. This would have the same mineral surfaces present, but not the ferrous iron involved in electron transfer. This experiment (blue data, Figure 3.12) showed low CL-20 degradation rate, essentially the same as observed for microbes alone (Figure 3.10). A second test of this hypothesis is described in detail in the following pages, and uses gibbsite (aluminum oxide) with high surface area (same surface area as the FRC sediment), but no ferrous iron for electron transfer. This experiment also showed a slow CL-20 degradation rate. In a third experiment, the ferrous iron of the bioreduced FRC sediment was stripped off the surface before the coupled experiment. This also resulted in a very slow CL-20 degradation rate (described in detail below). It was concluded from these experiments that the mineral surface was indeed involved in the coupled electron transfer reaction, and not just acting as a catalyst.

Hypothesis 3 was tested by calculating the affinity of ferrous iron for the FRC sediment surface in comparison to ferrous iron adsorption onto the microbial surface (Liu et al. 2001). This hypothesis was discounted because the ferrous iron affinity (i.e., adsorption strength) for the microbial surface is 4 orders of magnitude less than the FRC sediment, so the ferrous iron would not desorb from the mineral surface and adsorb on the microbial surface. In addition, for this hypothesis to work, the reactive phase would have to be adsorbed ferrous iron. Iron extractions of $1 \mathrm{M} \mathrm{CaCl}_{2}$ were done on the bioreduced FRC sediment, and did not produce any measurable adsorbed ferrous iron - in other words, the ferrous iron present in the bioreduced FRC sediment is predominantly structural in green rust and clays, as described earlier.

Additional coupled abiotic/ biotic experiments were conducted to confirm the results of the first experiment, and further confirm hypotheses. For coupled systems, degradation was examined for $3 \mathrm{~g}$ of bioreduced FRC sediment at three cell densities, 1.0E+7 (low), 4.6 and $4.7 \mathrm{E}+7$ (med) and $1.0 \mathrm{E}+8$ (high) CFU/ml (Figure 3.13). Excellent reproducibility was obtained as can be seen by comparing the points shown by solid squares and $\mathrm{x}$ 's for 4.6 and 4.7E+7 CFU (i.e., green vs. pink

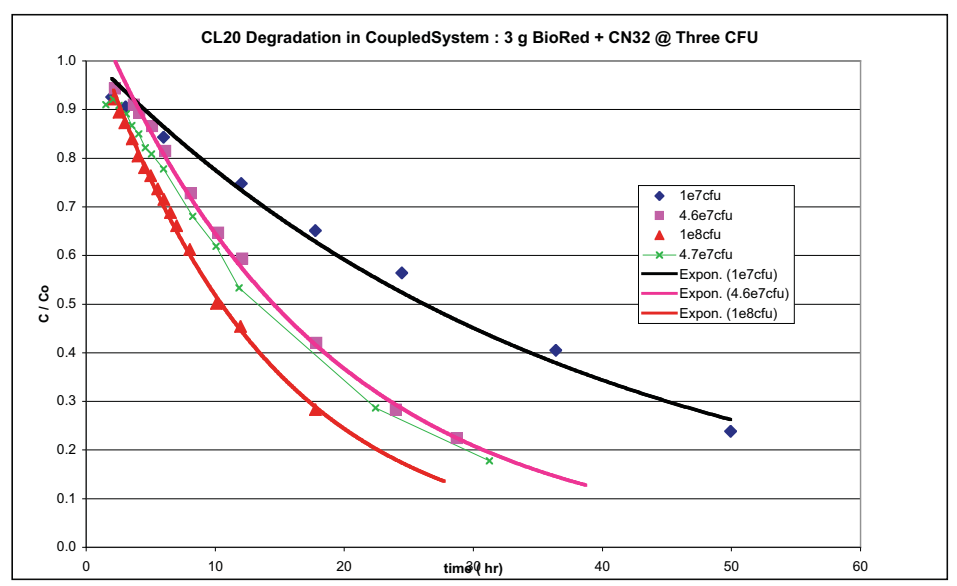

Figure 3.13. CL-20 coupled degradation data). The solid points at the three cell concentrations were inoculated from the same CN-32 starter culture, whereas points indicated by ' $x$ ' were from a prior experiment and inoculated with organisms grown up from a different but identical starter culture. Degradation half lives for the low, medium, and high cell concentrations were 26.6, 12.3 and $9.3 \mathrm{~h}$, respectively. The half live decreased by a factor of 3 for the factor of 10 increase in cell 
concentration. For the low, medium, and high cell concentrations the presence of the $\mathrm{Fe}$ (II) sediment increases the degradation rate by a factor of 21,9 , and 5 relative to that observed in homogeneous solution. This enhancement diminished with increasing cell density as shown by a plot of the degradation rate constant normalized by cfu, or $\mathrm{cfu}^{*} \mu \mathrm{mol}$ $\mathrm{Fe}(\mathrm{II})$ versus cell density. This indicates that cell density or microbial activity may be the factor controlling the synergistic enhancement in degradation.

\section{Model Prediction of CL-20} Degradation in Coupled System Based on Independent Biotic and Abiotic Degradation Rates. A simple conceptual model of degradation in the coupled system assumed that abiotic and biotic processes degrade CL-20 independently. Expressed mathematically this model of coupled degradation could be written as $\mathrm{Y}_{\mathrm{m}}=\mathrm{Y}(\mathrm{Fe}(\mathrm{II}) * \mathrm{Y}(\mathrm{CN}-32)$, where $\mathrm{Y}(\mathrm{Fe}(\mathrm{II})$ and $\mathrm{Y}(\mathrm{CN}-32)$ are the first order expressions observed above for individual abiotic and biotic processes, respectively. These expressions and rates are given in Tables 3.1. For example, for the low cell concentration in Figure 3.14, the predicted degradation would be given by the expression, $\mathrm{Ym}=$ $\left(0.96 * \mathrm{e}^{-0.013 * t}\right) *\left(0.98 * \mathrm{e}^{-0.0013^{*} t}\right)$. For all three cell concentrations examined this model under predicts the observed degradation (Figure 3.14a, b, c), yielding half lives for the low, medium, and high cell concentrations of $48.5,36.3$, and $24.6 \mathrm{~h}$, respectively. The observed half lives were factors of 1.8, 2.9, and 2.7 times greater, respectively, than predicted values (Table 3.1).
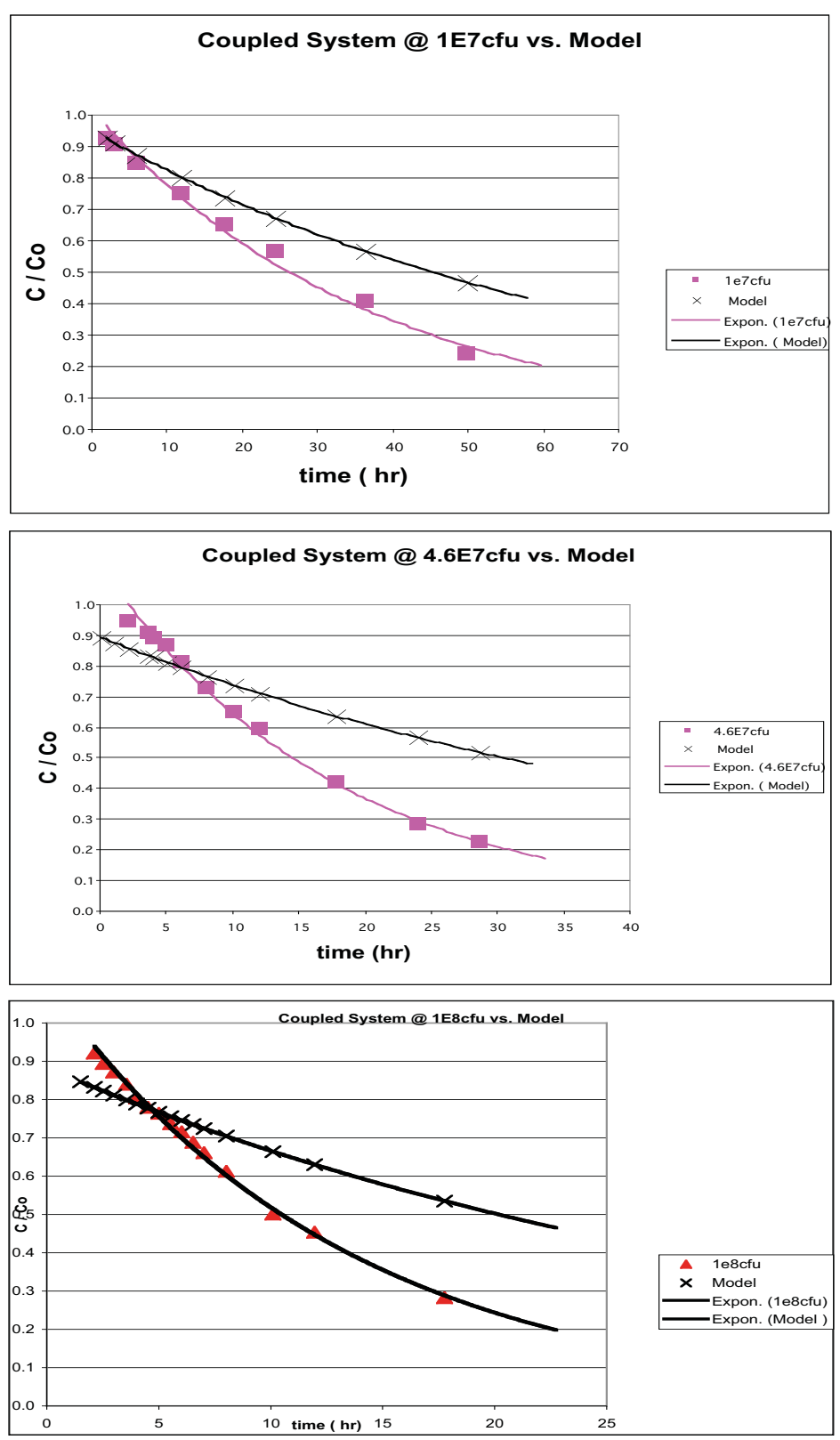

Figure 3.14. Comparison of observed coupled degradation to predicted degradation, based on independent processes 
Table 3.1. Rates of coupled CL-20 abiotic/biotic degradation

Coupled Systems (3 g BioRed FRC+CN-32@3 CFU + 2ppm CL-20

Fit Data with:
\begin{tabular}{|c|c|c|c|}
\hline & $\mathrm{Y}_{\mathrm{o}}=\mathrm{C} / \mathrm{Co}=\mathrm{A} * \exp (-\mathrm{b} * \mathrm{t})$ \\
\hline $\mathrm{cfu}$ & $1.00 \mathrm{E}+07$ & $4.60 \mathrm{E}+07$ & $1.00 \mathrm{E}+08$ \\
\hline $\mathrm{A}$ & 1.02 & 1.13 & 1.1 \\
\hline $\mathrm{b}$ & 0.0271 & 0.0564 & 0.0755 \\
\hline $\mathrm{R}^{*} 2$ & 0.984 & 0.997 & 0.997 \\
\hline $\mathrm{t}(1 / 2)(\mathrm{hr})$ & 26.6 & 12.3 & 9.2 \\
\hline$\mu \mathrm{mol} \mathrm{Fe}(\mathrm{II})$ & 214.5 & 214.5 & 214.5 \\
\hline $\mathrm{b} / \mu \mathrm{mol} \mathrm{Fe}(\mathrm{II})$ & $1.26 \mathrm{E}-04$ & $2.63 \mathrm{E}-04$ & $3.52 \mathrm{E}-04$ \\
\hline $\mathrm{b} / \mathrm{cfu}$ & $2.71 \mathrm{E}-09$ & $1.23 \mathrm{E}-09$ & $7.55 \mathrm{E}-10$ \\
\hline $\mathrm{b} / \mathrm{cfu} * \mu \mathrm{mol} \mathrm{Fe}(\mathrm{II})$ & $5.81 \mathrm{E}-07$ & $2.63 \mathrm{E}-07$ & $1.62 \mathrm{E}-07$ \\
\hline $\mathrm{b}($ coupled $) / \mathrm{b}(\mathrm{bugs})$ & 21 & 9 & 5 \\
\hline
\end{tabular}

Thus, in the coupled system, a significant synergistic enhancement of degradation occurs relative to the individual processes acting independently. Comparing the enhancement in half lives, degradation increased $60 \%$ with the $\mathrm{x} 5$ increase in cell density from 1 to $4.6 \times 10^{7} \mathrm{cfu}$, but remained relatively constant with another $\mathrm{x} 2$ increase to $1 \times 10^{8} \mathrm{cfu}$. This suggests again that the observed enhancement was biotic in nature and that cell density could be the rate-limiting factor responsible for enhancing degradation in this coupled system.

CL-20 Degradation in Coupled System Containing Gibbsite and CN-32. For a coupled system that was identical but contained gibbsite rather than bioreduced FRC (no bioreduced $\mathrm{Fe}(\mathrm{II})$ ), no synergistic enhancement in CL-20 degradation was observed (Figure 3.15). This system consisted of $6 \mathrm{~g}$ of the common $\mathrm{Al}$ oxide mineral gibbsite $\left(\mathrm{Al}(\mathrm{OH})_{3}\right), 2$ ppm CL-20 with a cell density of $4.6 \times 10^{7} \mathrm{cfu}$. Six grams of gibbsite provided the same surface area as $3 \mathrm{~g}$ of bioreduced FRC. The rate of CL-20 degradation was determined in the presence of $6 \mathrm{~g}$ gibbsite without CN-32 (Table 3.2.) Using this rate constant and the rate constant for biotic degradation at $4.6 \times 10^{7} \mathrm{cfu}$ in homogeneous solution in the predictive model of independent abiotic and biotic processes, the observed and predicted degradation agree almost exactly. Certainly, gibbsite was not a surrogate for bioreduced FRC without the Fe(II),

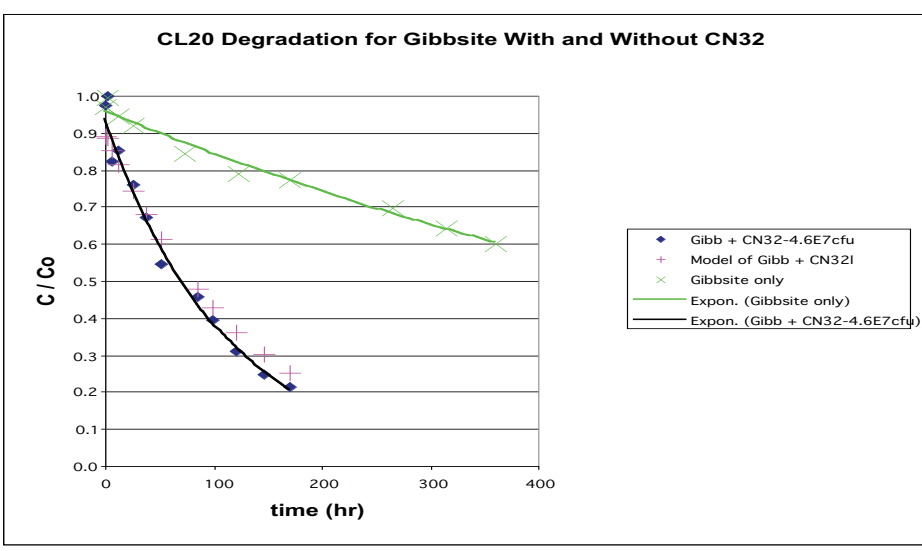

Figure 3.15. Degradation of CL-20 with gibbsite only and in a coupled system containing gibbsite and $\mathrm{CN}-32$ at $4.6 \times 10^{7} \mathrm{cfu} / \mathrm{ml}$. Gibbsite surface area is $16 \mathrm{~m}^{2} / \mathrm{g}$. 
but this result indicates that the synergistic enhancement observed for the bioreduced FRC was not due to the attachment of CN-32 to the solid of to proximity of sorbed CL-20 and attached CN-32.

Table 3.2. CL-20 coupled degradation in gibbsite

\begin{tabular}{|c|c|c|}
\hline \multicolumn{3}{|l|}{ Abiotic and Coupled Degradation for 6 g Gibbsite } \\
Fit Data with : $\quad \mathrm{Y}=\mathrm{C} / \mathrm{Co}=\mathrm{A}^{*} \exp (-\mathrm{b} * \mathrm{t})$ \\
$\mathrm{CL}-20=2 \mathrm{ppm}$ & Abiotic & \\
\hline & 0 & Coupled \\
\hline $\mathrm{CN}-32 \mathrm{cfu}$ & 0.96 & $0.60 \mathrm{E}+07$ \\
$\mathrm{~A}$ & 0.0013 & 0.038 \\
$\mathrm{~b}$ & 0.985 & 0.992 \\
$\mathrm{R}^{*} 2$ & 533 & 78 \\
$\mathrm{t}(1 / 2)(\mathrm{hr})$ & NA & $1.93 \mathrm{E}-10$ \\
$\mathrm{~b} / \mathrm{cfu}$ & &
\end{tabular}

CL-20 Degradation in Modified Coupled System. To examine the nature of the bioreduced Fe(II) in the FRC sediment and its role in the enhancement of CL-20 degradation in the coupled FRC-CN-32 system, extractions were performed on separate $3 \mathrm{~g}$ samples of bioreduced sediment to selectively remove Fe(II). Under anaerobic conditions, the first sediment was treated with $1 \mathrm{M} \mathrm{CaCl}_{2}$ in $30 \mathrm{mM}$ pipes buffer at $\mathrm{pH} 7$ for $1 \mathrm{~h}$, then centrifuged and rinsed pipes buffer three times. Operationally, this treatment removes ion exchangeable Fe(II) from the surface (Heron et al. 1994). A similar $1 \mathrm{~h}$ extraction with $0.5 \mathrm{M} \mathrm{HCl}$ was performed on a separate $3 \mathrm{~g}$ sample, then centrifuged and rinsed 7 times with $30 \mathrm{mM}$ pipes $(\mathrm{pH} 7)$ to achieve a final solution $\mathrm{pH}$ of 7 . The $\mathrm{CaCL}_{2}$ and $\mathrm{HCl}$ extractions removed $2 \%$ and $99 \%$ of the bioreduced $\mathrm{Fe}(\mathrm{II})$ reported for the $3 \mathrm{~g}$ sediments. These extracted sediments were then inoculated with $\mathrm{CN}-$ 32 to yield a cell density of $4.6 \mathrm{E} 7 \mathrm{cfu} / \mathrm{ml}$ to compare CL-20 degradation for these treatments (Table 3.3, Figure 3.16) and contrast this degradation to the untreated coupled system with the same cell density (Figure 3.14b).

Degradation of CL-20 in $\mathrm{CaCl}_{2}$ treated sediment was virtually identical to that observed for the untreated sediment suggesting that exchangeable Fe(II) is playing a negligible role in CL-20 degradation (compare rates constants and half lives in Table 3.2 and 3.3) and in its enhancement relative to model predictions (middle curve in Figure 3.16). The model prediction for the $\mathrm{CaCl}_{2}$ treatment (' $\mathrm{x}$ ' in Figure 3.16, $\mathrm{t}(1 / 2)=36.3 \mathrm{~h}$ ) is by definition identical to that in Figure 3.14b. The $\mathrm{HCl}$ extraction dramatically reduces degradation relative to that observed for the $\mathrm{CaCl}_{2}$ treated or untreated bioreduced sediment (Figures 3.14b and 3.16). The half-life for the $\mathrm{HCl}$ treatment is $55 \mathrm{~h}$ or a factor of 5 greater that that of the untreated bioreduced FRC with the same cell density. This result adds further support to our assertion that biogenic Fe(II) is the dominant abiotic reducing agent for CL-20 degradation. Although the $1 \mathrm{~h} \mathrm{HCl}$ extractable $\mathrm{Fe}(\mathrm{II})$ is considered to remove the most reactive of the biogenic Fe(II) without dissolution of Fe(III), additional biogenic Fe(II) remains as subsequent extractions and/or $2 \mathrm{~h}$ and $24 \mathrm{~h}$ extractions have demonstrated (data not shown). This residual Fe(II) may account for the difference between the degradation observed for the $\mathrm{HCl}$ treatment plus $\mathrm{CN}-32$ and that predicted for this system using 
an abiotic component consisting of untreated FRC ( $3 \mathrm{~g}$ sediment blank) and the biotic component for CN-32 degradation at $4.6 \mathrm{E} 7 \mathrm{cfu} / \mathrm{ml}$ (upper curve, $\mathrm{t}(1 / 2)=93.7 \mathrm{hr}$, Figure 3.16).

Table 3.3. Coupled CL-20 degradation in FRC sediment after iron extractions

Coupled System - Extracted Bioreduced FRC + CN-32

Fit Data with: $\quad \mathrm{Y}=\mathrm{C} / \mathrm{Co}=\mathrm{A} * \exp (-\mathrm{b} * \mathrm{t})$

$\mathrm{CL}-20=2 \mathrm{ppm}$

CN-32@4.6 E7 cfu/ml

\begin{tabular}{|c|c|c|}
\multirow{2}{*}{$\begin{array}{c}\text { Fe(II) Extractant } \\
\text { Conditions }\end{array}$} & $\mathrm{CaCl}_{2}$ & $\mathrm{HCl}$ \\
\cline { 2 - 3 } \% of Fe(II) removed [1] & $1 \mathrm{M} / 1 \mathrm{~h}$ & $0.5 \mathrm{M} / 1 \mathrm{~h}$ \\
$\mathrm{~A}$ & 2 & 99 \\
$\mathrm{~b}$ & & \\
$\mathrm{R}^{*} 2$ & 0.94 & 1.03 \\
$\mathrm{t}(1 / 2)(\mathrm{h})$ & 0.0487 & 0.0127 \\
$\mathrm{~b} / \mathrm{cfu}$ & 0.95 & 0.99 \\
& 14.2 & 54.6 \\
\hline
\end{tabular}

[1] Percentages are based on prior $0.5 \mathrm{M}, 1 \mathrm{~h}$ extractions of identical samples and are on a per gram basis.

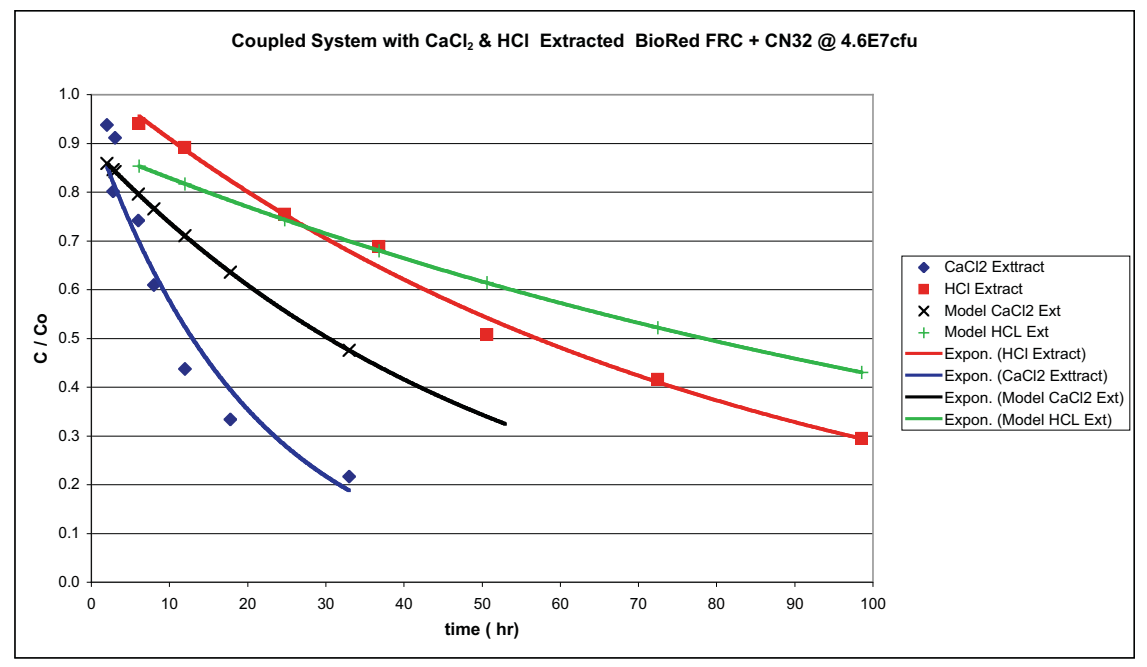

Figure 3.16. Degradation for coupled systems containing bioreduced FRC that were extracted with $1 \mathrm{M} \mathrm{CaCl}_{2}$ and $0.5 \mathrm{M}$ $\mathrm{HCl}$ for $1 \mathrm{~h}$ (solid points). The degradation half lives predicted with the independent process model for the $\mathrm{CaCL}_{2}$ and $\mathrm{HCl}$ treatments plus CN-32 were 36.3 and $93.7 \mathrm{~h}$, and shown by ' $\mathrm{x}$ ' and '+', respectively. 
Summary of Coupled Process Experiments. The following conclusions and hypotheses can be stated as follows. (1) The biogenic Fe(II) produced by $\mathrm{CN}-32$ under reducing conditions is a highly effective reducing agent promoting CL-20 degradation with $\mathrm{Fe}$ (II) oxidized to $\mathrm{Fe}$ (III). (2) The rates of degradation increase dramatically as the molar Fe(II):CL-20 ratio increases. (3) $\mathrm{CN}-32$ bacteria degrade CL-20, with degradation rates increasing with increasing cell density. However, at the lowest cell concentration examined $\left(1 \times 10^{7} \mathrm{cfu} / \mathrm{ml}\right)$, which is higher than typical subsurface population densities, degradation half lives are on the order of $533 \mathrm{~h}$, which is in the range observed under anaerobic conditions ( 433 to $693 \mathrm{~h}$ ) for degradation by FRC sediment which had not been bioreduced. (4) When $\mathrm{CN}-32$ is added to bioreduced FRC sediment, a synergistic enhancement in degradation was observed, that is the resultant rate of degradation was greater by up to a factor of 3 than that of the biotic and abiotic degradation processes acting independently. With $3 \mathrm{~g}$ of bioreduced sediment the degradation half life for the lowest cell concentration was $26 \mathrm{~h}$ (Figure 3.14a). This is a factor of 21 times faster than the CN-32 alone and a factor of 2 faster than degradation occurring with $3 \mathrm{~g}$ of bioreduced sediment alone. Thus, it is clear that the $\mathrm{CN}-32$ bacteria even at the lowest cell density are playing an important role in CL-20 degradation. (5) The synergistic enhancement is believed to be mediated by and rate limited by the $\mathrm{CN}-32$ bacteria. (6) It is our hypothesis that the enhancement is the result of rapid bacterial reduction of the Fe(III) resulting form CL-20 degradation by biogenic Fe(II). That is, $\mathrm{Fe}(\mathrm{II})-\mathrm{Fe}(\mathrm{III})$ cycling is being promoted by the bacteria on the FRC surface.

\section{Task 3.2 Aging}

Because CL-20 will sorb then degrade with sediments, it is important to address reactions that may occur in the natural environment, such as prolonged exposure of CL-20 to sediments. Aging was investigated by two different approaches: a) CL-20/RDX stop-flow column experiments, where desorption mass, desorption rate, and degradation was investigated as a function of contact time before desorption, and b) CL-20/RDX artificial aging using a supercritical fluid system.

Aging Column Experiments. A series of 24 column experiments were conducted to address the CL-20 and RDX aging with two different sediments. Each column aging experiment consists of a sorption phase, then lag phase from 1 to $2400 \mathrm{~h}$, after which the CL-20 and RDX is desorbed from the column (Figure 3.17 - sediment with organic carbon; Figure 3.18 - sediment with very low organic carbon). Putting all of the data together in single plots (Figure 3.19, only CL-20 shown) clearly shows very similar behavior for sorption limbs of the experiments (all should be the same), and vastly differing desorption behavior, depending upon the amount of aging time. In general, CL-20 desorbs much more slowly with greater aging time. All aging data is in Appendix I (RDX, CL-20).

It is hypothesized that:

a) additional binding to organic matter could occur leading to a desorption-resistant fraction (aging hypothesis for organic carbon)

b) CL-20 diffusion into microfractures in low organic carbon sediment will also lead to a desorption-resistant fraction (aging hypothesis for minerals

c) the degradation rate may be apparently slower due to no mixing. 
To address these hypotheses, the sorption and desorption data needs to be characterized for:

- desorption rate (model slope of the desorption limb versus adsorption $\operatorname{limb}$

- $\mathrm{K}_{\mathrm{d}}$ desorption versus adsorption limb from breakthrough curve mass balance and lag before the breakthrough

- the degradation rate from mass balance observed versus calculated mass balance using the aging time.

To collect this type of data, a significant amount of simulations were performed to fit the breakthrough curves. In addition to RDX and CL-20 data, modeling is also needed for the tracer data in order to determine the longitudinal dispersivity in the column system, which is needed. Parameters that were calculated from the data are in Appendix I, which includes tracer simulations (24), RDX simulations (22), and CL-20 simulations (46).

Aging and Sorption Mass. Both CL-20 and RDX showed smaller apparent desorption $\mathrm{K}_{\mathrm{d}}$ values with greater aging time (Figure 3.20). What is actually plotted is the ratio of desorption/ sorption $\mathrm{K}_{\mathrm{d}}$ observed in each experiment, so a value of $<1$ is a smaller $\mathrm{K}_{\mathrm{d}}$ value for desorption.

In these oxic, aseptic conditions of the experiments, RDX should not be degraded, so the smaller $\mathrm{K}_{\mathrm{d}}$ values for RDX reflect less mass desorption (i.e., a desorption resistant fraction). CL-20 will undergo some abiotic degradation, and a larger decrease in the desorption $\mathrm{K}_{\mathrm{d}}$ was observed.

Aging and Sorption Rate. Both CL-20 and RDX showed greater breakthrough curve tailing with more aging time, as shown in Figures 3.17 and 3.18. A model simulating breakthrough 


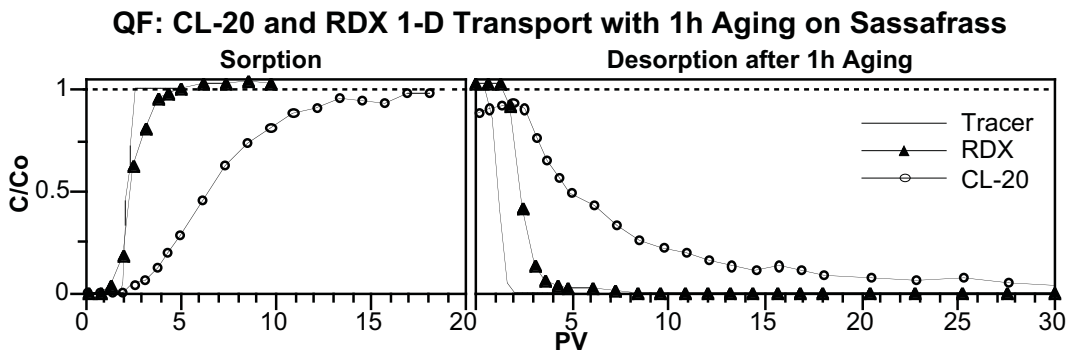

QH: CL-20 and RDX 1-D Transport with 112h Aging on Sassafrass

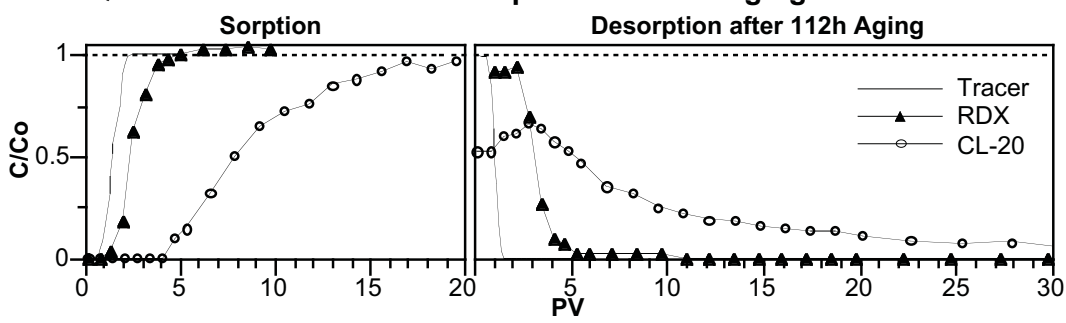

QJ: CL-20 and RDX 1-D Transport with 737h Aging on Sassafrass

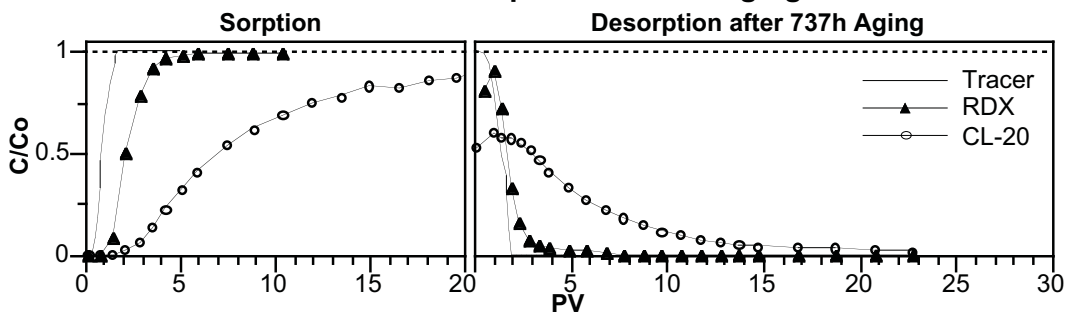

QN: CL-20 and RDX 1-D Transport with 2254h Aging on Sassafrass

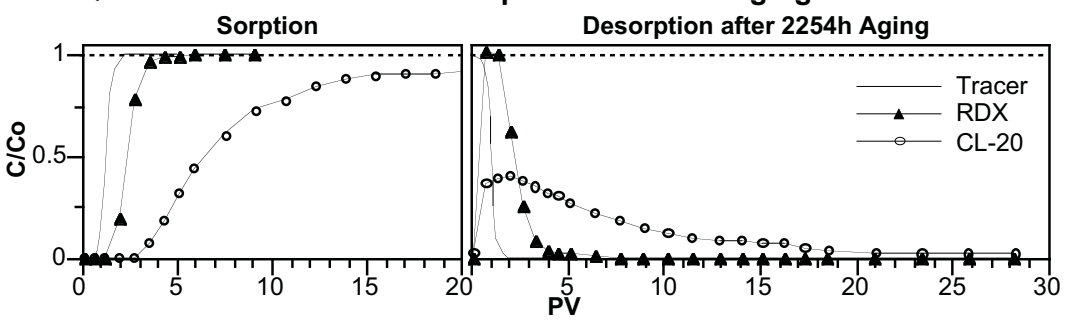

Figure 3.18. CL-20 sorption and desorption in aged columns for the Sassafrass sediment $(0.3 \%$ organic carbon $)$ with a first-order sorption and first-order mass loss was used to quantify the desorption rate. The CL-20 desorption rate showed a slight slowing with aging for the high $\mathrm{f}_{\text {oc }}$ sediment and no regular change for the low $f_{0 c}$ sediment (Figure 3.21a). In contrast, the RDX desorption rate showed a large decrease with aging time for the high $\mathrm{f}_{\mathrm{oc}}$ sediment, and no change for the low $\mathrm{f}_{\mathrm{oc}}$ sediment (Figure 3.21b).

This indicates that RDX and possibly CL-20 would desorb more slowly with long sediment/solute contact times for sediments with organic carbon possibly due to greater binding with the organic matter over time. The hypothesis of diffusion into microfractures for sediments with little or no organic matter was not apparent from any of the experimental data. While this hypothesis has been proven for small molecular weight organic compounds

(TCE, $\mathrm{CCl}_{4}$, for example), the somewhat larger size of the RDX and CL-20 molecules may preclude diffusion into microfractures, or the sediment chosen for this (Sassafrass silty loam) may have few microfractures.

Aging and the Abiotic Degradation Rate. CL-20 abiotically degraded slowly during the aging experiments. The CL-20 degradation rate was averaged for all sorption experiments (horizontal bar, Figure 3.21) and used to calculate the mass of CL-20 that should be degraded during the aging experiments. The observed rate of CL-20 degradation was calculated from the desorption experiments by mass balance eluted (i.e., water injection) and additionally during extraction using methanol. Surprisingly, there was greater CL-20 mass remaining than predicted. This indicated that the CL-20 degradation rate during the no-flow aging experiments showed apparent slowing with increasing aging time (Figure 3.22). 
CL-20 breakthrough during 1-D flow on West. (Sorb Phase)

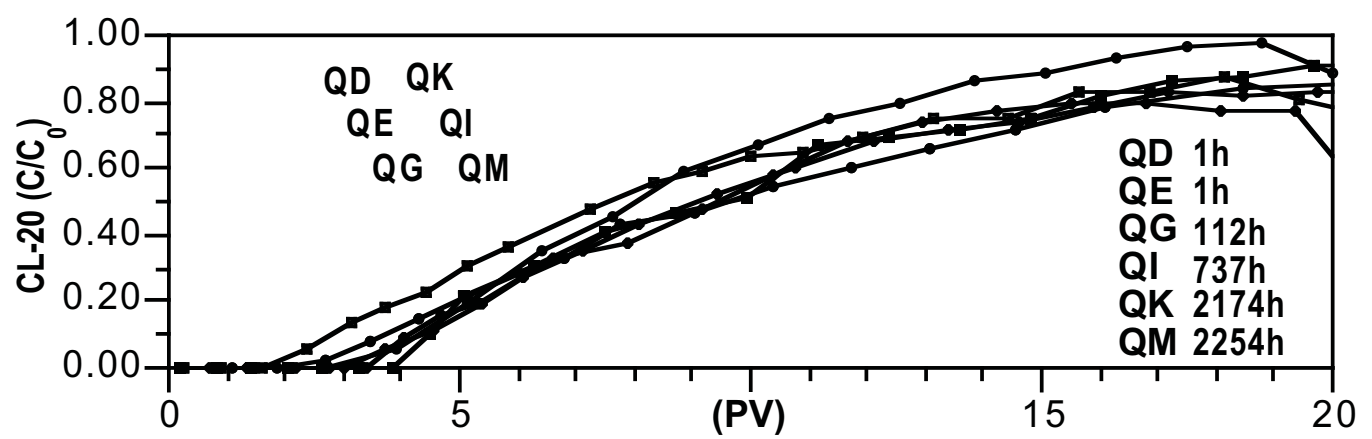

CL-20 breakthrough during 1-D flow on Sass. (Sorb Phase)

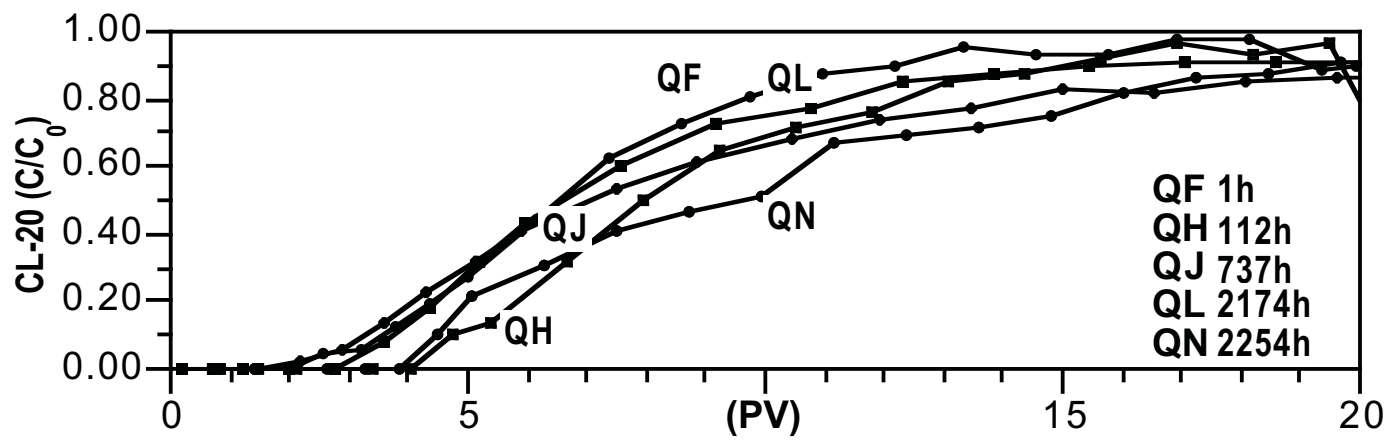

CL-20 breakthrough during 1-D flow on West. (DeSorb Phase)

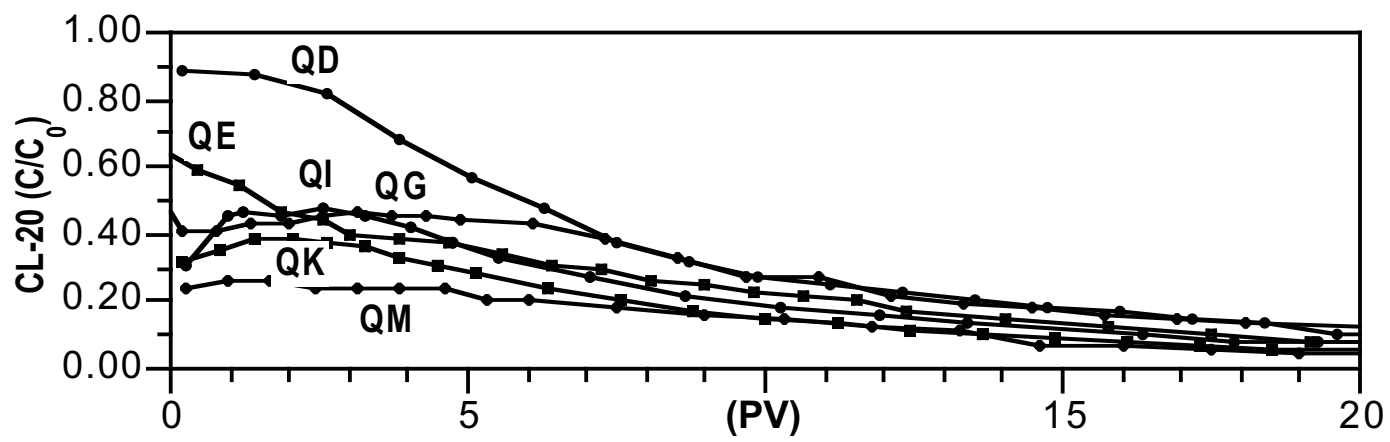

CL-20 breakthrough during 1-D flow on Sass. (DeSorb Phase)

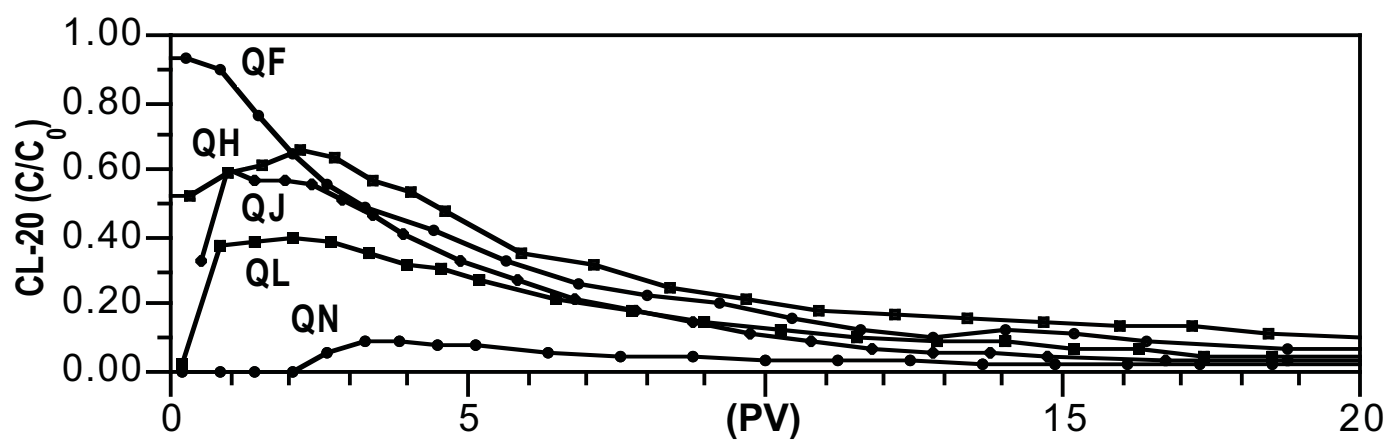

Figure 3.19. CL-20 sorption and desorption in aged columns 

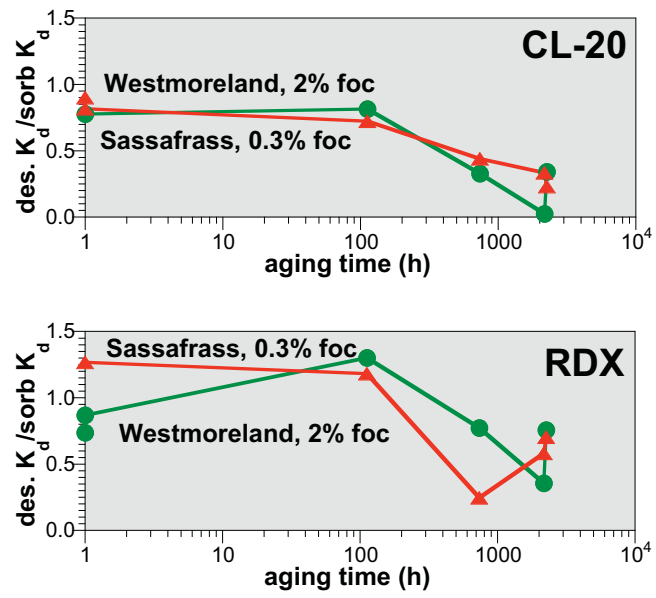

Figure 3.20. Change in desorption $\mathrm{K}_{\mathrm{d}} /$ sorption $\mathrm{K}_{\mathrm{d}}$ for a) CL-20 and b) RDX
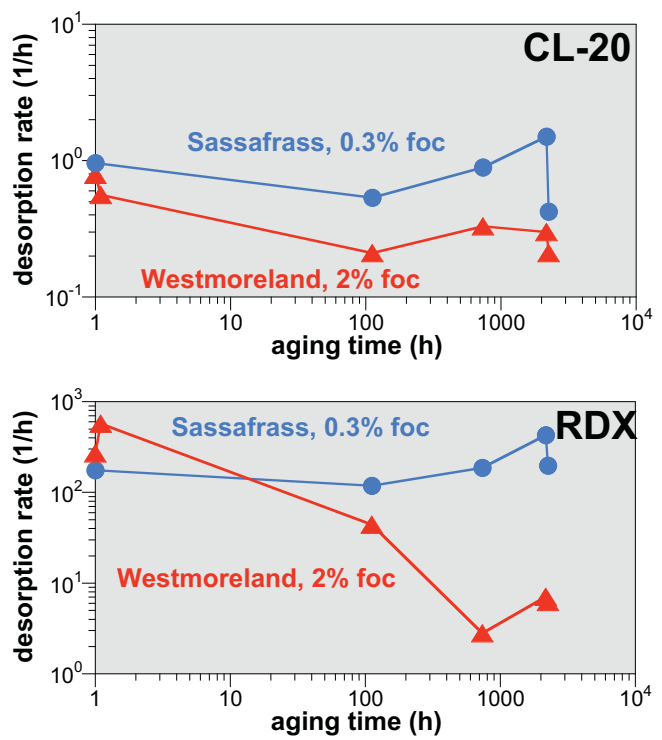

Figure 3.21. Change in desorption rate for a) CL-20 and b) RDX

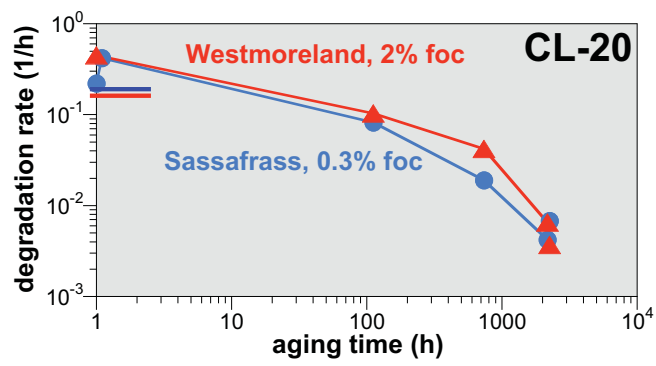

Figure 3.22. Change in degradation rate for CL-20 with aging time
It is hypothesized that CL-20 was relatively immobile on sorption sites and may not have had as much access to surface sites that could cause degradation (i.e., lack of flow meant that transport of CL-20 was limited to diffusion). The importance of a slower abiotic degradation rate is that CL-20 will be more persistent in soils and in groundwater than predicted with short time scale experiments.

Artificial Aging. This task was designed to study the potential effects of weathering of soils on the slow release and availability of CL-20 to the environment. To simulate the effects of aging on soils, a supercritical fluid system was used to load the CL-20 onto soils. Supercritical carbon dioxide has properties (Lee and Markides 1990) that allow chemical adsorption and equilibrium to occur on a time scale amenable to laboratory study. Stability experiments were conducted to determine the stability and recovery of CL-20 in circulating supercritical carbon dioxide. CL-20 was loaded into a stainless steel vessel and circulated in the supercritical fluid system for $1 \mathrm{~h}$. The circulating stream was monitored in real-time for changes in the magnitude of UV absorbance at $224 \mathrm{~nm}$. CL-20 and its potential degradation products were trapped in vials containing methanol, and the solutions were analyzed by HPLC. The stainless steel vessel was also extracted with methanol to determine CL-20 residue.

Supercritical carbon dioxide does not appear to be a good solvent for CL-20, as it degraded over several hours (Figure 3.23). When CL-20 is introduced, the initial CL-20 concentration, as measured by the UV absorption at $230 \mathrm{~nm}$ (Figure 3.23) is zero, but should increase to a steady concentration as the CL-20 is evenly distributed throughout the recirculating system. The real-time UV detector indicated that little CL-20 was circulating in the system, and the calculated recovery of the trapped material was $<1 \%$. The residue in the sample vessel was about $15 \%$ of the total mass added to the system. In an attempt to increase solubility, methanol was used as a cosolvent (approximately 10\% by volume) in the supercritical carbon dioxide. Recovery improved to $5 \%$; residue was $12 \%$. Additional CL-20 residue was 
collected from the system in subsequent blank runs. Consequently, a significant amount of CL-20 was not solubilized in the initial experiment. It is also likely that some degradation occurred based on the UV spectra of the trapped material and the residue.

Two experiments were conducted in which samples of Hanford soil were loaded with CL-20 for $4 \mathrm{~h}$. The first used 10\% methanol and the second used $10 \%$ acetonitrile as cosolvents in supercritical carbon dioxide. In each case, the soil was extracted with methanol using sonication. Recovery was poor, at $3 \%$ and $5 \%$, respectively. Based on UV spectra, there appears to be some degradation. Samples were analyzed by LC-MS, but sensitivity was insufficient to determine the presence of CL-20 or degradation products. 


\section{Task 4. Reactive Transport of CL-20}

The movement of CL-20 through natural sediments shows the relative importance of sorption and degradation on CL-20 mass and the potential for vadose zone and groundwater contamination. A total of 40 water-saturated columns were conducted (Appendix H, summarized below) with an additional 24 aging columns previously described (Appendix I, Task 3.2). A total of 30 unsaturated column experiments (PFBA, RDX, CL-20) at variable water saturation (Task 4.3, all data in Appendix J) were conducted.

\section{Task 4.1/4.2. CL-20 Transport in Water-Saturated Systems with Sorption and Abiotic/ Biotic Degradation.}

The relative velocity and mass of CL-20 transported through subsurface sediments depends on the influences of sorption and degradation relative to the transport time-scale. The relatively small $\mathrm{K}_{\mathrm{d}} \mathrm{s}$ for CL-20 in $2 \mathrm{~h}$ batch experiments (Task 1.1) suggest nearly unretarded transport. However, because degradation varied from near zero for the Kenoma sediment to $100 \%$ for Ocala, degradation may ultimately limit the extent of subsurface contamination. Reactive transport experiments were conducted to address CL-20 sorption and degradation behavior that would be observed at the high sediment/water ratios of natural subsurface systems (10x to 1000x greater than the sediment/water ratio in batch studies). These column experiments for CL-20 were conducted for a variety of sediments under oxic conditions (Figures 4.1 through 4.3 and Table 4.1) and showed a wide range of behaviors with differences in sorption (i.e., lag relative to the tracer) and rate of abiotic degradation (i.e., final steady-state CL-20 concentration).

Tracer breakthrough, which averaged $0.991 \pm 0.048$ pore volumes for all 40 column experiments, agreed well with the calculated porosity from dry and water-saturated sediment weight in the column. The CL-20 extracted from the sediment columns with methanol yielded $\mathrm{K}_{\mathrm{d}}$ values that averaged $22 \%$ greater (Table 4.1) than $\mathrm{K}_{\mathrm{d}}$ values from aqueous CL-20 breakthrough, although the difference was not statistically significant. The methanol extraction data yielded CL-20 degradation half-lives that agreed with mass balance from aqueous CL-20 breakthrough (Table 4, methanol extractions were $3.8 \%$ larger). Based on the agreement between the methanol extraction and breakthrough methods of estimating $\mathrm{K}_{\mathrm{d}} \mathrm{s}$ and degradation, we will refer to the breakthrough data (Table 4) in the discussion of $\mathrm{K}_{\mathrm{d}} \mathrm{s}$ and degradation below.

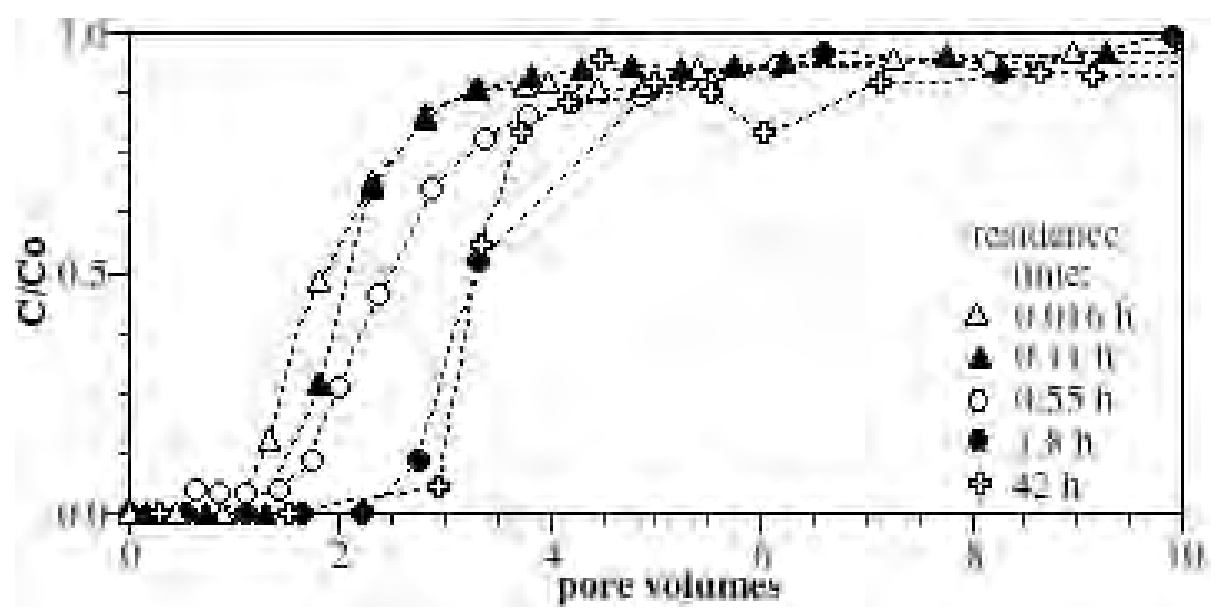

Figure 4.1. CL-20 reactive transport at five different velocities in Norborne sediment 

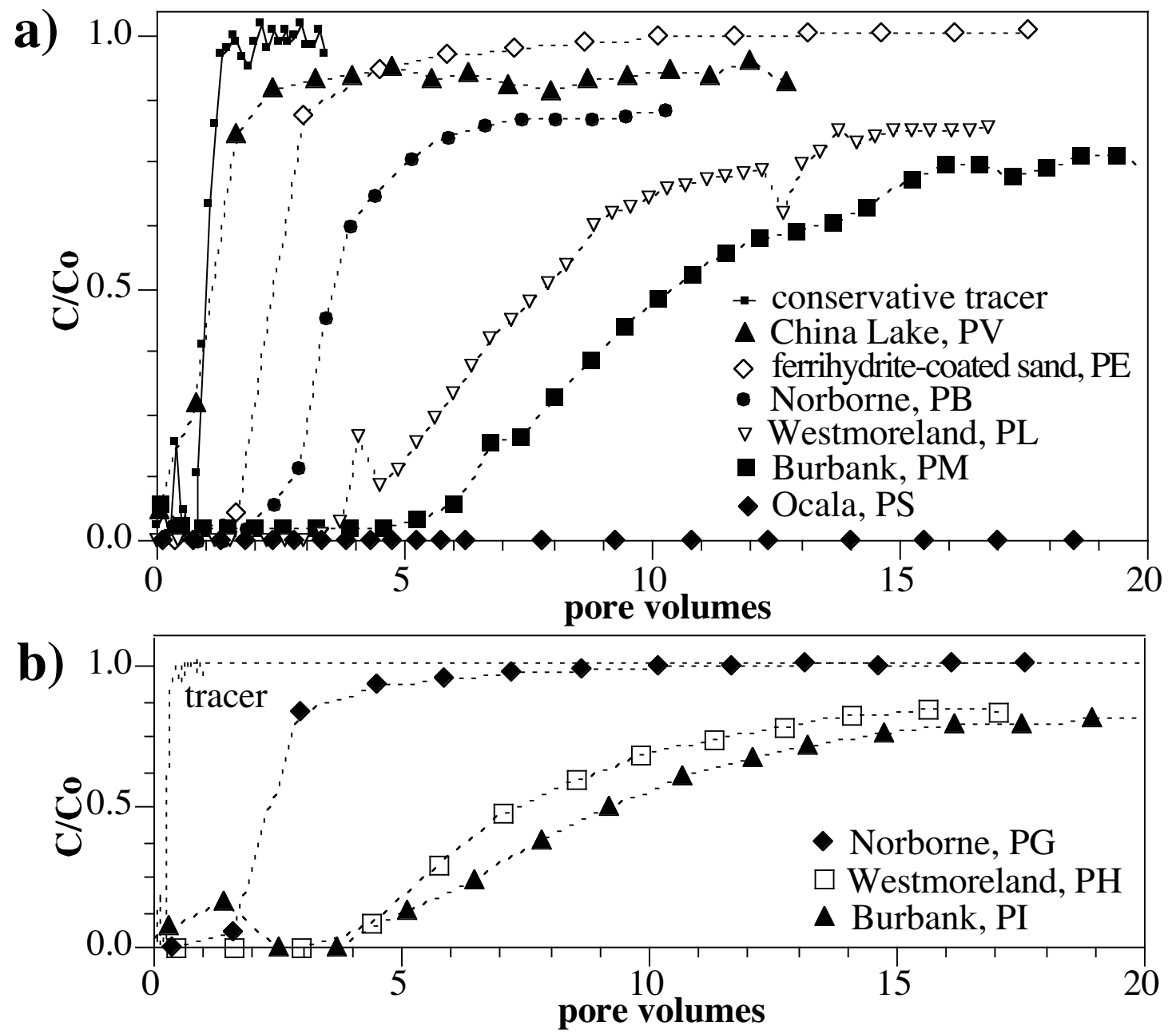

Figure 4.2. Reactive transport of CL-20 through oxic sediments illustrating differing sorption or lag relative to a tracer, and differing degradation rates, based on the steadystate concentration reached. Experiments shown differ in: a) sediment flow rate flow rate, and b) sediment type and at the same flow rate $(0.36 \mathrm{~h} / \mathrm{pv})$. Experiment label after sediment type (i.e., PV) in Table 4.1, where residence time and CL-20 influent concentration are given. 


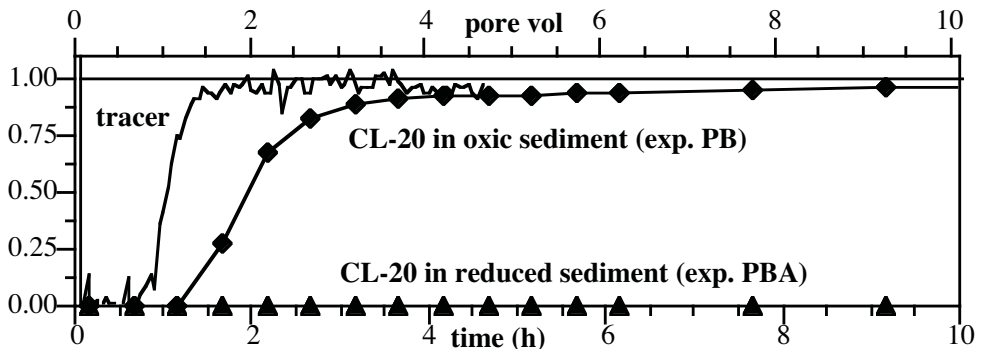

Figure 4.3. CL-20 degradation in oxic and reduced Norborne sediment columns during 1-D flow
For the Norborne sediment, which for batch conditions exhibited minimal degradation, experiments were conducted at five different velocities to assess the adsorption/desorption rate (Figure 4.1). Reactive transport simulations of these breakthrough curves using a model with a single first-order reversible reaction yielded an average sorption half-

life of 3.6 minutes $\left(\mathrm{k}_{\mathrm{f}}=15 \mathrm{~h}^{-1}\right.$; batch half-life $\left.0.8 \mathrm{~min}\right)$, an average desorption half-life of 22 minutes $\left(\mathrm{k}_{\mathrm{b}}=1.9 \mathrm{~h}^{-1}\right)$, and an average $\mathrm{K}_{\mathrm{d}}$ of $0.52 \pm 0.28 \mathrm{~cm}^{3} \mathrm{~g}^{-1}$ (Table 4.1). This $\mathrm{K}_{\mathrm{d}}$ derived from these column experiments is roughly comparable to the value of $0.22 \mathrm{~cm}^{3} \mathrm{~g}^{-1}$ obtained for 2 $\mathrm{h}$ batch experiments, whereas average column $\mathrm{K}_{\mathrm{d}}$ and the batch $\mathrm{K}_{\mathrm{d}}$ for the Westmoreland sediment are in closer agreement, $3.23 \pm 0.35$ and $3.83 \mathrm{~cm}^{3} \mathrm{~g}^{-1}$, respectively.

Table 4.1 CL-20 sorption mass and degradation rates in 1-D columns

\begin{tabular}{|c|c|c|c|c|c|c|}
\hline \multirow[b]{2}{*}{ Sediment } & \multirow[b]{2}{*}{ exp. } & \multirow[b]{2}{*}{$\begin{array}{c}\text { residence } \\
\text { time (h) }\end{array}$} & \multirow[b]{2}{*}{$\begin{array}{c}K_{d} \\
\left(\mathrm{~cm}^{3} \mathbf{g}^{-1}\right)\end{array}$} & \multicolumn{3}{|c|}{ CL-20 degradation } \\
\hline & & & & $\begin{array}{c}\text { mass loss } \\
(\%)\end{array}$ & $\begin{array}{l}\text { half-life } \\
\text { (h) }\end{array}$ & $\begin{array}{c}\text { rate } \\
\left(\mathrm{mol} \mathrm{h}^{-1} \mathrm{~g}^{-1}\right)\end{array}$ \\
\hline \multirow[t]{7}{*}{ Norborne C } & $\mathrm{PD}$ & 0.016 & 0.48 & 0.32 & 3.6 & $5.5 \mathrm{E}-07$ \\
\hline & $\mathrm{PC}$ & 0.11 & 0.44 & 2.3 & 3.2 & $6.3 \mathrm{E}-07$ \\
\hline & PG & 0.35 & 0.54 & 3.6 & 6.6 & $3.1 \mathrm{E}-07$ \\
\hline & PB & 0.55 & 0.72 & 7.4 & 4.9 & 4.1E-07 \\
\hline & $\mathrm{PA}$ & 1.8 & 1.01 & 17.2 & 6.7 & $3.0 \mathrm{E}-07$ \\
\hline & PK & 3.9 & 0.26 & 11.3 & 22.4 & $9.0 \mathrm{E}-08$ \\
\hline & $\mathrm{PP}$ & 42 & 0.19 & 22.1 & 118 & $1.7 \mathrm{E}-08$ \\
\hline \multirow[t]{3}{*}{ Westmoreland } & $\mathrm{PH}$ & 0.36 & 3.21 & 9.0 & 1.2 & $1.7 \mathrm{E}-06$ \\
\hline & PL & 4.0 & 3.26 & 35.3 & 6.4 & $3.2 \mathrm{E}-07$ \\
\hline & PQ & 34 & -- & 93.2 & 8.8 & $2.3 \mathrm{E}-07$ \\
\hline \multirow[t]{3}{*}{ Burbank } & PI & 0.38 & 2.21 & 2.21 & 2.2 & $9.1 \mathrm{E}-07$ \\
\hline & $\mathrm{PM}$ & 3.04 & 2.14 & 20.1 & 9.4 & $2.1 \mathrm{E}-07$ \\
\hline & PR & 31 & 1.43 & 54.4 & 27.4 & 7.4E-08 \\
\hline Ocala & PS & 32 & -- & 100 & -- & -- \\
\hline \multirow[t]{3}{*}{ China Lake } & PV & 2.0 & 0.32 & 0.25 & 550 & 3.7E-09 \\
\hline & PW & 2.5 & 0.16 & 0.38 & 450 & 4.5E-09 \\
\hline & PX & 1.8 & 0.27 & 0.16 & 780 & 2.6E-09 \\
\hline \multirow[t]{2}{*}{ Ferrihydrite sand } & $\mathrm{PF}$ & 0.13 & 0.47 & 2.5 & 3.6 & $5.6 \mathrm{E}-07$ \\
\hline & $\mathrm{PE}$ & 2.0 & 0.58 & 15 & 8.6 & $2.3 \mathrm{E}-07$ \\
\hline
\end{tabular}

In the reactive transport experiments for the other sediments (Figure 4.2a), the CL- 20 degradation rates for these oxic sediments ranged from a few hours to $780 \mathrm{~h}$, as defined by a pseudo-first-order reaction (Table 4.1). The China Lake sediments exhibited the slowest CL-20 
degradation rates ( $450 \mathrm{~h}$ to $780 \mathrm{~h}$ half-life), and with low sorption $\left(\mathrm{K}_{\mathrm{d}}\right.$ averaged $\left.0.25 \mathrm{~cm}^{3} \mathrm{~g}^{-1}\right)$ CL-20 could move considerable distances in the subsurface. The average CL-20 degradation rates were faster for the Burbank sediment (13 $\pm 13 \mathrm{~h}$ half-life), the Westmoreland sediment $(5.5 \pm 3.9 \mathrm{~h})$, the Norborne sediment $(5.0 \pm 1.6 \mathrm{~h})$, and the ferrihydrite-coated sand $(6.1 \pm 3.5 \mathrm{~h})$. For the Norborne, Burbank, and Westmoreland column experiments conducted at the same velocity the relative degradation rates are evident (Figure 4.2b), with respective CL-20 half-lives of 6.6, 2.2 and $1.2 \mathrm{~h}$. The average CL-20 K for all sediments tested in this study was $2.3 \mathrm{~cm}^{3} \mathrm{~g}^{-1}$. From this, we can conclude that sorption of CL-20 by sediments is relatively weak, which is consistent with hypothesis (i). For this study, the average $K_{d}$ for RDX was $2.0 \mathrm{~cm}^{3} \mathrm{~g}^{-1}$. RDX is a typical groundwater contaminant due to its low sorption and low oxic degradation, and migrates essentially unretarded in some groundwater systems (Spalding and Fulton 1988). Considering that CL-20 sorption is, on average, only 10\% greater than RDX, CL-20 has a high risk of becoming a groundwater contaminant, particularly for sediments with low degradation rates, such as the China Lake sediment.

A 1-D column experiment was conducted in which CL-20 (pH buffered to 7.0) was injected through Ocala sediment. Because this sediment has a high (33\%) clay content and is finegrained, water flow could not be achieved, so this sediment was mixed $50 \%$ with silica sand with a resultant residence time of $32 \mathrm{~h}$. This column was pre-equilibrated with 20 pore volumes of the $\mathrm{pH}$ buffer solution. During CL-20 injection, the average effluent $\mathrm{pH}$ was 9.2 , so hydrolysis was possibly a contributing mechanism in CL-20 degradation. Because 100\% of the injected CL-20 was degraded, a degradation rate could not be calculated (Table 4.1). The pressure was high (2600 psi), so faster flow rates could not be achieved.

A possible mechanism for CL-20 abiotic degradation in oxic columns (and in batch) may be an electron transfer reaction (e.g., ferrous iron may be an electron donor) or a catalytic reaction with clay surfaces. In columns with continuous CL-20 injection, a catalytic reaction would continue to occur, whereas an electron-transfer reaction would be limited by the mass of electron donor, e.g., Fe ${ }^{\mathrm{II}}$. To examine this, one column containing the Norborne sediment was used in four successive experiments (PB, PG, PK, and PP; Table 4) and was thus subjected to a significant mass of injected CL-20 (57 pore volumes). The later experiments showed slower degradation rates (PK, $22 \mathrm{~h}$ half-life; PP, $118 \mathrm{~h}$ half-life) than the initial experiment (PA). This is consistent with a limited electron donor capacity mechanism of degradation, although other possibilities exist. That the decrease in degradation rate was not an artifact of repeated methanol extractions of the Norborne sediment was shown by a companion experiment to PB in which a fresh sample of Norborne sediment was successively extracted three times with methanol prior to a repeat of experiment $\mathrm{PB}$. The $\mathrm{K}_{\mathrm{d}}$ and half-live of this companion experiment $\left(0.46 \mathrm{~cm}^{3} \mathrm{~g}^{-1}\right.$, $2.4 \mathrm{~h}$ ) agree quite well with that of experiment PB (Table 4.1). Thus, repeated methanol extractions did not contribute to the increase in half-life observed in the PB, PG, PK, and PP experimental sequence.

CL-20 Observed Degradation Products in 1-D Columns. CL-20 degradation products were identified for a limited number of batch and column experiments conducted under oxic conditions and for one dithionite-reduced sediment (Figures 4.2a and 4.3). The cage structure of the CL-20 molecule contains six nitro groups, with a total of 12 nitrogens and 6 carbons.

Because CL-20 degradation may produce small degradation products (such as nitrate) as well as 
high-molecular-weight compounds (partially degraded CL-20), both ion chromatography and LC-MS/MS were used to identify products. The appearance of six or less moles of nitrogen compounds per mole of CL-20 degraded is consistent with stepwise loss of nitro groups with the cage structure remaining in tact. The first step in breaking the CL-20 cage structure has been proposed to be the cleavage of the attic C-C bond (Figure 1.7) allowing CL-20 to unfold as a three ring heterocyclic compound (Monteil-Rivera et al. 2004; Qasim et al. 2004, 2005). If this is correct, the presence of carbon compounds (e.g., formate) in the suite of degradation products indicates that (i) the cage structure was broken, and (ii) cleavage of additional $\mathrm{C}-\mathrm{C}$ or $\mathrm{C}-\mathrm{N}$ bonds of the heterocyclic has occurred in the degradation processes. One previous study (Balakrishnan et al. 2004) showed that hydrolysis of CL-20 in aqueous solution at $\mathrm{pH} 10$ produced a total of 3.6 moles of nitrogen compounds per mole of CL-20 degraded, and 0.49 moles of formate per mole of CL-20 degraded (Table 1.4). The molar ratios of nitrogen and formate per mole of CL-20 degraded, designated in Table 1.3 as mol N mol ${ }^{-1} \mathrm{CL}-20$ and mol C mol ${ }^{-1} \mathrm{CL}-20$, will be referred to in the text as the molar $\mathrm{N}$ and $\mathrm{C}$ ratios. For the hydrolysis of CL-20 at $\mathrm{pH} 9.5$ in this study (Task 1.2), the molar $\mathrm{N}$ and $\mathrm{C}$ ratios (Table 1.4) compared favorably to those of Balakrishnan and others. The presence of the formate in both studies indicates that alkaline hydrolysis results in CL-20 cage breakage and subsequent ring cleavage.

Analysis of degradation products in the column effluent ( $\mathrm{pH}$ 9.2) of the high-pH Ocala sediment are shown in Figure 4.4. The $\mathrm{N}$ and $\mathrm{C}$ molar ratios for the Ocala (Table 1.4) are similar to those observed for aqueous hydrolysis, suggesting that hydrolysis is a contributing mechanism in CL-20 degradation by the Ocala sediment, as suggested in the previous section. Cleavage of the CL-20 cage structure is indicated by the presence of formate. In addition, trace quantities of 2-oxa-CL-20, and 2,12-dioxa-CL-20 were detected by HPLC. These two compounds have been tentatively identified by electrospray tandem mass spectrometry along with a third high-molecular-weight compound (MW $411 \mathrm{~g} \mathrm{~mol}^{-1}$ ), which appears to be CL-20 with $\mathrm{NHOH}$ substituted for two nitro groups (possibly 2,12hydroxylamino-4,6,8,10-tetranitro4,6,8,10-tetraazaisowurzitane).

CL-20 degradation in the presence of the Burbank and

a)

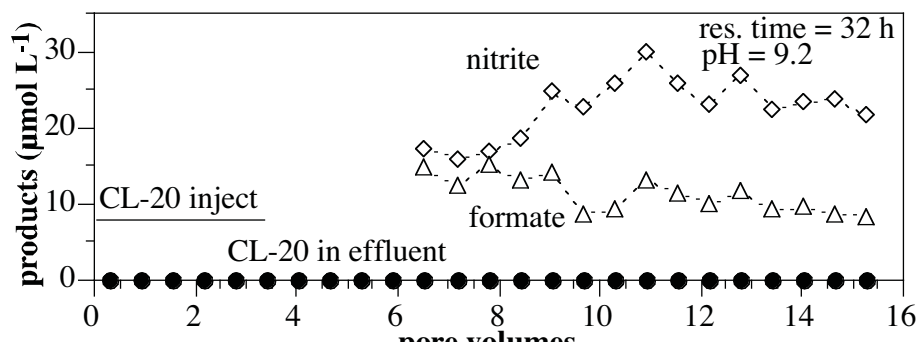

b)

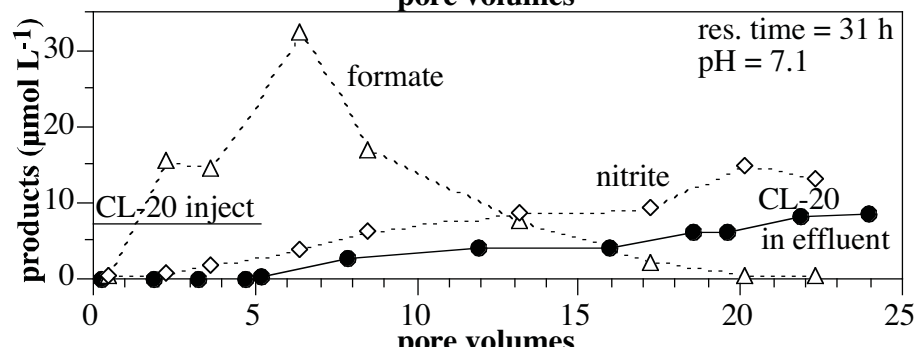

c)

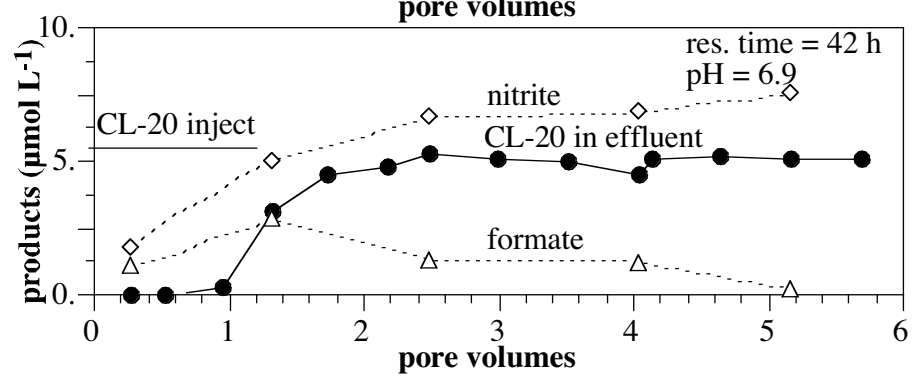

Figure 4.4. CL-20 degradation products during 1-D transport in oxic sediments: a) Norborne, b) Burbank), and c) Ocala 
Norborne sediments at neutral $\mathrm{pH}$ also shows the presence of nitrite and formate (Figure $10 \mathrm{~b}$ and 10c; Table 5). HPLC analysis of effluent samples of the Norborne sediment indicated trace quantities of 2-oxa-CL-20. The similarity between the molar $\mathrm{N}$ and $\mathrm{C}$ ratios for aqueous hydrolysis and for the Norborne and Burbank sediments suggests that the degradation pathway for oxic sediments at near neutral $\mathrm{pH}$ investigated here may have elements in common with the hydrolytic pathways under the highly basic conditions. By contrast, for both the dithionitereduced Norborne sediment and an oxic suspension of hectorite (2:1 clay) (Table 5), where complete CL-20 degradation occurred rapidly in batch experiments, the molar $\mathrm{N}$ ratios were a factor of 2 to 3 times greater than those of the Ocala, Burbank, and oxic Norborne while the molar $\mathrm{C}$ ratio was a factor of 2 (reduced sediment only) greater. This may simply reflect that degradation in the batch experiments had progressed further towards completion, which is consistent with the long half-life of the oxic Norborne (exp PP in Table 4.1) and the complete degradation within 3 minutes for the reduced Norborne. With significant quantities of formate resulting from the interaction of CL-20 with oxic and reduced sediments, and the clay examined in this study, and for aqueous CL-20 in contact with glass ( $\mathrm{SiO}_{2}$, Figure 1.6), it appears that the cage structure of aqueous CL-20 does not persist when in contact with sediment and mineral surfaces.

CL-20 degradation rates for these oxic sediments ranged from a few hours to $800 \mathrm{~h}$, as defined by a pseudo-first order reaction (Table 4.1). The China Lake sediments exhibited the slowest CL-20 degradation rates $\left(450 \mathrm{~h}\right.$ to $780 \mathrm{~h}$ half-life), and with low sorption $\left(\mathrm{K}_{\mathrm{d}}\right.$ averaged $0.25 \mathrm{~cm}^{3} \mathrm{~g}^{-1}$ ), CL-20 could move considerable distances in the subsurface. The average CL-20 degradation rates were faster for the Burbank sediment ( $13 \pm 13 \mathrm{~h}$ half-life), the Westmoreland sediment $(5.5 \pm 3.9 \mathrm{~h})$, the Norborne sediment $(5.0 \pm 1.6 \mathrm{~h})$, and the ferrihydrite-coated sand $(6.1 \pm 3.5 \mathrm{~h})$. 


\section{Task 4.3. Abiotic CL-20 Transport in Low Water-Saturation Columns}

Understanding chemical transport in unsaturated sediments is an essential component to understanding environmental fate. The current state of knowledge of the environmental fate and transport of CL-20 is limited (Bhushan et al. 2003; Geetha et al. 2003; Jenkins et al. 2003; Balakrishnan et al. 2004; Szecsody et al. 2004a). While research on the environmental fate of RDX is significant, a search of the literature did not provide data on the transport of RDX in unsaturated systems. The development of two-region flow (mobile-immobile water) in unsaturated systems is well-documented (Nielsen and Biggar 1961; van Genuchten and Wierenga 1976; Gaudet et al. 1977; De Smedt and Wierenga 1979, 1984; Wierenga and van Genuchten 1989). Previous research illustrates a significant decreases in the apparent soil-water distribution coefficient of the solute with decreasing water content in systems at very low water contents on the order of $10 \%$ to $20 \%$ moisture saturation (Gamerdinger and Kaplan 2001; Gamerdinger et al. 2001).

Hypothesis. Under unsaturated conditions, regions of stagnant or immobile water develop in soils. At very low water contents ( $10 \%$ to $20 \%$ moisture saturation), solutes can be excluded from a fraction of the pore space; this may be due to "isolated" water, or immobile regions where solutes cannot diffuse freely between the mobile and "isolated" domain. We hypothesize that under these hydrodynamic conditions that develop at very low water contents, sorptive solutes may experience decreased accessibility to sorptive surfaces, and therefore, a corresponding decrease in sorption will be observed. In addition, the sorption/desorption rate will decrease at low water content, due to increased fraction of immobile pore fluid. Finally, because abiotic degradation of CL-20 occurs at mineral surfaces, and because there will be decreased access to some mineral surfaces and slower access to mineral surfaces, we hypothesize that the $\underline{C L-20}$ abiotic degradation rate will decrease.

The primary objective of experiments in this task was to quantify the reactive transport of CL-20 in unsaturated soils, particularly at low water content. The transport of RDX was also investigated as a basis for comparison. A series of unsaturated transport experiments on three soils with different texture and surface properties were conducted. Water content was manipulated as a means for changing hydrodynamic conditions and evaluating changes in sorption with decreasing water content. The transport of pentafluorobenzoic acid, PFBA (conservative tracer), is used as the basis for assessing the sorption and degradation of RDX and CL-20. Three soils with varying texture and organic carbon content were used; properties are summarized in Table 4.2.

Table 4.2. Selected physical and chemical properties of soils

\begin{tabular}{|l|c|c|c|c|c|c|}
\hline Soil & & & & & $\begin{array}{c}\text { CEC } \\
(\text { meq/100g } \\
)\end{array}$ & pH \\
\hline MSC & 98 & $<1$ & 2 & 0.023 & 7.8 & 8.07 \\
Norborne & 15 & 72 & 13 & 0.23 & 9.1 & 7.10 \\
Burbank & 42 & 53 & 4 & 0.50 & 5.5 & 7.00 \\
\hline
\end{tabular}

Pentaflourobenzoic acid (PFBA) was used as a conservative tracer for assessing hydrodynamic conditions. CL-20 and RDX were evaluated as sorptive solutes. 
A centrifuge method that allows for highly controlled, steady-state unsaturated flow at low water contents was selected for conducting transport experiments (Gamerdinger and Kaplan 2000). Breakthrough curves for the non-sorptive pentafluorobenzoic acid (PFBA) tracer were used to quantify the ratio of mobile-immobile water at low water content ( $10 \%$ to $20 \%$ moisture saturation) and to characterize hydrodynamic conditions that ultimately determine CL-20 and RDX transport. The unsaturated column system includes two infusion pumps and an "unsaturated flow apparatus" (L8-UFA ${ }^{\circledR}$, Beckman Coulter, Inc., Fullerton, CA). A rotating seal is at the center of the rotor and is the conduit through which fluid is delivered from the pump to the columns by two independent flow paths. Miscible displacement or unsaturated transport experiments are initiated when the columns reach a steady-state average water content $(>10$ pore volumes). During operation of the UFA, centripetal acceleration forces water from the column, which is replaced by fluid delivery via the pump, with step inputs of solute-free and solute-laden fluid. Column effluent was collected and analyzed for RDX and CL-20 to determine the fluxaveraged effluent concentration.

The results for unsaturated transport experiments are summarized in Table 4.2. The trends for the sorption of CL-20 and RDX are similar across the three soil types. Data for the Burbank soil (sandy loam) are presented here to illustrate trends (Table 4.3). Figure 4.5 shows the transport of PFBA in Burbank soil at five water contents. At water contents greater than 50\% moisture saturation $(100 \%, 59 \%$, and 54\%), the breakthrough curves exhibit characteristics that are consistent with a single flow domain or one-region transport. Retardation factors are equal to one, as expected for a non-interactive tracer. At water contents less than $50 \%$ moisture saturation $(27 \%$ and $24 \%)$, the breakthrough curves show a dramatic shift to the left. Retardation factors range from 0.5 to 0.6 . Values less than one are characteristic of exclusion which means that the solute is not accessing some of the pore space that was available at higher water contents. 


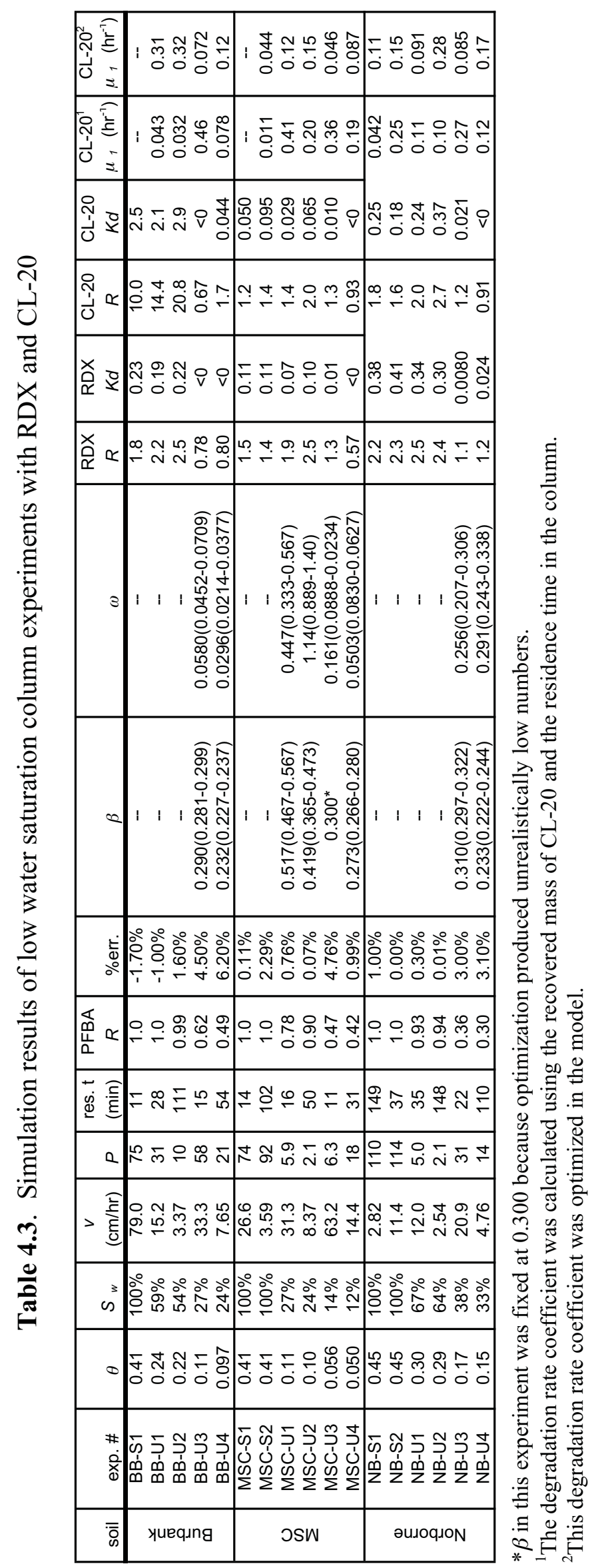




\section{PFBA in Burbank}

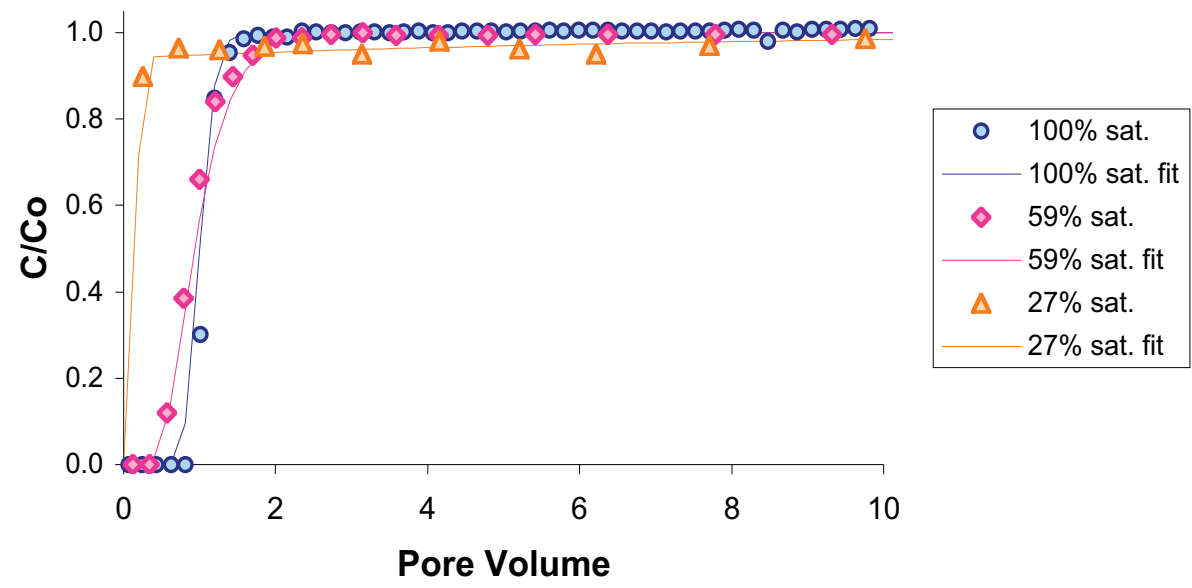

Figure 4.5. Conservative tracer PFBA transport in the Burbank sediment column at differing water content

Figure 4.6a shows the transport of RDX in Burbank soil for the same hydrodynamic conditions that were detailed above for PFBA. The effect of changing hydrodynamic conditions at low water content on sorption and retardation are apparent. The three curves at water contents greater than 50\% moisture saturation are clustered with retardation factors ranging from 1.8 to 2.5. If a simple distribution model for sorption in a simple one-region flow regime applied, we would expect greater retardation with decreasing moisture content. However, due to the dramatically different hydrodynamic condition at water contents less than $50 \%$ moisture saturation, retardation of RDX decreases. This is the first report of the exclusion of non-charged organic solutes at low water contents in unsaturated sediments.

Figure 4.6b shows the transport of CL-20 in the same soil; full breakthrough curves are illustrated to better illustrate the mass lost to degradation. At water contents greater than $50 \%$ moisture saturation, retardation of CL-20 is on the order of 10 to 20 . As shown for RDX, at the very low water contents of $24 \%$ and $27 \%$ moisture saturation, the CL-20 breakthrough curves are shifted to the left. Again the hydrodynamic conditions that resulted in solute exclusion of PFBA are influencing CL-20 sorption and transport, such that sorption is less than predicted.

Degradation of CL-20 was apparent in all soils at all water contents. Values for the first order degradation rate constants $\left(\mathrm{hr}^{-1}\right)$ were somewhat variable and were estimated based on mass lost during transport experiments. Estimates of ranged from 0.19 to 0.47 for the coarse sand, 0.03 to 0.5 for the sandy loam, and 0.1 to 0.3 for the silt loam.

In summary, the sorption and transport of RDX and CL-20 were greatly influenced by the hydrodynamic conditions of the system. When water content is greater than $50 \%$ moisture saturation, transport behavior is more consistent with predictions that are based on transport in saturated systems. At very low water contents that are less than $30 \%$ moisture saturation, the 
hydrodynamic conditions change such that solutes are excluded from a fraction of the pore space. This apparently decreases access of sorptive solutes to surfaces and sites for sorption. The result is accelerated transport.
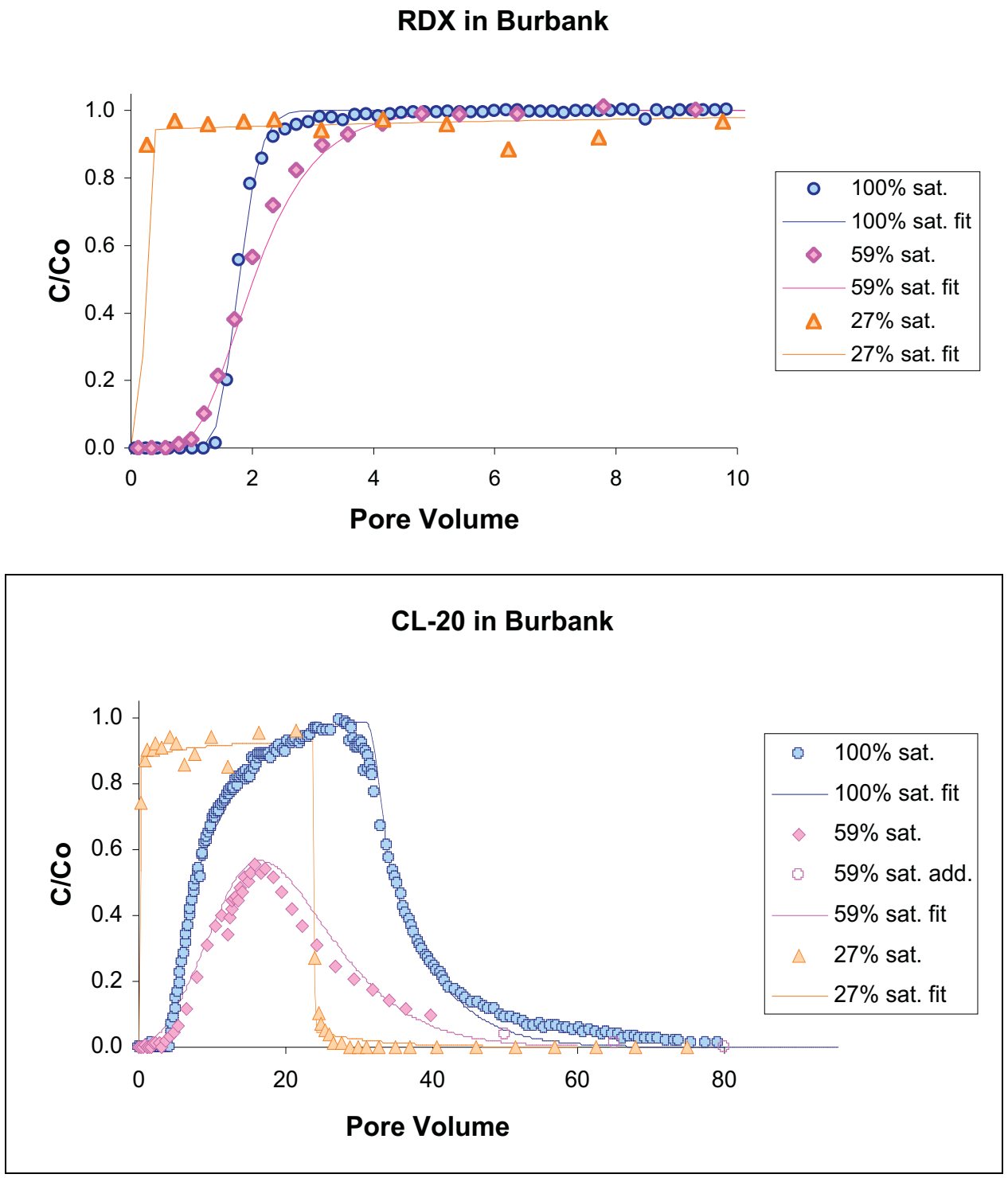

Figure 4.6. RDX and CL-20 transport in the Burbank sediment column at differing water content

Evaluation of Low Water Saturation Experiments. The sorption, transport, and degradation of CL-20 were evaluated in unsaturated laboratory columns to gain insight regarding transport processes in the vadose zone. Varying water content (percent moisture saturation) and hydrodynamic conditions were of particular interest. We hypothesized that the abiotic sorption and transport of CL-20 would change as a function of water content. Specifically, that immobile (stagnant) and possibly isolated water regimes would develop as water content was lowered to 
$30 \%$ or lower moisture saturation. We hypothesized that under these conditions, solutes would experience decreased accessibility to reactive surfaces. This would translate to:

- decreased sorption mass (i.e., $\mathrm{K}_{\mathrm{d}}$ ) relative to saturated systems

- increased sorption/desorption rate due to additional diffusion

- decreased CL-20 abiotic degradation.

The sorption and transport behavior of RDX was also evaluated. RDX has been extensively used in the past; however, little has been published regarding RDX transport in unsaturated systems. Three soils ranging from a low organic carbon coarse sand to a silt loam were evaluated. Sorption of RDX and CL-20 were greater on the sandy loam and silt loam compared with the coarse sand, but sorption was not directly correlated with increasing organic carbon content. Degradation of RDX was negligible under the conditions evaluated (relatively short residence times during unsaturated transport). For CL-20, firstorder degradation rate constants $\left(\mathrm{hr}^{-1}\right)$ were estimated based on mass lost during transport experiments. Estimates ranged from 0.19 to 0.47 for the coarse sand, 0.03 to 0.5 for the sandy loam, and 0.1 to 0.3 for the silt loam.

Consistent with our hypothesis, hydrodynamic conditions changed from a one-region flow regime (all water is in the mobile domain) at conditions where the moisture saturation was $50 \%$ or higher, to a two-region, or mobile-immobile water regime at water contents less than $50 \%$ moisture saturation (Figure 4.7) for both CL-20 and RDX and all three sediments. At very low water contents $(30 \%$ moisture saturation or less), solutes were excluded from a fraction of the pore space. Exclusion simply means that the solutes did not access all of the pore space that was available at conditions of higher water content. As hypothesized, a decrease in sorption was observed and
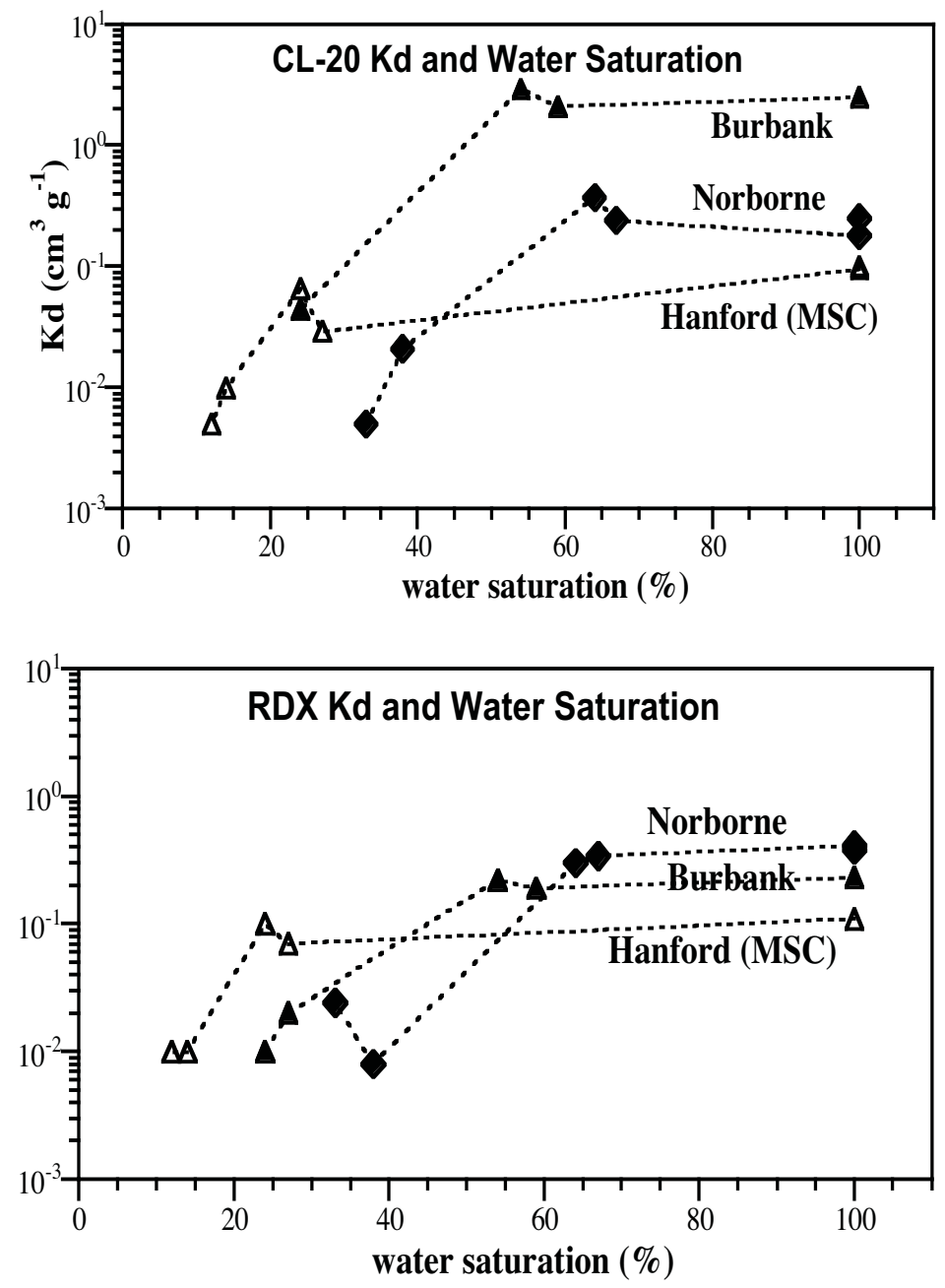

Figure 4.7. Relationship of CL-20 (a) and RDX (b) sorption to water saturation during transport. 
corresponded to the lower water content conditions. This result is important because previously, this effect has only been reported for anionic compounds.

The magnitude of the change in apparent distribution coefficient $\left(\mathrm{K}_{\mathrm{d}}\right)$ was greater than a factor of 5 for both RDX and CL-20. Although these results are for controlled laboratory systems, they should be considered when estimating solute transport in the vadose zone. The implications are most significant for arid soils where water contents approach $10 \%$ to $20 \%$ moisture saturation. It is important to consider that hydrodynamic conditions at low water contents may affect sorption behavior.

For the Burbank soil at $64 \%$ moisture saturation, sorption behavior is similar to what we would expect for a saturated sediment. Retardation of the conservative tracer, PFBA, is equal to one, indicating no sorption. There is some sorption of RDX and CL-20; the apparent distribution coefficient for CL-20 is ten times that for RDX. Degradation of CL-20 is greater than that for RDX (i.e., none), as evidenced by the decreased area under the BTC. Both the increased sorption and more rapid degradation of CL-20 suggest that CL-20 will migrate more slowly and will persist less compared with RDX. The degradation rate of CL-20 also appeared to decrease significantly from a half-life of $2.2 \mathrm{~h}$ (water saturated) to $49 \mathrm{~h}$ at $12 \%$ water saturation (Figure 4.8). This is consistent with Jenkins et al. (2003), who reported degradation half lives of CL-20 in sediments at low water saturation of 144 to 686 days.
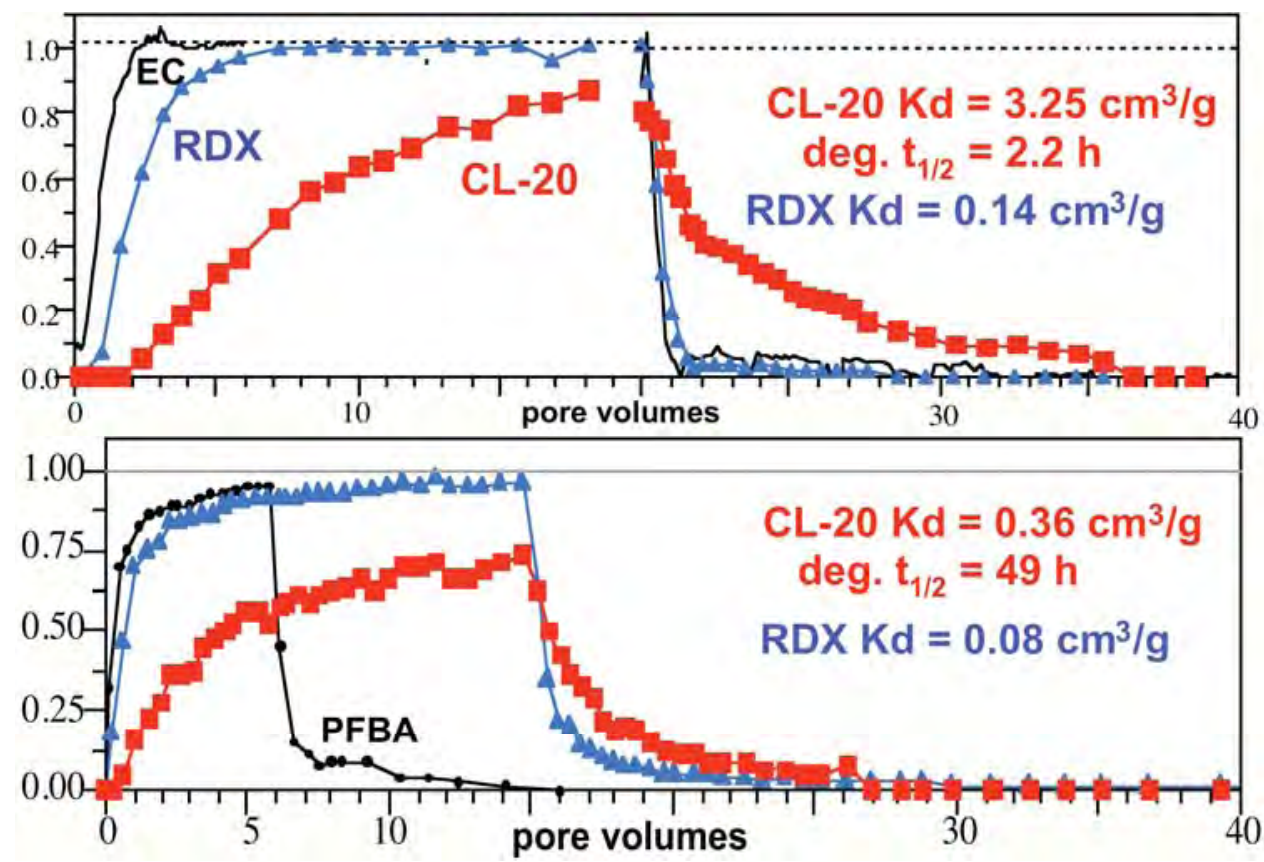

Figure 4.8. Reactive transport of RDX and CL-20 through Burbank sediment at: a) water saturated conditions and b) low (12\%) water saturation

The results for the Norborne soil at $70 \%$ to $73 \%$ moisture saturation and the MSC soil at $25 \%$ to $28 \%$ moisture saturation do not show the same differences in sorption that were observed for the Burbank soil. Sorption of RDX and CL-20 was similar on Norborne and MSC. 
When water content was lowered to $24 \%$ to $27 \%$ for Burbank, $28 \%$ to $40 \%$ for Norborne, and $12 \%$ to $14 \%$ for MSC, there was a significant decrease in retardation for all three tracers. When the retardation factor for the conservative tracer is less than one $(R<1)$, it suggests exclusion of the solutes from a fraction of the pore space. A corresponding decrease in sorption is usually observed. The experimental results require further analysis to determine the impact of immobile water on the process of sorption and degradation. Implications of these low water content experiments are that CL-20 will be more persistent and mobile in the vadose zone than predicted from saturated experiments. 



\section{Summary}

This SERDP-funded project was initiated to investigate the fate of CL-20 in the subsurface environment, with a focus on identification and quantification of geochemical and microbial reactions of CL-20, the effects of aging, and the influence of transport on these reactions in soils and subsurface sediments. This report represents results for the third year of this SERDP project. Results of this study show that CL-20 abiotic and biotic reactions occur, which significantly influence the fate of CL-20 in the subsurface environment from a surface spill. Sorption mass and degradation rate define the potential for deep migration of CL- 20.

CL-20/RDX Sorption Measurements and Prediction. CL-20 sorption in the subsurface below the 2-meter-thick soil zone (vadose zone, groundwater aquifers; organic carbon $<0.5 \%$ ) is small for CL-20 ( $\mathrm{K}_{\mathrm{d}}=0.02$ to $4.2 \mathrm{~cm}^{3} \mathrm{~g}^{-1}$; Table S1) and for RDX $\left(\mathrm{K}_{\mathrm{d}}=0.53\right.$ to $\left.6.5 \mathrm{~cm}^{3} \mathrm{~g}^{-1}\right)$, which results in only slight CL-20 retardation relative to water (i.e., $\mathrm{R}_{\mathrm{f}} 1.1$ to 12 ; Figure $\mathrm{S} 1 \mathrm{a}$ ). CL-20 sorption in subsurface (low $\mathrm{f}_{\mathrm{oc}}$ ) sediments has a good correlation to the mass of iron oxides in the sediment (not organic carbon nor clay, Figure S1a), whereas RDX sorption to subsurface sediments has a fair correlation with the mass of clay (not organic carbon nor mass of iron oxides, Figure S1c). While there will be some retention in sediments, realistically, both CL-20 and RDX can migrate deep in the subsurface. RDX is well documented as a groundwater contaminant (Spalding and Fulton, 1988, Spanggord et al., 1980; Boopathy and Manning, 2000), moving nearly unretarded. In contrast, for the shallow soil environment, surface soils $(<2 \mathrm{~m})$ in humid regions can have organic matter (fraction organic carbon or $f_{o c}$ ) as high as $40 \%$, whereas soils in arid regions have lower organic matter $(<5 \%)$. As predicted, CL-20 sorption to surface soils (high $\mathrm{f}_{\mathrm{oc}}$ ) is mainly to the organic matter so can be generally predicted from the organic carbon content (Figure $\mathrm{S} 1 \mathrm{~b}$ ), although differing types of organic matter adsorbed CL-20 more strongly (Monteil et al. 2004). Therefore, in more humid regions, CL-20 will be retained in the soil
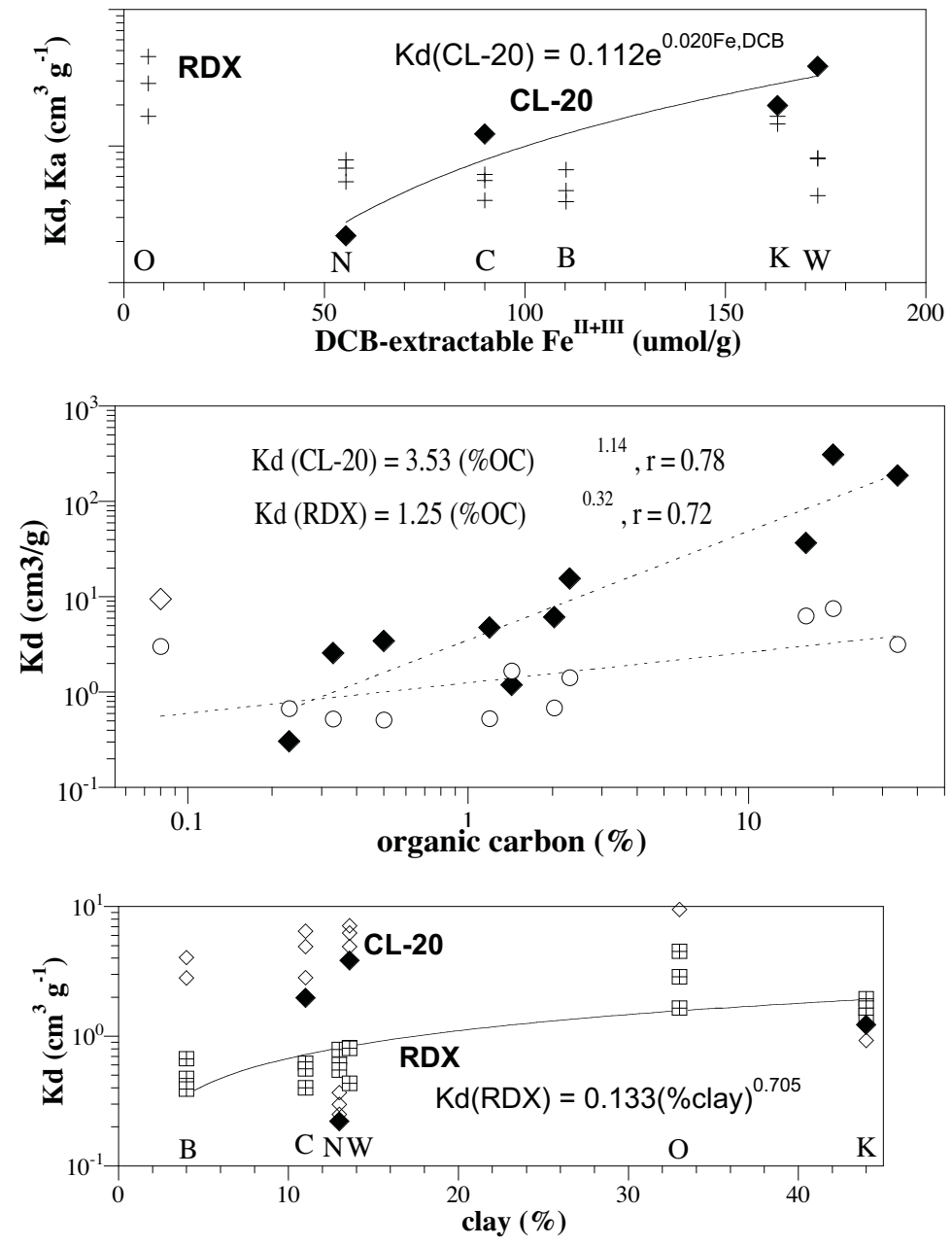

Figure S1. Geochemical factors influencing CL-20 adsorption in sediments: a) DCB-extractable iron, b) organic carbon (\%), and c) clay 

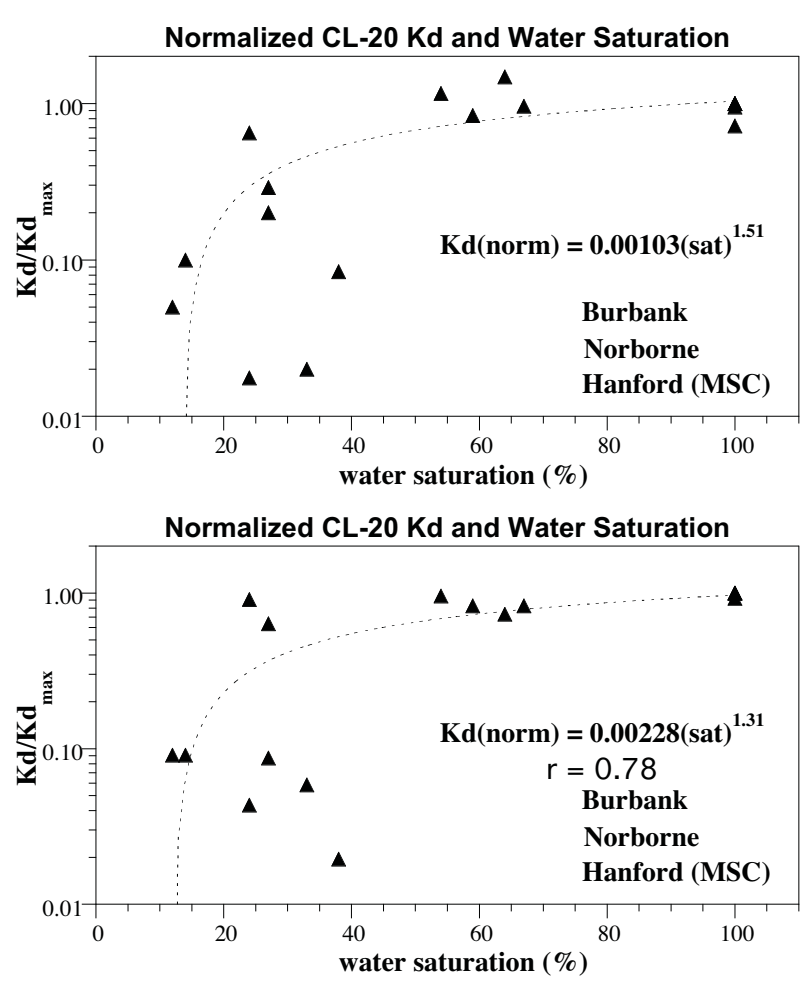

Figure S2. Influence of water saturation on a) CL-20 and b) RDX sorption, based on low water content column experiments conducted in a centrifuge zone where degradation (microbial, photodegradation) can occur. In contrast, RDX is more weakly correlated with fraction organic carbon relative to CL-20 (Figure S1b). For sediments in the vadose zone, the water content has a significant effect on decreasing the sorption of both CL-20 (Figure S2a) and RDX (Figure S2b), due to water traveling in only a portion of the sediment pores, so accessing less adsorption sites.

Based on the results of this study, CL-20 sorption can be estimated from sediment physical and geochemical properties. The correlations used for this empirical estimate are shown in Figures S1 and S2 (details in the Results Section 1.1), which include data. To estimate CL-20 sorption in surface and subsurface sediments, the following parameters are needed: a) fraction organic carbon, b) DCB-extractable Fe, c) percent clay, and d) water content. The $\mathrm{pH}$ of the sediment should be $<8.5$, as CL-20 degrades under alkaline ( $\mathrm{pH}>8.5$ ) conditions (described below). The correlation of sediment properties to CL-20 sorption is:

$$
\mathrm{K}_{\mathrm{d}}\left(\mathrm{cm}^{3} / \mathrm{g}, \mathrm{CL}-20\right)=\left[3.53(\% \mathrm{OC})^{1.14}+0.112 \mathrm{e}^{0.020 \mathrm{DCB}-\mathrm{Fe}}\right]\left[1.03 \mathrm{E}^{-3}(\% \mathrm{sat})^{1.51}\right]
$$

where $\% \mathrm{OC}$ is the percent organic carbon in the sediment, DCB-Fe is the DCB-extractable iron (Heron et al. 1994), and \%sat is the percent water saturation in the sediment. The correlation of sediment properties to RDX sorption is:

$$
\mathrm{K}_{\mathrm{d}}\left(\mathrm{cm}^{3} / \mathrm{g}, \mathrm{RDX}\right)=\left[1.25(\% \mathrm{OC})^{0.32}+0.133(\% \text { clay })^{0.705}\right]\left[2.28 \mathrm{E}^{-3}(\% \mathrm{sat})^{1.31}\right]
$$

where \%clay is the percent clay in the sediment. It should be noted that these correlations are empirical and estimates for new sediments should be within the bounds of measured values to be valid (Table S1).

CL-20 Factors Influencing Abiotic/Biotic Degradation Rates. While CL-20 subsurface transport is influenced only slightly by sorption, CL-20 degradation (abiotic, biotic) can have a major influence on the distribution of mass, and defines sediments with low degradation rates where there is great potential for subsurface migration (Figure S3). Abiotic and biotic degradation in oxic sediments is observed (hours to 1000s of $\mathrm{h}$ ), which limits migration. CL-20 will degrade in alkaline waters ( $\mathrm{pH}>9.5$; hydrolysis, Table $\mathrm{S} 2)$, and with in sediments the degradation rate is related to adsorbed $\mathrm{Fe}^{\mathrm{II}}$ or $2: 1$ clays. Of 15 sediments (varied properties), $7 \mathrm{had}$ very slow degradation (100 to $6400 \mathrm{~h}$ half-life), and so would be persistent in the subsurface for 
Table S1. RDX and CL-20 sorption and degradation in oxic subsurface sediments

\begin{tabular}{|c|c|c|c|c|c|c|c|c|c|}
\hline Saturated sediments & $\begin{array}{l}\text { epth } \\
(\mathrm{ft})\end{array}$ & $\mathrm{pH}$ & $\begin{array}{c}\text { properties } \\
\text { foc }(\%)\end{array}$ & ${ }^{*} \mathrm{Fe}_{\text {tot }}$ & $\begin{array}{c}\text { RDX } \\
K_{d}\left(\mathrm{~cm}^{3} / g\right)\end{array}$ & $\begin{array}{c}\text { RDX deg. } \\
\text { half-life }(h)\end{array}$ & $\begin{array}{c}\text { CL-20 } \\
K_{\mathrm{a}}\left(\mathrm{cm}^{3} \mathrm{~g}^{-1}\right)^{1}\end{array}$ & $\begin{array}{l}\text { CL-20 d } \\
\text { half-life (h) }\end{array}$ & $\begin{array}{l}\text { legradation } \\
\text { rate }\left(\mathrm{mol} \mathrm{h}^{-1} \mathrm{~g}^{-1}\right)\end{array}$ \\
\hline Burbank Ap & 0 & 7.00 & 0.50 & 21.7 & $6.5 \pm 0.14$ & $>1000$ & $1.93 \pm 0.38$ & $13 \pm 1.7$ & $2.1 \mathrm{E}-07$ \\
\hline Cloudland Ap, TN & 0 & 5.30 & 1.19 & 25.0 & $0.53 \pm 0.11$ & $>1000$ & $4.8 \pm 1.8$ & $200 \pm 40$ & $4.5 \mathrm{E}-09$ \\
\hline Kenoma Bt1, KS & 1 & 5.60 & 1.43 & 45.3 & $1.7 \pm 0.25$ & $>1000$ & $1.2 \pm 0.37$ & $3.5 \pm 1.2$ & $6.3 \mathrm{E}-07$ \\
\hline Norborne C, MO & 3 & 7.10 & 0.23 & 13.3 & $0.68 \pm 0.06$ & $>1000$ & $0.52 \pm 0.28$ & $5.0 \pm 1.6$ & $4.1 \mathrm{E}-07$ \\
\hline Ocala C4, NV & 2 & 10.70 & 0.08 & 72.3 & $3.0 \pm 1.4$ & $>1000$ & $0.51 \pm 0.22$ & 0.93 & $1.7 \mathrm{E}-05$ \\
\hline Westmoreland $\mathrm{A} 1$ & 0 & 4.90 & 2.03 & 35.2 & $0.69 \pm 0.22$ & $>1000$ & $3.23 \pm 0.35$ & $5.5 \pm 3.9$ & $3.2 \mathrm{E}-07$ \\
\hline China Lake, CA & 15 & 8.42 & 0.04 & & 0.16 & & $0.25 \pm 0.08$ & $600 \pm 170$ & $2.6 \mathrm{E}-09$ \\
\hline Ft Lewis, WA & 65 & 6.58 & 0.02 & & 0.382 & & 0.624 & 145 & $6.3 E+09$ \\
\hline Hanford, WA & 95 & 8.13 & 0.00 & 24.4 & 0.435 & $>1000$ & 0.566 & 82 & $9.1 \mathrm{E}-09$ \\
\hline Umatilla Depot, OR & 70 & 7.92 & 0.00 & 26.1 & 0.506 & & 0.421 & & \\
\hline Pantex, TX & 258 & 8.1 & 0.00 & 102 & 0.203 & $>1000$ & 0.35 & & \\
\hline Sassafrass Loam & 3 & 7.0 & 0.33 & & $0.35 \pm 0.11$ & $>2000$ & $1.87 \pm 0.33$ & 480 & 2.10E-09 \\
\hline \multicolumn{4}{|c|}{ Unsaturated sediments } & $\%$ sat. & & & & & \\
\hline Ft Greely, $\mathrm{AK}^{+}$ & 0 & 7.2 & 1.1 & 45 & 0.0 & 2260 & 1.7 & 1660 & 1.30E-09 \\
\hline 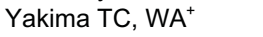 & 0 & 6.2 & 2.0 & 45 & 15 & 2350 & 2.1 & 6400 & 3.37E-10 \\
\hline Camp Guernsey, WY' & 0 & 7.7 & 1.6 & 45 & 4.2 & 3700 & 4.15 & 3460 & $6.70 \mathrm{E}-10$ \\
\hline Burbank Ap & 0 & 7.00 & 0.50 & 10 & 0.053 & $>1000$ & 0.050 & 49.5 & $4.40 \mathrm{E}-08$ \\
\hline Burbank Ap & 0 & 7.00 & 0.50 & 29 & 0.073 & $>1000$ & 0.36 & 48 & $4.20 \mathrm{E}-08$ \\
\hline Hanford, WA & 95 & 8.13 & 0.00 & 28 & 0.01 & $>1000$ & & & \\
\hline
\end{tabular}

years. At low water content, the CL-20 degradation rate decreased significantly. These changes are likely caused by more limited access to reactive surface sites at low water content (i.e., at low water content, water and solutes travel predominantly in larger pores next to larger mineral grains; smaller mineral grains tend to be more reactive). The implication of these low water content experiments is that CL- 20 will be more persistent and mobile in the vadose zone than predicted from saturated experiments. These results are consistent with low-water content batch experiments conducted by Jenkins et al. (2003) in which long-term studies showed CL-20 degradation half-lives ranging from 144 to 686 days (significantly longer than saturated experiments in this study).

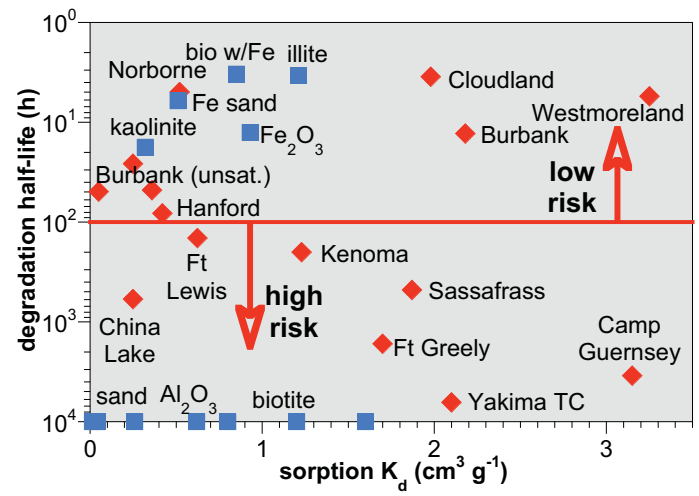

Figure S3. Potential for deep migration of CL-20 with different sediments and minerals, based on sorption and abiotic degradation rate

Long-term aging studies with CL-20 and RDX also showed change in the potential risk of deep migration. The smaller apparent $K_{d}$ values for both CL-20 and RDX indicate that some mass remains on the sediment (i.e., lower migration risk) with long contact time. However, the same aging studies also showed that CL-20 persisted much longer in water saturated conditions with no flow, with degradation rates 1 to 2 orders of magnitude slower than determined in flow experiments (i.e., greater migration risk).

Prediction of Abiotic CL-20/RDX Degradation. The rate of CL-20 abiotic degradation can be calculated in aqueous systems with pure minerals (Figure S4), but can be only poorly 
Table S2. CL-20 degradation factors

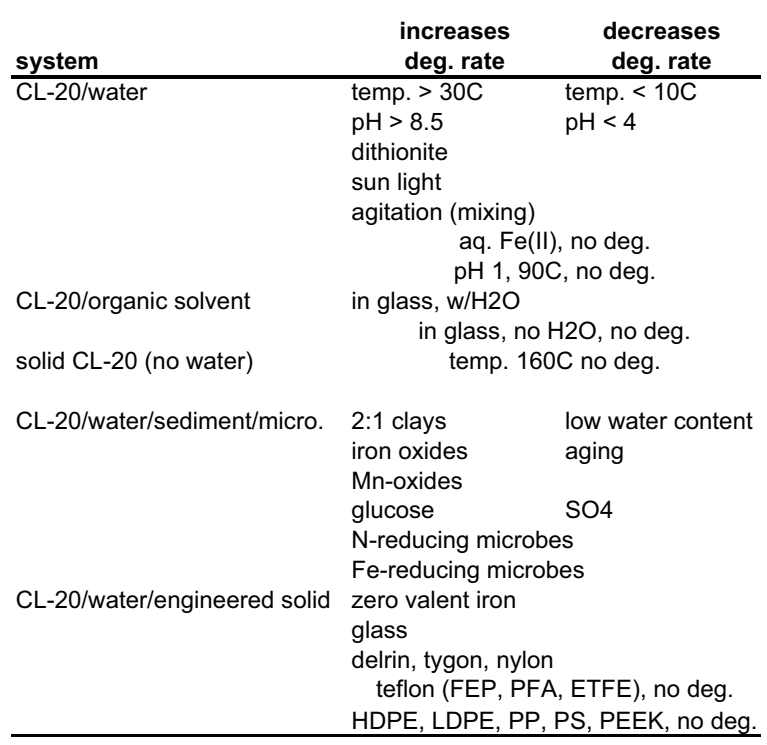

estimated in sediments, which are complex mixtures of minerals coated with other minerals and cations/anions. Results Section 1.2 describes a systematic sequence of CL-20 degradation experiments on minerals that contain no ferrous iron, minerals with structural ferrous iron, and minerals with adsorbed ferrous iron. CL-20 abiotic degradation in oxic sediments appears to via several different mechanisms, with slow rates (i.e., 10 s to 1000 s of hours) with a wide variety of minerals, but rapid rates (i.e., $<10 \mathrm{~h}$ ) to $2: 1$ clays or iron oxides with adsorbed ferrous iron and some 2:1 clays with no structural or surface ferrous iron. The CL-20 abiotic degradation rate in oxic systems can be estimated from:

CL-20 oxic abiotic degradation rate $(1 / h)=$

$\left[1.85 \mathrm{E}^{-4}(\mathrm{DCB} \mathrm{Fe})^{1.72}+3.26 \mathrm{E}^{-12}\left(\mathrm{~T}^{\circ} \mathrm{C}\right)^{6.17}+2.85 \mathrm{E}^{-36}(\mathrm{pH})^{36}\right]\left[0.043 \mathrm{e}^{0.031 \% \mathrm{sat}}\right][1-0.308 \log (\mathrm{t})]$

where $\mathrm{DCB} F e$ is the dithionite-citrate-bicarbonate extractable iron in the sediment, temperature is in Celsius, $\mathrm{pH}$ is the natural equilibrium $\mathrm{pH}$ of the sediment, \%sat is the percent water saturation, and $t$ is the contact time in hours (for a stagnant flow system, from Figure 3.22). If the calculation is intended for a flow system, use $t=0$. This estimate for CL-20 oxic abiotic degradation for natural subsurface systems shows most of the rate is correlated with the DCB iron (i.e., increased iron from iron oxides and clays promote CL-20 degradation, Figure S4a). There is an additional correlation of CL-20 degradation rate with a specific 2:1 smectite clay with no iron (hectorite, see Figure 1.16). Most groundwater temperatures are $10^{\circ} \mathrm{C}$ to $16^{\circ} \mathrm{C}$, so would not promote CL-20 degradation (temperatures above $30^{\circ} \mathrm{C}$ do promote degradation, Figure S4b). CL-20 hydrolysis occurs rapidly at a $\mathrm{pH}$ of 9 or above, but is very slow at lower $\mathrm{pH}$ (Figure S4c). CL-20 oxic, abiotic degradation is slower at lower water content (Figure S4d), so degradation rates in the vadose zone are less than the same water-saturated sediment. Given the number of measurements needed, it is likely more accurate to measure the CL-20 degradation rate for the specific sediment of interest and use the $\mathrm{pH}$, temperature, and lag time correlations. CL-20 is degraded rapidly in sediments under various reducing conditions. Abiotically, ironreducing conditions can be well characterized by a $0.5 \mathrm{M} \mathrm{HCl}, 1 \mathrm{~h}$ extraction for ferrous iron (described in Results Section 3.1 and Figure 1.5b), so the CL-20 abiotic degradation rate in ironreducing conditions can be calculated from:

CL-20 abiotic degradation rate, iron reducing conditions $(1 / h)=$ [5.66E-5 $\pm 1.59 \mathrm{E}-5]$ (ferrous iron, $0.5 \mathrm{M} \mathrm{HCl}, 1 \mathrm{~h}$ ) 

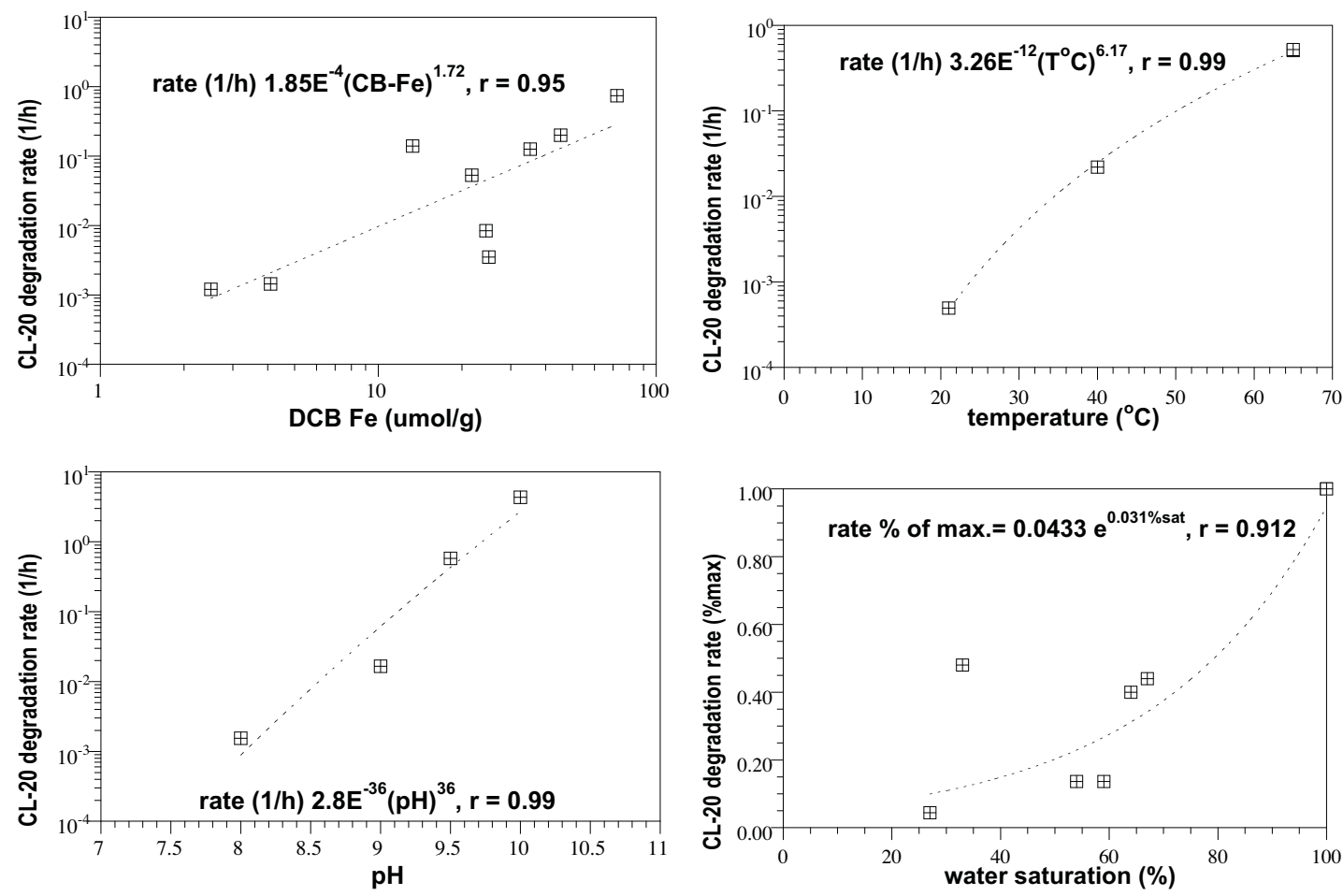

Figure S4. CL-20 degradation rate as correlated with: a) DCB-extractable iron, b) temperature, c) $\mathrm{pH}$, and d) water saturation

The CL-20 abiotic degradation rate can be as rapid as 4 minutes (half-life) for a sediment with $100 \mu \mathrm{mol} / \mathrm{g}$ of ferrous iron. In comparison, the RDX degradation rate in the same iron reducing conditions is more rapid:

$R D X$ abiotic degradation rate, iron reducing conditions $(1 / h)=$

[1.07E-2 $\pm 4.52 \mathrm{E}-3]$ (ferrous iron, $0.5 \mathrm{M} \mathrm{HCl}, 1 \mathrm{~h}$ )

CL-20 and RDX mineralization does not occur abiotically, although iron reducing conditions that promote rapid transformation to intermediates can then be biotically transformed to carbon dioxide (described in the next section, Table S3).

Prediction of Biotic and Coupled CL-20/RDX Degradation. The rate of degradation and mineralization of CL-20 by bacterial isolates, isolates in sediment, and natural microbial microbial communities in sediments (oxic, anaerobic, and various reducing conditions) vary considerably, depending on microbial and geochemical factors (Table S3). Prediction of the rates of energetic transformation can be estimated based on the results in this study, although it would be more accurate to measure abiotic and biotic degradation rates for the specific sediment of interest due to the highly variable nature of biodegradation. Degradation rates of CL-20 and RDX were divided into differing geochemical environments (Table S3) and can be estimated from Table S4:

CL-20 or $R D X$ biodegradation/mineralization rate $(1 / h)=$

(intrinsic rate, Table S4) (g sediment)(CFU/g) 
Table S3. CL-20 and RDX degradation/mineralization rates (as half-life)

\begin{tabular}{|c|c|c|c|c|c|c|}
\hline \multirow[b]{2}{*}{ system } & \multirow[b]{2}{*}{ reactant } & \multirow{2}{*}{$\begin{array}{c}\text { CL-20 } \\
\text { degradation } \\
\text { half-life }(\mathrm{h})\end{array}$} & \multicolumn{2}{|c|}{$\begin{array}{c}\text { CL-20 } \\
\text { mineralization }\end{array}$} & \multicolumn{2}{|c|}{$\begin{array}{cc}\text { RDX } & \text { RDX } \\
\text { degradation } & \text { mineralization }\end{array}$} \\
\hline & & & half-life (h) & maximum (\%) & & \\
\hline microbe & Shewanella putrefaciens, CN32, 1E7 & 533 & & & & \\
\hline \multirow[t]{5}{*}{+ water } & Shewanella putrefaciens, CN32, 4.6E7 & 114 & 1500 & $31 \%, 715 \mathrm{~h}$ & & \\
\hline & Shewanella putrefaciens, CN32, 1E8 & 45.6 & 1100 & $36 \%, 715 \mathrm{~h}$ & & \\
\hline & Shewanella putrefaciens, CN32, 5.7E8 & & 881 & $40 \%, 715 \mathrm{~h}$ & & \\
\hline & microbes, N-reducing & $1.5^{*}$ & & & & \\
\hline & microbes, China Lake isolate & $30-180$ & $120-430$ & & & \\
\hline sediments & oxic Ft Lewis sed., pH 7.0 & $5-700$ & 300 & $39 \%, 1000 \mathrm{~h}$ & 250 & 31000 \\
\hline w/microbes & oxic West. sed., pH 7, $2200 \mathrm{~h}$ aging & $340+$ & & & & \\
\hline \multirow[t]{21}{*}{+ water } & oxic Ft Lewis sed., $\mathrm{pH}$ 7, C addition & 30 & 320 & $69 \%, 1650 \mathrm{~h}$ & & 9900 \\
\hline & oxic Ft Lewis sed., $\mathrm{pH} 7$, nutrient addition & 30 & 270 & $51 \%, 1000 \mathrm{~h}$ & & 14400 \\
\hline & oxic China Lake sed, $1 \mathrm{E} 7 \mathrm{cfu} / \mathrm{g}$, no nutrients & $410-770$ & 790 & $51 \%, 1200 \mathrm{~h}$ & $110-16000$ & \\
\hline & oxic China Lake sed, 1E7 cfu/g, glucose & $41-100$ & $230-980$ & $56 \%, 1200 \mathrm{~h}$ & $36-1600$ & \\
\hline & oxic Ft Bliss sed. + glucose & $14-245$ & & & $115-980$ & \\
\hline & oxic Ft Polk sed. + glucose & 60 & & & & \\
\hline & oxic West., pH 7, $30 \%$ water sat..** & $50^{* * *}$ & & & & \\
\hline & oxic Ft Greely sed., unsat. & 1660 & & & 2260 & \\
\hline & oxic Yakima TC sed., unsat & 6400 & & & 2350 & \\
\hline & oxic Gamp Guernsey sed., unsat & 3460 & & & 3700 & \\
\hline & oxic, $\mathrm{pH} 7$, dry sediment & $>2000$ & & & & \\
\hline & oxic, high clay sediment, $\mathrm{pH} 9.5$ & 0.012 & & & & \\
\hline & anoxic Ft Lewis sed., pH 7.0 & 60 & & & & \\
\hline & anoxic Ft Lewis sed., $\mathrm{pH} 7, \mathrm{C}$ addition & 30 & 280 & $79 \%, 1650 \mathrm{~h}$ & & \\
\hline & anoxic Ft Lewis sed., pH 7, C, SO4 add. & & 780 & $48 \%, 1650 \mathrm{~h}$ & & \\
\hline & anoxic China Lake sed, 1E7 cfu/g, no nutrients & $278-415$ & & & 60 & \\
\hline & reducing China Lake sed, $1 \mathrm{E} 7 \mathrm{cfu} / \mathrm{g}$, fermentative & 79 & & $7.4 \%, 1000 \mathrm{~h}$ & & \\
\hline & NO3 reducing China Lake sed, 1E7 cfu/g, no nut. & 41 & & $13.3 \%, 1000 \mathrm{~h}$ & & \\
\hline & SO4 reducing China Lake sed, 1E7 cfu/g, no nut. & 86 & & $12.9 \%, 1000 \mathrm{~h}$ & & \\
\hline & reduced Ft Lewis sediment (Fe red.), $\mathrm{pH} 7$ & 0.0029 & & & 0.04 & 315 \\
\hline & reduced Ft Lewis sediment (Fe red.), nutr. add & & & & & 112 \\
\hline (coupled) & bioreduced FRC sed.+CN32 microbes 1E7 & 26.6 & & & & \\
\hline (coupled) & bioreduced FRC sed.+CN32 microbes $4.6 \mathrm{E} 7$ & 12.3 & & & & \\
\hline (coupled) & bioreduced FRC sed.+CN32 4.6E7, no ads Fell & 14.2 & & & & \\
\hline & bioreduced FRC sed.+CN32 4.6E7, Fell removed & 54.6 & & & & \\
\hline & bioreduced FRC sed.+CN32 microbes 1E8 & 9.2 & & & & \\
\hline & gibbsite $+\mathrm{CN} 32$ microbes $4.6 \mathrm{E} 7$ & 78 & & & & \\
\hline & reduced Ft Lewis sed., $\mathrm{pH} \mathrm{7,} \mathrm{C} \mathrm{addition}$ & & 180 & $52 \%, 1650 \mathrm{~h}$ & & 682 \\
\hline & reduced Ft Lewis sed., pH 7, C, SO4 add. & & 210 & $39 \%, 1650 \mathrm{~h}$ & & \\
\hline sediment & iron oxide (goethite, ferrihydrite) & $>1500$ & & & $>2000$ & \\
\hline component & iron oxide, adsorbed Fe(II) & 24 & & & $>2000$ & \\
\hline + water & bioreduced FRC sed., pH 7, Fe:CL20 =177 & 192 & & & & \\
\hline & bioreduced FRC sed., pH 7, Fe:CL20 =413 & 53 & & & & \\
\hline & bioreduced FRC sed., pH 7, Fe:CL20 =1834 & 11.2 & & & & \\
\hline & magnetite & 13 & & & $>2000$ & \\
\hline & $\mathrm{Al}_{2} \mathrm{O}_{3}$ & 1.3 & & & $>2000$ & \\
\hline & gibbsite & 533 & & & $>2000$ & \\
\hline & silica sand, no Fe(II) or ads. Fe(II) & $>1000$ & & & $>2000$ & \\
\hline & $1: 1$ clay, kaolinite & 3600 & & & $>2000$ & \\
\hline & 1:1 clay, kaolinite, adsorbed $\mathrm{Fe}(\mathrm{II})$ & 18 & & & $>2000$ & \\
\hline & 2:1 silicate, biotite (no ads. $\mathrm{Fe}(\mathrm{II})$ ) & 450 & & & $>2000$ & \\
\hline & $2: 1$ clay, illite & 3.4 & & & $>2000$ & \\
\hline & 2:1 clay, montmorillonite, $\mathrm{pH} 7$ & $>1000$ & & & $>2000$ & \\
\hline & 2:1 clay, montmorillonite, ads. Fe(II) & 0.06 & & & $>2000$ & \\
\hline & 2:1 clay, hectorite [no $\mathrm{Fe}(\mathrm{II})]$ & 330 & & & $>2000$ & \\
\hline & 2:1 clay, hectorite [ads. Fe(II)] & 0.1 & & & $>2000$ & \\
\hline & 2:1 clay, nontronite, no ads. $\mathrm{Fe}(\mathrm{II})$ & 0.55 & & & $>2000$ & \\
\hline aqueous & aqueous, $\mathrm{pH} 7,65 \mathrm{C}$ & 1.32 & $>2000$ & $<1 \%$ & $>2000$ & \\
\hline & aqueous, $\mathrm{pH} 8,22 \mathrm{C}$ & $120^{*}$ & & & $>2000$ & \\
\hline & aqueous, $\mathrm{pH} 9.5,22 \mathrm{C}$ & 0.8 & & & & \\
\hline & aqueous, $\mathrm{pH} 10,22 \mathrm{C}$ & 0.03 & $>2000$ & $<1 \%$ & & \\
\hline & aqueous, photosynthesis (sunlight) & 80 & & & & \\
\hline & aqueous, photosynthesis (fluorescent light) & $>2000$ & & & $>2000$ & \\
\hline & aqueous $\mathrm{Fe}(\mathrm{II})$ & $>2000$ & & & $>2000$ & \\
\hline & aqueous dithionite & 0.02 & & & 0.005 & \\
\hline engineered & glass, $22 \mathrm{C}$ & 380 & & & $>2000$ & \\
\hline solids & glass, $30 \mathrm{C}$ & 250 & & & & \\
\hline + water & delrin plastic, $22 \mathrm{C}$ & 36 & & & & \\
\hline & tygon plastic & 3 & & & & \\
\hline & nylon plastic & 20 & & & $>2000$ & \\
\hline & teflon: FEP, PFA, ETFE & $>1500$ & & & & \\
\hline & plastics: HDPE, LDPE, PP, PEEK, PS & $>1500$ & & & & \\
\hline & zero valent iron & 0.75 & & & & \\
\hline & less otherwise noted: & & & & & \\
\hline & various published and unpublished & & & & & \\
\hline **Jenkins, $\mathrm{T}$ & tolini, C., Ranney, T., 2003, Stability of CL-20, TNAZ, HI & X, NG, & & & & \\
\hline and PETN in & ist, Unsaturated Soil, U.S. Army Corps of Engineers, ER & REEL TR- & & & & \\
\hline *Burbank Ap & diment saturated deg. half-life $13 \mathrm{~h}$ (slowed to $50 \mathrm{~h}$ at 30 & uration) & & & & \\
\hline
\end{tabular}


Table S4. Intrinsic rates of CL-20 and RDX biotransformation

\begin{tabular}{lcccccc} 
& \multicolumn{6}{c}{ biotransformation rate per microbe $\left(\mathbf{h}^{-1} \mathbf{C F U}^{-1}\right)$} \\
\multicolumn{1}{c}{ environment } & $\mathbf{C L - 2 0} \mathbf{~ d e g .}$ & $\mathbf{n}$ & $\mathbf{m i n} / \mathbf{m a x}$ & $\mathbf{C L}-\mathbf{2 0} \mathbf{m i n}$ & $\mathbf{R D X}$ deg. & RDX min. \\
\hline oxic & $8.7 \pm 9.5 \mathrm{E}-10$ & 16 & $5 \mathrm{E}-11 / 7 \mathrm{E}-9$ & $7.7 \pm 5.2 \mathrm{E}-11$ & $1.1 \pm 0.4 \mathrm{E}-10$ & $2.3 \pm 1.2 \mathrm{E}-12$ \\
anaerobic & $6.7 \pm 4.9 \mathrm{E}-10$ & 4 & $2 \mathrm{E}-10 / 1 \mathrm{E}-9$ & $8.2 \pm 5.4 \mathrm{E}-11$ & $1.2 \pm 0.5 \mathrm{E}-9$ & $1.4 \pm 2.3 \mathrm{E}-11$ \\
reducing & $7.7 \pm 5.4 \mathrm{E}-10$ & 3 & $2 \mathrm{E}-10 / 3 \mathrm{E}-9$ & $1.2 \pm 0.1 \mathrm{E}-9$ & & \\
reducing, coupled & $1.6 \pm 0.8 \mathrm{E}-9$ & 3 & $1 \mathrm{E}-9 / 3 \mathrm{E}-9$ & & $5.8 \pm 3.2 \mathrm{E}-6$ & $1.1 \pm 0.9 \mathrm{E}-9$ \\
\hline
\end{tabular}

CL-20 biotransformation was fastest in reducing/coupled systems (i.e., combination of abiotic and biotic processes) by less than an order of magnitude, so the environment (i.e., oxic or reducing) had only a slight influence on CL-20 biodegradation. In contrast, RDX biodegradation was three orders of magnitude more rapid in reducing systems relative to oxic systems.

The intrinsic CL-20 oxic transformation rate only varied by two orders of magnitude (first line, Table S4), although the rate in sediment will vary further. The microbial population density in soils ( 0 to $2 \mathrm{~m}$ depth) varies from $10^{6}$ to $10^{9} \mathrm{CFU} / \mathrm{g}$ (greater in humid soils), whereas subsurface sediments vary from $10^{3}$ to $10^{6} \mathrm{CFU} / \mathrm{g}$, so the actual observed CL-20 biodegradation rates variability appears to be predominantly due to the differences in microbial population. For comparison, the most rapid abiotic CL-20 transformation processes (reduced sediment, zero valent iron, alkaline hydrolysis) are 4 to 5 orders of magnitude more rapid than CL-20 biotransformation.

Mineralization of CL-20 in sediments can only occur in systems with microbes (or other biotic systems not addressed in this study). Mineralization of CL-20 was 1.5 orders of magnitude more rapid in reducing systems relative to oxic systems, as compared with 3 orders of magnitude for RDX. CL-20 mineralization rates were roughly an order of magnitude slower than the initial CL-20 degradation rate.

RDX degradation rate data in a sediment can be used to estimate the rate of CL-20 degradation or mineralization (with Table S4). In the same system, the RDX initial degradation rates were 6 to 9x slower in oxic systems, but 3 orders of magnitude more rapid in reduced systems. For mineralization, RDX was 3 orders of magnitude slower in oxic systems relative to CL-20, but about the same rate in reducing systems. Therefore, comparisons of RDX to CL-20 biotransformation rates are specific to the geochemical environment (CL-20 can be degraded in oxic systems, RDX generally is not; both are degraded in reducing systems).

CL-20 Degradation Mechanism and Pathway. Activation energies calculated from different CL-20 degradation systems in this project indicate that there may be two different degradation pathways. Activation energies for the first step(s) of CL-20 transformation by: a) electron transfer reactions (CL-20/reduced sediment $=35.2 \mathrm{~kJ} / \mathrm{mol} ; \mathrm{RDX} /$ reduced sediment $=$ $52.2 \mathrm{~kJ} / \mathrm{mol}$ ), and b) non-electron transfer reactions (CL-20/hot H2O $=133 \mathrm{~kJ} / \mathrm{mol} ; \mathrm{CL}-20$ / glass $+\mathrm{H}_{2} \mathrm{O}=146 \mathrm{~kJ} / \mathrm{mol}$ ). These results may imply two different initial transformation steps. In addition, the activation energies of CL-20 transformation by other processes can also imply electron transfer. For example, CL-20 degradation by hectorite $(43 \mathrm{~kJ} / \mathrm{mol})$ may involve electron 
transfer. CL-20 transformation by oxic sediment $(14.1 \mathrm{~kJ} / \mathrm{mol})$ is very low and in the range of diffusion control (i.e., diffusion may be rate controlling rather than the chemical reaction).

Molecular modeling predicted that the roof C-C bond of the CL-20 molecule should cleave (Qasim et al. 2004), based on calculations on the strained cage structure (Figure 1.7). Intermediates and end products were first identified by Jalal Hawari (2003 and 2004, SERDP project CP 1256) that include high molecular weight compounds 3 and 5 (Figure 1.7), glyoxal, nitrate, nitrite, $\mathrm{N}_{2} \mathrm{O}, \mathrm{NH}_{3}$, and formate. The current proposed CL-20 degradation pathway for hydrolysis and zero valent iron (J. Hawari) involves removal of two $\mathrm{NO}_{2}$ groups before the roof C-C bond breaks (as shown in Figure 1.7). More recent molecular modeling indicates that two intermediates might form (compounds 6 and 7). The presence of an aromatic compound during degradation with reduced sediment and hydrolysis is consistent with these proposed compounds.

Carbon and nitrogen mass balance is useful in demonstrating that only part of the pathway has been identified, and further identification is needed. The CL-20 molecule contains 6 carbon atoms and 12 nitrogen atoms. Carbon mass balance of intermediates that include formate, glyoxal, and glycolic acid (Figure 1.8a) in the $<100 \mathrm{~h}$ time frame account for $<50 \%$ of the $\mathrm{C}$ mass, although mass balance is actually better than shown since high molecular weight degradation products (Figure 1.7, compounds 3, 5, 6, 7) are not quantified. Beyond $200 \mathrm{~h}$, the carbon mass balance increases to a high of $79 \%$ (by $1600 \mathrm{~h}$ ), as the main product is carbon dioxide. All of these mineralization experiments involve microbes. The remaining carbon mass may be incorporated into the microbial biomass or form other low molecular weight compounds such as methane. It is clear that abiotic processes alone, while rapidly degrading CL-20 1 to 5 orders of magnitude faster than biotic processes, result in only minor $(<30 \%)$ of the CL-20 carbon mass becoming smaller molecular weight (identified) $\mathrm{C}$ products. Microbial degradation of the initial CL-20 or intermediates is apparently necessary to break some of the bonds.

Nitrogen mass balance is slightly better than carbon, with as high as $80 \%$ mass balance at $>100 \mathrm{~h}$ for reduced sediment and hydrolysis (Figure 3b). As with carbon mass balance, low $\mathrm{N}$ balance at early times $(<100 \mathrm{~h})$ do not include high molecular weight degradation products, which contain considerable $\mathrm{N}$ mass. Low molecular weight $\mathrm{N}$ products include nitrate, nitrite, and nitrous oxide. Further work here using a combination of the uniformly N-15-labeled CL-20 and ring only N-15-labeled CL-20 molecules could help distinguish that early time scale N products may be from nitroso functional groups, whereas late time scale $\mathrm{N}$ products are hypothesized from the heterocyclic ring intermediates.

The most significant general conclusion about CL-20 degradation in the subsurface environment is by comparison to other energetics such as RDX. Most energetics, including RDX, are degraded/mineralized more rapidly in reducing environments. Although natural or engineered abiotic electron transfer reactions (reduced sediment, zero valent iron) can rapidly transform energetics to intermediates, microbes are necessary for one or more steps for mineralization (i.e., microbes are necessary for mineralization of all energetics). Despite similarities in chemical structure, CL-20 and RDX soil biodegradation differs. RDX-degrading aerobes (Rhodococcus $11 Y$, DN22) cannot use CL-20 as an N source. In addition, a CL-20 degrading aerobic isolate from China Lake sediment (Gordonia, $\mathrm{g}+$ ) cannot use RDX as a sole $\mathrm{N}$ source. CL-20 
degradation in oxic systems is 10x more rapid than RDX, and CL-20 mineralization is 3 orders of magnitude more rapid than RDX (i.e., generally RDX is degraded/mineralized in reducing systems).

Reducing systems may differ; an iron degrading isolate, Shewanella putrefaciens (CN-32) can readily degrade and mineralize both CL-20 and RDX. In these reducing systems, RDX degradation is 2 to 3 orders of magnitude more rapid than CL-20. Mineralization of CL-20 was only slightly faster in reduced systems, compared with oxic or anaerobic systems. The most rapid RDX mineralization rates occur in reduced sediments with sequential (series) abiotic reactions (first five transformation reactions) followed by final one or more biotic mineralization reactions. In contrast, CL-20 mineralization is slower in the presence of excess ferrous iron (i.e., more highly reduced sediments), likely due to a lower microbial population. The most rapid CL-20 mineralization rates occur in coupled reduced systems, where a coupled abiotic/biotic reaction appears to be occurring. More specifically, ferrous iron in 2:1 smectite clays and/or green rust is degrading CL-20 (producing ferric iron), and iron-degrading bacteria is then rapidly reducing the ferric iron.

Implications to CL-20 Release in Surface and Subsurface Sediments. Results of this study show that CL-20 will exhibit differing behavior in different parts of the subsurface terrestrial environment:

1. CL-20 on the sediment surface (from manufacture spills, UXO, or low-order detonations at firing ranges/deployment) will photodegrade and may interact with plants/animals (results described in other SERDP projects CU 1254, 1256). CL-20 will exhibit greater adsorption in humid sediments (to organic matter). Transport will be solubility limited (i.e., low CL-20 aqueous solubility).

2. CL-20 in soils $(<2 \mathrm{~m})$ from spills will again be subjected to sorption dependent mainly on the organic matter content of the sediment, and may exhibit low to high biodegradation rates (depending on the microbial population; greater in humid environment). Degradation rates in soils with a reasonably high microbial population can degrade aqueous CL-20 within weeks to months.

3. CL-20 in the vadose zone ( $>2 \mathrm{~m}$, above water table) will, in most cases, exhibit low sorption and low degradation rates with subsurface sediments, so would persist in the subsurface environment and be at risk for deep migration. Sorption is dependent mainly on the presence of iron oxides ( since the fraction organic carbon is $<0.5 \%$ ). Low water content (in more arid regions) results in a decrease in both sorption and degradation rate of CL-20. Measured degradation rates in unsaturated sediments leave CL-20 in soils for years.

4. CL-20 in groundwater will be subjected to some sorption but likely very slow degradation rates. With higher water content, CL-20 sorption will be greater than RDX. Because the microbial population in deep subsurface systems are extremely small, biodegradation rates are likely minimal. Natural iron and other transition metal reducing environments do occur, so some natural subsurface CL-20 degradation can occur. 
Although CL-20 will more rapidly through some of these sediments in the subsurface environment, subsurface remediation of a wide variety of types based upon simple mechanisms described in this report can be utilized for cleanup. Transformation of CL-20 to intermediates can be rapidly accomplished under: a) reducing conditions (CL-20 $4.1 \mathrm{~min}$. half-life, RDX 18 min. half-life), b) alkaline ( $\mathrm{pH}>10$ ) conditions, and c) bioremediation with added nutrients. CL-20 degradation to intermediates may be insufficient to mitigate environmental impact, as the toxicity of many of these compounds is unknown. Biostimulation in oxic to reducing systems by carbon and nutrient addition can mineralize CL-20, with the most rapid rates occurring under reducing conditions. 


\section{References}

Adrian, N. R. and A. Lowder. 1999. "Biodegradation of RDX and HMX by a Methanogenic Enrichment Culture." In In Situ and On-Site Bioremediation, Vol 5(7), pp. 1-6, Alleman and Leeson (eds.), Battelle Press, Columbus, OH.

Ainsworth, C., S. Harvey, and J. Szecsody. 1993. "Relationship between the leachability characteristics of unique energetic compounds and soil properties." Report 91PP1800, U.S. Army Medical Research.

Amonette, J. 2000. "Iron Redox Chemistry of Clays and Oxides: Environmental Applications." Book chapter in A. Fitch (ed.) Electrochemistry of Clays, CMS Workshop Lectures, Clay Minerals Society, Aurora, CO, in review.

Alexander, M. 2000. "Aging, Bioavailability, and Overestimation of Risk from Environmental Pollutants." Environ. Sci. Technol. 34(20):4259-4265.

Ausubel, F. M., R. Brent, R. E. Kingston, D. D. Moore, J. G. Seidman, J. A. Smith, and K. Struhl. 1989. Current protocols in molecular biology. Greene Publishing Associates-Wiley Interscience, New York, N.Y.

Autenrieth, R. L., M. D. Jankowski, J. S. Bonner, and M. Kodikanti. 1999. "Optimizing the Biotransformation of RDX and HMX." In In Situ and On-Site Bioremediation, Vol 5(7), pp. 21-26, Alleman and Leeson (eds.), Battelle Press, Columbus, OH.

Balakrishnan, V. K., F. Monteil-Rivera, M.A. Gautier, and J. Hawari. 2004. "Sorption and stability of the polycyclic nitramine explosive CL-20 in soil." J. Environ. Qual. 33:1362-1368.

Balkwill, D.L., F.R. Leach, J.T. Wilson, J.F. McNabb, and D.C. White. 1988. Equivalence of microbial biomass measures based on membrane lipid and cell wall components, adenosine triphosphate, and direct counts in subsurface sediments. Microb. Ecol. 16:7384.

Bazaki, H., S. Kawabe, and H. Miya. 1998. "Synthesis and sensitivity of CL-20." Propellants Explosives Pyrotechnics 23(6):333-336.

Bhushan, B., L. Paquet, J. C. Spain, and J. Hawari. 2003a. "Biotransformation of 2,4,6,8,10,12Hexanitro-2,4,6,8,10,12-Hexaazaisowurtzitane (CL-20) by Denitrifying Pseudomonas sp. Strain FA1." Applied Environmental Microbiology 69:5216-5221.

Bhushan, B., S. Trott, J. C. Spain, A. Halasz, L. Paquet, and J. Hawari. 2003b. "Biotransformation of Hexahydro-1,3,5-Trinitro-1,3,5-Triazine (RDX) by a Rabbit Liver Cytochrome P450: Insight into the Mechanism of RDX Biodegradation by Rhodococcus sp. Strain DN22." Applied Environmental Microbiology 69:1347-1351. 
Binks, P. R., S. Nicklin, and N. C. Bruce. 1995. "Degradation of Hexahydro-1,3,5-trinitro1,3,5-triazine (RDX) by Stenotrophomonas maltophilia PB1.” Appl. Env. Microbiol. 61(4):1318-1322.

Boopathy, R. and Manning, J. F. 2000. "Laboratory Treatability Study on Hexahydro-1,3,5Trinitro-1,3,5-Triazine (RDX) Contaminated Soil from the Iowa Army Ammunition Plant, Burlington, IA". Water Env. Res. 72(2):238-242.

Brannon, J. M., Adrian, D. D., Pennington, J. C., and Myers, T. E. 1992. "Slow Release of PCB, TNT, and RDX from Soils and Sediments." Technical Report Y-78-11, US Army Engineer Research and Development Center, Waterways Experiment Station, Vicksburg, MS.

Bryce, A., W. Kornicker, and A. Elzerman, Nickel adsorption to hydrous ferric oxide in the presence of EDTA: effects of component addition sequence, Environmental Science and Technology, 28 (13), 2353-2359, 1994.

Burgisser, C., M. Cernik, M. Borkovec, and H. Sticher, Determination of nonlinear isotherm from column experiments: an alternative to batch studies, Environmental Science and Technology, 27 (5), 943-948, 1993.

Burton, D. T. and Turley, S. D. 1995. "Reduction of Hexahydro-1,3,5-trinitro-13,5-triazine (RDX) Toxicity to the Cladoceran ceriodaphnia dubia following photolysis in sublight." Bull. Env. Contam. Tocicol. 55:89-95.

Cassada, D. A., S. J. Monson, D. D. Snow, and R. F. Spalding. 1999. Sensitive determination of RDX and other munitions in groundwater y solid-phase extraction and isotope dilution liquid chromatography-atmospheric pressure chemical ionization mass spectrometry. J. of Chromatogr. 844:87-95.

Chen, Y., L. Abriola, J. Alvarez, P. Anid, and T. Vogel, Modeling transport and biodegradation of benzene and toluene in sandy aquifer material: comparisons with experimental measurements, Water Resources Research, 28 (7), 1833-1847, 1992.

Coates, J. and A. Elzerman, 1986, Desorption kinetics for selected PCB congers from river sediments. J. Cont. Hydrol., 1, 191-210.

Coleman, N., D. Nelson, and T. Duxbury. 1998. "Aerobic Biodegradation of RDX as a Nitrogen Source by a Rhodococcus Sp. Strain DN22." Soil Biology and Biochemistry 30(8/9):1159-1167.

Deng, B., Burris, D.R. and Campbell, T.J., 1999. Reduction of Vinyl Chloride in Metallic Iron Water Systems. Environmental Science and Technology, 33(15): 2651-2656.

De Smedt, F. and P. J. Wierenga. 1979. "Mass transfer in porous media with immobile water." J. Hydrol. 41:59-67. 
De Smedt, F. and P. J. Wierenga. 1984. "Solute transfer through columns of glass beads." Water Resour. Res. 20:225-232.

EPA, 1997, Nitroaromatics and Nitramines by High Performance Liquid Chromatography (HPLC), pp. 1-21, Environmental Protection Agency, Washington, DC.

Freedman, D. L. and K. W. Sutherland. 1998. "Biodegradation of Hexahydro-1,3,5-Trinitro1,3,5-Triazine (RDX) under Nitrate-Reducing Conditions.” Water Sci. Technol. 38(7):33-40.

Funk, S. B., Roberts, D. J., Crawford, D. L., and Crawford, R. L. 1993. "Initial Phase Optimization for Bioremediation of Munition Compound-Contaminated Soils". Appl. Env. Microbiol. 59:2171-2177.

Gamerdinger, A. P., D. I. Kaplan, C. T. Resch. 1998. Uranium(VI) sorption and transport in unsaturated, subsurface Hanford Site sediments -- Effect of moisture content and sediment texture: Final Report for subtask 2b, PNNL-11975, Pacific Northwest National Laboratory, Richland, Washington.

Gamerdinger, A. P. and D. I. Kaplan. 2000. "Application of a continuous-flow centrifugation method for solute transport in disturbed, unsaturated sediments and illustration of mobileimmobile water." Water Res. Research 36.7:1747-1755.

Gamerdinger, A. P. and D. I. Kaplan. 2001. "Physical and chemical determinants of colloid transport and deposition in water-unsaturated sand and Yucca Mountain tuff material." Environ. Sci. Technol. 35:2497-2504.

Gamerdinger, A. P., D. I. Kaplan, D. M. Wellman, and R. J. Serne. 2001. “Two-region flow and decreased sorption of uranium (VI) during transport in Hanford groundwater and unsaturated sands." Water Resour. Res. 37:3155-3162.

Gassman, P. R. Yamaguchi, G. Koser, 1982, Photosynthesized conversion of Norbornadiene and quadricyclane, J. Org. Chem, 43, 4392

Gaudet, J. P., H. Jegat, G. Vachuad, and P. J. Wierenga. 1977. "Solute transfer, with exchange between mobile and stagnant water, through unsaturated sand." Soil Sci. Soc. Am. J. 41:665-671.

Geetha, M, U. R. Nair, D. B. Sarwade, G. M. Gore, S. N. Asthana, and H. Singh. 2003. "Studies on CL-20: The most powerful high energy material." J. Therm. Anal. Calor. 73:913-922.

Greer, C. W., J. G. Godbout, B. Zilber, S. Labelle, G. Sunahara, J. Hawari, G. Ampleman, S. Thiboutot, and C. Dubois. 1999. "Bioremediation of RDX/HMX-Contaminated Soil: From Flask to Field." In In Situ and On-Site Bioremediation, Vol 5(7), pp. 393-398, Alleman and Leeson (eds.), Battelle Press, Columbus, OH. 
Guiot, S. R., C. F. Shen, J. A. Hawari, G. Ampleman, and S. Thiboutot. 1997. "Bioremediation of Nitramine Explosive Contaminated Soils." In W. D. Tedder (ed.), Proc. American Chemical Society Conference on Emerging Technologies in Hazardous Waste Management IX, Pittsburgh, PA, September 15-17, pp. 341-344.

Guiot, S. R., C. F. Shen, L. Paquet, J. Breton, and J. Hawari. 1999. "Pilot-Scale Anaerobic Bioslurry Remediation of RDX- and HMX-Contaminated Soils." In In Situ and On-Site Bioremediation, Vol 5(7), pp. 15-20, Alleman and Leeson (eds.), Battelle Press, Columbus, $\mathrm{OH}$.

Haderlein, S.B., K.W. Weissmahr, and R.P. Schwarzenbach, Specific Adsorption of Nitroaromatic Explosives and Pesticides to Clay Minerals, Environ. Sci. Technol., 30 (2), 612-622, 1996.

Halasz, A., J. Spain, L. Paquet, C. Beaulieu, and J. Hawari. 2002. Insights into the formation and degradation mechanisms of methylenedinitramine during the incubation of RDX with anaerobic sludge. Environ. Sci. Technol. 36:633-638.

Hallgarth, M. R., Jankowski, M. D., Autenrieth, R. L. 1999. "Biodegradation of RDX and HMX'. in 'In Stu and On-Site Bioremediation', Vol 2, p. 17-22. Alleman and Leeson (Eds). Battelle Press, Columbus, $\mathrm{OH}$.

Hawari, J. 2000. "Biodegradation of RDX and HMX: From Basic Research to Field Application." In Biodegradation of Nitroaromatic Compounds and Explosives. J. C. Spain, J. B. Hughes, and H. Knackmuss (eds.), Lewis Publishers, Boca Raton, FL.

Hawari, J., Beaudet, S., Halasz, A., Thiboutot, S., and Ampleman, G. 2000a. Microbial degradation of explosives:biotransformation versus mineralization. Appl. Microbiol. Biotechnol. 54:605-618.

Hawari, J., A. Halasz, T. Sheremata, S. Beaudet, C. Groom, L. Paquet, C. Rhofir, G. Ampleman, and S. Thiboutot. 2000b. Characterization of metabolites during biodegradation of hexahydro1,3,5-trinitro-1,3,5-triazine (RDX) with municipal anaerobic sludge. Appl. Environ. Microbiol. $66: 2652-2657$.

Hawari, J. Halasz, A., Beaudet, S., Paquet, L., Ampleman, G., and Thiboutot, S. 2001. Biotransformation routes of octahydro-1,3,5,7-tetranitro-1,3,5,7-tetrazocine by municipal anaerobic sludge. Environ Sci. Technol. 35:70-75.

Hawari, J., S. Deschamps, C. Beaulieu, L. Paquet, and A. Halasz. 2004. Photodegradation of CL20: insights into the mechanisms of initial reactions and environmental fate. Water Res. 38:4055-4064. 
Hawari, J., A. Halasz, C. Groom, S. Deschamps, L. Paquet, C. Beaulieu, and C. A., Photodegradation of RDX in aqueous solution: a mechanistic probe for biodegradation with Rhodococcus Sp., Enviromental Science and Technology, 36 (23), 5117-5123, 2002.

Heilmann, H. M., Wiesmann, U., and Stenstrom, M. K. 1996. "Kinetics of the Alkaline Hydrolysis of High Explosives RDX and HMX in aqueous Solution and Adsorbed to activated Carbon”. Env. Sci. Technol. 30:1485-1492.

Heron, G., Christensen, T.H. and Tjell, J.C., 1994a. Oxidation Capacity of Aquifer Sediments. Environmental Science and Technology, 28: 153-159.

Heron, G., Crouzet, C., Bourg, A.C. and Christensen, T.H., 1994b. Speciation of Fe(II) and $\mathrm{Fe}(\mathrm{III})$ in contaminated aquifer sediments using chemical extraction techniques. Environmental Science and Technology, 28: 1698-1705.

Hofstetter, T.B., C.G. Heijman, S.B. Haderlein, C. Holliger, and R.P. Schwarzenbach, Complete Reduction of TNT and Other (Poly)nitroaromatic Compounds under Iron-Reducing Subsurface Conditions, Environmental Science and Technology, 33 (9), 1479-1487, 1999.

Holmen, B.A., and P.M. Gschwend, Estimating sorption rates of hydrophobic organic compounds in a iron oxide- and alumosilicate clay-coated aquifer sands, Environmental Science and Technology, 31 (1), 105-113, 1997.

Holtz, E., D. Ornellas, M. Foltz, and J. Clarkson. 1994. "The solubility of $\square$-CL-20 in Selected Materials." Propellants Explosives Pyrotechnics 19:206-212.

Huang, K., N. Toride, and M. van Genuchten, Experimental Investigation of Solute Transport in Large, Homogeneous and Heterogeneous, Saturated Soil Columns, Transport in Porous Media, 18, 283-302, 1995.

Jenkins, T., C. Bartolini, and T. Ranney. 2003. "Stability of CL-20, TNAZ, HMX, RDX, NG, and PETN in Moist, Unsaturated Soil." U.S. Army Corps of Engineers, Cold Regions Research and Engineering Laboratory, ERDC/CREEL TR-03-7.

Kaplan, D.I., Bertsch, P.M., Adriano, D.C. and Orlandini, K.A., 1994. Actinide Association with Groundwater Colloids in a Coastal Plain Aquifer. Radiochimica Acta, 66/67: 181-187.

Karickhoff, S., D. Browh, T, Scott, 1979, Sorption of hydrophobic pollutants on natural sediments, Water Resour, Res., 13, 241-248.

Karickhoff, S., 1984, Organic pollutant sorption in aquatic systems, J. Hyd. Eng., 110, 707-733.

Kim, J., J. Park, Y. Yim, and J. Han. 1998. "Crystallization behavior of CL-20 at 298K and quantitative analysis of mixtures of its polymorphs by FTIR." J. Chemical Eng. of Japan 31(3):478-481. 
Kitts, C. L., Cunningham, D. P., and Unkefer, P. J. 1994. "Isolation of Three Hexahydro-1,3,5trinitro-1,3,5-triazine Degrading Species of the Familie Enterobacteriaceae from Nitramine Explosive Contaminated Soil.” Appl. Environ. Microbiol., 60(12):4608-4711.

Klausen, J., Trober, S.P., Haderlein, S.B. and Schwarzenbach, R.P., 1995. Reduction of substituted nitrobenzenes by iron(II) in aqueous mineral suspensions. Environmental Science and Technology, 29(9): 2396-2404.

Knezovich, J. P. and J. I. Daniels. 1991. "Biodegradation of HE.” Water Environ. Technol. $3(3): 34$.

Kukkadapu, R. J. Zachara, J. Fredrickson, D. Kennedy, A. Dohnalkova, D. Mccready, 2005, Ferrous hydroxycarbonate is a stable transformation product of biogetic magnetite, Am. Mineralogist, 29:510-515.

Lane, D. J., G. Pace, G. J. Olsen, D. A. Stahl, M. L. Sogin, and N. R. Pace. 1985. Rapid determination of $16 \mathrm{~S}$ ribosomal RNA sequences for phylogenetic analyses. Proc. Natl. Acad. Sci. USA 82:6955-6959.

Larson, S., D. Felt, L. Escalon, J. Davis, and L. Hansen. 2001. "Analysis of CL-20 in Environmental Matrices: Water and Soil.” U.S Army Corps of Engineers, Engineer Research and Development Center, ERDC/EL TR-01-21, Vicksburg, MS.

Lee, M. L. and K. E. Markides. 1990. “Analytical Supercritical Fluid Chromatography and Extraction.” Chromatography Conferences, Inc., Provo UT.

Leehneer, J. and J. Ahlrichs, 1971. A kinetic and equilibrium study of the adsorption of carbarl and parathion upon soil organic natter, Soil Science Society of America, Proceedings, 35, 700-705.

Liu, C., C. Szecsody, J. Zachara, and W. Ball. 2000. "Use of the generalized integral transform method for solving equations of solute transport in porous media." Adv. Water Resour. 23:483-492.

Liu, C., J. Zachara, Y. Gorby, J. Szecsody, and C. Brown, 2001, Microbial reduction of Fe(III) and sorption/precipitation of Fe(II) on Shewanella putrefaciens Strain CN32, Environmental Science and Technology, 35, 1385-1393.

Luthy, R.G., G. A. Aiken, M. L. Brusseau, S. D. Cunningham, P. M. Gschwend, J. J. Pignatello, M. Reinhard, S. J. Traina, W. J. Weber, Jr., and J. C. Westall. 1997. "Sequestration of Hydrophobic Organic Contaminants by Geosorbents." Environ. Sci. Technol. 31(12)3341-3347.

Ma, L., and H. Selim, Predicting the transport of atrazine in soils: second-order and multireaction approaches, Water Resources Research, 30 (12), 3489-3498, 1994. 
McCarthy, P. M. Reinhard, and B. Rittmann, 1981. Trace organics in groundwater, Environmental Science and Technology, 15(1), 40-51.

McCormick, N. G., J. H. Cornell, and A. M. Kaplan. 1981. "Biodegradation of Hexahydro1,3,5-trinitro-1,3,5-triazine.” Appl Env. Microbiol. 42(5):817-823.

Monteil-Rivera, F., L. Paquet, S. Deschamps, V.K. Balakrishnan, C. Beaulieu, and J. Hawari. 2004. Physico-chemical measurements of CL-20 for environmental applications. Comparison with RDX and HMX. J. Chromatogr. A 1025:125-132.

Nedelko V.V., N. V. Chukanov, A. V. Raevskii, and B. L. Korsounskii. 2000. "Comparative investigation of thermal decomposition of modifications of CL-20." Propellants Explosives Pyrotechnics 25:(5)255-259.

Nielsen, A., A. Chafin, S. Christian, D. Moore, M. Nadler, R. Nissan, and D. Vanderah. 1998. "Synthesis of Polyazapolycyclic Caged Polynitramines." Tetrahedron 54:11793-11812.

Nielson, A.T., D. Vanderah, M. Nadler, N. R., and R. Yee, Polynitropolyaza Caged Explosives Part 8 (U), Naval Air Warfare Center Weapons Division, China Lake, CA, 1994.

Nielson, A.T., M. Chan, K. Kraeutle, C. Lowe-Ma, R. Hollins, M. Nadler, N. R., W. Norris, D. Vanderah, and R. Yee, Polynitropolyaza Caged Explosives Part 7 (U), Naval Weapons Center, China Lake, CA, 1989.

Nielsen, D. R. and J. W. Biggar. 1961. "Miscible displacement in soils. I. Experimental information." Soil Sci. Soc. Am. Proc. 25:1-5.

Nikolic, S., Medic-Seric, M., Rendic, S., and Trinajstic, N. 1994. "Toxic Effects and Structural Property Study of Organic Explosives, Propellants and Related Compounds.” Drug Metabolism Rev. 26:717-738.

Ogden, K. L., Byrnes, C. M., Hanners, J. L., Unkefer, P. J. 1994. “Combination of Aerobic and Anoxic Conditions to Increase the Rate of RDX Biodegradation." University of Arizona, Tuscon, AZ (Unpublished).

Ohnuki, T., 1993. Sorption Characteristics of Strontium on Sandy Soils and Their Components. Env. Sci Technol. 237-245.

Pennington, J. C., Myers, T. E., Davis, W. M., Olin, T. J., McDonald, T. A., Hayes, C. A., and Townsend, D. M. 1995. "Impacts of Sorption on In-Situ Bioremediation of ExplosivesContaminated Soils.” Technical Report IRRP-95-1, US Army Engineer Research and Development Center, Waterways Experiment Station, Vicksburg, MS.

Pignatello, J. J. and B. Xing. 1996. "Mechanisms of Slow Desorption of Organic Chemicals to Natural Particles.” Environ. Sci. Technol. 30(1):1-11. 
Qasim, M., H. Fredrickson, Honea, P., J. Leszczynski, S. Okovyty, J., Szecsody, 2005, Prediction of CL-20 chemical degradation pathways depend on competing modes of reaction with theoretical and experimental evidence, Structural Chemistry, accepted.

Qasim, M. M., J. S. Furey, H. L. Fredrickson, J. Szecsody, C. McGrath, and R. Bajpai. 2004. "Semiempirical Predictions of Chemical Degradation Reaction Mechanisms of CL-20 Other Cyclic Nitramines as Related to Their Molecular Structures.” Structural Chemistry 15(5).

Riley, R. G., C. J. Thompson, M. H. Huesemann, Z. Wang, B. Peyton, T. Fortman, and M. J. Truex. 2000. "Evidence of Pore Effects Influencing Slow Desorption of Phenanthrene." Env. Sci. Tech. (submitted).

Ronen, Z., A. Brenner, and A. Abeliovich. 1998. "Biodegradation of RDX-Contaminated Wastes in a Nitrogen-Deficient Environment." Water Sci. Technol. 38(4-5):219-224.

SAS Institute. 2003. SAS \STAT User's Guide Release 9.1 Ed., SAS Inst., Cary, NC.

Schilling, A. B. 1966. "The Analysis of Explosives Constituents and Metabolites with Electrospray and APCI LC/MS." In Proceedings of the 44th ASMS Conference on Mass Spectrometry and Allied Topics, May 12-16, 1966, Portland, OR, p. 196.

SERDP. 1993. "An approach to estimation of volumes of contaminated soil and groundwater for selected army installations." Labat-Anderson Inc. Prepared for the Executive Director, Strategic Environmental Research and Development Program.

Seth-Smith, H.M.B., S. J. Rosser, A. Basran, E. R. Travis, E. R. Dabbs, S. Nicklin, and N. C. Bruce. 2002. "Cloning, Sequencing, and Characterization of the Hexahydro-1,3,5Trinitro-1,3,5-Triazine Degradation Gene Cluster from Rhodococcus rhodocrous." Applied Environmental Microbiology 68:4764-4771.

Shah, M.M. and J.A. Campbell. 1997. Transformation of Nitrobenzene by Ferredoxin NADP Oxidoreductase from Spinach Leaves. Biochem. Biophys. Res. Com., 241, 794-798.

Shelton, D.R., and J.M. Tiedje. 1984. General method for determining anaerobic biodegradation potential. Appl. Environ. Microbiol. 47:850-857.

Sheremata, T. W. and J. Hawari. 2000. "Mineralization of RDX by the White Rot Fungus Phanerochaetre chrysosporium to Carbon Dioxide and Nitrous Oxide." Env. Sci. Technol. 34(16):3384-3388.

Singh, J., S. D. Comfort, and P. J. Shea. 1998a. "Long-Term RDX Sorption and Fate in Soil." J. Environ. Qual. 27:572-577.

Singh, J., S. D. Comfort, and P. J. Shea. 1998b. "Remediating RDX-Contaminated Water and Soil Using Zero-Valent Iron.” J. Environ. Qual. 27:1240-1245. 
Singh, J., S. D. Comfort, and P. J. Shea. 1999. "Iron-Mediated Remediation of RDXContaminated Water and Soil Under Controlled Eh/pH.” Environ. Sci. Technol. 33:1488-1494.

Spalding, R. F. and J. W. Fulton. 1988. "Groundwater Munition-Residues and Nitrate Near Grand Island, Nebraska, USA.” J. Contaminant Hydrol. 2:139-153.

Spanggord, R. J., Mill, T., Chou, T.-W., Mabey, W. R., Smith, J. H., and Lee, S. 1980. "Environmental Fate Studies on Certain Munition Wastewater Constituents, Final Report.". SRI Project No. LSU-7934, SRI International, Menlo Park, CA.

Sposito, G., and G. Dagan, Predicting solute plume evolution in heterogeneous porous formations, Water Resources Research, 30 (2), 585-589, 1994.

Spurlock, G., K. Huang, and M.T. van Genuchten, Isotherm nonlinearity and nonequilibrium sorption effects on transport of Fenuron and Monuron in soil columns, Environmental Science and Technology, 29, 1000-1007, 1995.

Stucki, J.W., Golden, D.C. and Roth, C.B., 1984. Preparation and handling of dithionite-reduced smectite suspensions. Clays and Clay Minerals, 32(3): 191-197.

Szecsody, J., D. Girvin, J. Campbell, and B. Devary. 2004a. "Sorption and Oxic Degradation of the Explosive CL-20 During Transport in Subsurface Sediments." Chemosphere 56:593-610.

Szecsody, J., M. Williams, J. Fruchter, V. Vermeul, and D. Sklarew. 2004b. "In Situ Reduction of Aquifer Sediments for Remediation: Enhancement of Reactive Iron Phases." Environmental Science and Technology 38:4656-4663.

Szecsody, J., W. Pavalko, and G. Streile. 1992. "Transport of Aminonaphthalene with a SiteLimited Transformation Reaction.” Env. Sci. \& Tech 27(2):356-365.

Szecsody, J. E., F. J. Brockman, G. P. Streile, and M. J. Truex. 1993. "Transport and Biodegradation of Quinoline in Horizontally Stratified Porous Media." J. Contaminant Hydrology 15:277-304.

Szecsody, J., A. Chilikapati, J. Zachara, P. Jardine, and A. Ferrency. 1998. "Importance of Flow and Particle-Scale Heterogeneity on CoII/IIIEDTA Reactive Transport." J. Hydrology 209(1-4):112-136.

Szecsody, J., M. Williams, J. Fruchter, V. Vermeul, and J. Evans. 2000a. "Influence of sediment reduction on TCE degradation", Remediation of Recalcitrant Compounds G. Wickramanayake (ed.), C2-6, 369-376.

Szecsody, J., J. Fruchter, D. Sklarew, and J. Evans. 2000b. In situ redox manipulation of subsurface sediments from Fort Lewis, Washington: iron reduction and TCE dechlorination mechanisms, Pacific Northwest National Laboratory, PNNL-13178, Richland, Washington. 
Trott, S., S. F. Nishino, J. Hawari, and J. C. Spain. 2003. "Biodegradation of the Nitramine Explosive CL-20.” Applied Environmental Microbiology 69:1871-1874.

van Genuchten, M. Th. and P. J. Wierenga. 1976. "Mass transfer studies in sorbing porous media I. Analytical solutions." Soil Sci. Soc. Am. J. 40:473-480.

Vermeul, V., M. Williams, J. Evans, J. Szecsody, B. Bjornstad, and T. Liikala. 2000. In situ redox manipulation proof-of-principle test at the Fort Lewis Logistics Center: final report, Pacific Northwest National Laboratory PNNL-13357, Richland, Washington.

Waisner, S. A., H. Fredrickson, L. Hansen, M. Zappi, G. Myrick, and S. Banerji. 2000. "Removal of RDX from a Contaminated Groundwater by In-Situ Bioremediation." U.S. Army Research and Development Center, Waterways Experiment Station Report \#ERDC/EL TR-00-14.

Wang, Z., D. M. Friedrich, M. R. Beversluis, S. L. Hemmer, A. G. Joly, M. H. Huesemann, M. J. Truex, R. G. Riley, and C. J. Thompson. 2000. "A Spectroscopic Study of Phenanthrene Sorption on Porous Silica." Environmental Science and Technology, 36, 15501559.

White, D.C., and D.B. Ringelberg, 1998. Signature lipid biomarker analysis. p. 255-272. In R.S. Burlage et al. (ed.) Techniques in microbial ecology. Oxford Univ. Press, New York.

Wierenga, P. J. and M. Th. Van Genuchten. 1989. "Solute transport through small and large unsaturated soil columns." Ground Water 27:35-42.

Winget, P., C. Cramer, and D. Truhlar, Prediction of soil sorption coefficients using a universal solvation model, Enviromental Science and Technology, 34 (22), 4733-4740, 2000.

Wise, W. R. 1993. Effects of laboratory-scale variability upon batch and column determinations of nonlinearity sorptive behavior in porous media, Water Resour. Res., 29(9): 2983-2992.

Wu, S., and P. Gschwend, 1986, Sorption kinetics of hydrophobic organic compounds to natural sediments and soils, Environmental Science and Technology, 20, 717-725.

Young, D. M., Unkefer, P. J., and Ogden, K. L. 1997. "Biotransformation of Hexahydro-1,3,5trinitro-1,3,5-triazine (RDX) by a Prospective Consortium and its Most Effective Isolate Serratia marcescens.” Biotechnol. Bioeng. 53(5):515-522.

Zepp, R., and P. Schlotzhauer, 1981. Comparison of photochemical behavior of various humic substances in water. Geochemica Cosmo. Acta, 58, 553-566.

Zhao, J.-S., A. Halasz, L. Paquet, C. Beaulieu, and J. Hawari. 2002. Biodegradation of hexahydro-1,3,5-trinitro-1,3,5-triazine and its mononitroso derivative hexahydro-1-nitroso- 
3,5-dinitro-1,3,5-triazine by Klebsiella pneumoniae strain SCZ-1 isolated from an anaerobic sludge. Appl. Environ. Microbiol. 68:5336-5341. 


\section{Attachment 1: Publications and Presentations*}

$* 100 \%$ funded by this project unless otherwise noted

\section{Journal Publications}

Campbell, J., J. Szecsody, B. Devary, B. Valenzuela, 2005, Electrospray Ionization Mass Spectrometry of CL-20, J. Chromatography, in preparation

Crocker, F., Szecsody, J., H. Fredrickson, 2005, Biodegradation of 2,4,6,8,10,12-hexanitro2,4,6,8,10,12-hexaazaisowurtzitane (CL-20) under anaerobic conditions. Applied Environmental Microbiology, submitted.

Crocker, F.H., K.T. Thompson, J.E. Szecsody, and H.L. Fredrickson. 2005. Biotic and Abiotic Degradation of Hexanitrohexaazaisowurtzitane (CL-20) and Hexahydro-1,3,5-trinitro-1,3,5triazine (RDX) in Soils. Journal of Environmental Quality, accepted, in press.

Girvin, D., Szecsody, J. Kennedy, D., 2005, Enhancement of CL20 Degradation in a sediment by the iron reducing bacteria Shewanella putrefaciens (strain CN32), Geochimica, in preparation.

LeMond, L., A. Gamerdinger, J. Szecsody, 2005, Fate and transport of energetics CL-20 and RDX in unsaturated laboratory columns, Water Resour. Res., in preparation.

Qasim, M., H. Fredrickson, Honea, P., J. Leszczynski, S. Okovyty, J., Szecsody, 2005, Prediction of CL-20 chemical degradation pathways depend on competing modes of reaction with theoretical and experimental evidence, Structural Chemistry, accepted, partial funding by this project.

Qasim, M.M., J.S. Furey, H.L. Fredrickson, J. Szecsody, C. McGrath, R. Bajpai, 2004. Semiempirical Predictions of Chemical Degradation Reaction Mechanisms of CL-20 Other Cyclic Nitramines as Related to Their Molecular Structures. Structural Chemistry, 15(5), partially funded by this project.

Szecsody, J., Devary, B., Girvin, D., Zhang, L., 2005, Influence of Aging on Energetics RDX and CL-20 adsorption and degradation in subsurface sediments, J. Contaminant Hydrol.,in preparation.

Szecsody, J. Girvin, D., Campbell, Devary, B., 2004, Sorption and Oxic Degradation of the Explosive CL-20 during transport in subsurface sediments, Chemosphere, 56: 593-610.

Szecsody, J., Williams, M., J. Fruchter, V Vermeul, D. Sklarew, 2004. In Situ Reduction of Aquifer Sediments for Remediation: Enhancement of Reactive Iron Phases, Environmental Science and Technology, 38:4656-63. 


\section{Conference Papers and Presentations}

Crocker, F.H., D.C. McCullum, K.T. Thompson, and H.L. Fredrickson. 2004. Biodegradation of the Nitramine Explosive, CL-20. Annual Meeting of the South Central Branch of the American Society for Microbiology, 5-6 Nov. 2004, partially funded by this project.

LeMond, L. A., 2005, Fate and Transport of the Explosives RDX and CL-20 in Unsaturated Laboratory Columns, MS Thesis, Department of Soil, Water, and Environmental Science, University of Arizona.

LeMond, L., A. Gamerdinger, J. Szecsody, 2005, Fate and Transport of CL-20 and RDX in Unsaturated Laboratory Columns, American Geophysical Union, 2005 AGU Joint Assembly, New Orleans, LA.

McCullum, D.C., F.H. Crocker, K.T. Thompson, and H.F. Fredrickson. 2005. Anaerobic Biodegradation of the Nitramine Explosives, CL-20 and RDX, in Soil. 105th General Meeting of the American Society for Microbiology, 04-09 June 2005, partially funded by this project.

Qasim, Mo., Fredrickson, H., Furey, J., Honea, P. Szecsody, J., 2004, CL-20 Chemical Degradation pathways depend on competing modes of reaction. SERDP Annual Conference, December 2004, partially funded by this project.

Qasim, Mo, Herbert L. Fredrickson, John Furey; Ray Castellane, Jim Szecsody, 2005, Cyclic nitramines: spectrophotometry supports theoretical predictions of chemical transformations, Third International Conference on Remediation of Contaminated Sediments, New Orleans, LA, January 24-27, 2005, partially funded by this project.

Qasim, Mo, Herbert L. Fredrickson; John Furey; Ray Castellane1; Chris McGrath; Jim Szecsody, 2004, Using CL-20 as the Model, UV/VIS/FTIR Spectrophotometry Supports Theoretical Predictions as to Initial/Intermediate Steps in Chemical Transformation of Cyclic and Cage Cyclic Nitramines, 4th Annual Southern School on Computational Chemistry Conference, NSF-EPSCOR Computational Chemistry Cluster and Army High Performance Computing Research Center, Pensacola, Florida, partially funded by this project.

Szecsody, J., Campbell, F. Crocker, H. Fredrickson, 2003, Fate of CL-20 in the Natural Subsurface Environment, JAANAF Special Conference on CL-20, Ogden, Utah, May 2003.

Szecsody, J., J. Campbell, B. Devary, M. Qasim, H. Fredrickson, 2003, Sorption and oxic degradation of Cl-20 during transport in subsurface sediments, SERDP Annual Conference, Washington DC, December 2003.

Szecsody, J., J. Campbell, H. Fredrickson, F. Crocker, R. Riley, M McKinley, 2002, Factors affecting the fate and transport of CL-20 in the Vadose Zone and Groundwater, SERDP Annual Conference, Washington DC, December 2002. 
Szecsody, J., D. Girvin, M. Qasim, Cl-20 Reactivity in the subsurface environment and potential for migration, JANNAF 21st Safety and Environmental Protection Subcommittee \& 32nd Propellent Subcommittee Joint Meeting, July 27, 2004, Seattle, WA.

Szecsody, J., Girvin, D., Qasim, M., 2004, Degradation of the explosive CL-20 and influence of aging in subsurface environments, SERDP Annual Meeting, December 2004.

Szecsody, J., Girvin, D., Devary, B., Qasim, M., Crocker, F., Fredrickson, H., 2004, Transport and degradation of the explosive CL-20 in subsurface sediments, Fourth Annual Society of Environmental Toxicology and Chemistry (SETAC) World Congress, Portland, Oregon, November 14-18, 2004, Session A12 Fate and Effects of Energetic Compounds, Portland, Oregon.

Thompson, K.T., F.H. Crocker, T.L. Acuff, J.E. Szecsody, and H.L. Fredrickson. 2003. Biodegradation of the Explosives CL-20 and RDX in Surface and Subsurface Soils. 103rd General Meeting of the American Society for Microbiology, 18-22 May 2003, Washington, D.C. 
Appendix A.1; CL-20 Reactions with Laboratory Materials

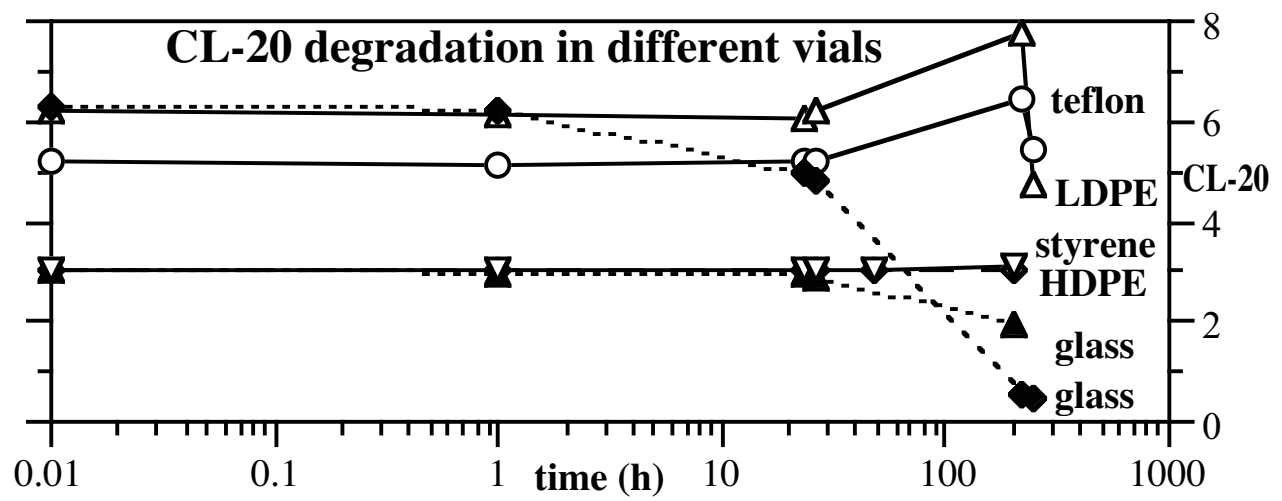

NXF/NXG: CL-20 degradation cosolvent and pH buffer effect
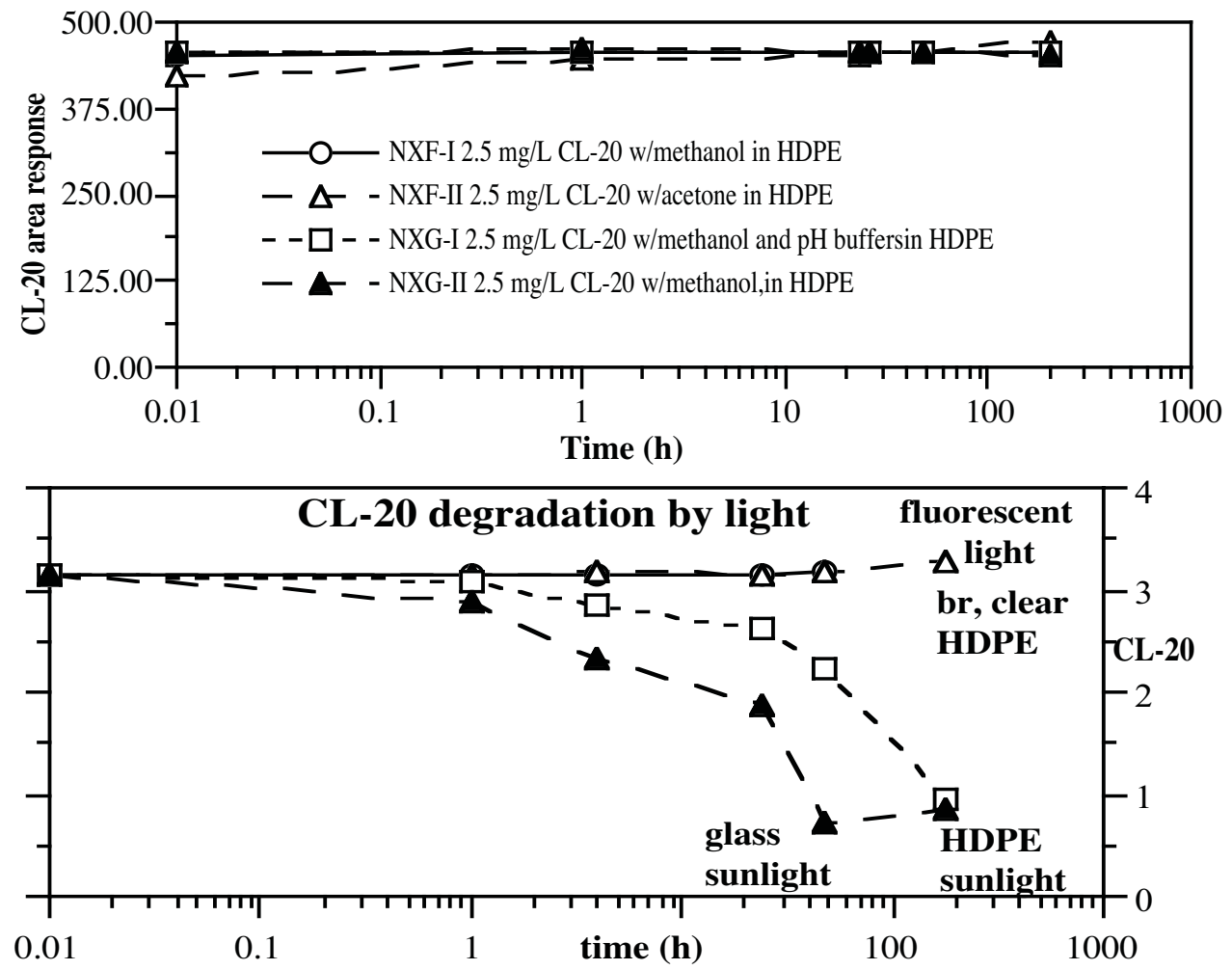

\begin{tabular}{|c|c|c|c|c|c|c|c|c|c|c|c|c|}
\hline \multicolumn{2}{|c|}{ Injections } & \multicolumn{10}{|c|}{ HPLC an al y sis: uni denti fied peak s ( $r$ et. ti me, min) } & \multirow{2}{*}{$\begin{array}{l}\text { CL-20 } \\
8.8 \mathrm{~min}\end{array}$} \\
\hline Des cription & Name & $\begin{array}{l}1.7 \\
\min \end{array}$ & $\begin{array}{l}2.4 \\
\mathrm{~min}\end{array}$ & $\begin{array}{l}3.7 \\
\min \end{array}$ & $\begin{array}{r}4.4 \\
\mathrm{~min}\end{array}$ & $\begin{array}{l}4.9 \\
\mathrm{~min}\end{array}$ & $\begin{array}{l}5.6 \\
\mathrm{~min}\end{array}$ & $\begin{array}{l}6.1 \\
\mathrm{~min}\end{array}$ & $\begin{array}{l}6.7 \\
\mathrm{~min}\end{array}$ & $\begin{array}{l}8.0 \\
\mathrm{~min}\end{array}$ & $\begin{array}{l}8.5 \\
\mathrm{~min}\end{array}$ & \\
\hline $\begin{array}{l}\mathrm{CL}-20,2.5 \mathrm{mg} / \mathrm{L} \\
\text { Standard }\end{array}$ & NXL-1 Oh & & & & & & & & & & & 455.7 \\
\hline $\begin{array}{l}\mathrm{CL}-20,2.5 \mathrm{mg} / \mathrm{L} \\
\text { through Teflon filter }\end{array}$ & NXL-7 & & & & & & & & & & & 455.6 \\
\hline $\begin{array}{l}\mathrm{CL}-20,2.5 \mathrm{mg} / \mathrm{L} \\
\text { through nylon filter }\end{array}$ & $\mathrm{NXL}-2 \mathrm{Oh}$ & & 127.4 & 138.0 & 427.9 & 114.3 & 41.0 & & 15.2 & 10.3 & 7.2 & \\
\hline $\begin{array}{l}\text { MeOH through clean } \\
\text { nylon filter }\end{array}$ & $\begin{array}{l}\mathrm{MeOH} \\
\text { nylon filt }\end{array}$ & & 333.2 & 201.9 & 174.9 & & & 71.3 & & & & \\
\hline $\begin{array}{l}\text { MeOH rinsed through } \\
\text { used nylon filter to } \\
\text { recover } \mathrm{CL}-20\end{array}$ & $\begin{array}{l}\mathrm{MeOH} \text { filt } \\
\text { rinse }\end{array}$ & 68.8 & 137.9 & 150.5 & 110.9 & & & 38.7 & & 15.0 & & $\begin{array}{c}379.7 \\
(80.5 \%)\end{array}$ \\
\hline
\end{tabular}


APPENDIX A.2; CL-20 AQUEOUS DEGRADATION
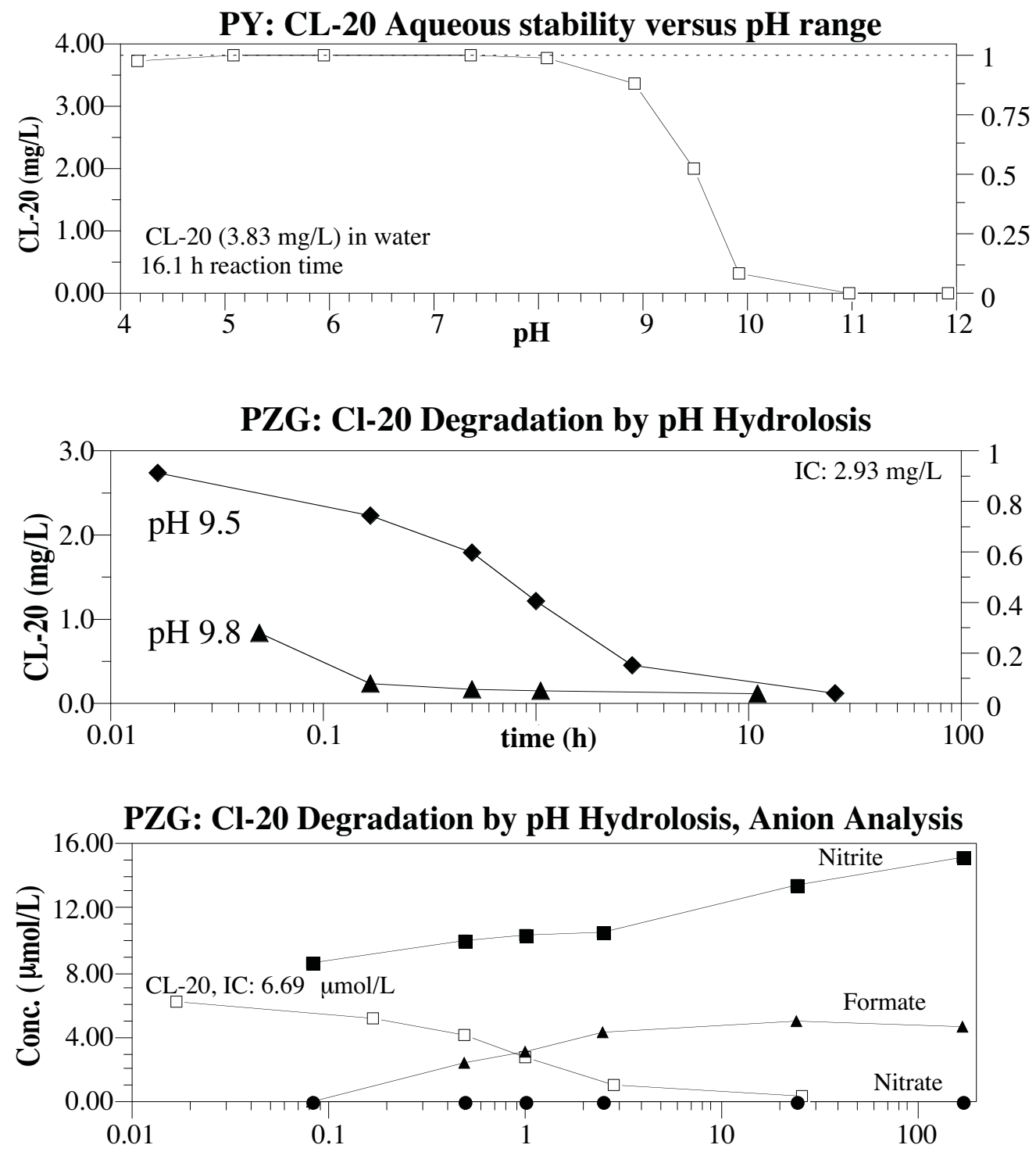

\begin{tabular}{|l|c|c|c|c|c|c|c|}
\hline Sample & $\begin{array}{c}\text { Time } \\
\text { (h) }\end{array}$ & $\begin{array}{c}\text { CL-20 } \\
(\mathbf{m m o l} / \mathbf{L})\end{array}$ & $\begin{array}{c}\text { Formate } \\
\text { (mmol/L) })\end{array}$ & $\begin{array}{c}\text { Nitrite } \\
(\mathbf{m m o l} / \mathbf{L})\end{array}$ & $\begin{array}{c}\text { Nitrate } \\
(\mathbf{m m o l} / \mathbf{L})\end{array}$ & $\begin{array}{c}\text { Formate/ } \\
\text { CL-20 }\end{array}$ & $\begin{array}{c}\text { Nitrite/ } \\
\text { CL-20 }\end{array}$ \\
\hline PZG-C1 & 0.08 & 5.09 & 0.00 & 8.67 & 0.00 & 0.000 & 1.296 \\
\hline PZG-C2 & 0.50 & 4.09 & 2.35 & 9.94 & 0.00 & 0.351 & 1.486 \\
\hline PZG-C3 & 1.00 & 2.77 & 3.09 & 10.32 & 0.00 & 0.461 & 1.543 \\
\hline PZG-C4 & 2.50 & 1.04 & 4.23 & 10.45 & 0.00 & 0.632 & 1.562 \\
\hline PZG-C5 & 24.00 & 0.28 & 5.00 & 13.45 & 0.00 & 0.747 & 2.010 \\
\hline PZG-C6 & 168.0 & & 4.73 & 15.13 & 0.00 & 0.707 & 2.262 \\
\hline
\end{tabular}


PZ: CL-20 stability with aqueous dithionite (anaerobic)

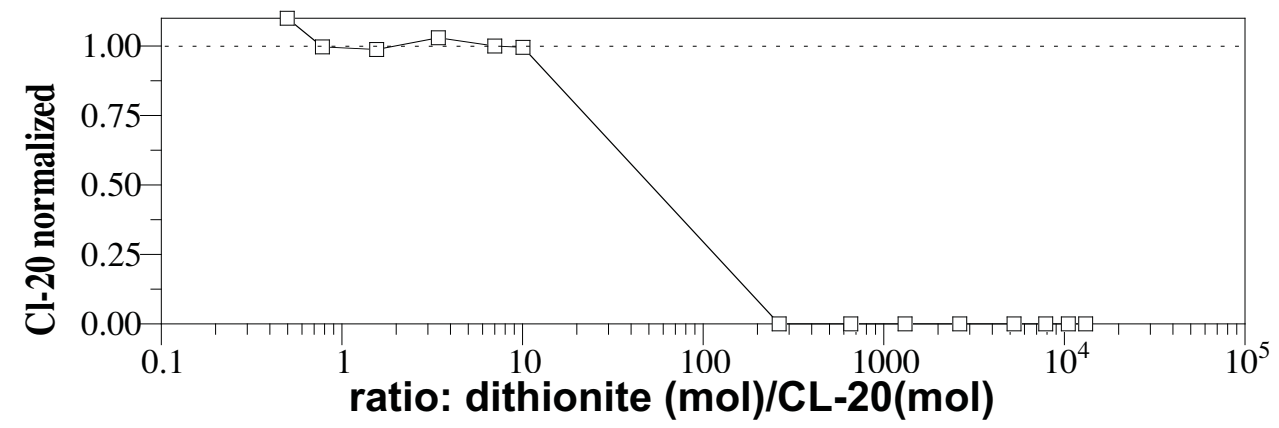

PZ: Cl-20 degradation rate with aqueous dithionite
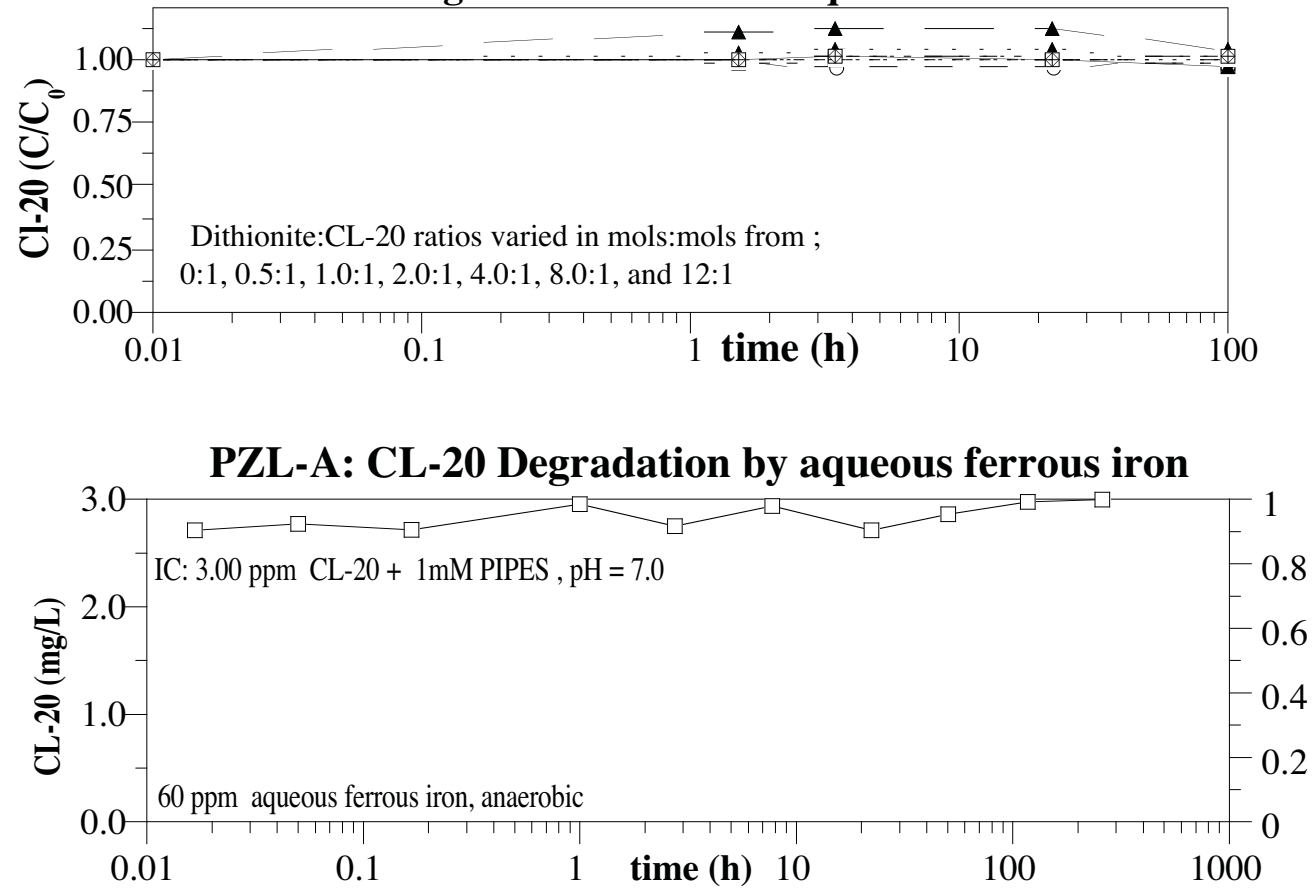

PZC-St. : CL-20 and RDX Aqueous Stability at $65{ }^{\circ} \mathrm{C}$

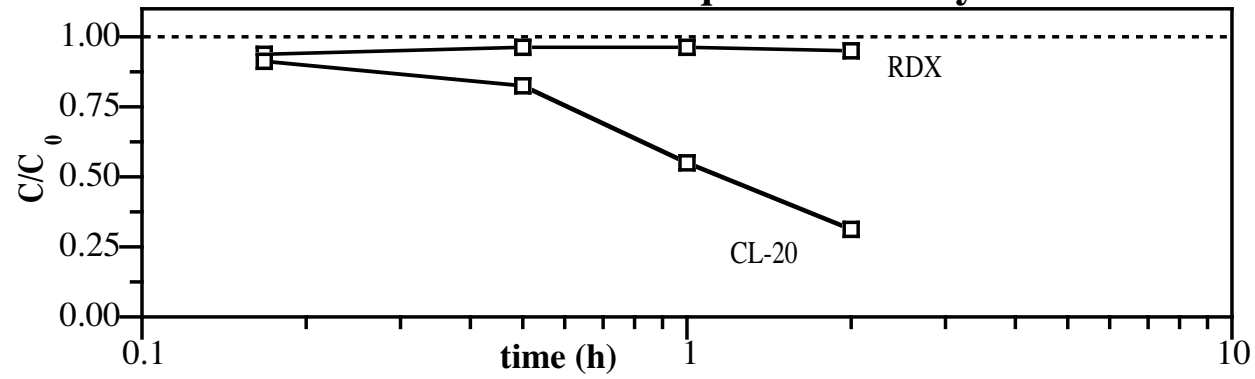


PZZB: CL-20 Aqueous Stability vs. temp. $40{ }^{\circ} \mathrm{C}$

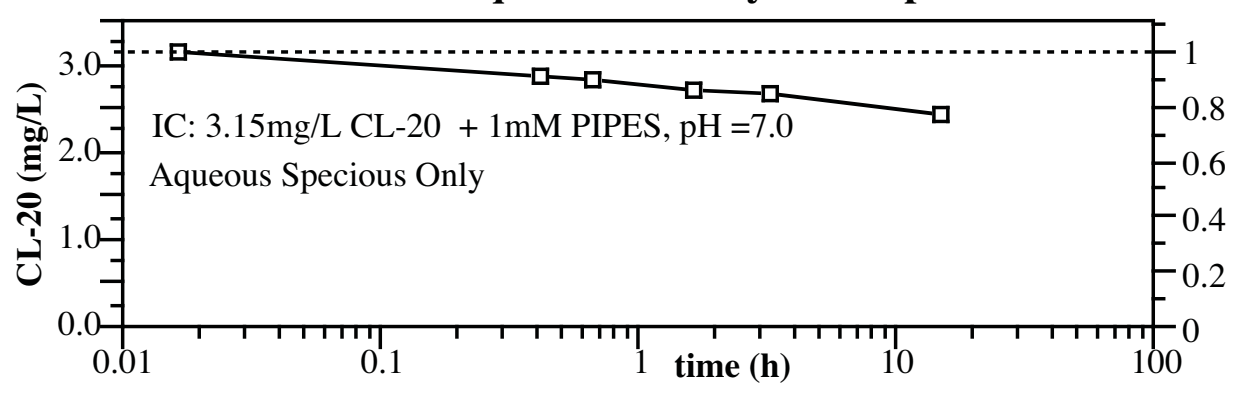

RDX Aqueous Stability vs. Temp (5mg/L)

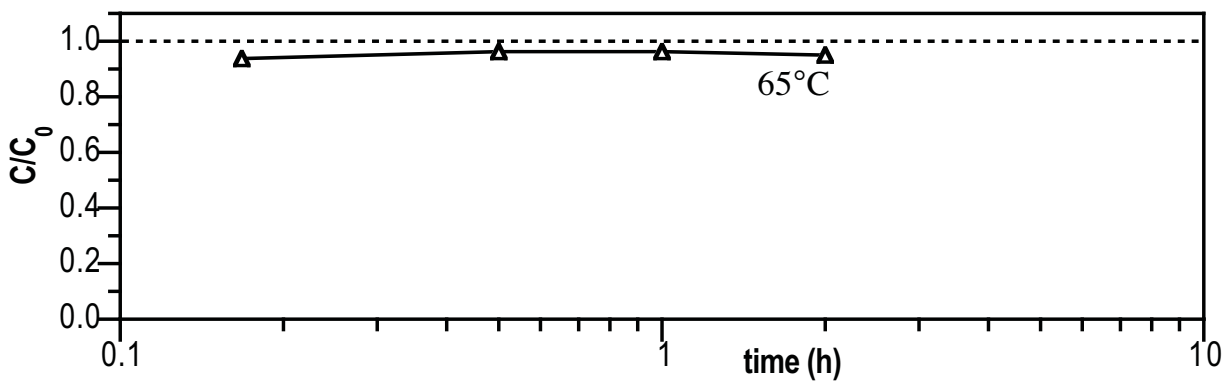

CL-20 Aqueous Stability vs. Temp (3mg/L)

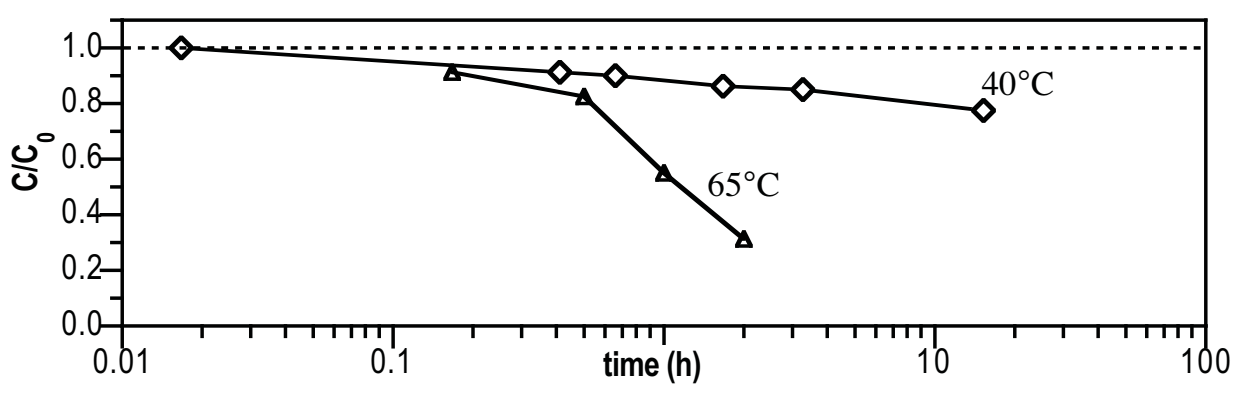

Q6-9: CL-20 Aqueous Degradation vs Temperature

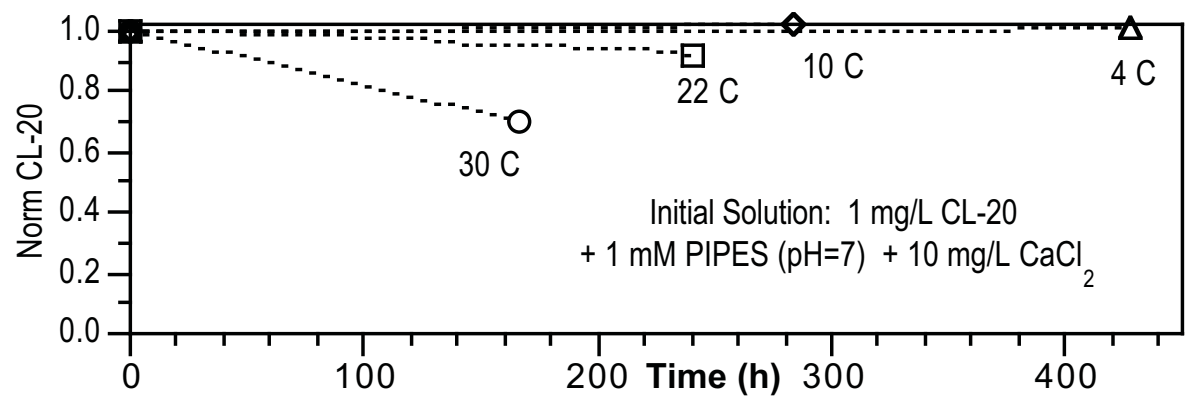


APPENDIX B. CL-20 DEGRADATION BY MINERALS W, W/O ADSORBED AND/OR STRUCTURAL IRON

Appendix B.1; CL-20 Degradation by Natural Minerals with no adsorbed Fe ${ }^{\text {II }}$ 
PZH-A: CL-20 Degradation by High Surface Area Silica
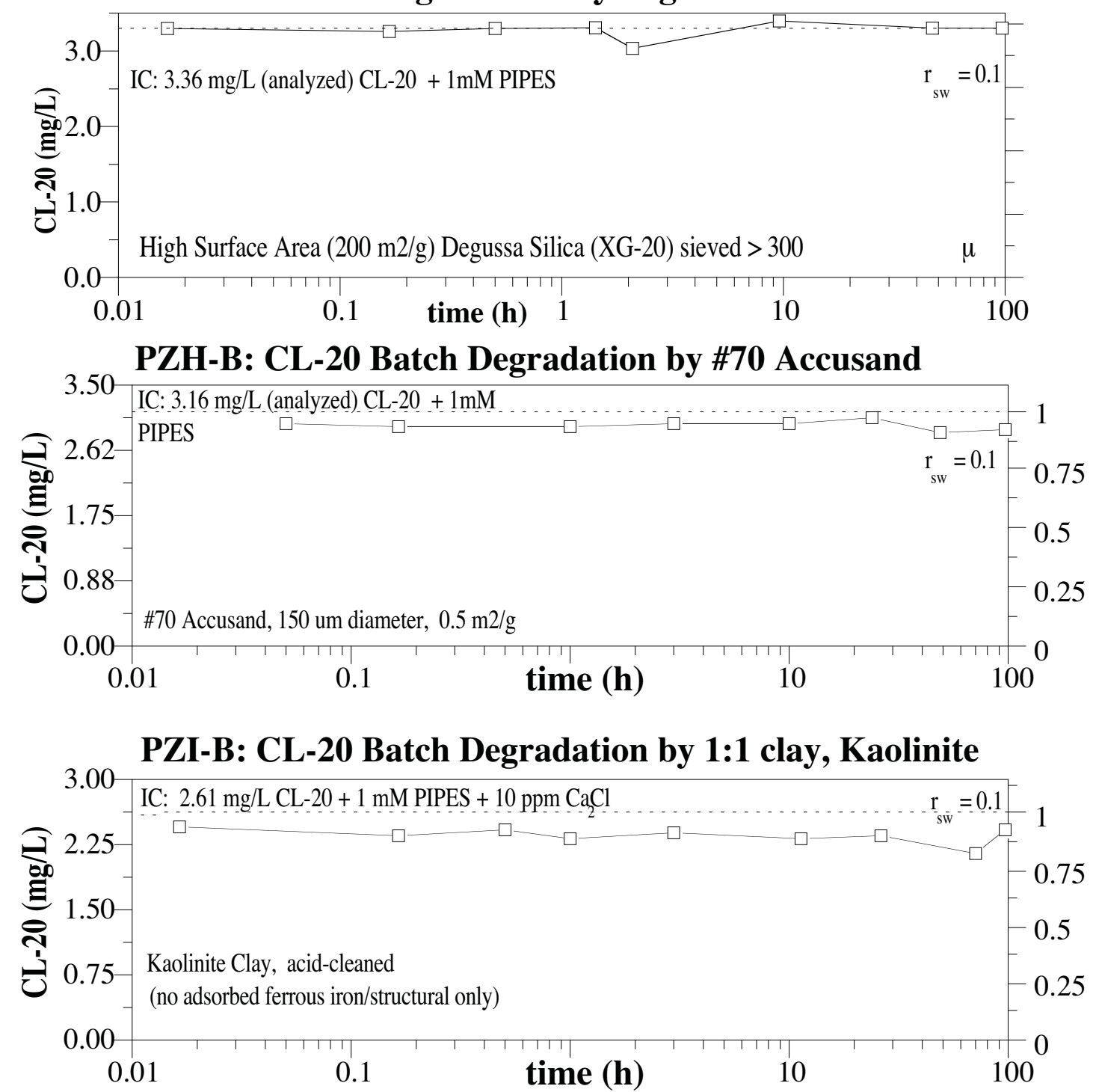

PZT: CL-20 Degradation by 1:1 clay, Kaolinite [no ads. Fe(II)]

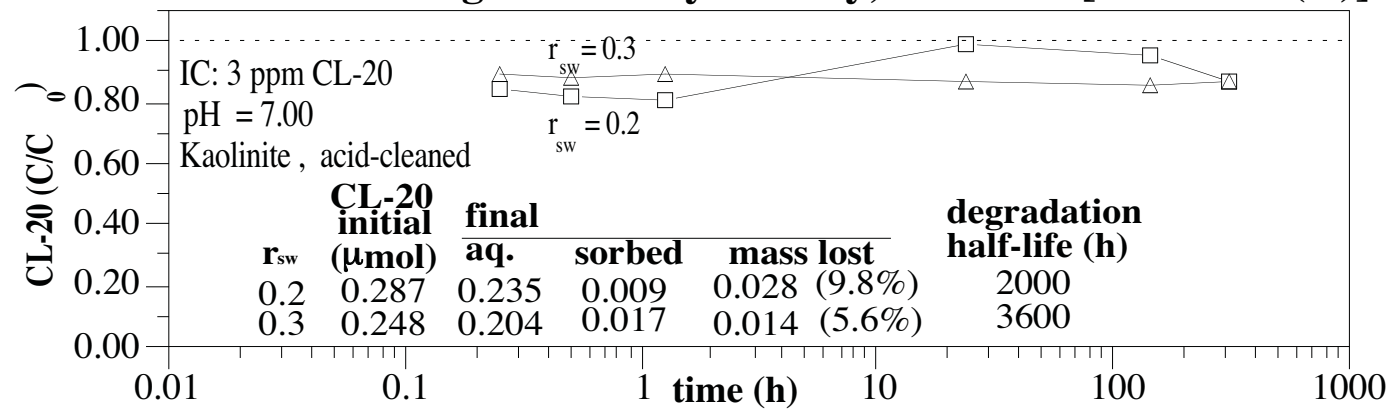




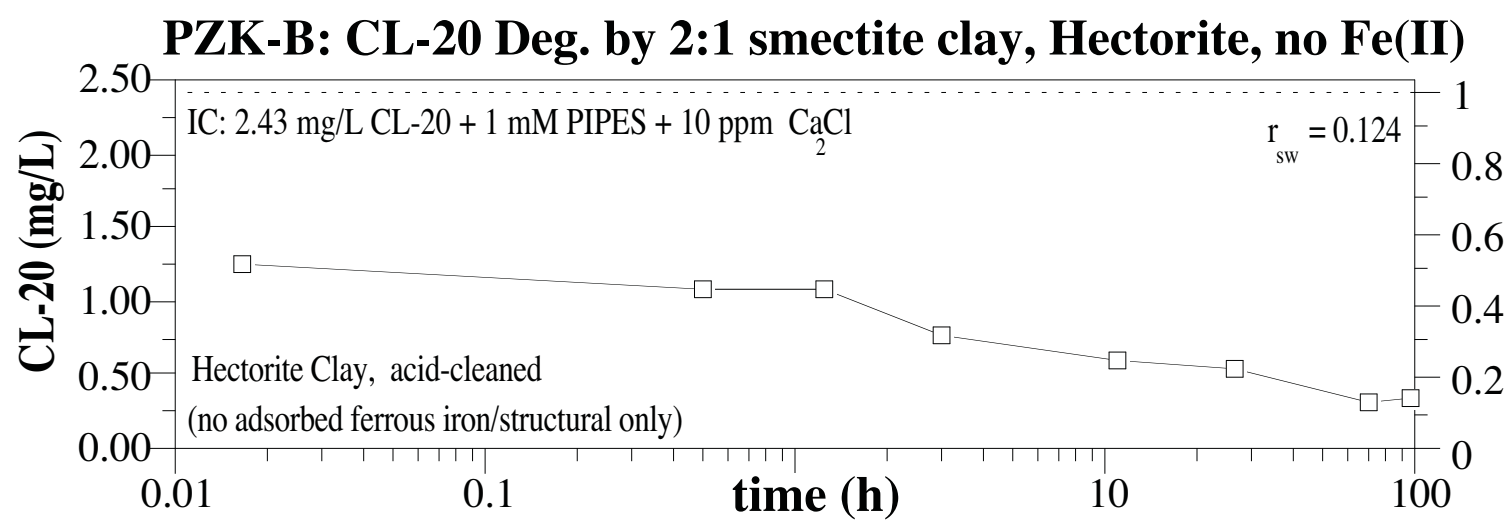

PZK: CL-20 Degradation by 2:1 smectite clay, anion analysis

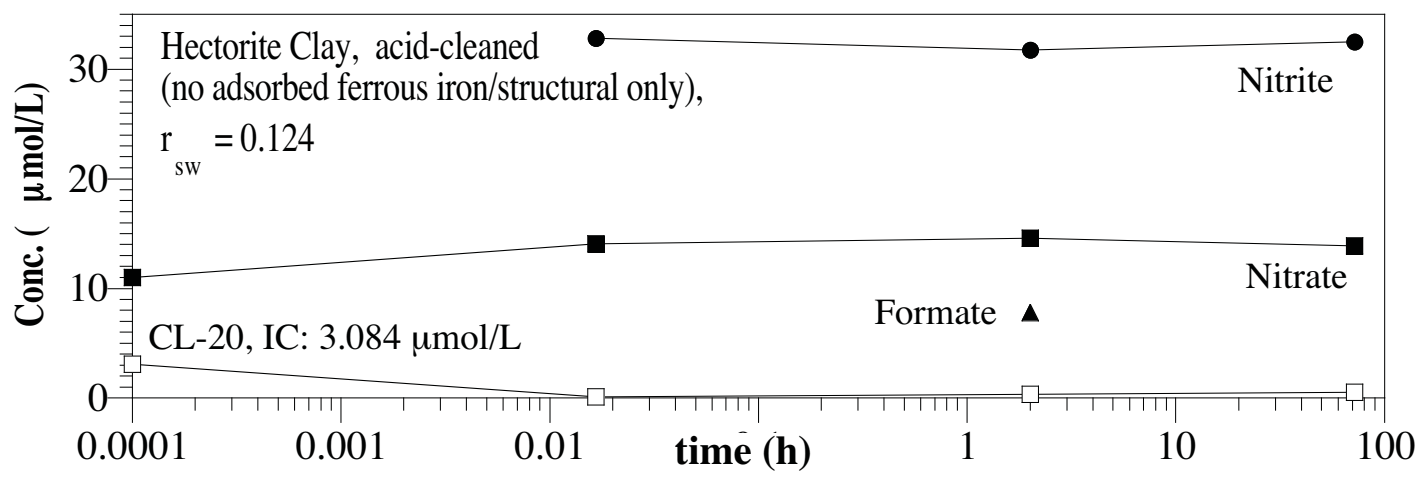

\begin{tabular}{|c|c|c|c|c|c|c|c|}
\hline $\begin{array}{c}\text { Elapsed Time } \\
\text { (h) }\end{array}$ & $\begin{array}{c}\text { CL-20 } \\
(\mu \mathrm{mol} / \mathrm{L})\end{array}$ & $\begin{array}{l}\text { Formate } \\
(\mu \mathrm{mol} / \mathrm{L})\end{array}$ & $\begin{array}{c}\text { Nitrite } \\
(\mu \mathrm{mol} / \mathrm{L})\end{array}$ & $\begin{array}{c}\text { Nitrate } \\
(\mu \mathrm{mol} / \mathrm{L})\end{array}$ & $\begin{array}{c}\text { Formate/ } \\
\text { Cl-20 }\end{array}$ & $\begin{array}{l}\text { Nitrite/ } \\
\text { CL-20 }\end{array}$ & $\begin{array}{c}\text { Nitrate/ } \\
\text { CL-20 }\end{array}$ \\
\hline IC & 3.084 & 0.000 & 11.011 & 0.000 & 0.00 & 3.57 & 0.00 \\
\hline 0.02 & 0.109 & 540.904 & 14.072 & 32.832 & 175.37 & 4.56 & 10.64 \\
\hline 2.00 & 0.329 & 7.782 & 14.547 & 31.770 & 2.52 & 4.72 & 10.30 \\
\hline 72.00 & 0.513 & 221.809 & 13.867 & 32.480 & 71.91 & 4.50 & 10.53 \\
\hline
\end{tabular}

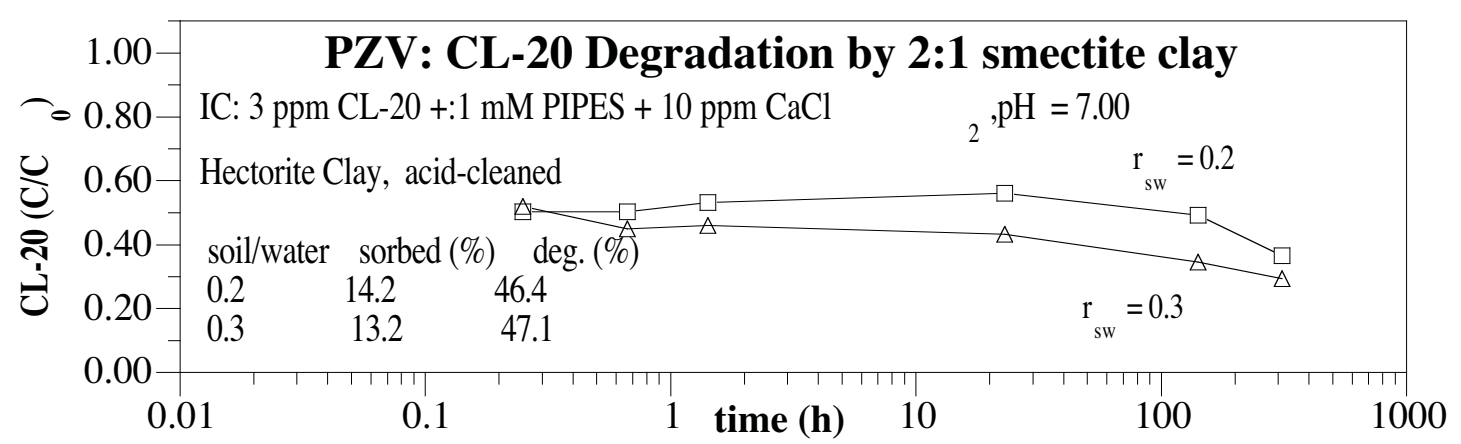


Appendix B.2; CL-20 Degradation by Natural Minerals with no adsorbed FeII (i.e. acid-washed Hectorite) vs. Temperature 
Q9: CL-20 sorption/degradation by acid-washed Hectorite Clay, $r_{s w}=0.5,4 C$

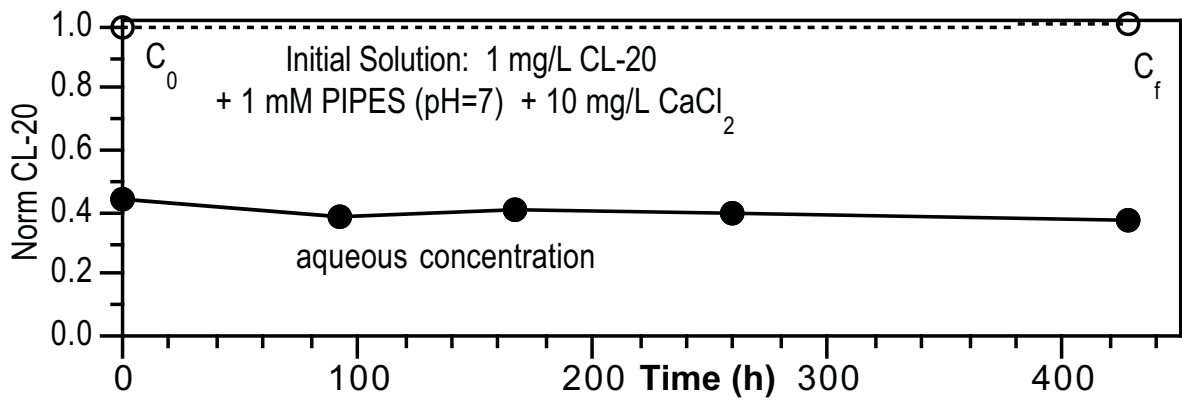

\begin{tabular}{|r|c|c|}
\hline CL-20 & (umol) & norm. \\
\hline Initial Aqe. & 0.034 & 1 \\
\hline Final Aqe. & 0.012 & 0.353 \\
\hline Sorbed & 0.013 & 0.379 \\
\hline Degraded & 0.009 & 0.268 \\
\hline
\end{tabular}

(2.5\% mass removed during sampling)

Q8: CL-20 sorption/degradation by acid-washed Hectorite Clay, $r_{\mathrm{sw}}=0.5,10 \mathrm{C}$

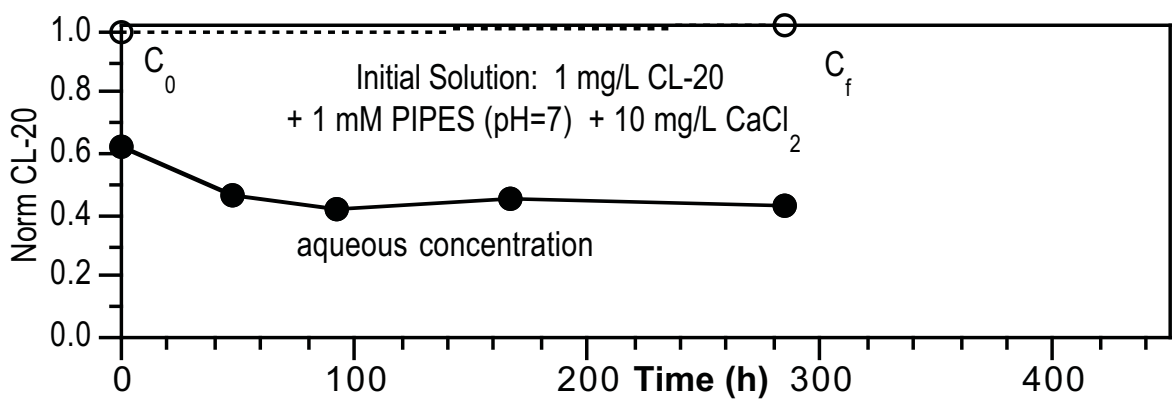

\begin{tabular}{|r|c|c|}
\hline CL-20 & (umol) & norm. \\
\hline Initial Aqe. & 0.0336 & 1 \\
\hline Final Aqe. & 0.0137 & 0.409 \\
\hline Sorbed & 0.0132 & 0.392 \\
\hline Degraded & 0.0067 & 0.199 \\
\hline
\end{tabular}

(3.0\% mass removed during sampling)

Q6: CL-20 sorption/degradation by acid-washed Hectorite Clay, $r_{\mathrm{sw}}=0.5,22 \mathrm{C}$

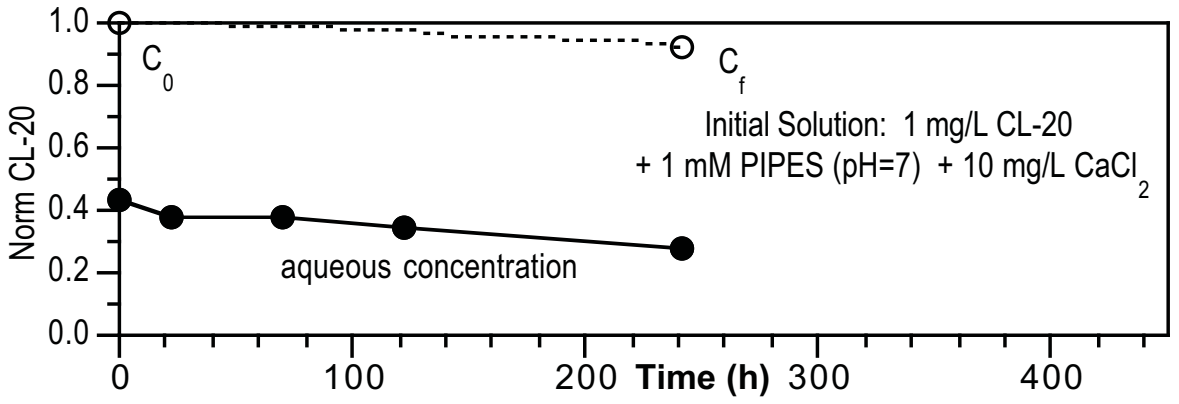

\begin{tabular}{|r|c|c|}
\hline CL-20 & (umol) & norm. \\
\hline Initial Aqe. & 0.036 & 1 \\
\hline Final Aqe. & 0.0096 & 0.266 \\
\hline Sorbed & 0.0094 & 0.262 \\
\hline Degraded & 0.017 & 0.472 \\
\hline
\end{tabular}

(2.3\% mass removed during sampling)

Q7: CL-20 sorption/degradation by acid-washed Hectorite Clay, $r_{\mathrm{sw}}=\mathbf{0 . 5}, 30 \mathrm{C}$

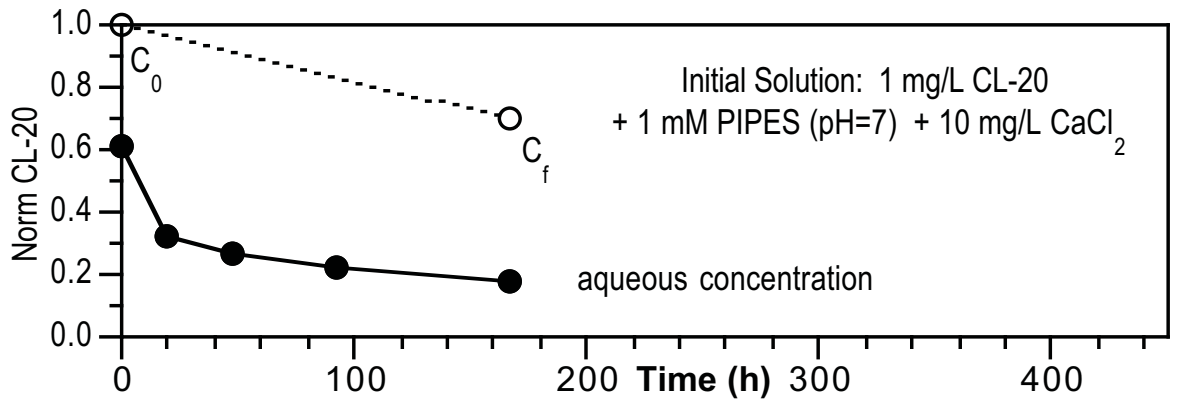

\begin{tabular}{|r|c|c|}
\hline CL-20 & (umol) & norm. \\
\hline Initial Aqe. & 0.0332 & 1 \\
\hline Final Aqe. & 0.0057 & 0.172 \\
\hline Sorbed & 0.0015 & 0.045 \\
\hline Degraded & 0.026 & 0.783 \\
\hline
\end{tabular}

(1.9\% mass removed during sampling) 
Q5: CL-20 Sorb/Deg by A/W Hectorite Clay, $r_{s w}=0.25,22 C$

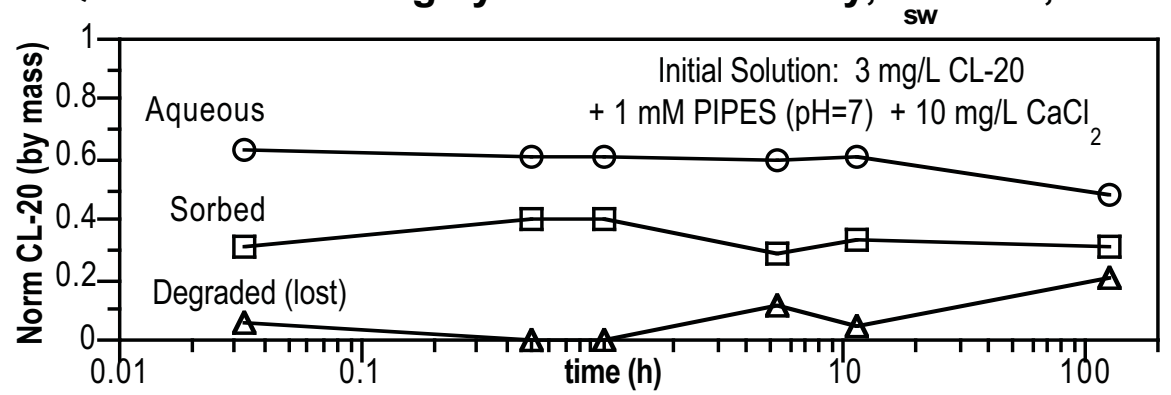

Q6-9: CL-20 Sorption/Degradation by A/W Hectorite clay

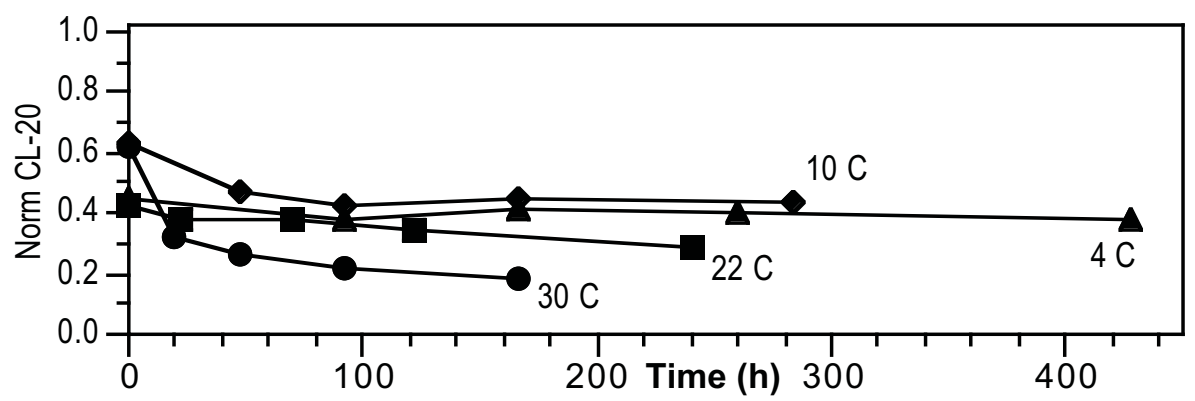

Q6-9: CL-20 Deg/Sorb by A/W Hectorite clay vs. temp

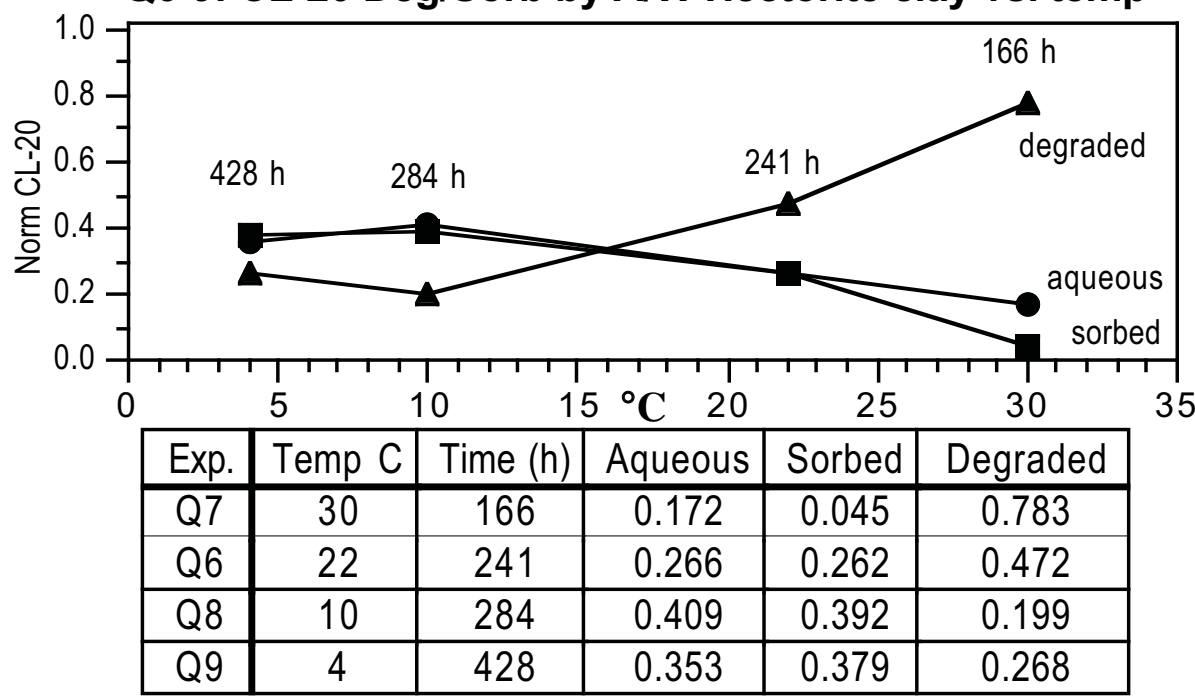


Appendix B.3; CL-20 Degradation by Minerals with adsorbed, but no structural, Fe ${ }^{\text {II }}$
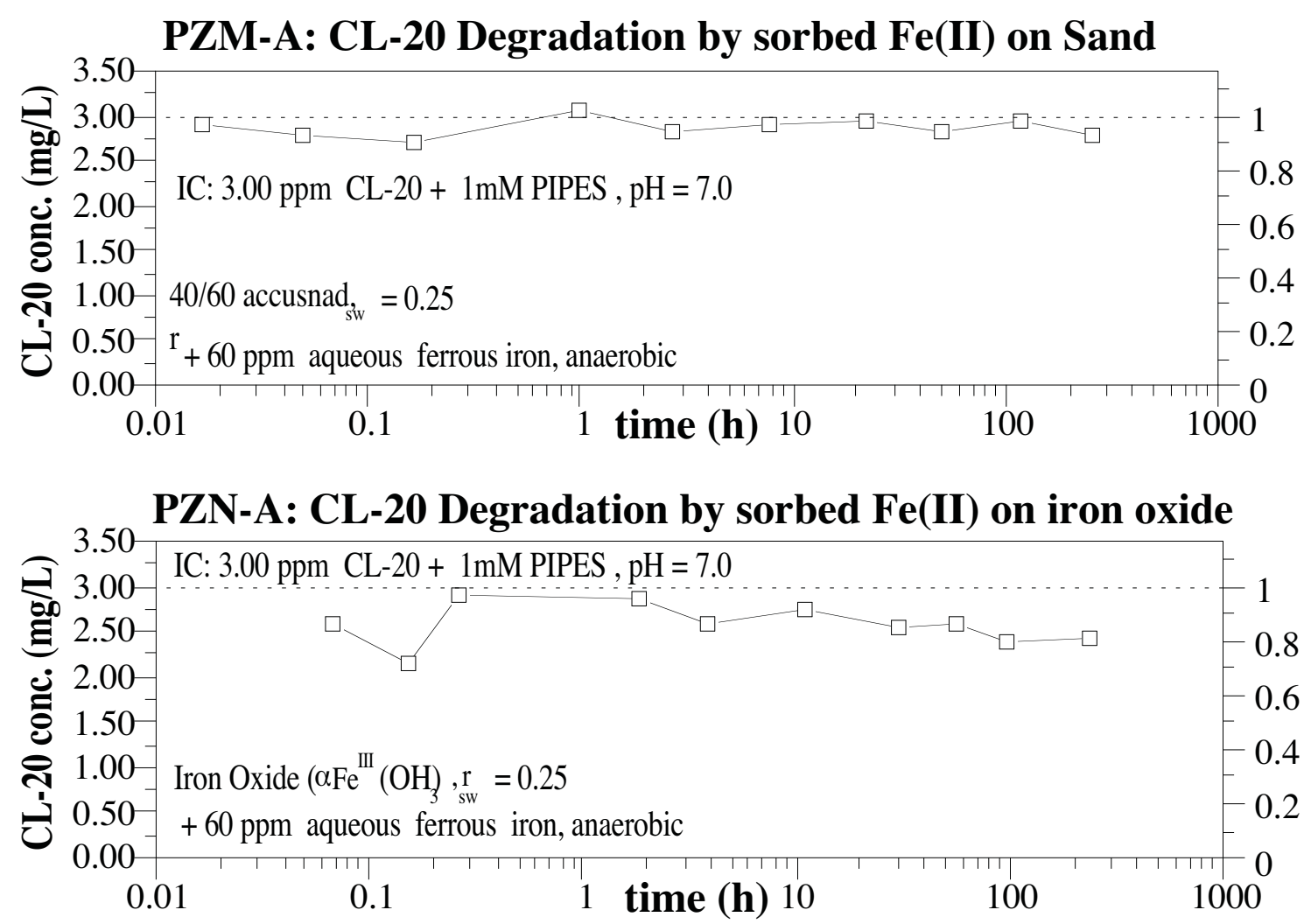

PZW: CL-20 deg. by ferrous iron sorbed to iron oxide

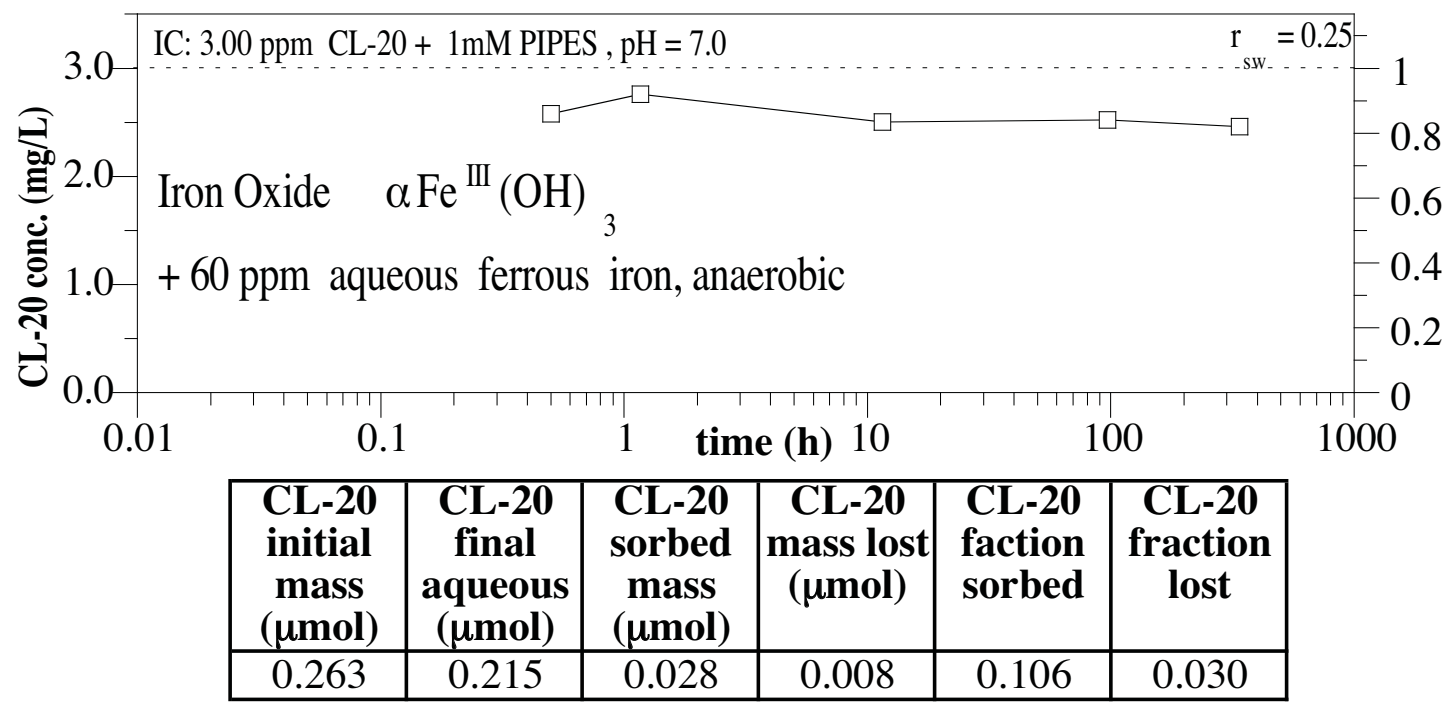


PZX: CL-20 deg. by sorbed ferrous iron on iron oxide

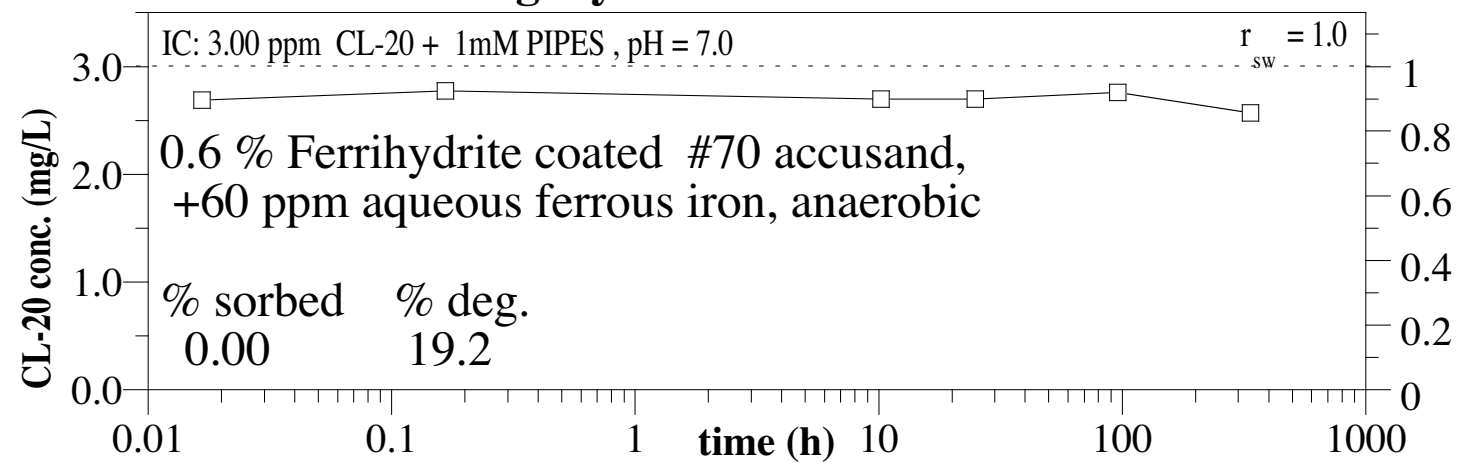

PZO-A: CL-20 Deg. by sorbed ferrous iron on 2:1 clay
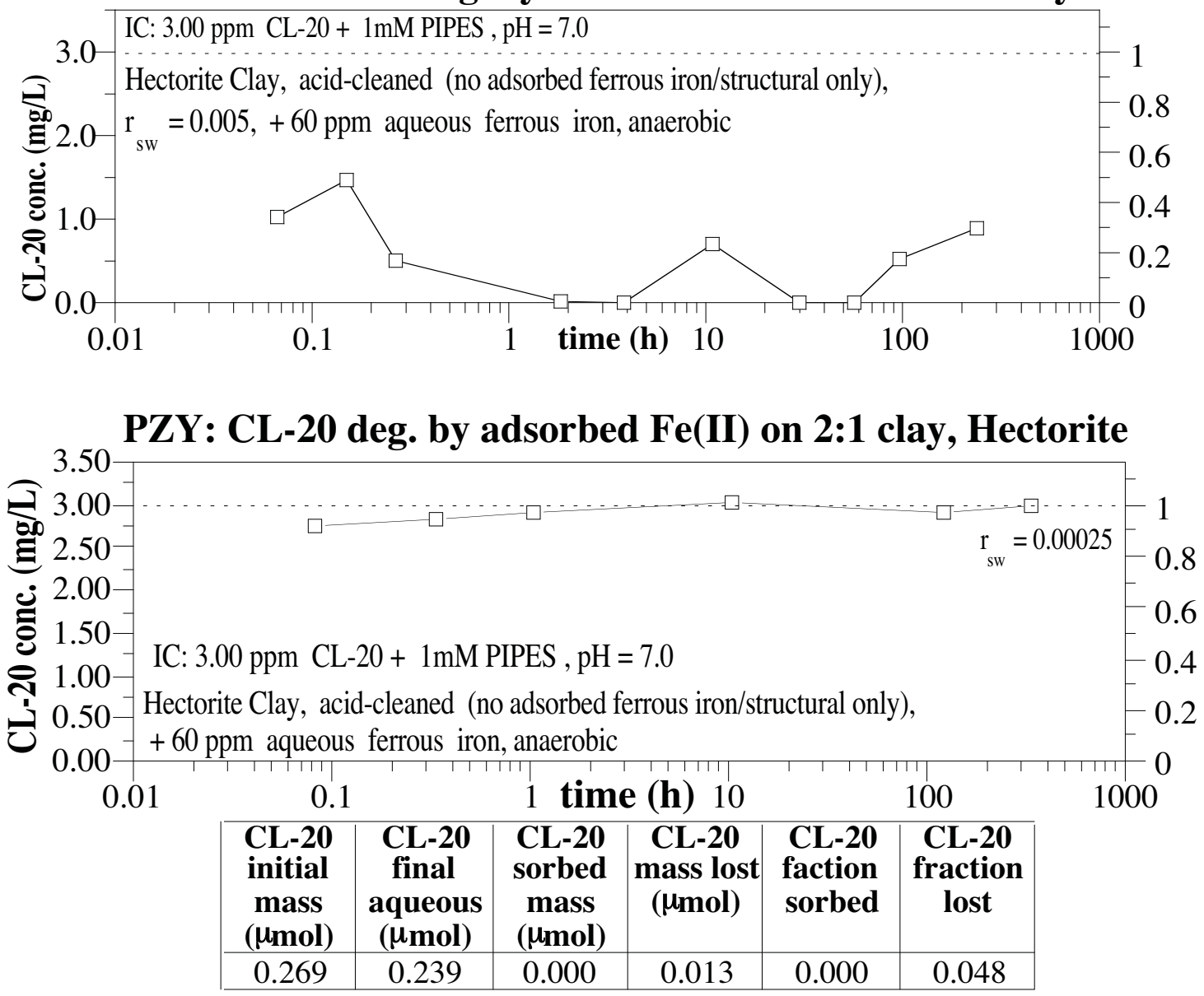


\section{Appendix B.4; CL-20 Degradation by Minerals with Structural, but no Adsorbed, Fe ${ }^{\text {II }}$}
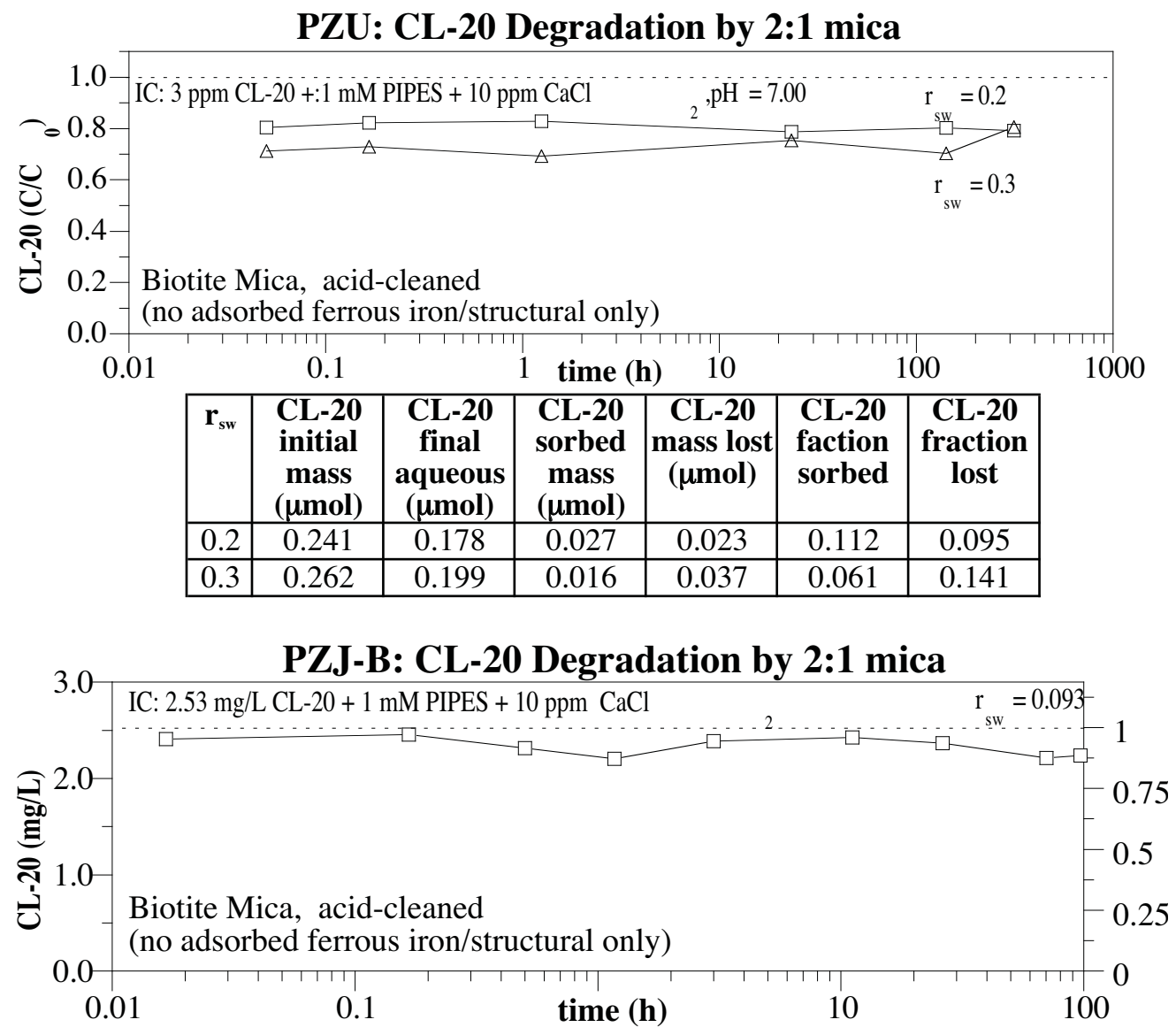

PZQ-A: CL-20 Deg. by 2:1 clay (Mont.) with structural Fe(II) only

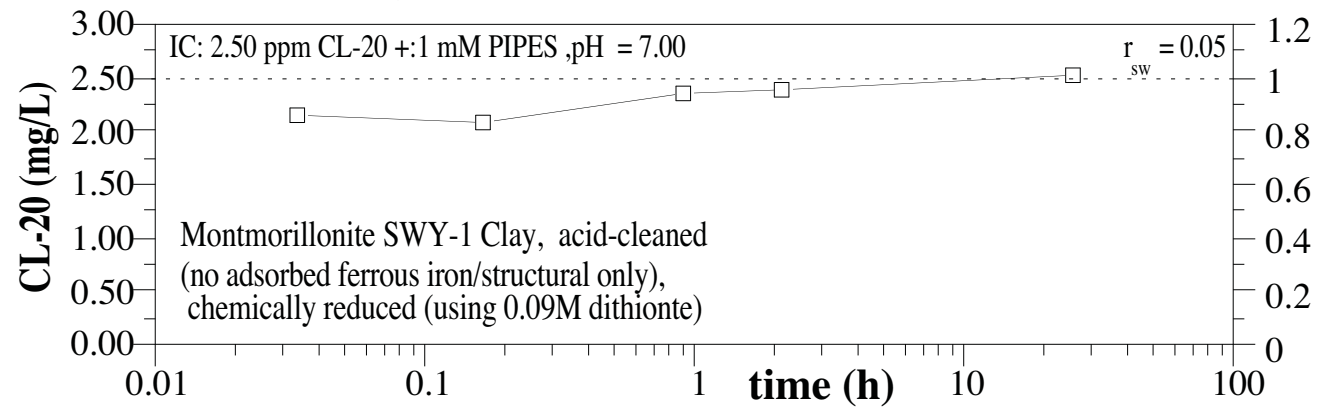



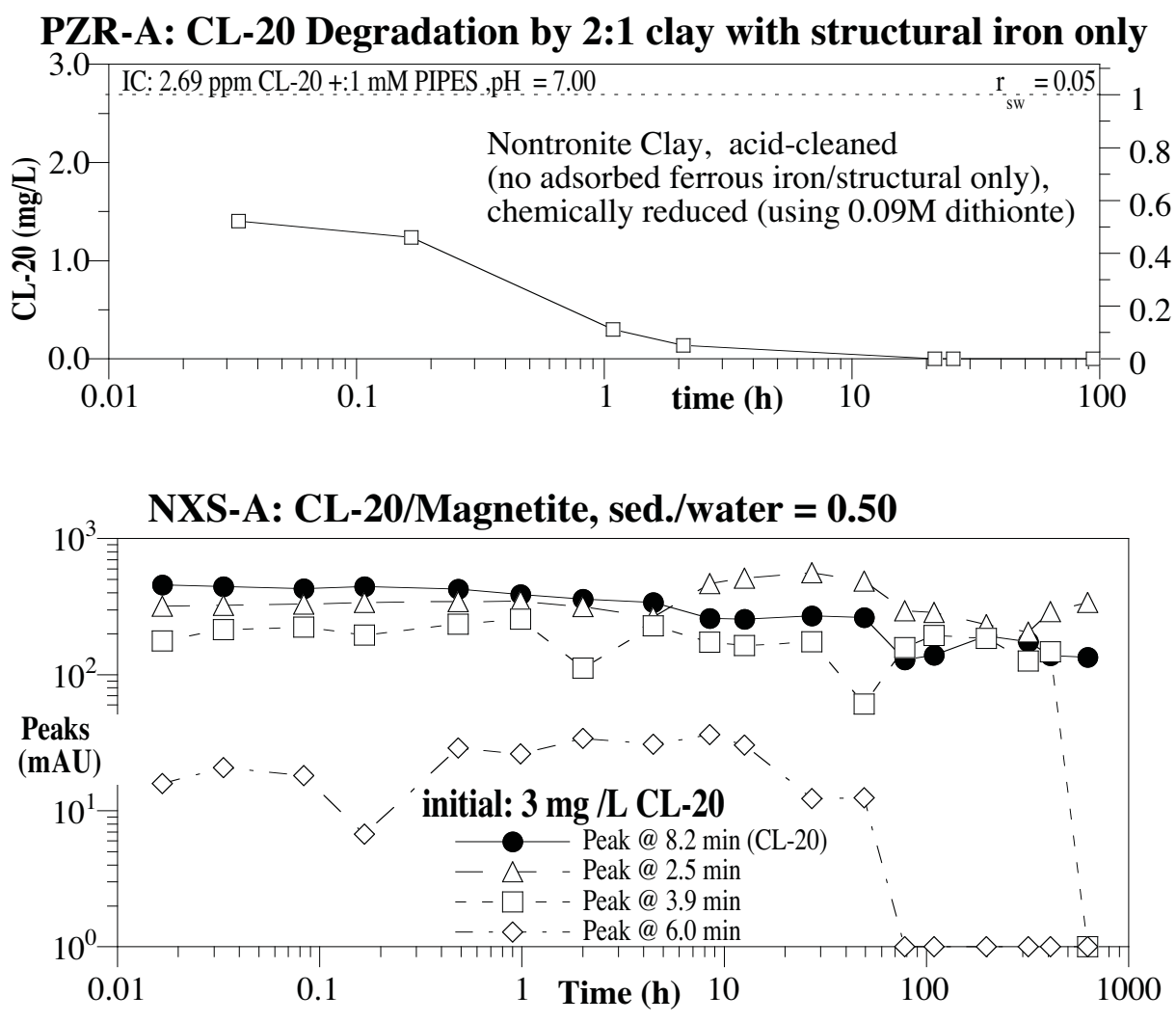


\section{Appendix B.5; CL-20 Degradation by Minerals with Structural and Adsorbed Fe ${ }^{\mathrm{II}}$}
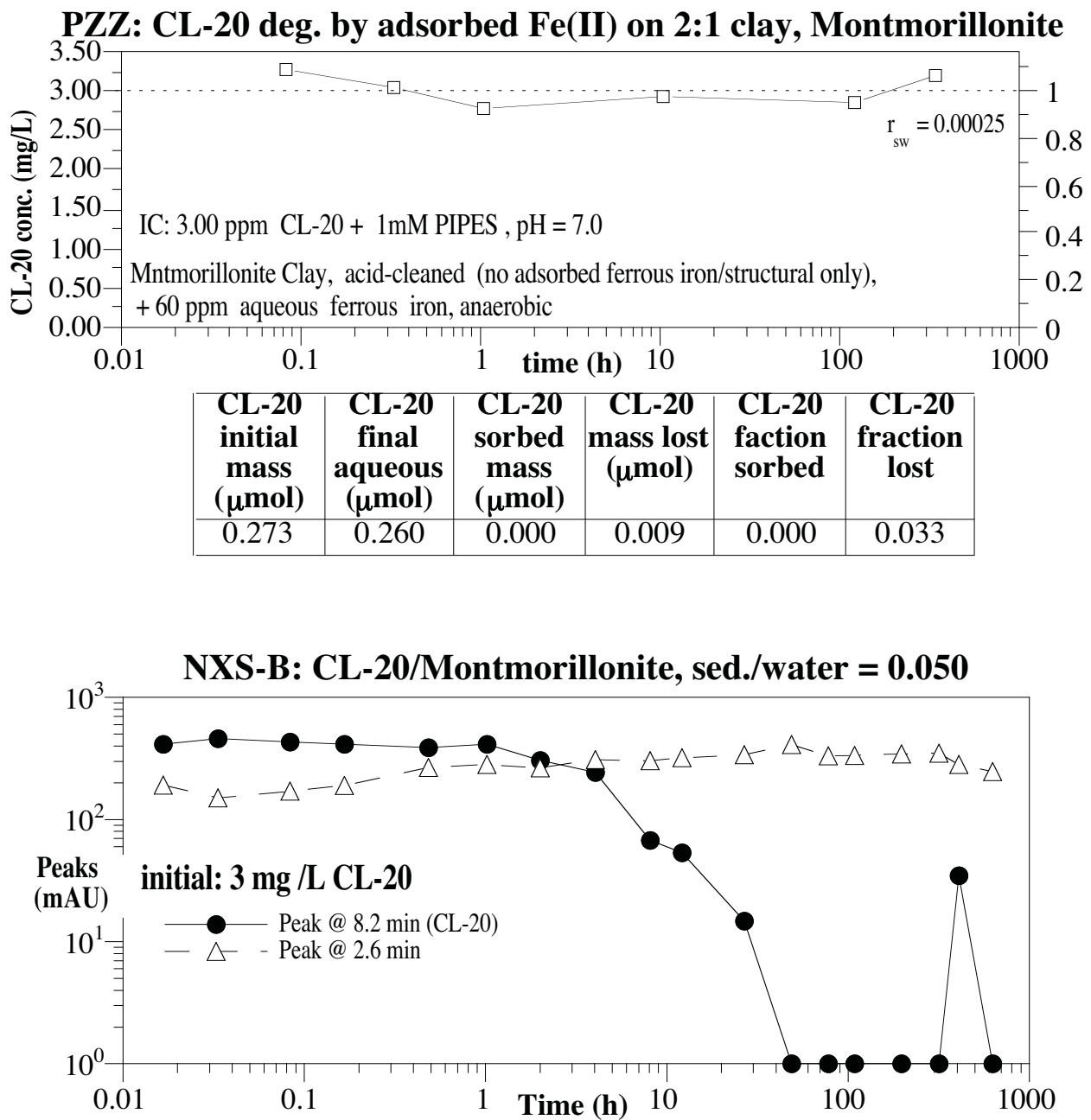


\section{APPENDIX C. CL-20 DEGRADATION AND SORPTION BY OXIC SEDIMENTS AND MINERALS BATCH EXPERIMENTS}

Appendix C.1; CL-20 Sorption to Natural Sediments 

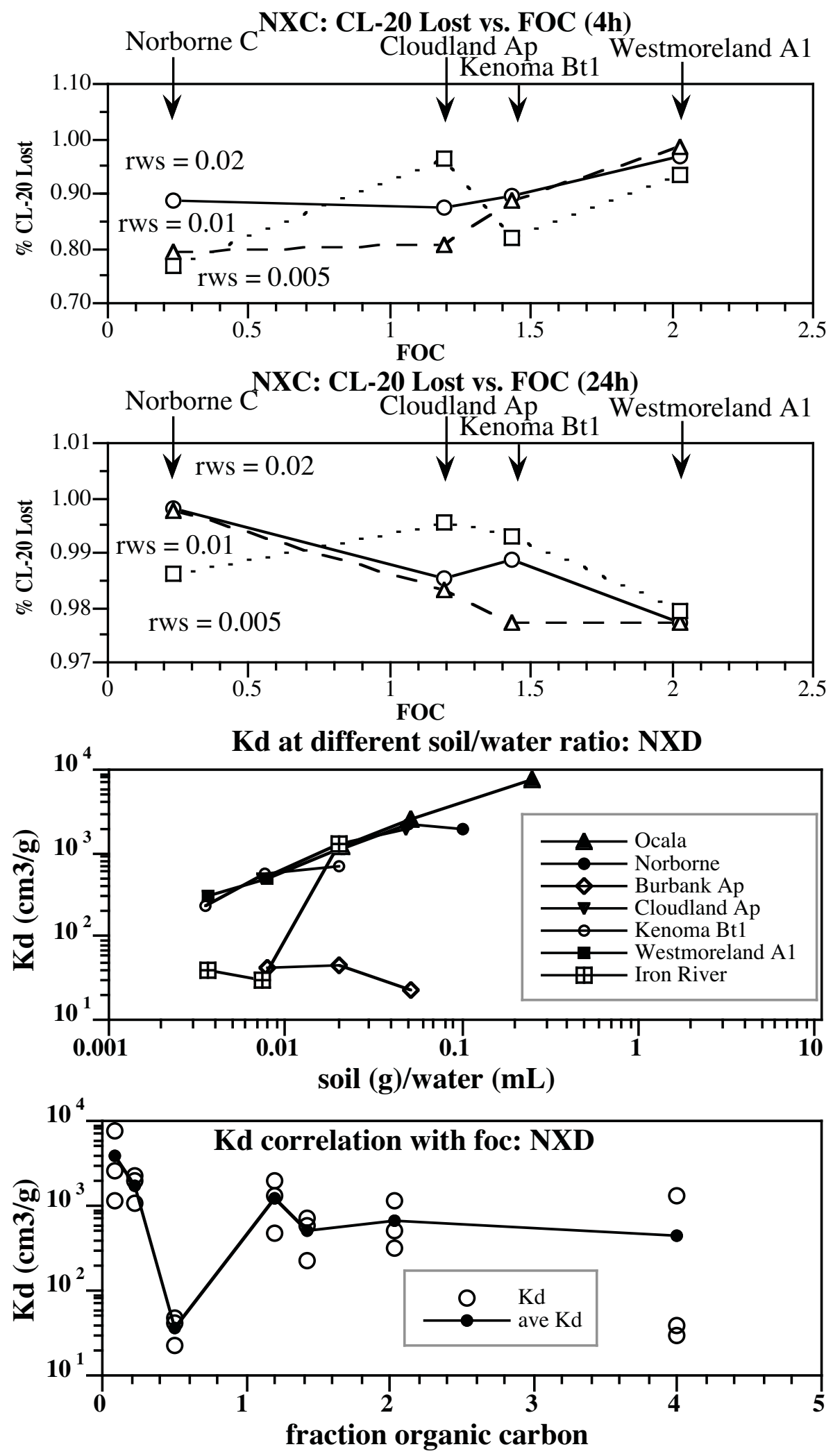

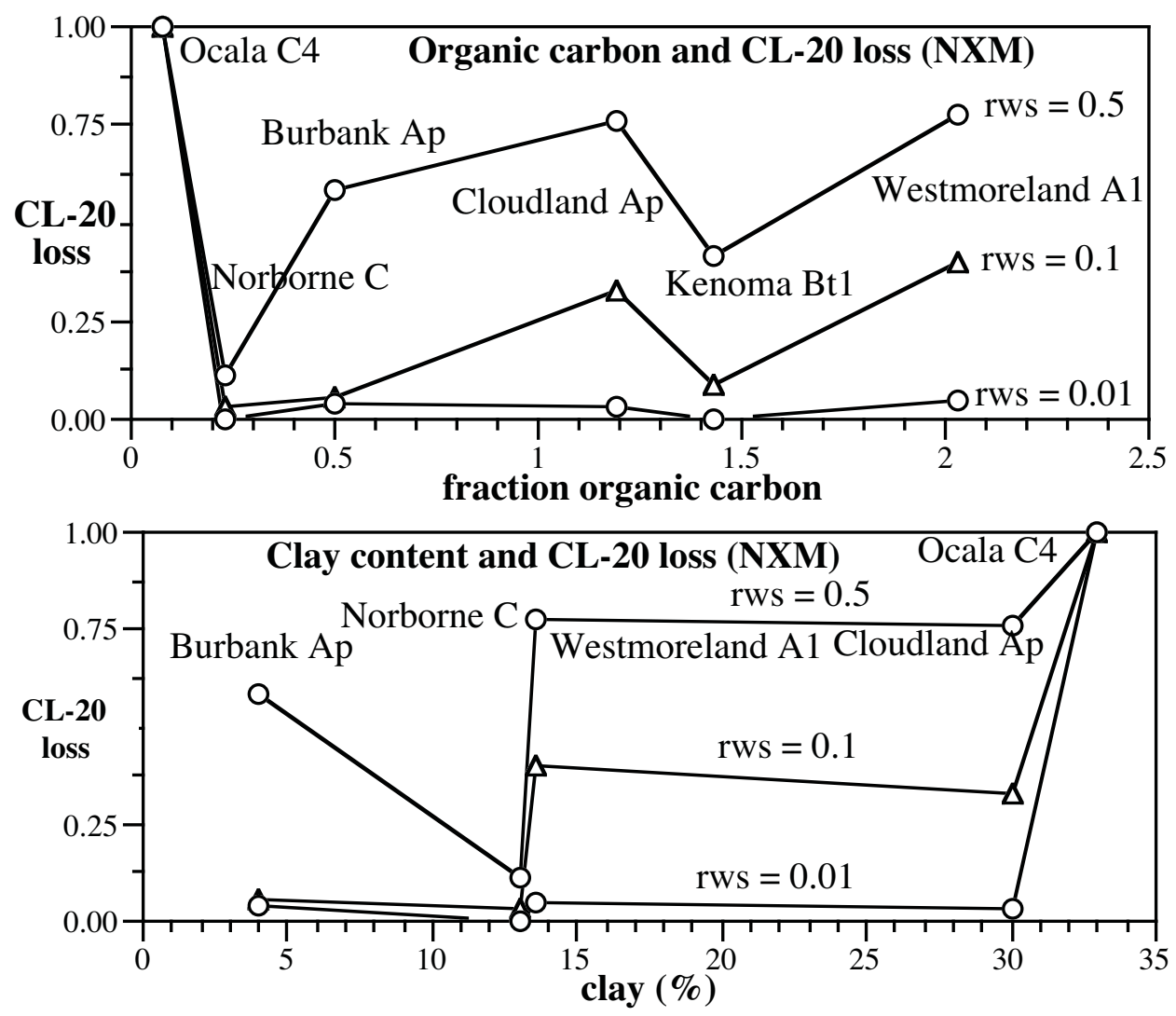

Ferrous iron content and CL-20 loss
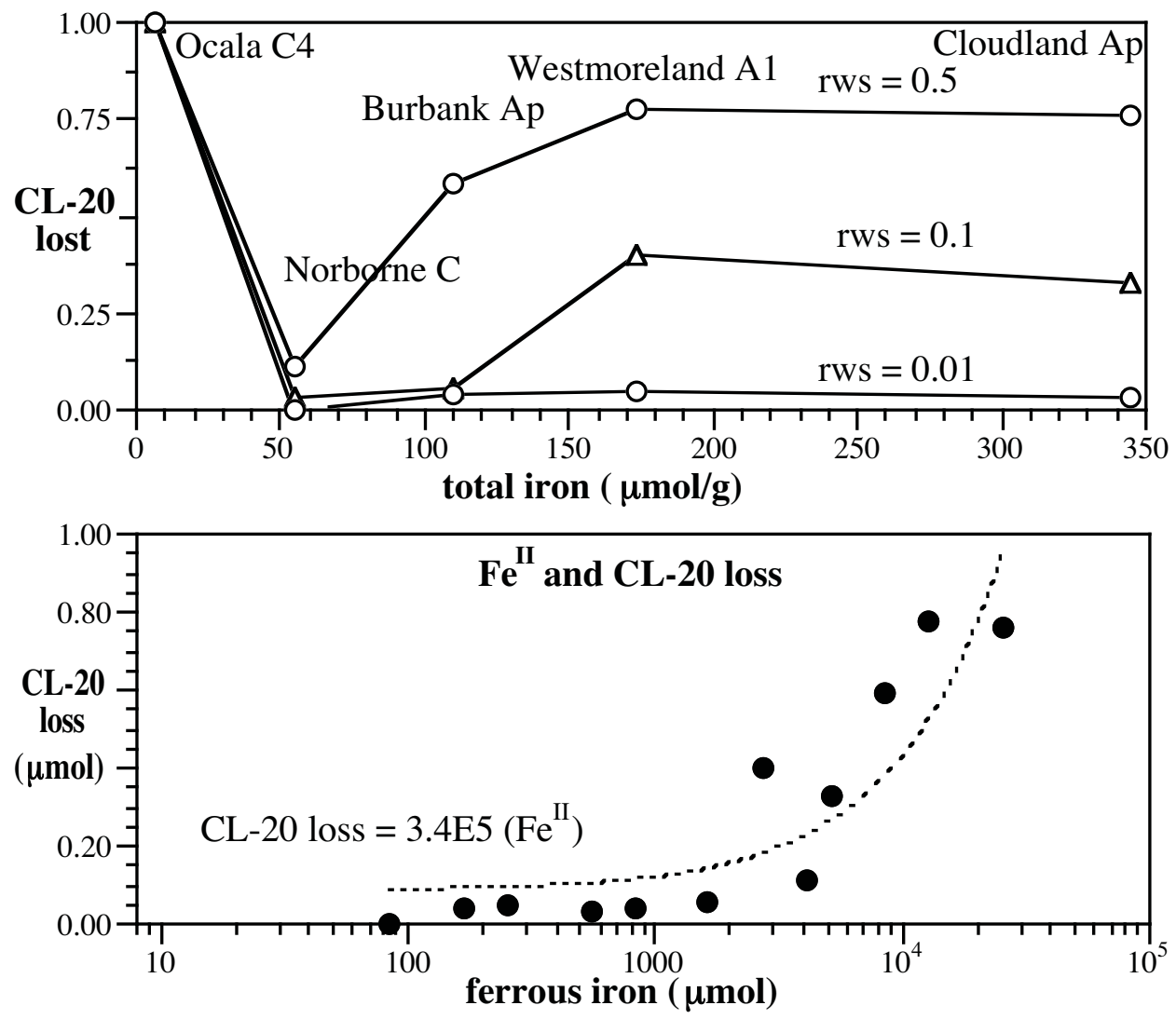
Appendix C.2; CL-20 Sorption Characterization to Minerals and Natural Sediments
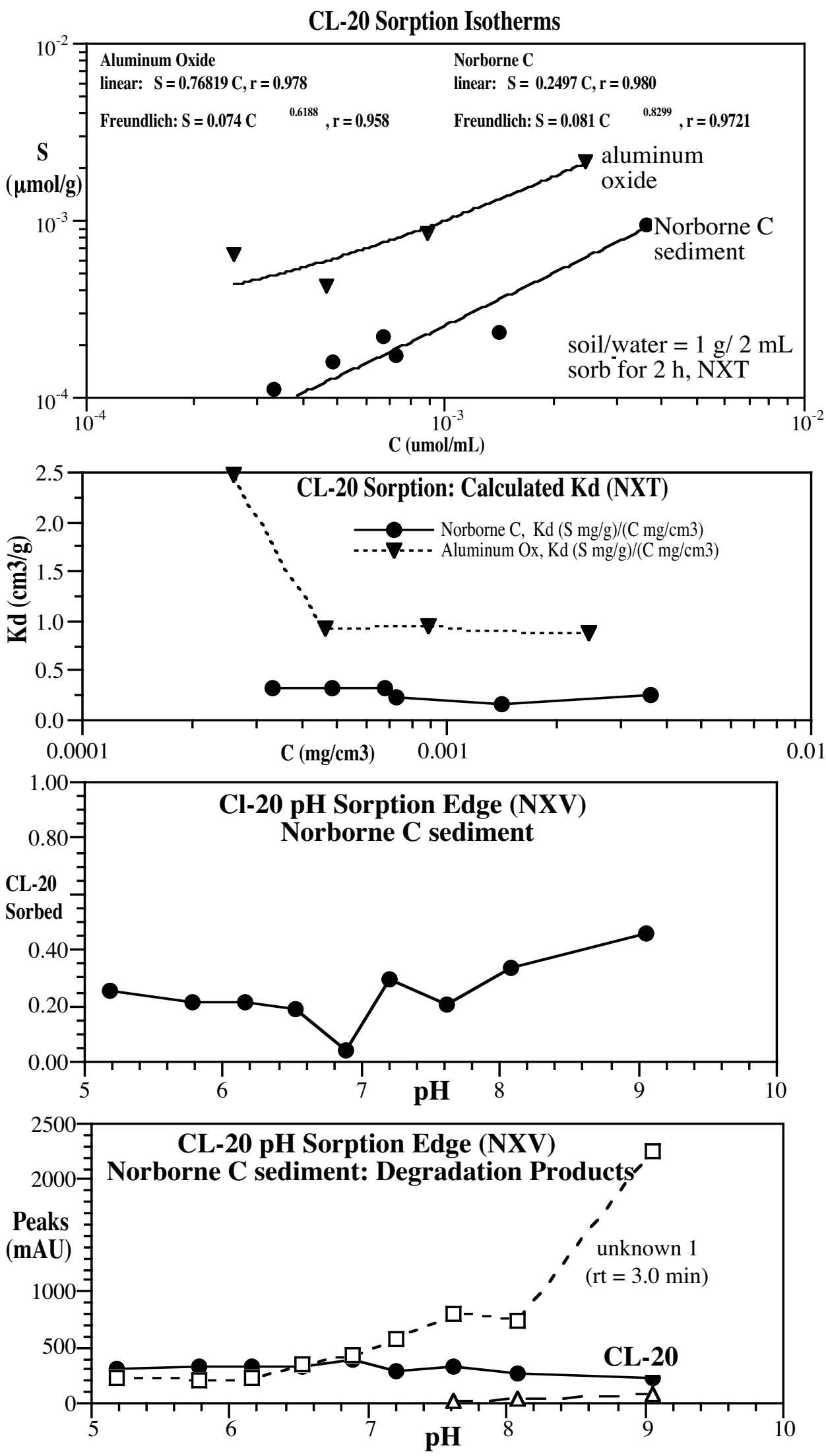
Appendix C.3; CL-20 Degradation by Oxic Sediments Rate Characterization
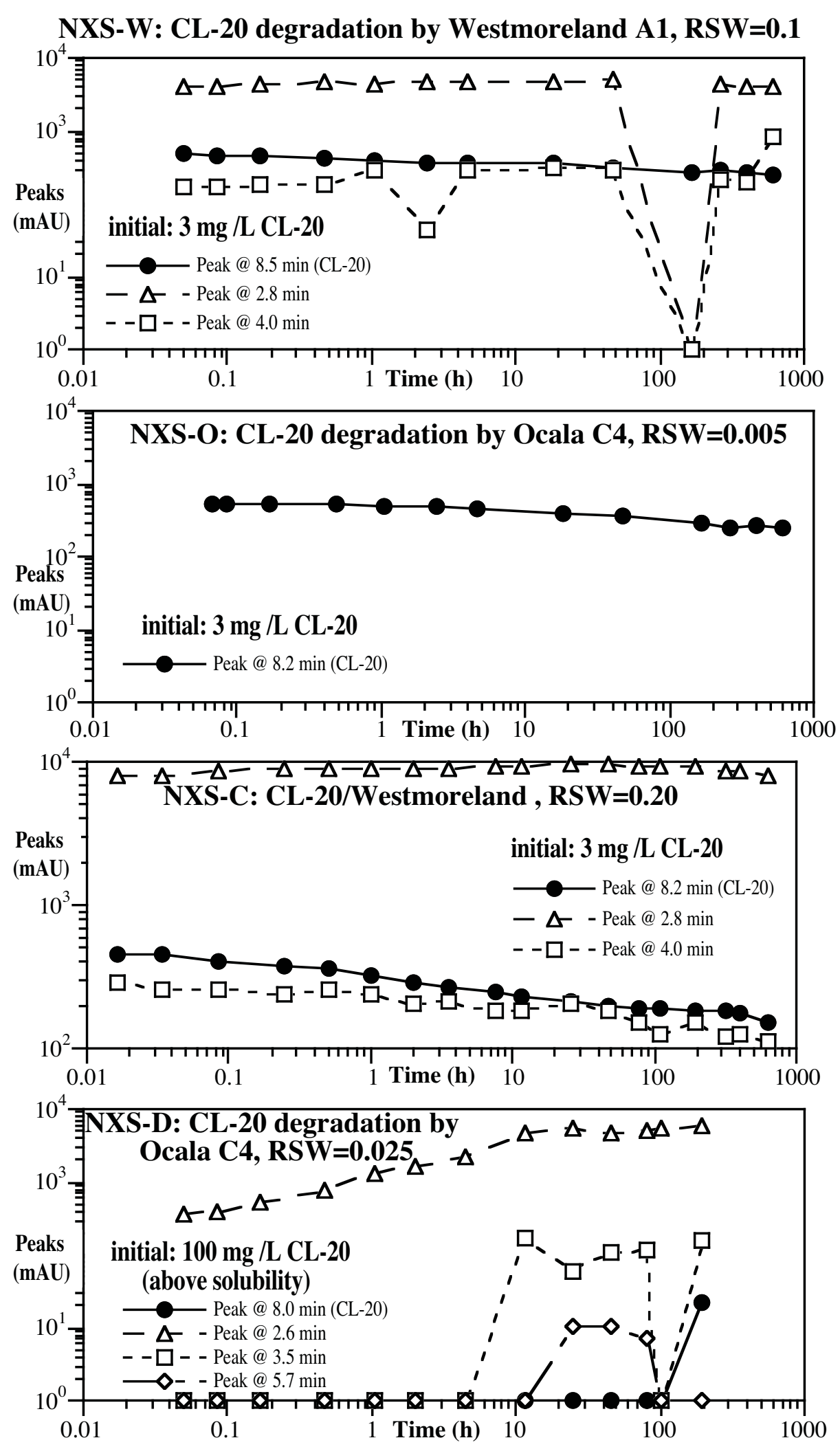

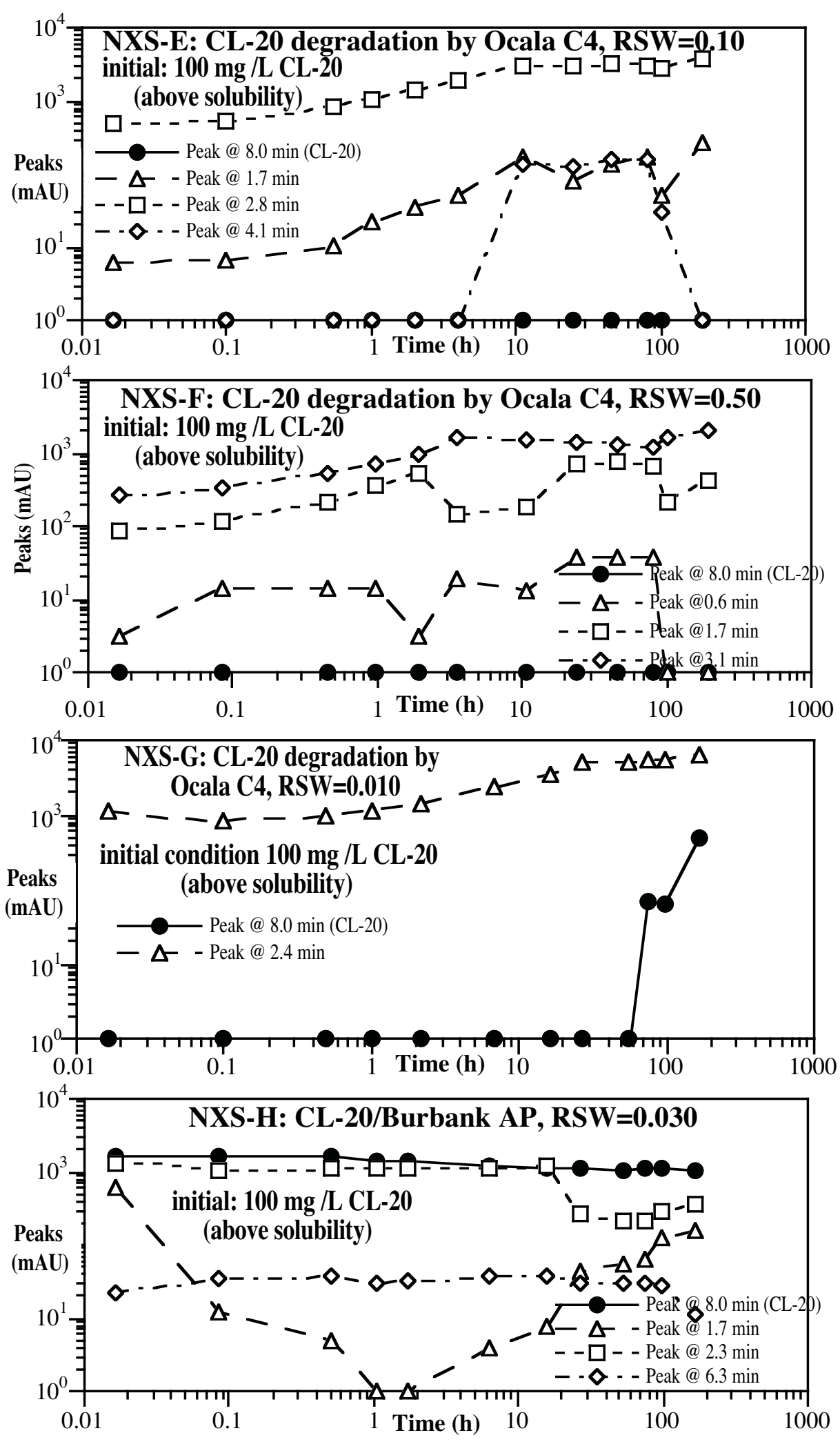

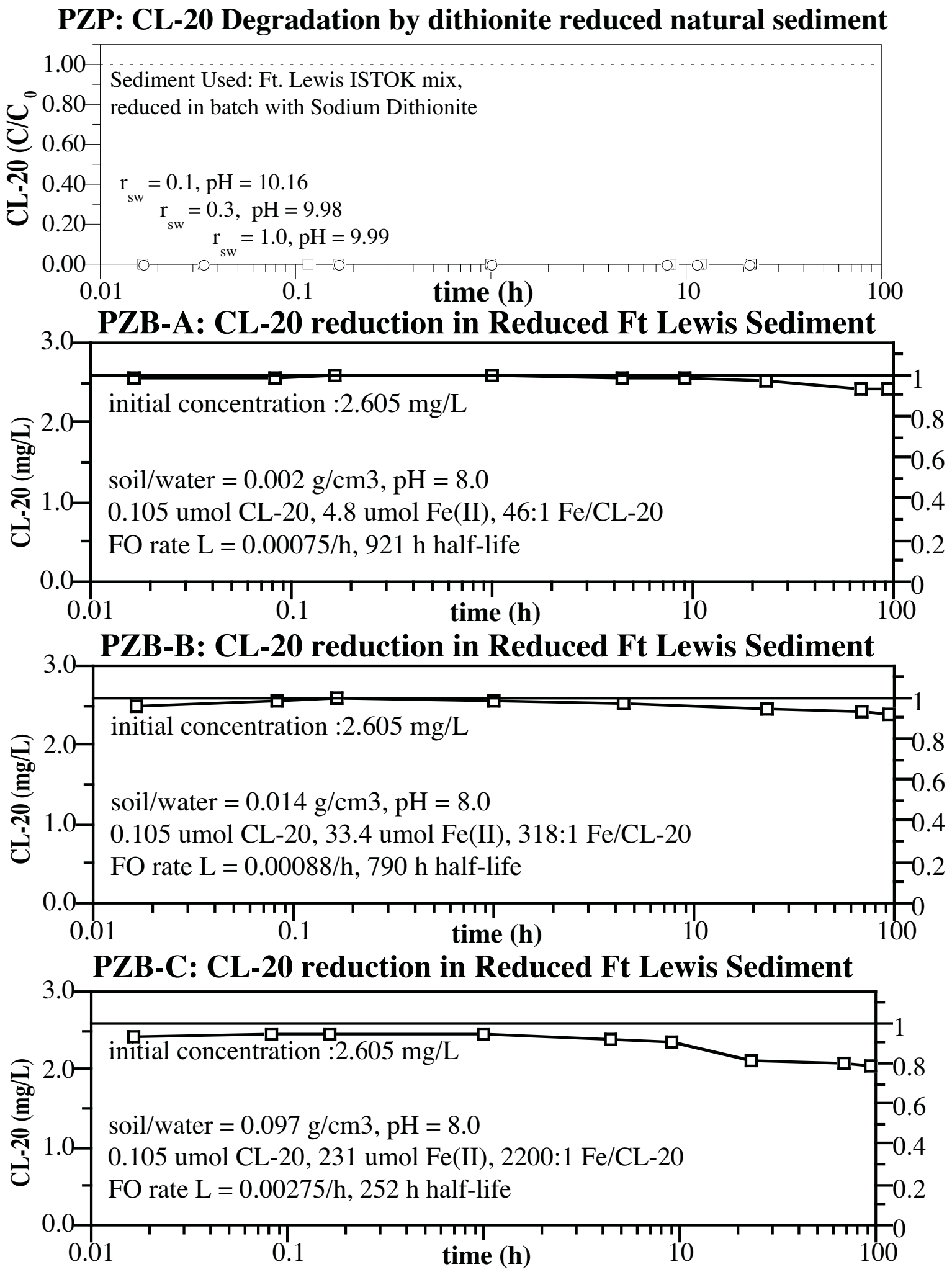
PZA: CL-20 stability with zero valent iron, \#40 mesh

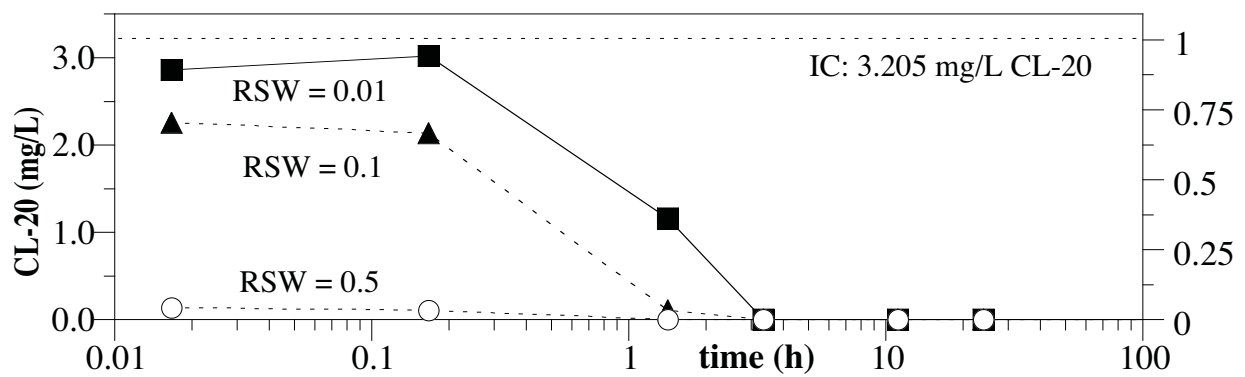

PZS: CL-20 degradation by dithionite reduced natural sediment

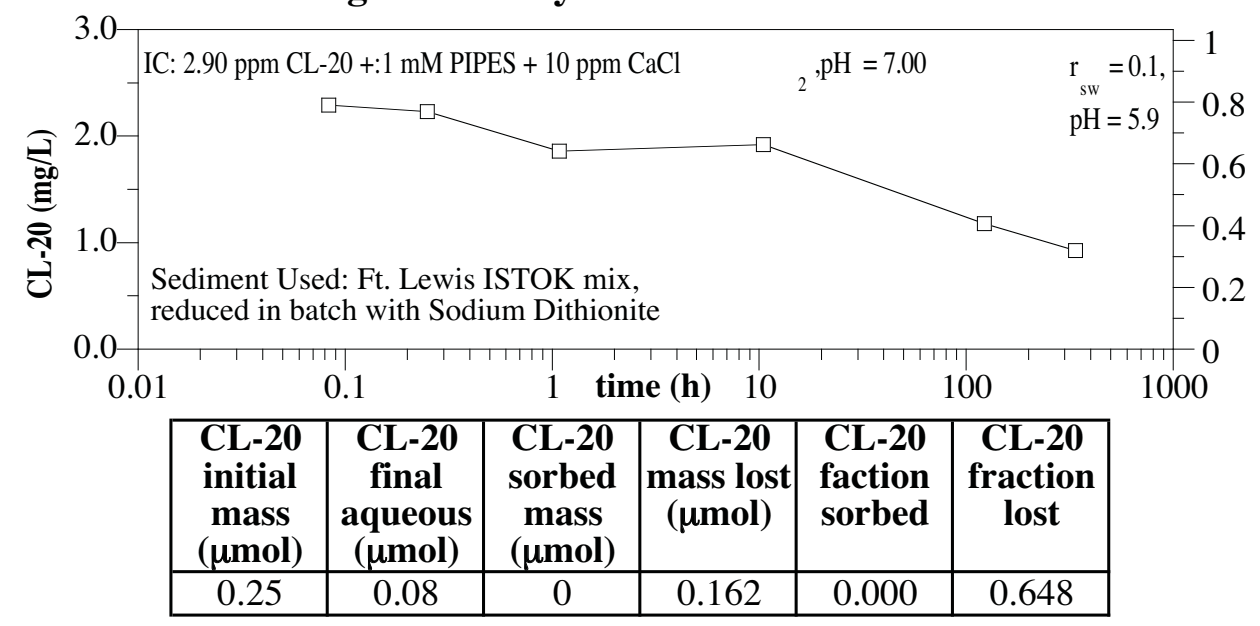

Comparison of CL-20 degradation rates

$\underline{\text { reductant first-order half-life }(\mathrm{h}) \quad \text { soil/water } \quad \text { first-order rate; soil/water }=5.0}$

zero valent iron $\quad 0.75 \mathrm{~h}$

0.01

0.1

$0.1 \mathrm{~min}$

reduced Ft. Lewis $252 \mathrm{~h}$

2.74

$300 \mathrm{~min}$

reduced Norborne $0.067 \mathrm{~h}$

(.74

$2.2 \mathrm{~min}$ 
APPENDIX E. CL-20 AND RDX DEGRADATION AND SORPTION TO OXIC AND REDUCED SEDIMENTS VS. TEMPERATURE BATCH EXPERIMENTS Appendix E.1; CL-20 Degradation and Sorption by Oxic Sediments vs. Temperature

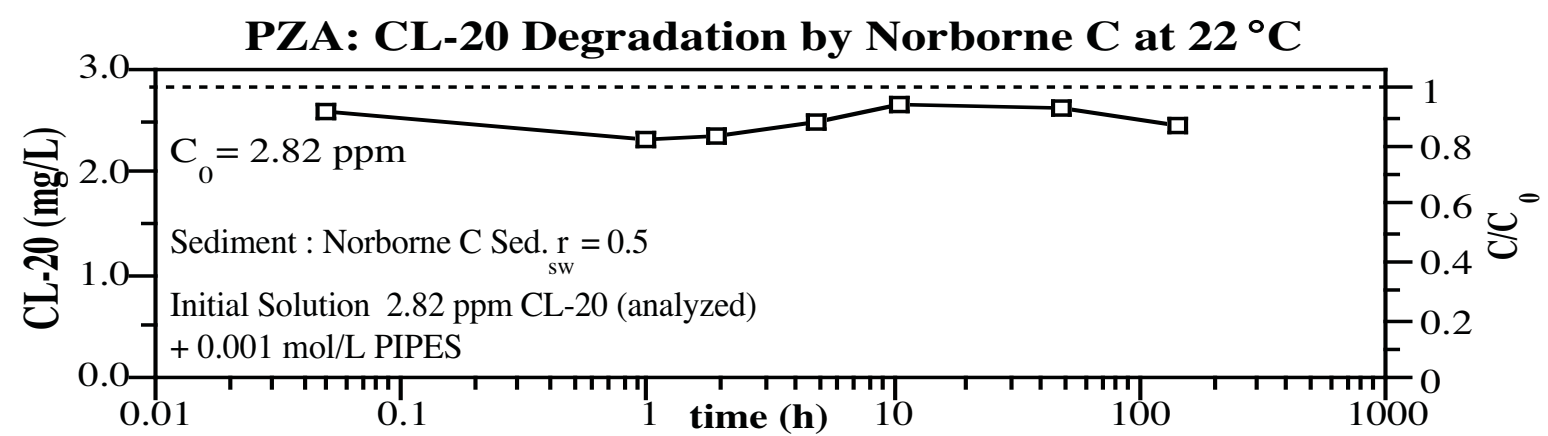

PZZL-A: CL-20 Sorption/Degradation by Norborne at 22C

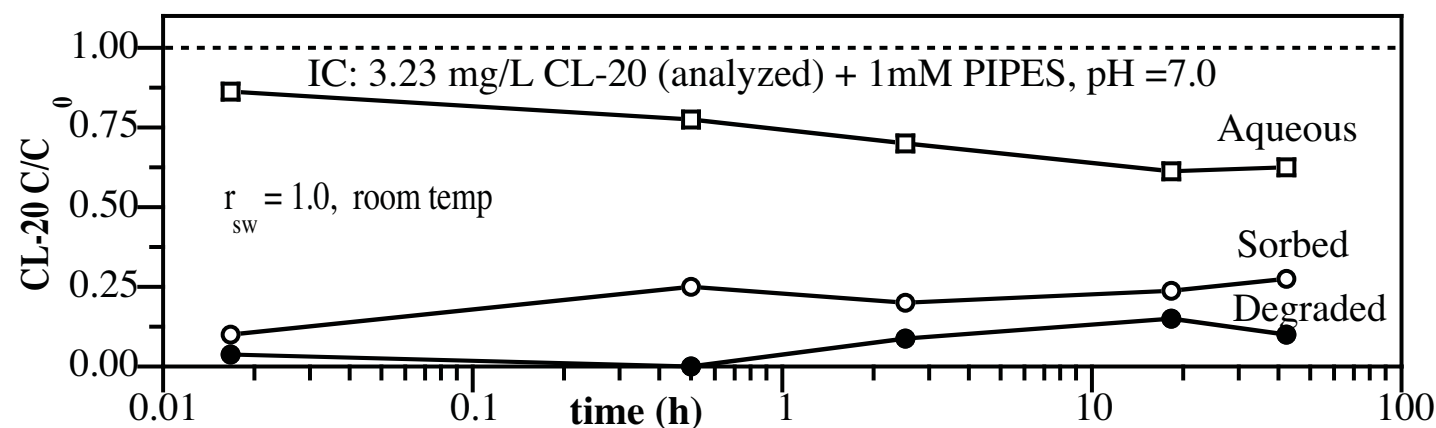

PZZL-B: CL-20 Sorption/Degradation by Westmoreland at 22C

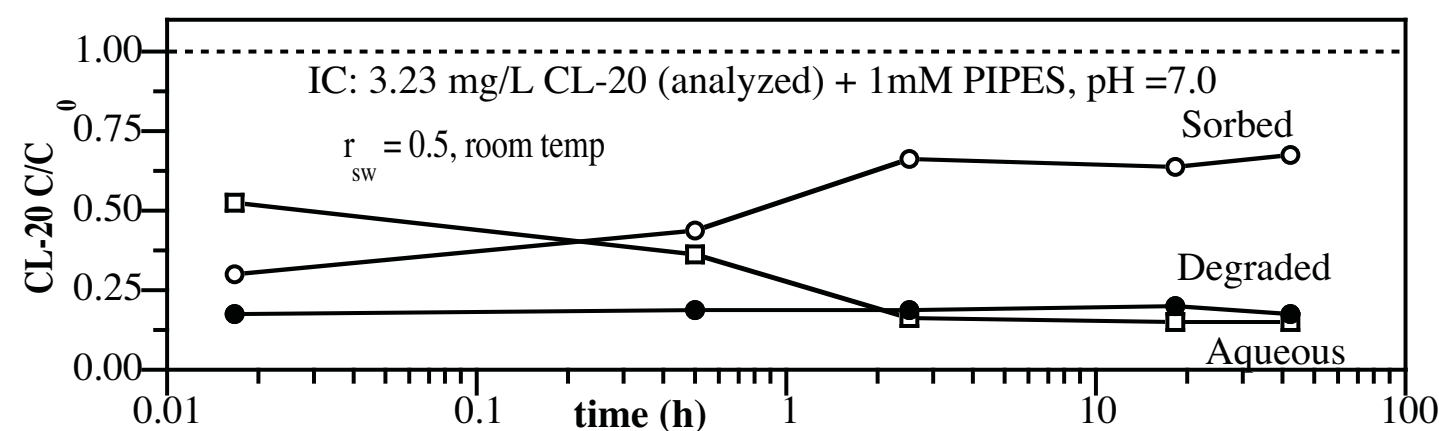

PZZL-C: CL-20 Sorption/Degradation by Kenoma at 22C

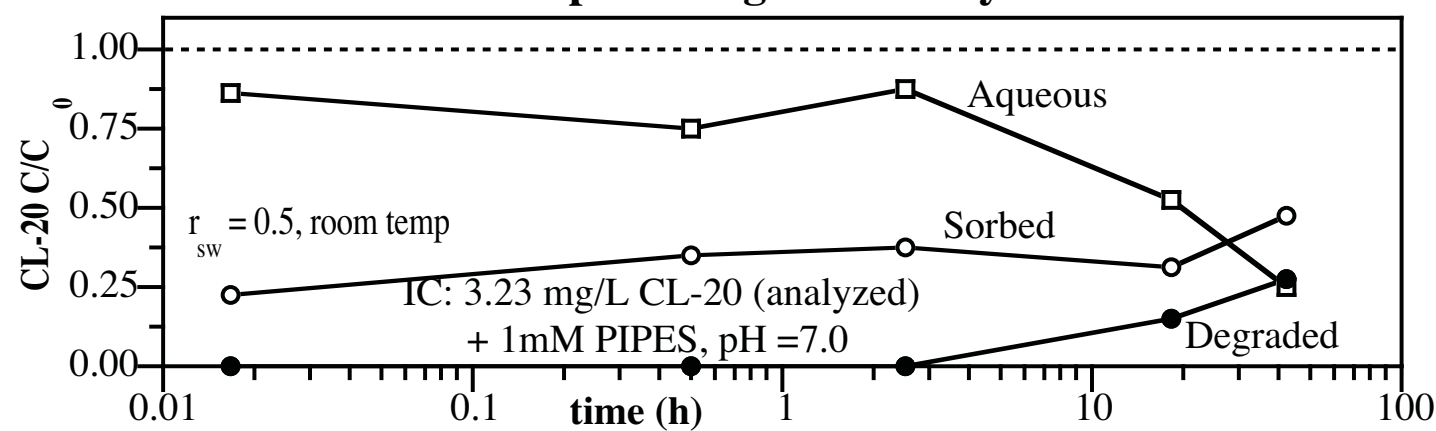




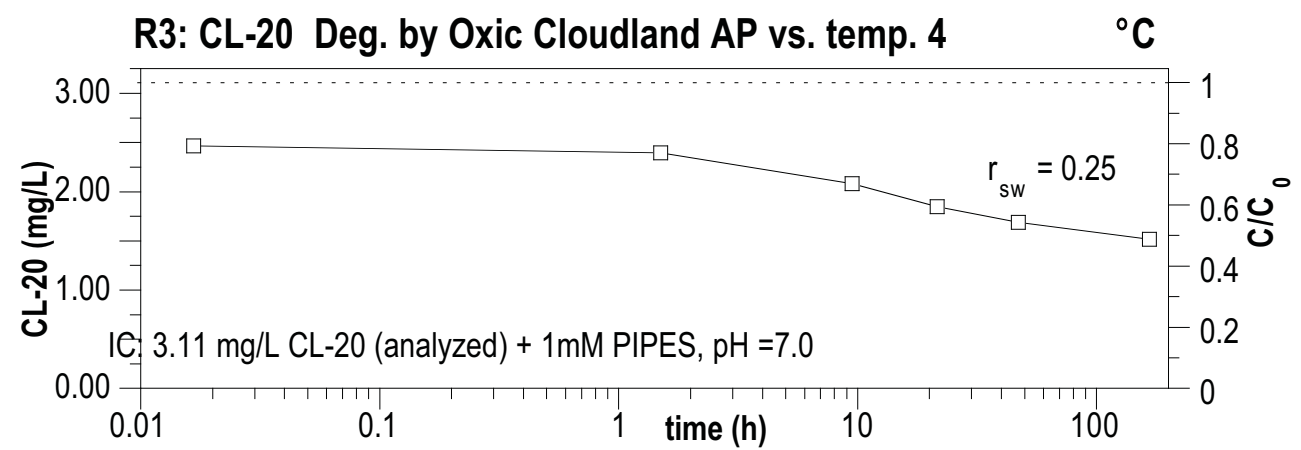

PZK-B: CL-20 Degradation by 2:1 smectite clay

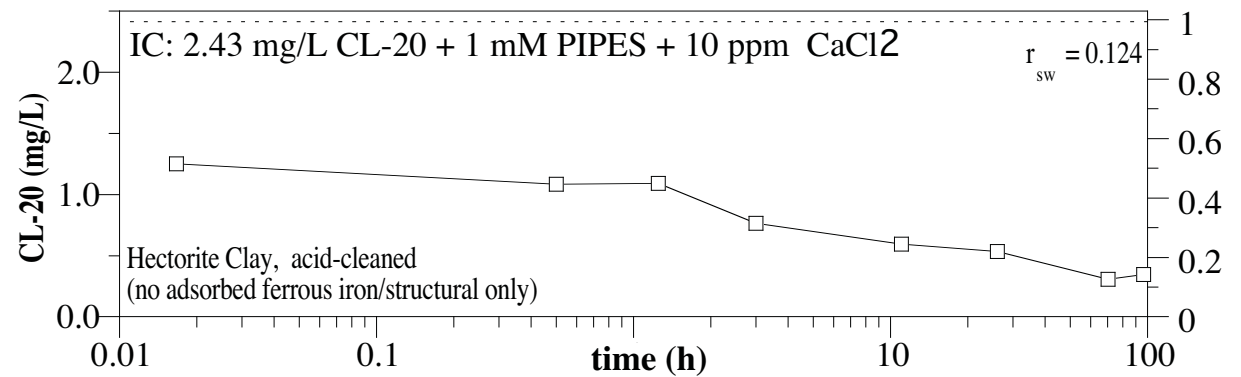

PZB-A: CL-20 Deg. by Cloudland Sed. at $40{ }^{\circ} \mathrm{C}$

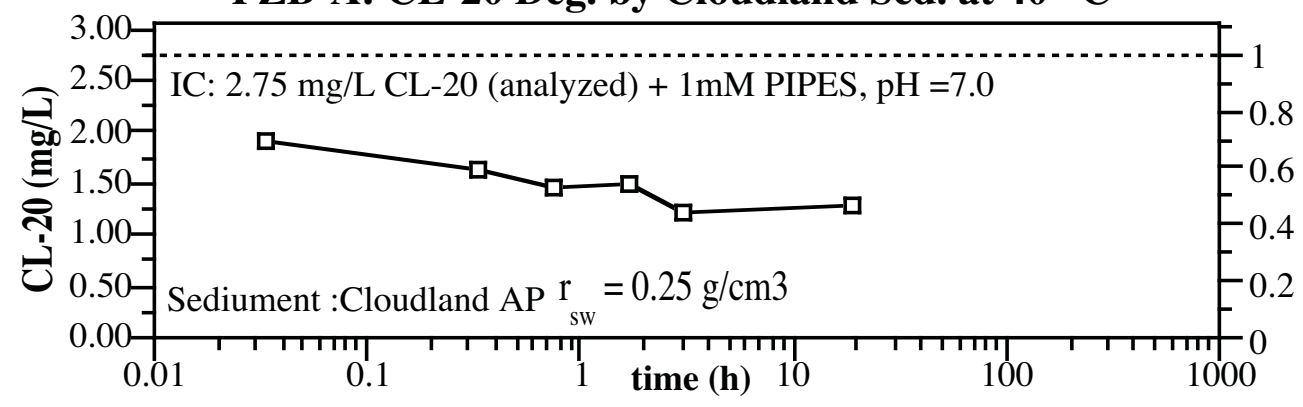




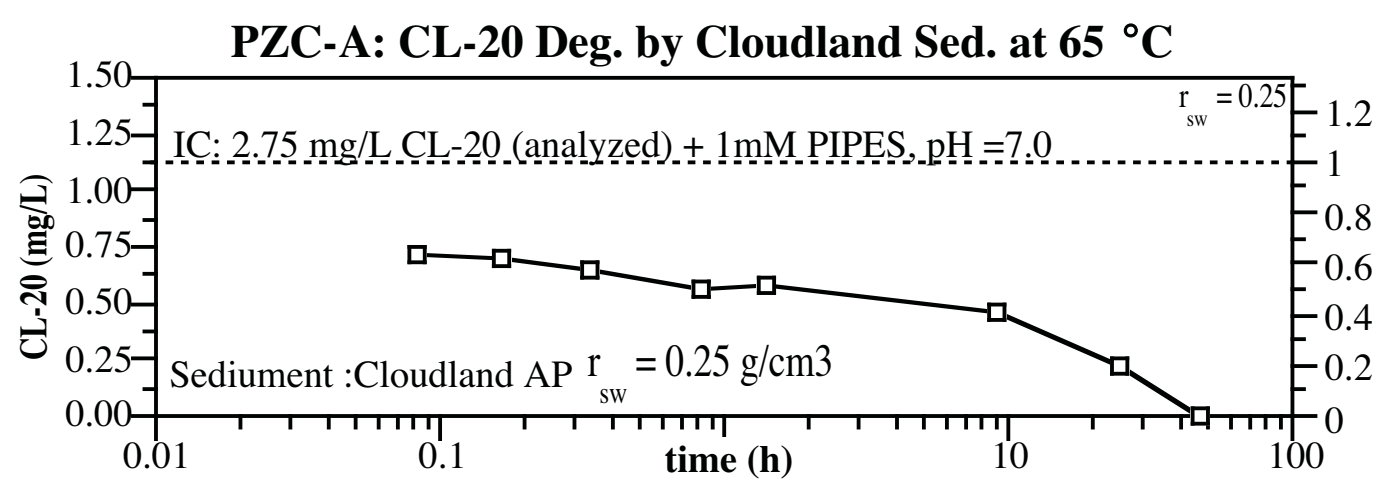

PZK-B: CL-20 Deg. by Cloudland Sed. at $22^{\circ} \mathrm{C}$

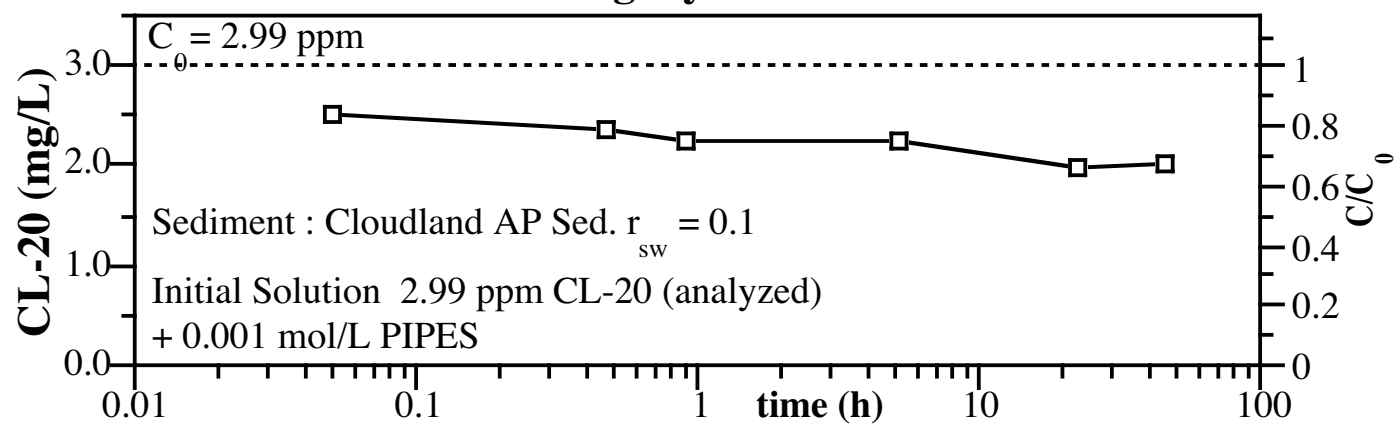

\begin{tabular}{|c|c|c|c|}
\hline $\begin{array}{c}\text { Mas s- } \\
\text { Balan ce }\end{array}$ & $\begin{array}{c}\mathrm{CL}-20 \\
(\mathrm{mg} / \mathrm{L})\end{array}$ & $\begin{array}{c}\mathrm{CL}-20 \\
(\mu \mathrm{mol})\end{array}$ & $\mathrm{C} / \mathrm{C}$ \\
\hline Initial Mas & 2.99 & 0.273 & 1.000 \\
\hline $\begin{array}{c}\text { Final } \\
\text { Aq ueous }\end{array}$ & 2.01 & 0.183 & 0.672 \\
\hline $\begin{array}{c}\text { So rbed } \\
\text { Mass }\end{array}$ & 1.35 & 0.053 & 0.195 \\
\hline $\begin{array}{c}\text { Deg raded } \\
\text { (Lost) }\end{array}$ & N/A & 0.04 & 0.132 \\
\hline
\end{tabular}

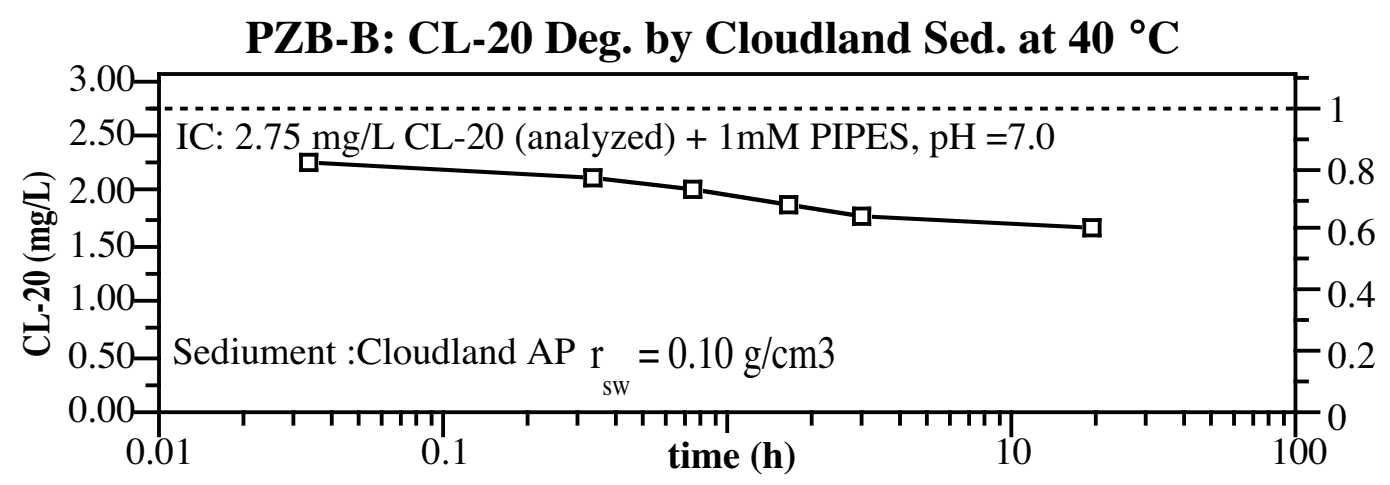


PZC-B: CL-20 Deg. by Cloudland Sed. at $65^{\circ} \mathrm{C}$

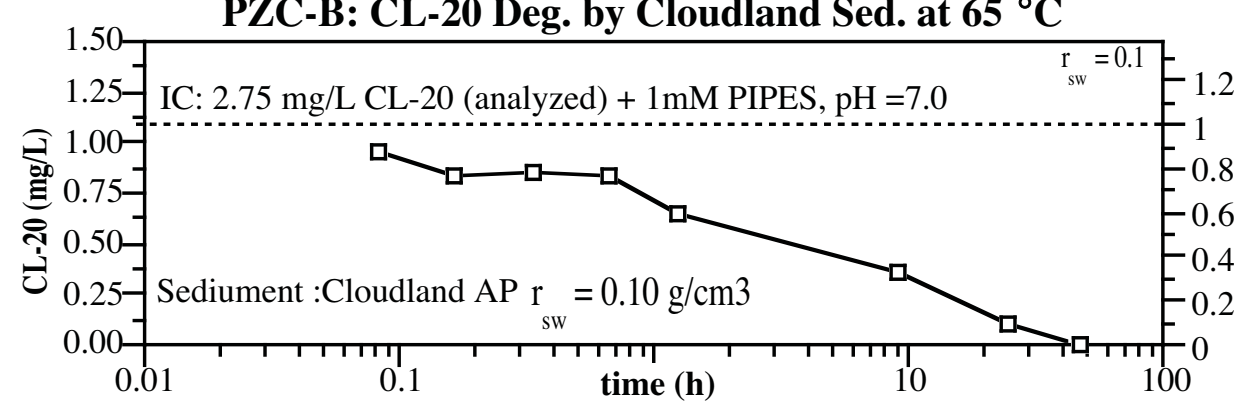

CL-20 Deg. by Oxic Cloudland sed. vs. temp, $r_{s w}=0.1$

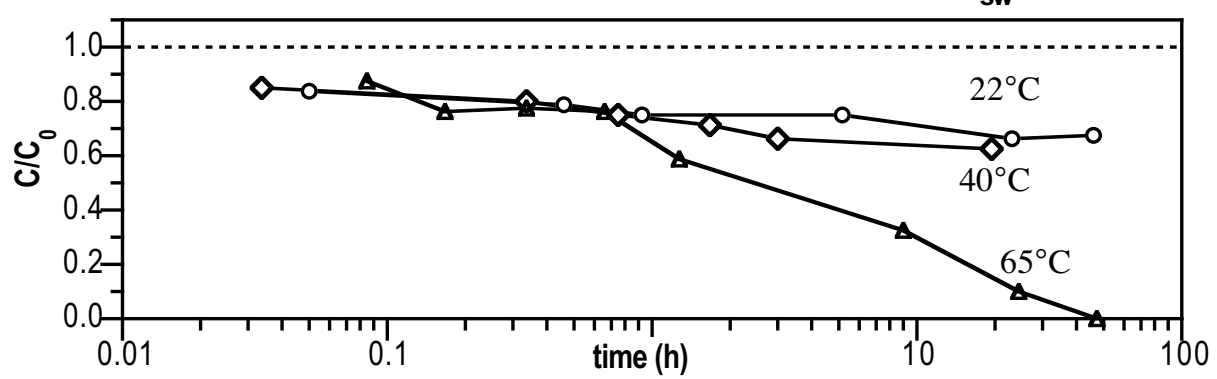

CL-20 Deg. by Oxic Cloudland sed. vs. temp, $r_{s w}=0.25$

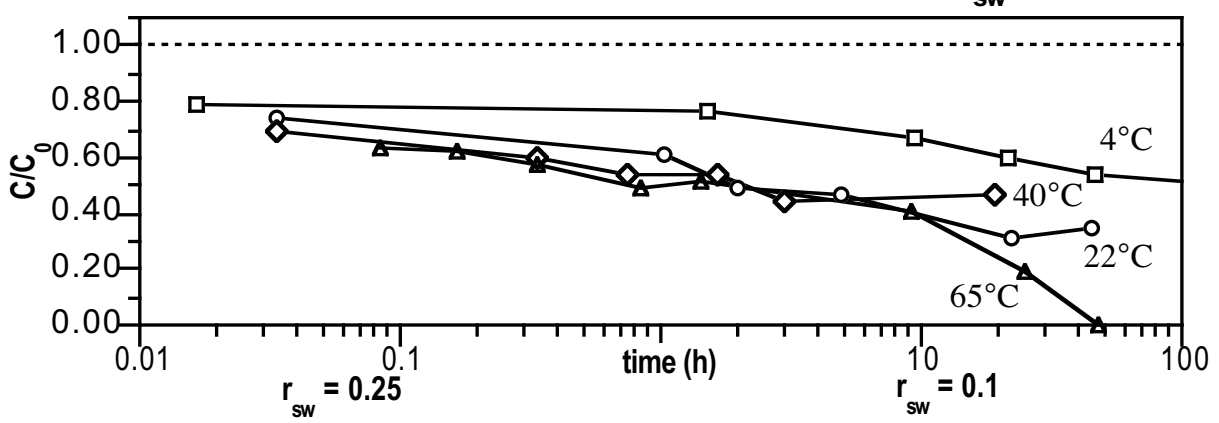

\begin{tabular}{|l|l|c|c|}
\hline Exp & temp & \% sorb & \% deg \\
\hline R3 & $4{ }^{\circ} \mathrm{C}$ & 48.7 & 2.5 \\
\hline PZZK & $22^{\circ} \mathrm{C}$ & 38.7 & 26.4 \\
\hline PZZB & $40^{\circ} \mathrm{C}$ & 36.9 & 16.9 \\
\hline PZZC & $65^{\circ} \mathrm{C}$ & 11.9 & 88.1 \\
\hline
\end{tabular}

\begin{tabular}{|l|c|c|c|}
\hline Exp & temp & \% sorb & \% deg \\
\hline PZZK & $22^{\circ} \mathrm{C}$ & 19.5 & 13.2 \\
\hline PZZB & $40^{\circ} \mathrm{C}$ & 13.6 & 23.9 \\
\hline PZZC & $65^{\circ} \mathrm{C}$ & 0.0 & 100.0 \\
\hline
\end{tabular}


PZZJ: CL-20 and RDX Deg. by Red. Norbornce C vs. temp. $22^{\circ} \mathrm{C}$

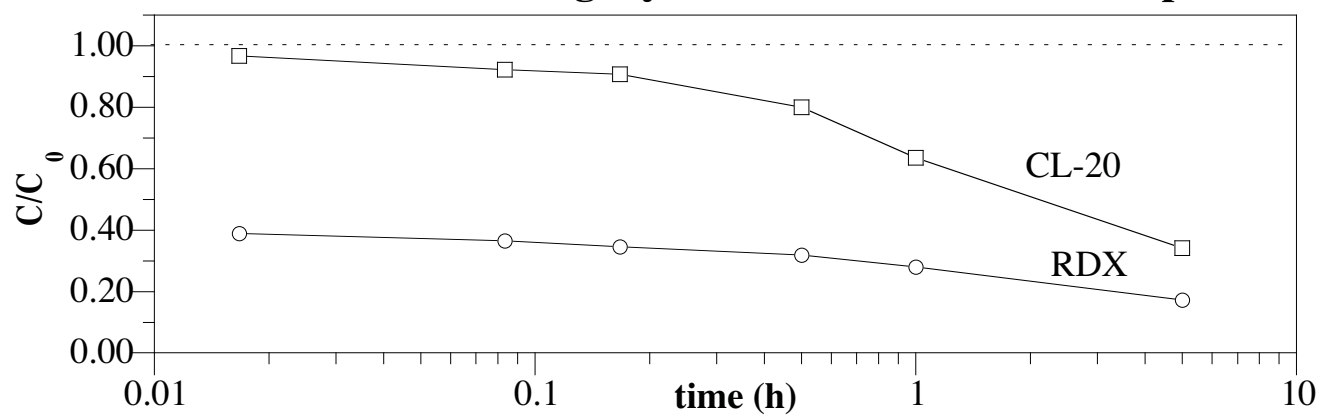

R4: CL-20 and RDX Deg. by Red. Norborne C vs. temp. $4^{\circ} \mathrm{C}$

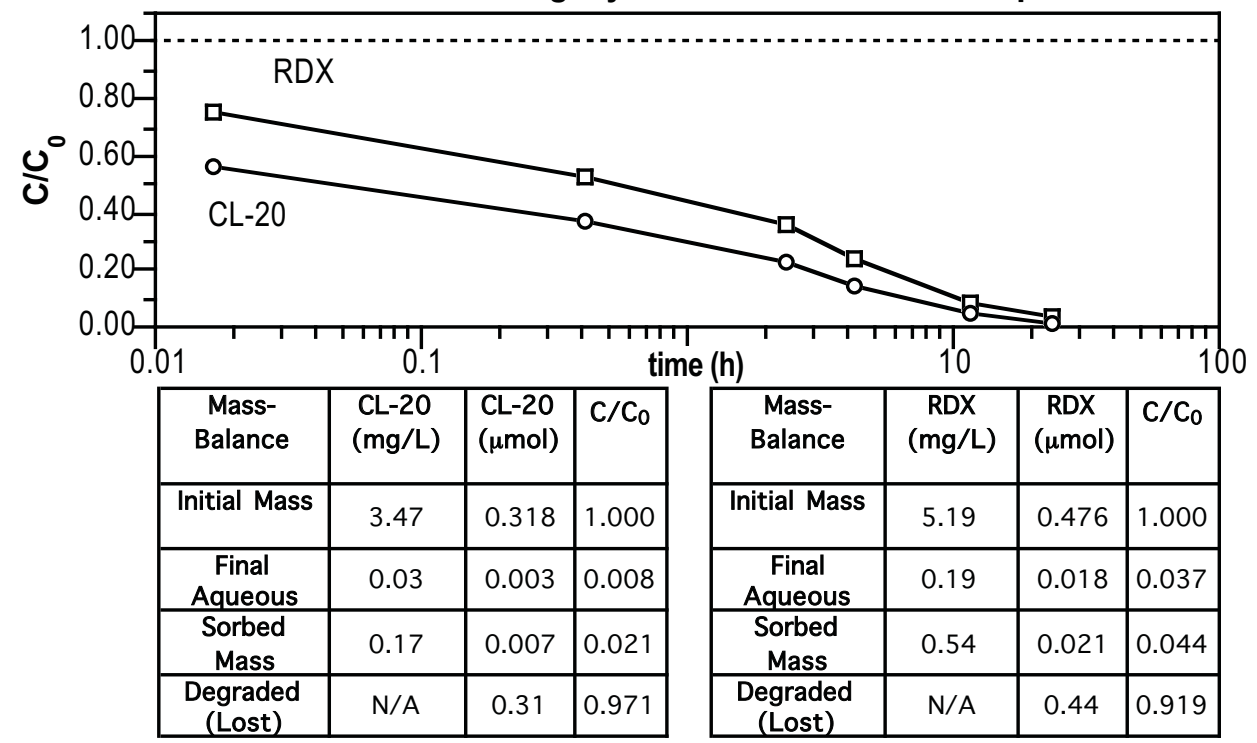

R2: CL-20 and RDX Deg. by Red. Norborne C vs. temp. $12^{\circ} \mathrm{C}$

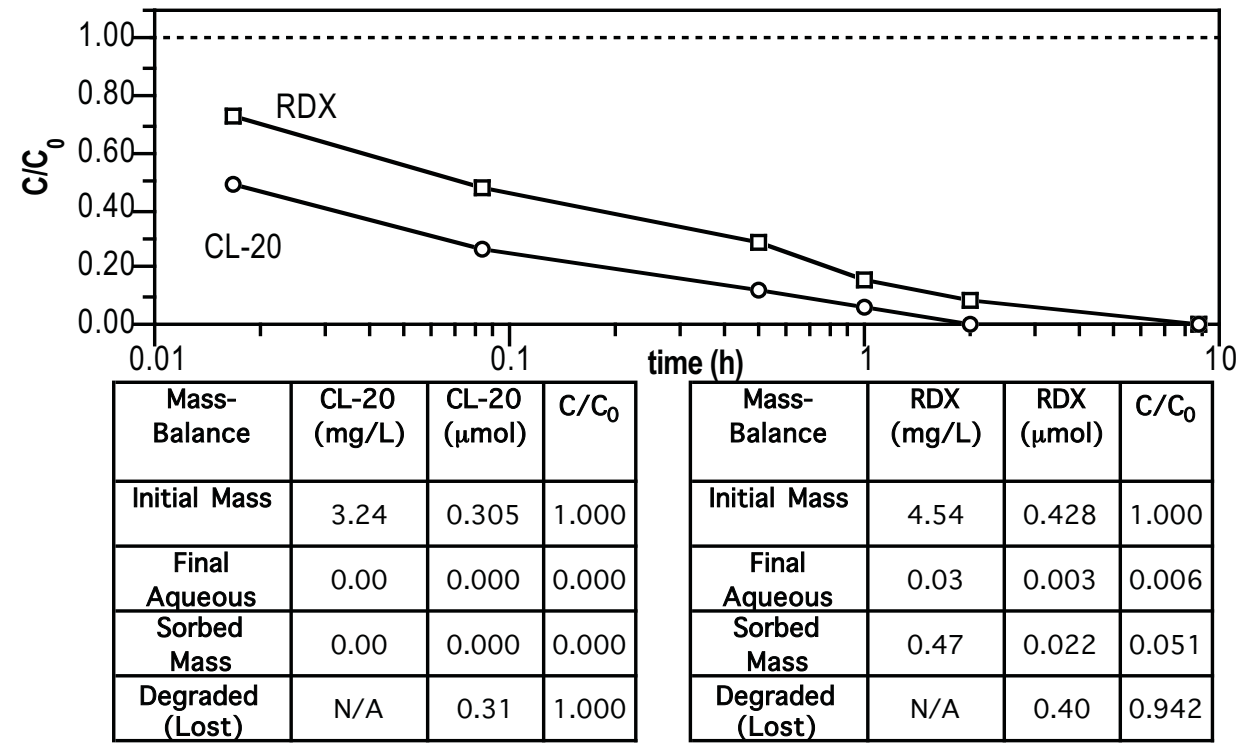




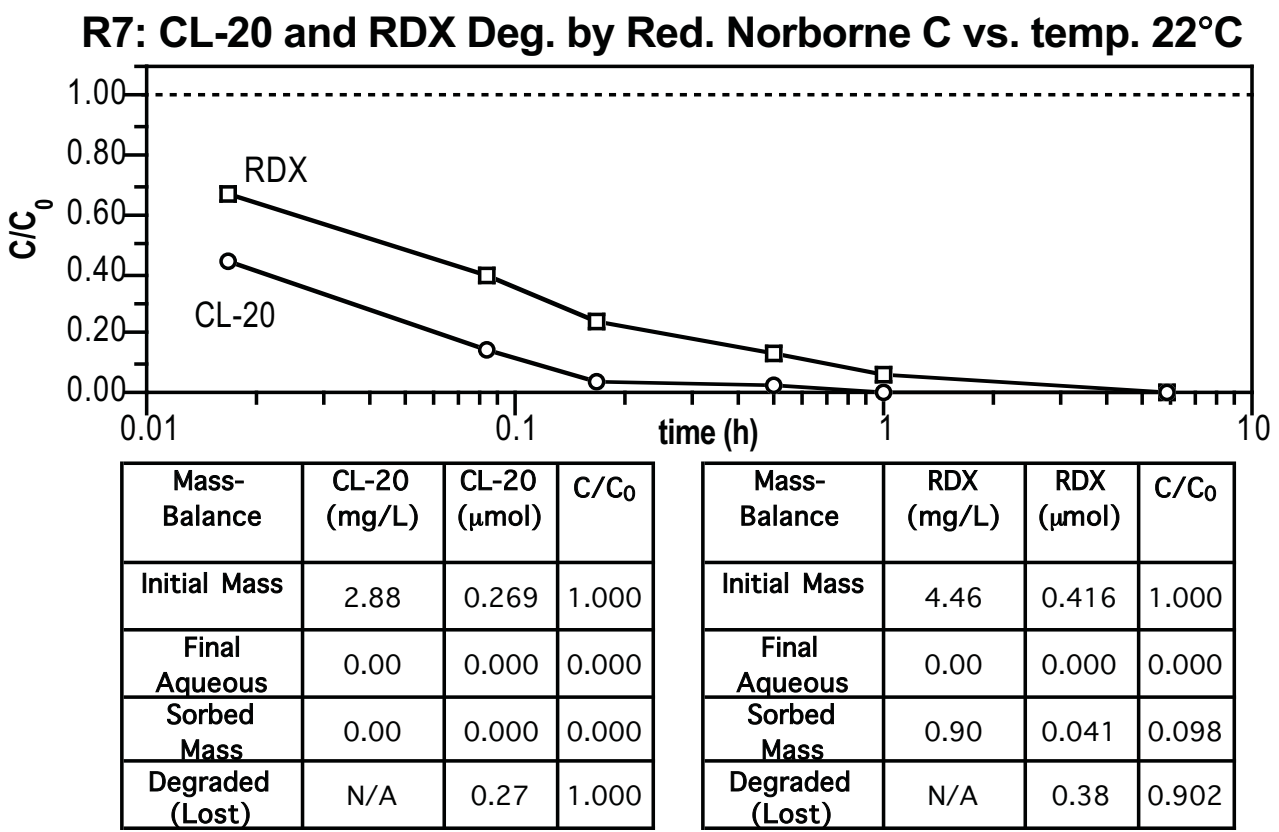

R1: CL-20 and RDX Deg. by Red. Norborne C vs. temp. $32^{\circ} \mathrm{C}$

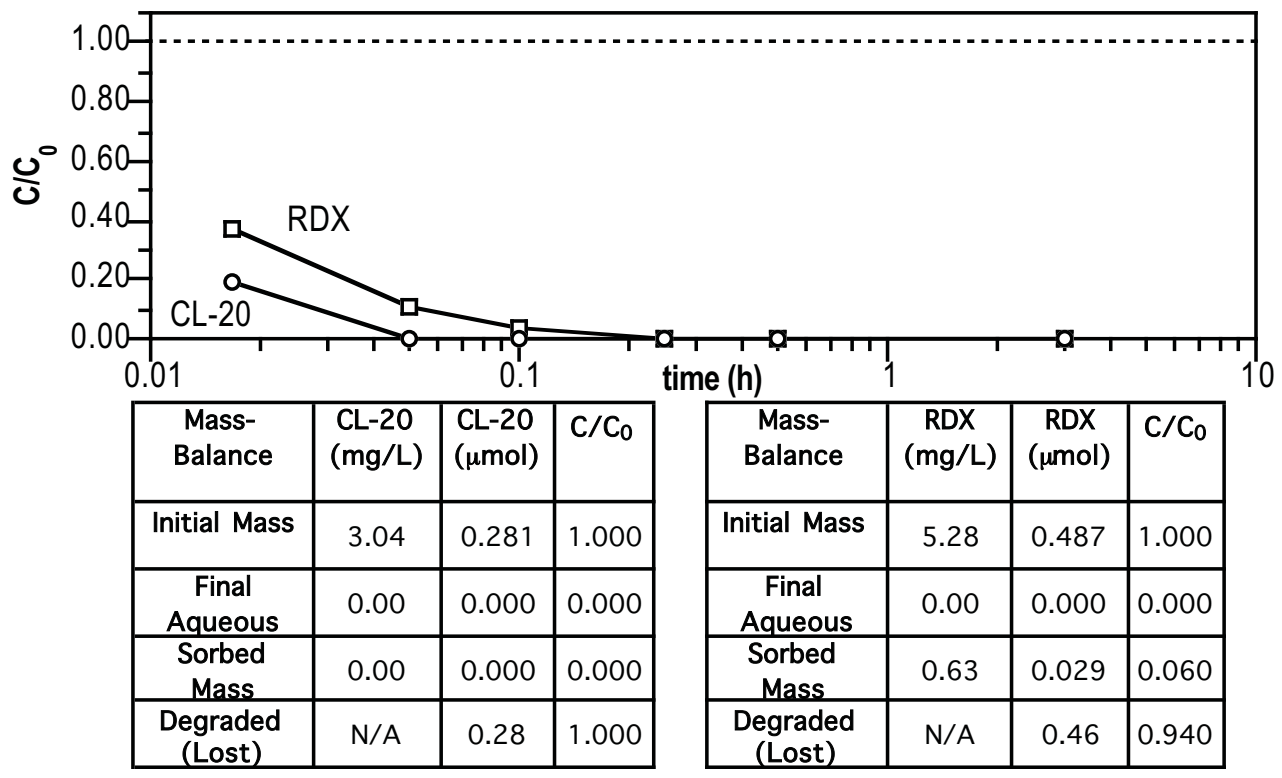


RDX Degradation by Red Norb C sed vs. temp, $r_{s w}=0.1$

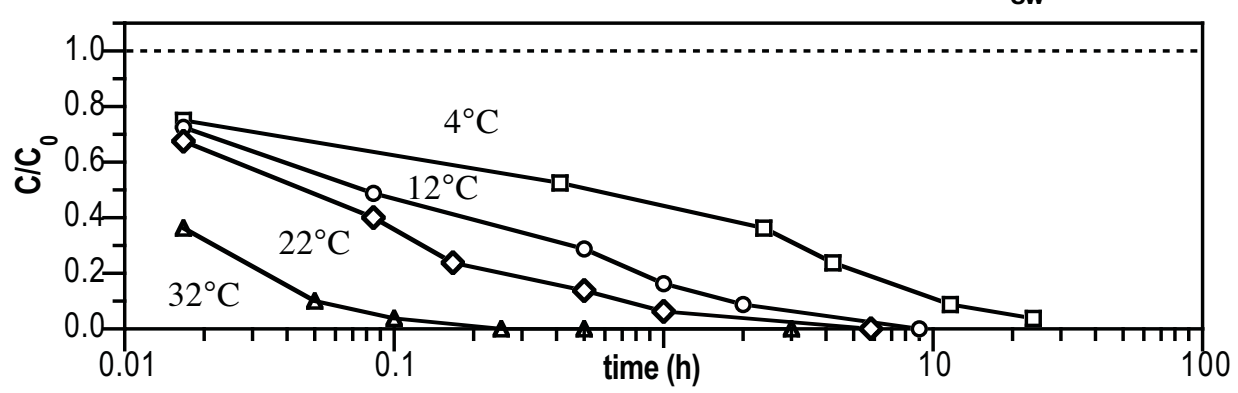

CL-20 Degradation by Red Norb C sed vs. temp, $r_{s w}=0.1$

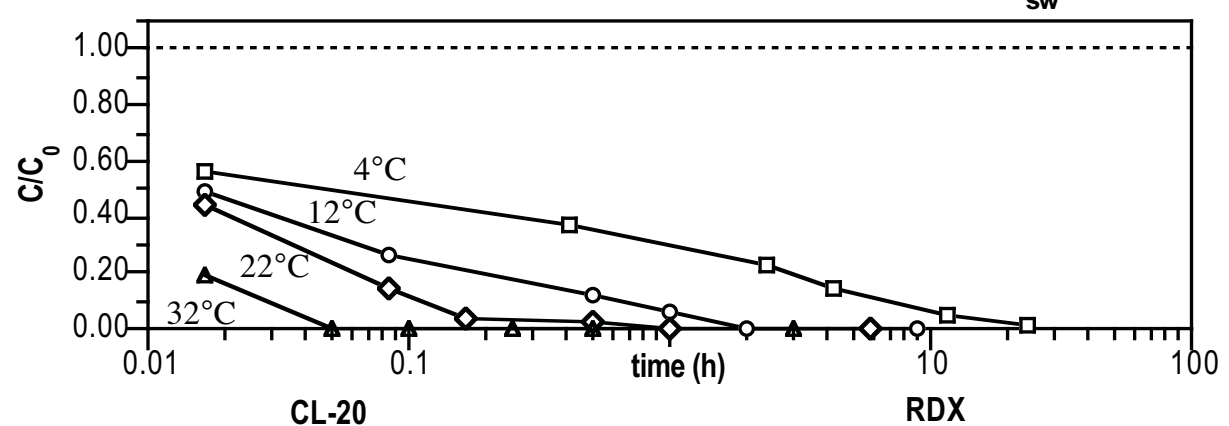

\begin{tabular}{|l|l|c|c|}
\hline Exp & temp & \% sorb & \% deg \\
\hline $\mathrm{R} 4$ & $4^{\circ} \mathrm{C}$ & 2.1 & 97.1 \\
\hline $\mathrm{R} 2$ & $12^{\circ} \mathrm{C}$ & 0.0 & 100.0 \\
\hline $\mathrm{R} 7$ & $22^{\circ} \mathrm{C}$ & 0.0 & 100.0 \\
\hline $\mathrm{R} 1$ & $32^{\circ} \mathrm{C}$ & 0.0 & 100.0 \\
\hline
\end{tabular}

\begin{tabular}{|l|l|c|c|}
\hline Exp & temp & \% sorb & \% deg \\
\hline $\mathrm{R} 4$ & $4^{\circ} \mathrm{C}$ & 4.4 & 91.9 \\
\hline $\mathrm{R} 2$ & $12^{\circ} \mathrm{C}$ & 5.1 & 94.2 \\
\hline $\mathrm{R} 7$ & $22^{\circ} \mathrm{C}$ & 9.8 & 90.2 \\
\hline $\mathrm{R} 1$ & $32^{\circ} \mathrm{C}$ & 6.0 & 94.0 \\
\hline
\end{tabular}




\section{APPENDIX F. CL-20 COUPLED ABIOTIC/BIOTIC DEGRADATION BATCH EXPERIMENTS}

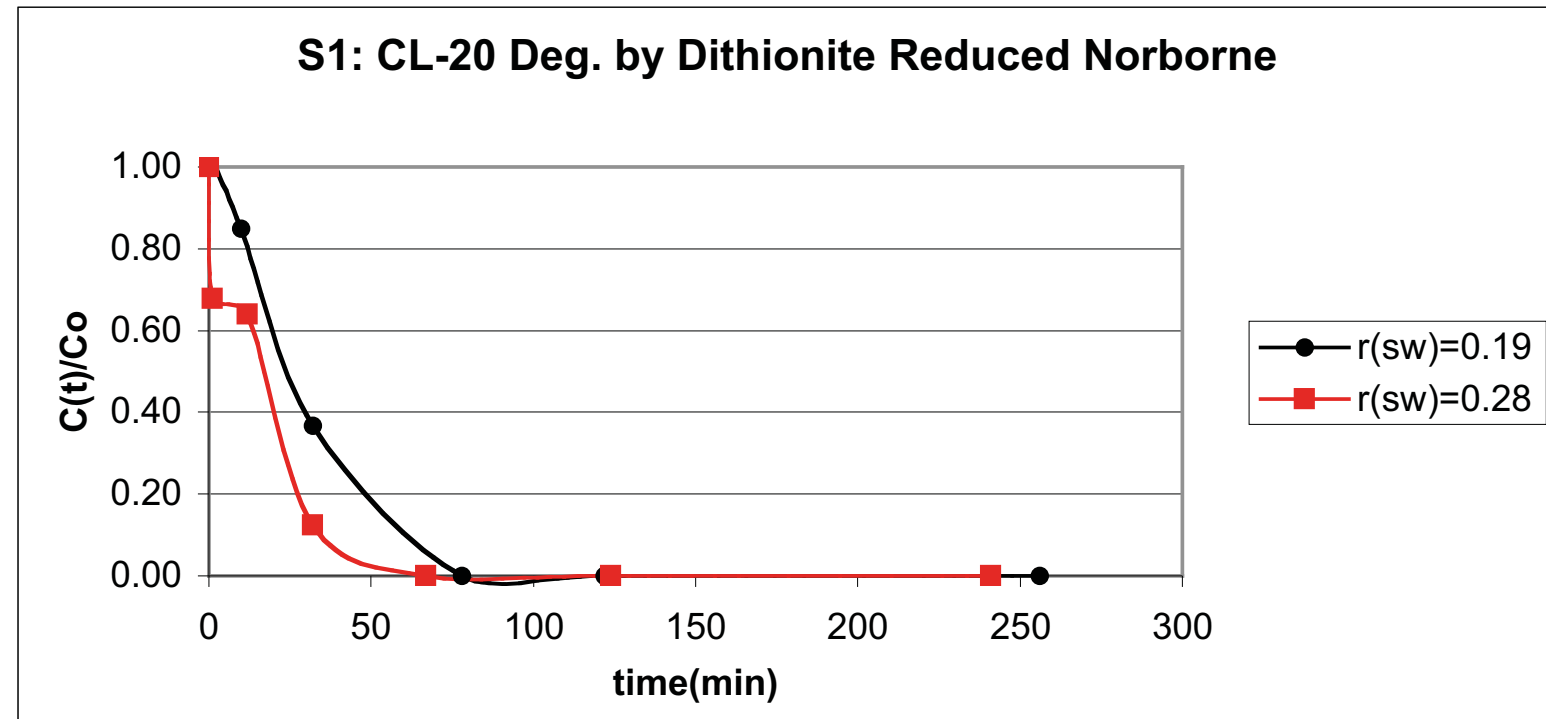

S2: CL-20 Deg. by Reduced FRC

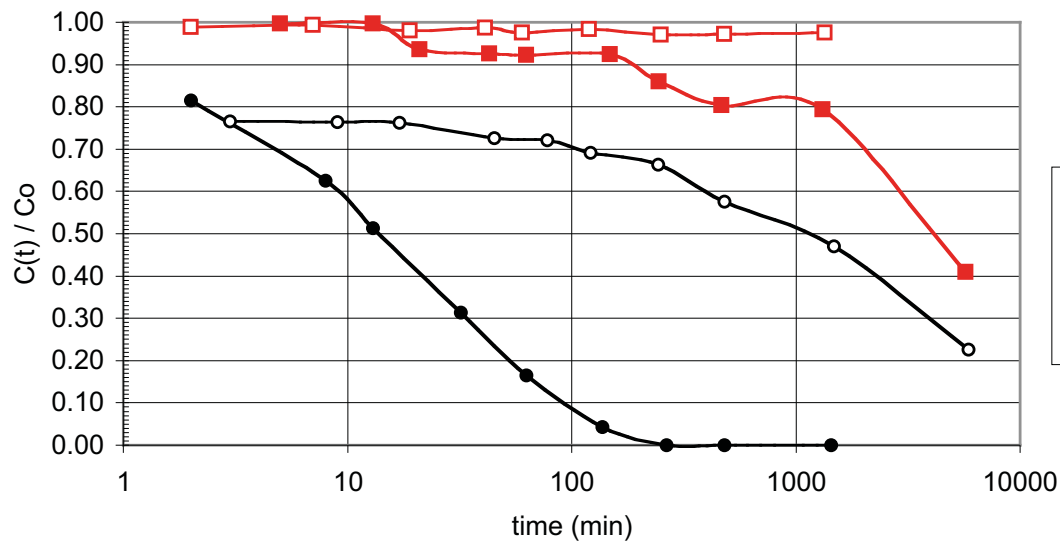

Not Normalized to Fe(II)

Initial CL-20 approx $3 \mathrm{mg} /$ 30 mM Pipes @ pH 7 $100 \mathrm{ml}$ tot vol

$\longrightarrow$ Dith. Rsw $=0.013 \mathrm{~g} / \mathrm{ml}$

—-Dith. Rsw $=0.003 \mathrm{~g} / \mathrm{ml}$

$\longrightarrow$ BioR. Rsw $=0.01 \mathrm{~g} / \mathrm{ml}$

$\longrightarrow$-BioR. Rsw $=0.002 \mathrm{~g} / \mathrm{ml}$ 
S3: CL-20 Deg. by Bio Red FRC; Orig Glass Tube vs Plastic

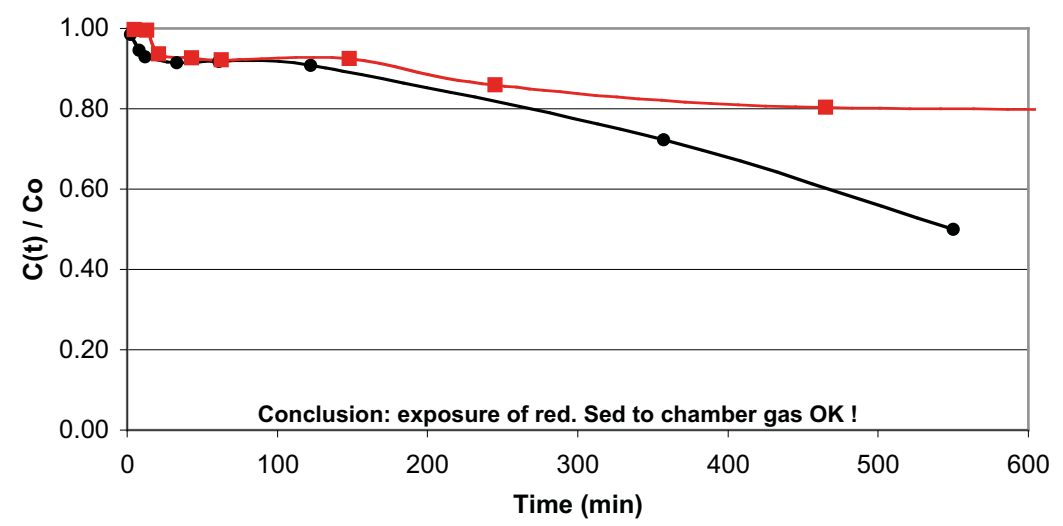

Exp S3: Initial CL-20 $=5.6 \mathrm{mg} / \mathrm{L}$ 30 mM Pipes@pH 7,30 ml vol Deg after $1 \mathrm{hr}$ prob due to glass

Exp S2c : initial CL-20 $=3 \mathrm{mg} / \mathrm{L}$ 3 mM Pipes@ @ pH7, 100 ml vol

$\longrightarrow$ Exp S3; Rsw $=0.034$

$\longrightarrow$ Exp S2C: Rsw $=0.01$

S5: CL-20 Degradation with FRC

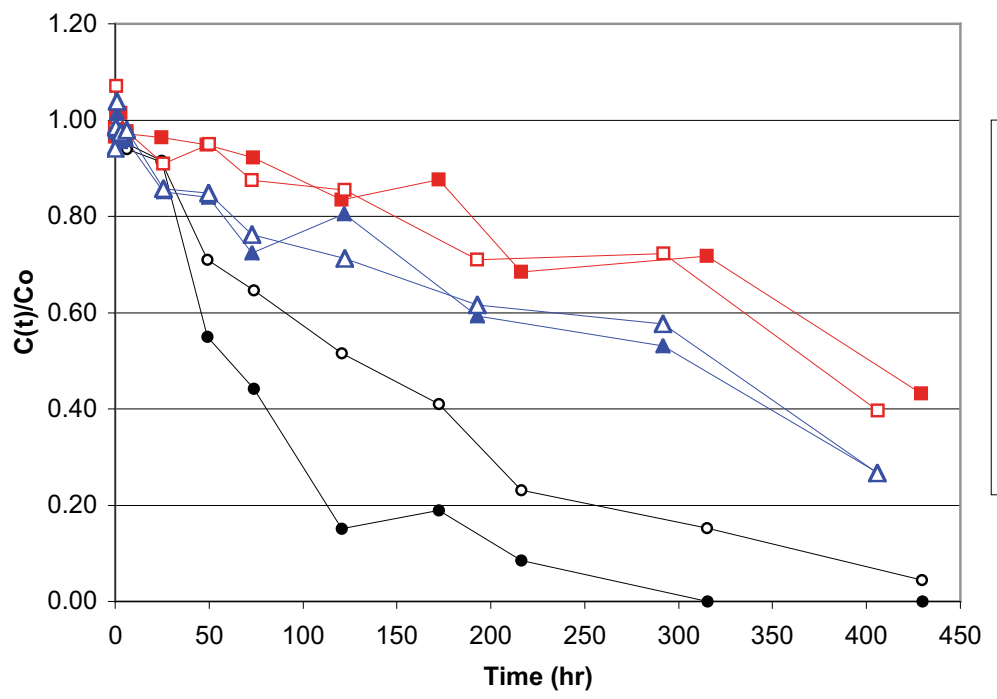

-S5A-BioRed Sed, CN32, Lac.

$\multimap$ S5B-BioRed Sed, CN32, Lac.

- S5C-Non-Red Sed, Lac.

$\multimap-$ S5D-Non-Red Sed, Lac.

$\_$S5E-Fresh Sed

$\triangle$ S5F-Fresh Sed 


\section{S6: CL-20 with CN $32 @ 4.2$ E08 cfu}

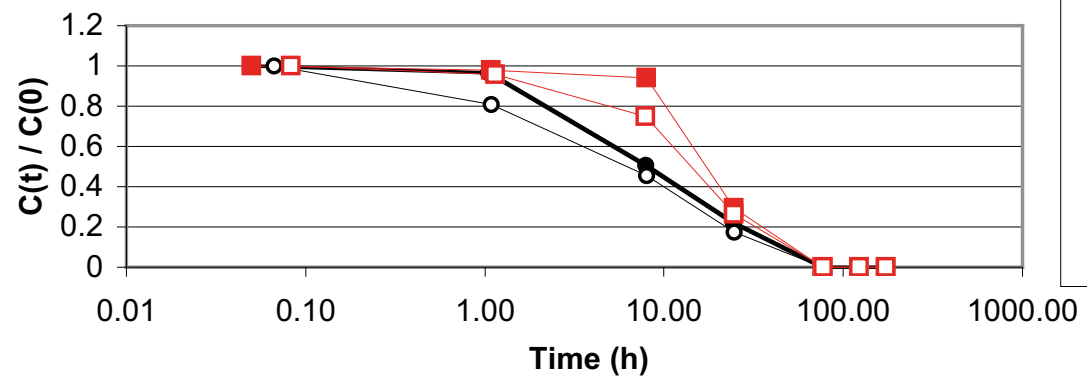

$\longrightarrow$ S6A1: CN32, Lac

o- S6A2: CN32, Lac

- S6B1: CN32

$\square-\mathrm{S} 6 \mathrm{~B} 2: \mathrm{CN} 32$

S6: CL-20 with CN $32 @ 4.2$ E08 cfu

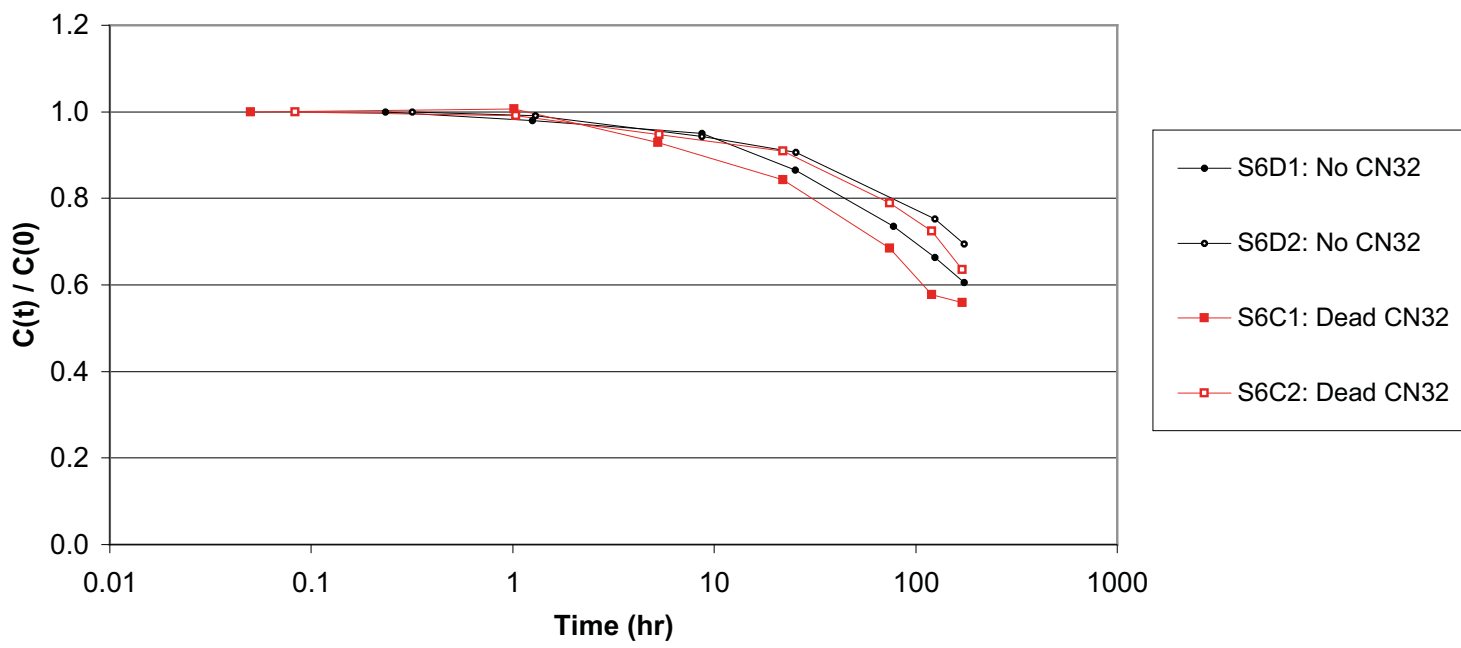




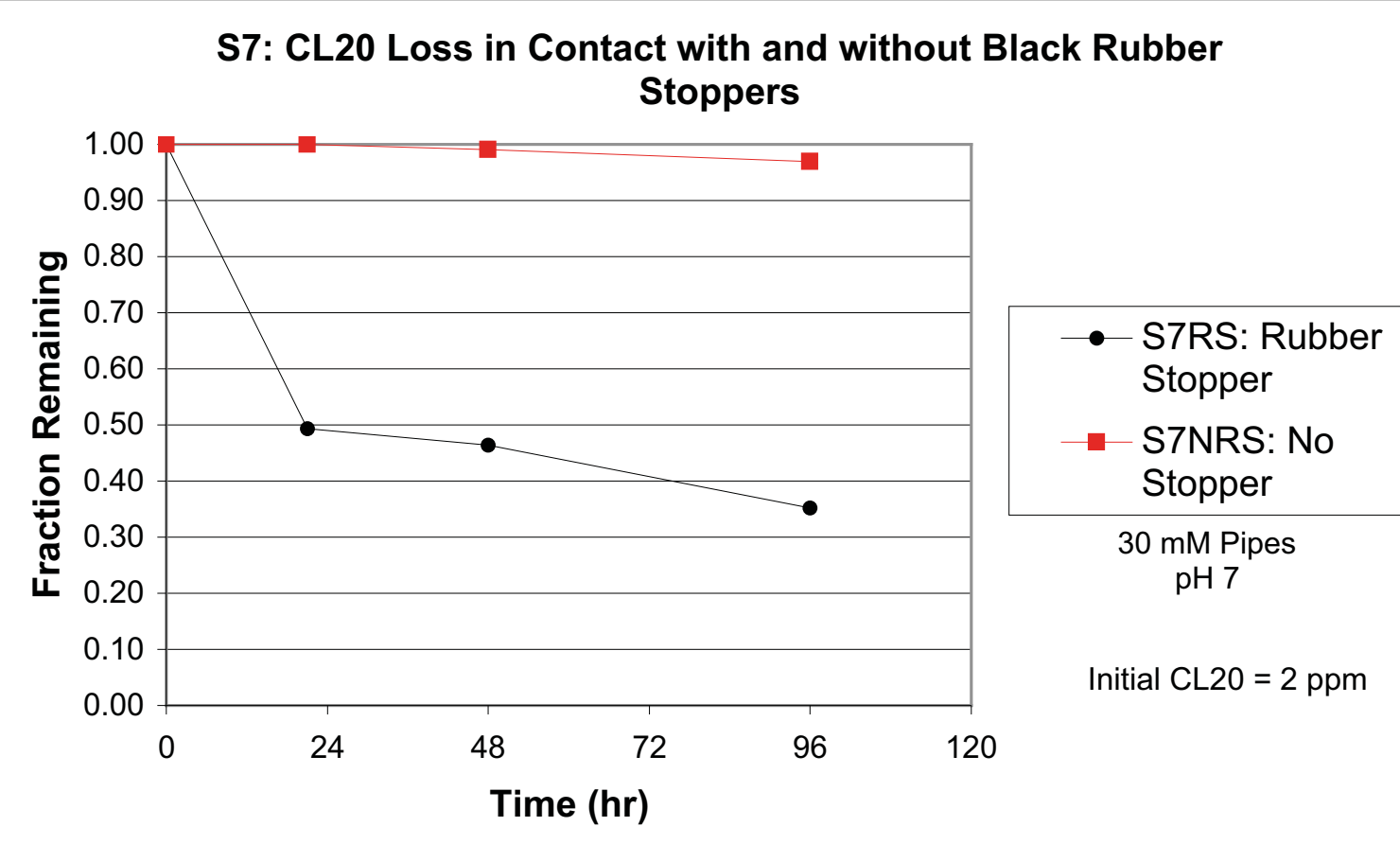

S7: CL20 Loss in Contact with Black Rubber Stoppers with and without Lactate

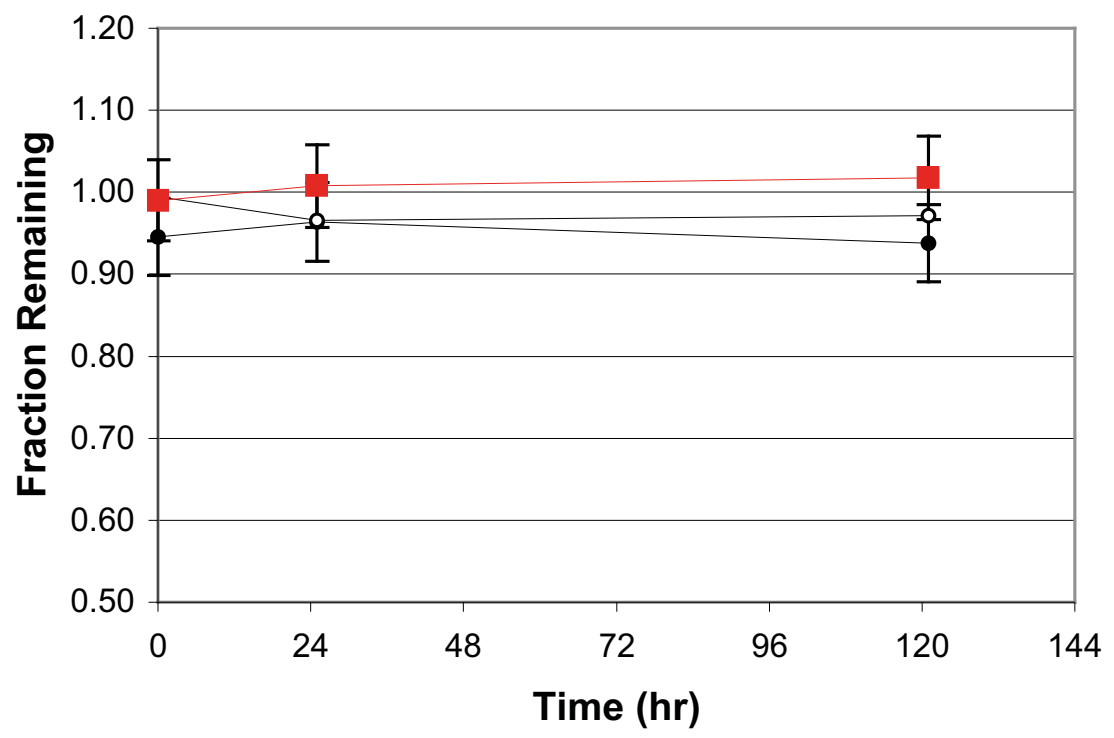

- S7C1: w/Lac.

$\multimap$ S7C2: w/Lac.

- S7D1: No Lac. 30 mM Pipes $\mathrm{pH} 7$

Initial CL20 = 1.8 ppm Analytical error 5\% 
S8: CL20 Loss in the presence of CN32

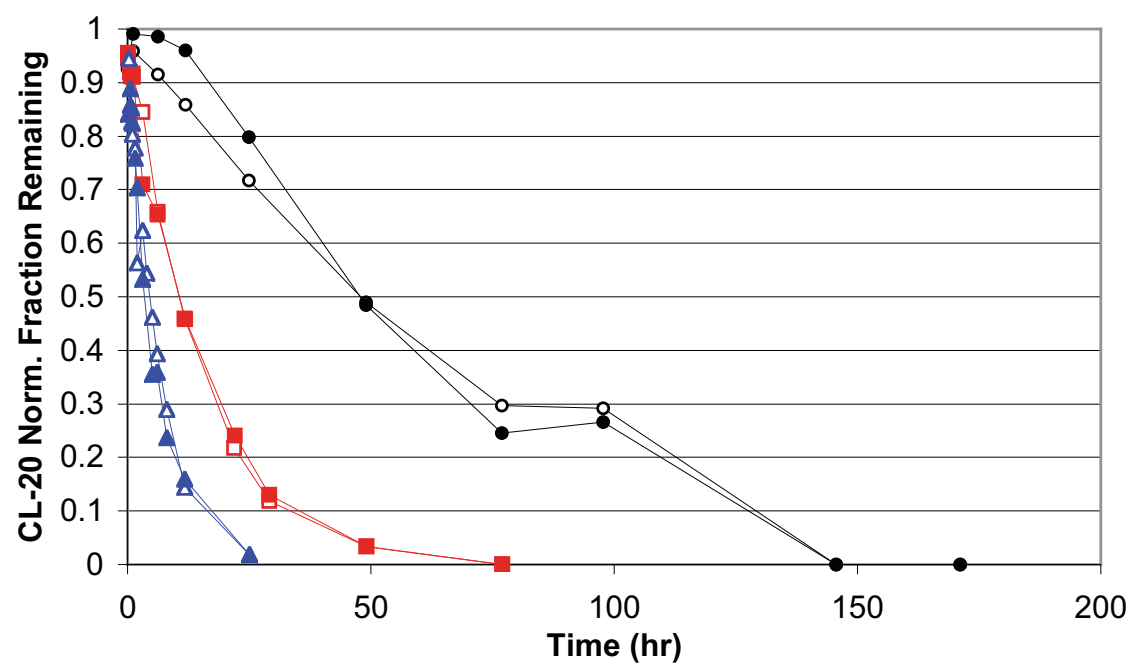

CN32 Concentration $(\mathrm{CFU} / \mathrm{mL})$

—- S8A1: 4.77E+07

- S8A2: 4.77E+07

—- S8B1: $3.27 E+08$

— S8B2: 3.27E+08

$\triangle$ S8C1: 7.40E+08

$\_$S8C2: $7.40 \mathrm{E}+08$

Blank Corrected

30 mM Pipes $\mathrm{pH} 7$

$10 \mathrm{mM}$ Lactate

S8: CL20 Loss with and without Sterilized CN32

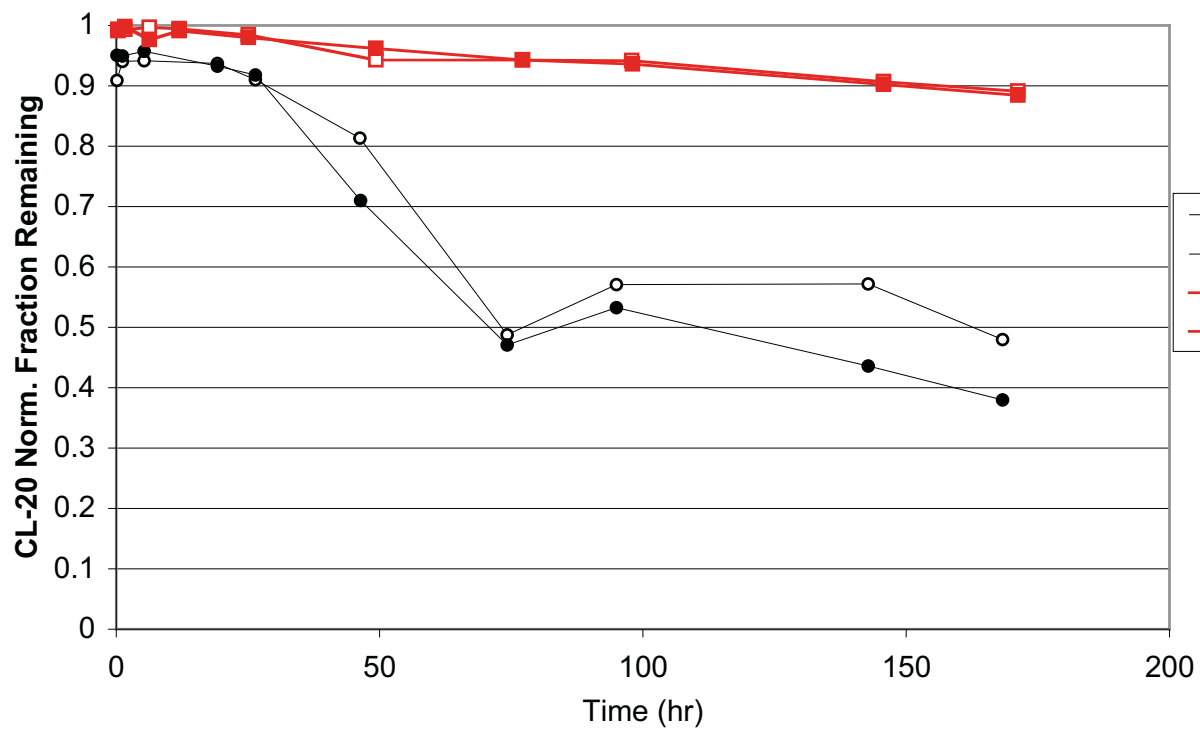

CN32

Concentration

(CFU/mL)

- - S8D1: Ster. CN32

- S8D2: Ster. CN32

$-\square-S 8 E 1:$ No CN32

$\rightarrow-S 8 E 2:$ No CN32

Blank Corrected

30 mM Pipes $\mathrm{pH} 7$

$10 \mathrm{mM}$ Lactate 


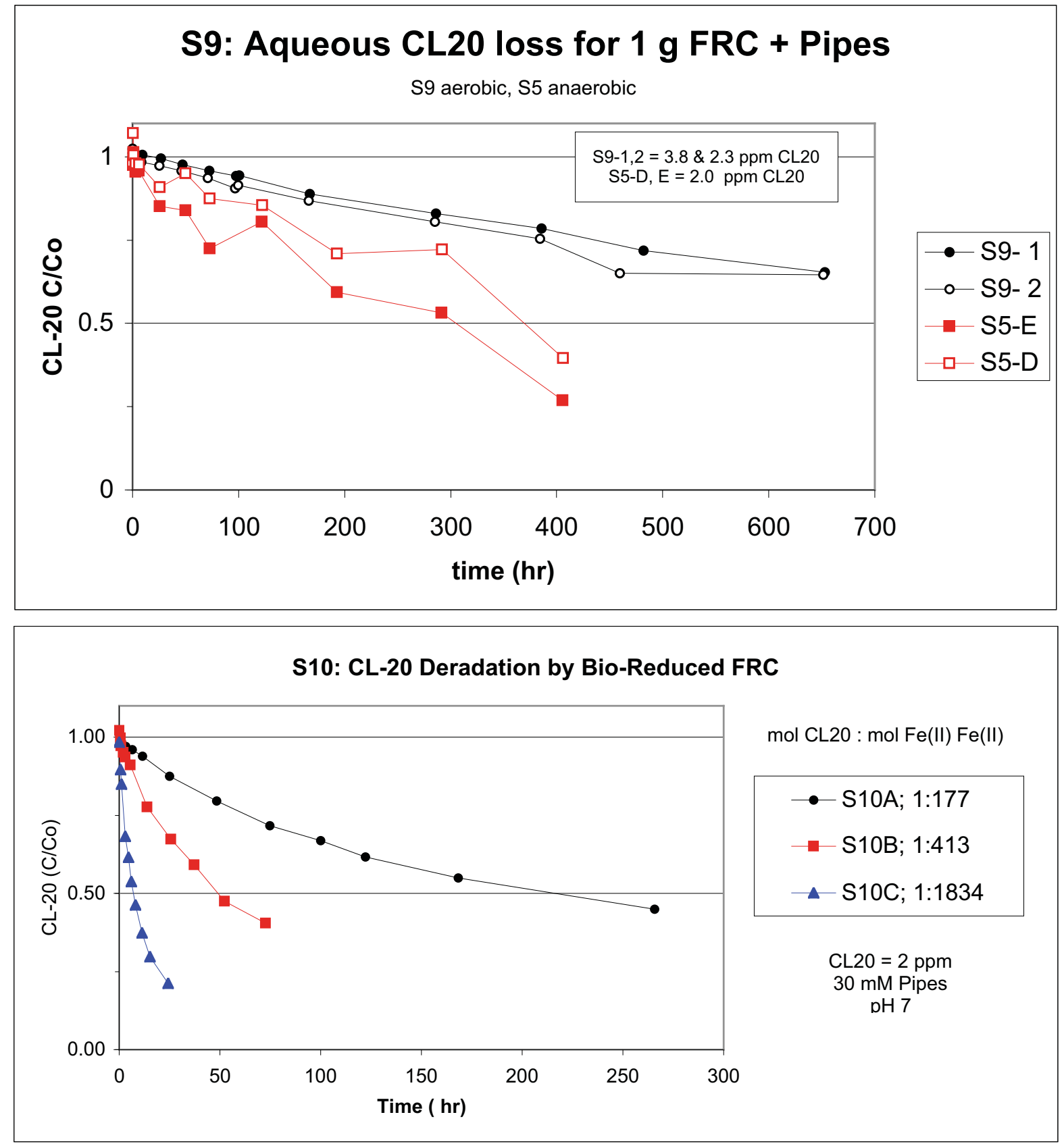


S11: CL20 Aqueous Degradation with and without Gluteraldehyde

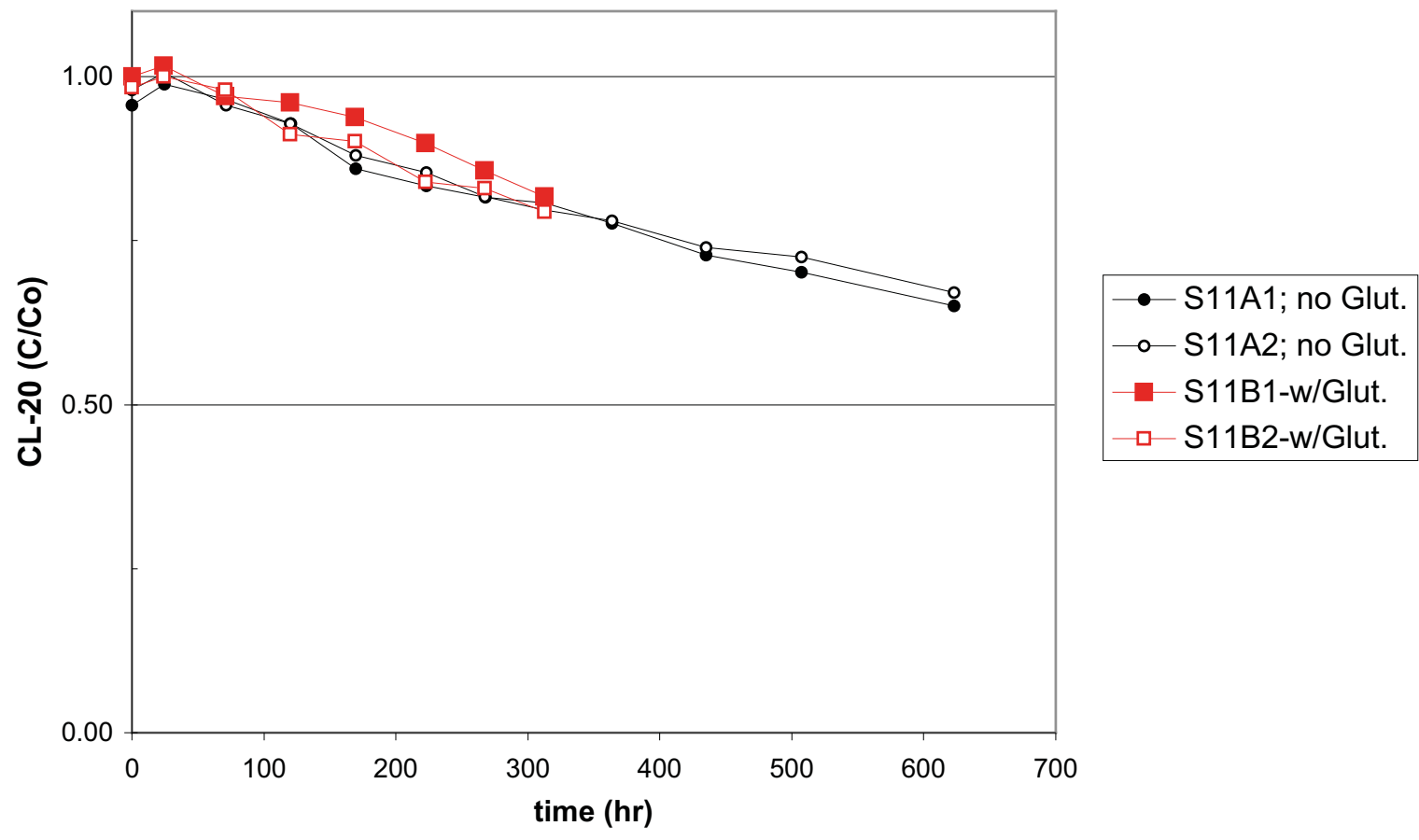

S12; CL20 loss with 1g FRC + Gluterhaldehyde

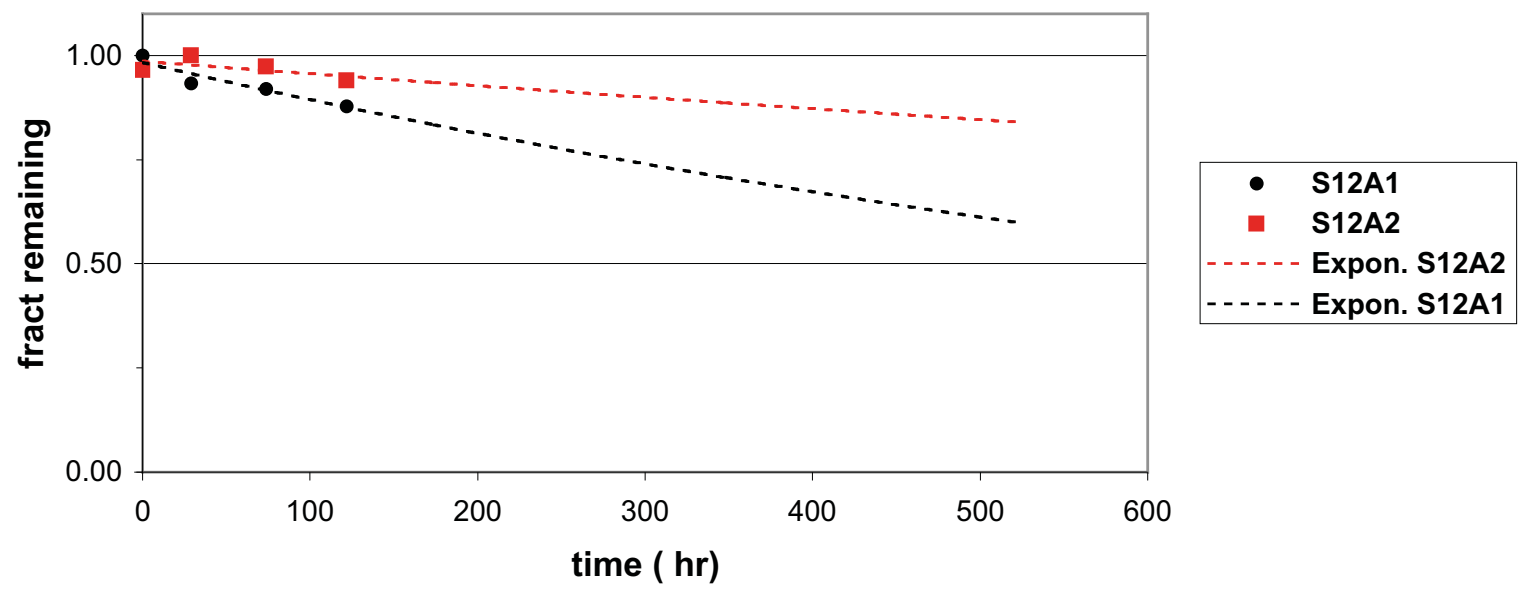


S13: CL20 Deg. with 1g BioRed FRC : molar Fe(II) : CL20 = $177: 1$

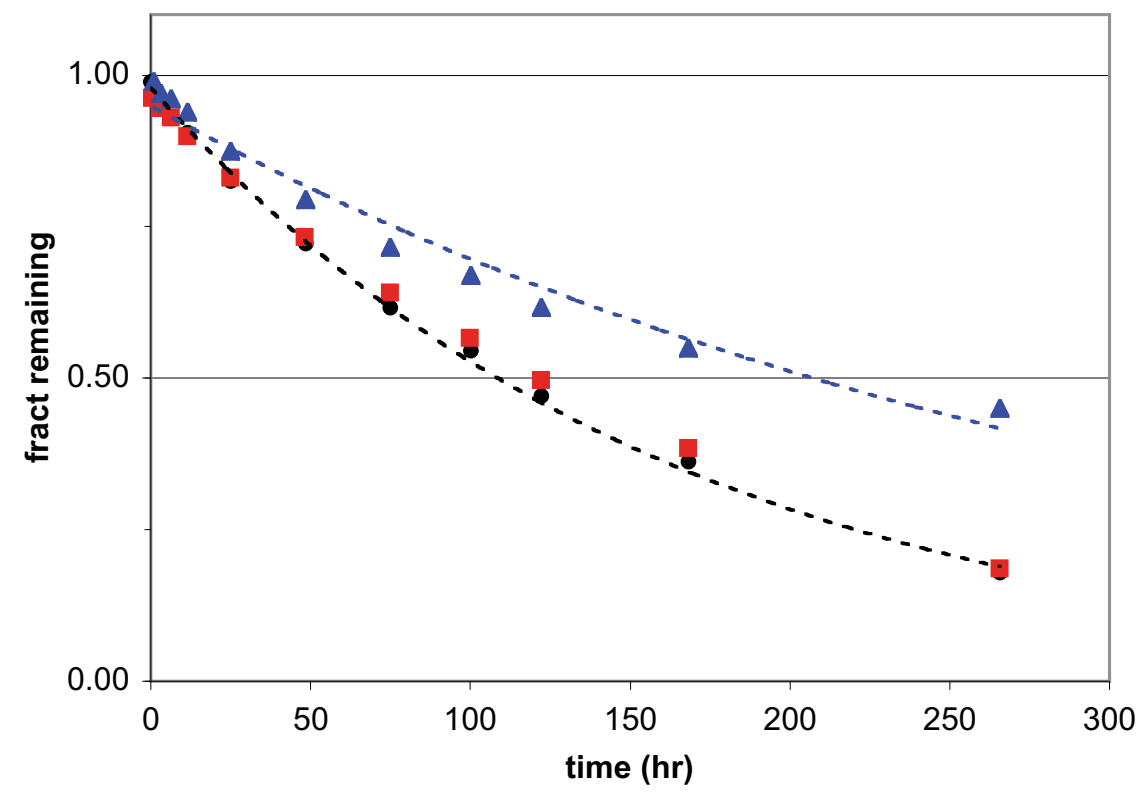

Note S13 blank correction is for $550 \mathrm{hr}$ of blank data

- $\quad$ S13A1

- S13A2

- S13 Blank

- - - - - Expon. S13A1

- - - - - Expon. S13 Blank

$t(1 / 2)=193 h$

S14: CL20 Degradation with $10 \mathrm{~g}$ of FRC : Fresh \& Control

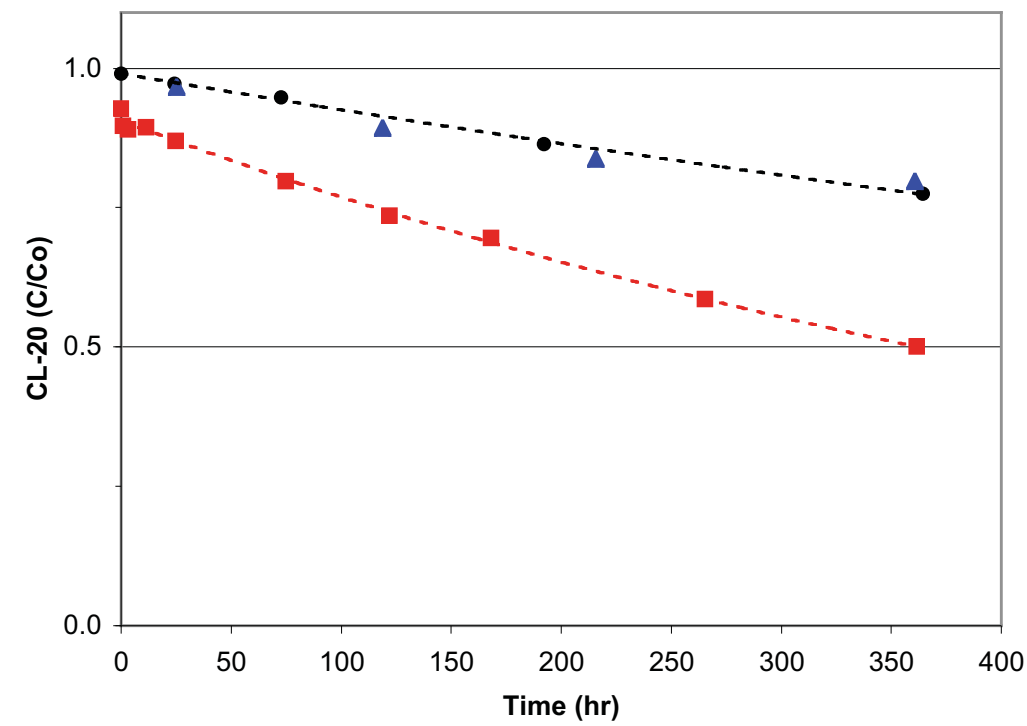

- $\quad$ S14A; Fresh FRC, 2.0 ppm

- S14B; Control FRC, 2.3ppm

$\Delta \quad$ S14C; Extractions Expon. (S14B; Control FRC, 2.3ppm)

Expon. (S14A; Fresh FRC, 2.0 ppm) 


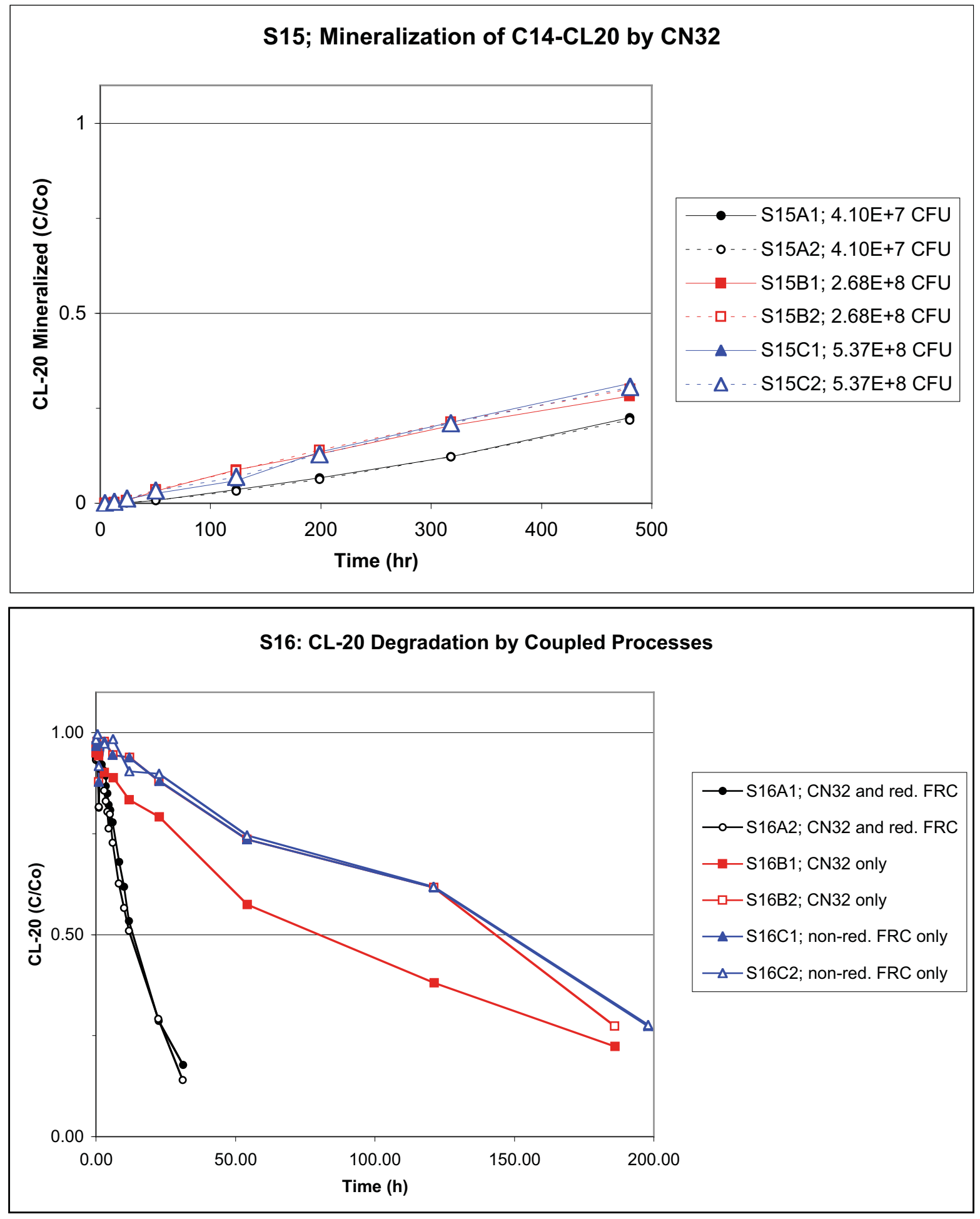



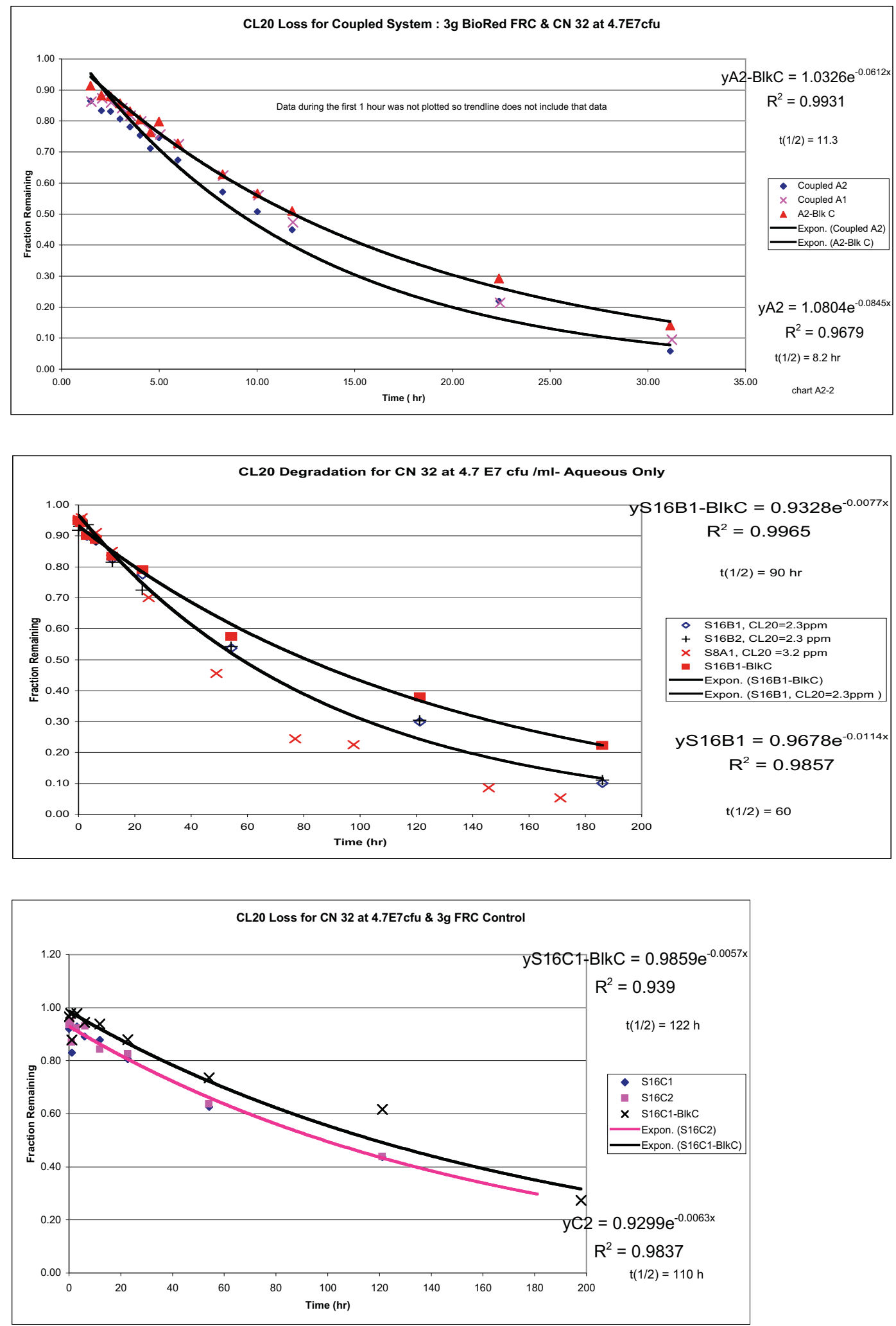


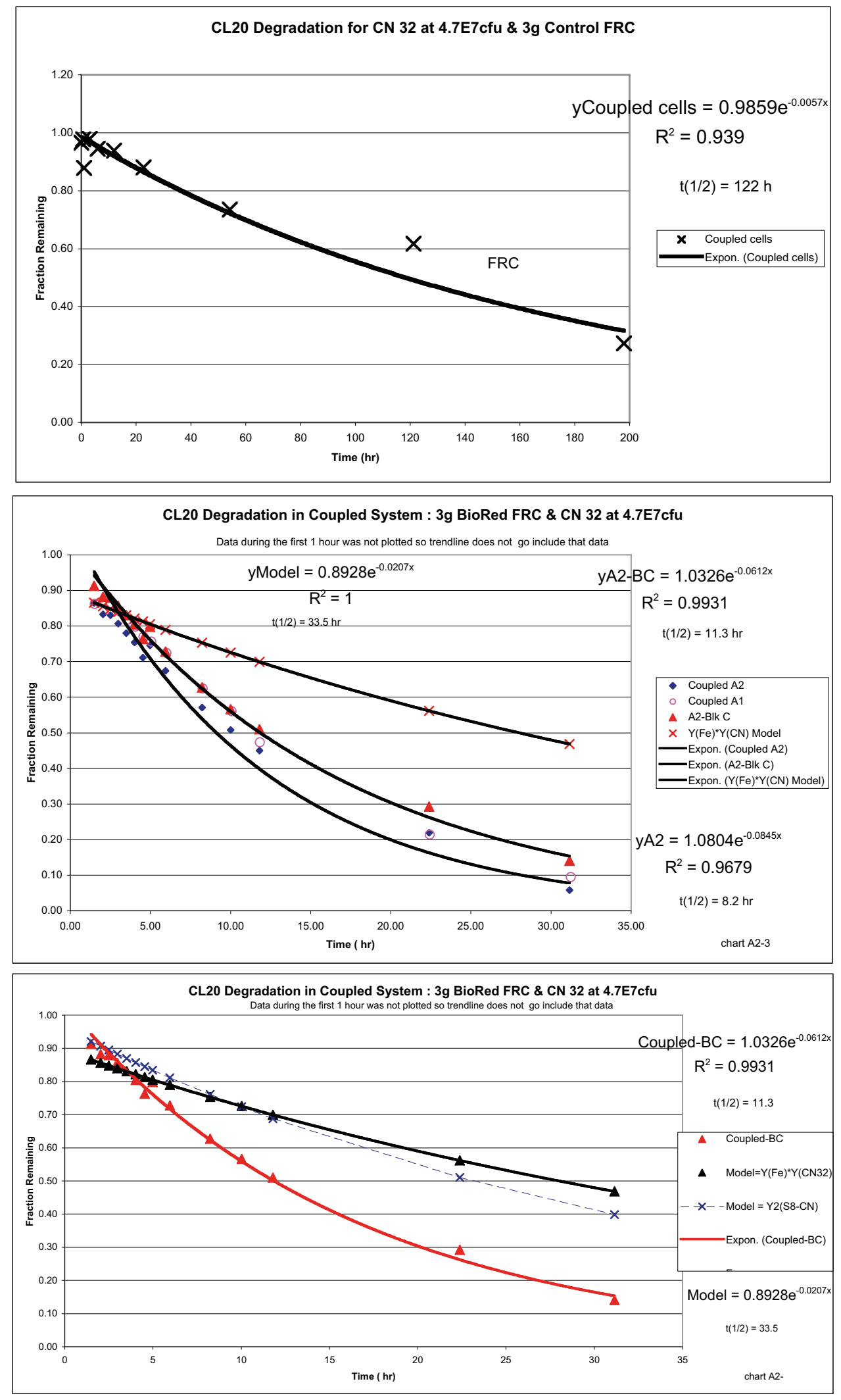



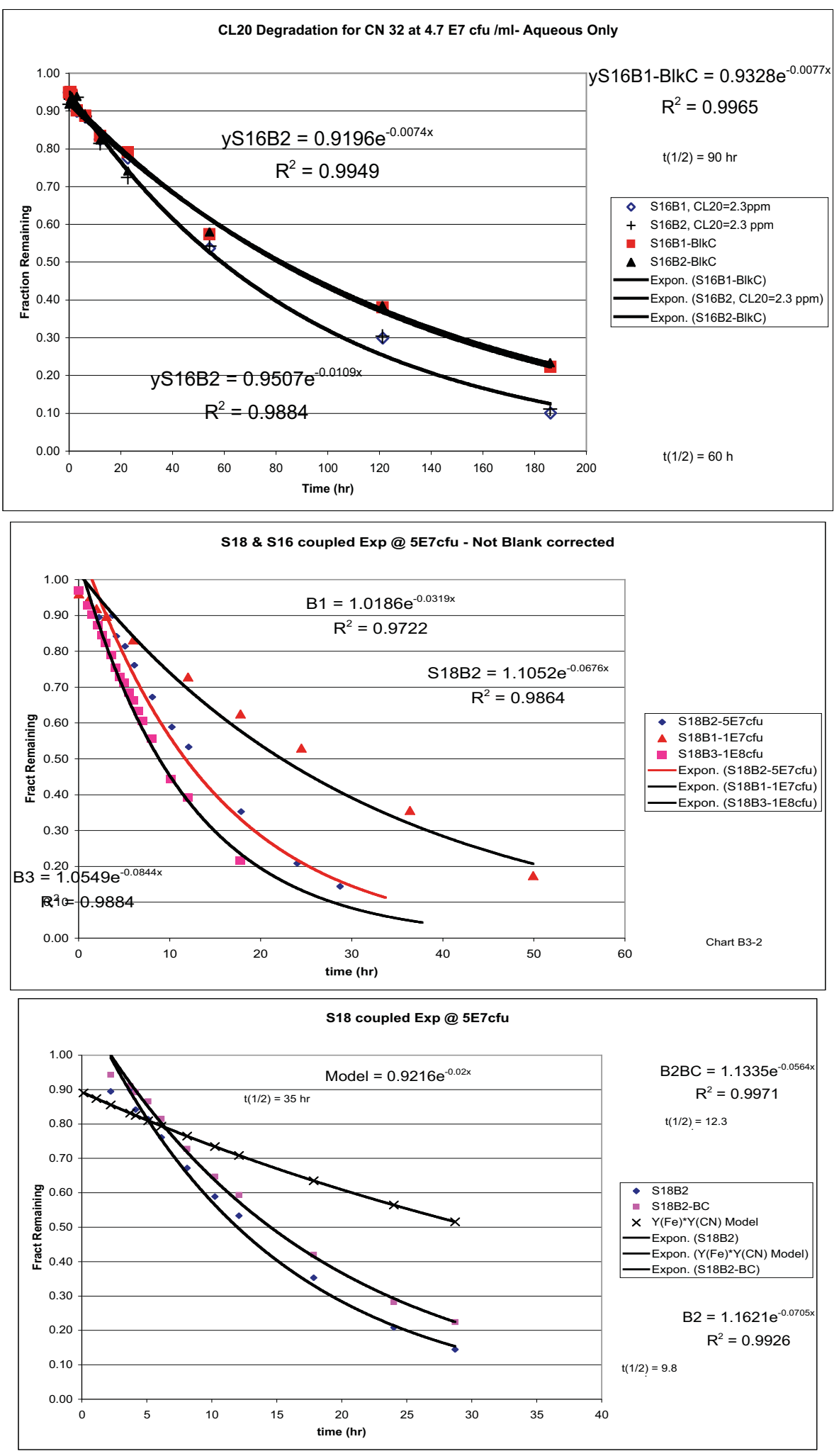


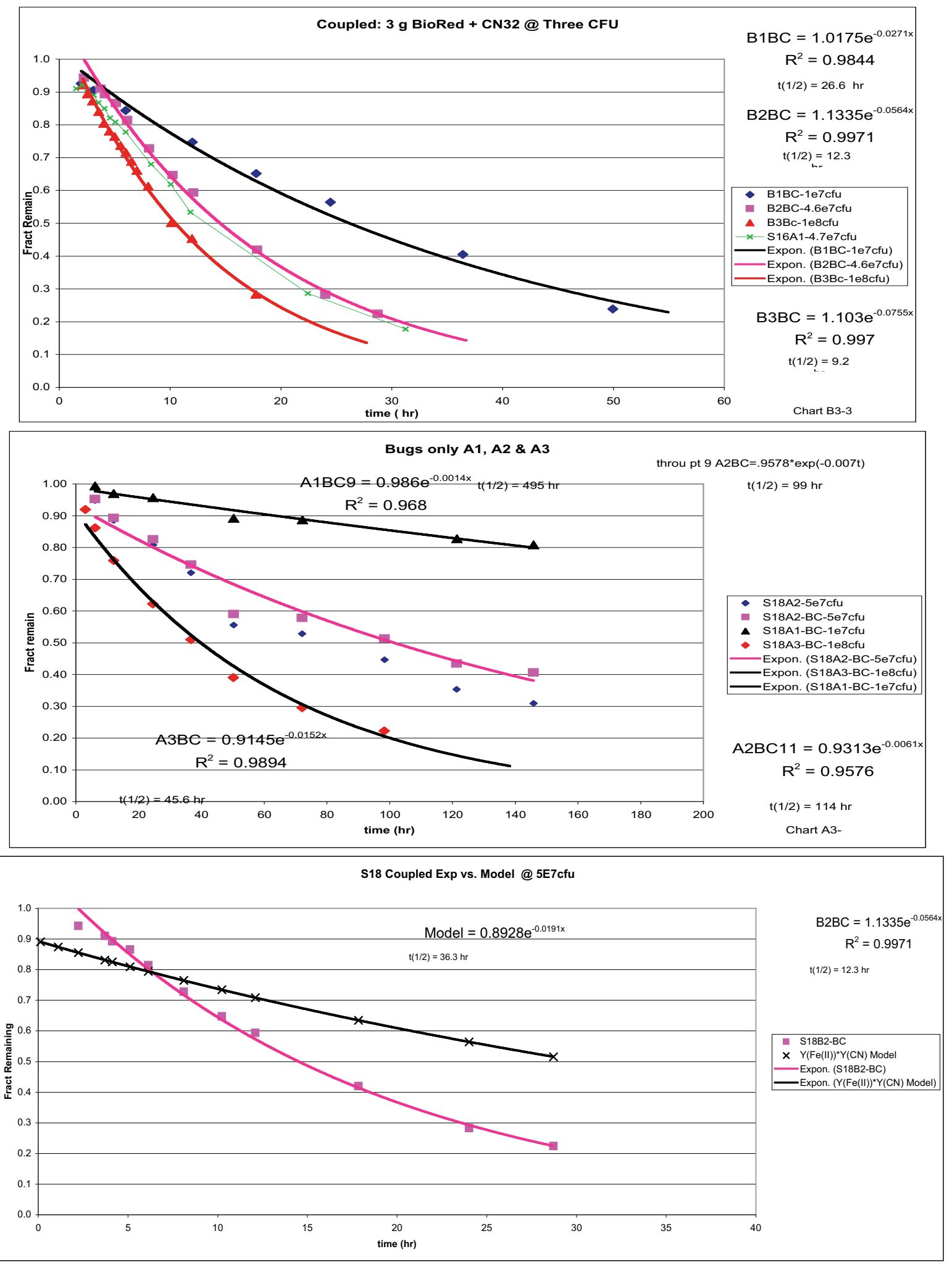



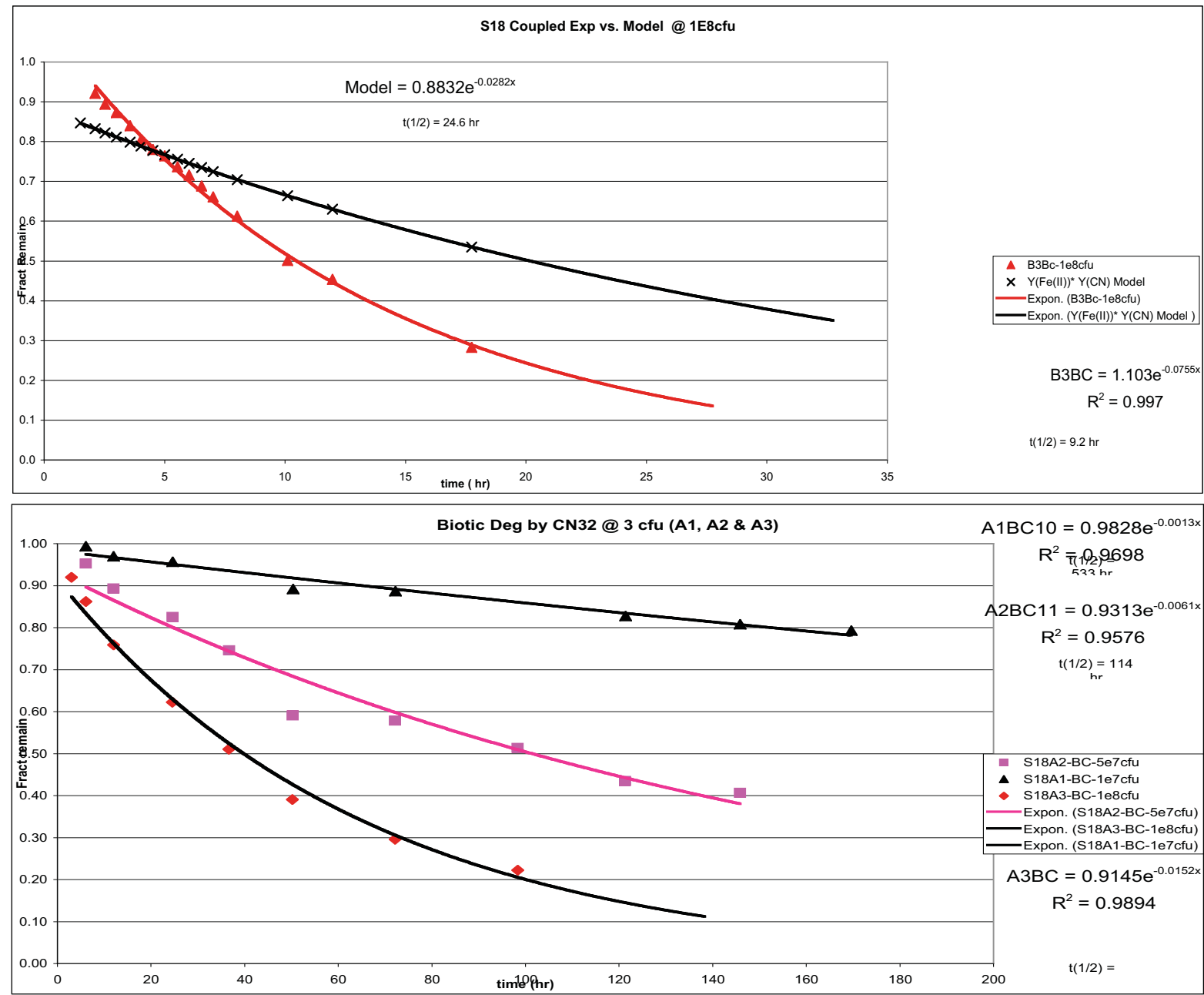

$\mathrm{CaCl} 2 \& \mathrm{HCl}$ Extractions of $3 \mathrm{~g} \mathrm{BioRed}+5 \mathrm{E} 7 \mathrm{cfu}$

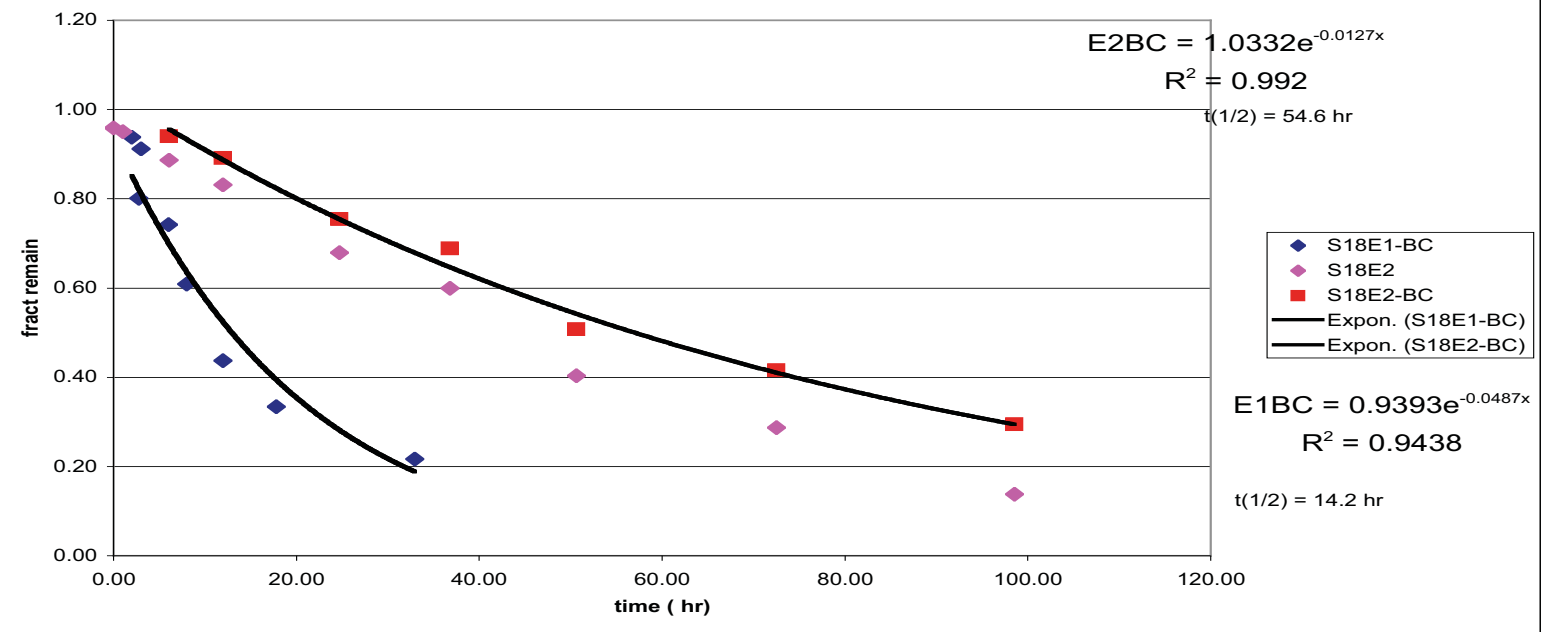




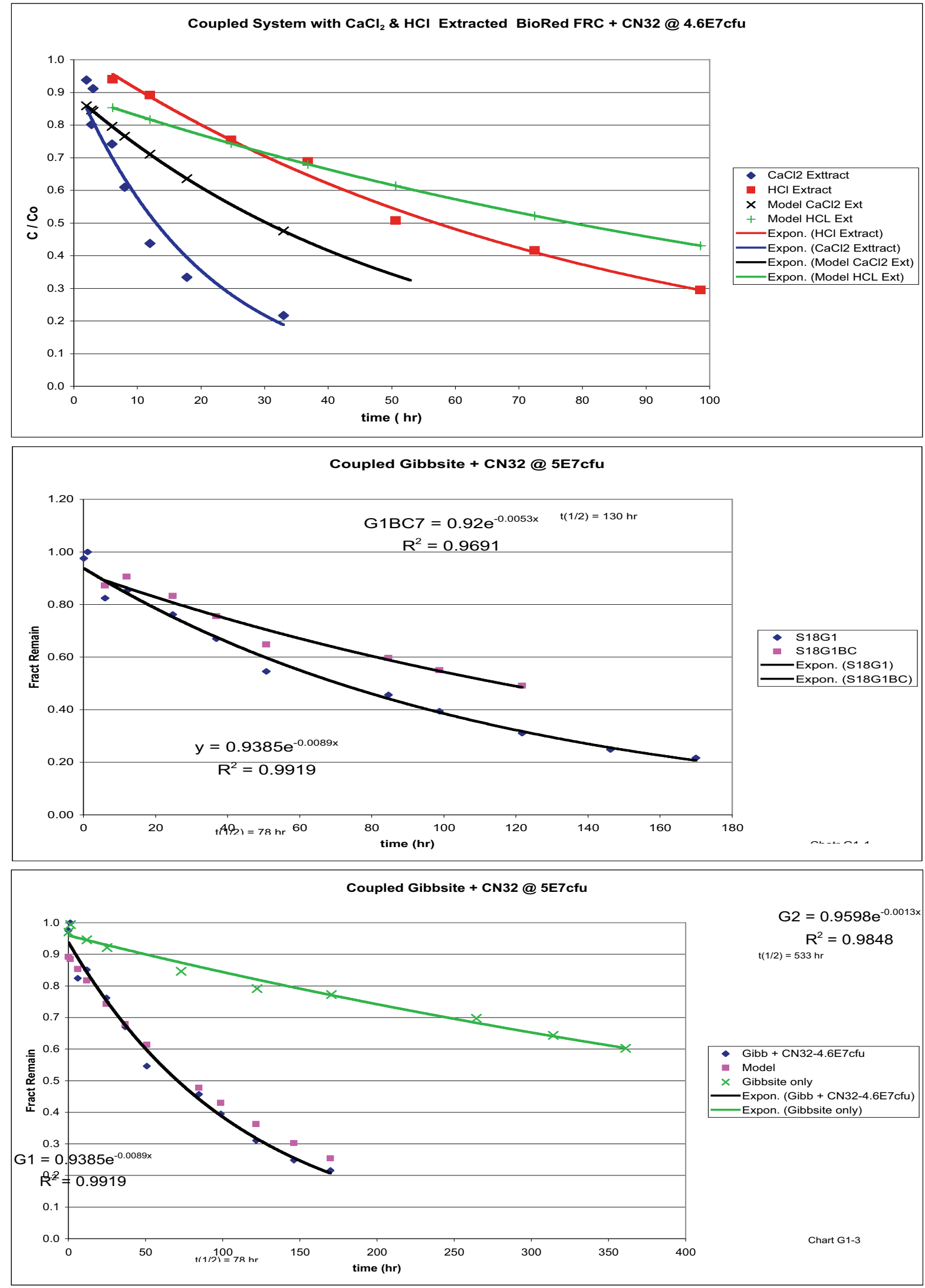


S18 G2 - 6g Gibbsite Blank, No CN32

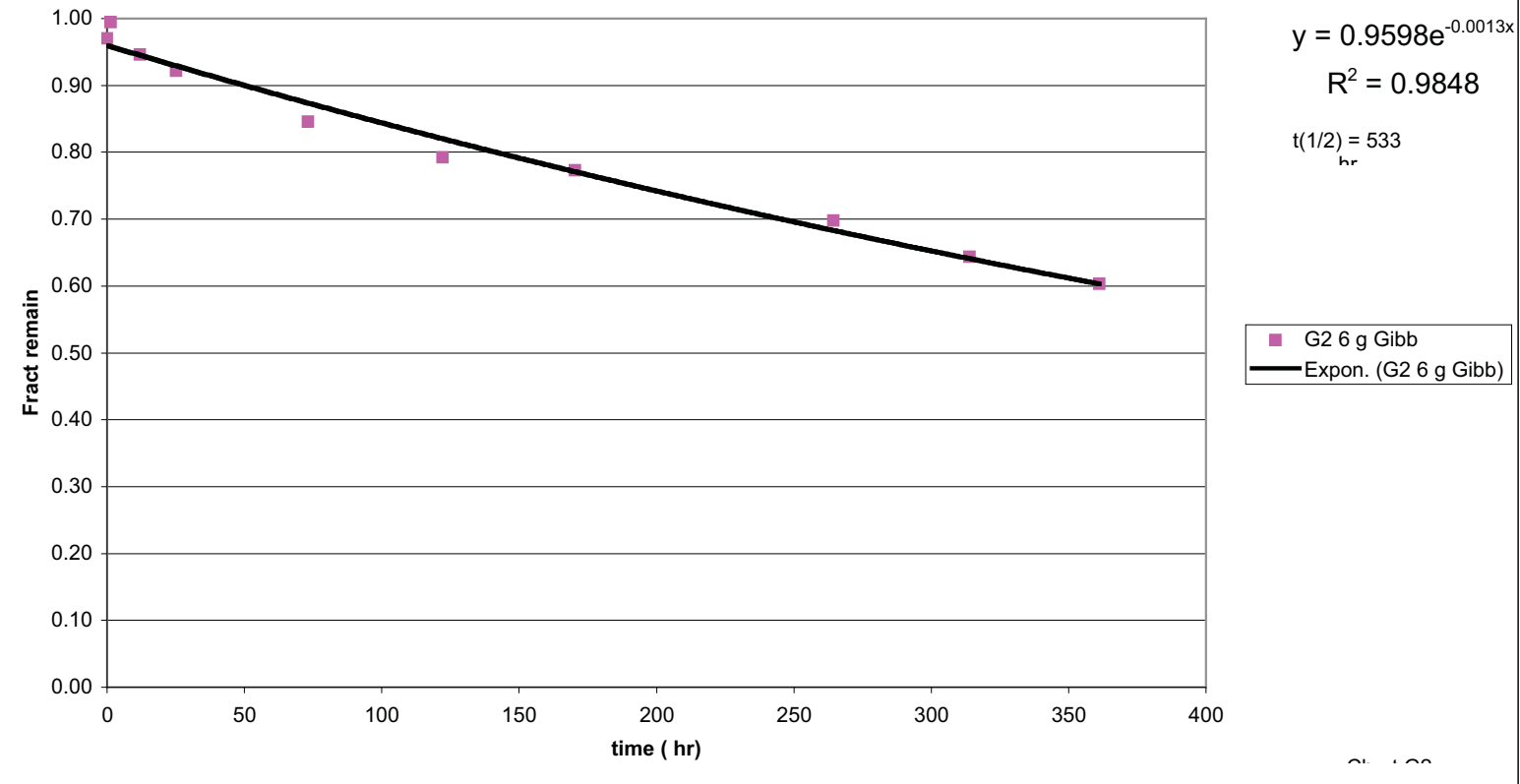


Appendix G. CL-20 Mineralization in Aqueous and by Reduced and Oxic Sediment
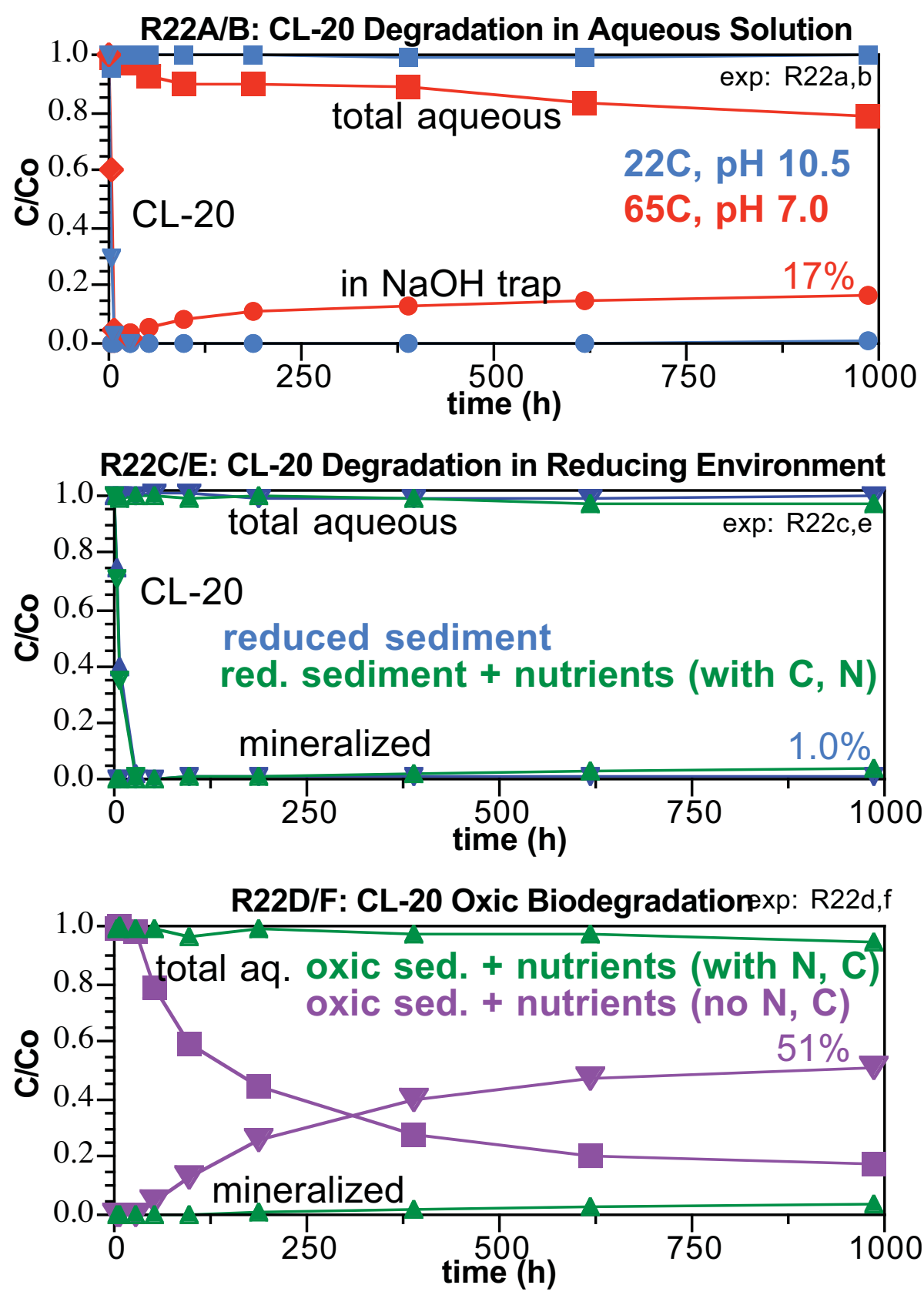

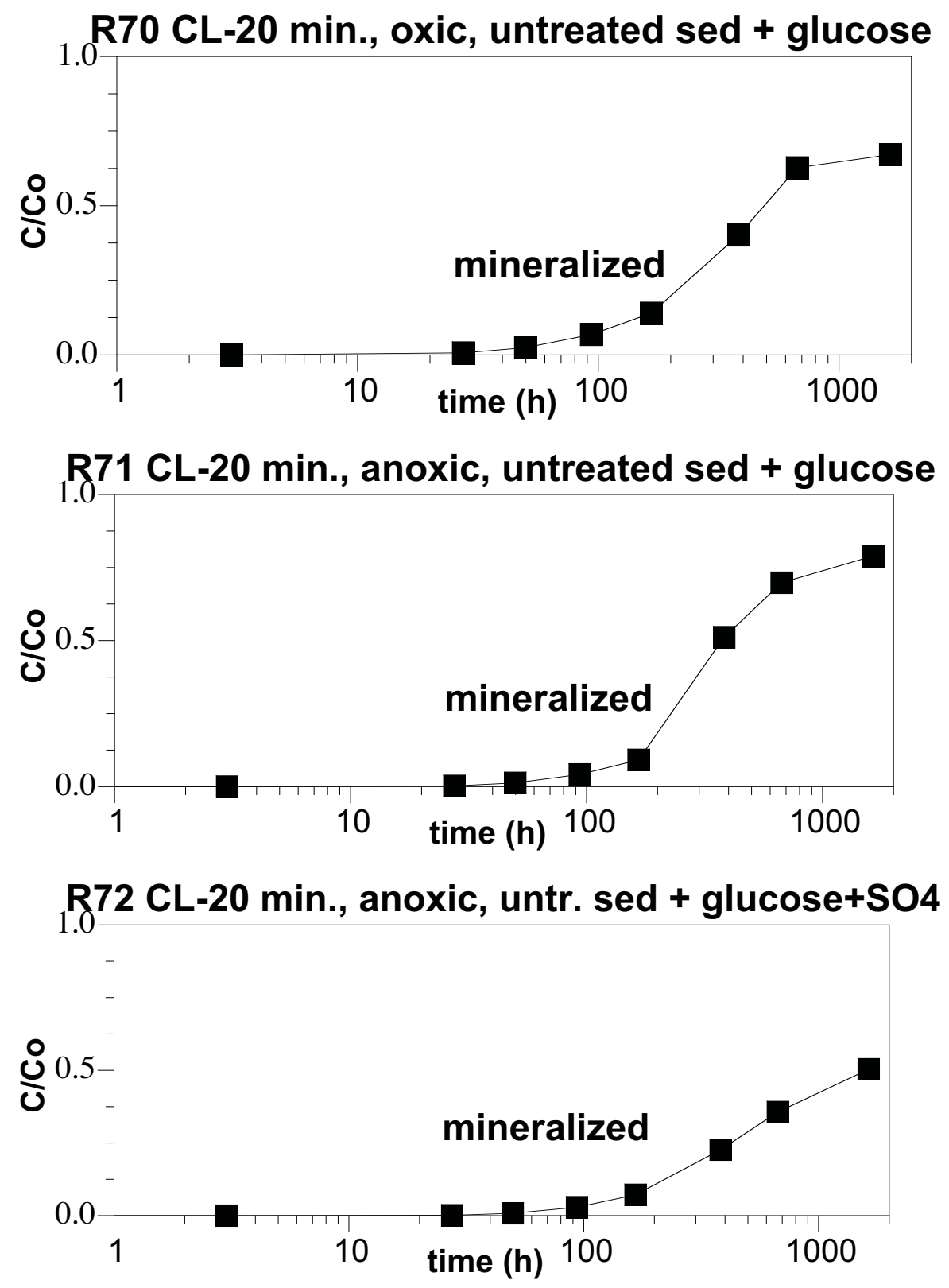

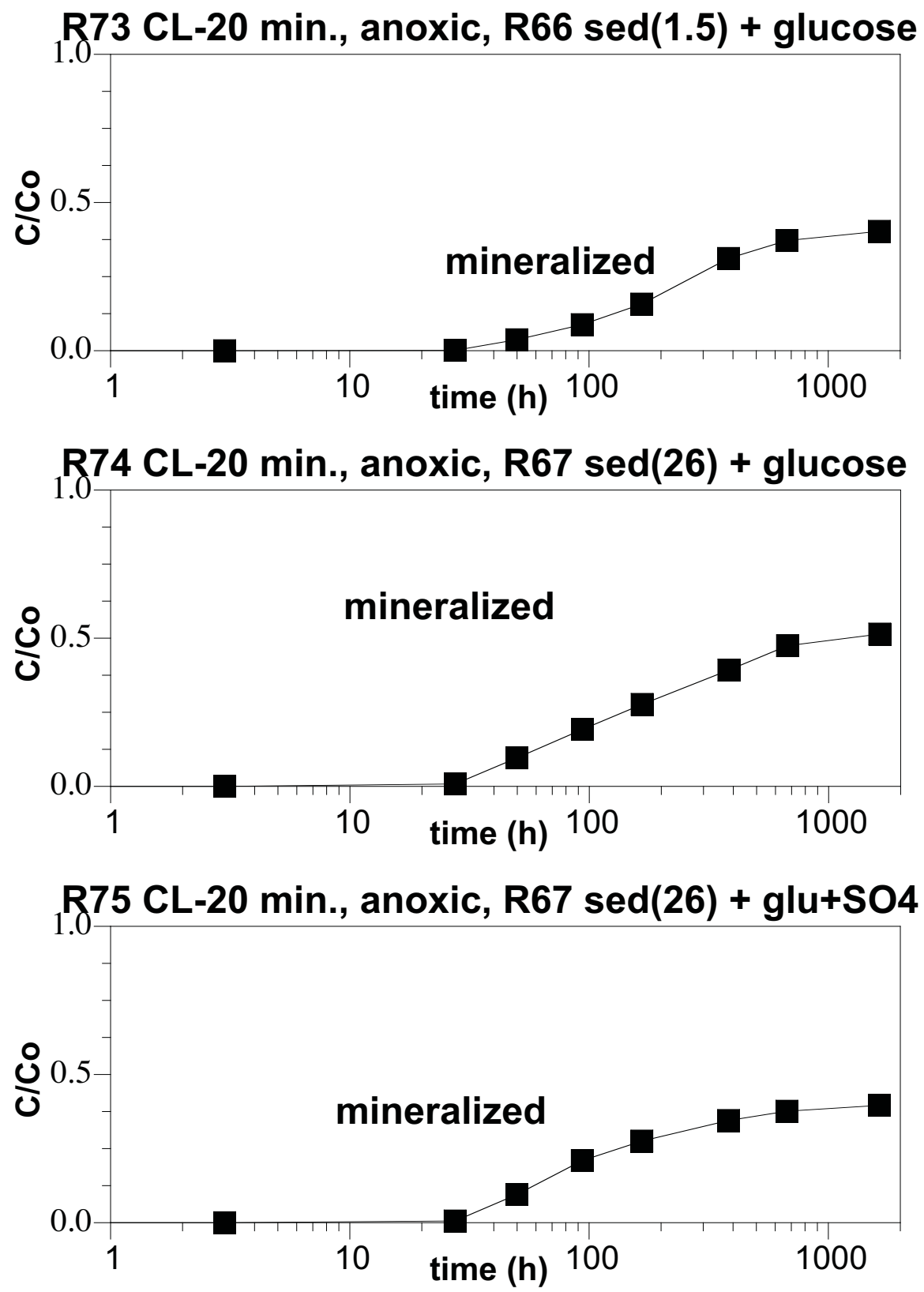
APPENDIX H. CL-20 SORPTION AND DEGRADATION DURING 1-D FLOW

Appendix H.1; CL-20 Sorption and Degradation to Oxic Norborne C 

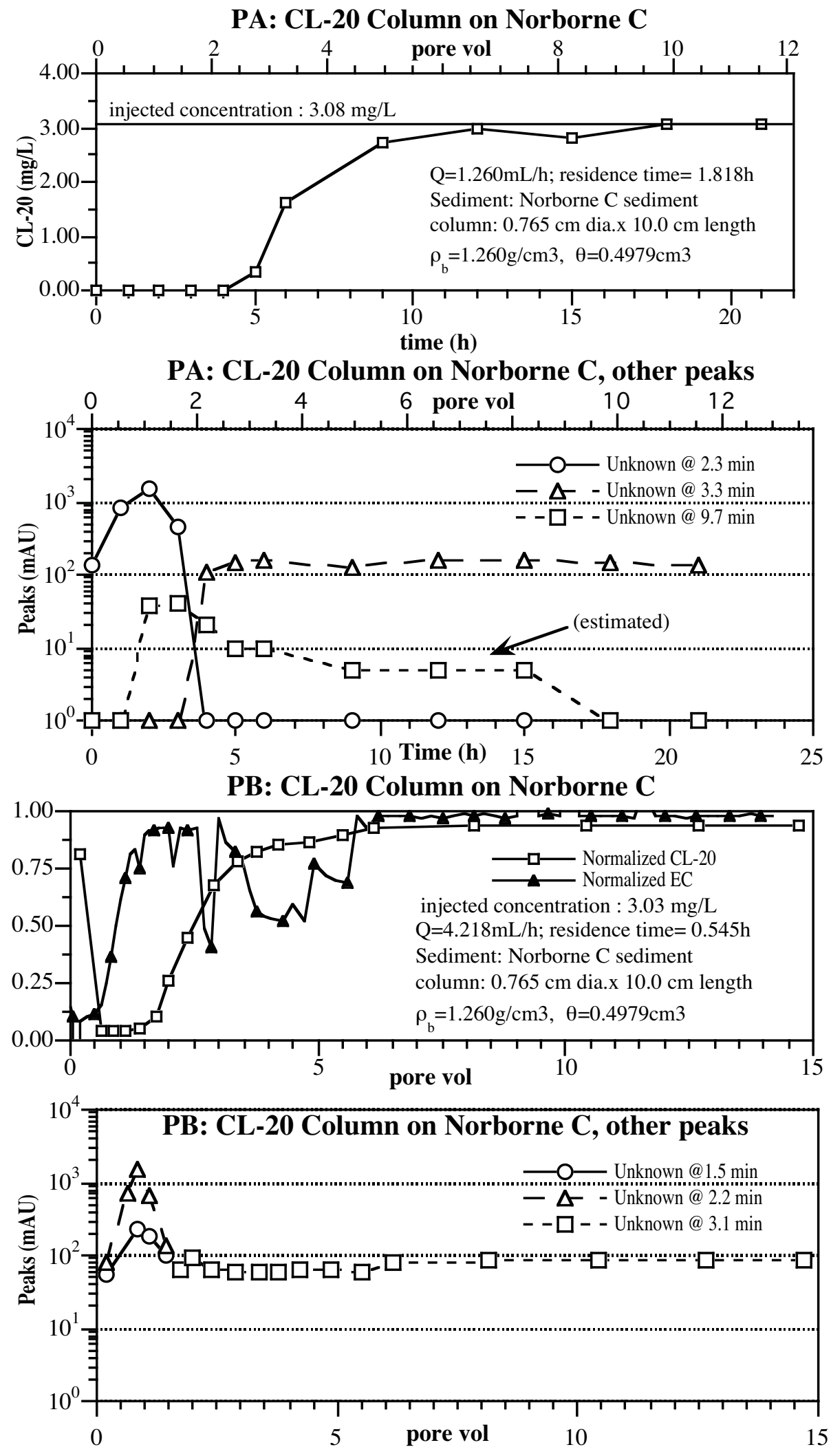


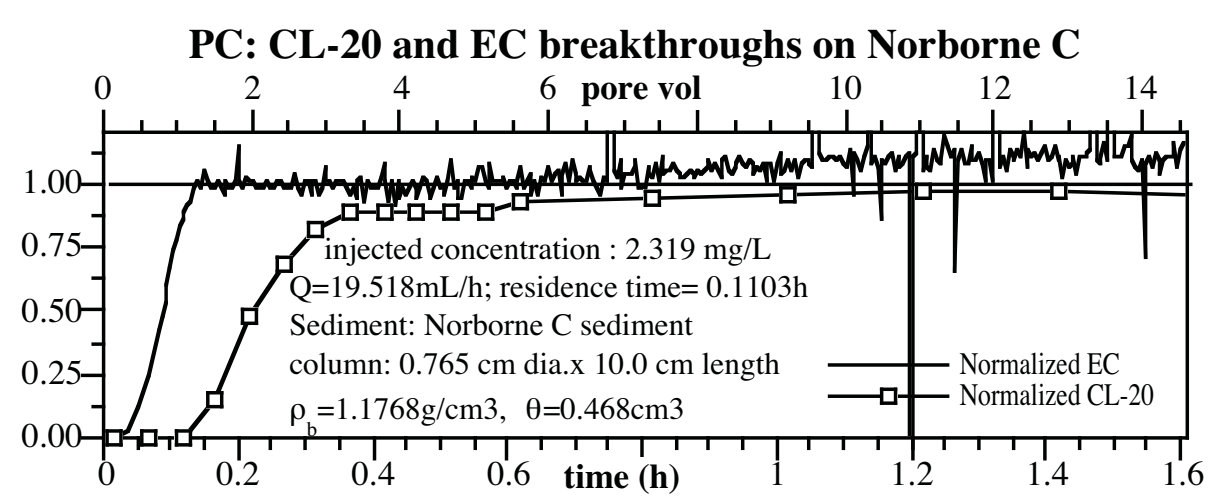

PC: CL-20 on Norborne $C$, other peaks

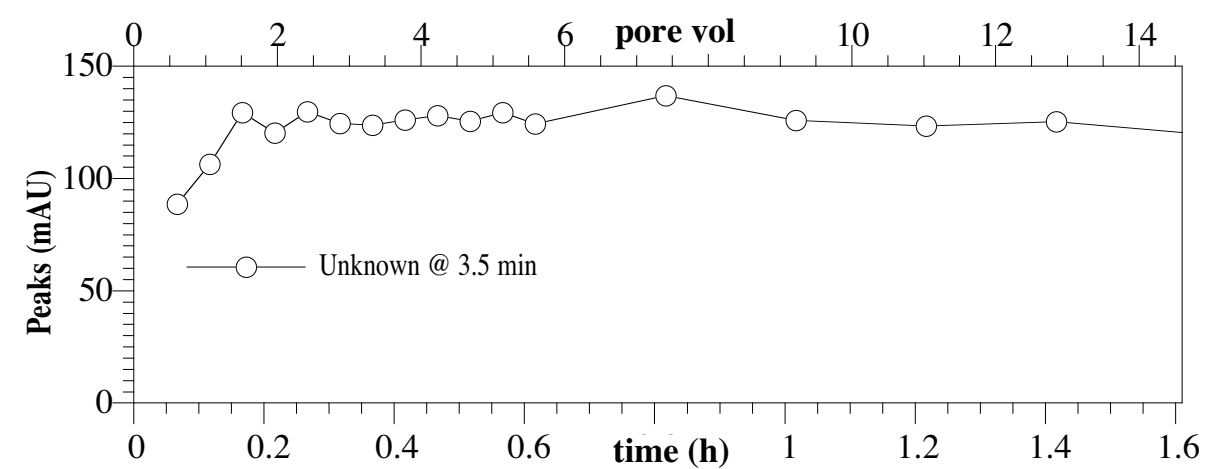

PD: CL-20 and EC breakthroughs on Norborne C

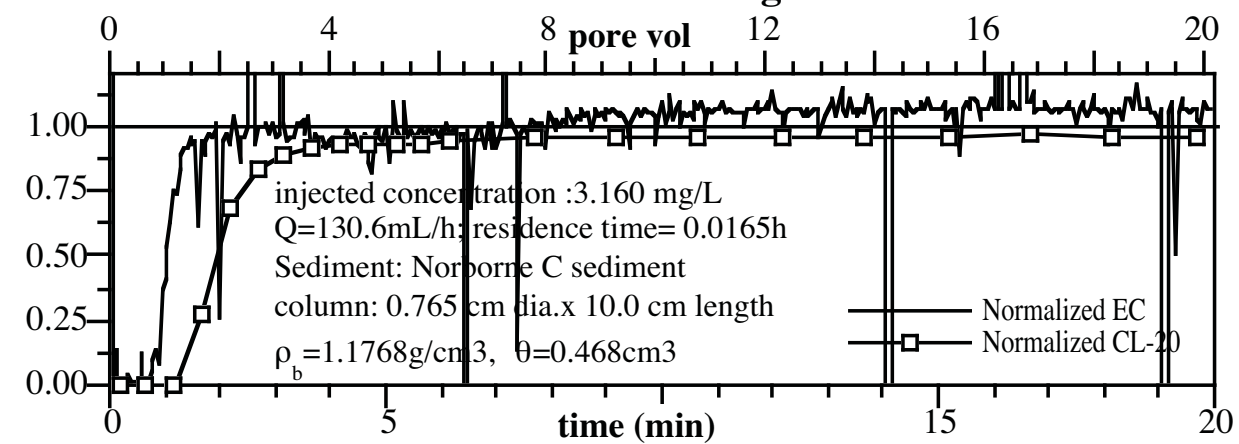

PD: CL-20 on Norborne C, other peaks

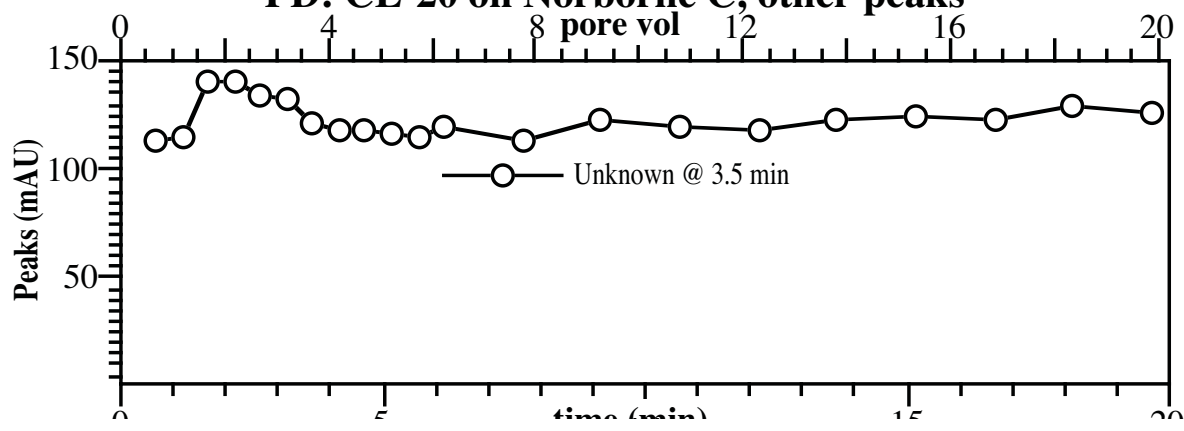




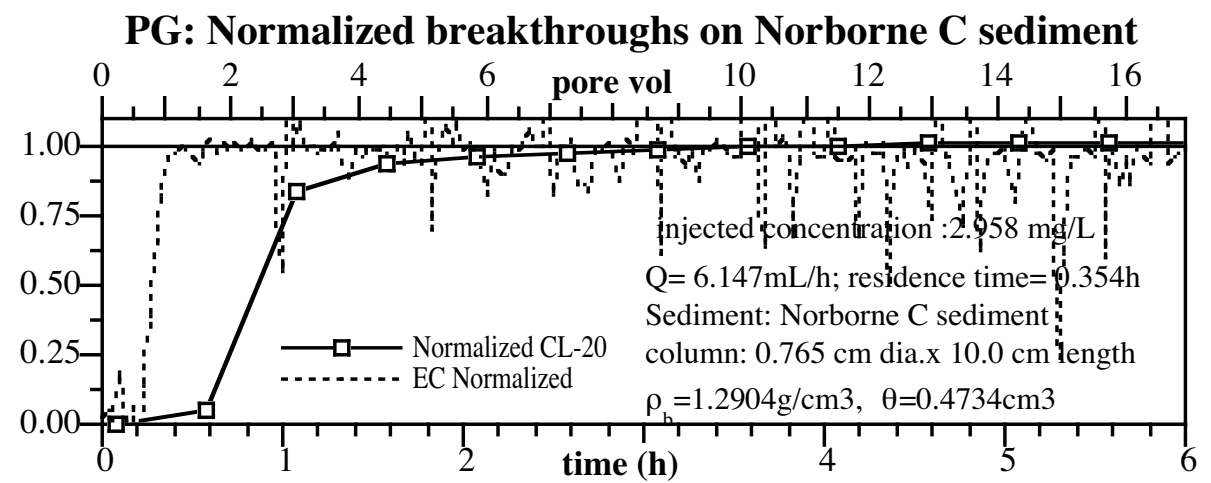

PG: CL-20 on Norborne C, other peaks

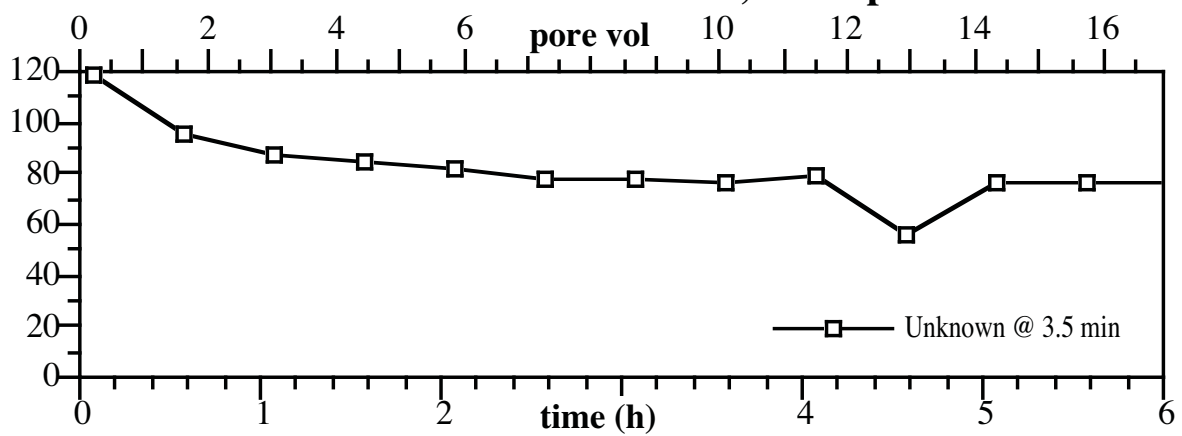

PK: CL-20 1-D Breakthough in Norborne C Sediment

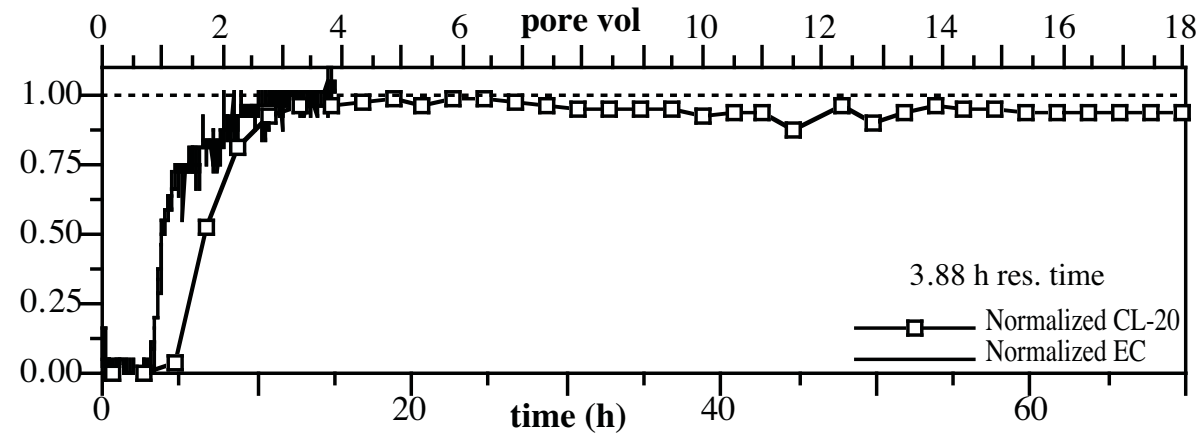


PP: CL-20 1-D Breakthrough in Norborne C Sediment

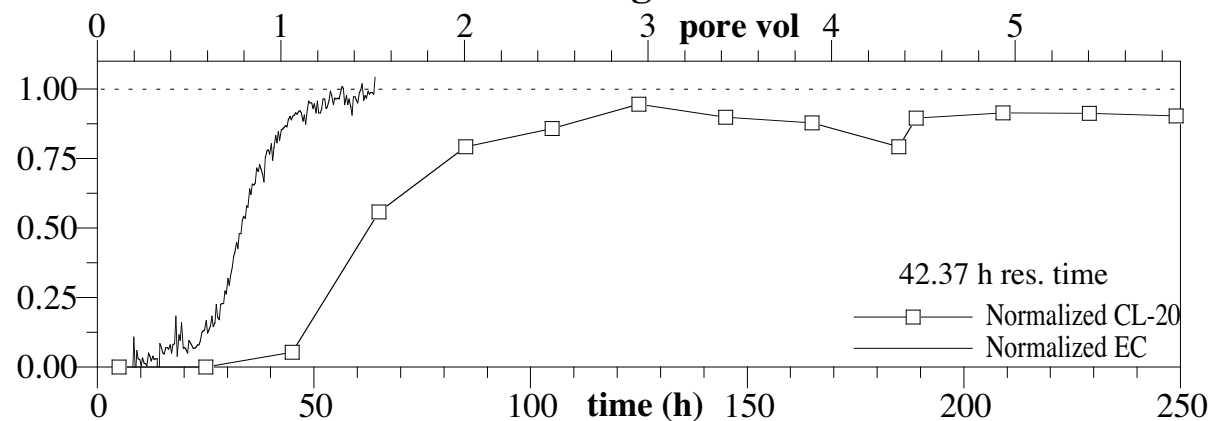

PP: CL-20 and Anions 1-D Breakthrough in Norborne C Sediment

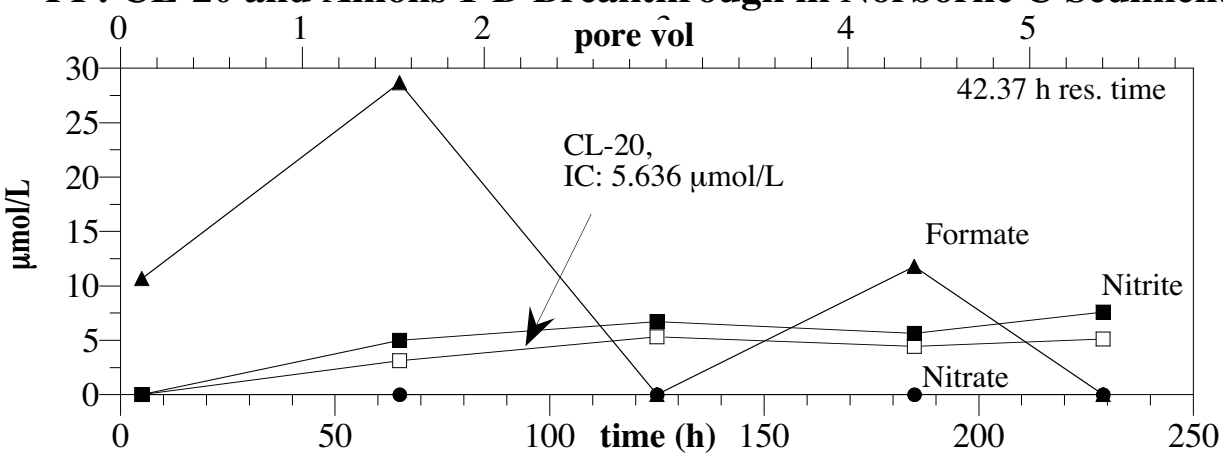

\begin{tabular}{|c|c|c|c|c|c|c|c|}
\hline $\begin{array}{l}\text { Elapsed } \\
\text { Time (h) }\end{array}$ & $\begin{array}{c}\text { CL-20 } \\
(\mu \mathrm{mol} / \mathrm{L})\end{array}$ & $\begin{array}{l}\text { Formate } \\
(\mu \mathrm{mol} / \mathrm{L})\end{array}$ & $\begin{array}{c}\text { Nitrite } \\
(\mu \mathrm{mol} / \mathrm{L})\end{array}$ & $\begin{array}{c}\text { Nitrate } \\
(\mu \mathrm{mol} / \mathrm{L})\end{array}$ & $\begin{array}{c}\text { Formate/ } \\
\text { CL-20 }\end{array}$ & $\begin{array}{c}\text { Nitrite/ } \\
\text { CL-20 }\end{array}$ & $\begin{array}{c}\text { Nitrate/ } \\
\text { CL-20 }\end{array}$ \\
\hline 5 & 0.00 & 10.67 & 1.74 & 0.00 & 1.89 & 0.31 & 0.00 \\
\hline 65 & 3.13 & 28.67 & 5.00 & 0.00 & 5.09 & 0.89 & 0.00 \\
\hline 125 & 5.32 & 2.51 & 6.74 & 0.00 & 0.45 & 1.20 & 0.00 \\
\hline 185 & 4.46 & 11.78 & 5.65 & 0.00 & 2.09 & 1.00 & 0.00 \\
\hline 229 & 5.13 & 2.51 & 7.61 & 0.00 & 0.45 & 1.35 & 0.00 \\
\hline
\end{tabular}


PZZO: Norm. 1-D Breakthroughs on Norborne $C$ sed.

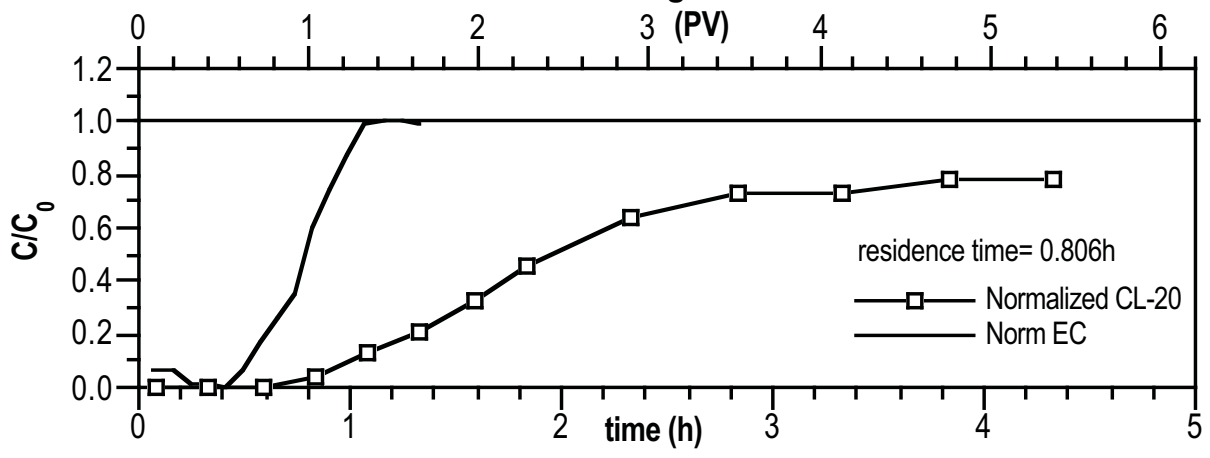

\begin{tabular}{|c|c|c|c|c|}
\hline & $\begin{array}{c}\text { Aqueous } \\
\text { CL-20 } \\
\text { Inject }\end{array}$ & $\begin{array}{c}\text { Aqueous } \\
\text { CL-20 } \\
\text { Effluent }\end{array}$ & $\begin{array}{c}\text { CL-20 } \\
\text { Sorbed }\end{array}$ & $\begin{array}{c}\text { CL-20 } \\
\text { Degraded }\end{array}$ \\
\hline$\mu \mathrm{mol}$ & 0.0567 & 0.0322 & 0.0208 & 0.0038 \\
\hline fraction $\mathbf{C}_{\mathbf{0}}$ & $\mathrm{N} / \mathrm{A}$ & 0.568 & 0.366 & 0.066 \\
\hline
\end{tabular}

PZZP: Norm. 1-D Breakthroughs on $\mathrm{MeOH}$ treated Norborne $\mathrm{C}$ sed.

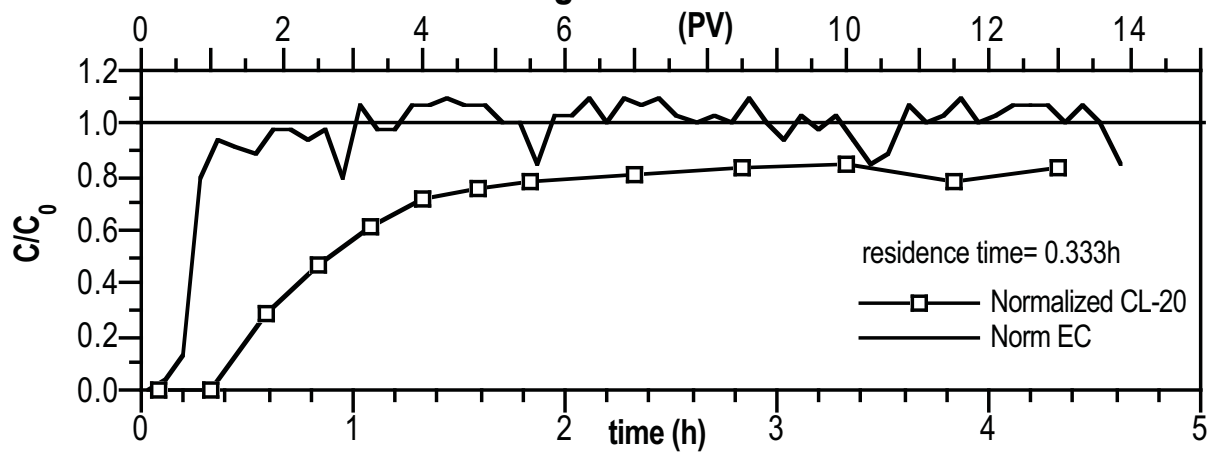

\begin{tabular}{|c|c|c|c|c|}
\hline & $\begin{array}{c}\text { Aqueous } \\
\text { CL-20 } \\
\text { Inject }\end{array}$ & $\begin{array}{c}\text { Aqueous } \\
\text { CL-20 } \\
\text { Effluent }\end{array}$ & $\begin{array}{c}\text { CL-20 } \\
\text { Sorbed }\end{array}$ & $\begin{array}{c}\text { CL-20 } \\
\text { Degraded }\end{array}$ \\
\hline$\mu \mathrm{mol}$ & 0.1243 & 0.0818 & 0.0263 & 0.0162 \\
\hline fraction $\mathbf{C}_{\mathbf{0}}$ & $\mathrm{N} / \mathrm{A}$ & 0.658 & 0.212 & 0.130 \\
\hline
\end{tabular}


Appendix H.2; CL-20 Sorption and Degradation to Reduced Norborne C
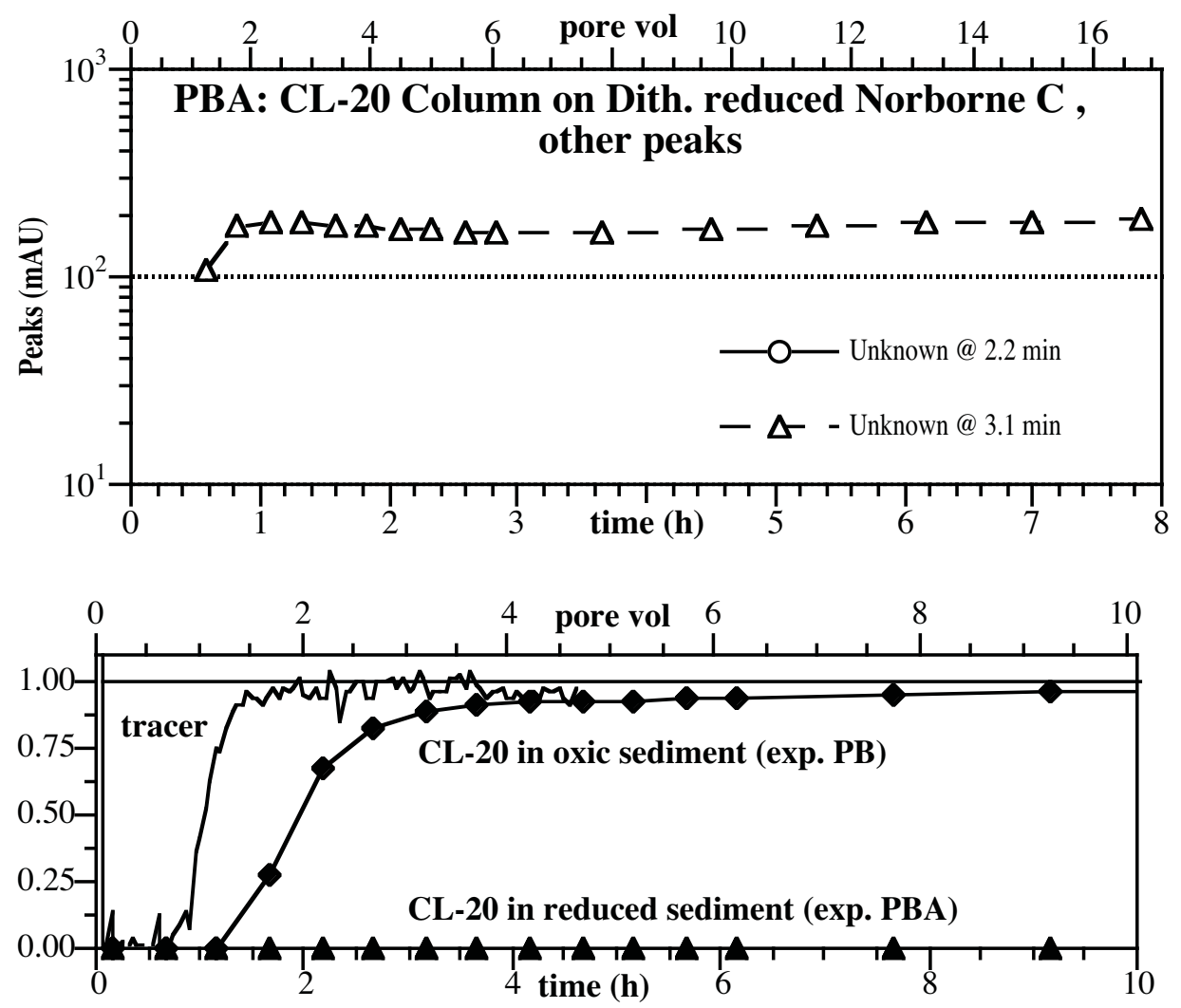


\section{Appendix H.3; CL-20 Sorption and Degradation to Westmoreland A1}

PH: Normalized breakthoughs on Westmoreland A1 sediment

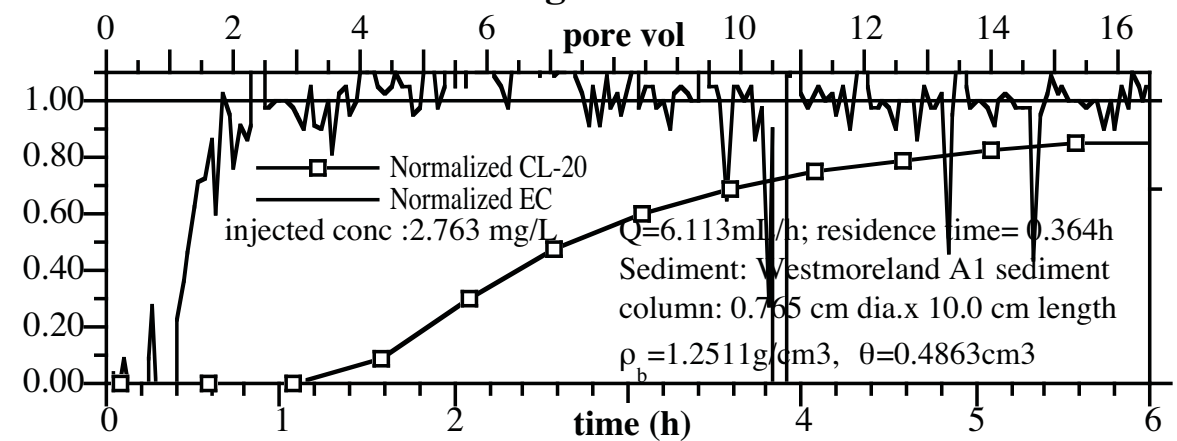

PH: CL-20 on Westmoreland A1, other peaks

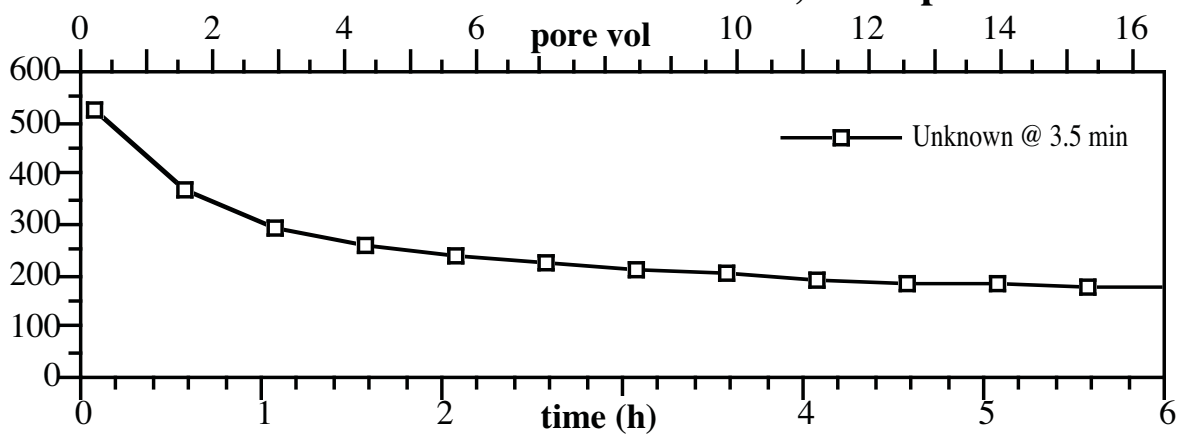

PL: CL-20 1-D Breakthrough in Wesmoreland A1 Sediment

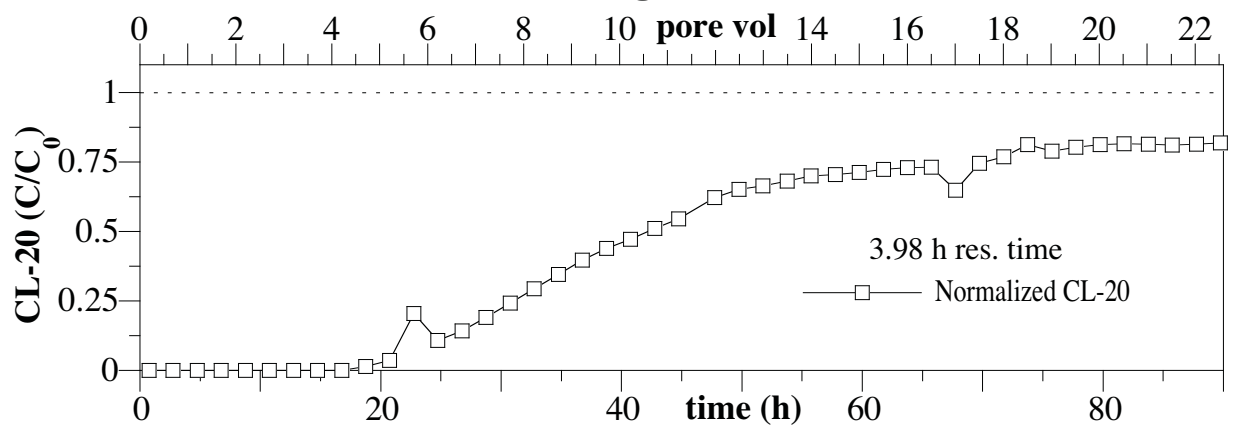

PL: CL-20 on Westmoreland A1; Other Peaks

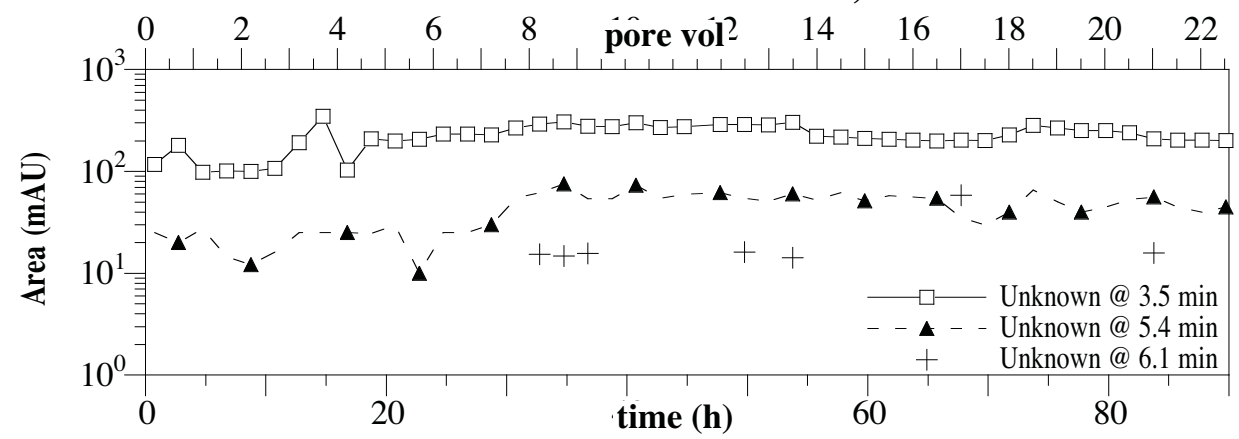


PQ: CL-20 1-D Breakthrough in Westmoreland A1 Sediment

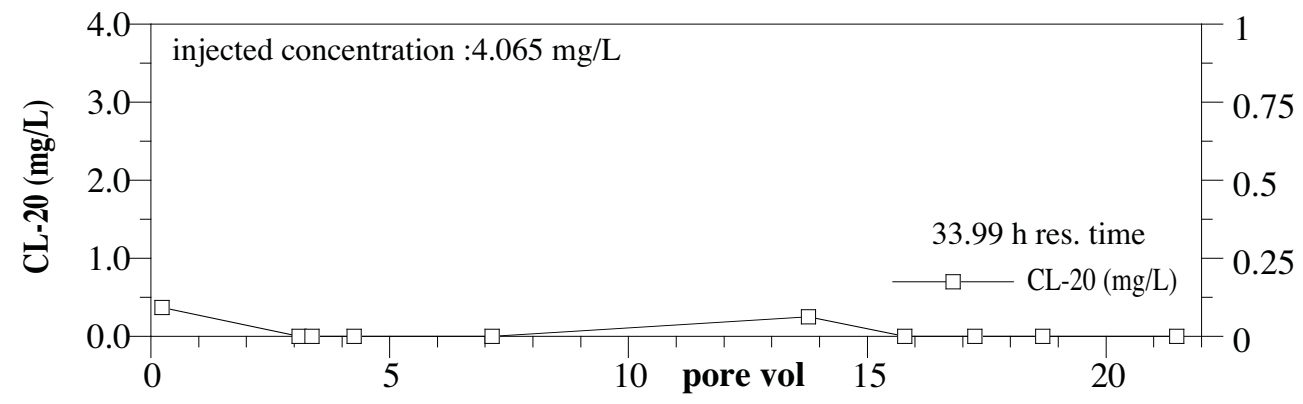

PQ: CL-20 and Anions 1-D Breakthrough in Westmoreland A1 Sed.

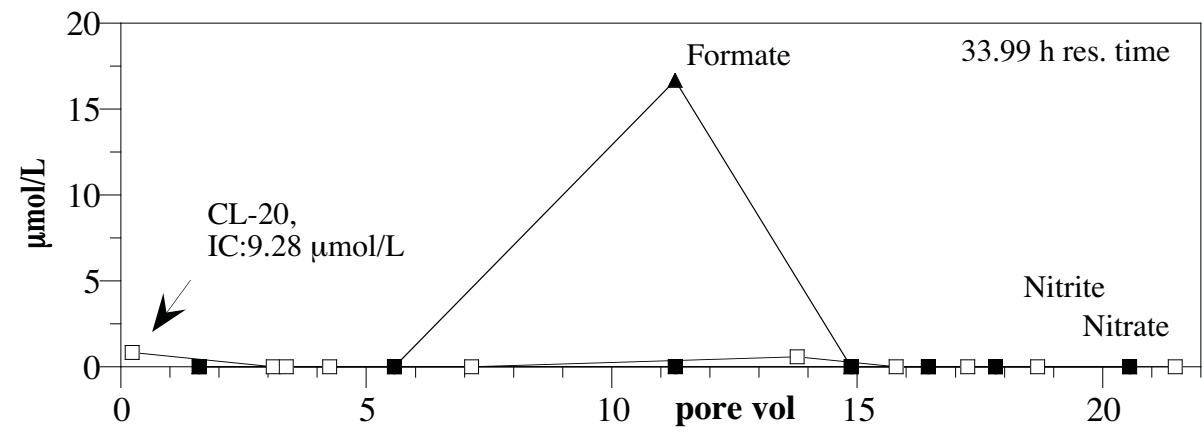

\begin{tabular}{|c|c|c|c|c|c|c|c|}
\hline $\begin{array}{c}\text { Elapsed } \\
\text { PV }\end{array}$ & $\begin{array}{c}\mathbf{C L}-20 \\
(\mu \mathrm{mol} / \mathrm{L})\end{array}$ & $\begin{array}{l}\text { Formate } \\
(\mu \mathrm{mol} / \mathrm{L})\end{array}$ & $\begin{array}{c}\text { Nitrite } \\
(\mu \mathrm{mol} / \mathrm{L})\end{array}$ & $\begin{array}{c}\text { Nitrate } \\
(\mu \mathrm{mol} / \mathrm{L})\end{array}$ & $\begin{array}{c}\text { Formate/ } \\
\text { CL-20 }\end{array}$ & $\begin{array}{c}\text { Nitrite/ } \\
\text { CL-20 } \\
\end{array}$ & $\begin{array}{c}\text { Nitrate/ } \\
\text { CL-20 }\end{array}$ \\
\hline 1.59 & 0.85 & 0.00 & 0.00 & 0.00 & 0.00 & 0.00 & 0.00 \\
\hline 5.57 & 0.00 & 0.00 & 0.00 & 0.00 & 0.00 & 0.00 & 0.00 \\
\hline 11.29 & 0.00 & 16.67 & 0.00 & 0.00 & 1.80 & 0.00 & 0.00 \\
\hline 14.87 & 0.58 & 0.00 & 0.00 & 0.00 & 0.00 & 0.00 & 0.00 \\
\hline 16.45 & 0.00 & 0.00 & 0.00 & 0.00 & 0.00 & 0.00 & 0.00 \\
\hline 17.82 & 0.00 & 0.00 & 0.00 & 0.00 & 0.00 & 0.00 & 0.00 \\
\hline 20.55 & 0.00 & 0.00 & 0.00 & 0.00 & 0.00 & 0.00 & 0.00 \\
\hline
\end{tabular}


Appendix H.4; CL-20 Sorption and Degradation to Burbank AP

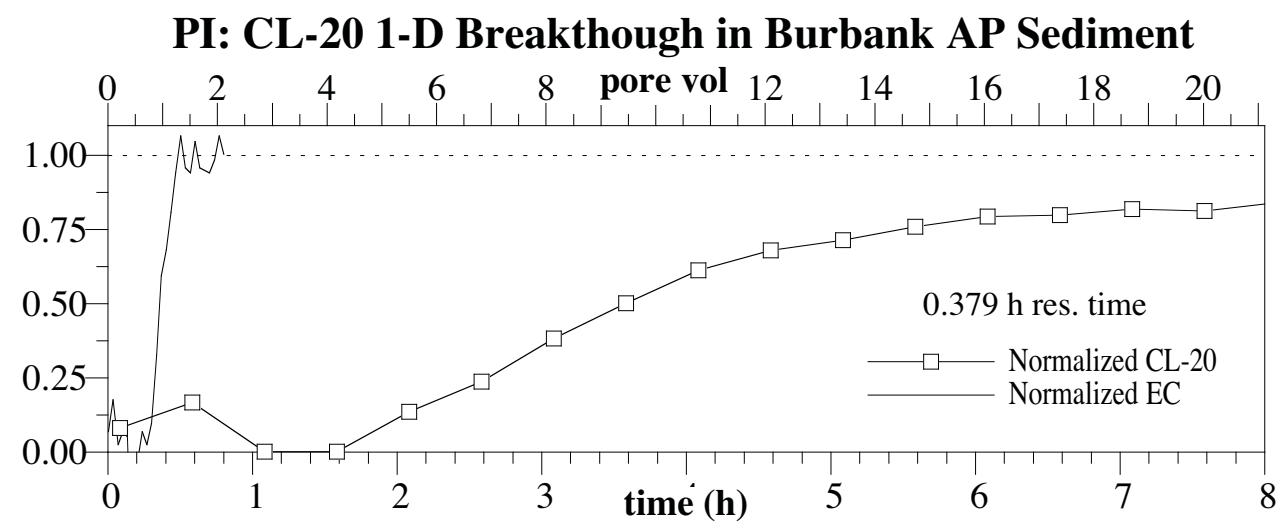

PI: CL-20 on Burbank AP, other peaks

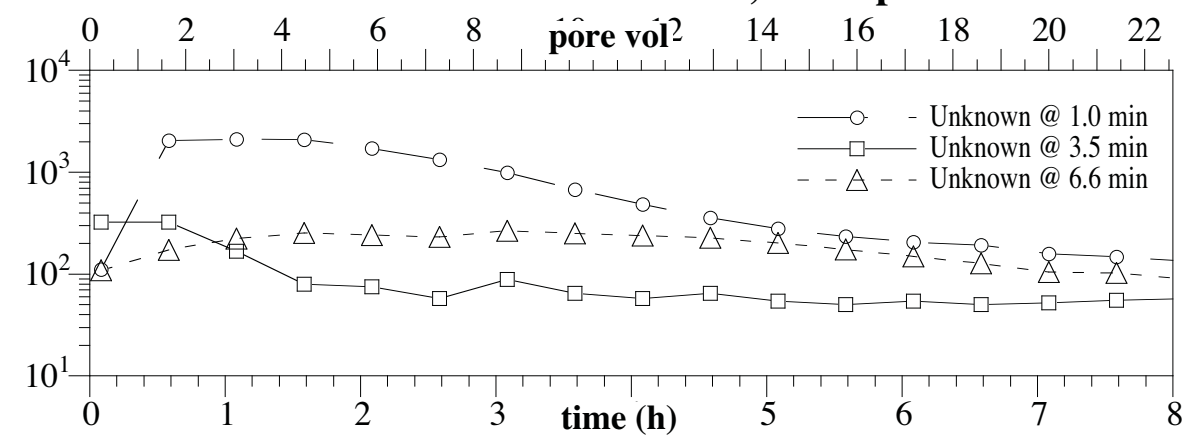

PM: CL-20 1-D Breakthrough in Burbank AP Sediment

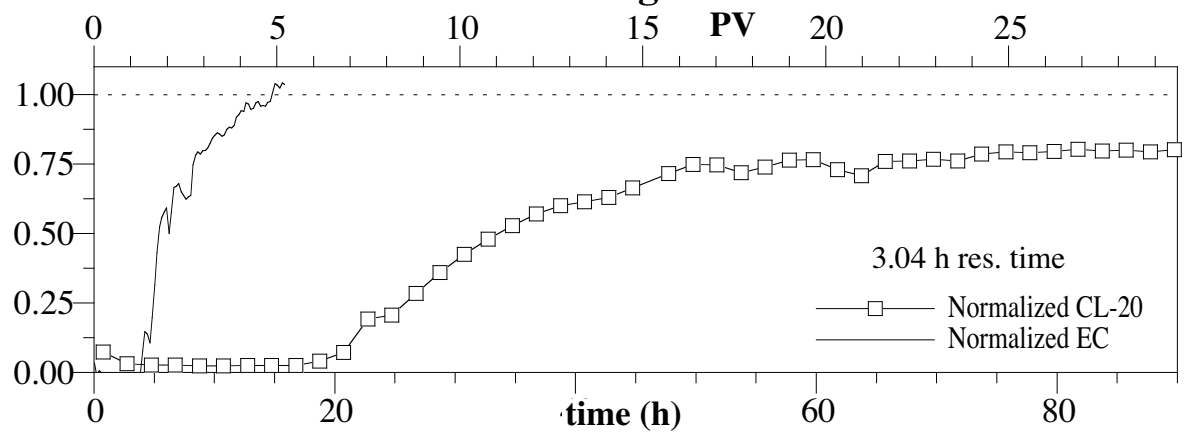

PM: CL-20 on Burbank AP, other peaks

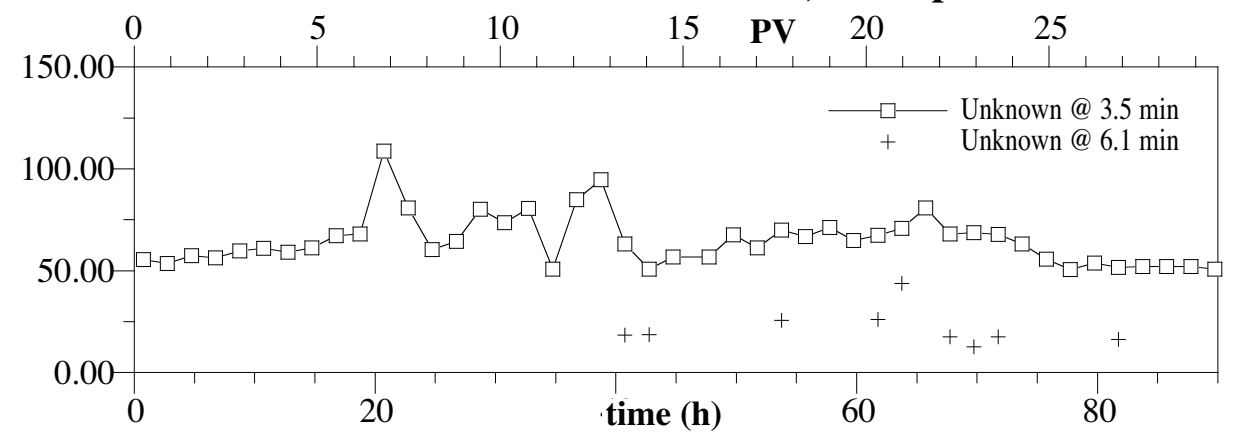


PR: CL-20 1-D Breakthrough in Burbank AP Sediment

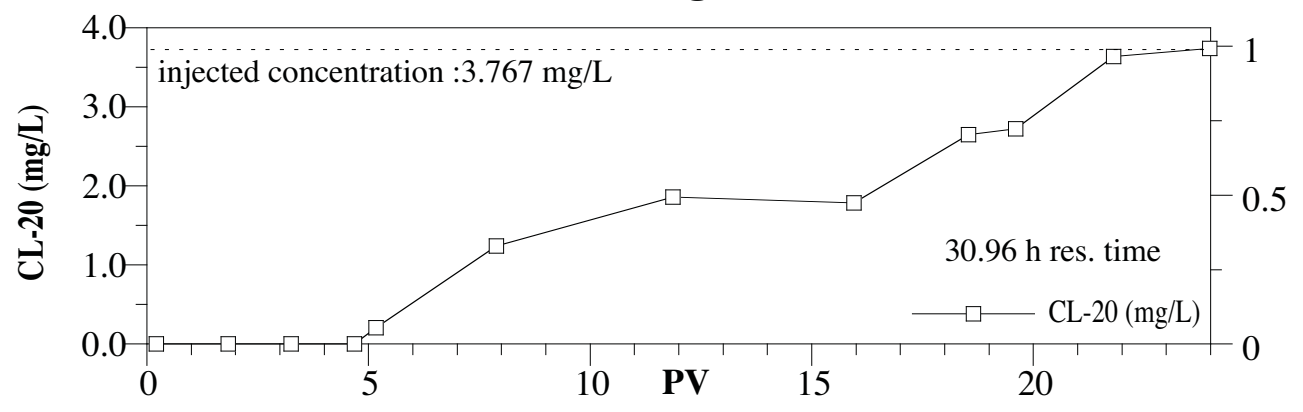

PR: CL-20 and Anions 1-D Breakthrough in Burbank AP Sediment

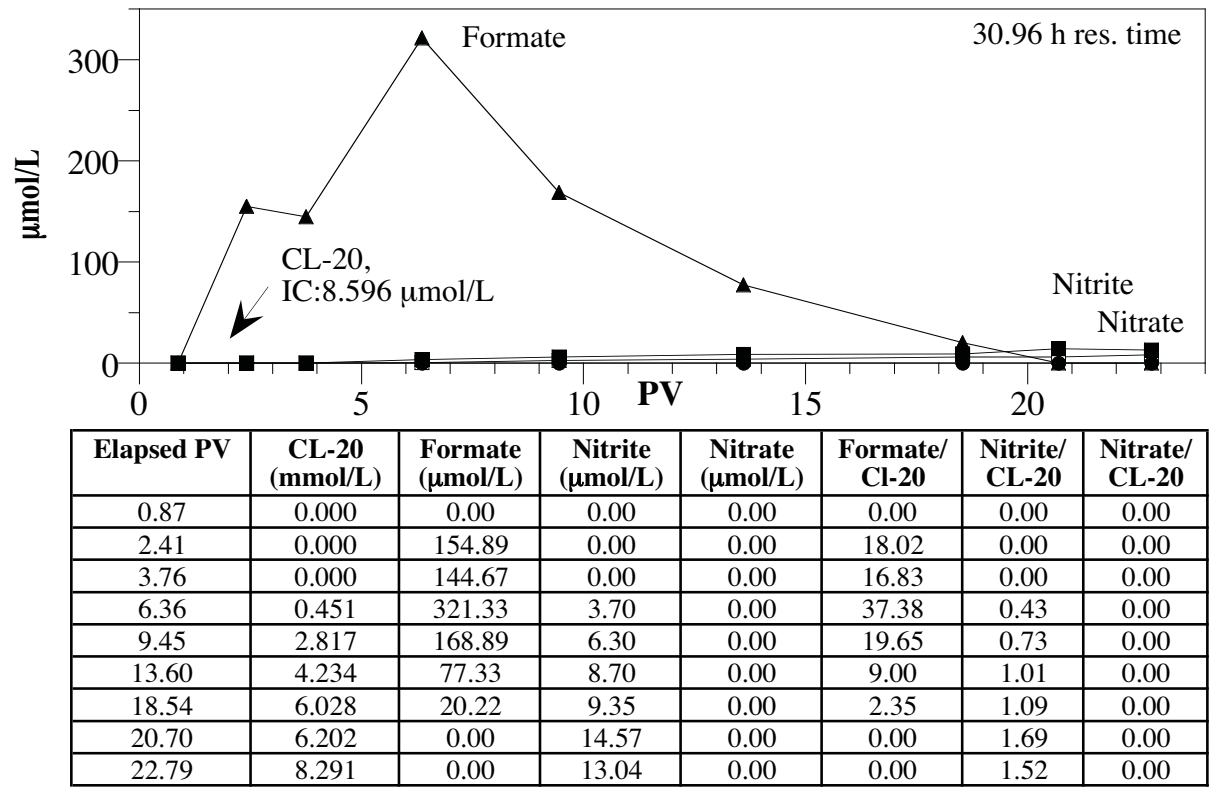


Appendix H.5; CL-20 Sorption and Desorption to Other Sediments and Natural Minerals

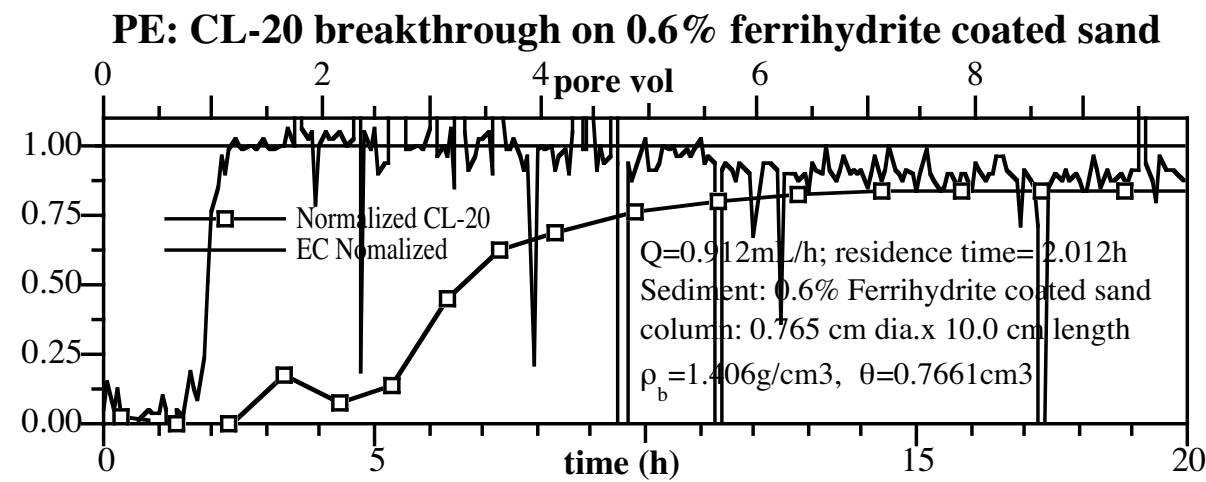

PE: CL-20 on 0.6\% ferrihydrite coated sand, other peaks

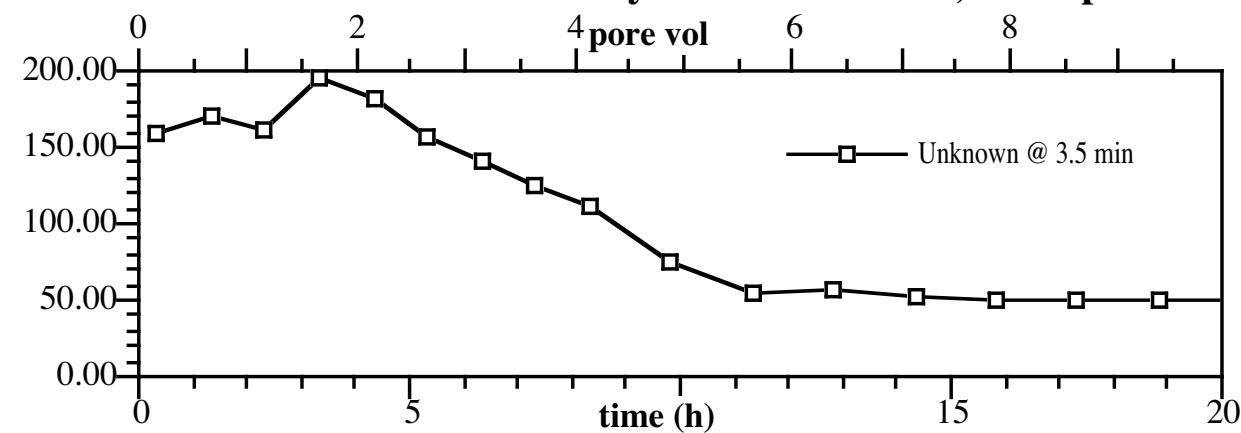

PF: CL-20 breakthrough on $0.6 \%$ ferrihydrite coated sand

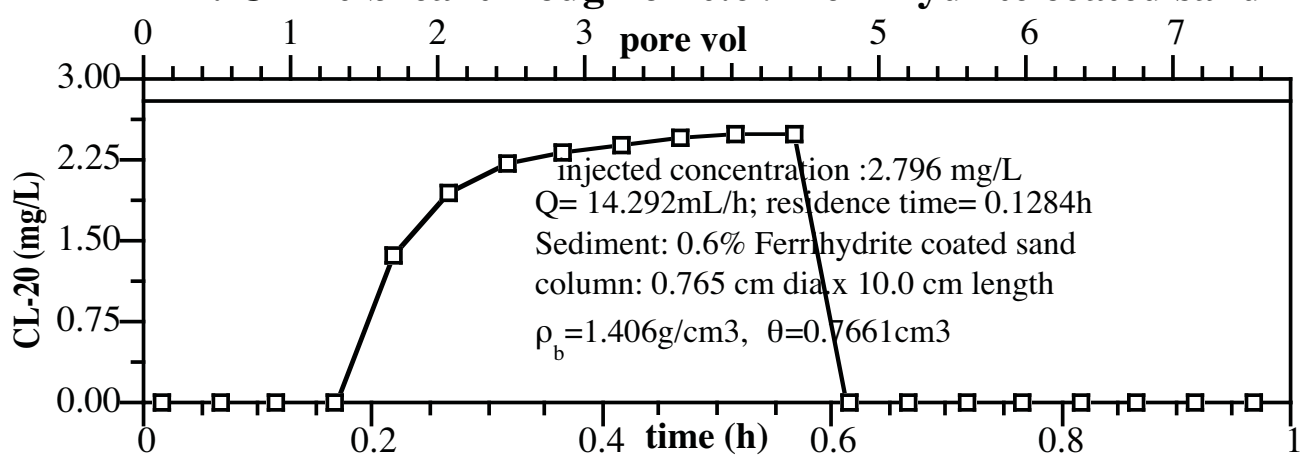

PF: CL-20 on $0.6 \%$ ferrihydrite coated sand, other peaks

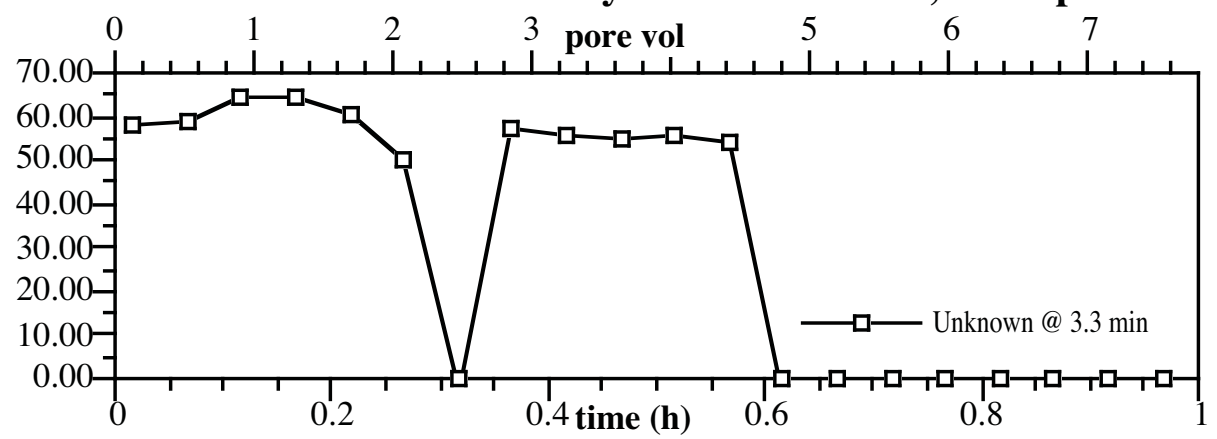




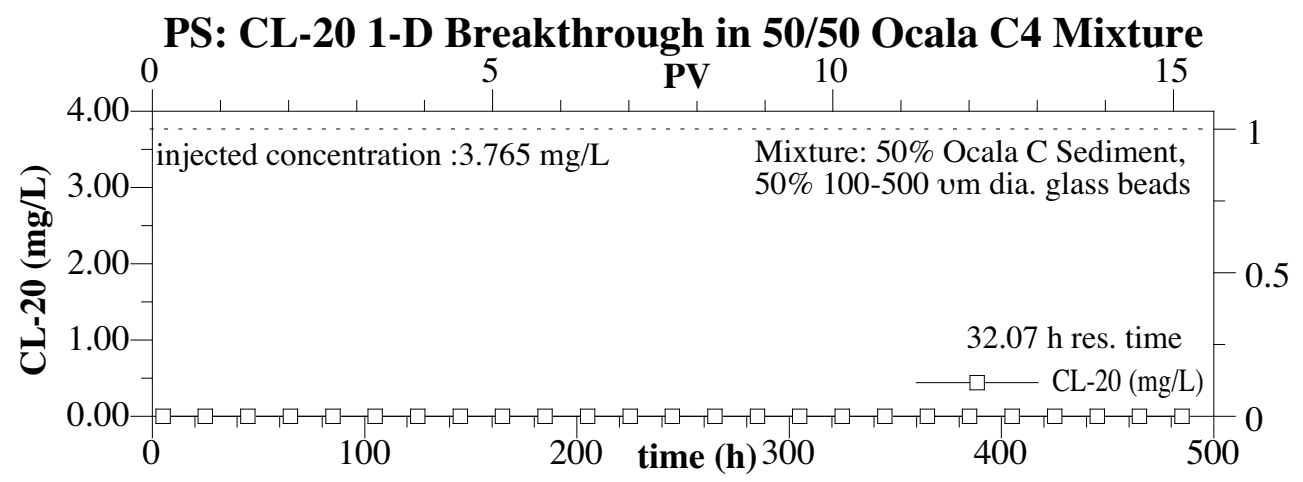

PS: CL-20 and Anions 1-D Breakthrough in 50/50 Ocala C Mixture

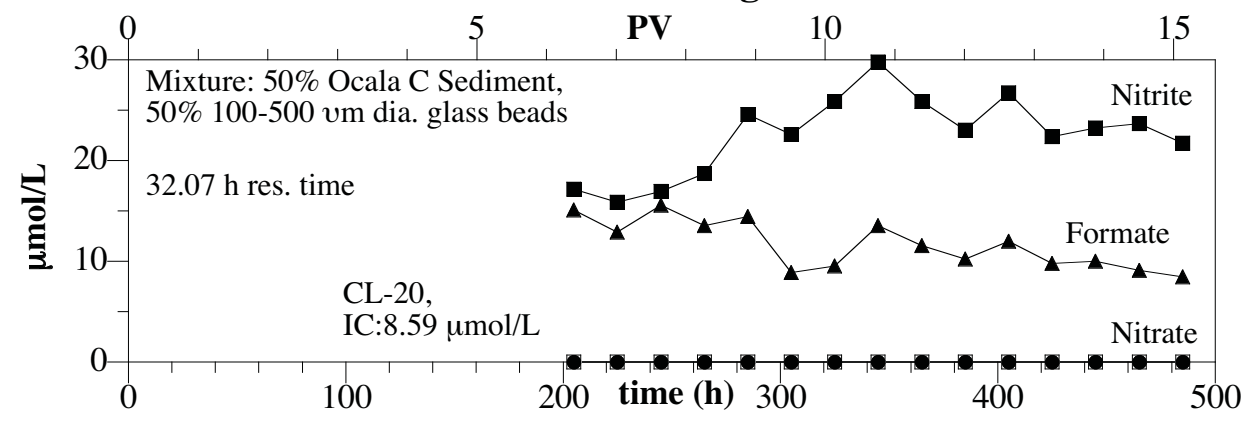

\begin{tabular}{|c|c|c|c|c|c|c|c|}
\hline $\begin{array}{c}\text { Elapsed } \\
\text { Time }(\mathbf{h})\end{array}$ & $\begin{array}{c}\mathbf{C L}-20 \\
(\boldsymbol{\mu m o l} / \mathbf{L})\end{array}$ & $\begin{array}{c}\text { Formate } \\
(\boldsymbol{\mu} \mathbf{m o l} / \mathbf{L})\end{array}$ & $\begin{array}{c}\text { Nitrite } \\
(\boldsymbol{\mu} \mathbf{m o l} / \mathbf{L})\end{array}$ & $\begin{array}{c}\text { Nitrate } \\
(\boldsymbol{\mu} \mathbf{m o l} / \mathbf{L})\end{array}$ & $\begin{array}{c}\text { Formate/ } \\
\mathbf{C L}-20\end{array}$ & $\begin{array}{c}\text { Nitrite/ } \\
\mathbf{C L}-20\end{array}$ & $\begin{array}{c}\text { Nitrate/ } \\
\mathbf{C L}-20\end{array}$ \\
\hline 205 & 0.00 & 15.11 & 17.17 & 0.00 & 1.76 & 2.00 & 0.00 \\
\hline 265 & 0.00 & 13.56 & 18.70 & 0.00 & 1.58 & 2.18 & 0.00 \\
\hline 285 & 0.00 & 14.44 & 24.57 & 0.00 & 1.68 & 2.86 & 0.00 \\
\hline 325 & 0.00 & 9.56 & 25.87 & 0.00 & 1.11 & 3.01 & 0.00 \\
\hline 345 & 0.00 & 13.56 & 29.78 & 0.00 & 1.58 & 3.47 & 0.00 \\
\hline 385 & 0.00 & 10.22 & 23.04 & 0.00 & 1.19 & 2.68 & 0.00 \\
\hline 405 & 0.00 & 12.00 & 26.74 & 0.00 & 1.40 & 3.11 & 0.00 \\
\hline 445 & 0.00 & 10.00 & 23.26 & 0.00 & 1.16 & 2.71 & 0.00 \\
\hline 485 & 0.00 & 8.44 & 21.74 & 0.00 & 0.98 & 2.53 & 0.00 \\
\hline
\end{tabular}

PZC: CL-20 1-D Breakthrough in 50/50 Ocala Mixture

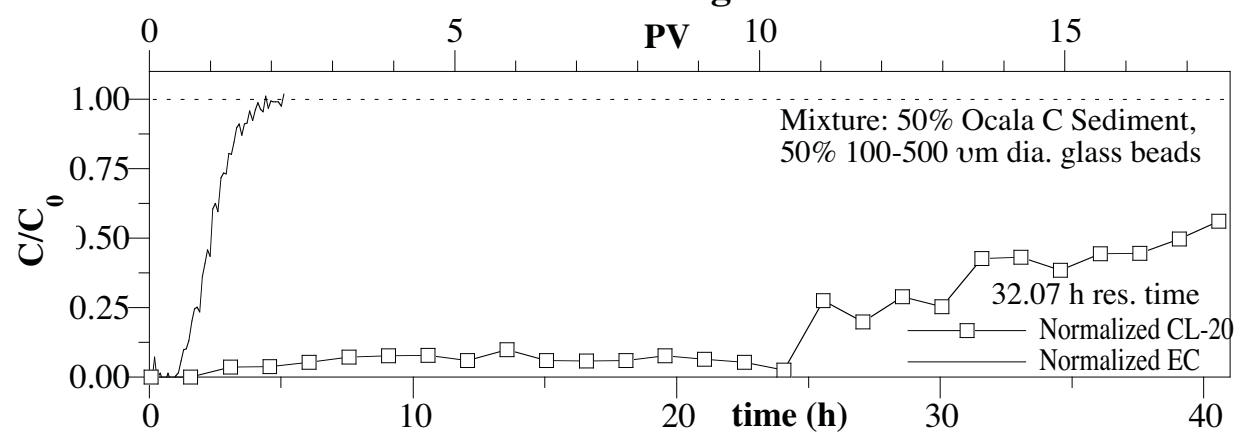


PU: CL-20 1-D Breakthough in Sassafrass Sediment

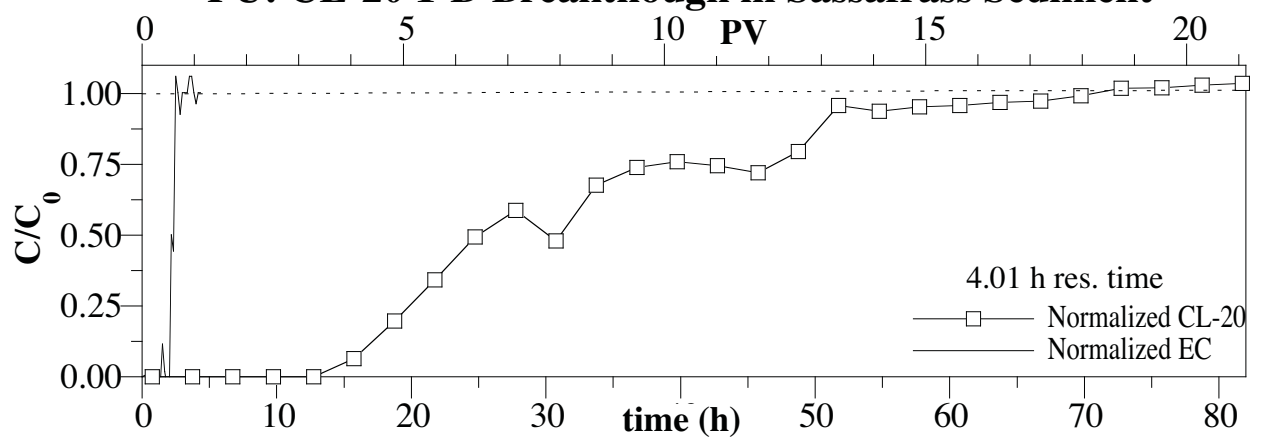

PV: CL-20 1-D Brakthrough in China Lake Sed. Site 7, 15' bgs

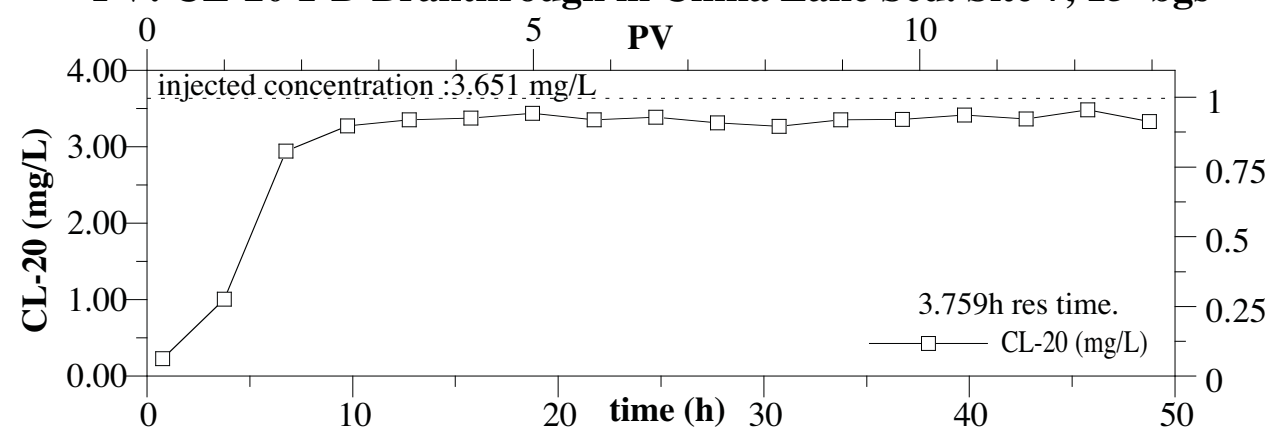

PW: CL-20 1-D Breakthrough in China Lake Sed. 55' bgs

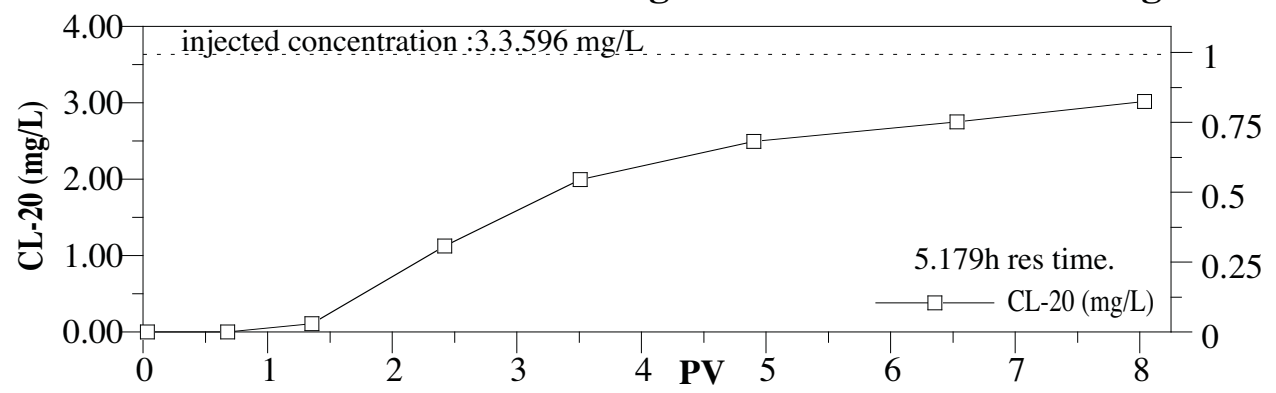

PX: CL-20 1-D Breakthrough in China Lake Sed. Site 8, wash

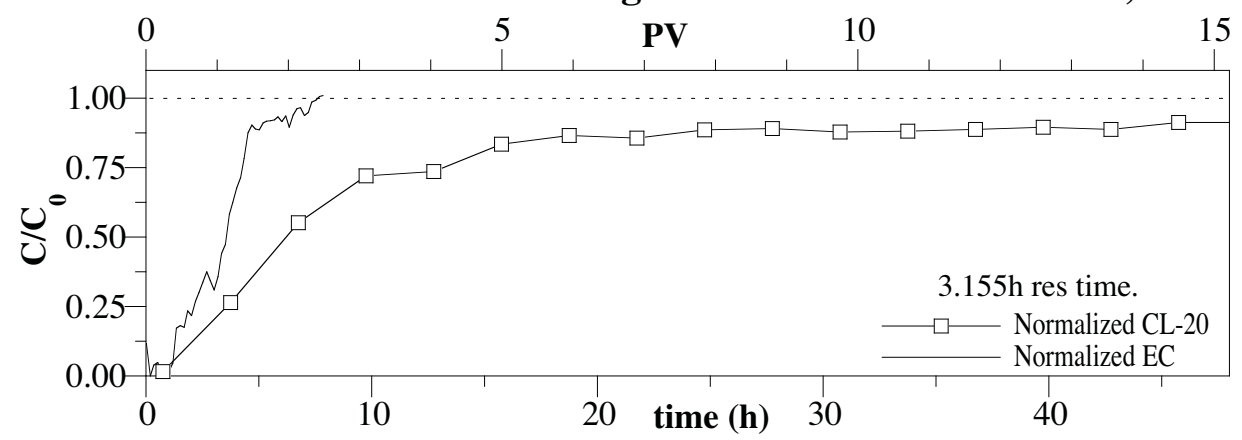




\section{ON OXIC SEDIMENTS}

Appendix I.1; CL-20 and RDX Degradation and Sorption/Degradation on Westmoreland A1

QD: CL-20 and RDX 1-D Transport with 1h Aging on Westmoreland A1

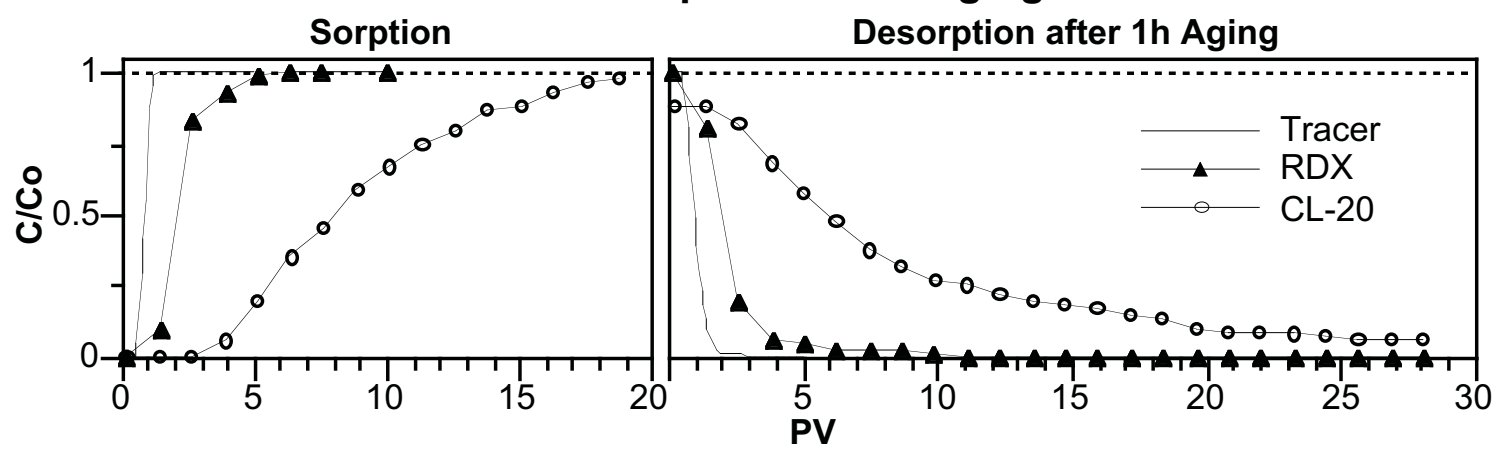

QE: CL-20 and RDX 1-D Transport with 1h Aging on Westmoreland A1

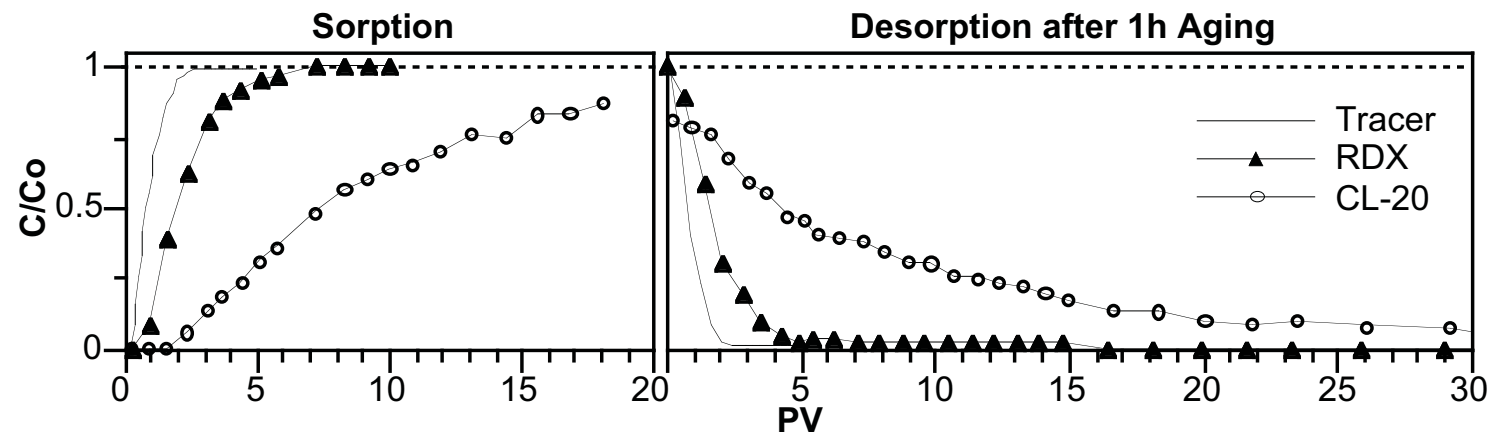

QG: CL-20 and RDX 1-D Transport with 112h Aging on Westmoreland A1

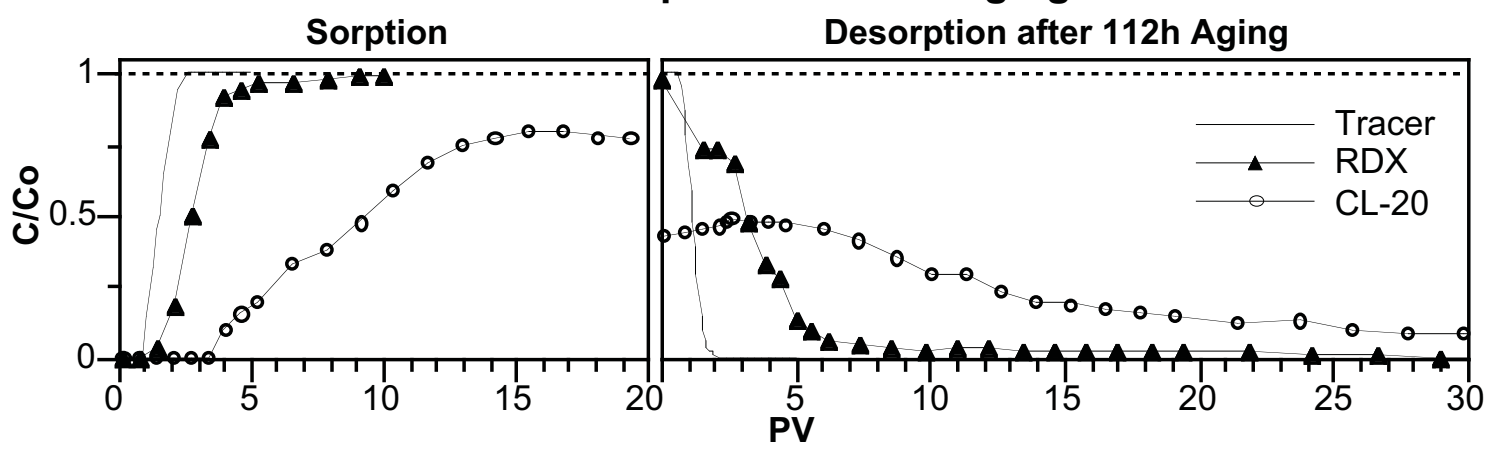

QI: CL-20 and RDX 1-D Transport with 737h Aging on Westmoreland A1

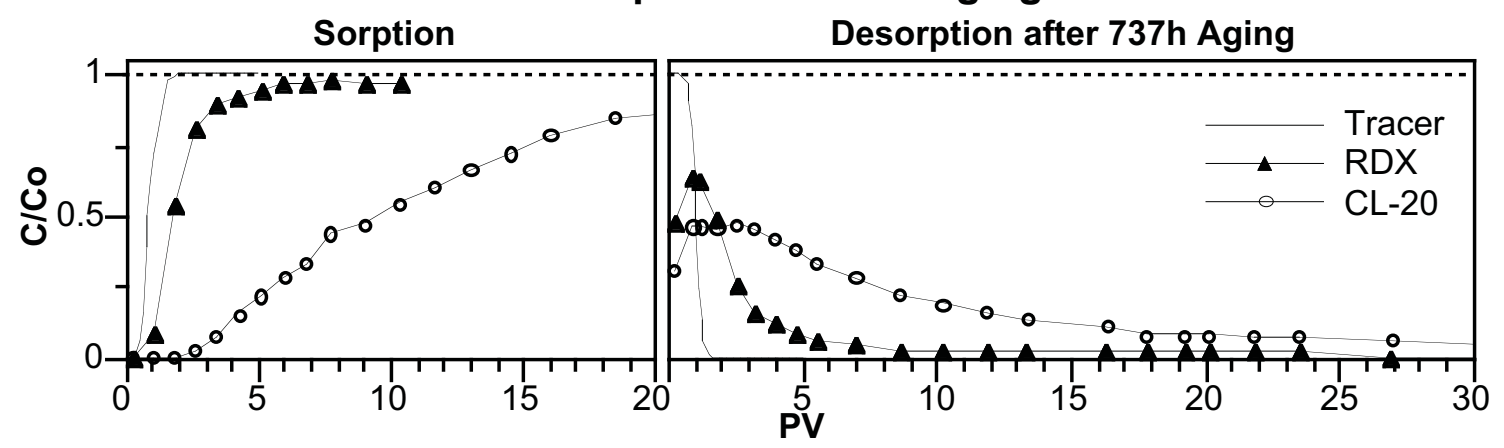


QK: CL-20 and RDX 1-D Transport with 2174h Aging on Westmoreland A1

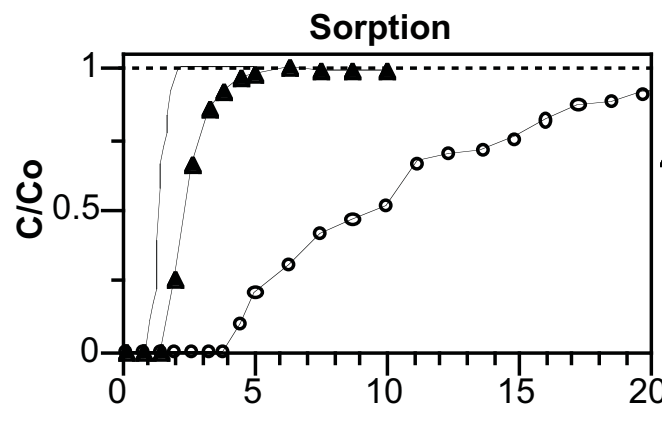

Desorption after 2174h Aging

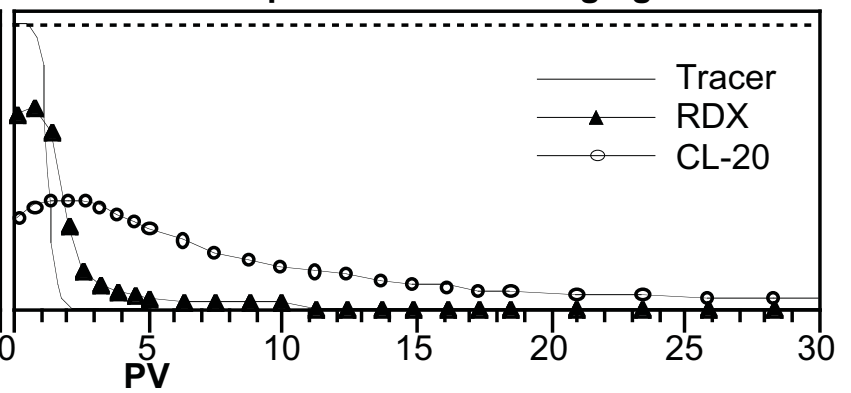

QM: CL-20 and RDX 1-D Transport with 2254h Aging on Westmoreland A1

Sorption

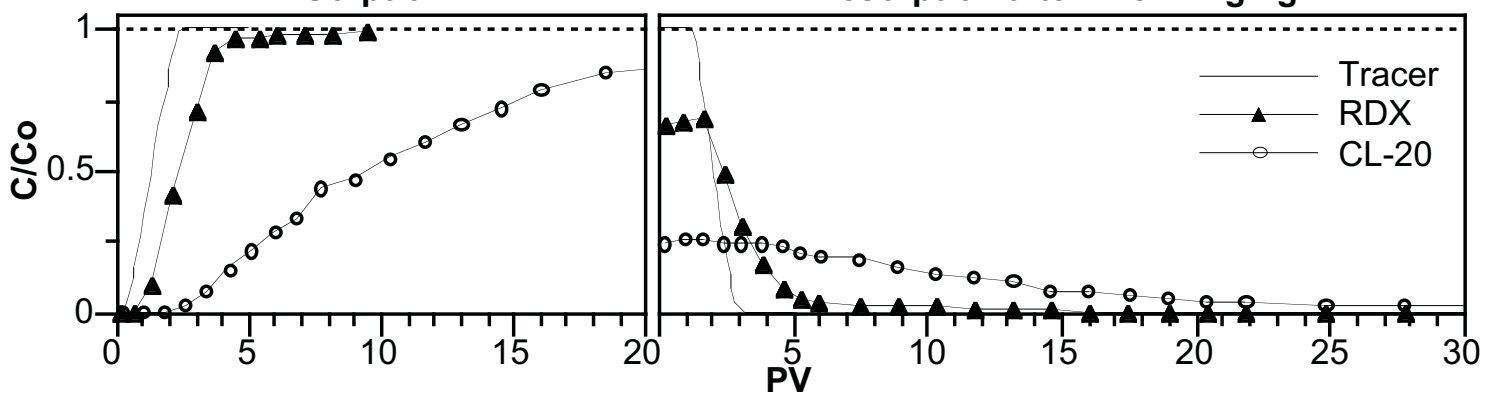

EC breakthrough during 1-D flow on West. (Sorb Phase)

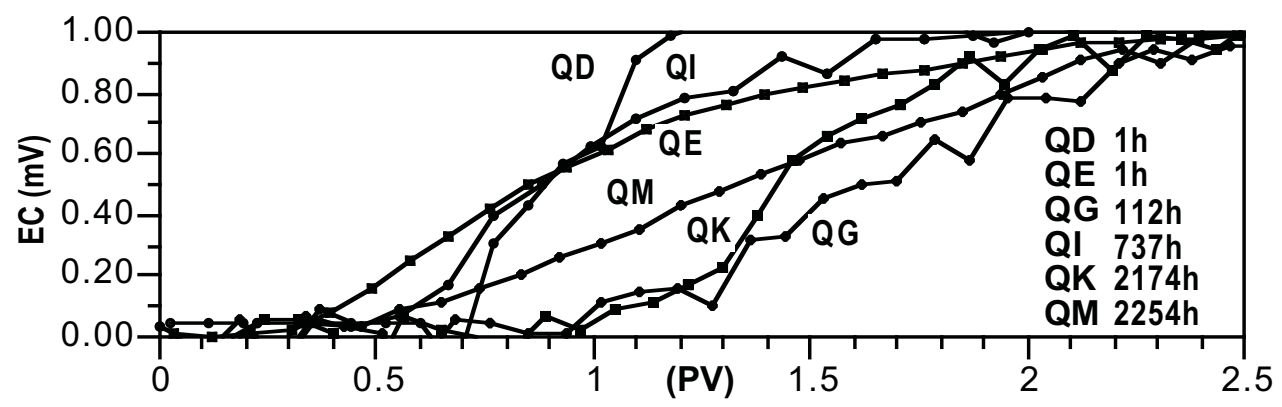

EC breakthrough during 1-D flow on West. (DeSorb Phase)

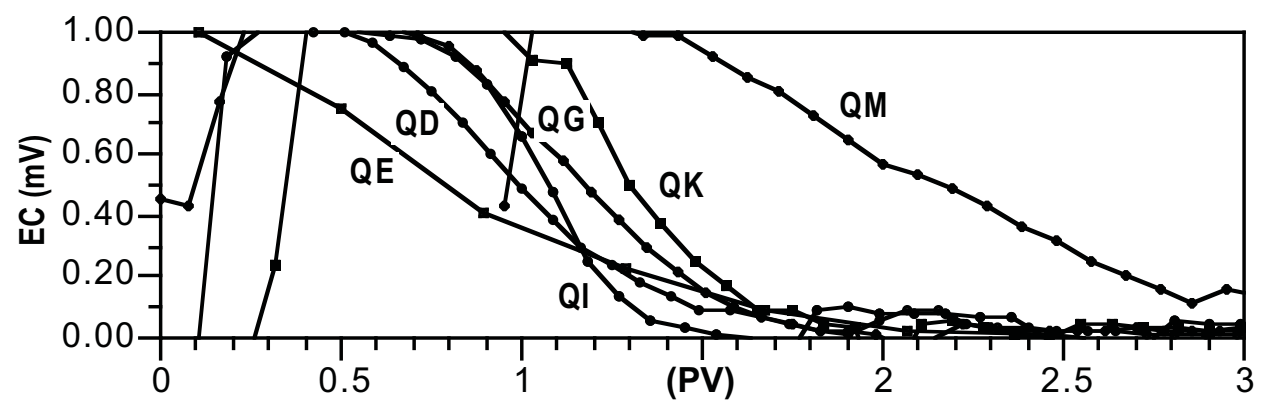


RDX breakthrough during 1-D flow on West. (Sorb Phase)

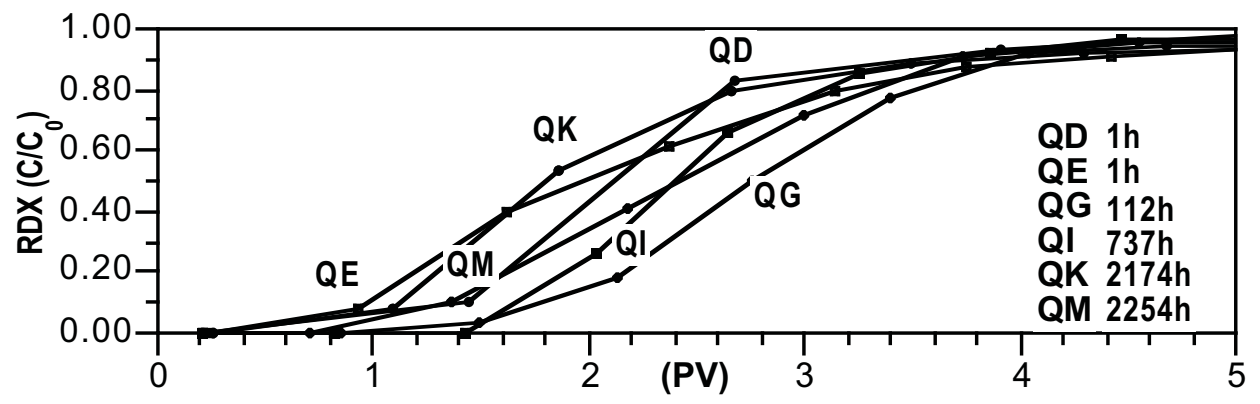

RDX breakthrough during 1-D flow on West. (DeSorb Phase)

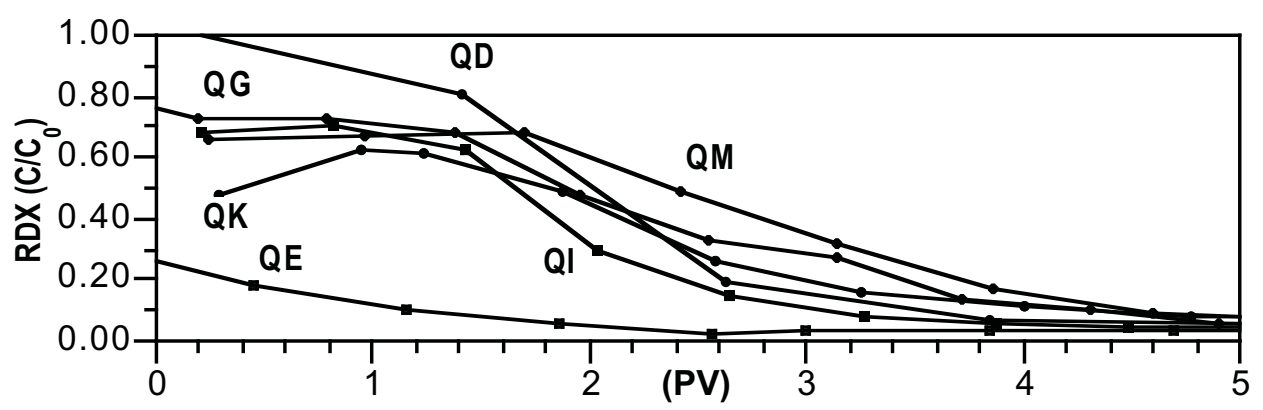

CL-20 breakthrough during 1-D flow on West. (Sorb Phase)

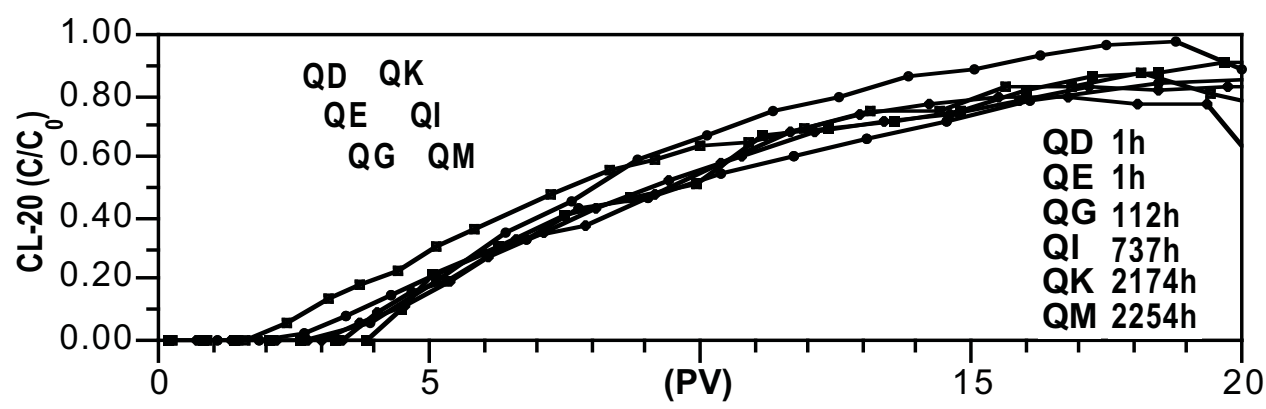

CL-20 breakthrough during 1-D flow on West. (DeSorb Phase)

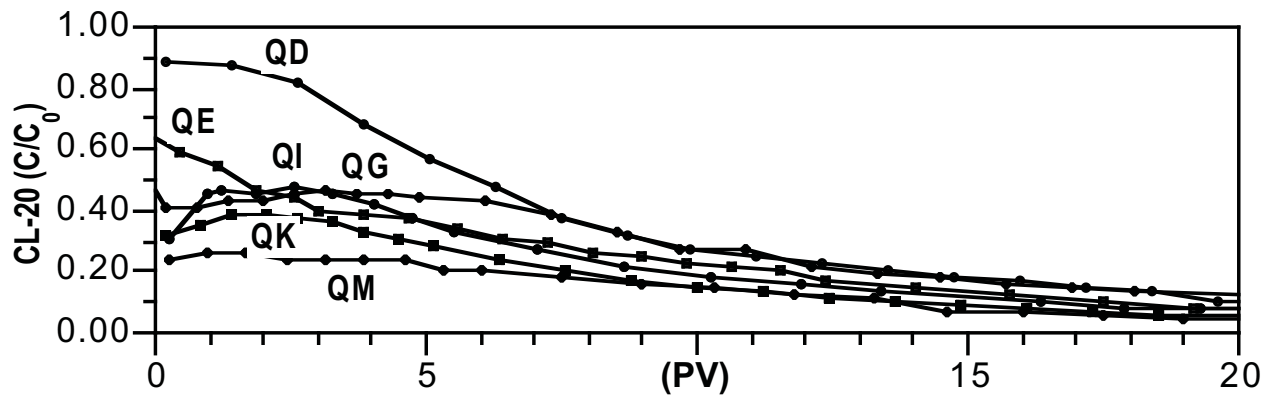


Appendix I.2; CL-20 and RDX Degradation and Sorption/Degradation on Sassafrass Silty Loam

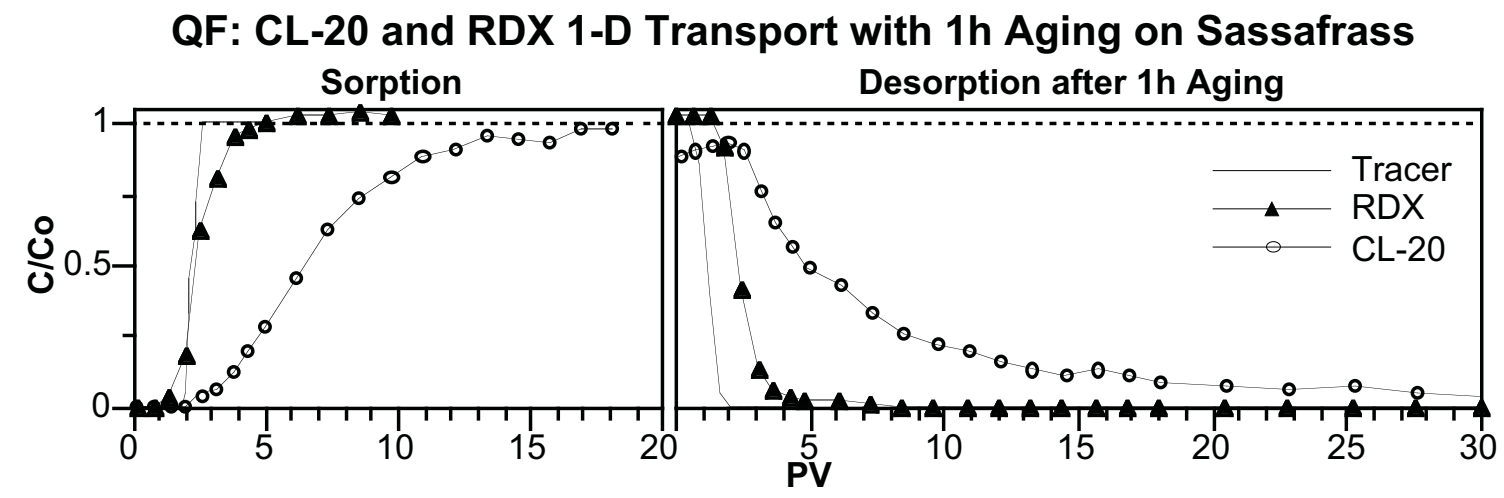

QH: CL-20 and RDX 1-D Transport with 112h Aging on Sassafrass

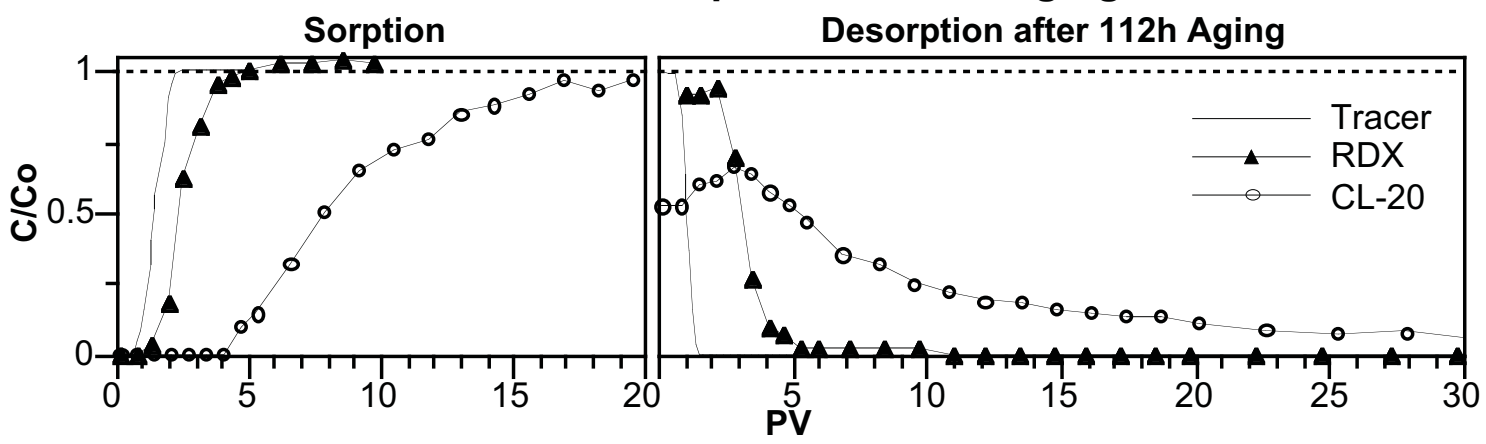

QJ: CL-20 and RDX 1-D Transport with 737h Aging on Sassafrass

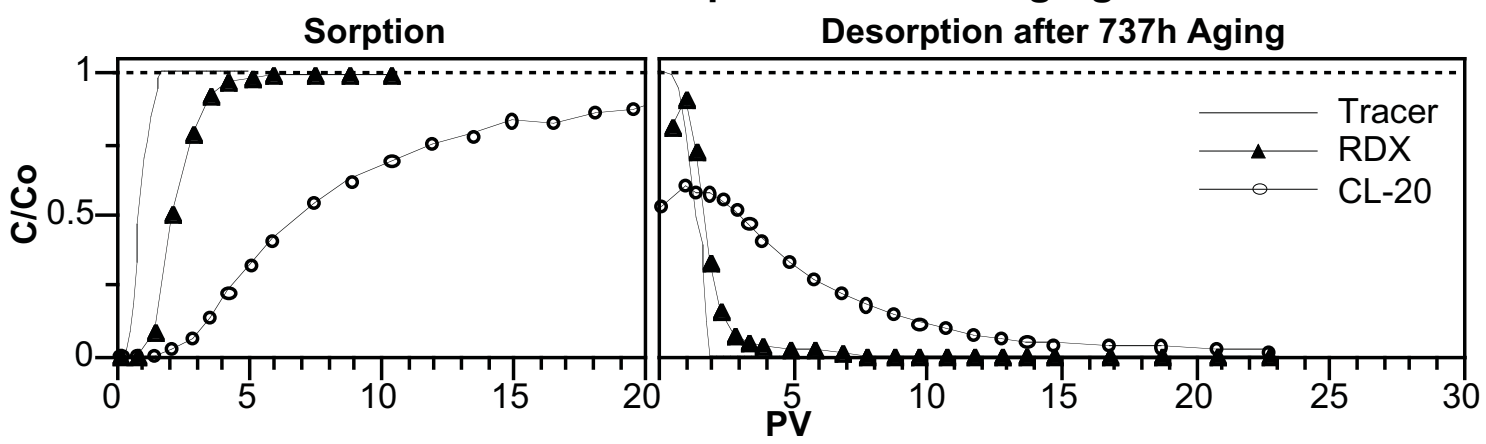

QL: CL-20 and RDX 1-D Transport with 2174h Aging on Sassafrass

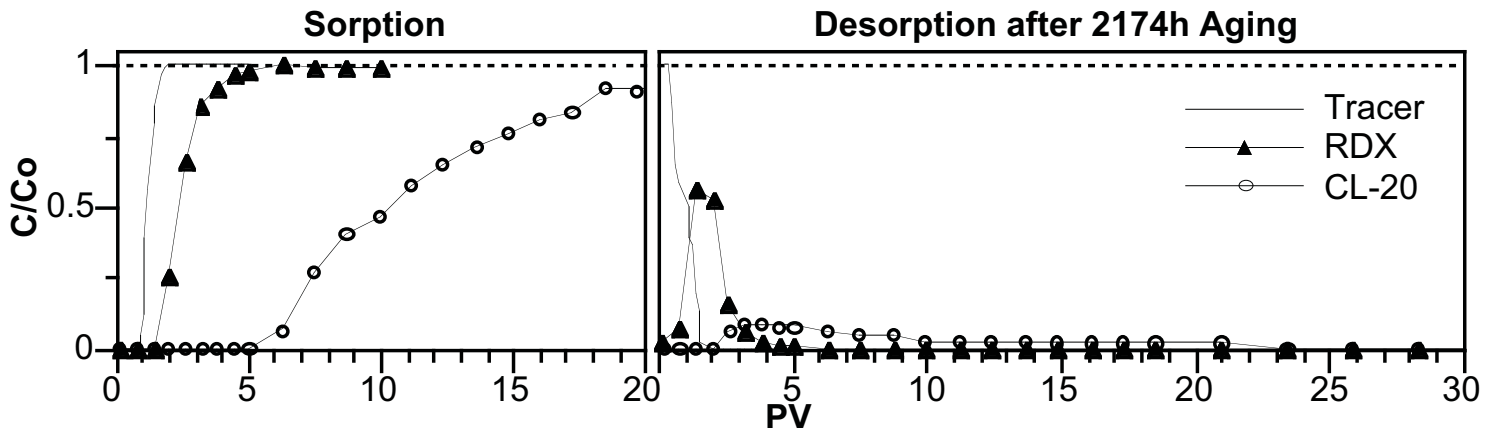


QN: CL-20 and RDX 1-D Transport with 2254h Aging on Sassafrass

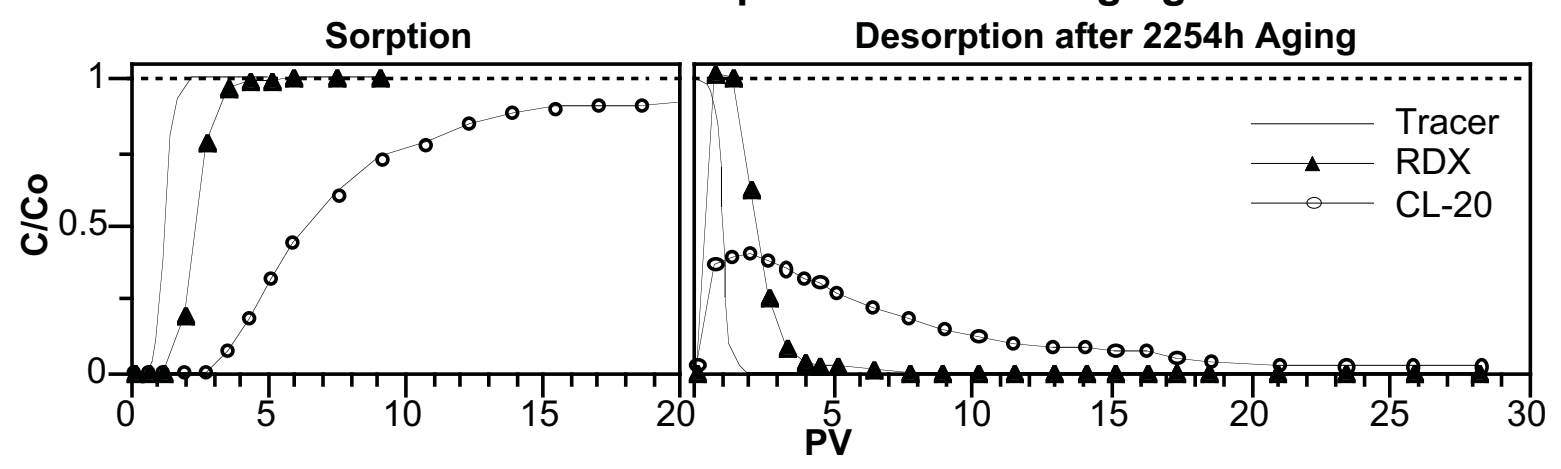

EC breakthrough during 1-D flow on Sass. (Sorb Phase)

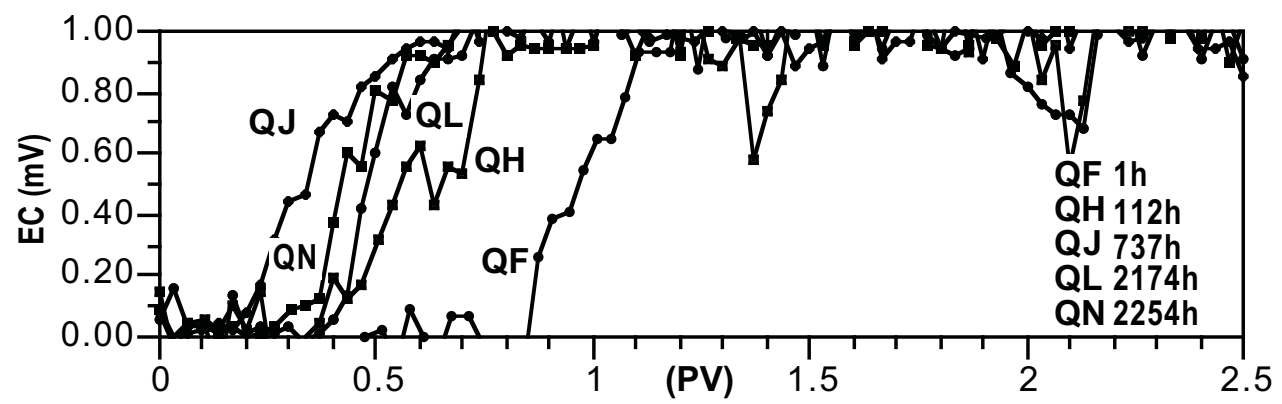

EC breakthrough during 1-D flow on Sass. (Sorb Phase)

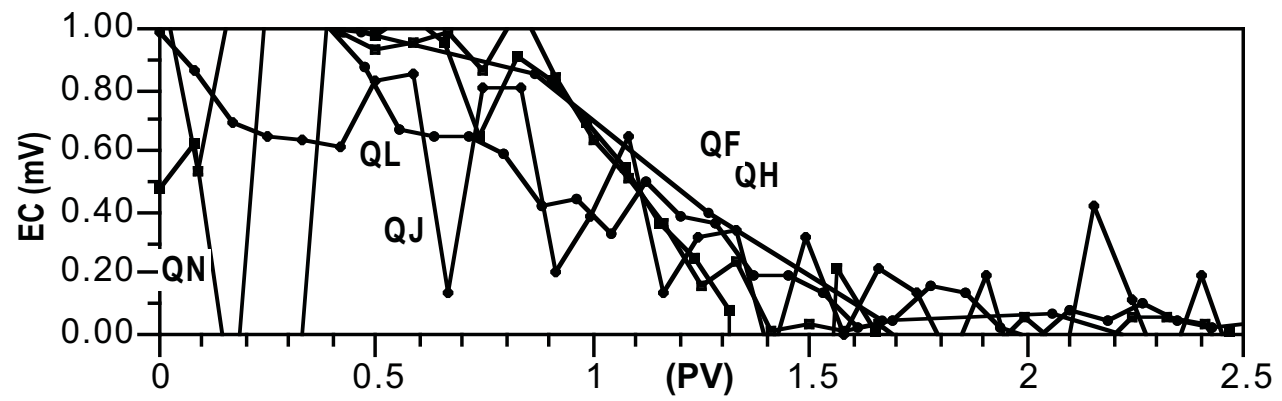


RDX breakthrough during 1-D flow on Sass. (Sorb Phase)

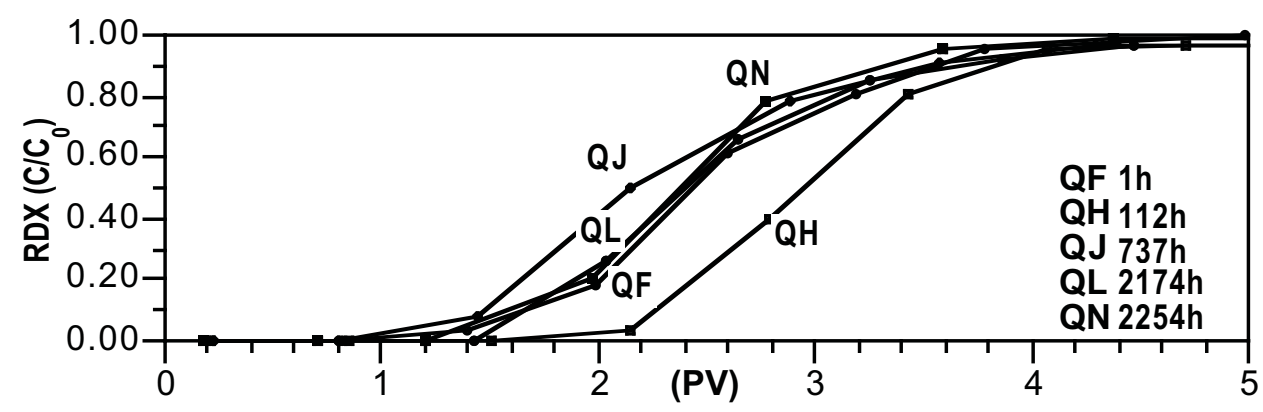

RDX breakthrough during 1-D flow on Sass. (DeSorb Phase)

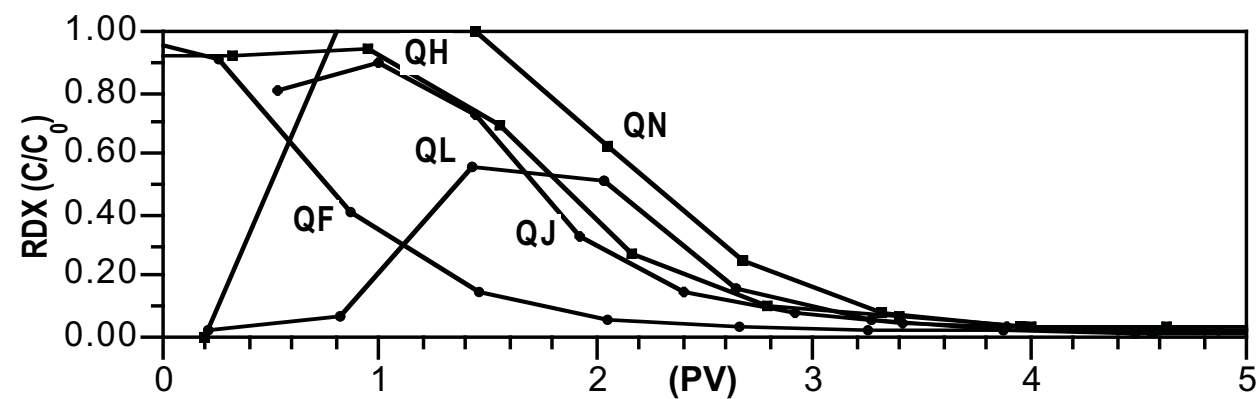

CL-20 breakthrough during 1-D flow on Sass. (Sorb Phase)

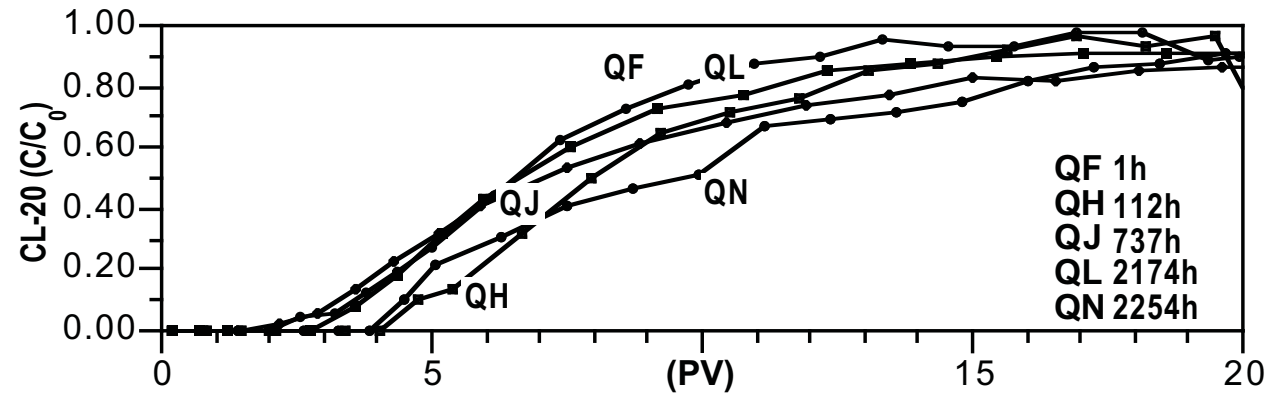

CL-20 breakthrough during 1-D flow on Sass. (DeSorb Phase)

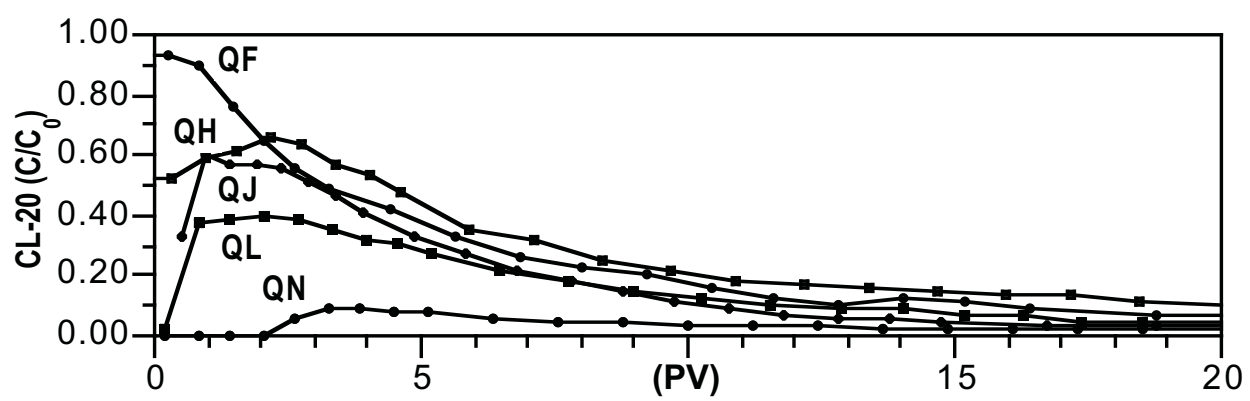


Appendix I.3; Calculated and Modeled Results of Aging on CL-20 and RDX Sorption/Desorption During 1-D Flow
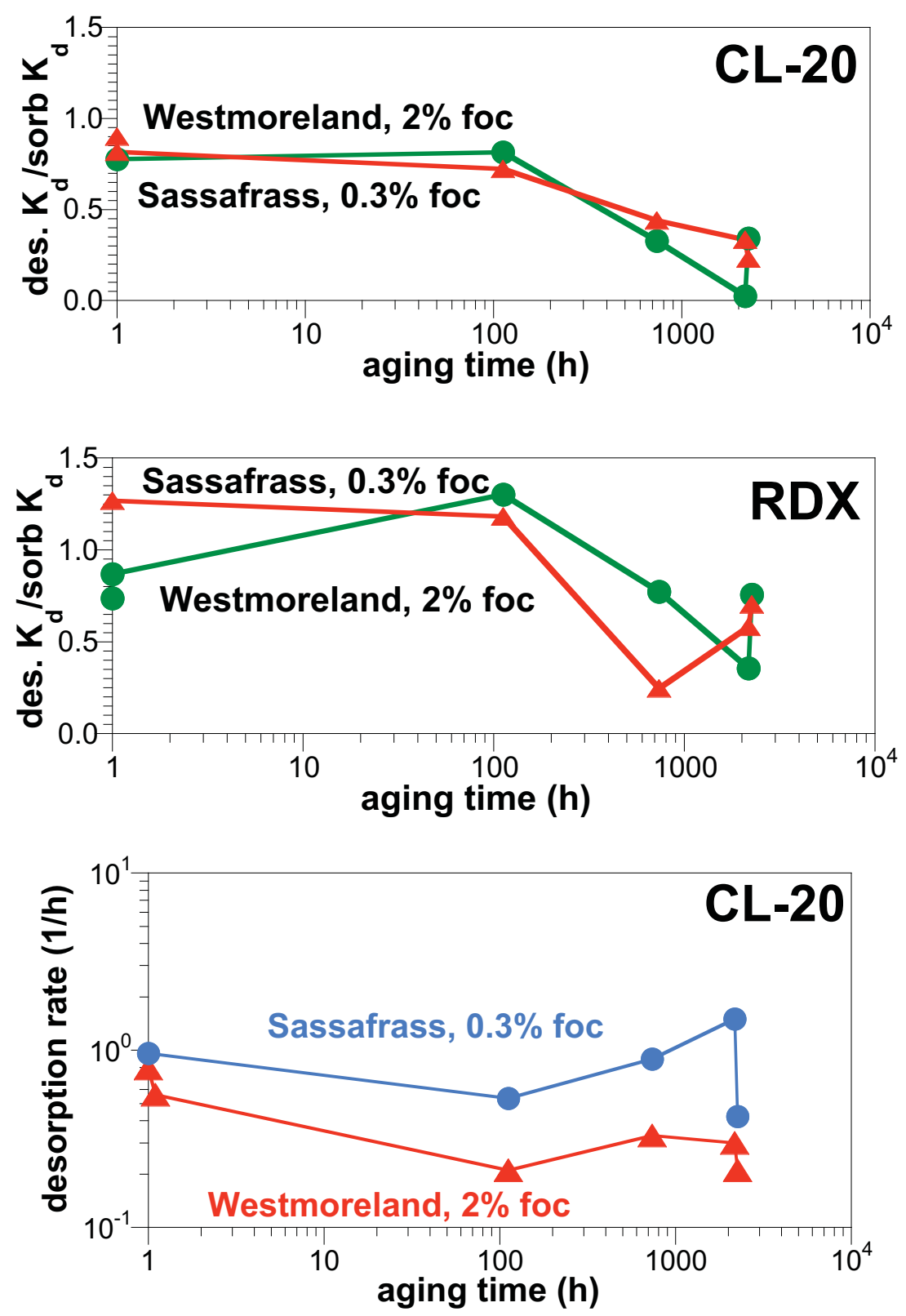

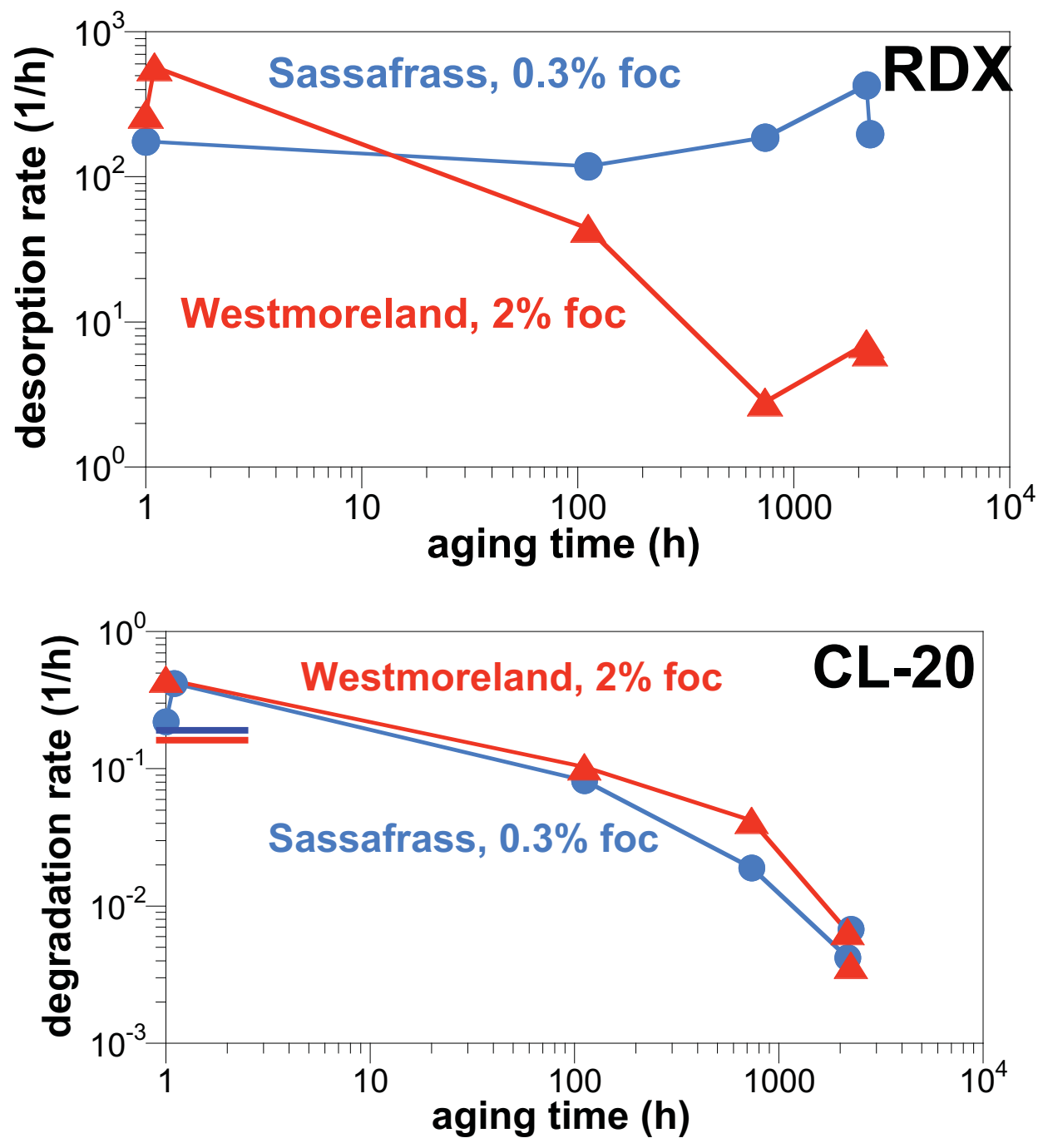
Appendix I.4; Impact on BTC Shape by Varying Model Parameters for Inverse fitting CL-20 and RDX Aging Column Experiments

CXTfit Testing for var. $R_{f}$ model

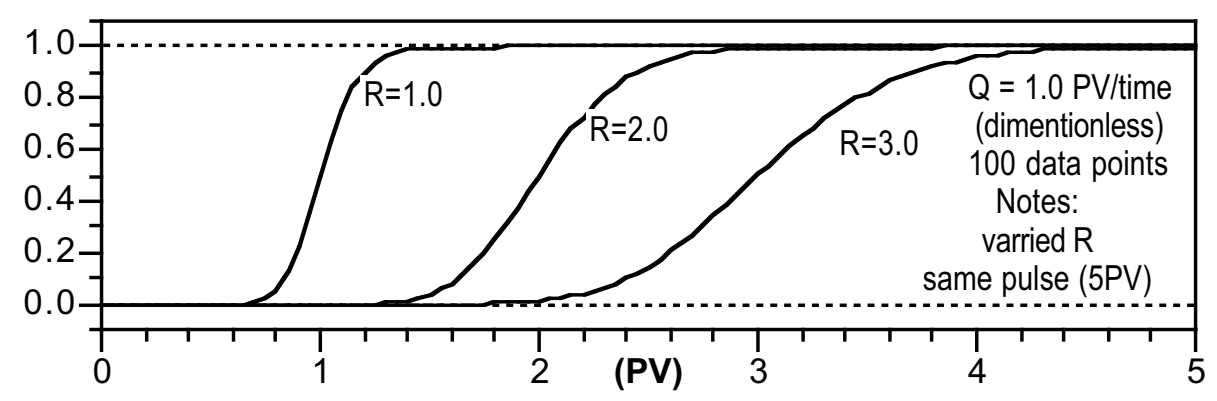

Area ahead of curve

\begin{tabular}{|c|c|}
\hline $\mathrm{R}$ & area \\
\hline 1.0 & 1.010 \\
\hline 2.0 & 2.020 \\
\hline 3.0 & 3.030 \\
\hline
\end{tabular}

(up to $5.0 \mathrm{PV}$ )

CXTfit Testing for var. $R_{f}$ model

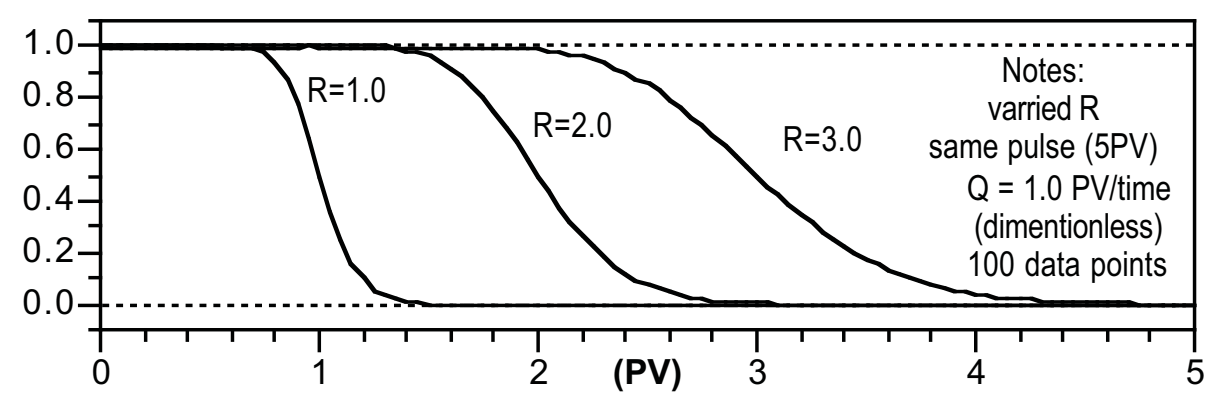

Area ahead of curve

\begin{tabular}{|c|c|}
\hline $\mathrm{R}$ & area \\
\hline 1.0 & 1.010 \\
\hline 2.0 & 2.020 \\
\hline 3.0 & 3.030 \\
\hline
\end{tabular}

(up to $5.0 \mathrm{PV}$ )

CXTfit Testing for var. $R_{f}$ model

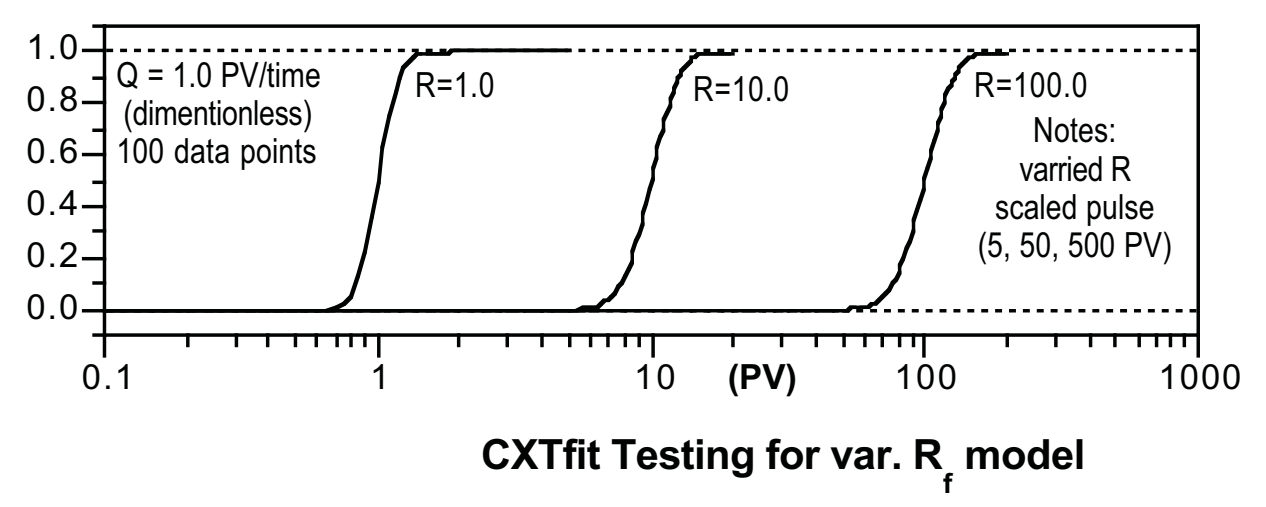

Area ahead of curve

\begin{tabular}{|c|c|}
\hline$R$ & area \\
\hline 1.0 & 1.010 \\
\hline 10.0 & 10.10 \\
\hline 100.0 & 101.0 \\
\hline
\end{tabular}

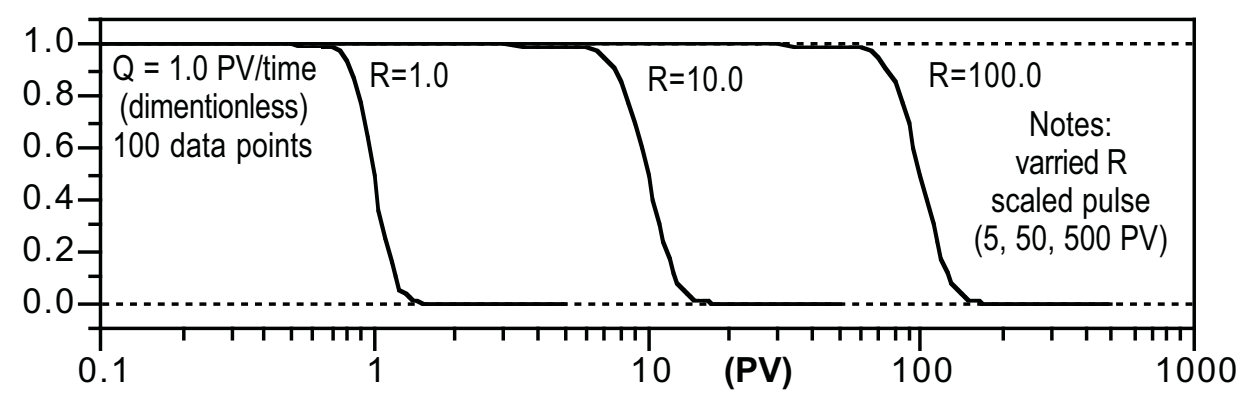

Area ahead of curve

\begin{tabular}{|c|c|}
\hline $\mathrm{R}$ & area \\
\hline 1.0 & 1.010 \\
\hline 10.0 & 10.10 \\
\hline 100.0 & 101.0 \\
\hline
\end{tabular}


CXTfit Testing for var. Disp. model

Comments:

all values reset for $\mathrm{ZL}=1$

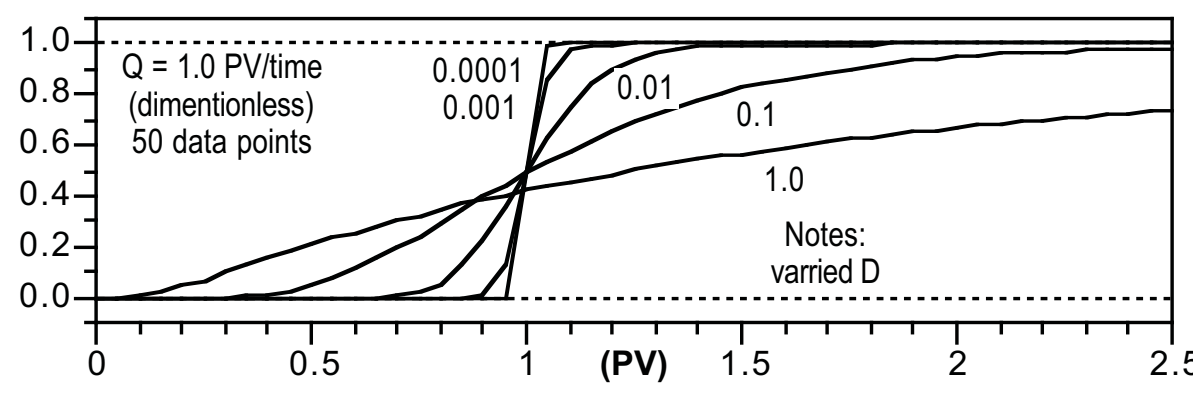

Area ahead of curve

\begin{tabular}{|c|c|}
\hline$D$ & area \\
\hline 0.0001 & 1.000 \\
\hline 0.001 & 1.001 \\
\hline 0.01 & 1.010 \\
\hline 0.1 & 1.095 \\
\hline 1.0 & 1.385 \\
\hline
\end{tabular}

(up to $2.5 \mathrm{PV}$ )

Comments:

CXTfit Testing for var. Disp. model all values reset for $\mathrm{ZL}=1$ injection pulse $=10$

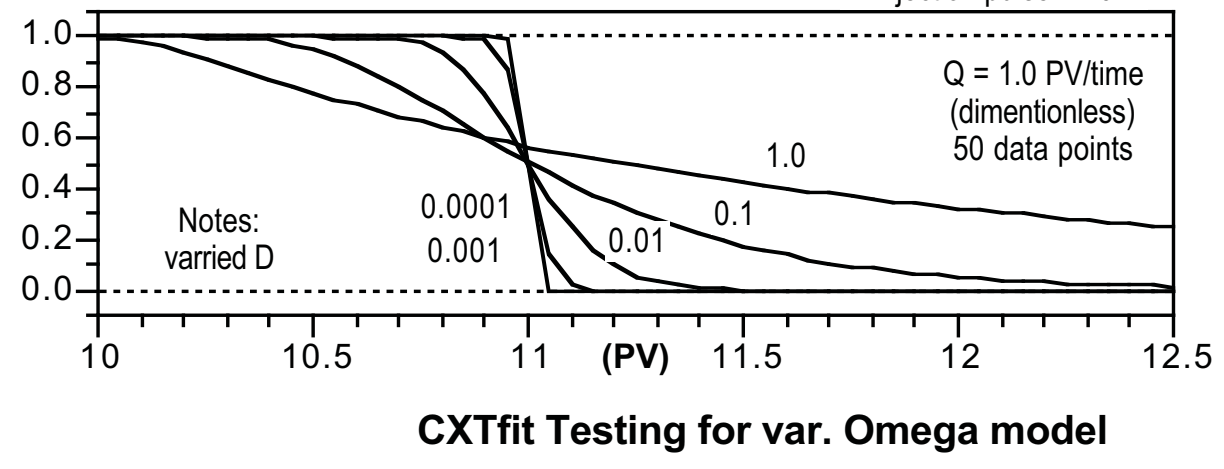

Area ahead of curve

\begin{tabular}{|c|c|}
\hline$D$ & area \\
\hline 0.0001 & 1.000 \\
\hline 0.001 & 1.001 \\
\hline 0.01 & 1.010 \\
\hline 0.1 & 1.095 \\
\hline 1.0 & 1.385 \\
\hline
\end{tabular}

(up to $2.5 \mathrm{PV}$ )

CXTfit Testing for var. Omega model

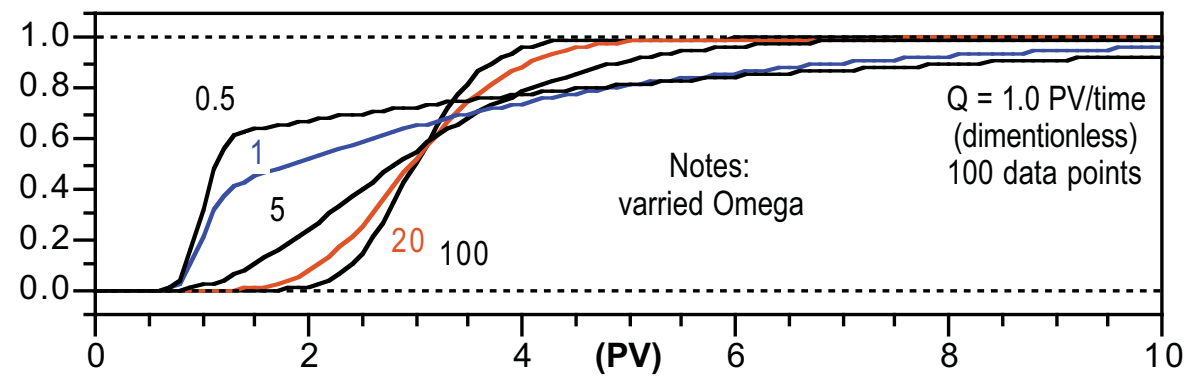

CXTfit

Variables

\begin{tabular}{|c|c|}
\hline $\mathrm{V}$ & 1 \\
\hline $\mathrm{D}$ & 0.01 \\
\hline $\mathrm{R}$ & 3.0 \\
\hline beta & 0.333 \\
\hline omega & $\mathrm{X}$ \\
\hline $\mathrm{DZ}$ & 1 \\
\hline pulse & 10 \\
\hline
\end{tabular}


QD: EC BTC during 1-D flow on West. (Sorb Phase)

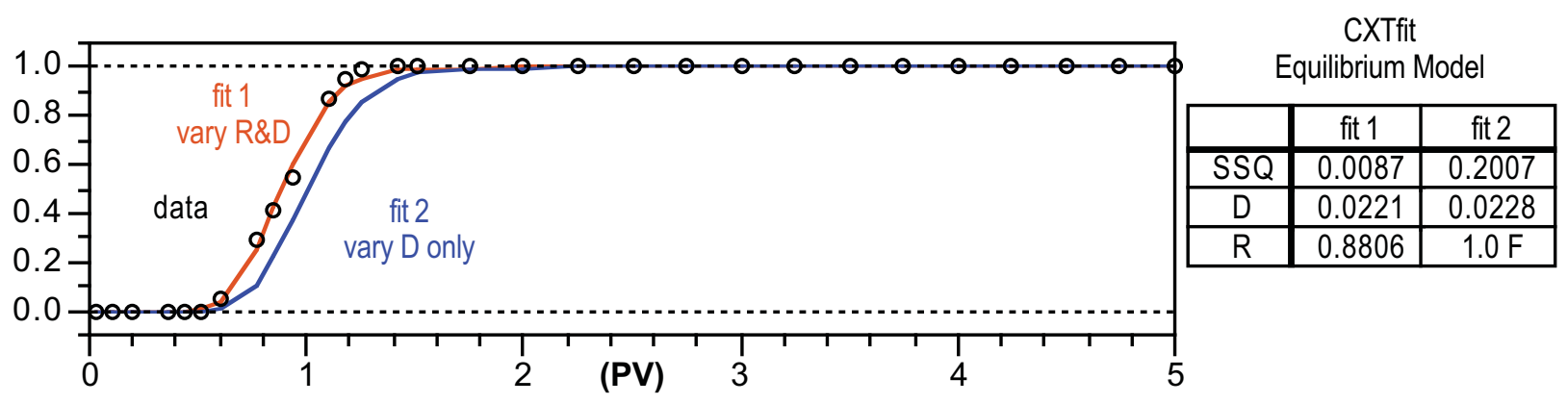

QE: EC BTC during 1-D flow on West. (Sorb Phase)

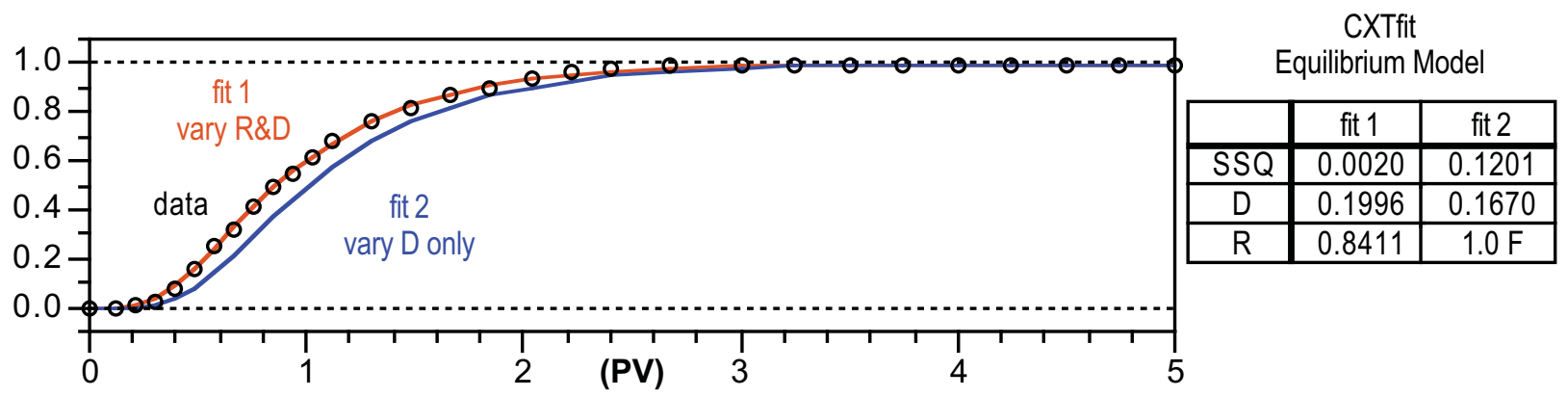

QG: EC BTC during 1-D flow on West. (Sorb Phase)

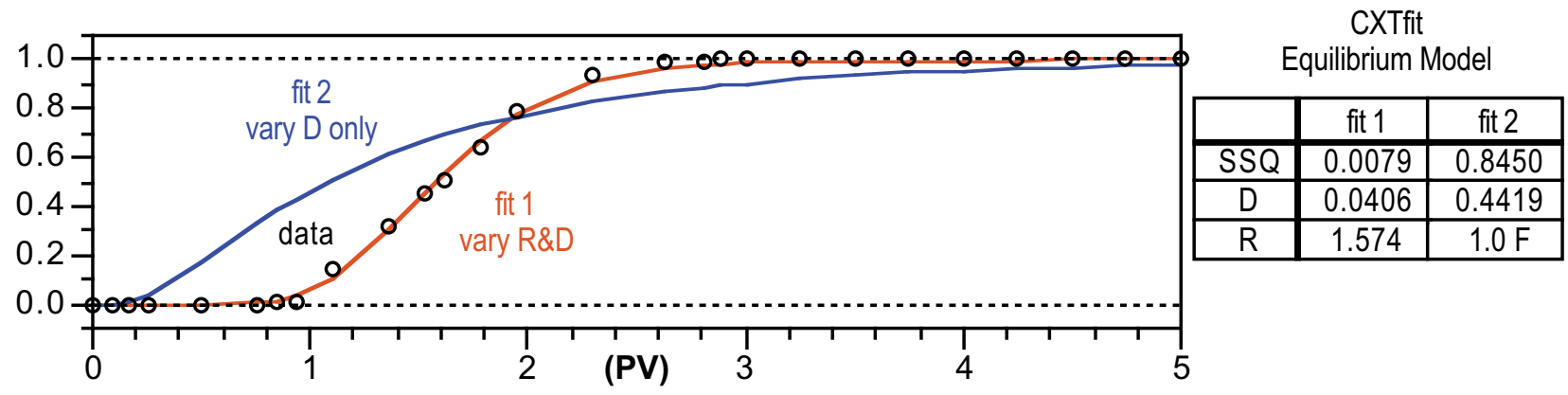

QI: EC BTC during 1-D flow on West. (Sorb Phase)

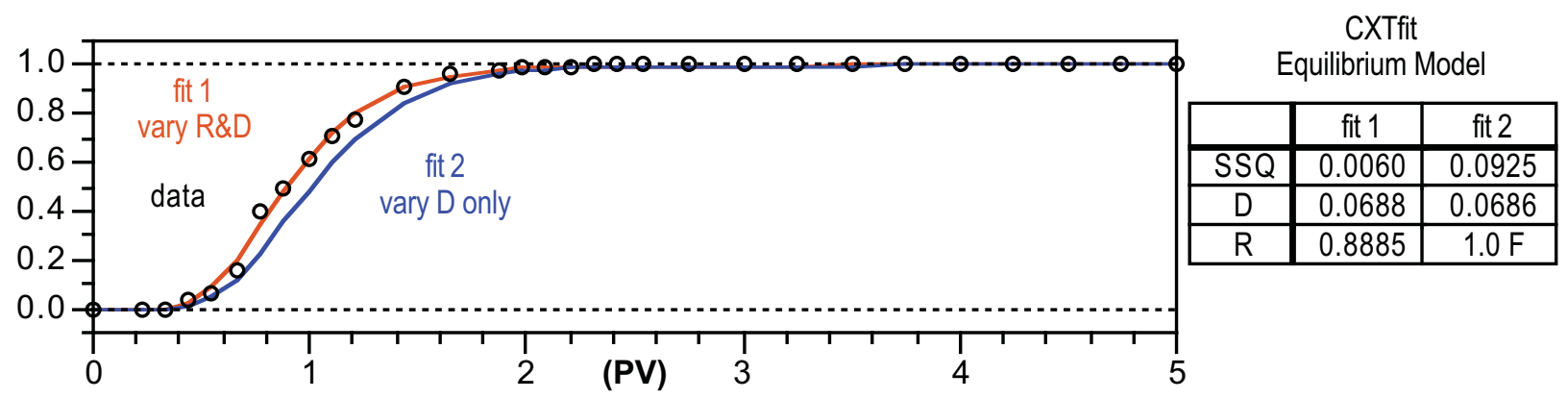


QK: EC BTC during 1-D flow on West. (Sorb Phase)

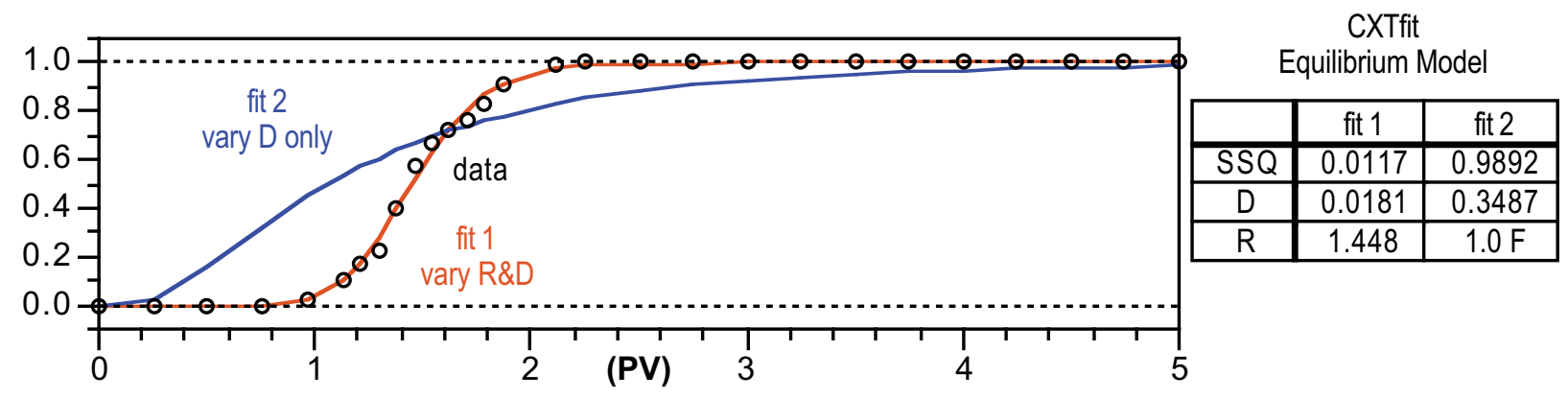

QM: EC BTC during 1-D flow on West. (Sorb Phase)

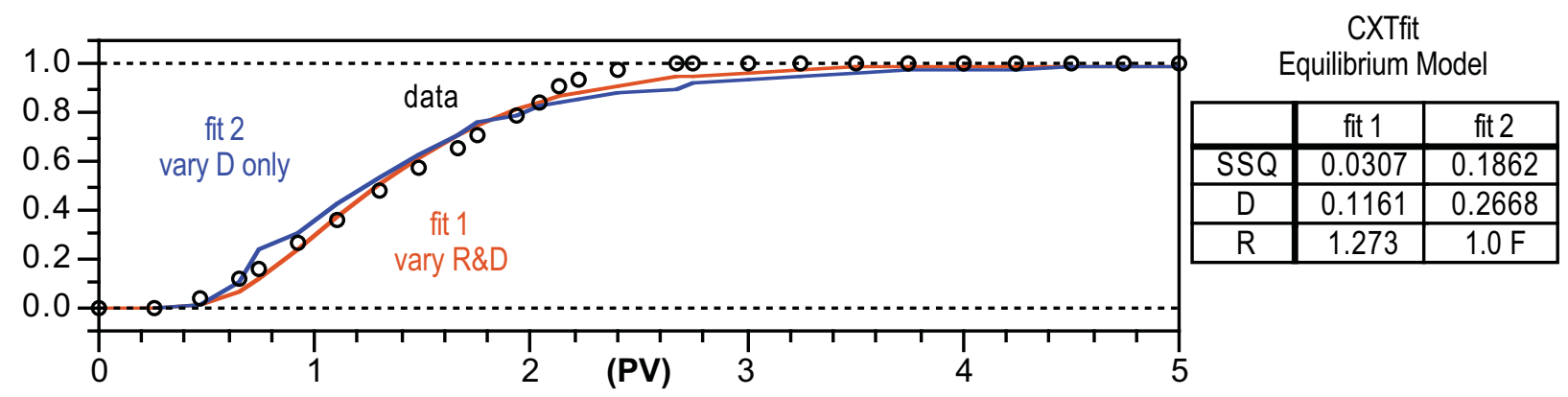

QD: EC BTC during 1-D flow on West. (Desorb Phase) w/1h aging

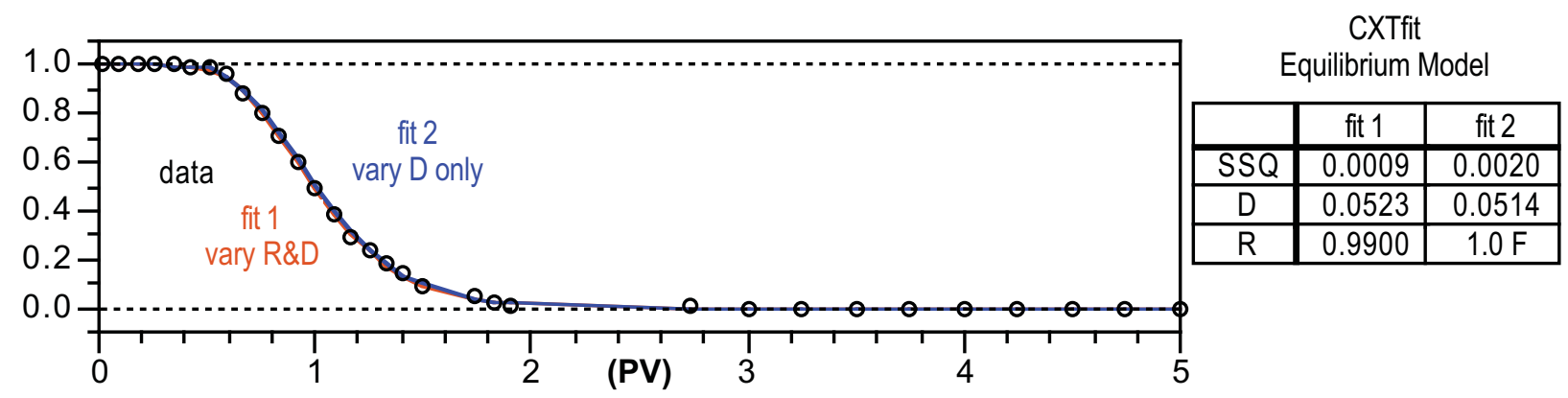

QE: EC BTC during 1-D flow on West. (Desorb Phase) w/1h aging

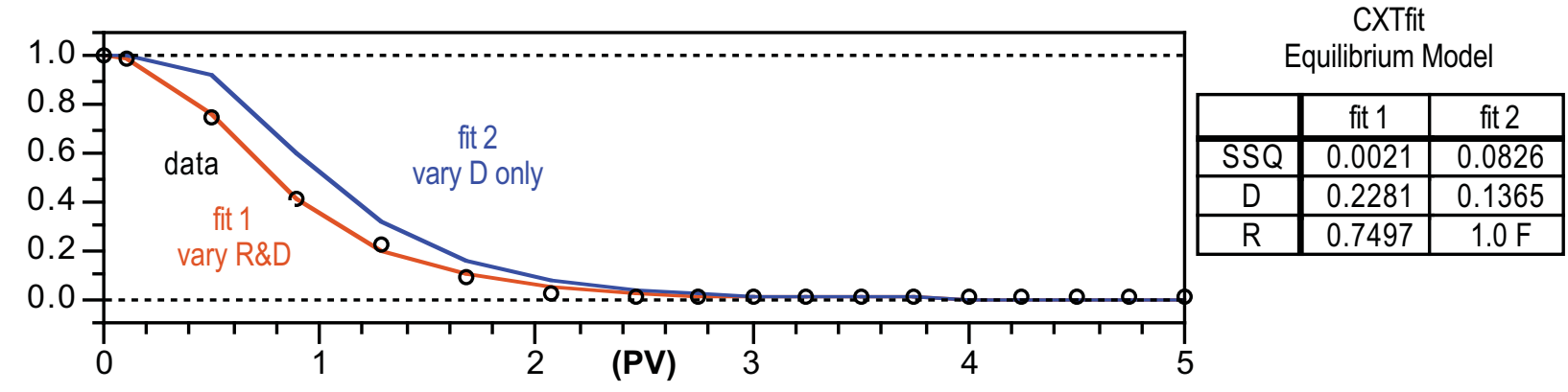


QG: EC BTC during 1-D flow on West. (Desorb Phase) w/112h aging

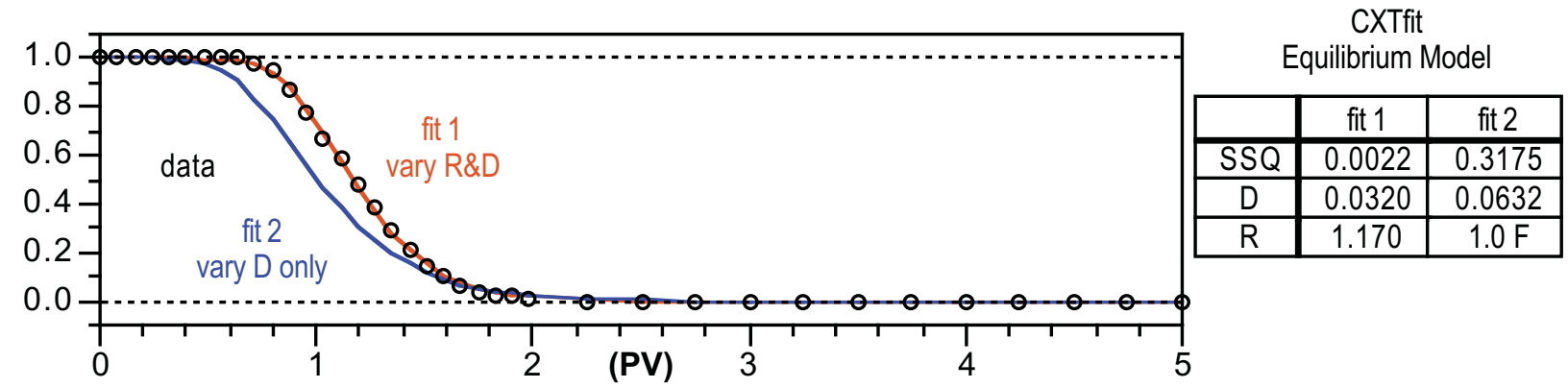

QI: EC BTC during 1-D flow on West. (Desorb Phase) w/737h aging

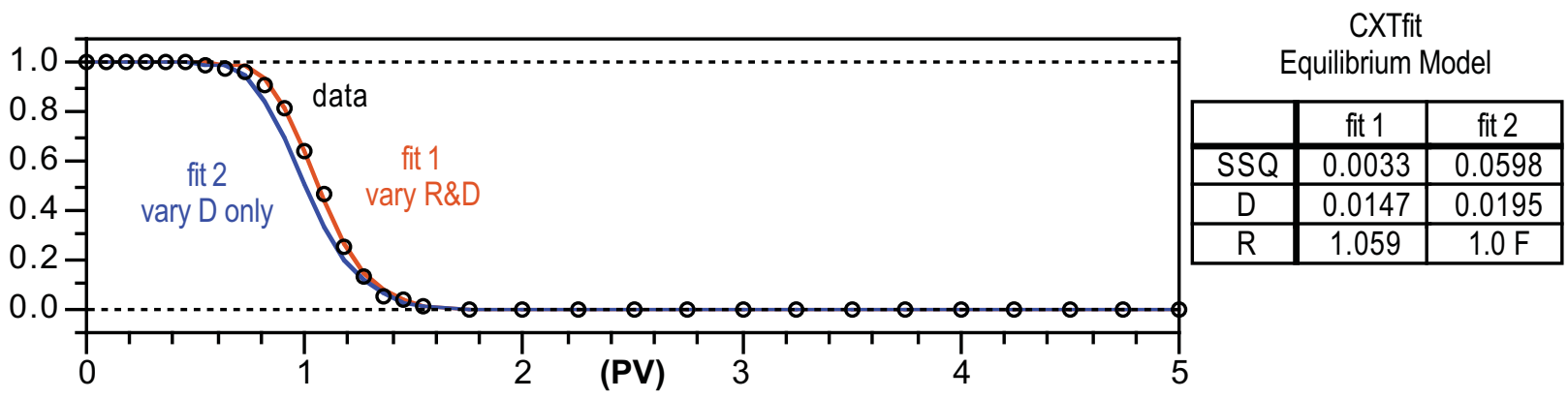

QK: EC BTC during 1-D flow on West. (Desorb Phase) w/2174h aging

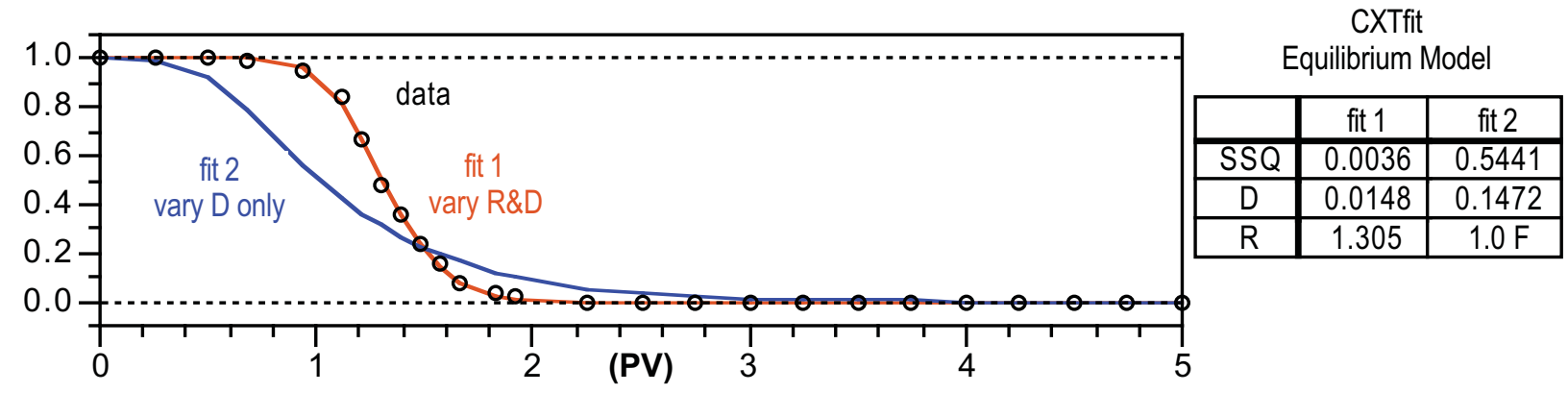

QM: EC BTC during 1-D flow on West. (Desorb Phase) w/2254h aging

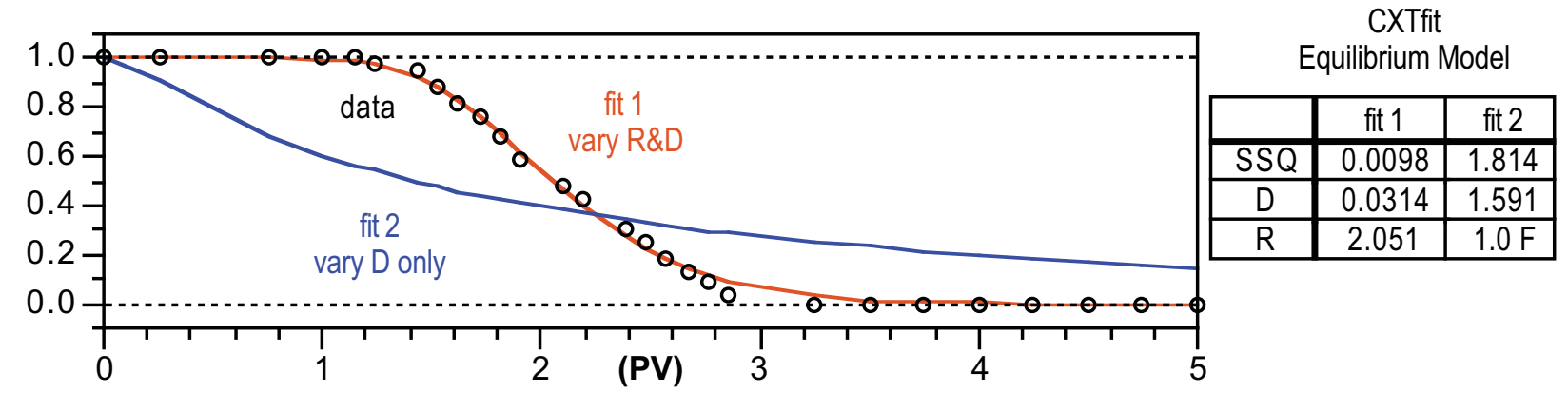


Appendix I.6; Conservative Tracer BTC Fitting on Sassafrass Column Aging Experiments

QF: EC BTC during 1-D flow on Sass. (Sorb Phase)

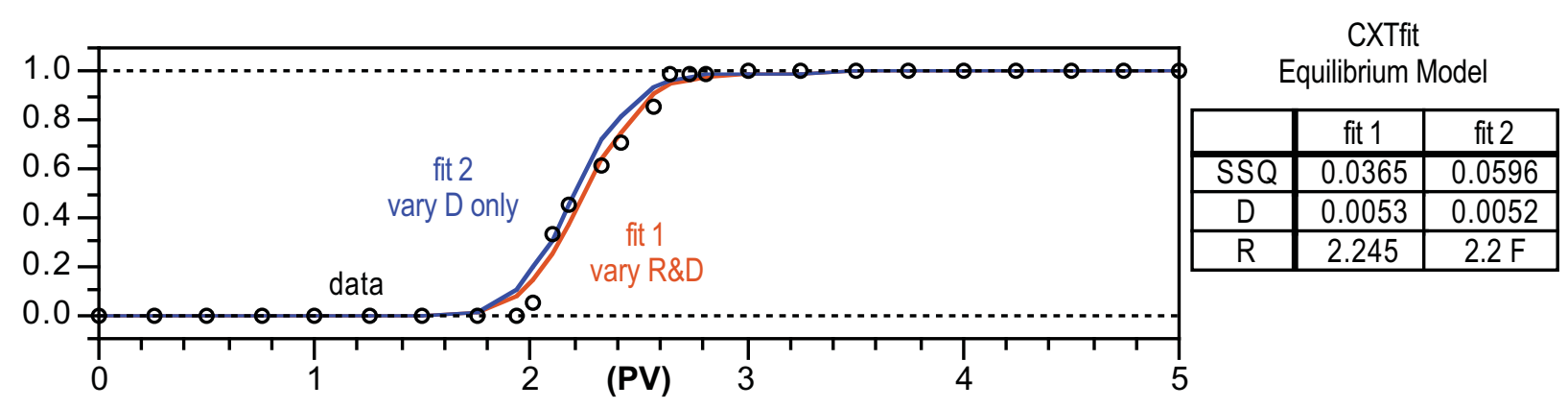

QH: EC BTC during 1-D flow on Sass. (Sorb Phase)

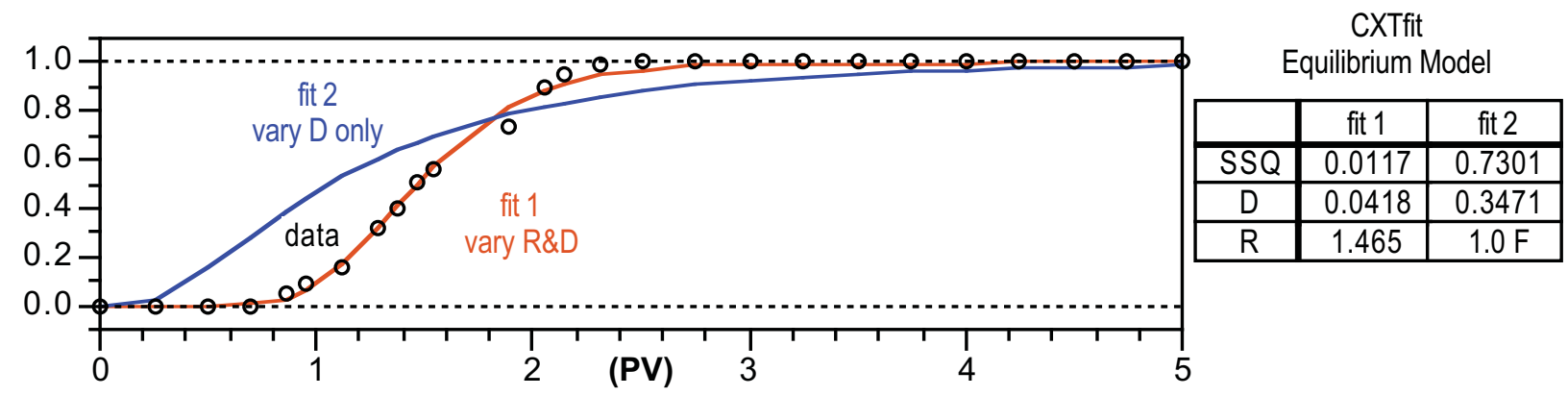

QJ: EC BTC during 1-D flow on Sass. (Sorb Phase)

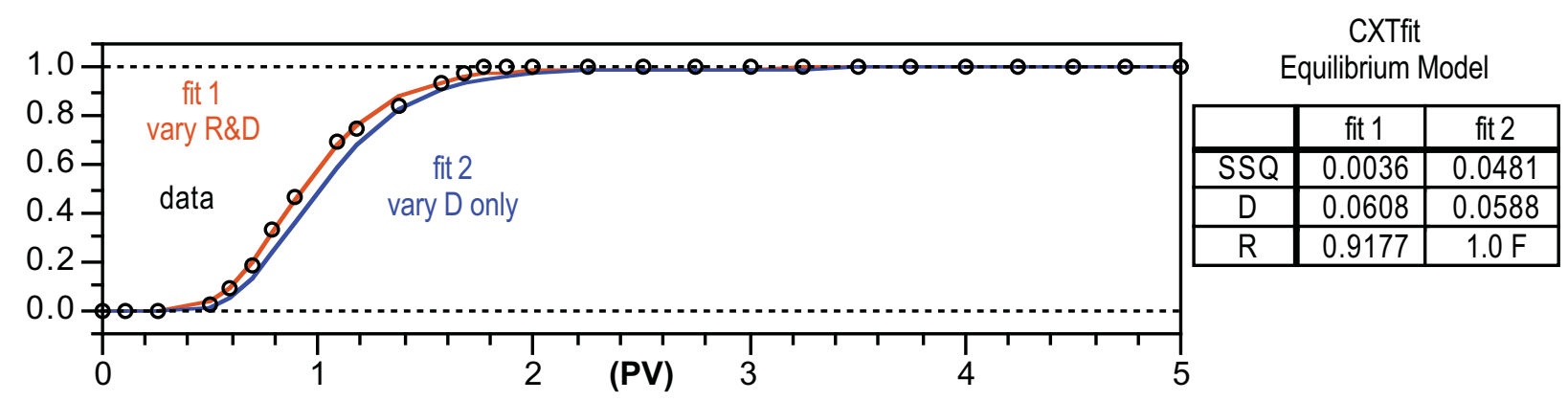

QL: EC BTC during 1-D flow on Sass. (Sorb Phase)

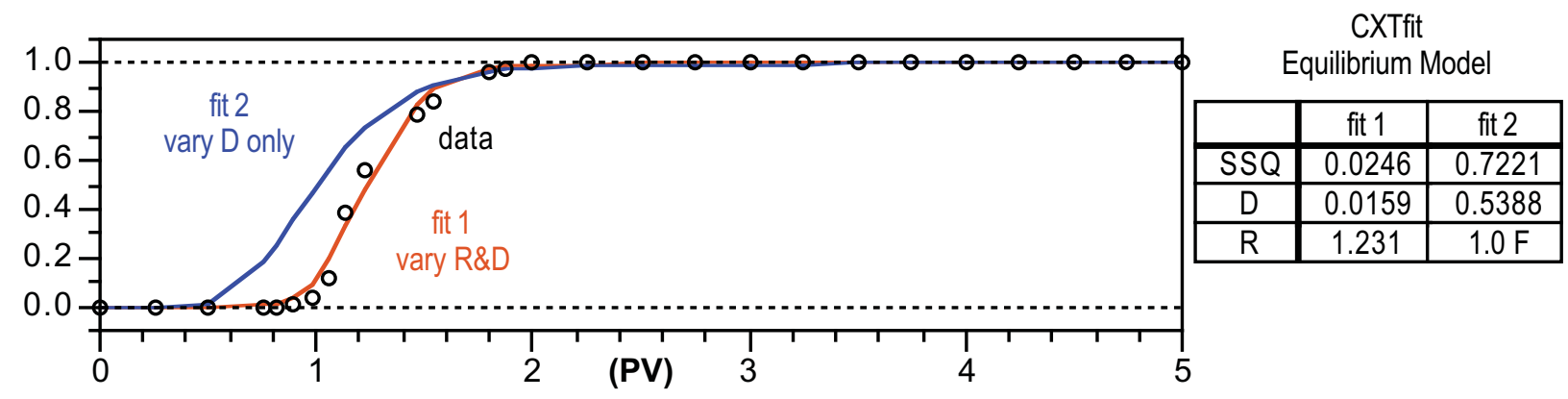


QN: EC BTC during 1-D flow on Sass. (Sorb Phase)

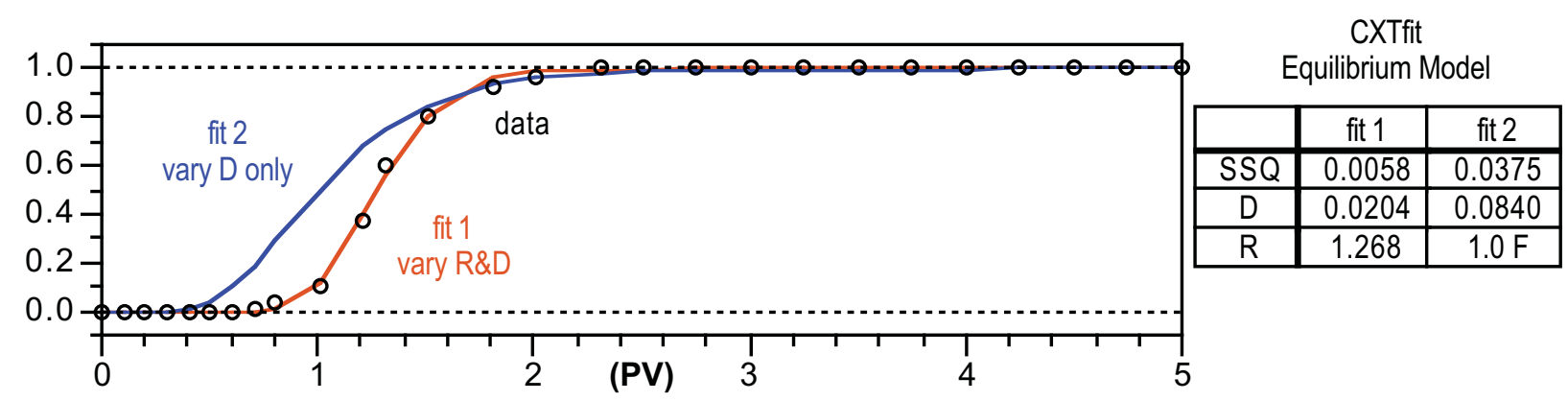

QF: EC BTC during 1-D flow on Sass. (Desorb Phase) w/1h aging

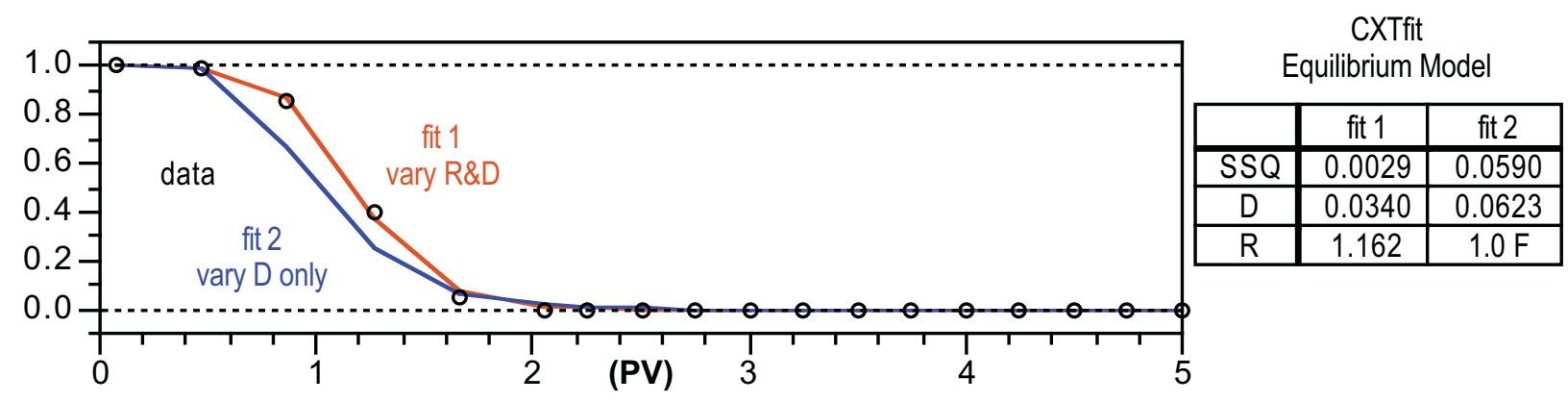

QH: EC BTC during 1-D flow on Sass. (Desorb Phase) w/112h aging

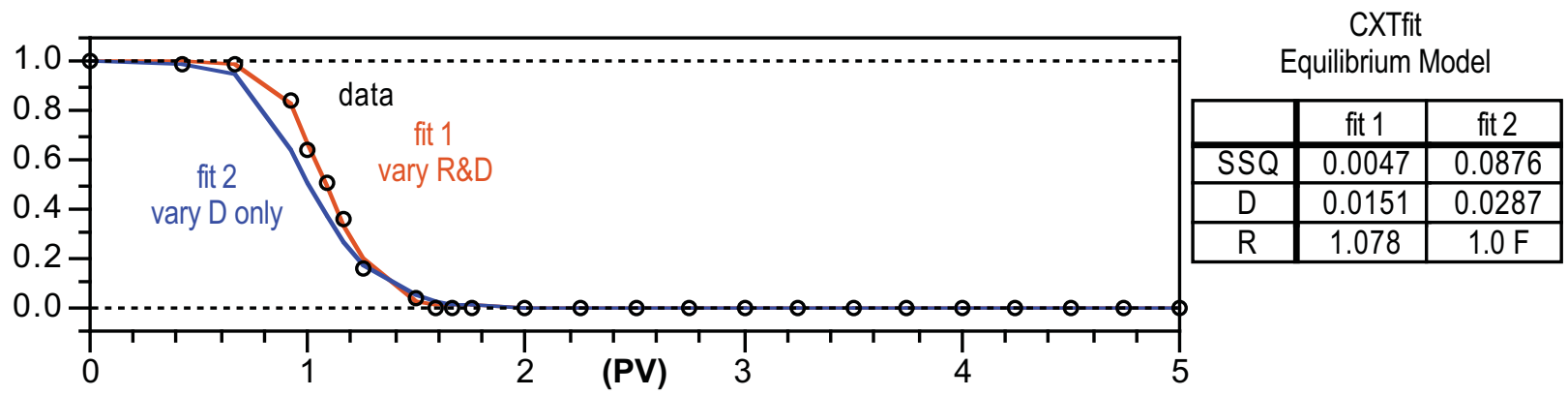

QJ: EC BTC during 1-D flow on Sass. (Desorb Phase) w/737h aging

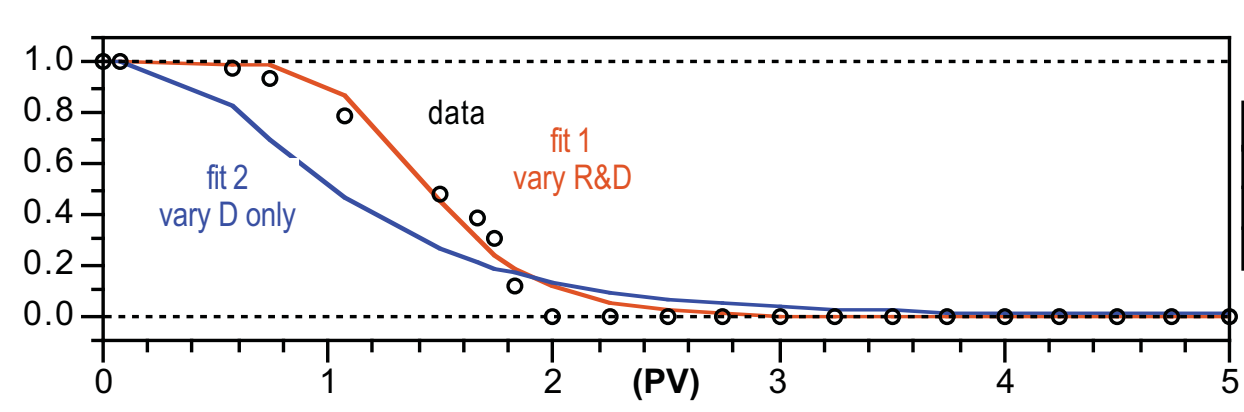

CXTfit

Equilibrium Model

\begin{tabular}{|c|c|c|}
\hline & fit 1 & fit 2 \\
\hline SSQ & 0.0413 & 0.3257 \\
\hline D & 0.0349 & 0.2192 \\
\hline R & 1.453 & $1.0 \mathrm{~F}$ \\
\hline
\end{tabular}


QL: EC BTC during 1-D flow on Sass. (Desorb Phase) w/2174h aging

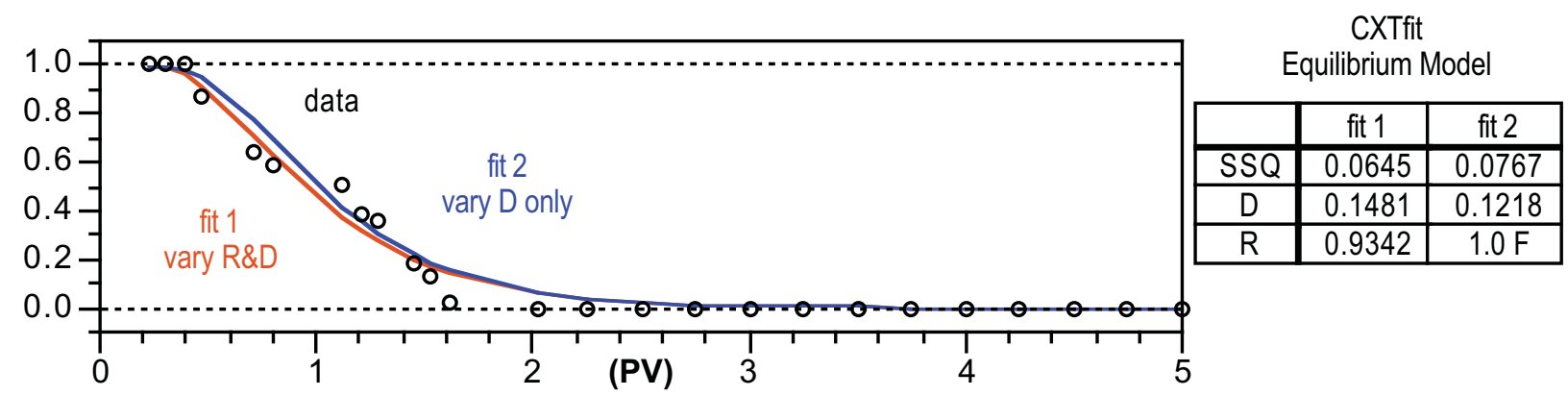

QN: EC BTC during 1-D flow on Sass. (Desorb Phase) w/2254h aging

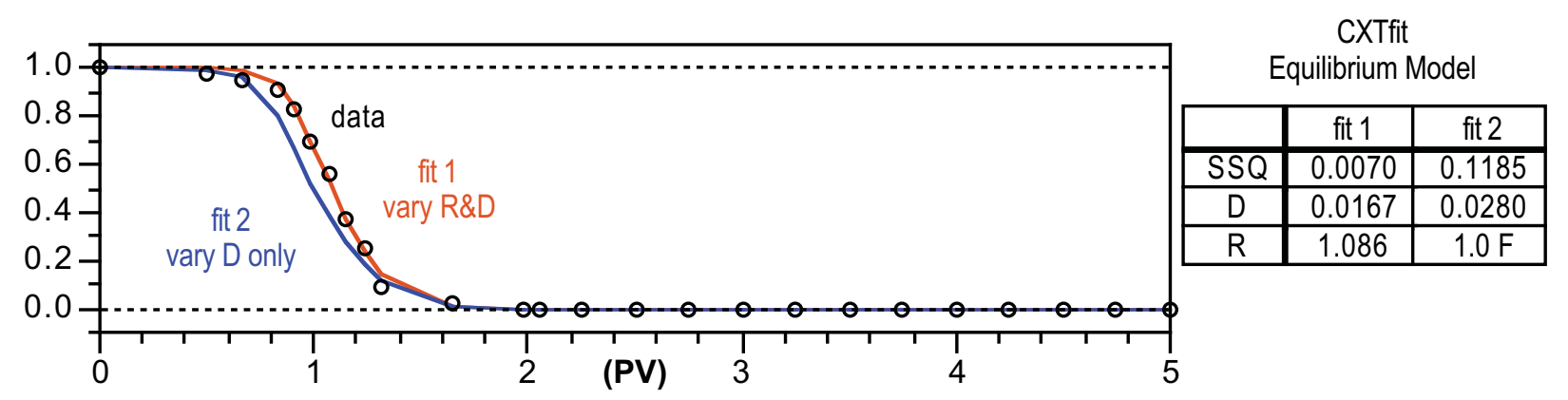

QD: RDX BTC during 1-D flow on West. (Sorb Phase)

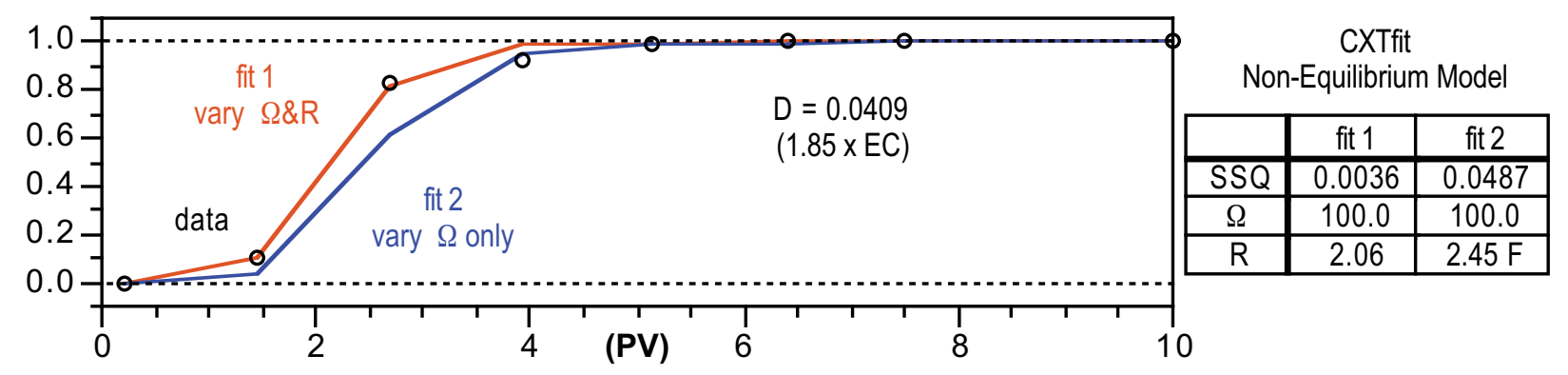

\section{QE: RDX BTC during 1-D flow on West. (Sorb Phase)}

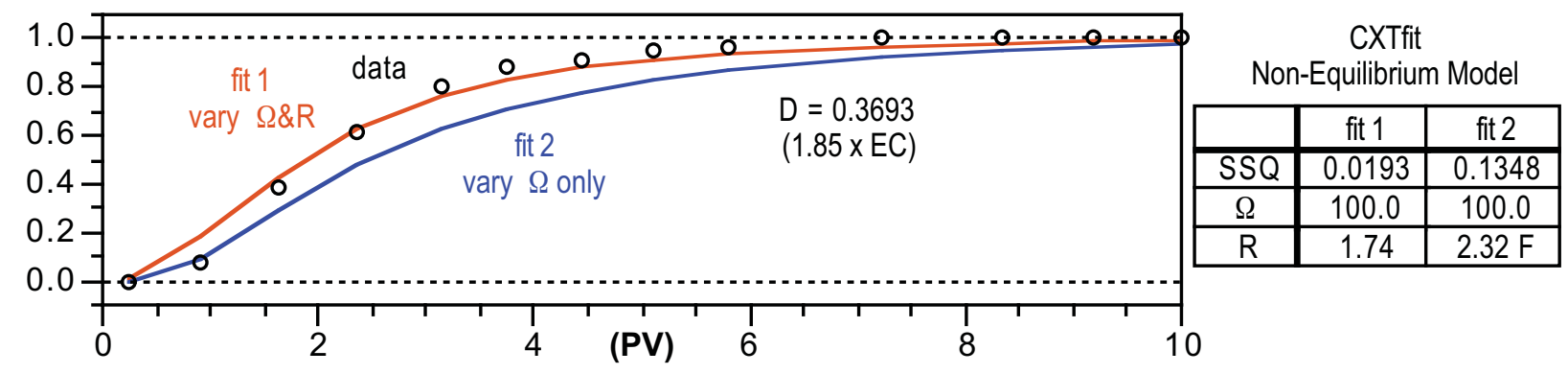




\section{Appendix I.7; RDX BTC Fitting on Westmoreland A1 Column Aging Experiments}

QD: RDX BTC during 1-D flow on West. (Sorb Phase)

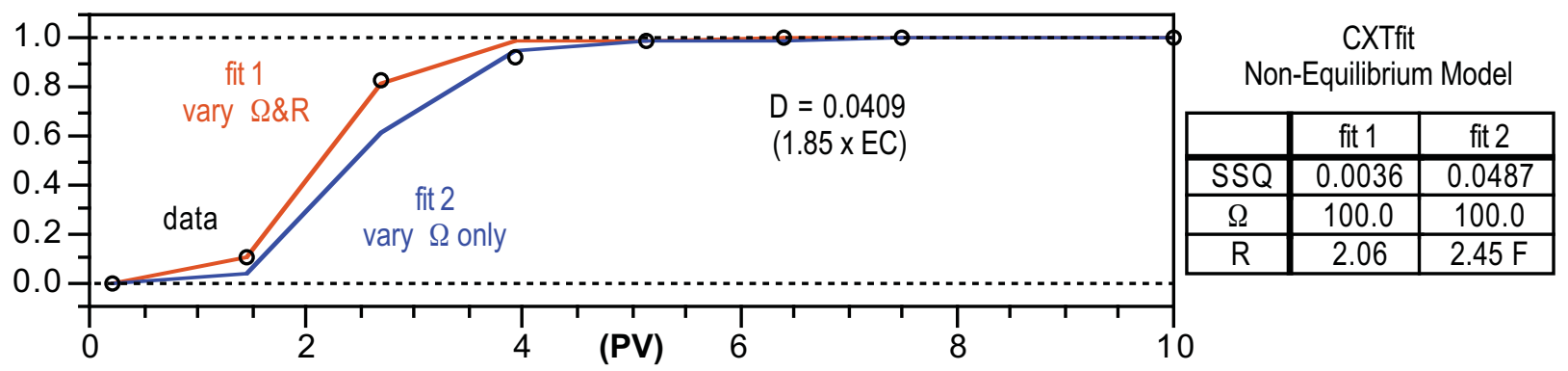

QE: RDX BTC during 1-D flow on West. (Sorb Phase)

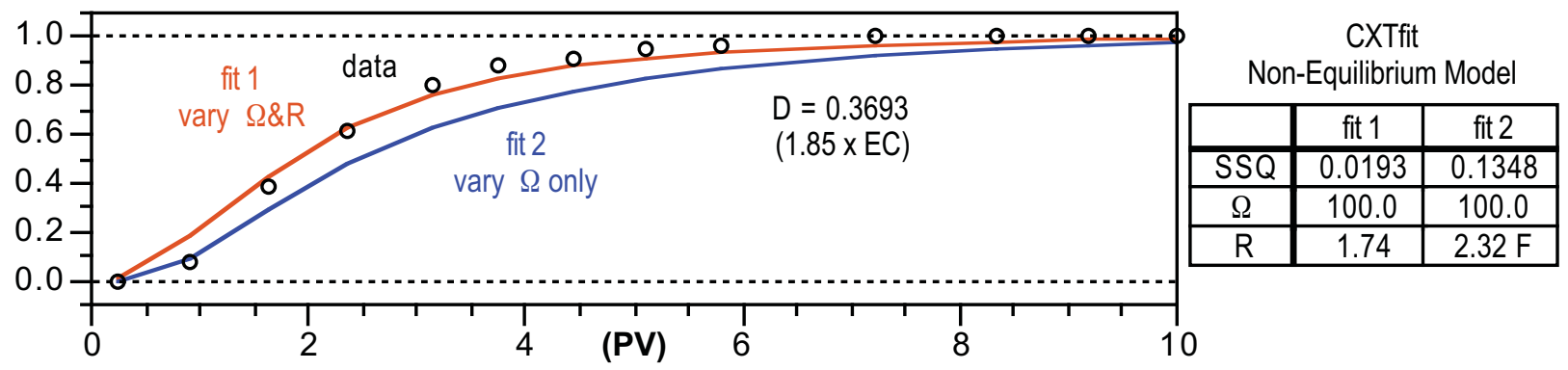

QG: RDX BTC during 1-D flow on West. (Sorb Phase)

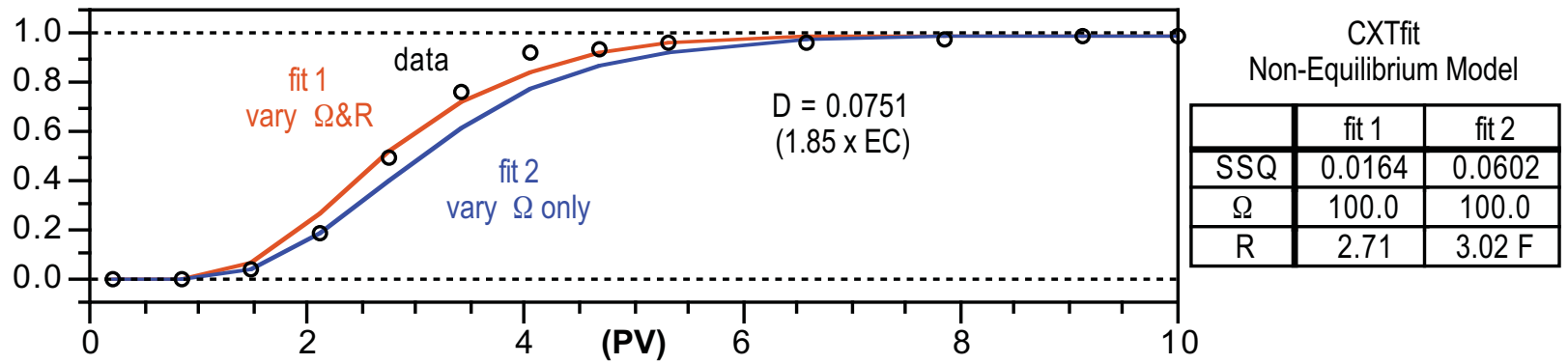

QI: RDX BTC during 1-D flow on West. (Sorb Phase)

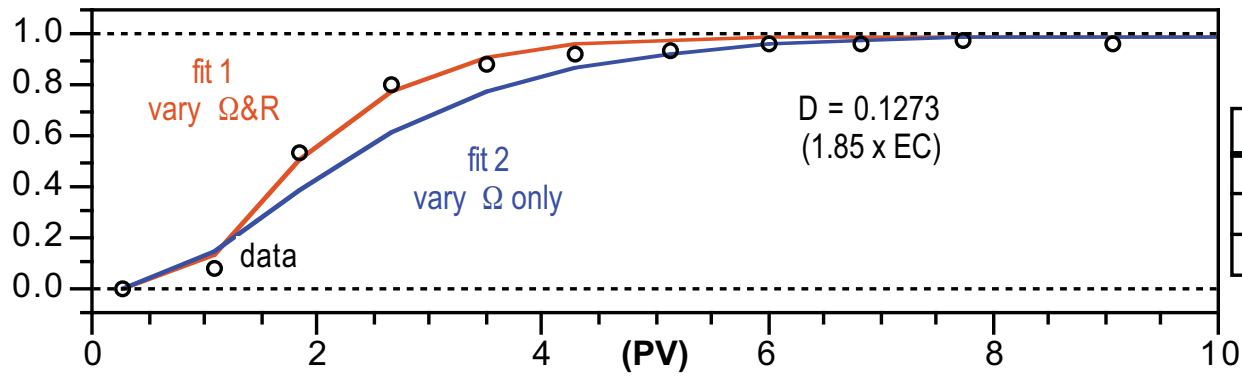

CXTfit

Non-Equilibrium Model

\begin{tabular}{|c|c|c|}
\hline & fit 1 & fit 2 \\
\hline$S S Q$ & 0.0144 & 0.0836 \\
\hline$\Omega$ & 100.0 & 4.14 \\
\hline$R$ & 1.82 & $2.28 \mathrm{~F}$ \\
\hline
\end{tabular}


QK: RDX BTC during 1-D flow on West. (Sorb Phase)

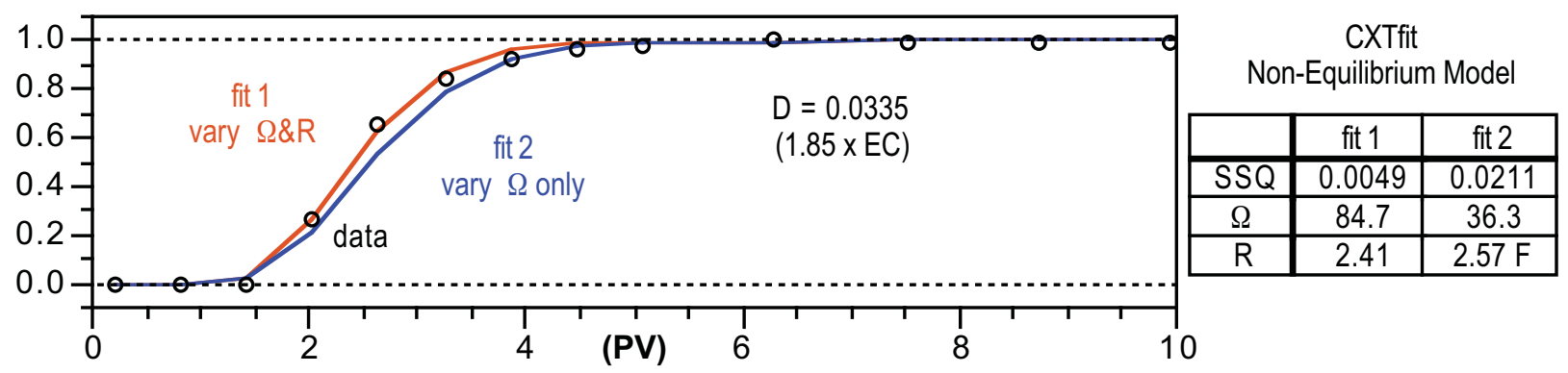

QM: RDX BTC during 1-D flow on West. (Sorb Phase)

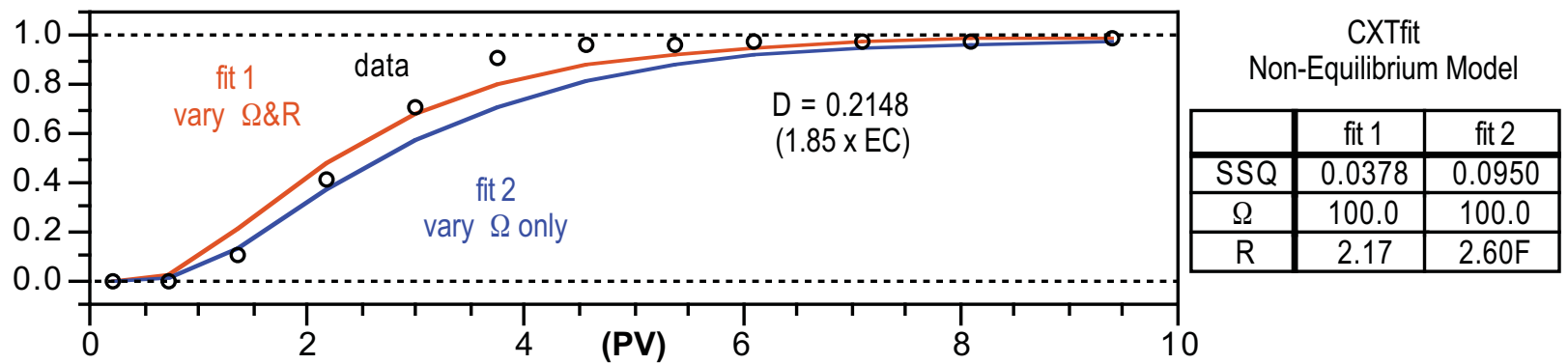

QD: RDX BTC during 1-D flow on West. (Desorb Phase) after $1 \mathrm{~h}$ aging

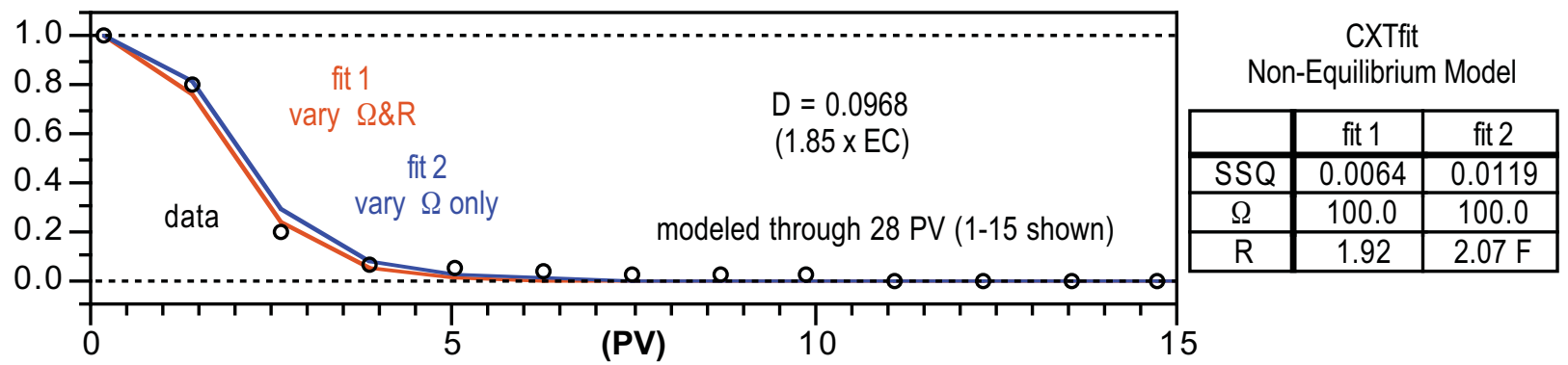

QE: RDX BTC during 1-D flow on West. (Desorb Phase) after $1 \mathrm{~h}$ aging

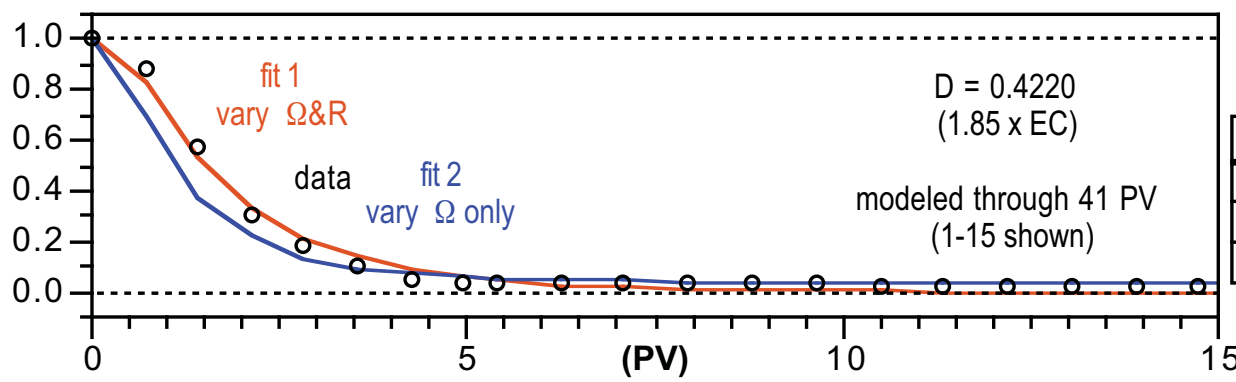

Non-Equilibrium Model

\begin{tabular}{|c|c|c|}
\hline & fit 1 & fit 2 \\
\hline SSQ & 0.0150 & 0.0960 \\
\hline$\Omega$ & 100.0 & 3.34 \\
\hline$R$ & 1.41 & $2.15 \mathrm{~F}$ \\
\hline
\end{tabular}


QG: RDX BTC during 1-D flow on West. (Desorb Phase) after 112h aging

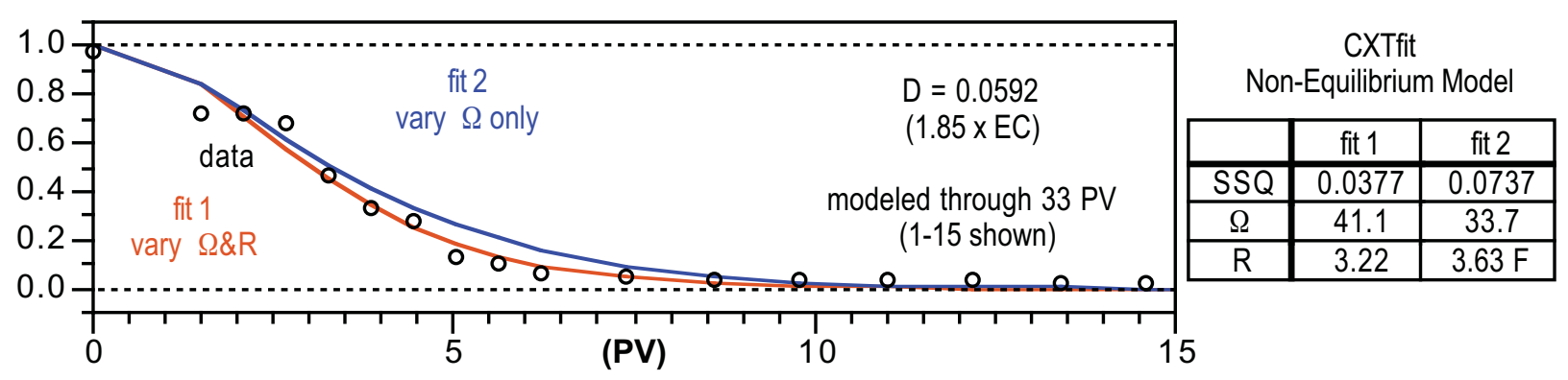

QI: RDX BTC during 1-D flow on West. (Desorb Phase) after 737h aging

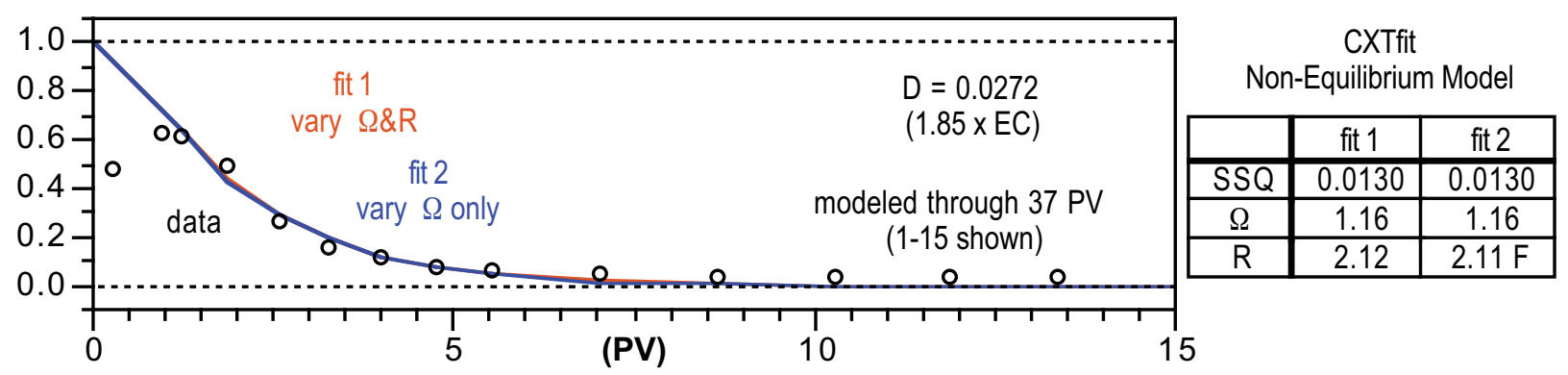

QK: RDX BTC during 1-D flow on West. (Desorb Phase) after 2174h aging

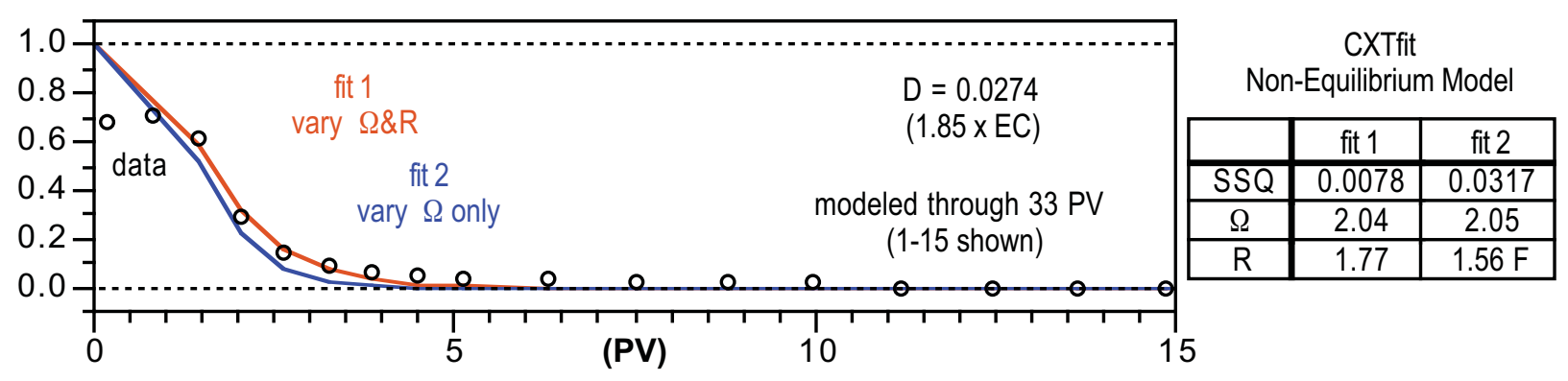

QM: RDX BTC during 1-D flow on West. (Desorb Phase) after 2254h aging

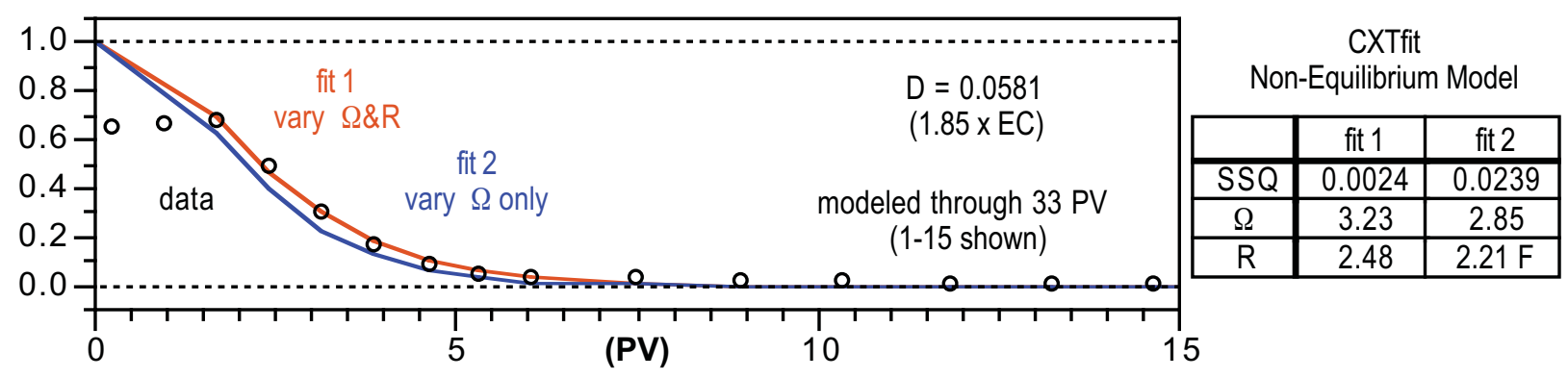




\section{Appendix I.8; RDX BTC Fitting on Sassafrass Column Aging Experiments}

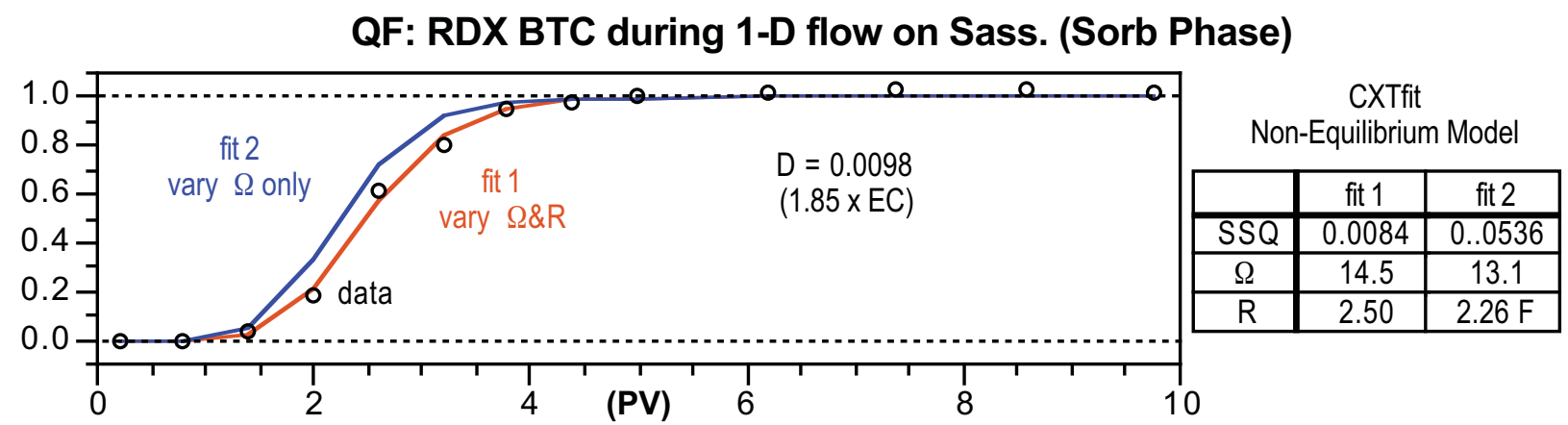

QH: RDX BTC during 1-D flow on Sass. (Sorb Phase)

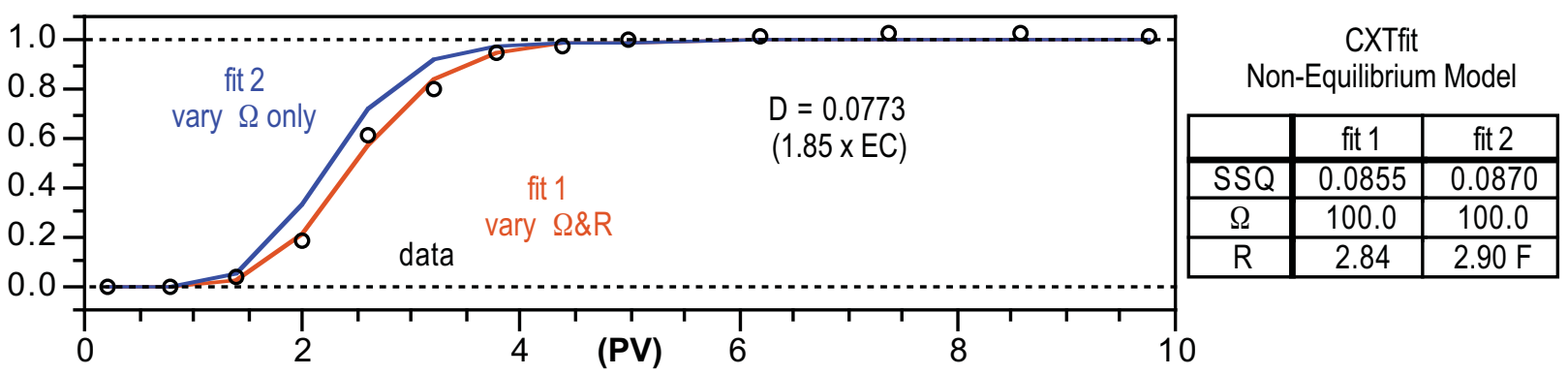

\section{QJ: RDX BTC during 1-D flow on Sass. (Sorb Phase)}

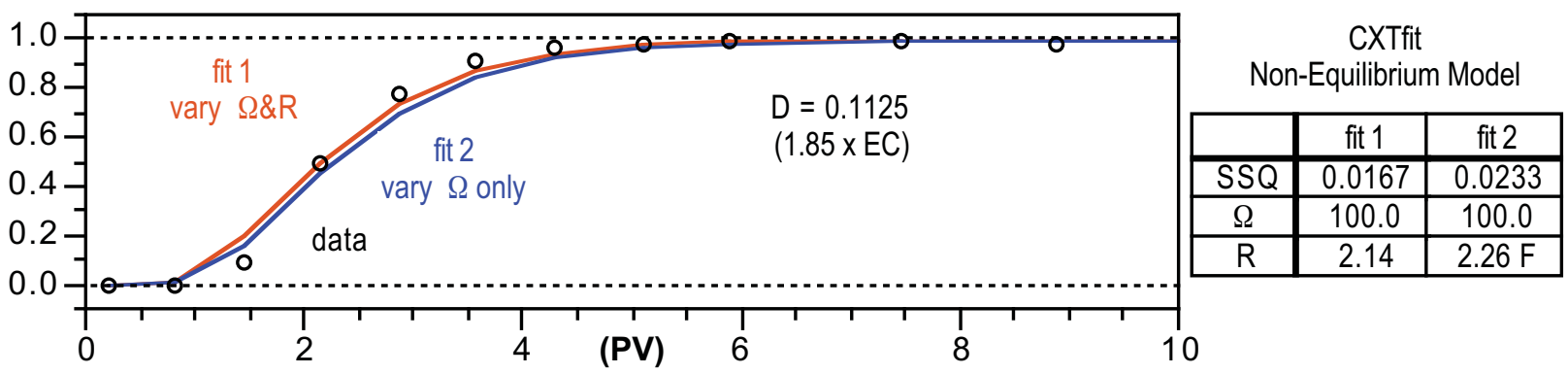

\section{QL: RDX BTC during 1-D flow on Sass. (Sorb Phase)}

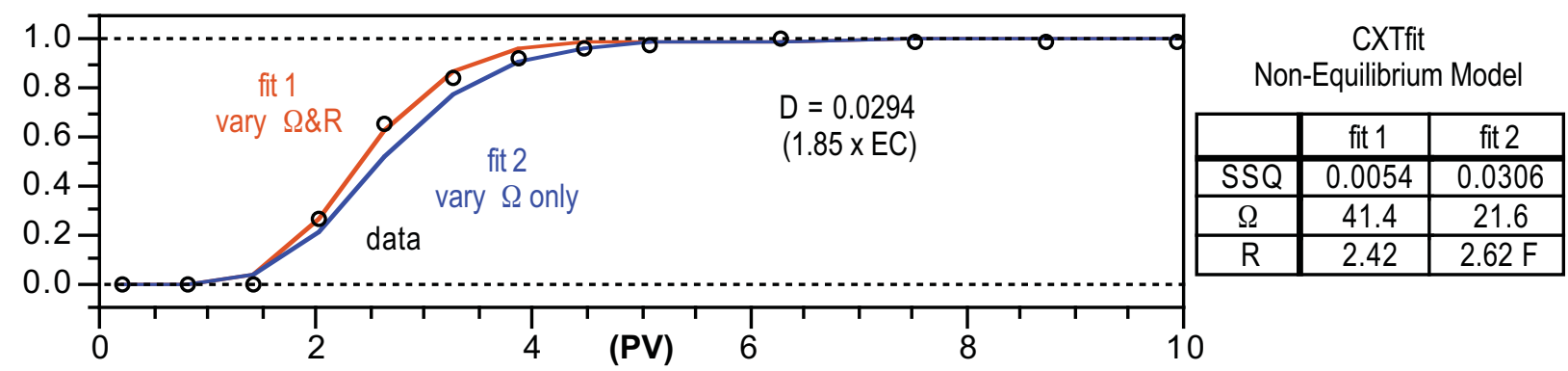


QN: RDX BTC during 1-D flow on Sass. (Sorb Phase)

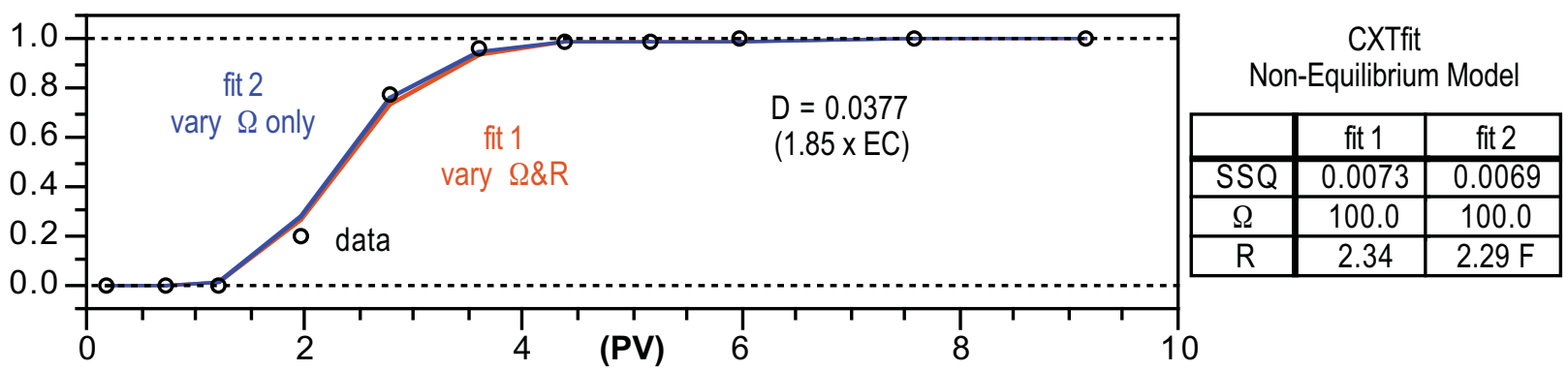

QF: RDX BTC during 1-D flow on Sass. (Desorb Phase) after $1 \mathrm{~h}$ aging

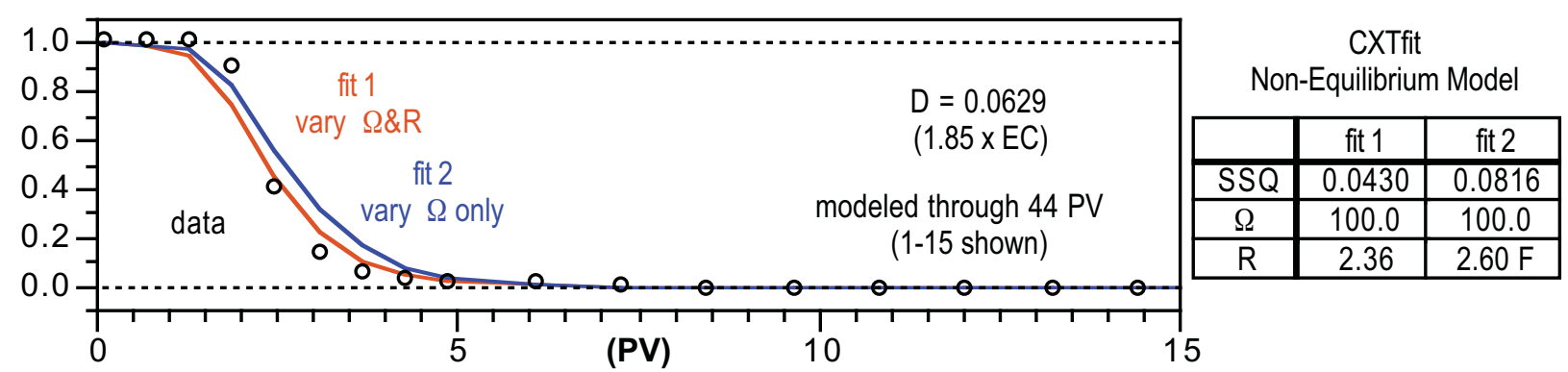

QH: RDX BTC during 1-D flow on Sass. (Desorb Phase) after 112h aging

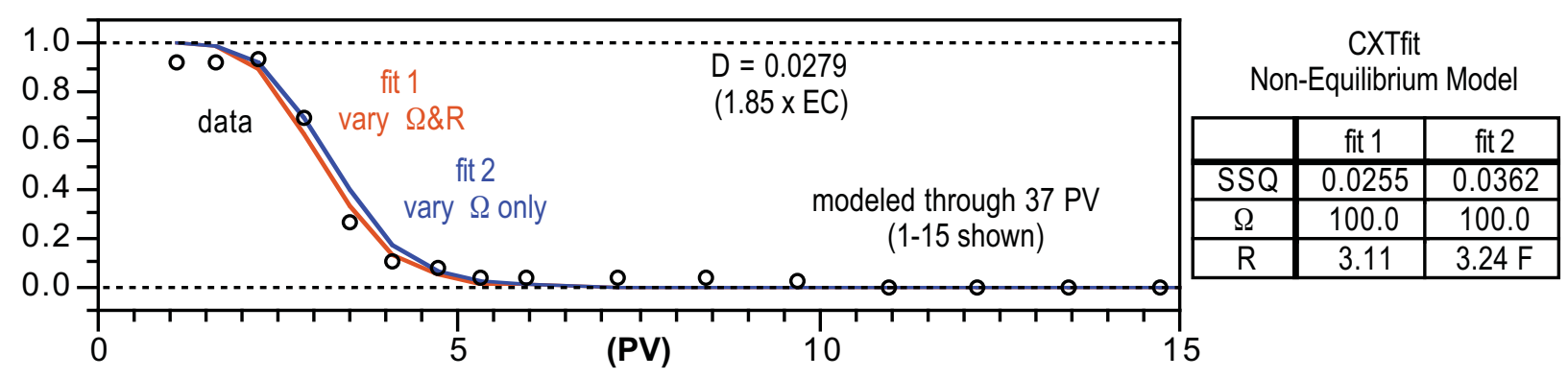

QJ: RDX BTC during 1-D flow on Sass. (Desorb Phase) after 737h aging

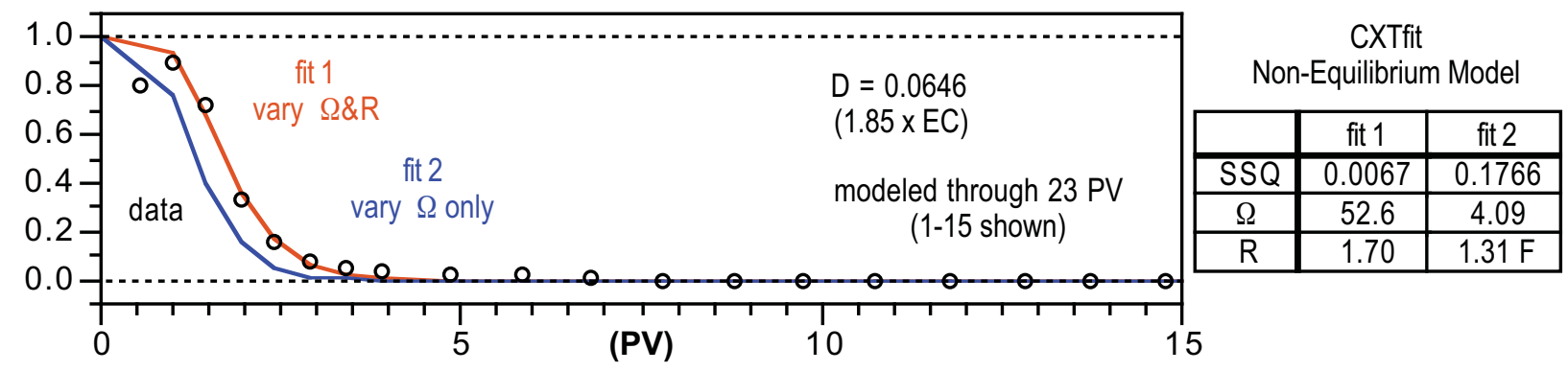


QL: RDX BTC during 1-D flow on Sass. (Desorb Phase) after 2174h aging

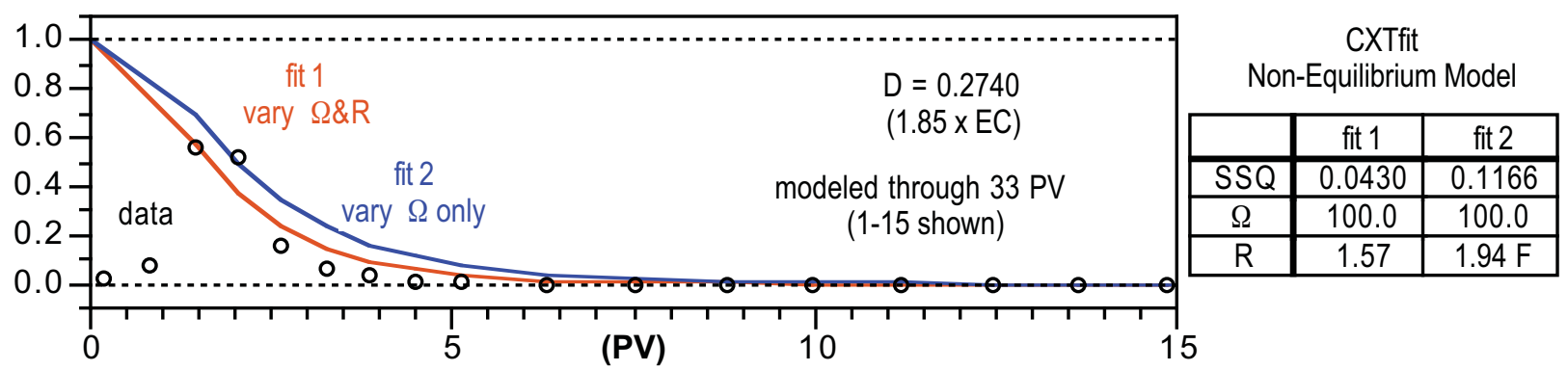

QN: RDX BTC during 1-D flow on Sass. (Desorb Phase) after 2254h aging

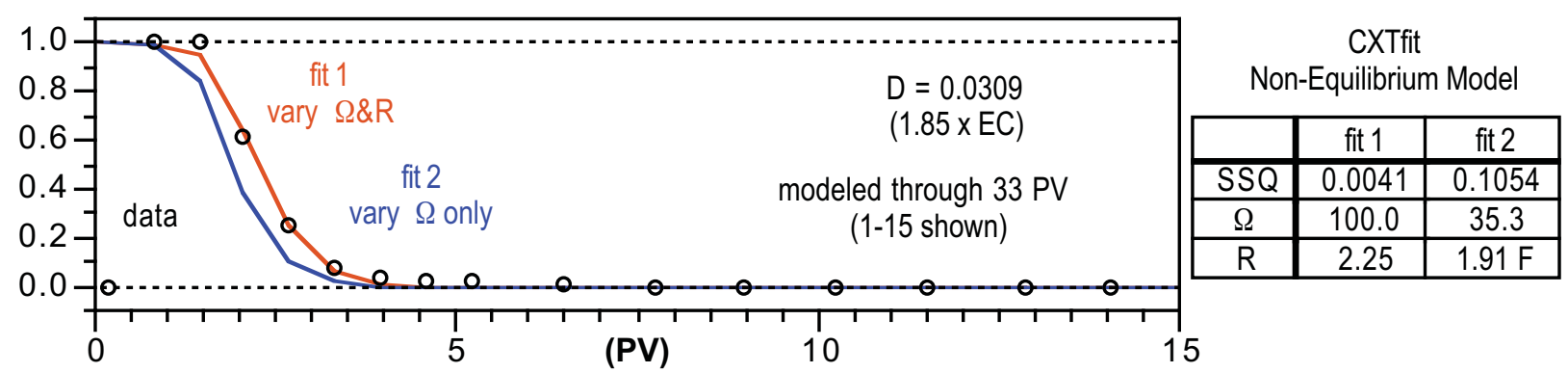


Appendix I.9; CL-20 BTC Fitting on Westmoreland A1 Column Aging Experiments

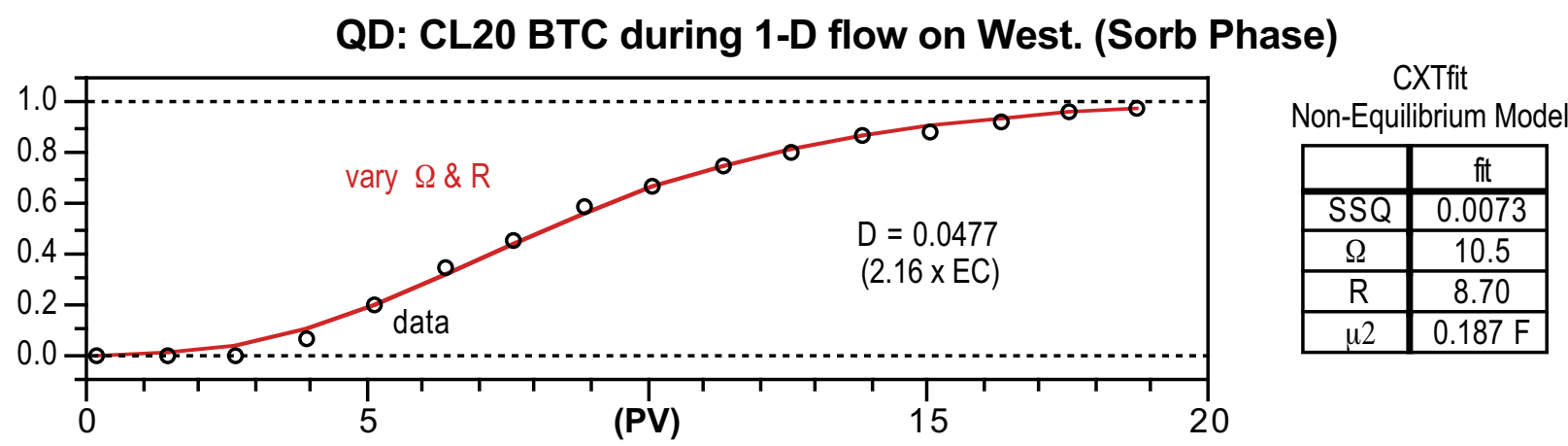

QE: CL20 BTC during 1-D flow on West. (Sorb Phase)

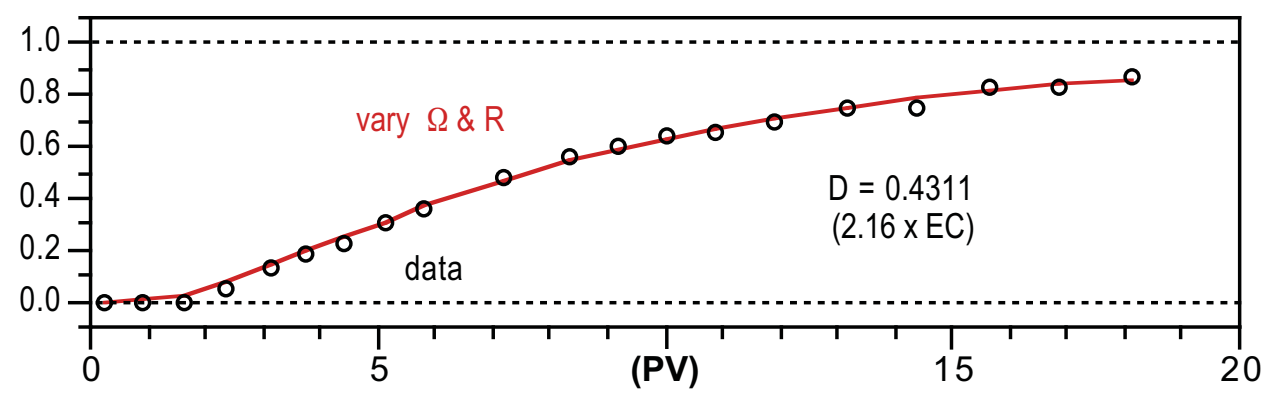

CXTfit

Non-Equilibrium Model

\begin{tabular}{|c|c|}
\hline & fit 1 \\
\hline$S S Q$ & 0.0047 \\
\hline$\Omega$ & 100.0 \\
\hline$R$ & 7.11 \\
\hline$\mu 2$ & $0.187 \mathrm{~F}$ \\
\hline
\end{tabular}

QG: CL20 BTC during 1-D flow on West. (Sorb Phase)

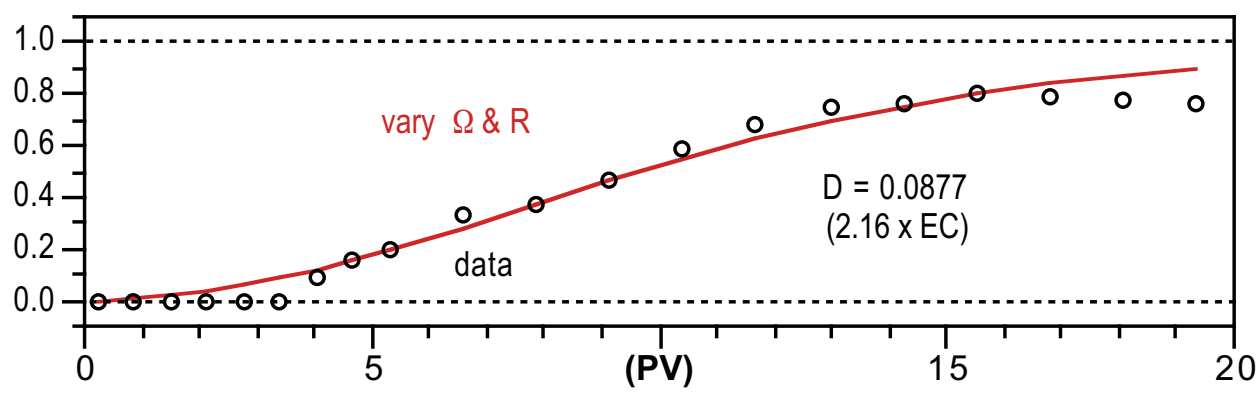

CXTfit

Non-Equilibrium Model

\begin{tabular}{|c|c|}
\hline & fit 1 \\
\hline SSQ & 0.0522 \\
\hline$\Omega$ & 8.36 \\
\hline$R$ & 10.4 \\
\hline$\mu 2$ & $0.187 \mathrm{~F}$ \\
\hline
\end{tabular}

QI: CL20 BTC during 1-D flow on West. (Sorb Phase)

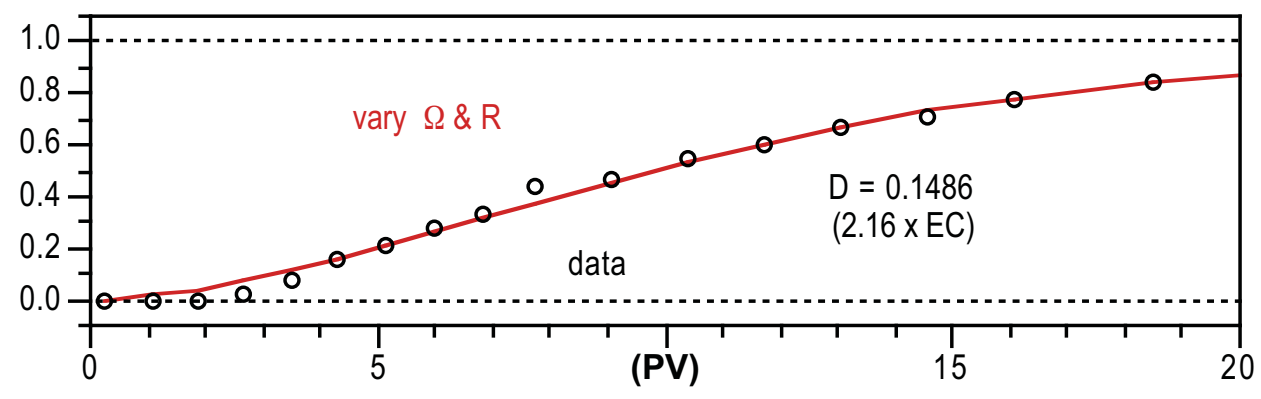

CXTfit Non-Equilibrium Model

\begin{tabular}{|c|c|}
\hline & fit 1 \\
\hline SSQ & 0.0107 \\
\hline$\Omega$ & 8.23 \\
\hline$R$ & 10.2 \\
\hline$\mu 2$ & $0.187 \mathrm{~F}$ \\
\hline
\end{tabular}




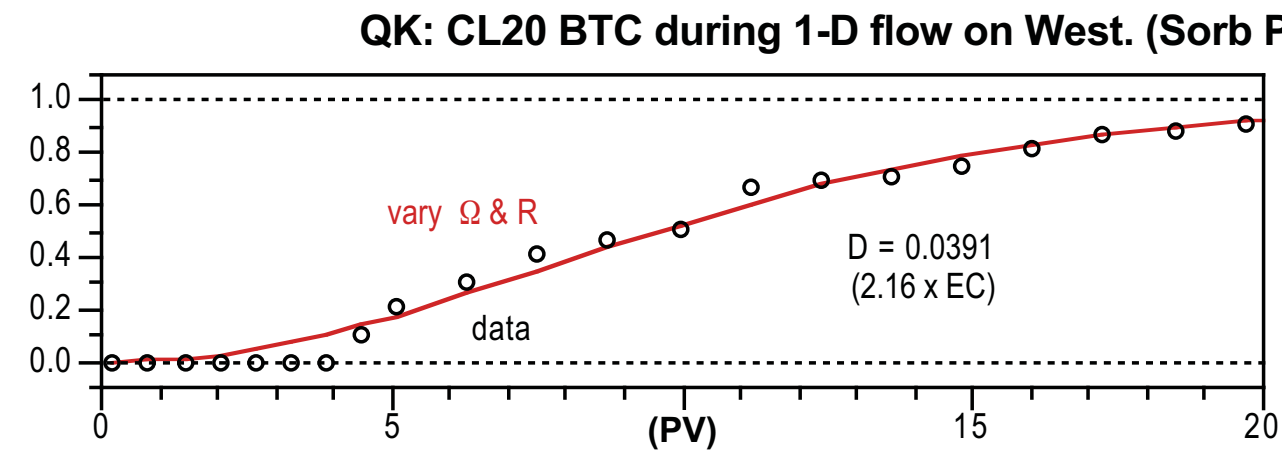

Phase)

CXTfit

Non-Equilibrium Model

\begin{tabular}{|c|c|}
\hline & fit \\
\hline$S S Q$ & 0.0396 \\
\hline$\Omega$ & 6.86 \\
\hline$R$ & 10.60 \\
\hline$\mu 2$ & $0.187 \mathrm{~F}$ \\
\hline
\end{tabular}

QM: CL20 BTC during 1-D flow on West. (Sorb Phase)

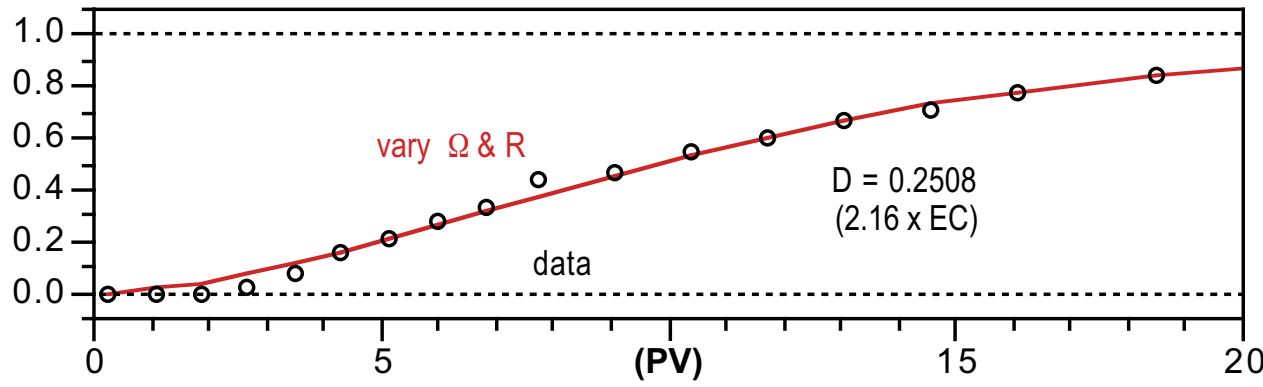

CXTfit

Non-Equilibrium Model

\begin{tabular}{|c|c|}
\hline & fit \\
\hline$S S Q$ & 0.0133 \\
\hline$\Omega$ & 100.0 \\
\hline$R$ & 9.09 \\
\hline$\mu 2$ & $0.187 \mathrm{~F}$ \\
\hline
\end{tabular}

QD: CL20 BTC during 1-D flow on West. (Desorb Phase) after $1 \mathrm{~h}$ aging

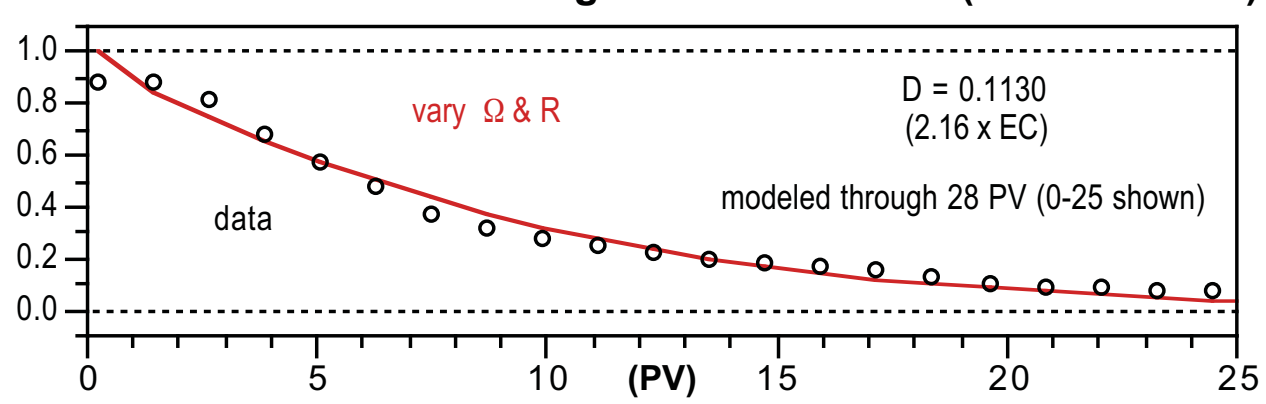

CXTfit Non-Equilibrium Model

\begin{tabular}{|c|c|}
\hline & fit \\
\hline$S S Q$ & 0.0028 \\
\hline$\Omega$ & 2.51 \\
\hline$R$ & 8.90 \\
\hline$\mu 2$ & $0.187 \mathrm{~F}$ \\
\hline
\end{tabular}

QE: CL20 BTC during 1-D flow on West. (Desorb Phase) after 1h aging

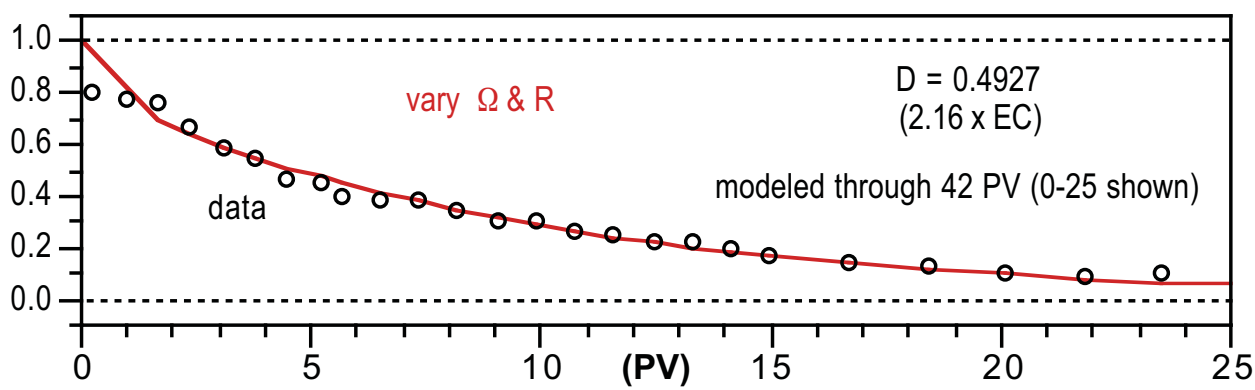

Non-Equilibrium Model

\begin{tabular}{|c|c|}
\hline & fit \\
\hline$S S Q$ & 0.0034 \\
\hline$\Omega$ & 1.46 \\
\hline$R$ & 7.16 \\
\hline$\mu 2$ & $0.187 \mathrm{~F}$ \\
\hline
\end{tabular}


QG: CL20 BTC during 1-D flow on West. (Desorb Phase) after 112h aging

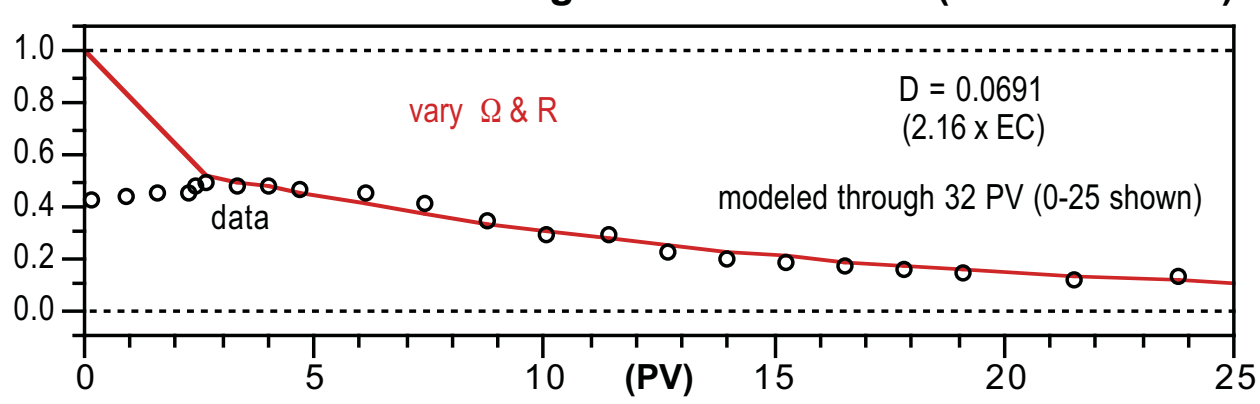

CXTfit

Non-Equilibrium Model

\begin{tabular}{|c|c|}
\hline & fit \\
\hline$S S Q$ & 0.0094 \\
\hline$\Omega$ & 0.886 \\
\hline$R$ & 10.9 \\
\hline$\mu 2$ & $0.187 \mathrm{~F}$ \\
\hline
\end{tabular}

QI: CL20 BTC during 1-D flow on West. (Desorb Phase) after 737h aging

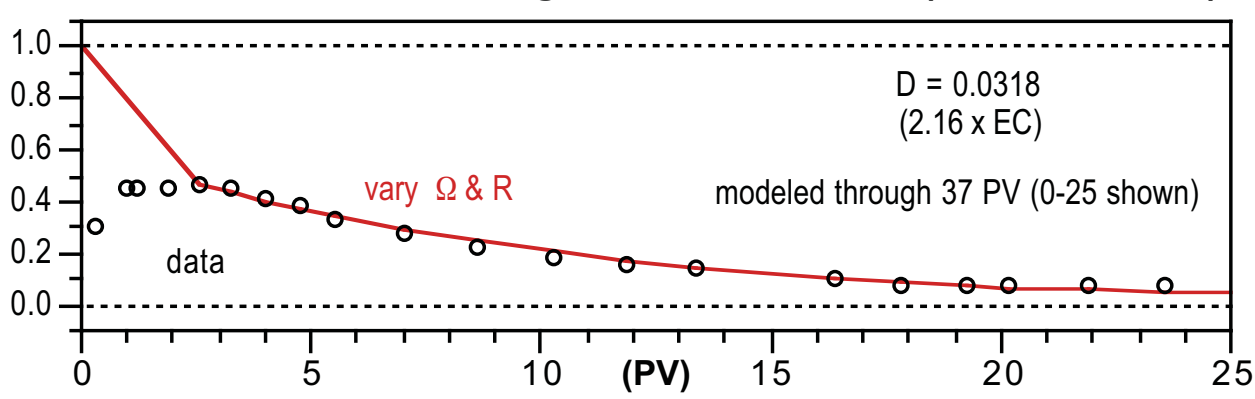

CXTfit

Non-Equilibrium Model

\begin{tabular}{|c|c|}
\hline & fit \\
\hline$S S Q$ & 0.0048 \\
\hline$\Omega$ & 0.812 \\
\hline$R$ & 7.67 \\
\hline$\mu 2$ & $0.187 \mathrm{~F}$ \\
\hline
\end{tabular}

QK: CL20 BTC during 1-D flow on West. (Desorb Phase) after 2174h aging

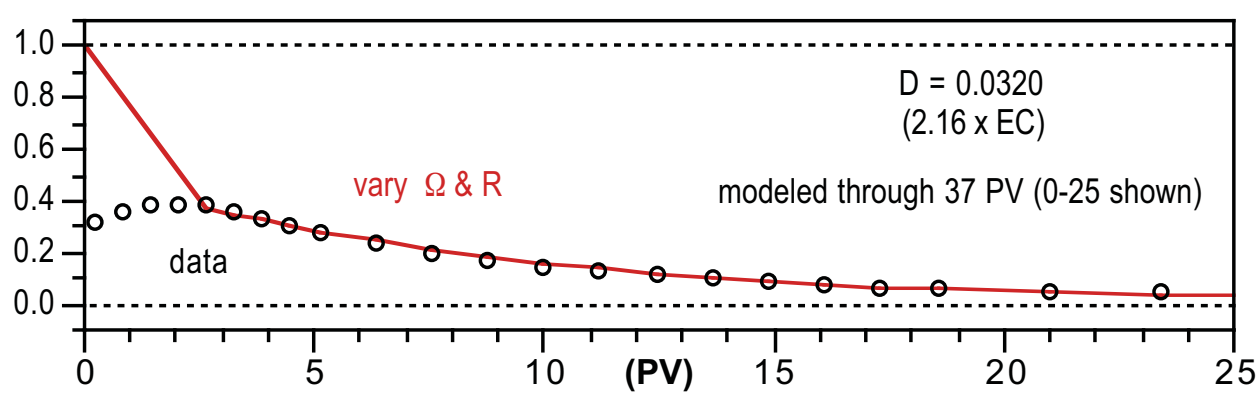

CXTfit

Non-Equilibrium Model

\begin{tabular}{|c|c|}
\hline & fit \\
\hline$S S Q$ & 0.0026 \\
\hline$\Omega$ & 0.610 \\
\hline$R$ & 6.34 \\
\hline$\mu 2$ & $0.187 \mathrm{~F}$ \\
\hline
\end{tabular}

QM: CL20 BTC during 1-D flow on West. (Desorb Phase) after 2254h aging

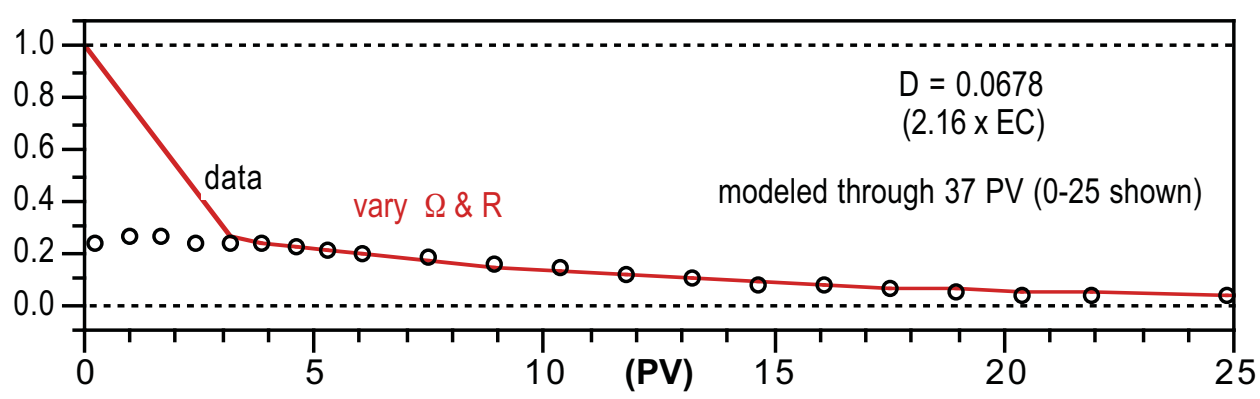

Non-Equilibrium Model

\begin{tabular}{|c|c|}
\hline & fit \\
\hline$S S Q$ & 0.0017 \\
\hline$\Omega$ & 0.368 \\
\hline$R$ & 6.12 \\
\hline$\mu 2$ & $0.187 \mathrm{~F}$ \\
\hline
\end{tabular}




\section{Appendix I.10 CL-20 BTC Fitting on Sassafrass Column Aging Experiments}

QF: CL20 BTC during 1-D flow on Sass. (Sorb Phase)

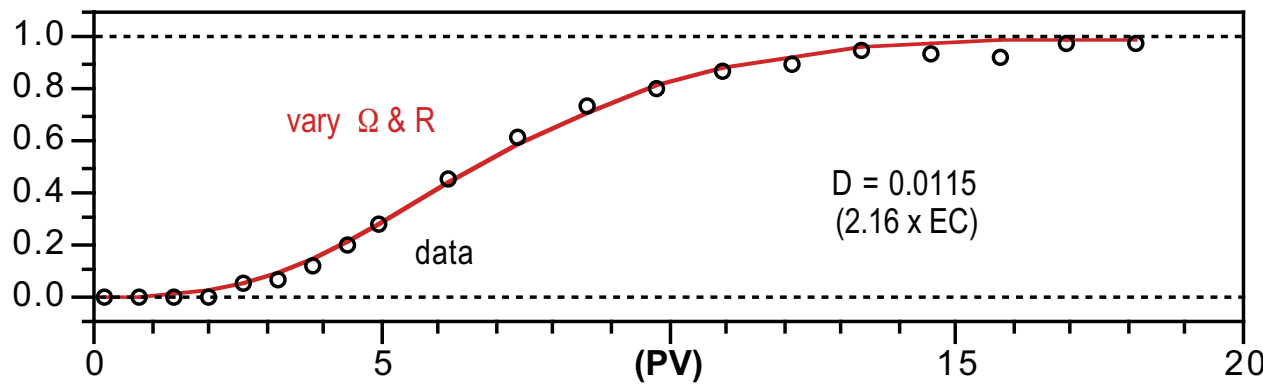

CXTfit

Non-Equilibrium Model

\begin{tabular}{|c|c|}
\hline & fit \\
\hline$S S Q$ & 0.0098 \\
\hline$\Omega$ & 8.30 \\
\hline$R$ & 7.07 \\
\hline$\mu 2$ & $0.0557 \mathrm{~F}$ \\
\hline
\end{tabular}

QH: CL20 BTC during 1-D flow on Sass. (Sorb Phase)

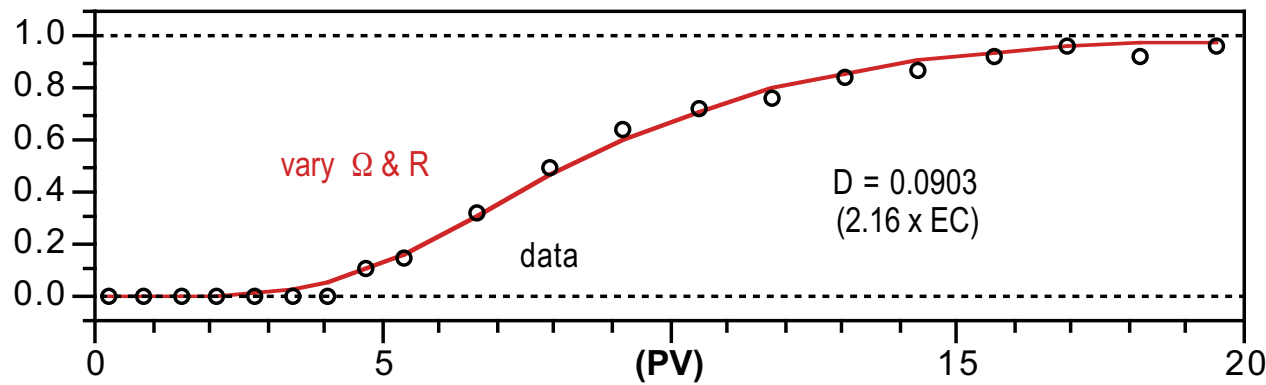

CXTfit

Non-Equilibrium Model

\begin{tabular}{|c|c|}
\hline & fit \\
\hline SSQ & 0.0126 \\
\hline$\Omega$ & 100.0 \\
\hline$R$ & 8.21 \\
\hline$\mu 2$ & $0.0557 \mathrm{~F}$ \\
\hline
\end{tabular}

QJ: CL20 BTC during 1-D flow on Sass. (Sorb Phase)

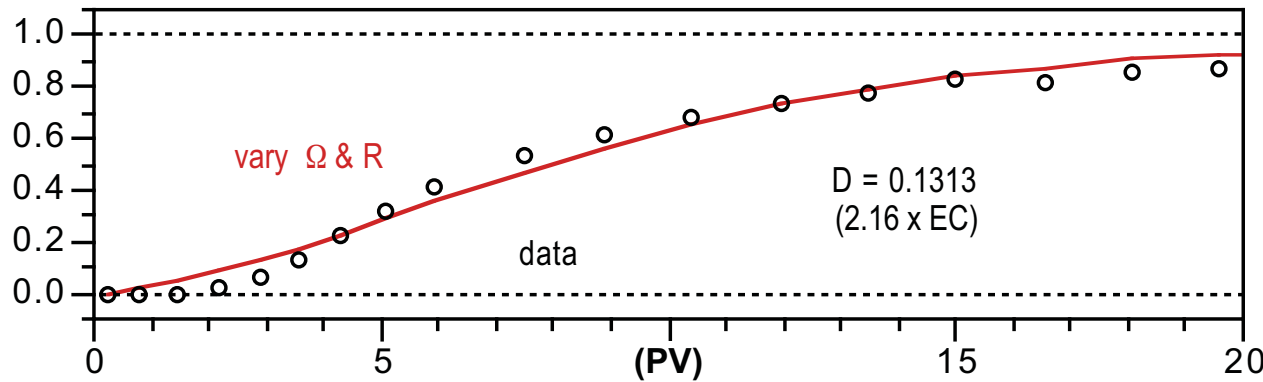

CXTfit

Non-Equilibrium Mode

\begin{tabular}{|c|c|}
\hline & fit \\
\hline$S S Q$ & 0.0417 \\
\hline$\Omega$ & 6.01 \\
\hline$R$ & 8.26 \\
\hline$\mu 2$ & $0.0557 \mathrm{~F}$ \\
\hline
\end{tabular}

QL: CL20 BTC during 1-D flow on Sass. (Sorb Phase)

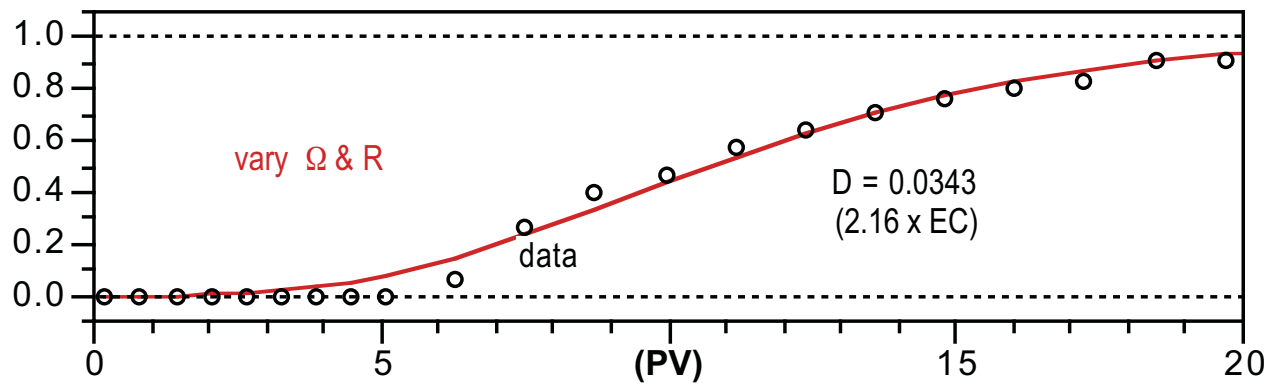

CXTfit

Non-Equilibrium Model

\begin{tabular}{|c|c|}
\hline & fit \\
\hline SSQ & 0.0336 \\
\hline$\Omega$ & 13.0 \\
\hline$R$ & 11.1 \\
\hline$\mu 2$ & $0.0557 \mathrm{~F}$ \\
\hline
\end{tabular}


QN: CL20 BTC during 1-D flow on Sass. (Sorb Phase)

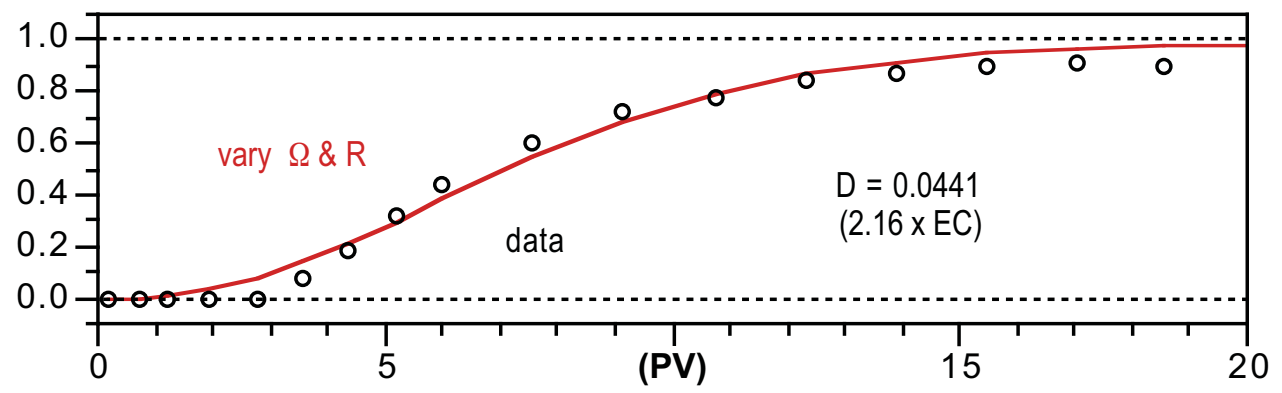

CXTfit

Non-Equilibrium Model

\begin{tabular}{|c|c|}
\hline & fit \\
\hline$S S Q$ & 0.0507 \\
\hline$\Omega$ & 7.53 \\
\hline$R$ & 7.51 \\
\hline$\mu 2$ & $0.0557 \mathrm{~F}$ \\
\hline
\end{tabular}

QF: CL20 BTC during 1-D flow on Sass. (Desorb Phase) after $1 \mathrm{~h}$ aging

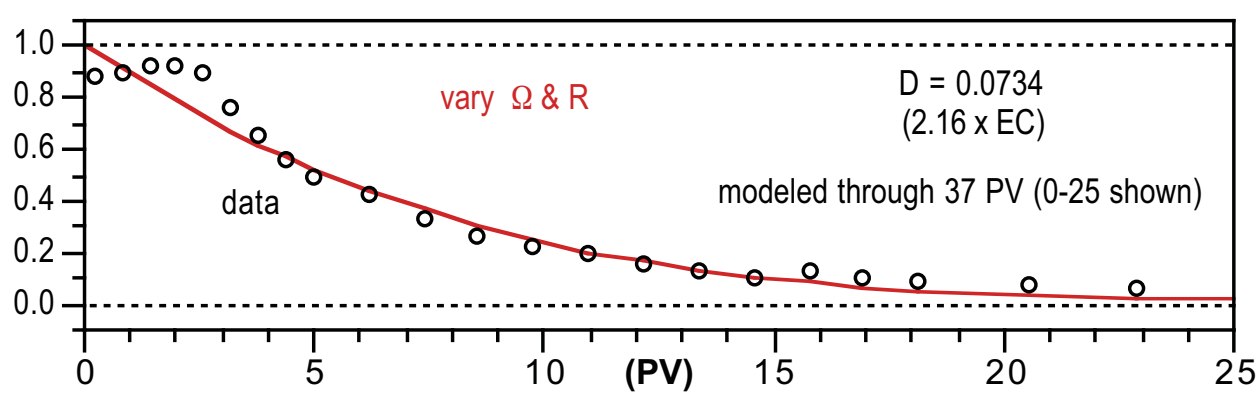

CXTfit

Non-Equilibrium Model

\begin{tabular}{|c|c|}
\hline & fit \\
\hline$S S Q$ & 0.0288 \\
\hline$\Omega$ & 2.31 \\
\hline$R$ & 6.77 \\
\hline$\mu 2$ & $0.0557 \mathrm{~F}$ \\
\hline
\end{tabular}

QH: CL20 BTC during 1-D flow on Sass. (Desorb Phase) after 112h aging

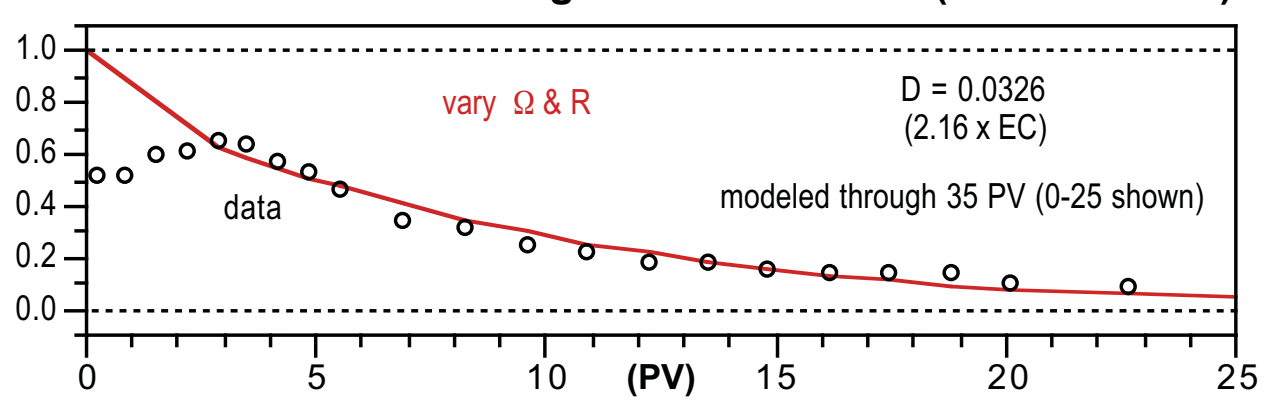

CXTfit

Non-Equilibrium Model

\begin{tabular}{|c|c|}
\hline & fit \\
\hline$S S Q$ & 0.0239 \\
\hline$\Omega$ & 1.48 \\
\hline$R$ & 7.89 \\
\hline$\mu 2$ & $0.0557 \mathrm{~F}$ \\
\hline
\end{tabular}

QJ: CL20 BTC during 1-D flow on Sass. (Desorb Phase) after 737h aging

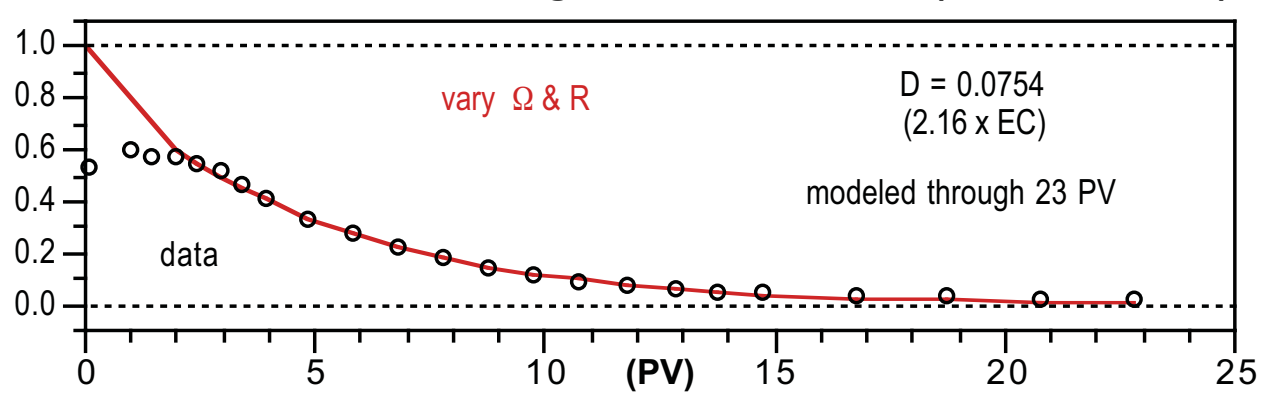

CXTfit

Non-Equilibrium Model

\begin{tabular}{|c|c|}
\hline & fit \\
\hline SSQ & 0.0025 \\
\hline$\Omega$ & 1.24 \\
\hline$R$ & 4.46 \\
\hline$\mu 2$ & $0.0557 \mathrm{~F}$ \\
\hline
\end{tabular}


QL: CL20 BTC during 1-D flow on Sass. (Desorb Phase) after 2174h aging

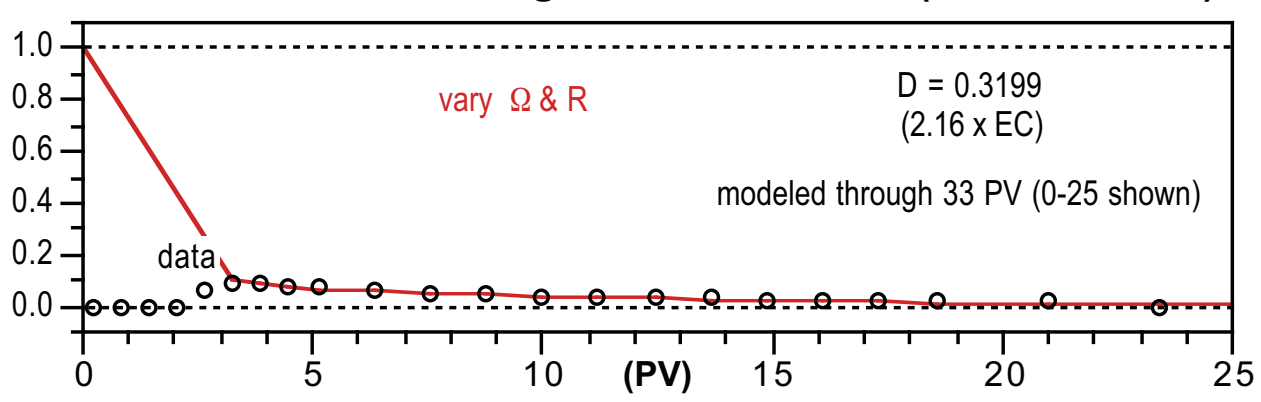

CXTfit

Non-Equilibrium Model

\begin{tabular}{|c|c|}
\hline & fit \\
\hline$S S Q$ & 0.0103 \\
\hline$\Omega$ & 0.736 \\
\hline$R$ & 2.20 \\
\hline$\mu 2$ & $0.0557 \mathrm{~F}$ \\
\hline
\end{tabular}

QN: CL20 BTC during 1-D flow on Sass. (Desorb Phase) after 2254h aging

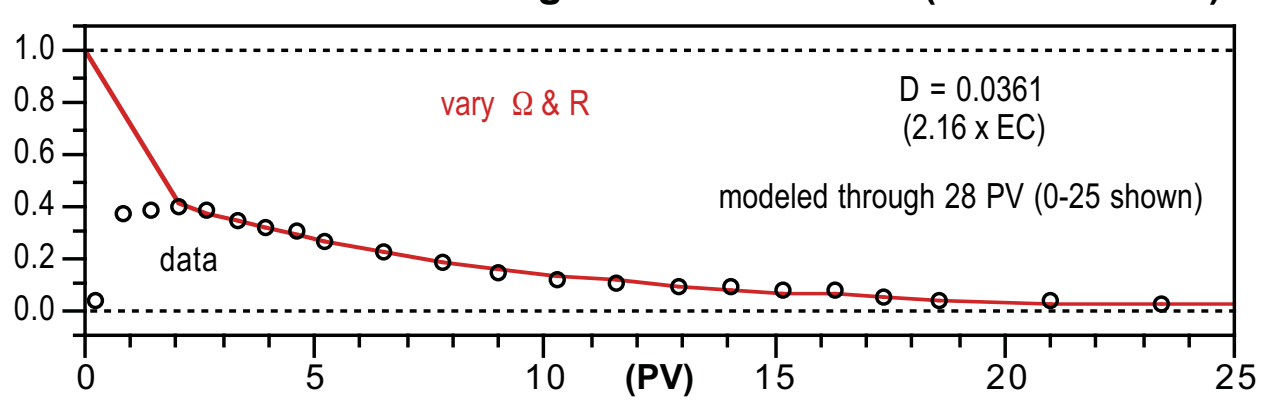

CXTfit

Non-Equilibrium Model

\begin{tabular}{|c|c|}
\hline & fit \\
\hline SSQ & 0.0014 \\
\hline$\Omega$ & 0.632 \\
\hline$R$ & 4.68 \\
\hline$\mu 2$ & $0.0557 \mathrm{~F}$ \\
\hline
\end{tabular}




\section{Appendix J1. Unsaturated Flow Experiments in the Hanford MSC sediment}

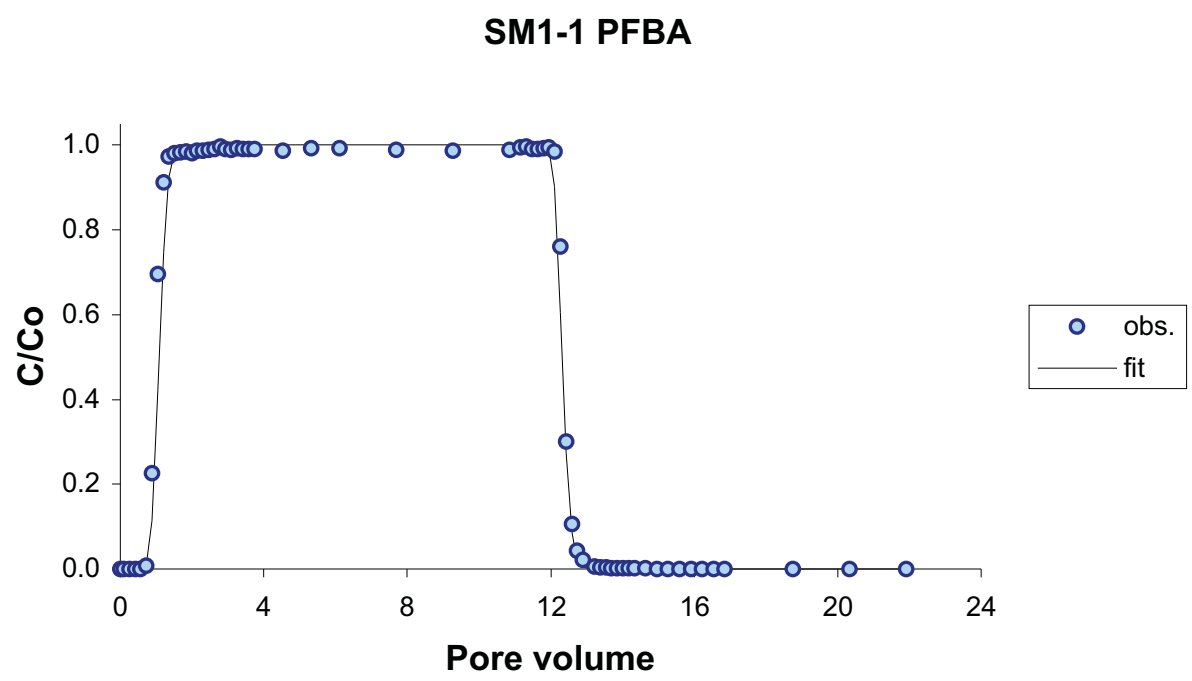

Figure J.1 Breakthrough curve and modeling result for PFBA in saturated MSC soil. $\left(\theta=0.41 ; \mathrm{v}=65.2 \mathrm{~cm} \mathrm{hr}^{-1} ; \mathrm{D}=13.3 \mathrm{hr}^{-1} ; \mathrm{R}=1.1\right)$ 


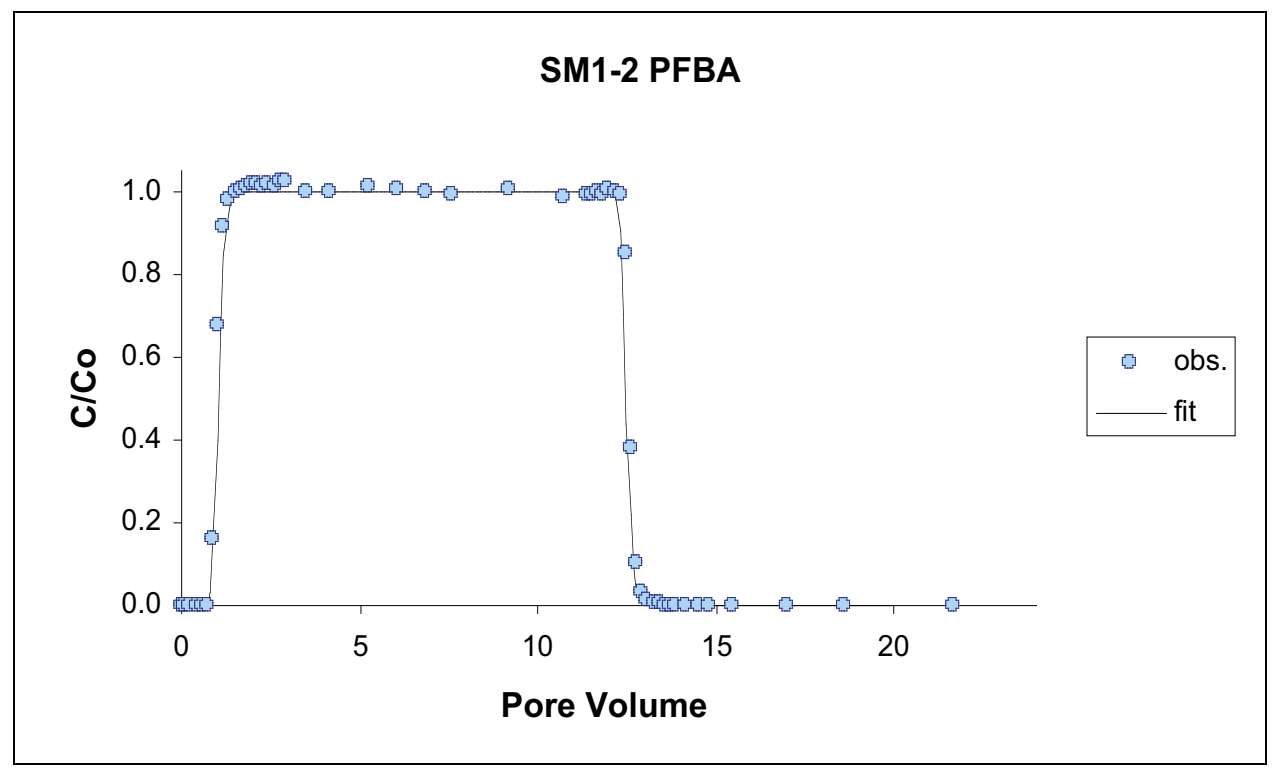

Figure J.2 Breakthrough curve and modeling result for PFBA in saturated MSC soil. $\left(\theta=0.41 ; v=8.81 \mathrm{~cm} / \mathrm{hr} ; D=1.36 \mathrm{hr}^{-1} ; R=1.1\right)$

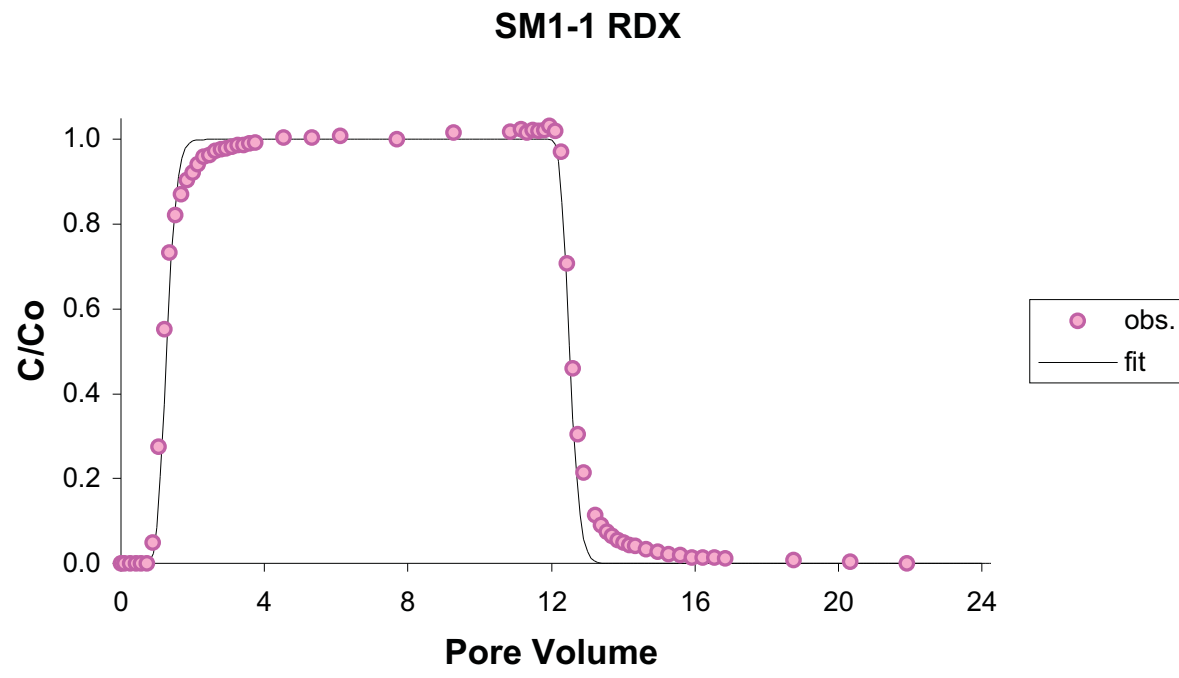

Figure J.3 Breakthrough curve and modeling results for RDX in saturated MSC.

$\left(\theta=0.41 ; v=65.2 \mathrm{~cm} \mathrm{hr}^{-1} ; D=13.3 \mathrm{hr}^{-1} ; R=1.3 ; K_{d-a p p}=0.073\right)$ 


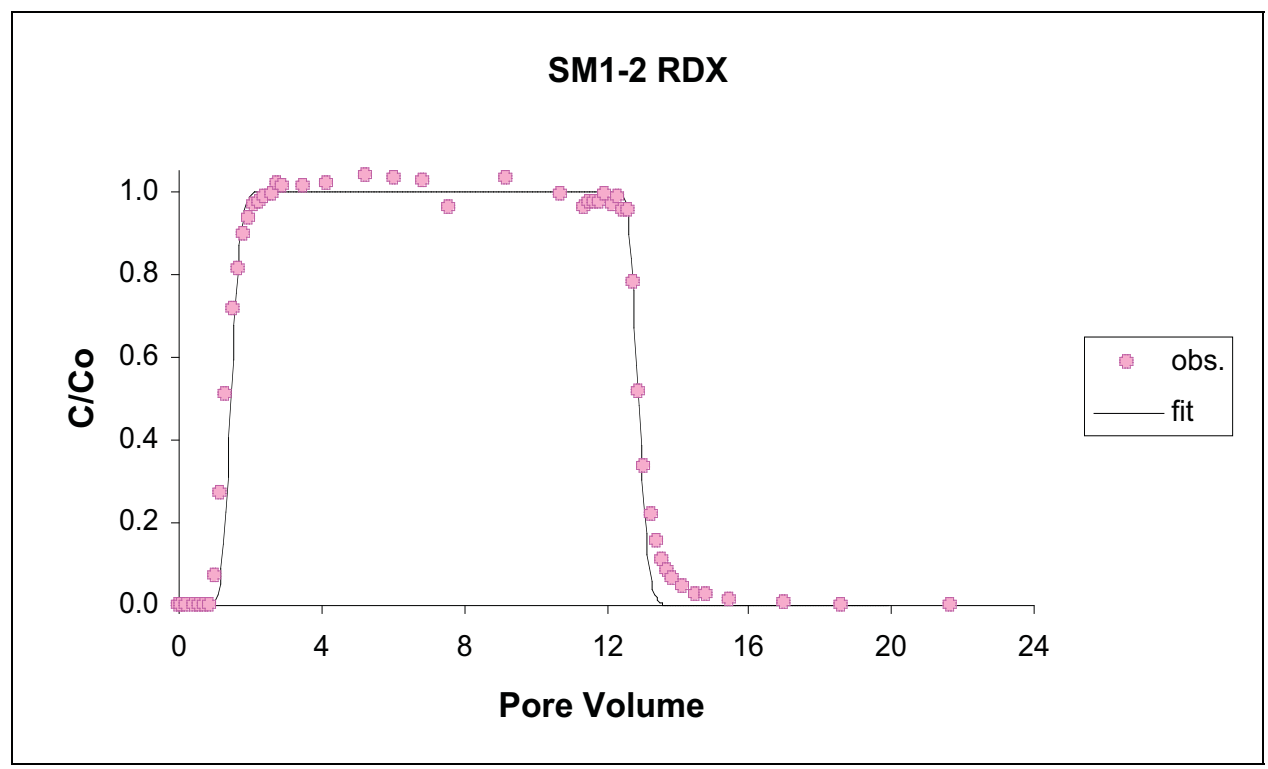

Figure J.4 Breakthrough curve and modeling results for RDX in saturated MSC. $\left(\theta=0.41 ; v=8.81 \mathrm{~cm} / \mathrm{hr} ; D=1.36 \mathrm{hr}^{-1} ; R=1.5 ; K_{d-\text { app }}=0.12\right)$

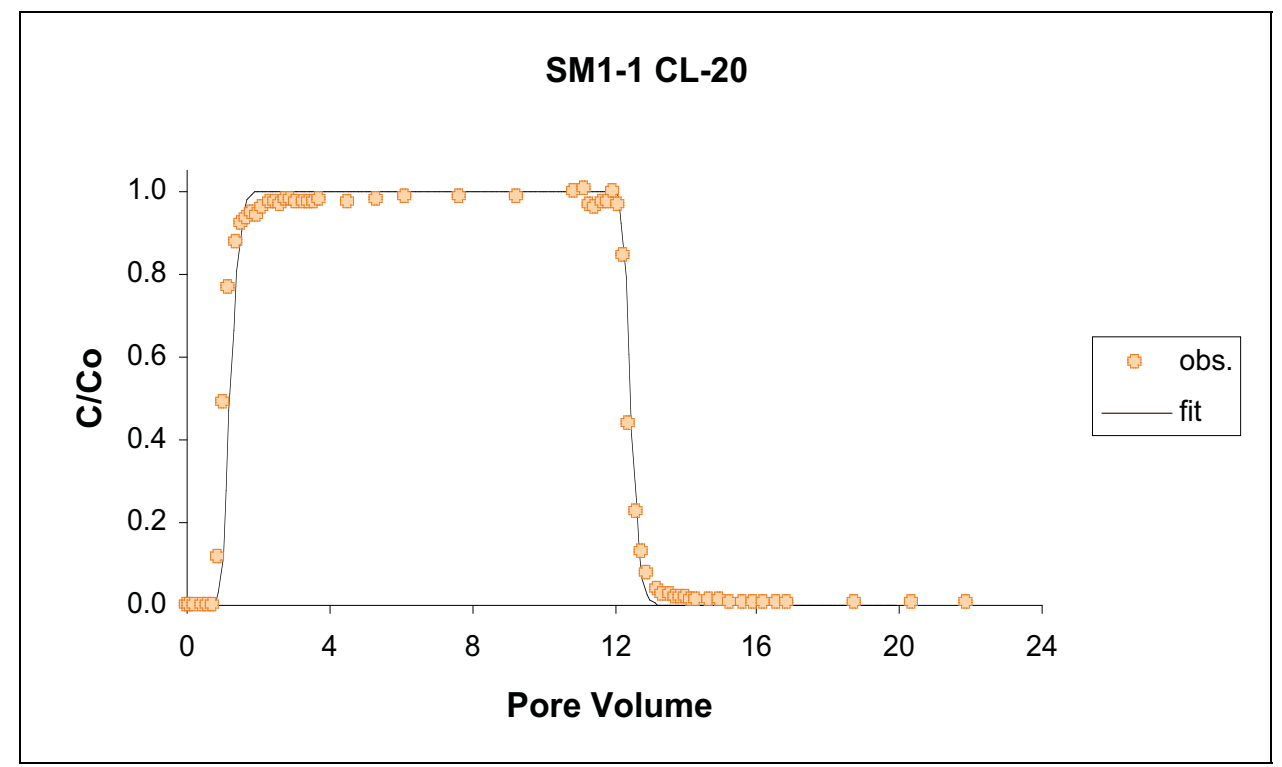

Figure J.5 Breakthrough curve and modeling results of CL-20 in saturated MSC soil. CL-20 recovery was calculated to be $101 \%$, so transport was modeled without using the degradation term.

$\left(\theta=0.41 ; v=65.2 \mathrm{~cm} \mathrm{hr}^{-1} ; D=13.3 \mathrm{hr}^{-1} ; R=1.2 ; K_{d-a p p}=0.060\right)$ 


\section{SM1-2 CL-20}

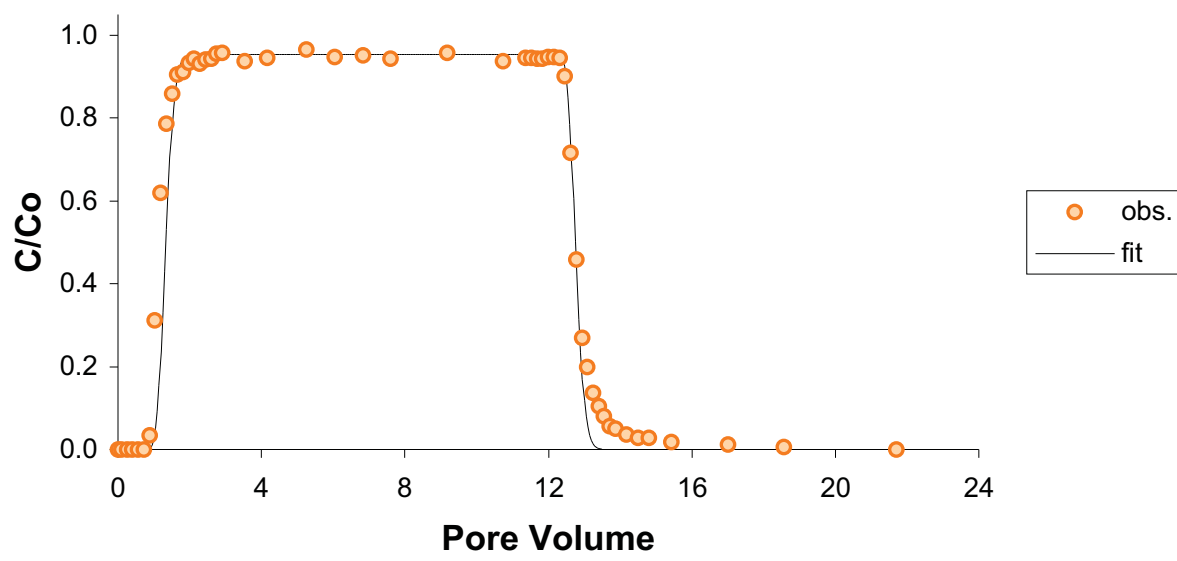

Figure J.6 Breakthrough curve and modeling results of CL-20 in saturated MSC soil. $\left(\theta=0.41 ; v=8.81 \mathrm{~cm} / \mathrm{hr} ; D=1.36 \mathrm{hr}^{-1} ; R=1.3 ; K_{d-a p p}=0.080 ; \mu_{1}=0.0435 \mathrm{hr}^{-1}\right)$

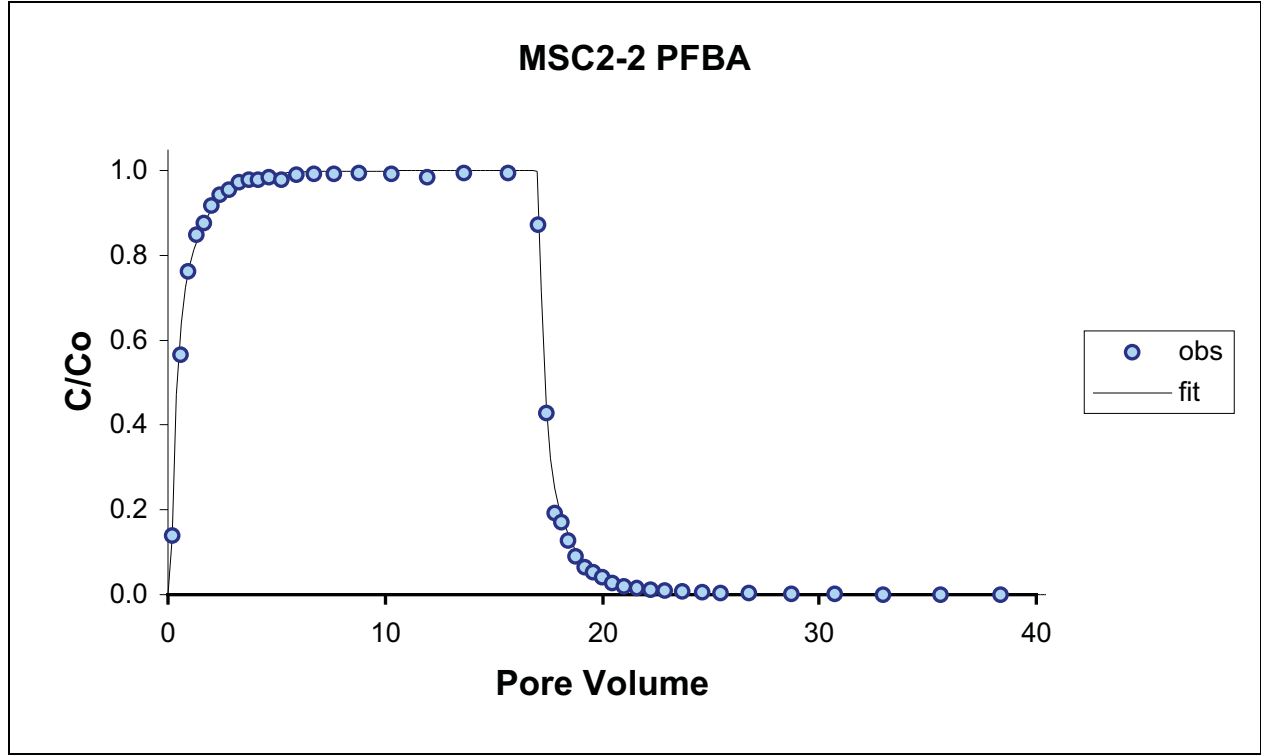

Figure J.7 Breakthrough curve and modeling results for PFBA in unsaturated MSC soil. $\left(\theta=0.11 ; v=31.3 \mathrm{~cm} / \mathrm{hr} ; D=31.75 \mathrm{hr}^{-1} ; \beta=0.517 ; \omega=0.447 \mathrm{hr}^{-1} ; R=0.78\right)$ 


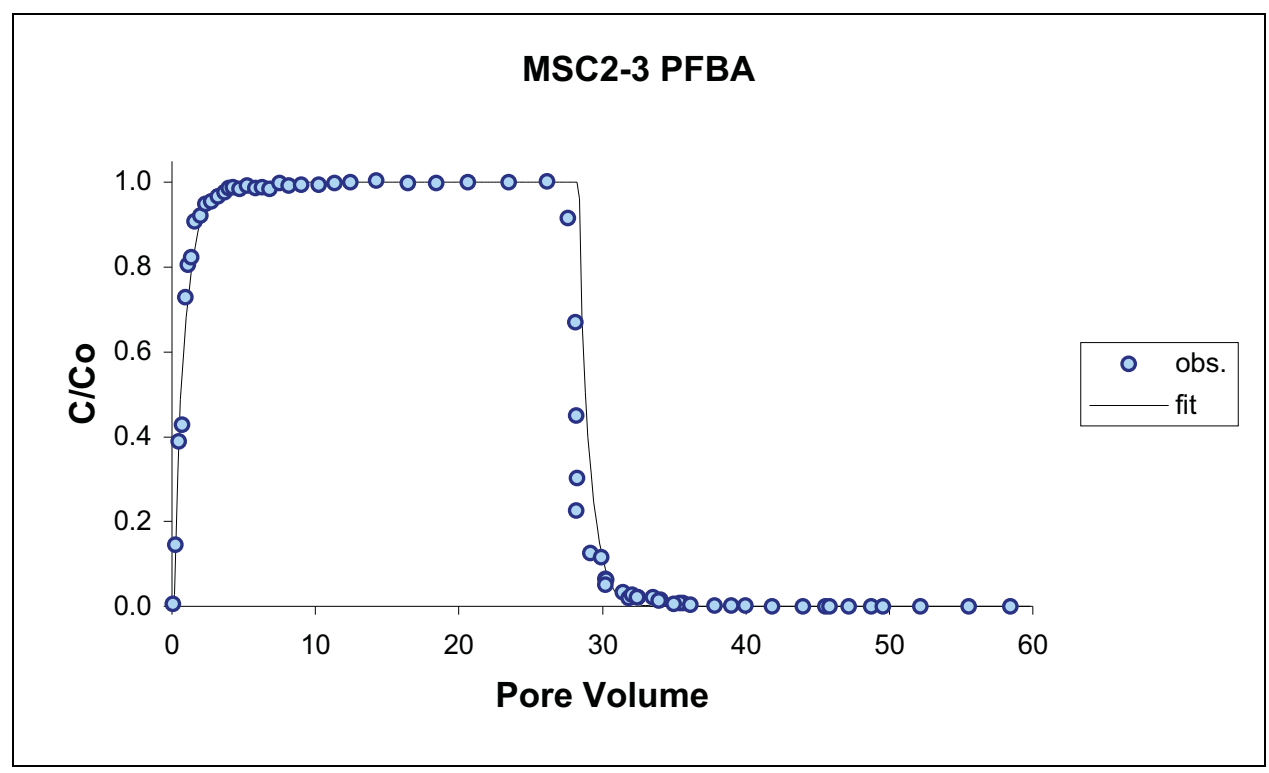

Figure J.8 Breakthrough curve and modeling results for PFBA in unsaturated MSC soil. $\left(\theta=0.10 ; v=8.37 \mathrm{~cm} / \mathrm{hr} ; D=23.81 \mathrm{hr}^{-1} ; \beta=0.419 ; \omega=1.14 \mathrm{hr}^{-1} ; R=0.90\right)$

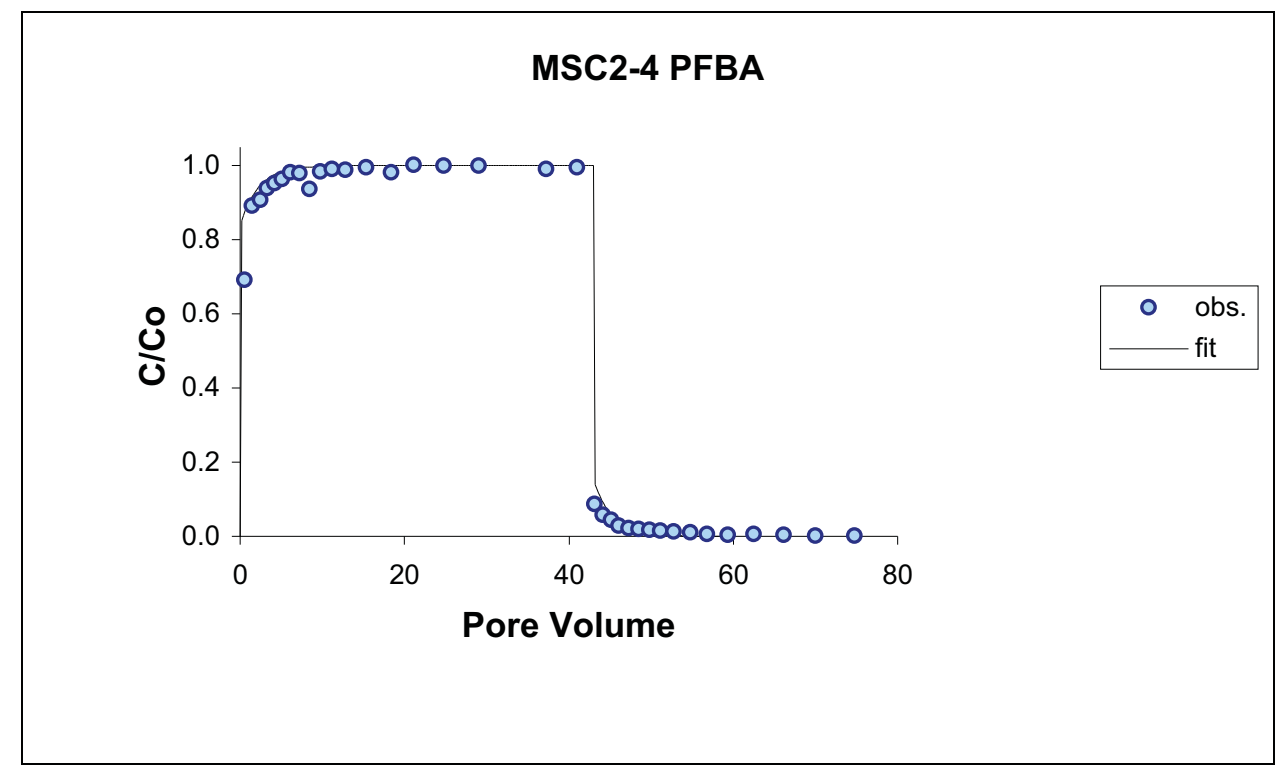

Figure J.9 Breakthrough curve and modeling results for PFBA in unsaturated MSC soil. $\left(\theta=0.056 ; v=63.2 \mathrm{~cm} / \mathrm{hr} ; D=60.32 \mathrm{hr}^{-1} ; \beta=0.300 ; \omega=0.161 \mathrm{hr}^{-1} ; R=0.47\right)$ 


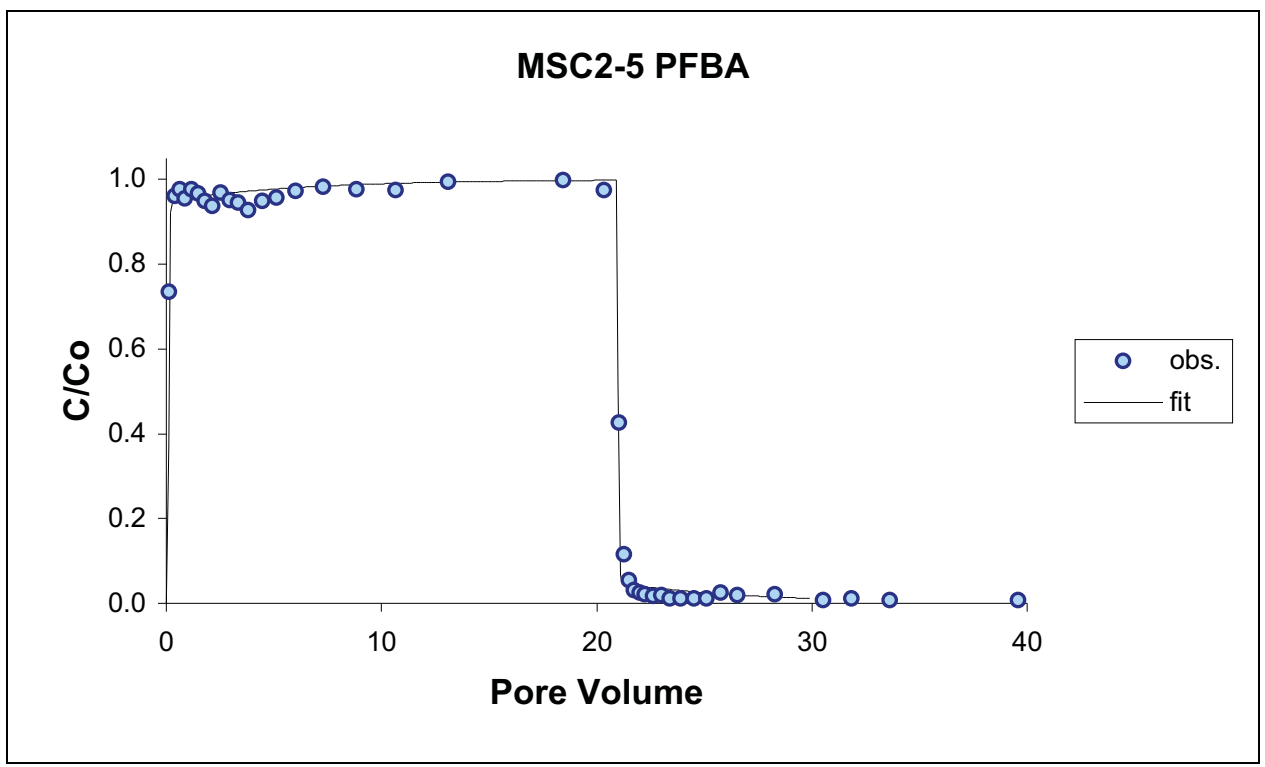

Figure J.10 Breakthrough curve and modeling results for PFBA in unsaturated MSC soil. $\left(\theta=0.050 ; v=14.4 \mathrm{~cm} / \mathrm{hr} ; D=4.79 \mathrm{hr}^{-1} ; \beta=0.273 ; \omega=0.503 \mathrm{hr}^{-1} ; R=0.42\right)$

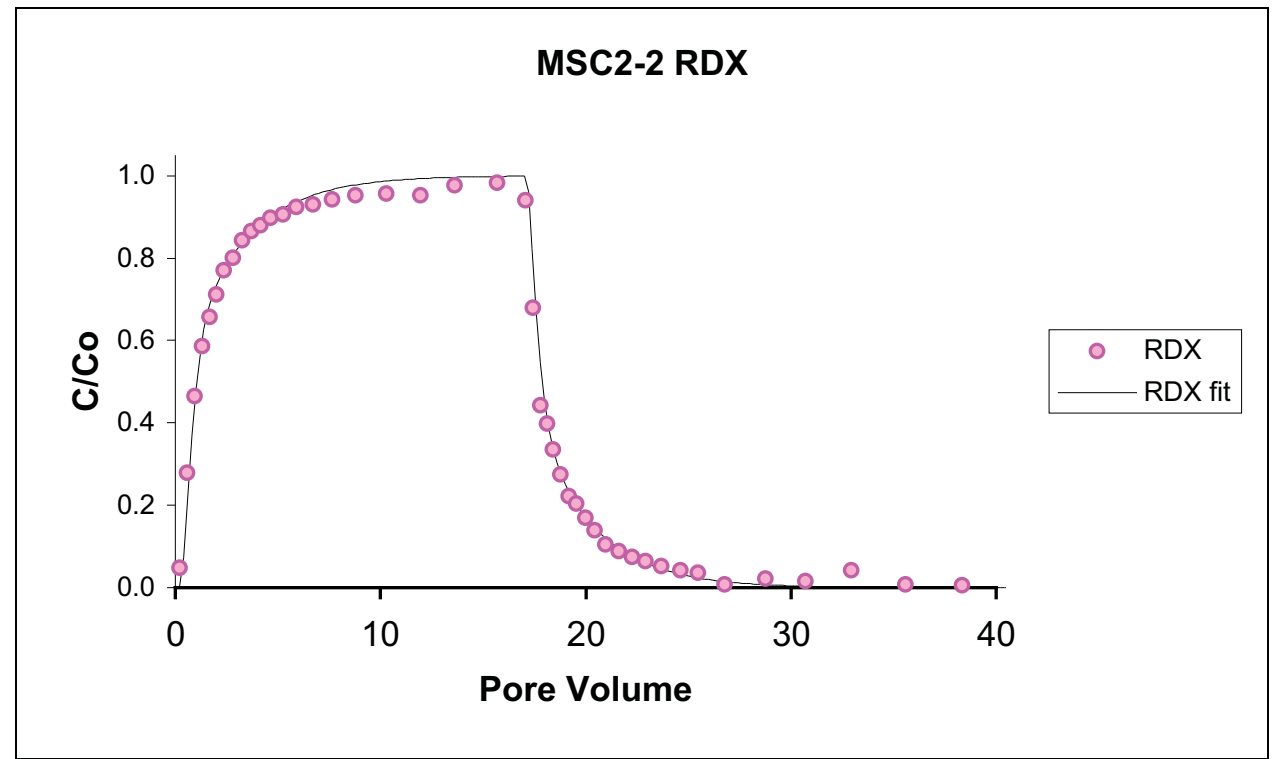

Figure J.11 Breakthrough curve and modeling results for RDX in unsaturated MSC soil. $\left(\theta=0.11 ; v=31.3 \mathrm{~cm} / \mathrm{hr} ; D=31.75 \mathrm{hr}^{-1} ; \beta=0.517 ; \omega=0.447 \mathrm{hr}^{-1} ; R=1.9 ; K_{d-\text { app }}=0.068\right)$ 


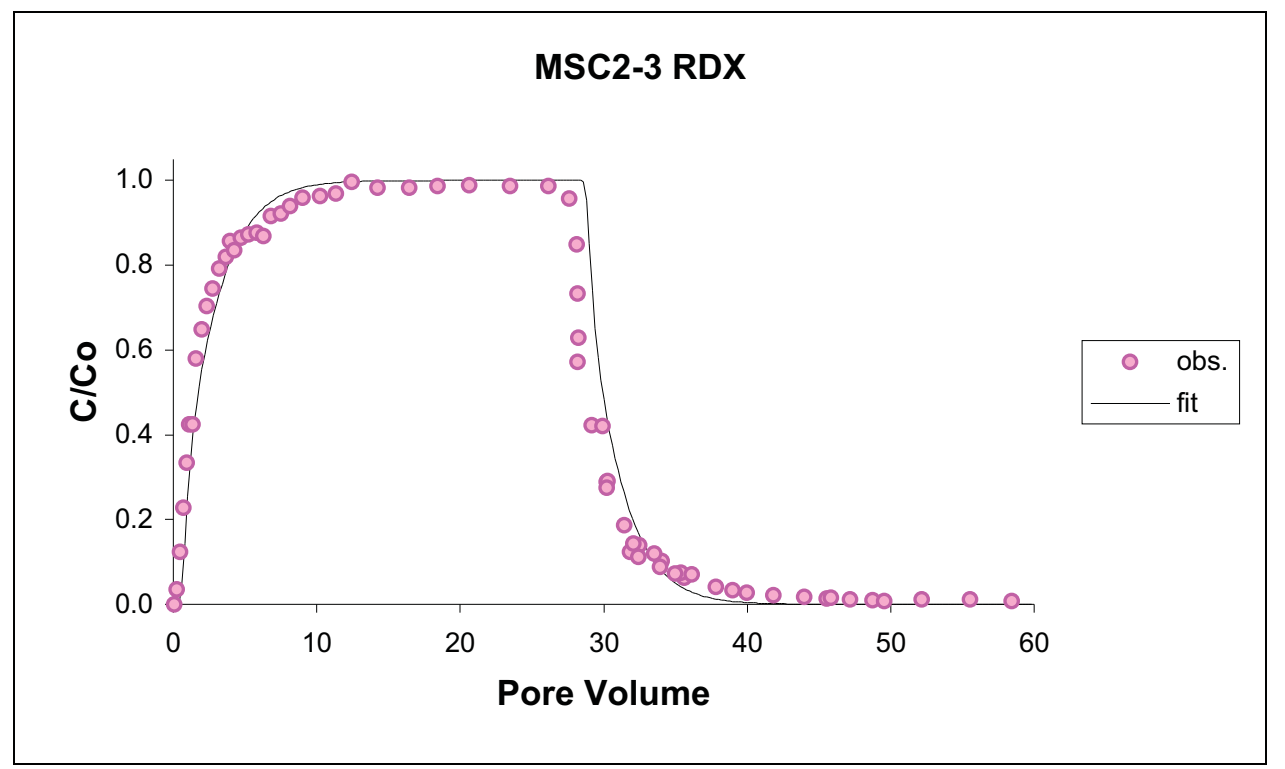

Figure J.12 Breakthrough curve and modeling results for RDX in unsaturated MSC soil. $\left(\theta=0.10 ; v=8.37 \mathrm{~cm} / \mathrm{hr} ; D=23.81 \mathrm{hr}^{-1} ; \beta=0.419 ; \omega=1.14 \mathrm{hr}^{-1} ; R=2.5 ; K_{d \text {-app }}=0.10\right)$

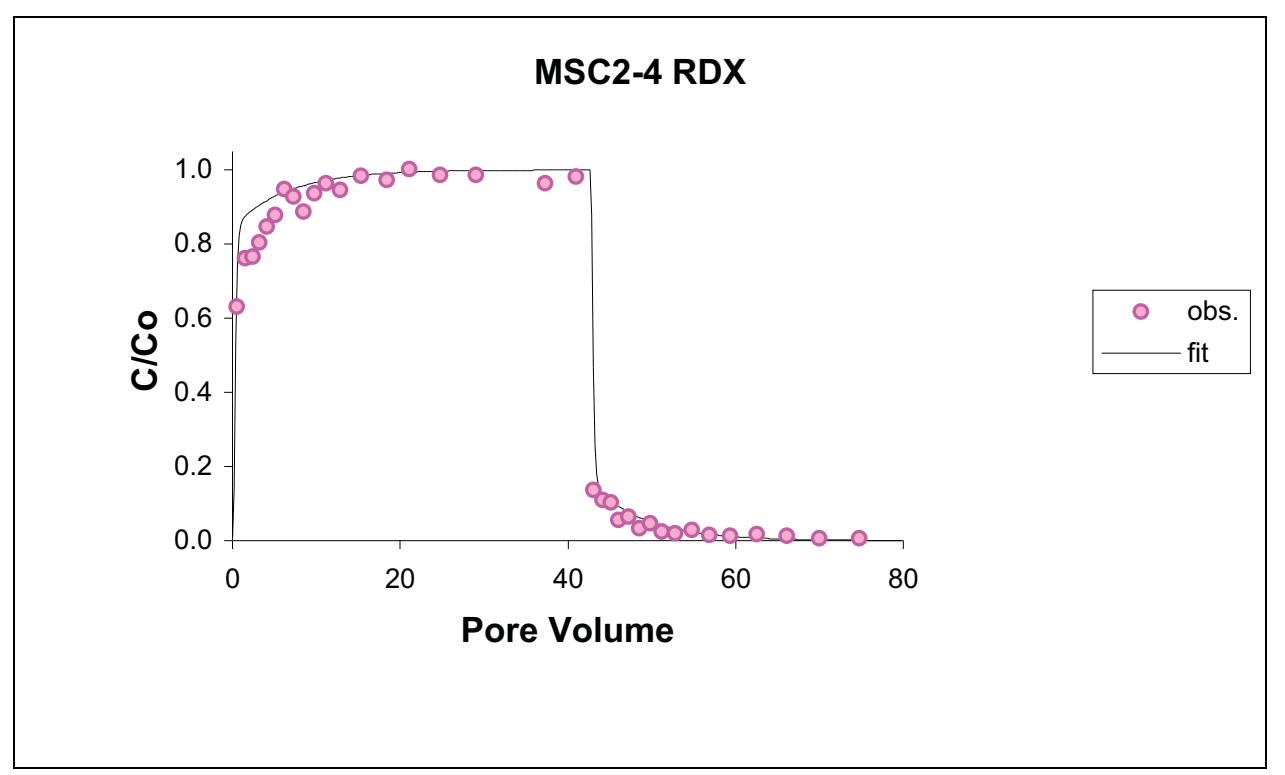

Figure J.13 Breakthrough curve and modeling results for RDX in unsaturated MSC soil. $\left(\theta=0.056 ; v=63.2 \mathrm{~cm} / \mathrm{hr} ; D=60.32 \mathrm{hr}^{-1} ; \beta=0.300 ; \omega=0.161 \mathrm{hr}^{-1} ; R=1.3 ; K_{d \text {-app }}=0.012\right)$ 


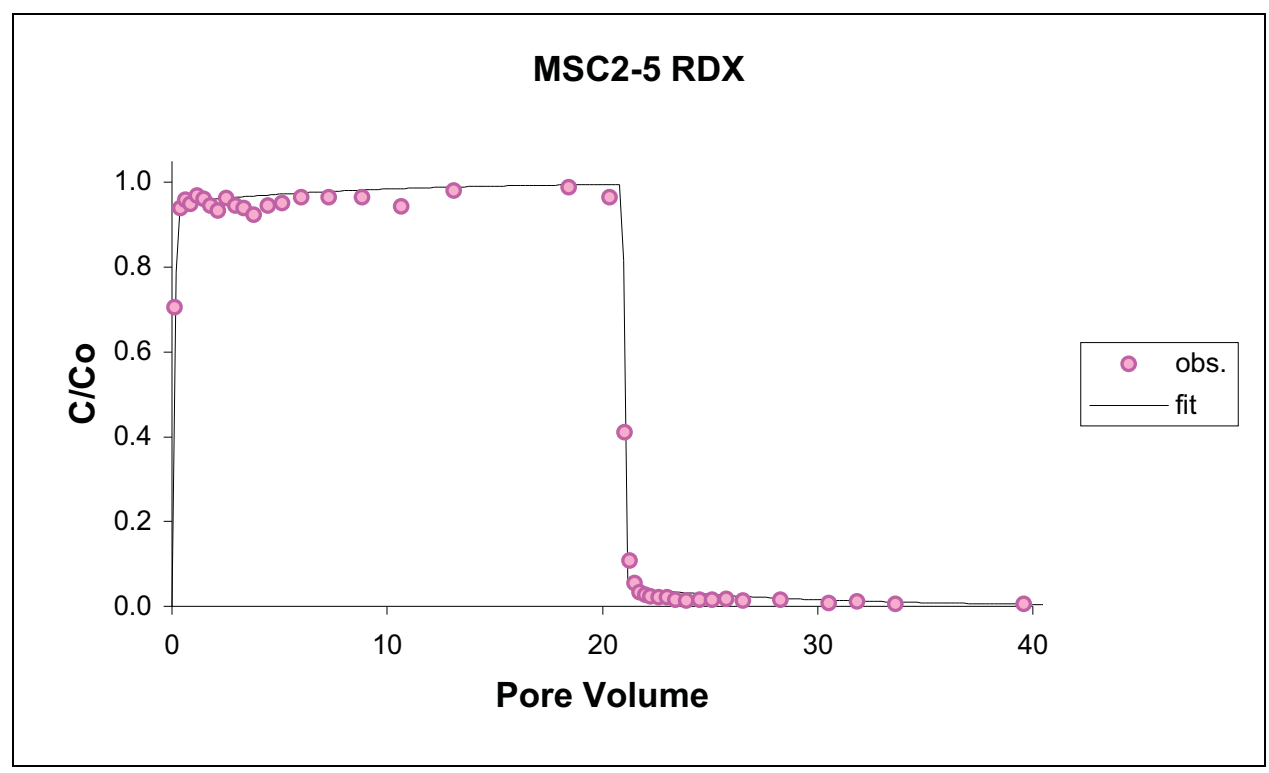

Figure J.14 Breakthrough curve and modeling results of RDX in unsaturated MSC soil. $\left(\theta=0.050 ; v=14.4 \mathrm{~cm} / \mathrm{hr} ; D=4.79 \mathrm{hr}^{-1} ; \beta=0.273 ; \omega=0.503 \mathrm{hr}^{-1} ; R=0.57 ; K_{d-a p p}<0\right)$

MSC2-2 CL-20

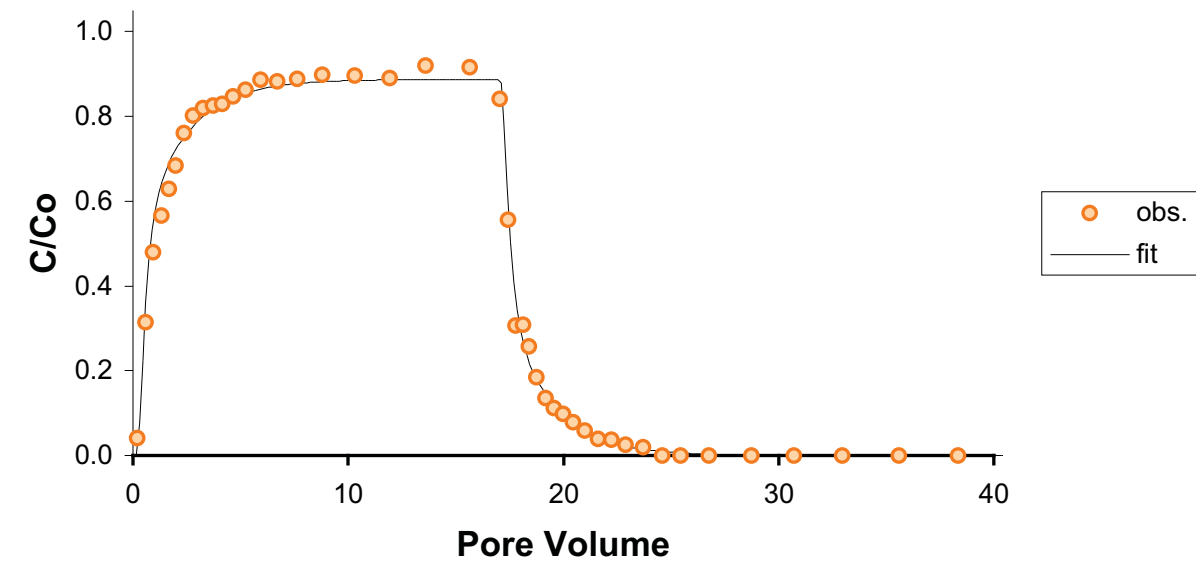

Figure J.15 Breakthrough curve and modeling results for CL-20 in unsaturated MSC soil. $\left(\theta=0.11 ; v=31.3 \mathrm{~cm} / \mathrm{hr} ; D=31.75 \mathrm{hr}^{-1} ; \beta=0.517 ; \omega=0.447 \mathrm{hr}^{-1} ; R=1.4 ; K_{d-a p p}=0.029\right.$; $\left.\mu_{1}=0.121 \mathrm{hr}^{-1}\right)$ 


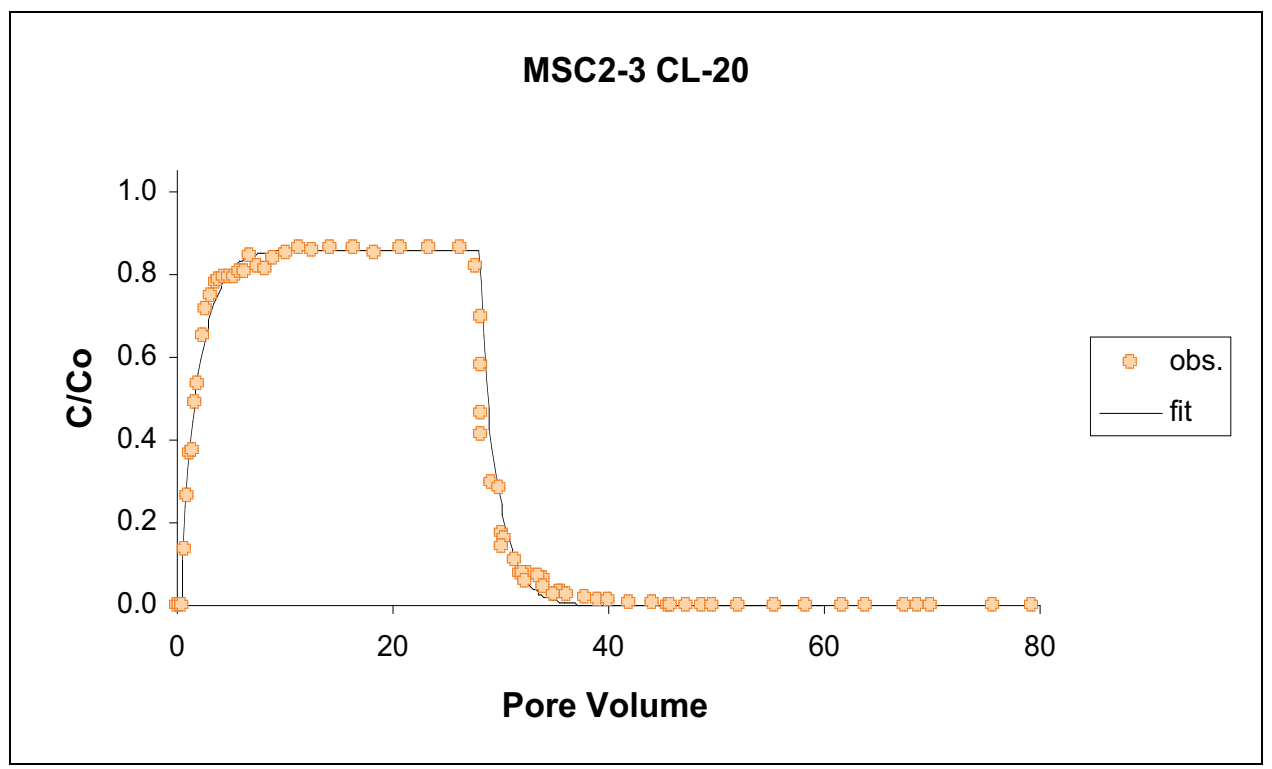

Figure J.16 Breakthrough curve and modeling results for CL-20 in unsaturated MSC soil. ( $\theta=0.10 ; v=$ $8.37 \mathrm{~cm} / \mathrm{hr} ; D=23.81 \mathrm{hr}^{-1} ; \beta=0.419 ; \omega=1.14 \mathrm{hr}^{-1} ; R=2.0 ; K_{d-a p p}=0.066 ; \mu_{1}=0.153 \mathrm{hr}^{-1}$ )

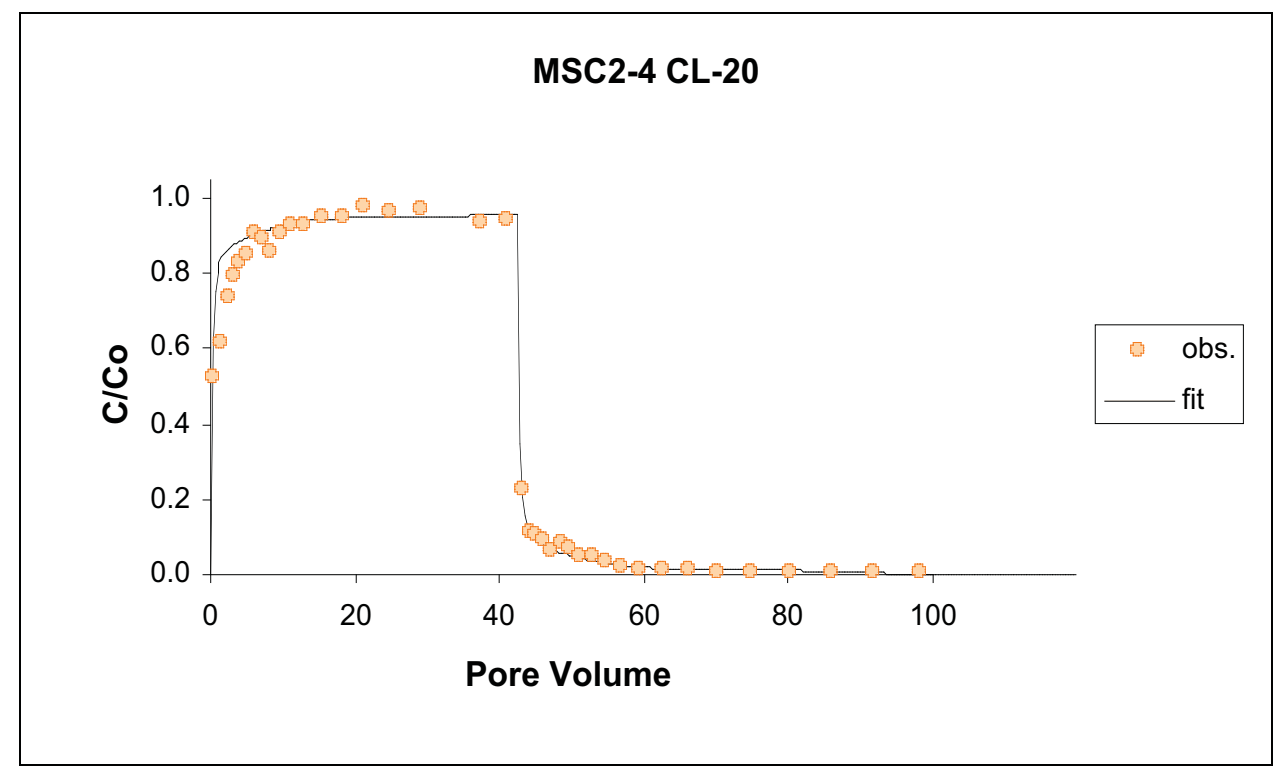

Figure J.17 Breakthrough curve and modeling results for CL-20 in unsaturated MSC soil. $(\theta=0.056 ; v=$ $\left.63.2 \mathrm{~cm} / \mathrm{hr} ; D=60.32 \mathrm{hr}^{-1} ; \beta=0.300 ; \omega=0.161 \mathrm{hr}^{-1} ; R=1.3 ; K_{d-a p p}=0.010 ; \mu_{1}=0.0458 \mathrm{hr}^{-1}\right)$ 


\section{MSC2-5 CL-20}

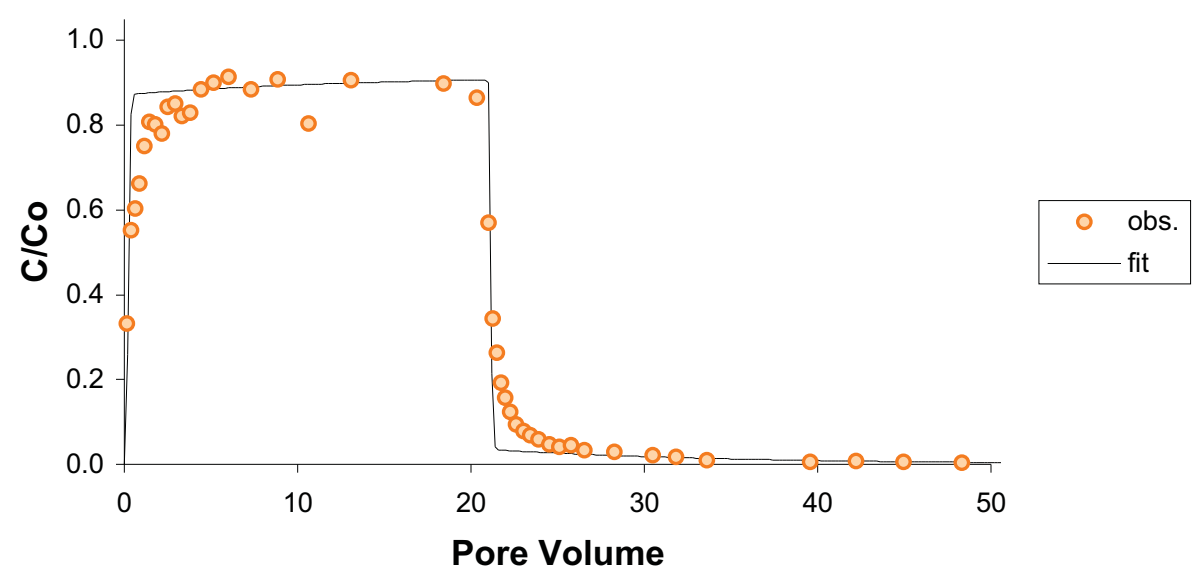

Figure J.18 Breakthrough curve and modeling results for CL-20 in unsaturated MSC soil. ( $\theta=0.050 ; v=$ $14.4 \mathrm{~cm} / \mathrm{hr} ; D=4.79 \mathrm{hr}^{-1} ; \beta=0.273 ; \omega=0.503 \mathrm{hr}^{-1} ; R=0.93 ; K_{d-a p p}<0 ; \mu_{1}=0.0867 \mathrm{hr}^{-1}$ )

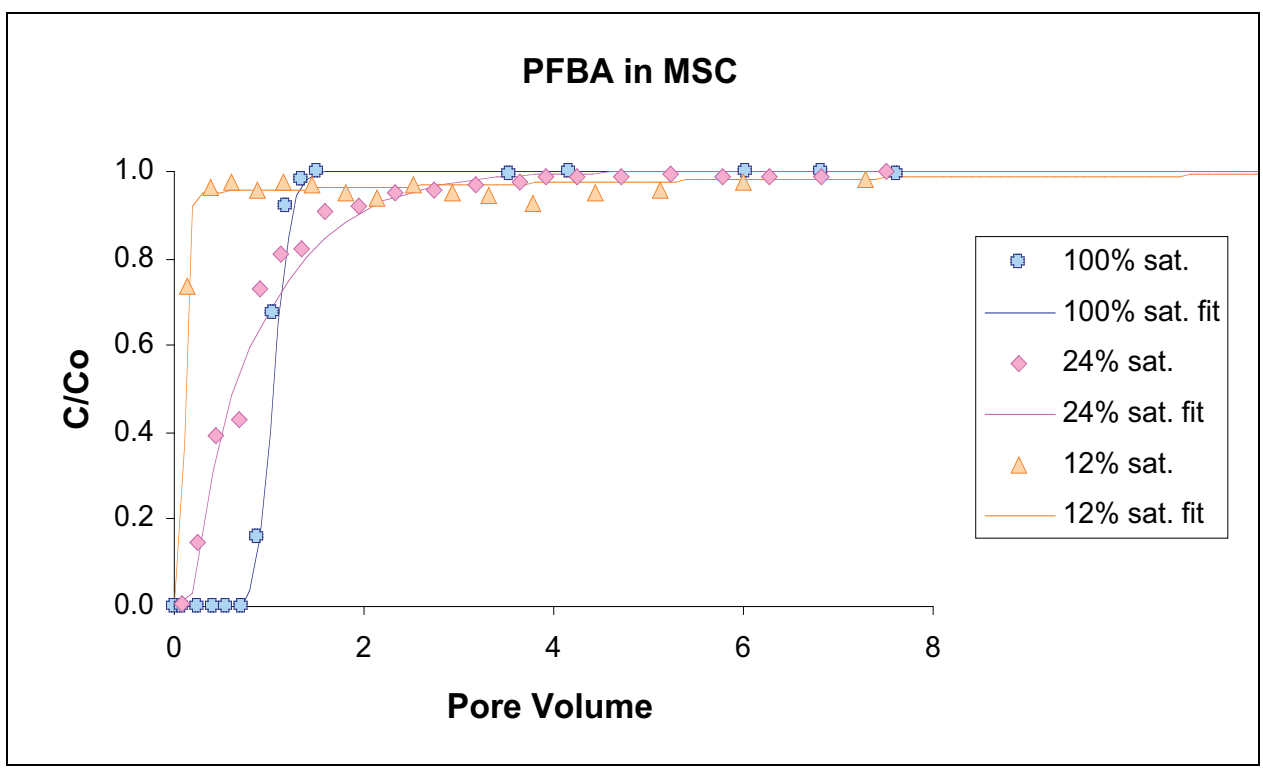

Figure J.19 Comparison of PFBA behavior with changing water content. Note that breakthrough accelerates with decreasing water content. (SM1-2; MSC2-3; MSC2-5) 


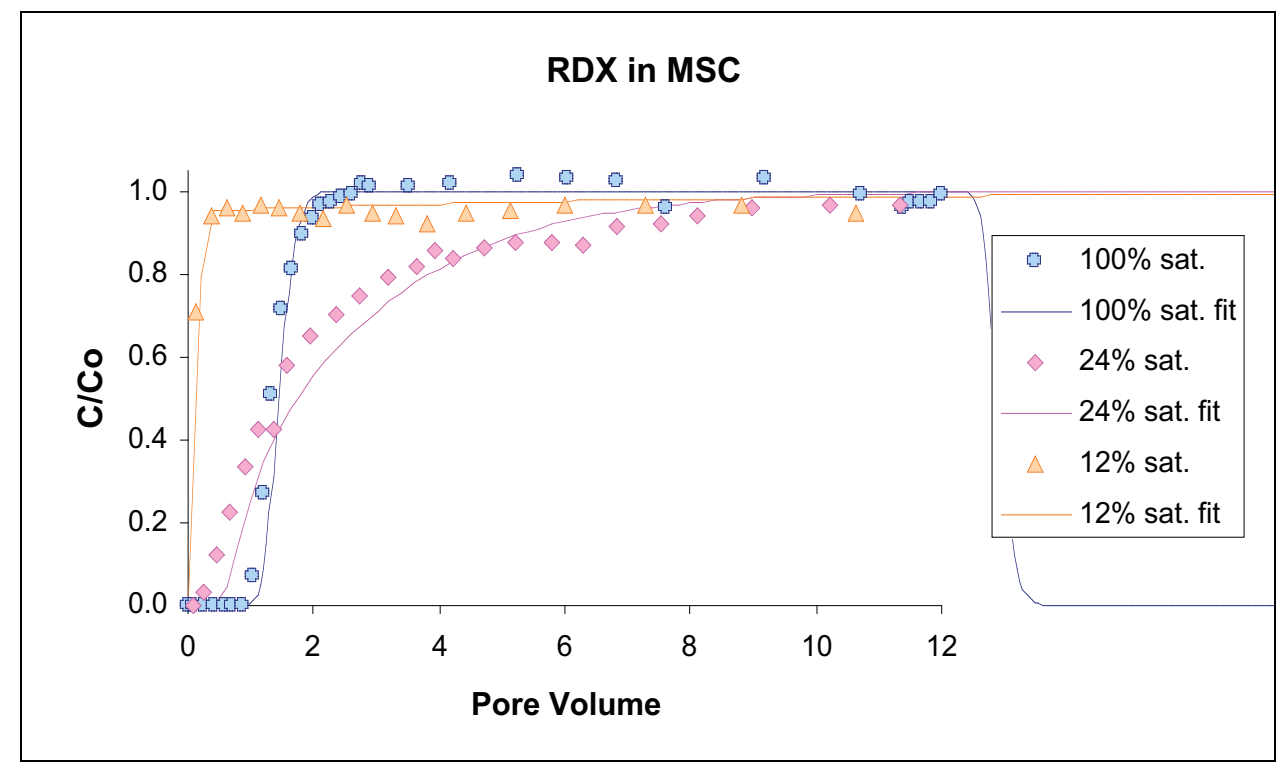

Figure J.20 Comparison of RDX behavior with changing water content. Note that breakthrough accelerates with decreasing water content. (SM1-2; MSC2-3; MSC2-5)

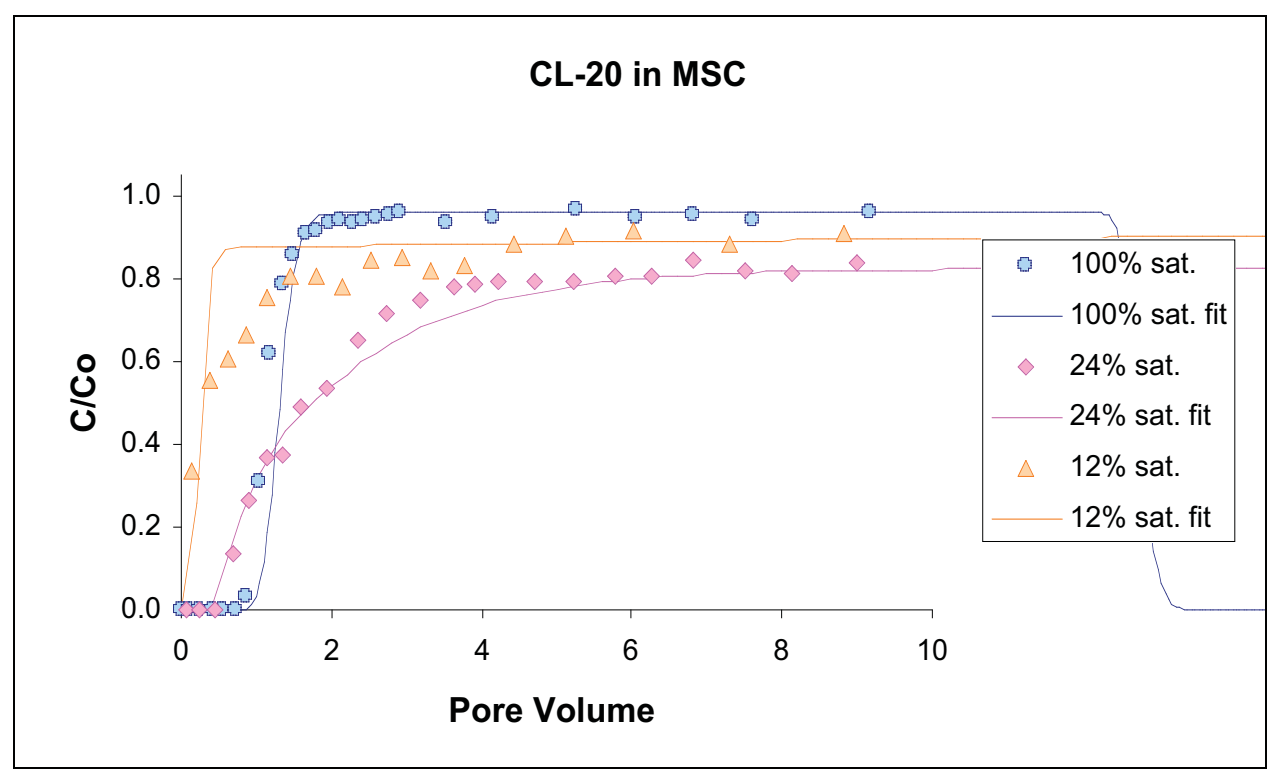

Figure J.21 Comparison of CL-20 transport in Burbank soil with changing water content. Not that breakthrough accelerates with decreasing water content. (SM1-2; MSC2-3; MSC2-5) 


\section{Appendix J2. Unsaturated Flow Experiments in the Norborne sediment}

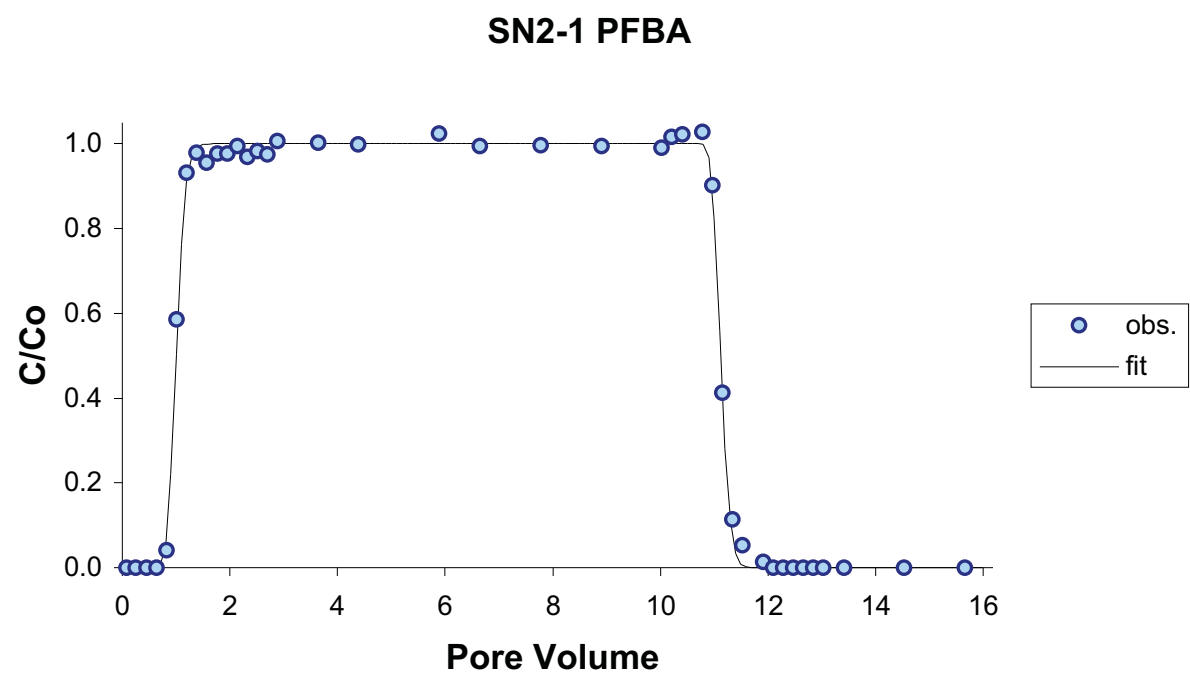

Figure J.22 Breakthrough curve and modeling results for PFBA in saturated Norborne soil. $\left(\theta=0.45 ; v=2.82 \mathrm{~cm} / \mathrm{hr} ; D=0.179 \mathrm{hr}^{-1} ; R=1.0\right)$

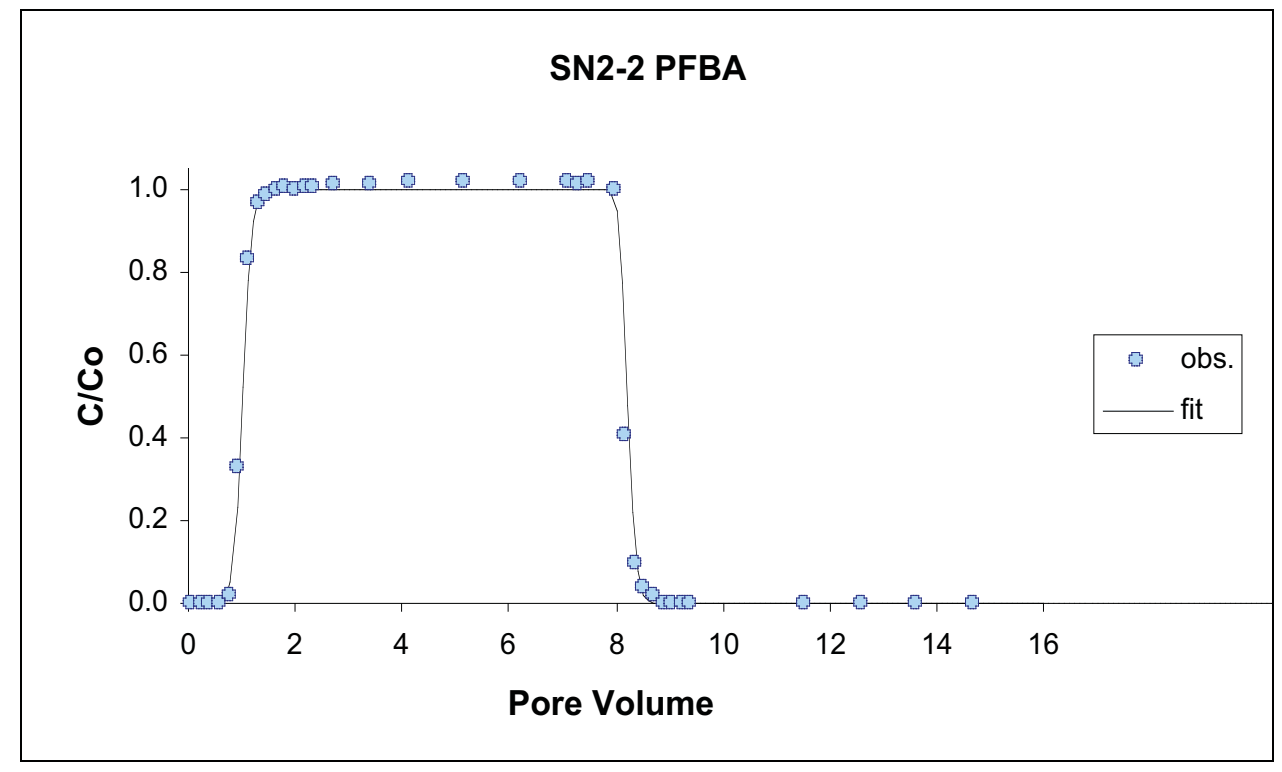

Figure J.23 Breakthrough curve and modeling results from PFBA in saturated Norborne soil. $\left(\theta=0.45 ; v=11.4 \mathrm{~cm} / \mathrm{hr} ; D=0.700 \mathrm{hr}^{-1} ; R=1.0\right)$ 


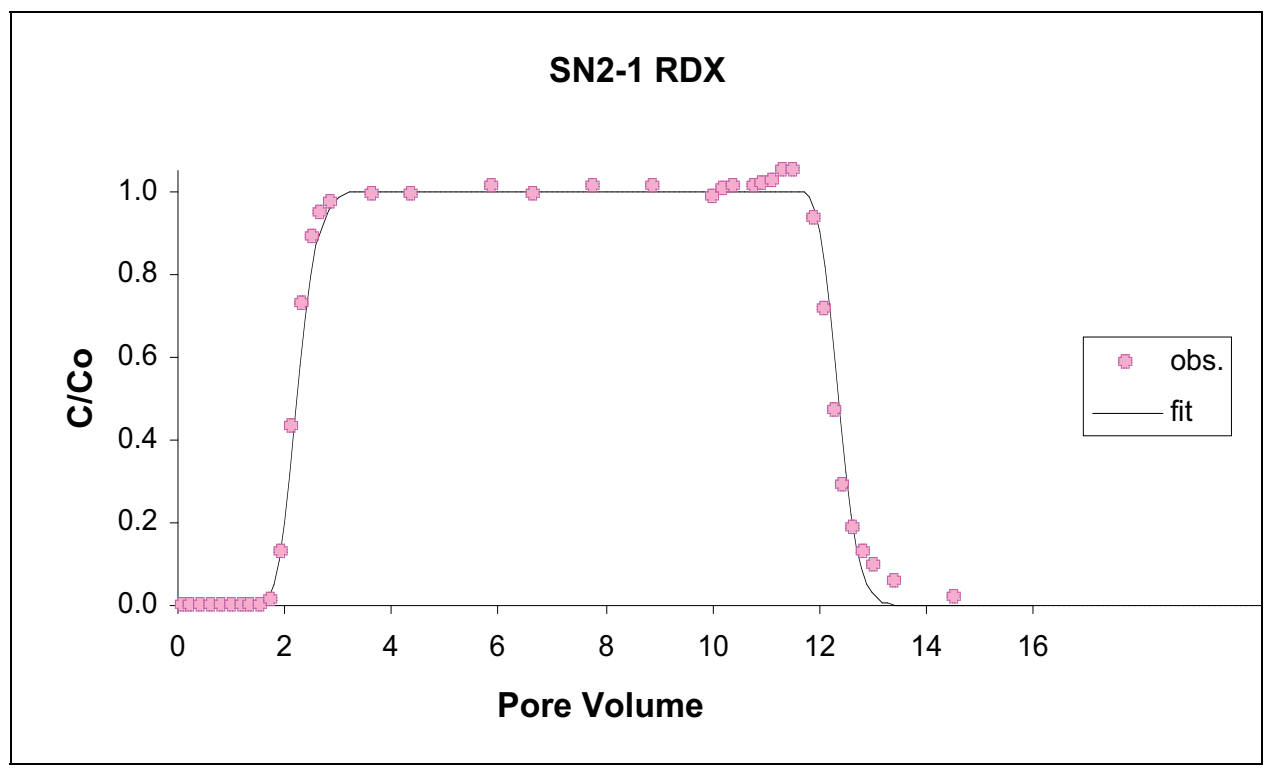

Figure J.24 Breakthrough curve and modeling results for RDX in saturated Norborne soil. $\left(\theta=0.45 ; v=2.82 \mathrm{~cm} / \mathrm{hr} ; D=0.179 \mathrm{hr}^{-1} ; R=2.2 ; K_{d \text {-app }}=0.38\right)$

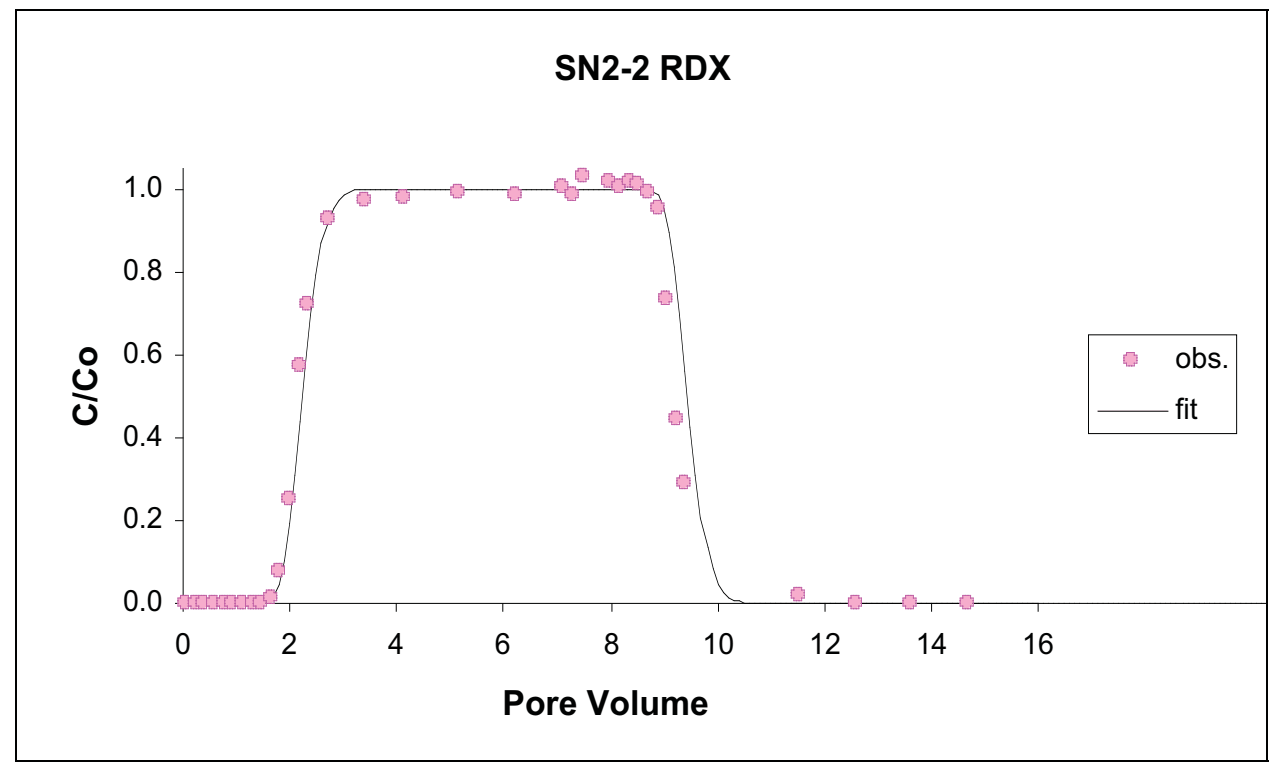

Figure J.25 Breakthrough curve and modeling results for RDX in saturated Norborne soil. $\left(\theta=0.45 ; v=11.4 \mathrm{~cm} / \mathrm{hr} ; D=0.700 \mathrm{hr}^{-1} ; R=2.3 ; K_{\text {d-app }}=0.41\right)$ 
SN2-1 CL-20

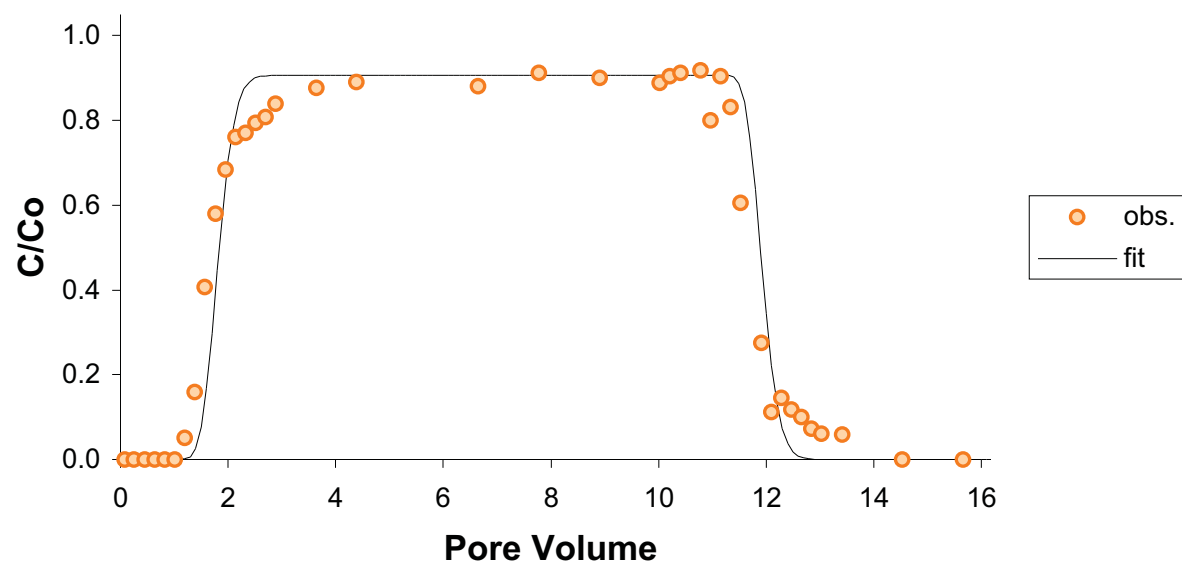

Figure J.26 Breakthrough curve and modeling results for CL-20 in saturated Norborne soil. $\left(\theta=0.45 ; v=2.82 \mathrm{~cm} / \mathrm{hr} ; D=0.179 \mathrm{hr}^{-1} ; R=1.8 ; K_{d-a p p}=0.25 ; \mu_{1}=0.156 \mathrm{hr}^{-1}\right)$

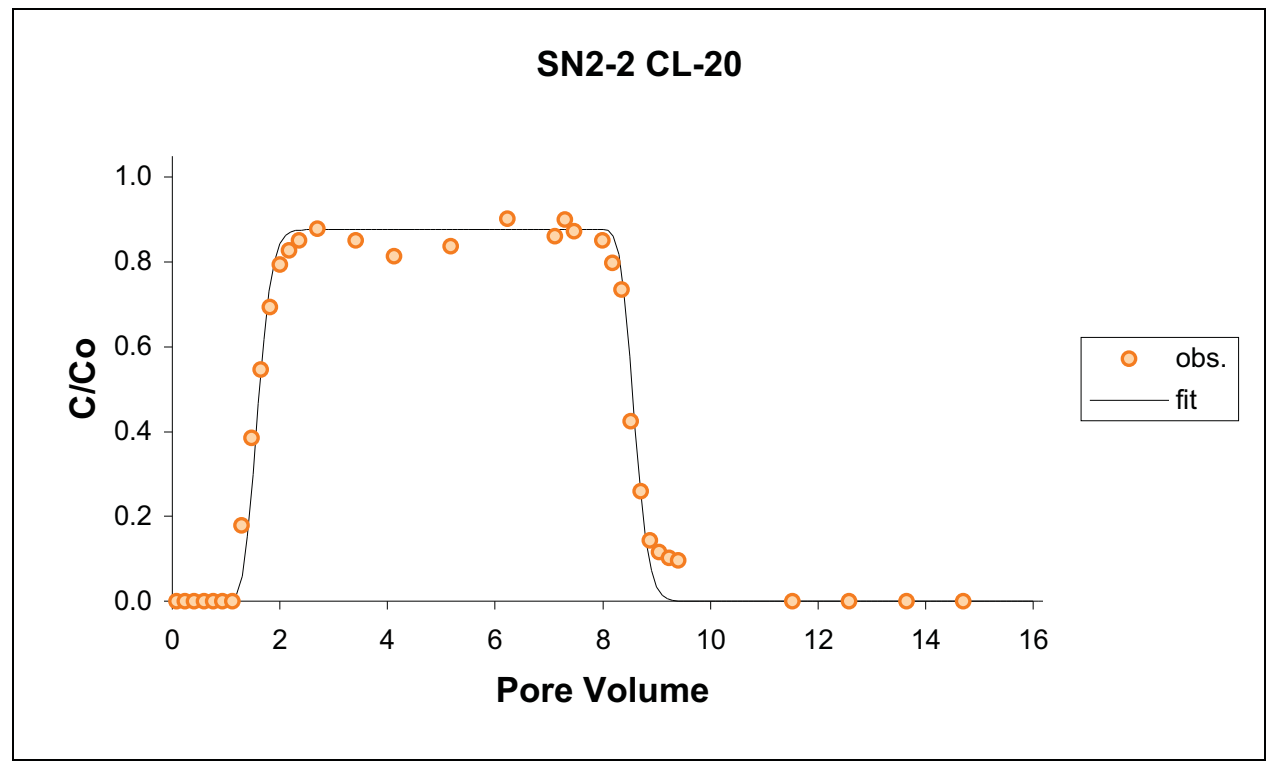

Figure J.27 Breakthrough curve and modeling results for CL-20 in saturated Norborne soil. $\left(\theta=0.45 ; v=11.4 \mathrm{~cm} / \mathrm{hr} ; D=0.700 \mathrm{hr}^{-1} ; R=1.6 ; K_{d-a p p}=0.18 ; \mu_{1}=0.209 \mathrm{hr}^{-1}\right)$ 


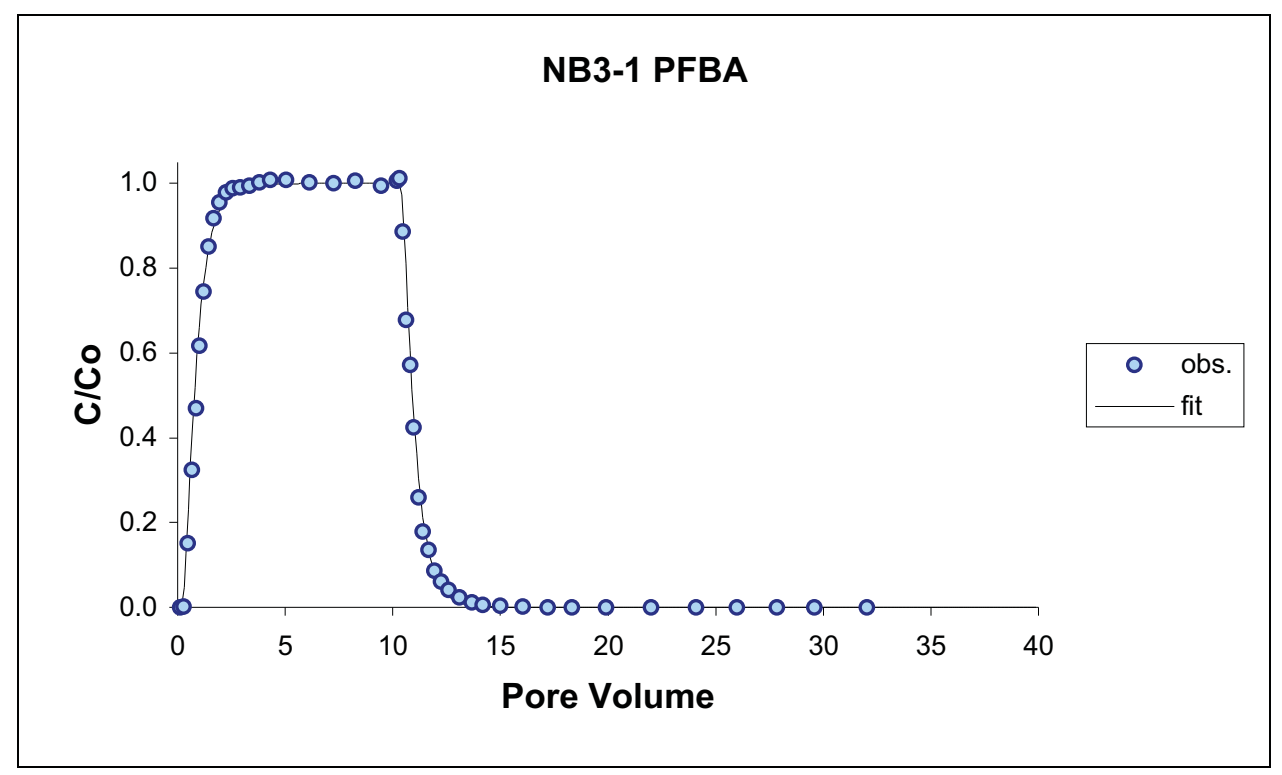

Figure J.28 Breakthrough curve and modeling results for PFBA in unsaturated Norborne soil. $\left(\theta=0.30 ; v=12.0 \mathrm{~cm} / \mathrm{hr} ; D=14.4 \mathrm{hr}^{-1} ; R=0.93\right)$

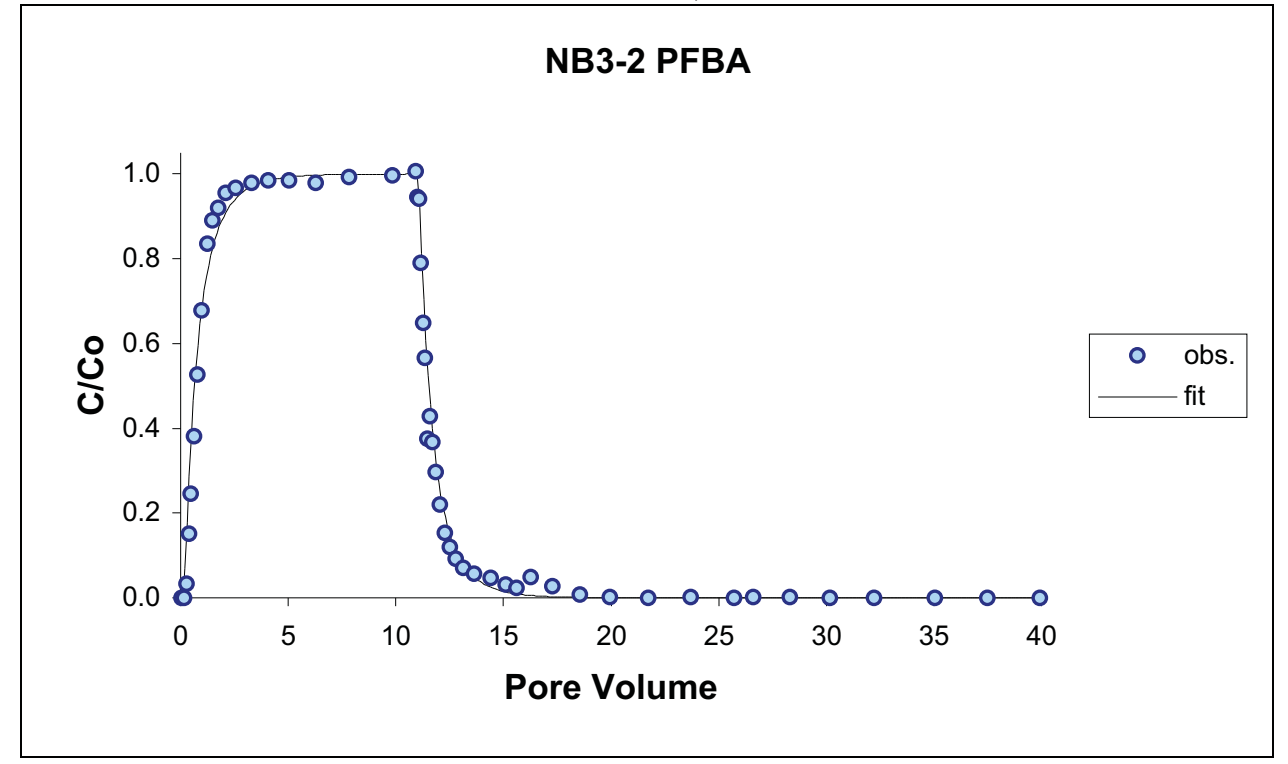

Figure J.29 Breakthrough curve and modeling results for PFBA in unsaturated Norborne soil. $\left(\theta=0.29 ; v=2.54 \mathrm{~cm} / \mathrm{hr} ; D=7.24 \mathrm{hr}^{-1} ; R=0.94\right)$ 


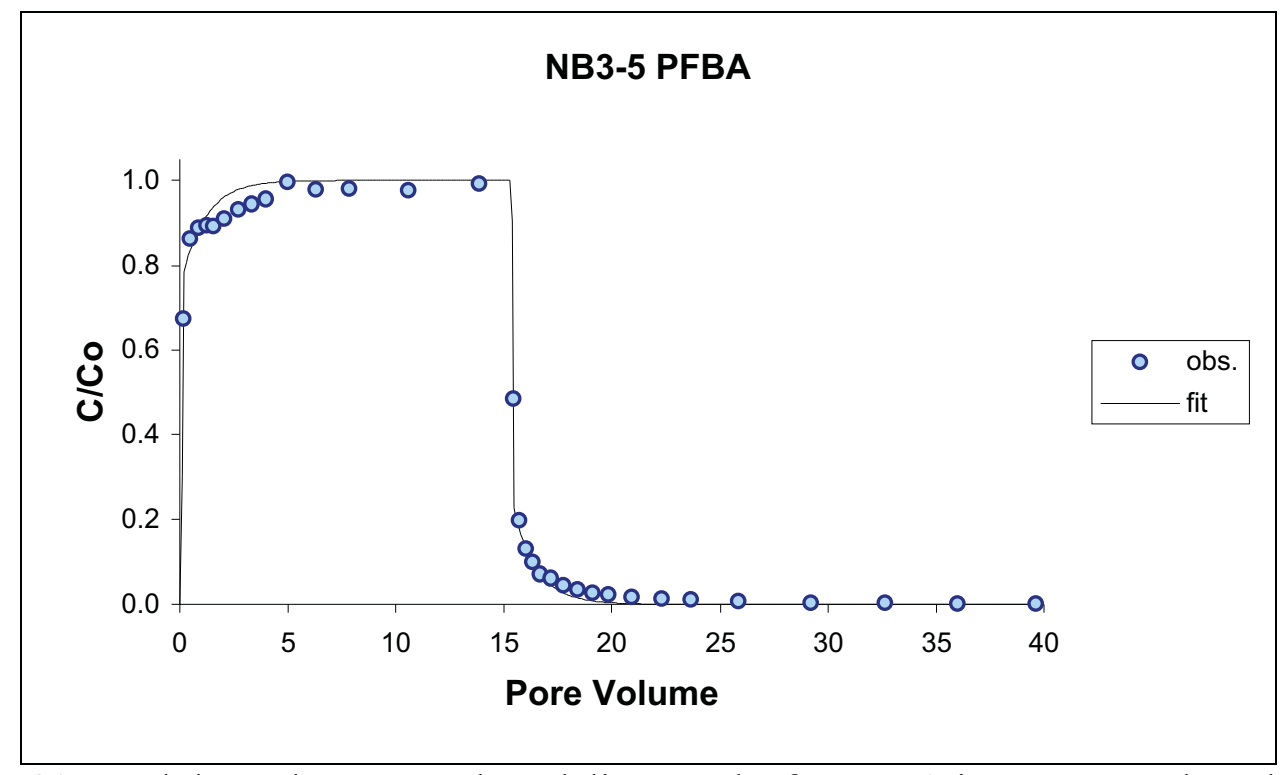

Figure J.30 Breakthrough curve and modeling results for PFBA in unsaturated Norborne soil. $\left(\theta=0.17 ; v=20.9 \mathrm{~cm} / \mathrm{hr} ; D=4.02 \mathrm{hr}^{-1} ; \beta=0.310 ; \omega=0.256 ; R=0.36\right)$

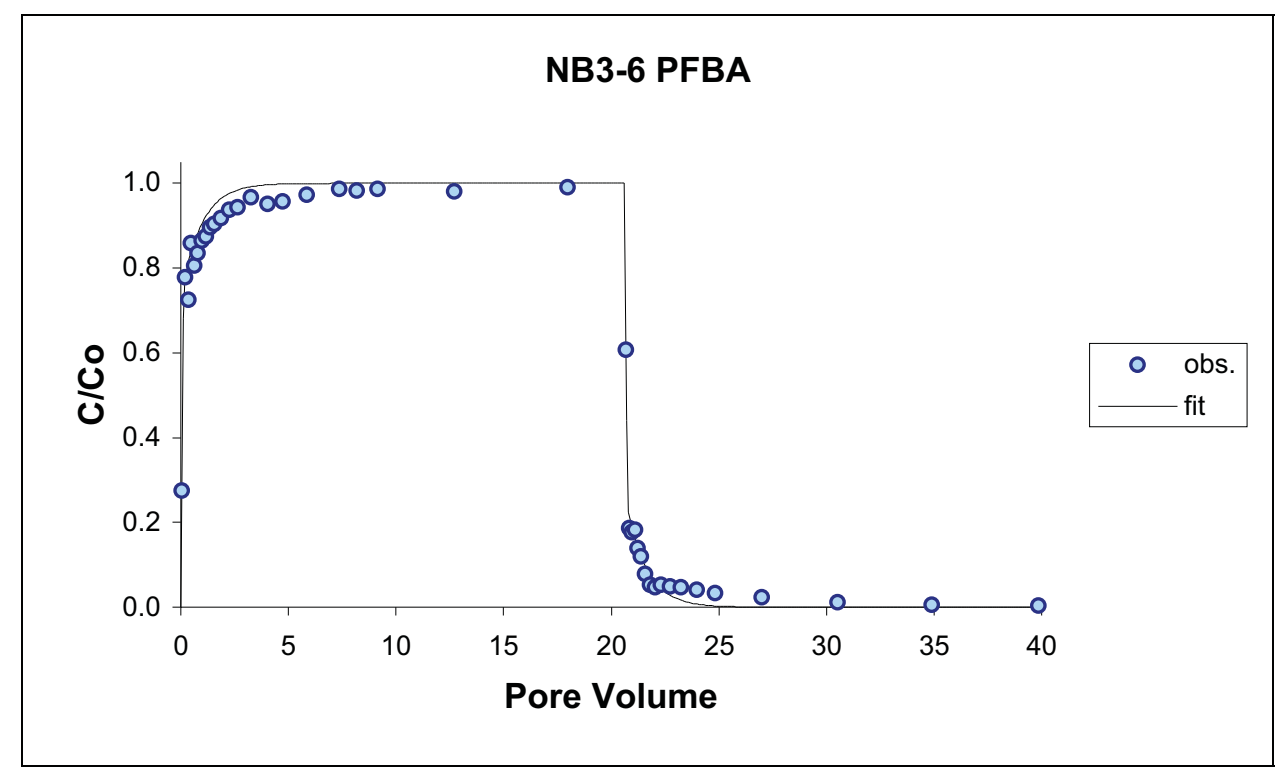

Figure J.31 Breakthrough curve and modeling results for PFBA in unsaturated Norborne soil. $\left(\theta=0.15 ; v=4.76 \mathrm{~cm} / \mathrm{hr} ; D=2.00 \mathrm{hr}^{-1} ; \beta=0.233 ; \omega=0.291 ; R=0.30\right)$ 


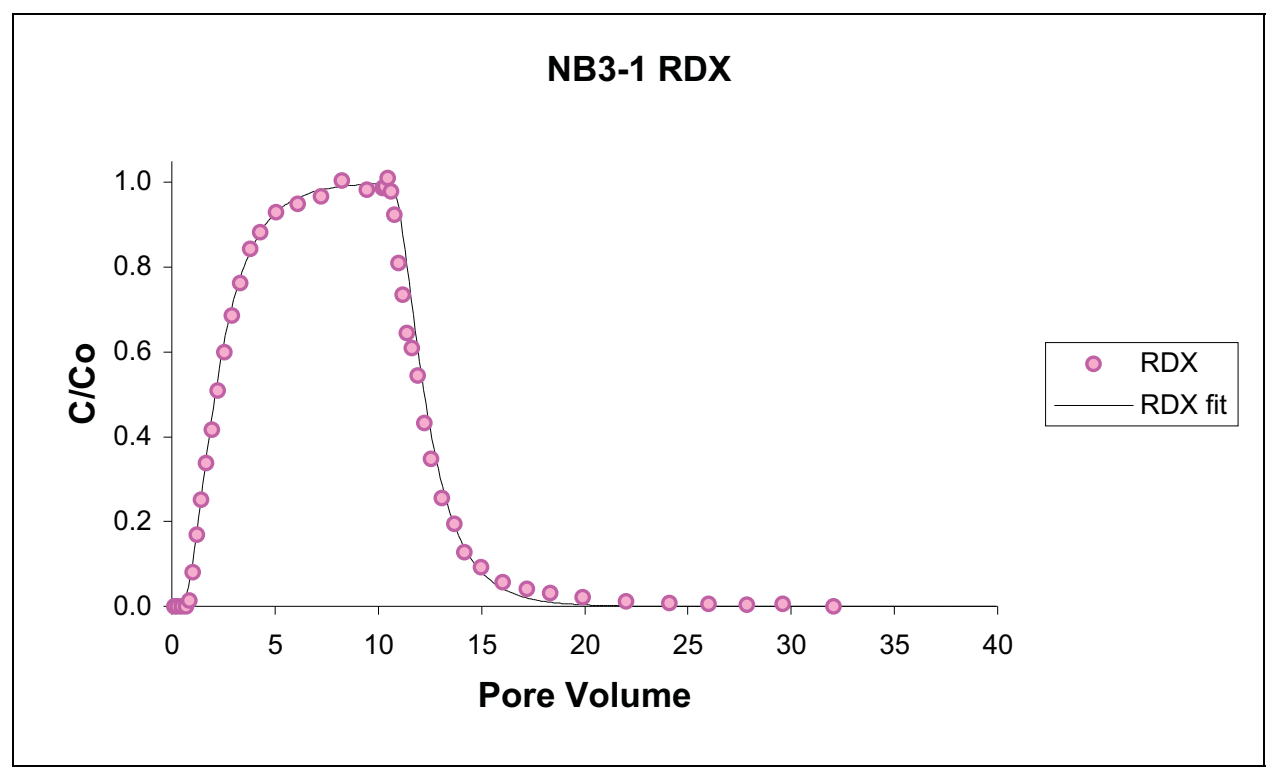

Figure J.32 Breakthrough curve and model output for RDx in unsaturated Norborne soil. $\left(\theta=0.30 ; v=12.0 \mathrm{~cm} / \mathrm{hr} ; D=14.4 \mathrm{hr}^{-1} ; R=2.5 ; K_{d-a p p}=0.34\right)$

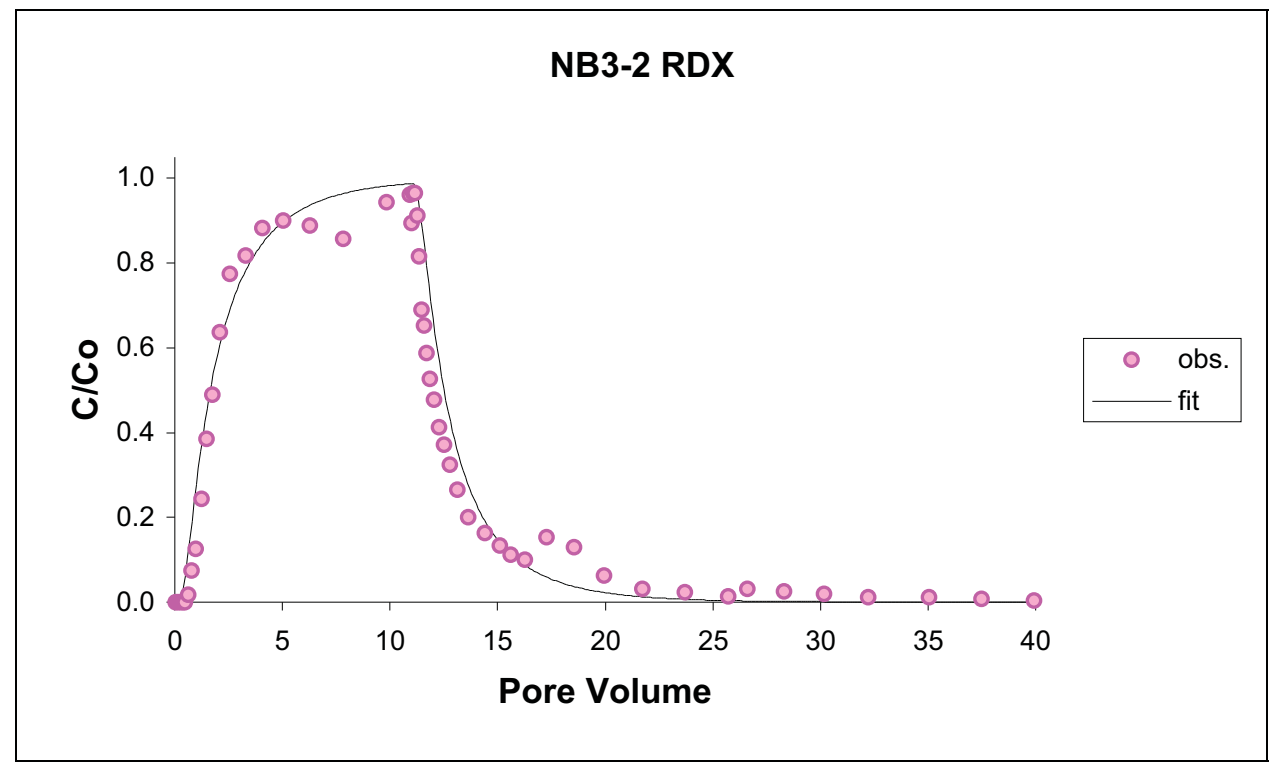

Figure J.33 Breakthrough curve and model output for RDX in unsaturated Norborne soil. $\left(\theta=0.29 ; v=2.54 \mathrm{~cm} / \mathrm{hr} ; D=7.24 \mathrm{hr}^{-1} ; R=2.4 ; K_{\text {d-app }}=0.30\right)$ 


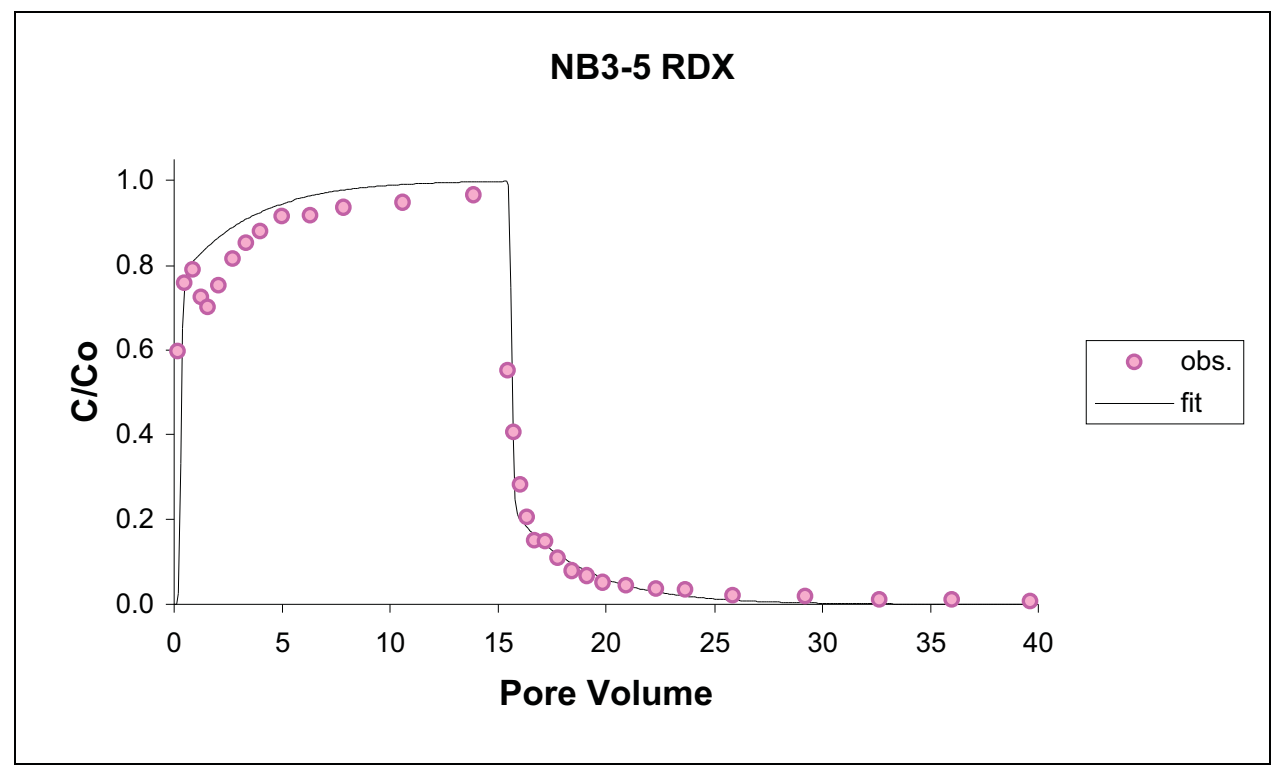

Figure J.34 Breakthrough curve and model output for RDX in unsaturated Norborne soil. $\left(\theta=0.17 ; v=20.9 \mathrm{~cm} / \mathrm{hr} ; D=4.02 \mathrm{hr}^{-1} ; \beta=0.310 ; \omega=0.256 ; R=1.1 ; K_{d-a p p}=0.008\right)$

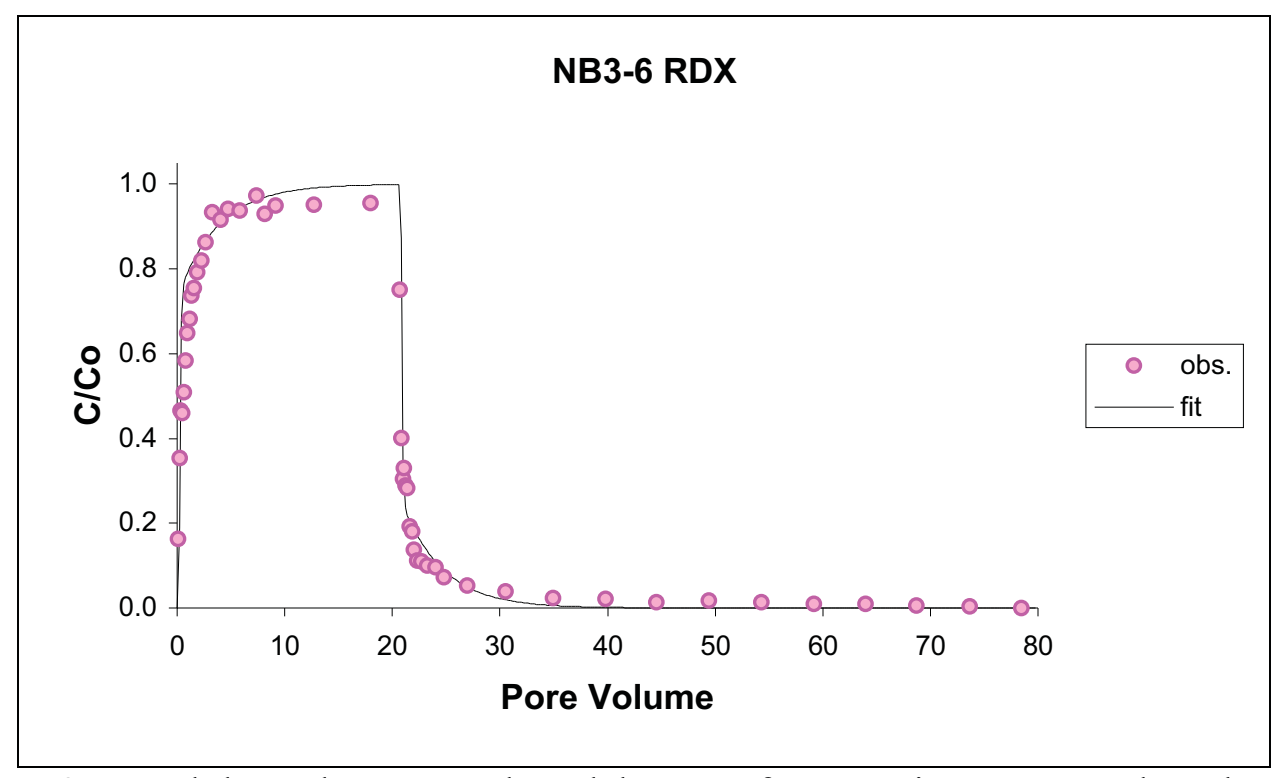

Figure J.35 Breakthrough curve and model output for RDX in unsaturated Norborne soil. $\left(\theta=0.15 ; v=4.76 \mathrm{~cm} / \mathrm{hr} ; D=2.00 \mathrm{hr}^{-1} ; \beta=0.233 ; \omega=0.291 ; R=1.2 ; K_{d-a p p}=0.024\right)$ 


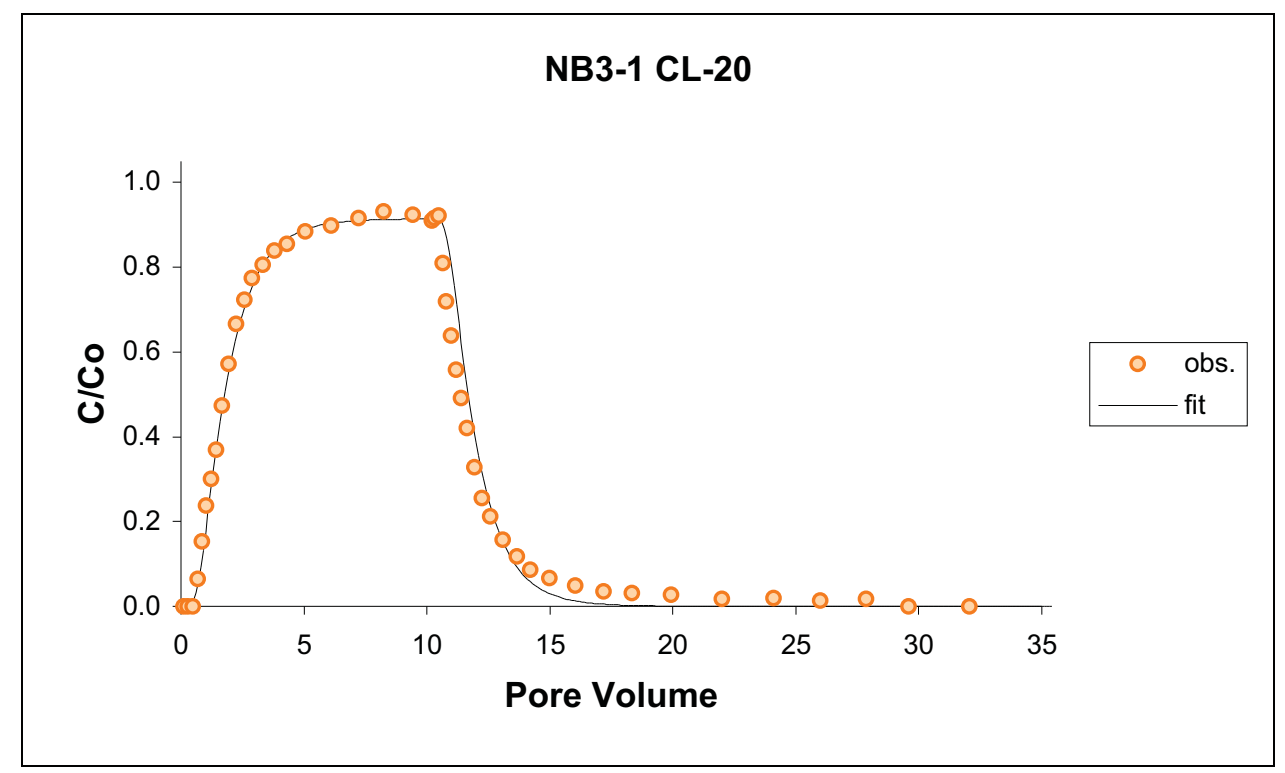

Figure J.36 Breakthrough curve and model output for CL-20 in unsaturated Norborne soil. $\left(\theta=0.30 ; v=12.0 \mathrm{~cm} / \mathrm{hr} ; D=14.4 \mathrm{hr}^{-1} ; R=2.0: K_{d-\text { app }}=0.024 ; \mu_{1}=0.141 \mathrm{hr}^{-1}\right)$

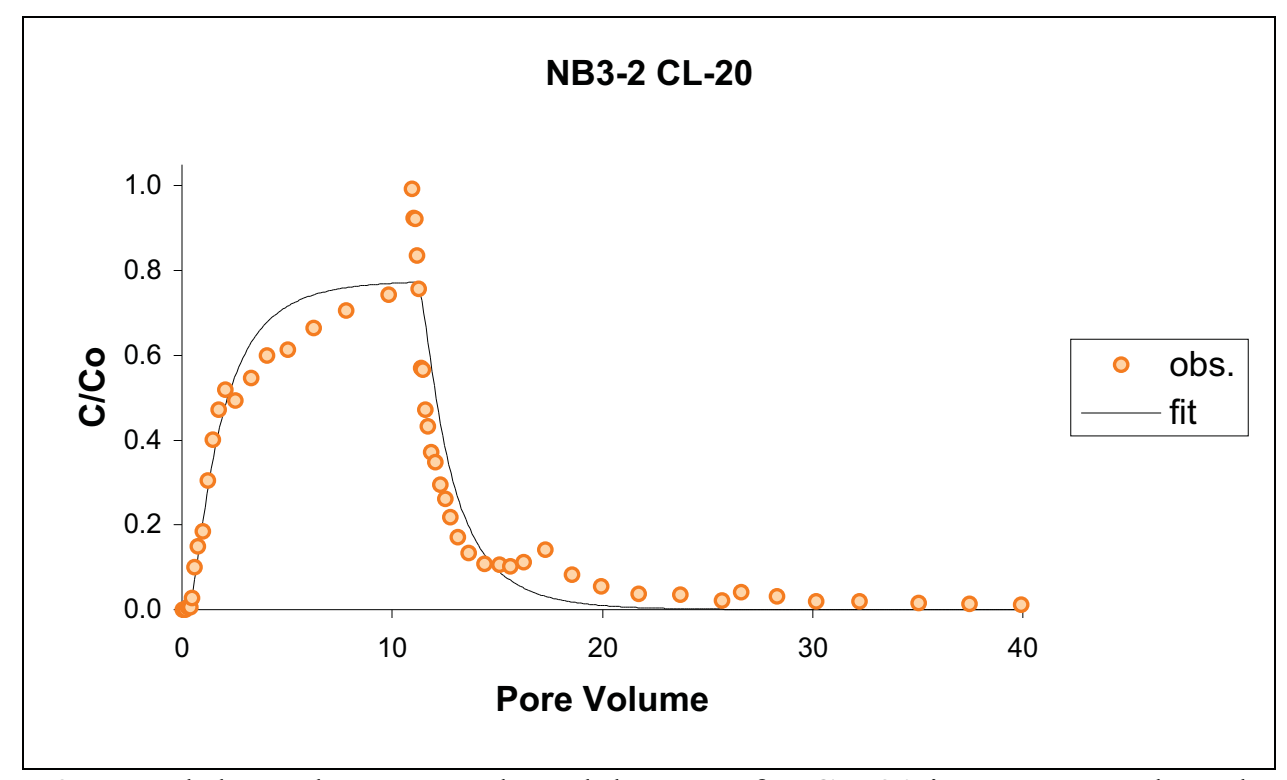

Figure J.37 Breakthrough curve and model output for CL-20 in unsaturated Norborne soil. $\left(\theta=0.29 ; v=2.54 \mathrm{~cm} / \mathrm{hr} ; D=7.24 \mathrm{hr}^{-1} ; R=2.7 ; K_{d-a p p}=0.37 ; \mu_{1}=0.516 \mathrm{hr}^{-1}\right)$ 


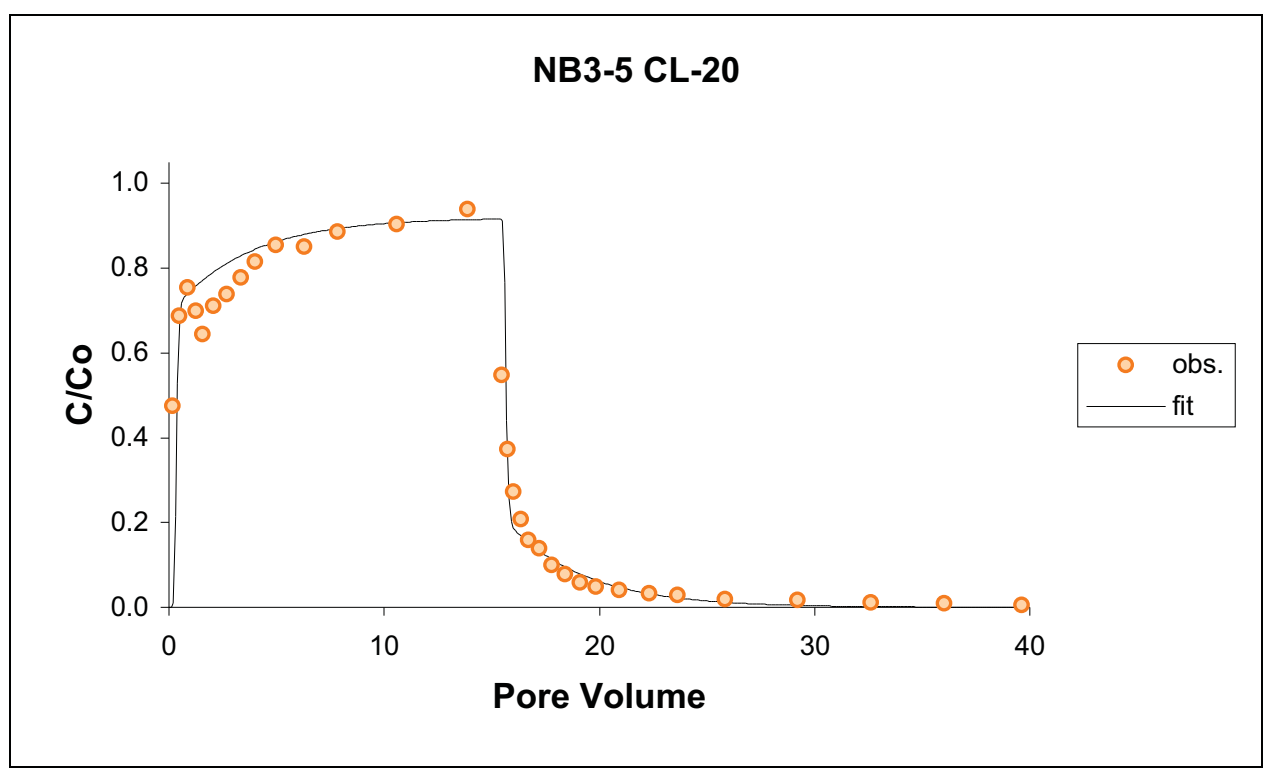

Figure J.38 Breakthrough curve and model output for CL-20 in unsaturated Norborne soil. $\left(\theta=0.17 ; v=20.9 \mathrm{~cm} / \mathrm{hr} ; D=4.02 \mathrm{hr}^{-1} ; \beta=0.310 ; \omega=0.256 ; R=1.2 ; K_{d-a p p}=0.021 ; \mu_{1}=0.151 \mathrm{hr}^{-1}\right)$

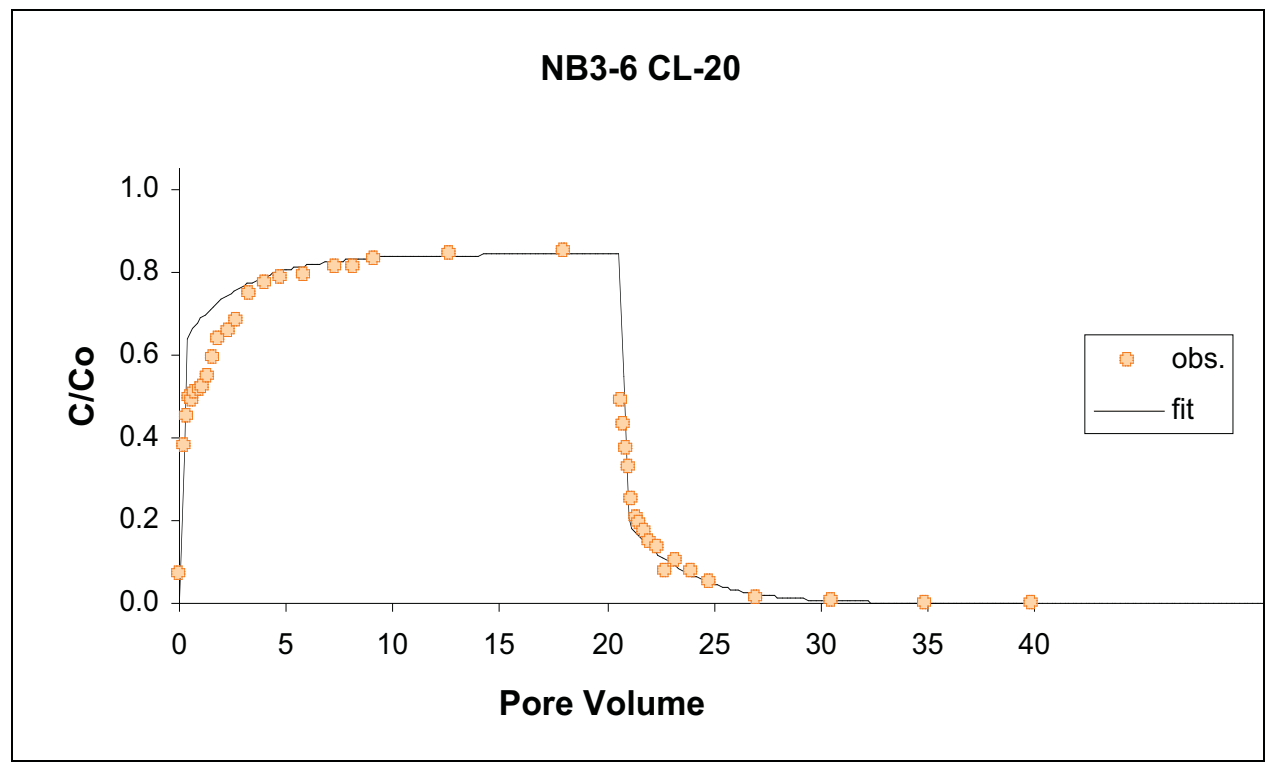

Figure J.39 Breakthrough curve and model output for CL-20 in unsaturated Norborne soil. $\left(\theta=0.15 ; v=4.76 \mathrm{~cm} / \mathrm{hr} ; D=2.00 \mathrm{hr}^{-1} ; \beta=0.233 ; \omega=0.291 ; R=0.91 ; K_{d-a p p}<0 ; \mu_{1}=0.290 \mathrm{hr}^{-1}\right)$ 


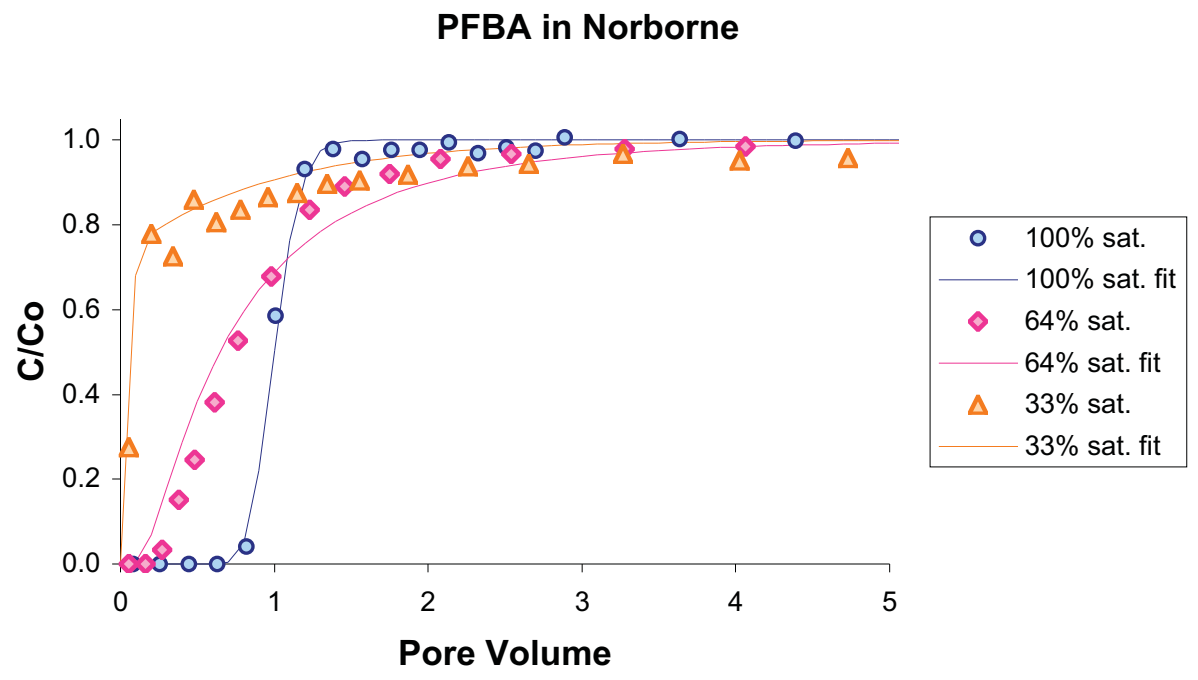

Figure J.40 Comparison of PFBA transport in Norborne soil with changing water content. Note that breakthrough accelerates with decreasing water content. (SN2-1; NB3-2; NB3-6)

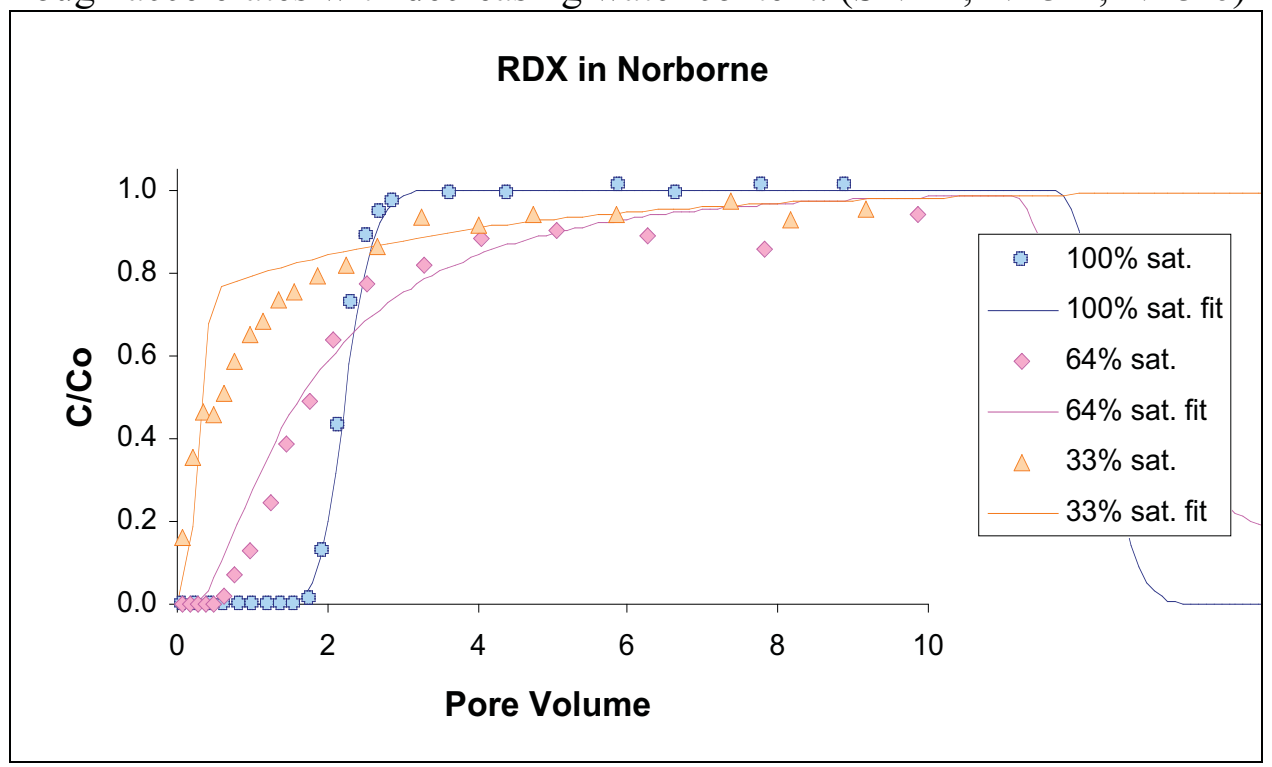

Figure J.41 Comparison of RDX transport in Norborne soil with changing water content. Note that breakthrough accelerates with decreasing water content. (SN2-1; NB3-2; NB3-6) 


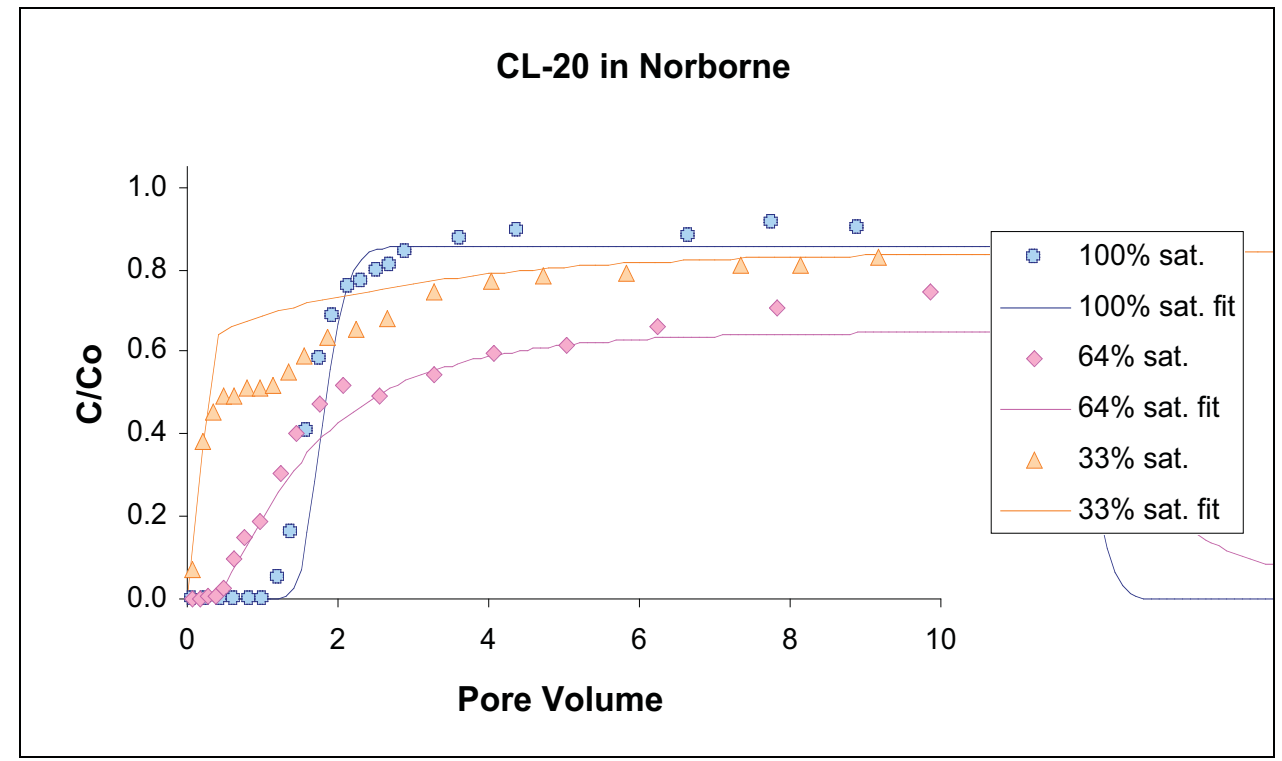

Figure J.42 Comparison of Cl-20 transport in Norborne soil with changing water content. Note that breakthrough accelerates with decreasing water content. (SN2-1; NB3-2; NB3-6) 


\section{Appendix J3. Unsaturated Flow Experiments in the Burbank sediment}

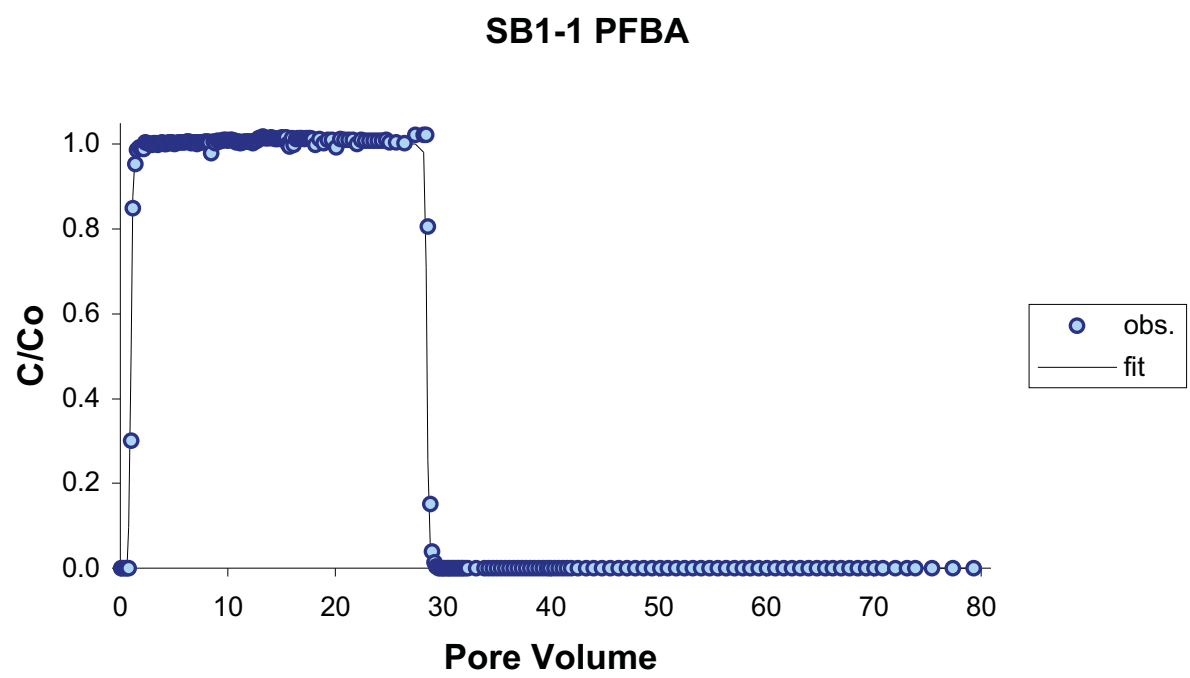

Figure J.43 Breakthrough curve and model output for PFBA in saturated Burbank soil. $\left(\theta=0.41 ; v=79.0 \mathrm{~cm} / \mathrm{hr} ; D=13.3 \mathrm{hr}^{-1} ; R=1.0\right)$

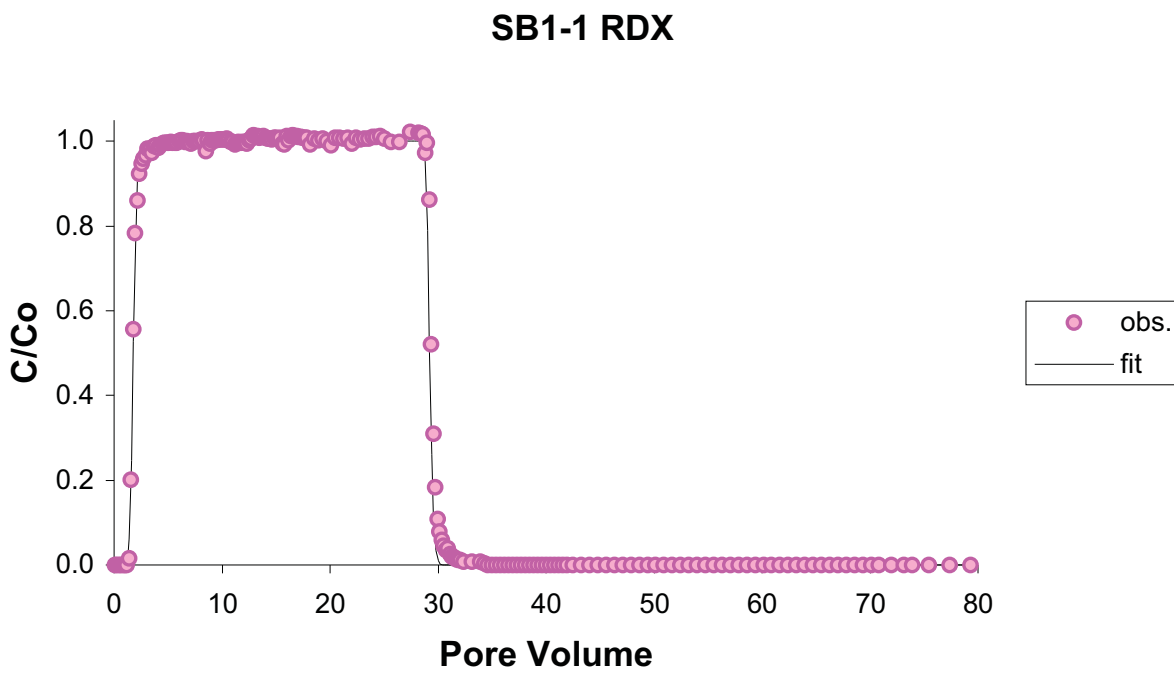

Figure J.44 Breakthrough curve and model output for RDX in saturated Burbank soil. $(\theta=0.41 ; v=$ $79.0 \mathrm{~cm} / \mathrm{hr} ; D=13.3 \mathrm{hr}^{-1} ; R=1.8 ; K_{\text {d-app }}=0.23$ ) 


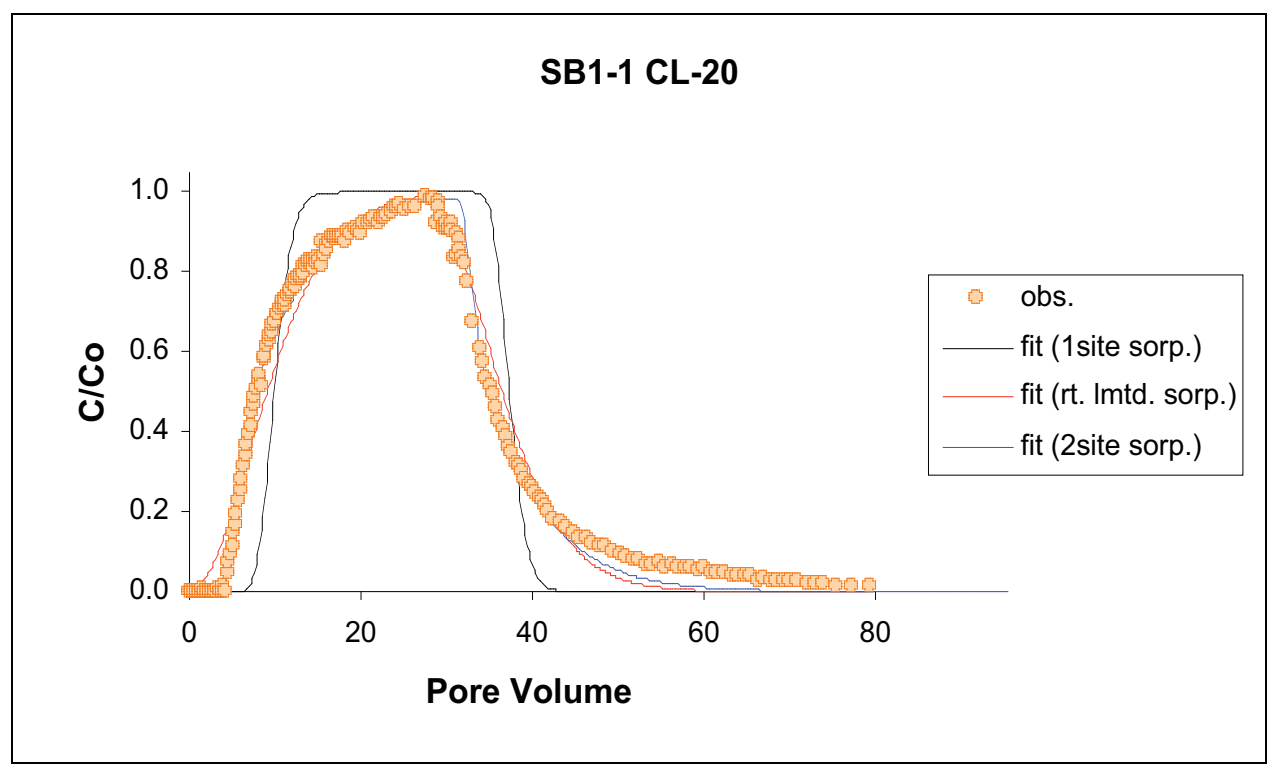

Figure J.45 Breakthrough curve and model output of CL-20 in saturated Burbank soil. The one-site sorption model (1 site sorp.) assumes ideal, instantaneous sorption behavior at one type of sorption site. The rate-limited sorption model (rt. lmtd. sorp.) assumes sorption occurs at one type of sorption site by a rate limited reaction. The two-site sorption model assumes that sorption occurs at two unique types of sorption sites. $\left(\theta=0.41 ; v=79.0 \mathrm{~cm} / \mathrm{hr} ; D=13.3 \mathrm{hr}^{-1} ; \beta=0.553 ; \omega=0.108 \mathrm{hr}^{-1} ; R=10 ; K_{d-a p p}=2.5\right)$

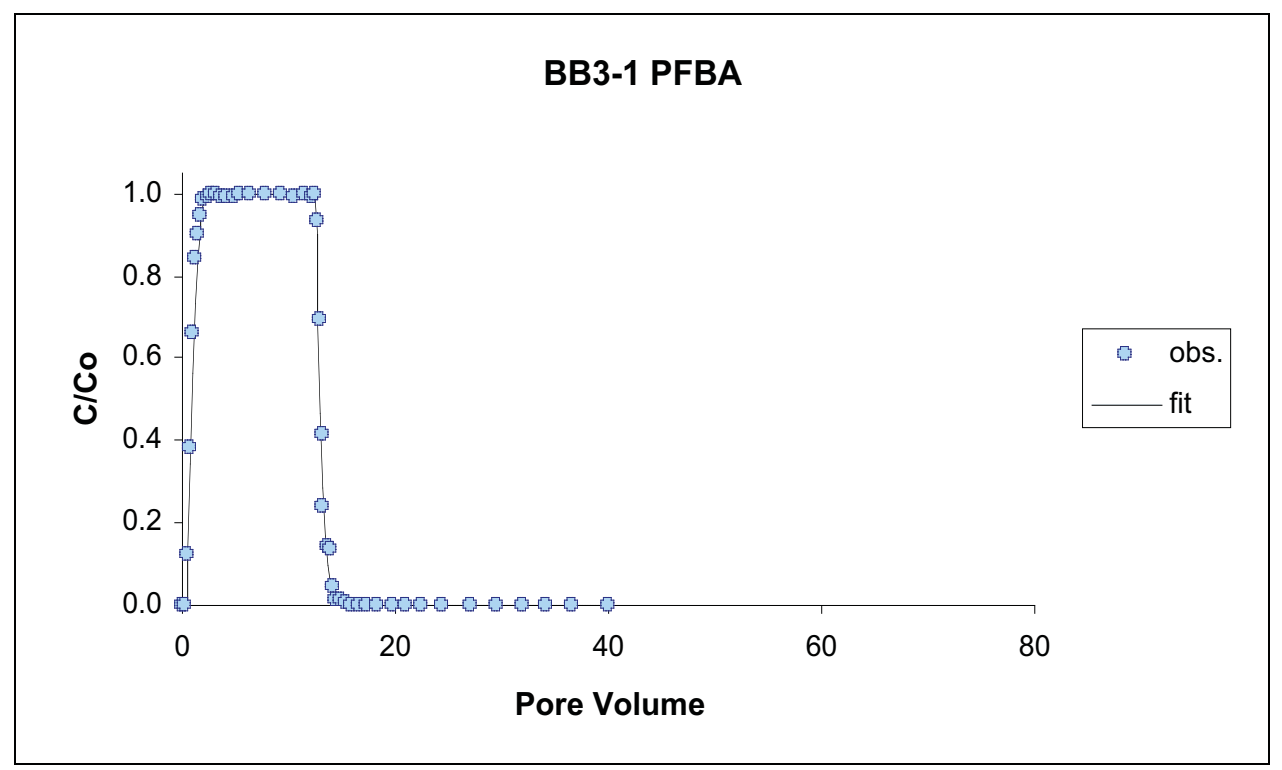

Figure J.46 Breakthrough curve and model output for PFBA in unsaturated Burbank soil. $\left(\theta=0.24 ; v=15.2 \mathrm{~cm} / \mathrm{hr} ; D=7.40 \mathrm{hr}^{-1} ; R=1.0\right)$ 
BB3-2 PFBA

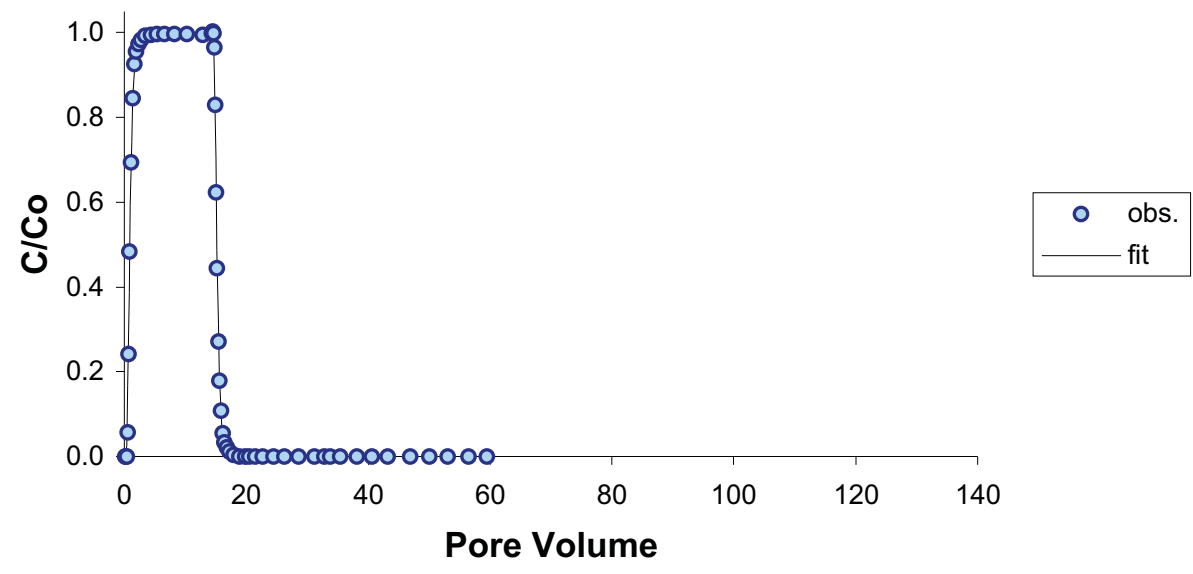

Figure J.47 Breakthrough curve and model output for PFBA in unsaturated Burbank soil. $\left(\theta=0.22 ; v=3.37 \mathrm{~cm} / \mathrm{hr} ; D=2.00 \mathrm{hr}^{-1} ; R=0.99\right)$

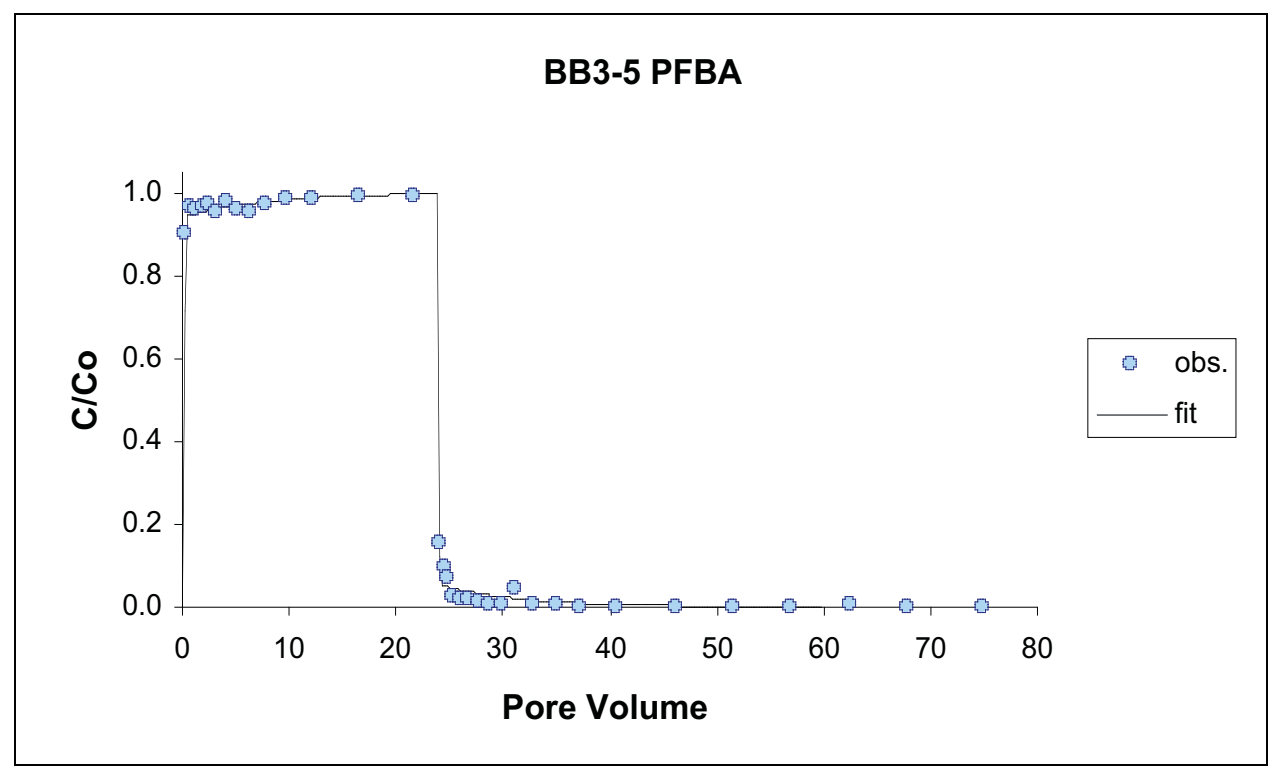

Figure J.48 Breakthrough curve and model output for PFBA in unsaturated Burbank soil. $\left(\theta=0.11 ; v=33.3 \mathrm{~cm} / \mathrm{hr} ; D=3.43 \mathrm{hr}^{-1} ; \beta=0.290 ; \omega=0.0580 \mathrm{hr}^{-1} ; R=0.62\right)$ 


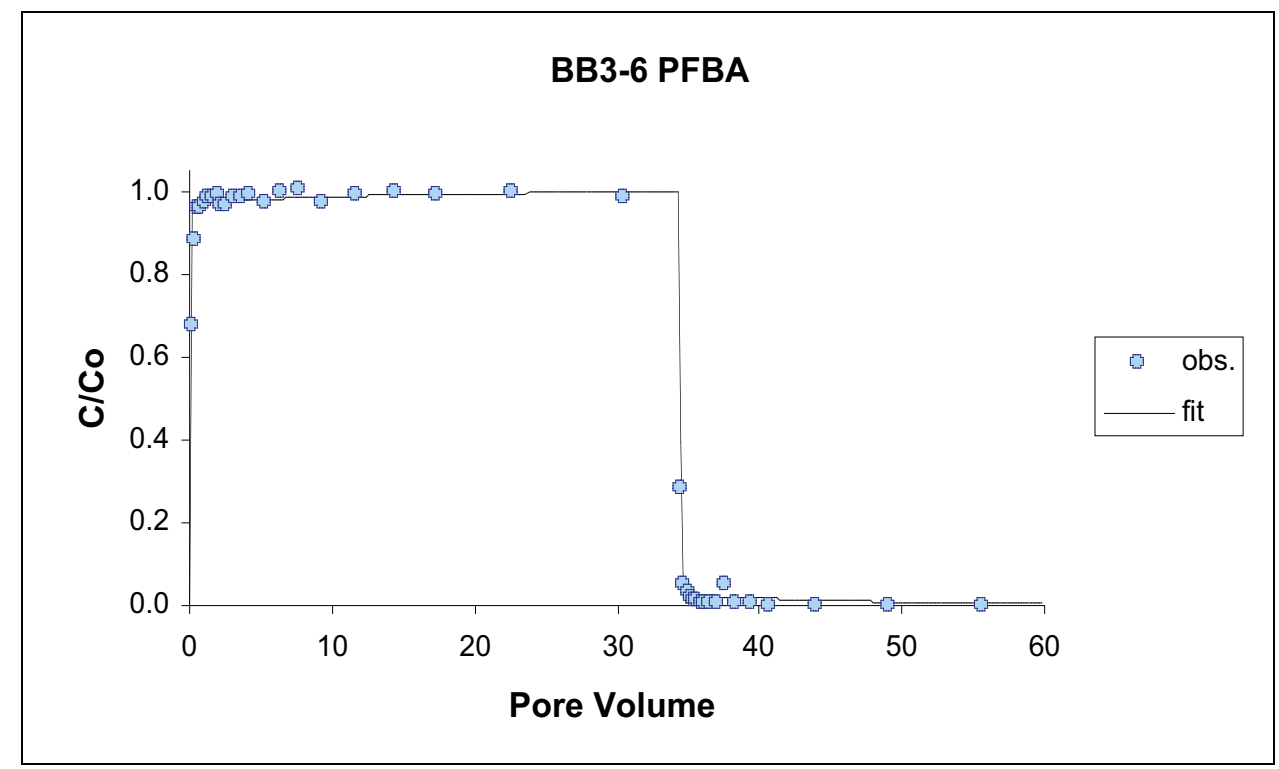

Figure J.49 Breakthrough curve and model output for PFBA in unsaturated Burbank soil. $\left(\theta=0.0 .097 ; v=7.65 \mathrm{~cm} / \mathrm{hr} ; D=2.17 \mathrm{hr}^{-1} ; \beta=0.232 ; \omega=0.296 \mathrm{hr}^{-1} ; R=0.49\right)$

\section{BB3-2 PFBA}

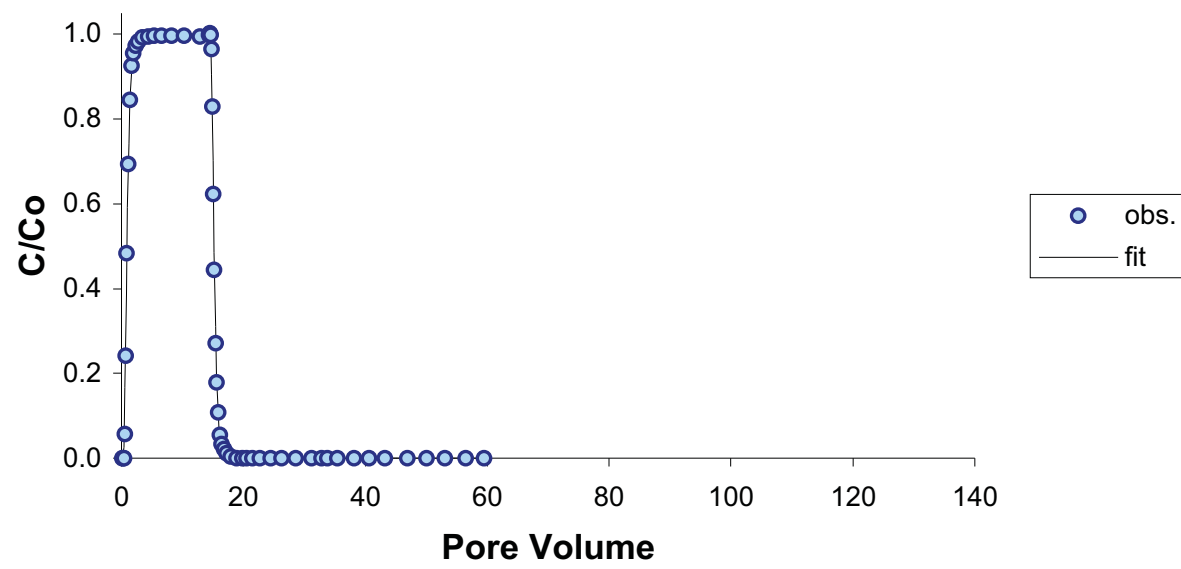

Figure J.50 Breakthrough curve and model output for RDX in unsaturated Burbank soil. $\left(\theta=0.24 ; v=15.2 \mathrm{~cm} / \mathrm{hr} ; D=7.40 \mathrm{hr}^{-1} ; R=2.2 ; K_{d-a p p}=0.19\right)$ 


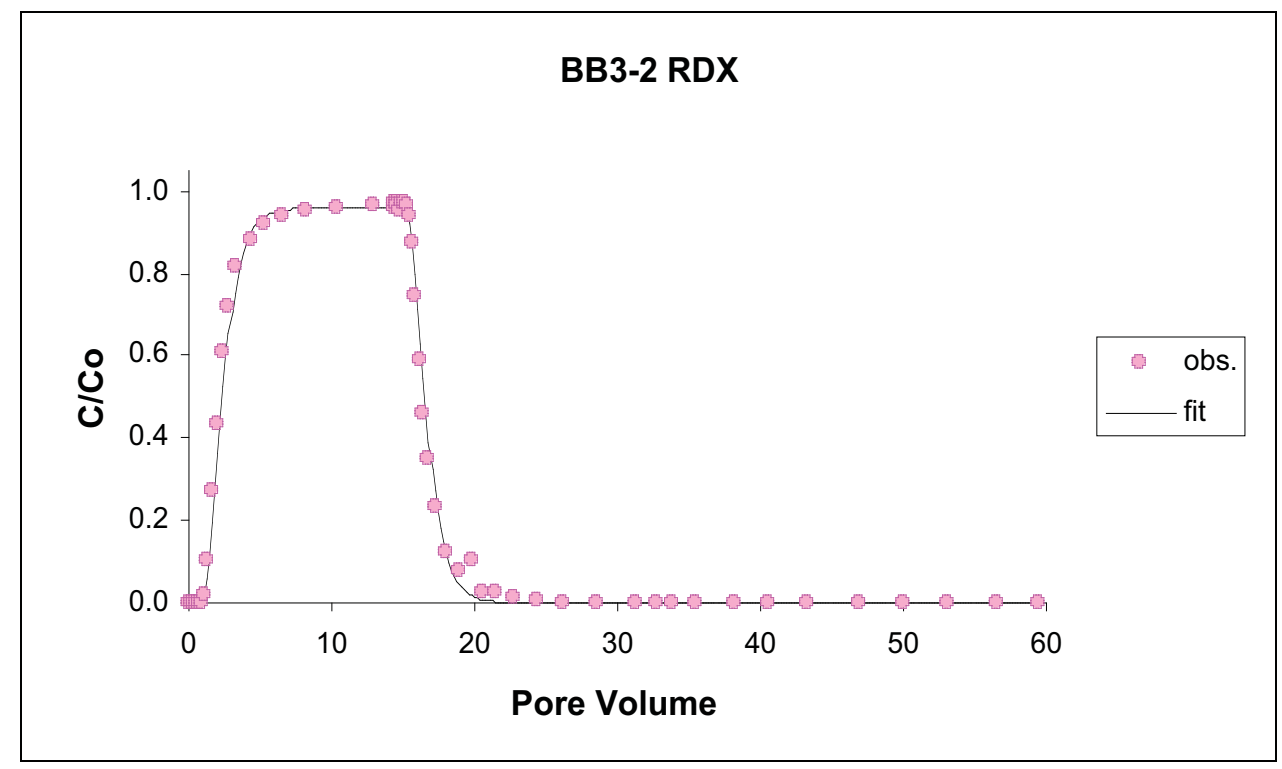

Figure J.51 Breakthrough curve and model output for RDX in unsaturated Burbank soil. $\left(\theta=0.22 ; v=3.37 \mathrm{~cm} / \mathrm{hr} ; D=2.00 \mathrm{hr}^{-1} ; R=2.5 ; K_{d-a p p}=0.22\right)$

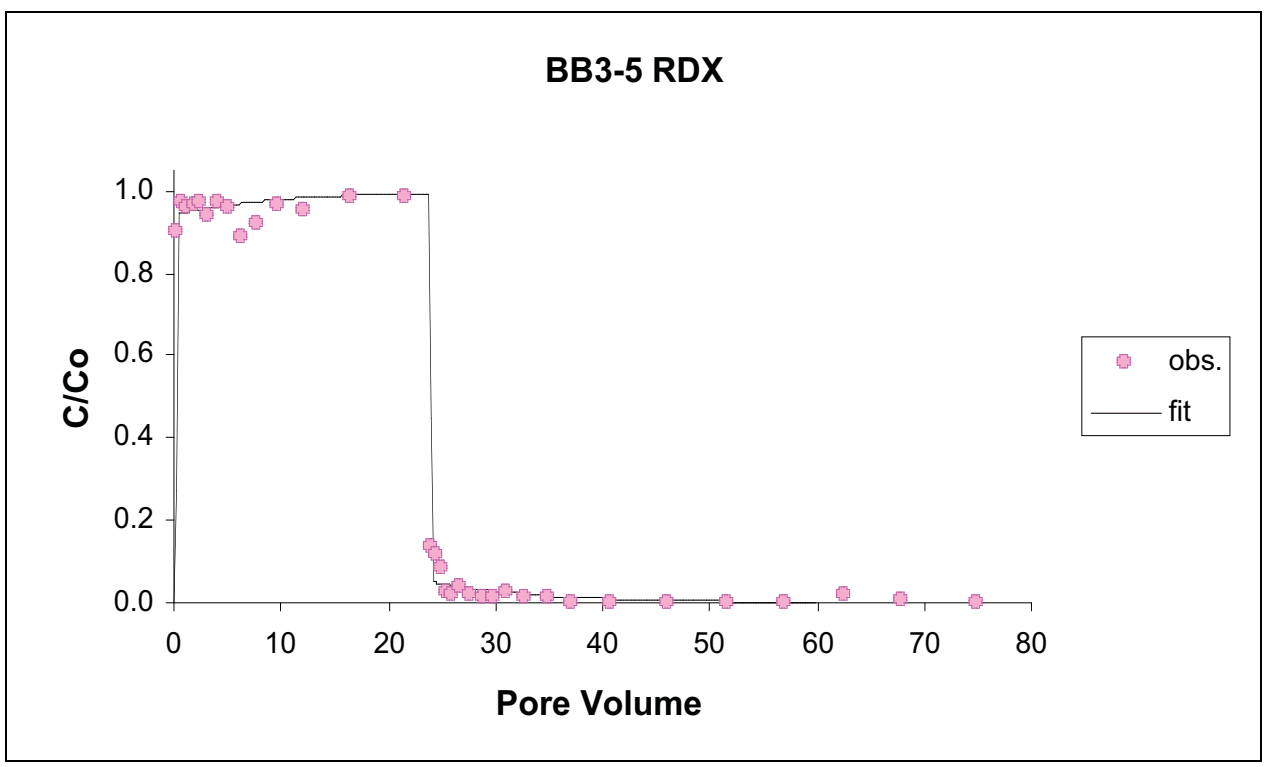

Figure J.52 Breakthrough curve and model output for RDX in unsaturated Burbank soil. $\left(\theta=0.11 ; v=33.3 \mathrm{~cm} / \mathrm{hr} ; D=3.43 \mathrm{hr}^{-1} ; \beta=0.290 ; \omega=0.0580 \mathrm{hr}^{-1} ; R=0.78 ; K_{\text {d-app }}<0\right)$ 


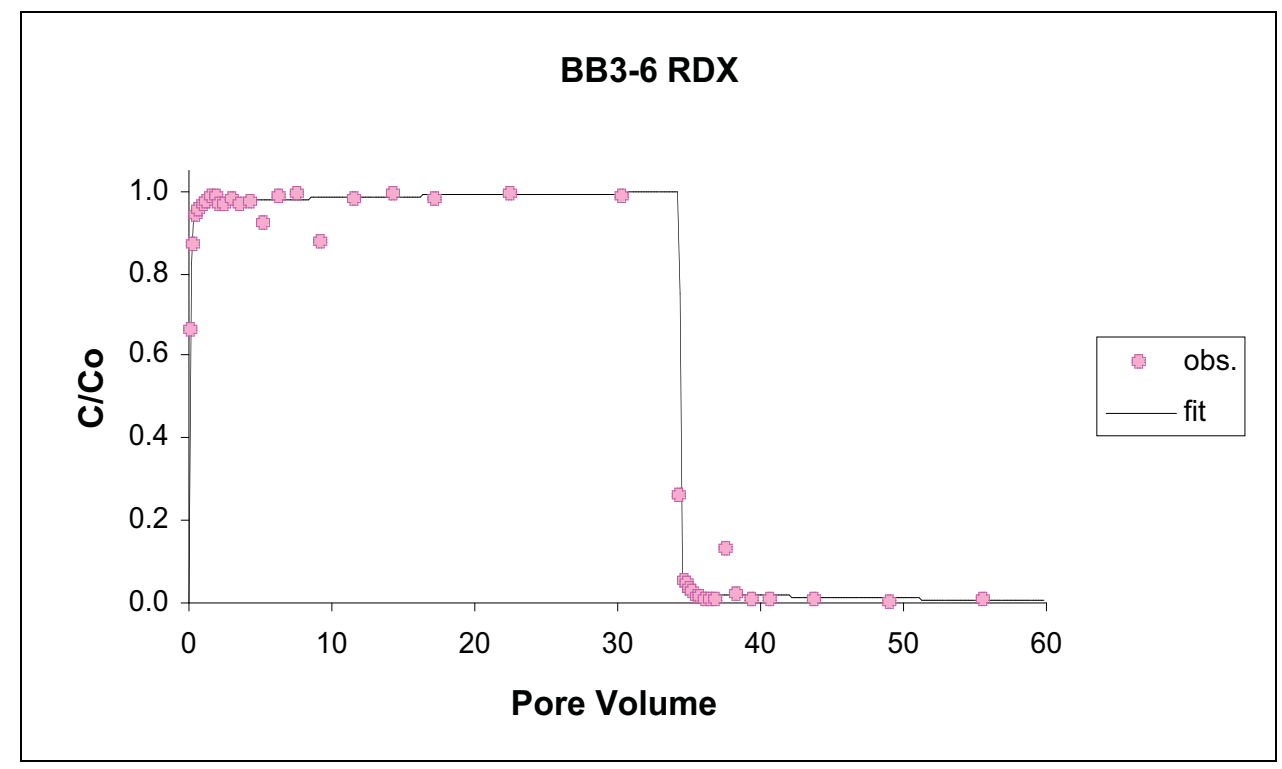

Figure J.53 Breakthrough curve and model output for RDX in unsaturated Burbank soil. $\left(\theta=0.0 .097 ; v=7.65 \mathrm{~cm} / \mathrm{hr} ; D=2.17 \mathrm{hr}^{-1} ; \beta=0.232 ; \omega=0.296 \mathrm{hr}^{-1} ; R=0.80 ; K_{\text {d-app }}<0\right)$

\section{BB3-1 CL-20}

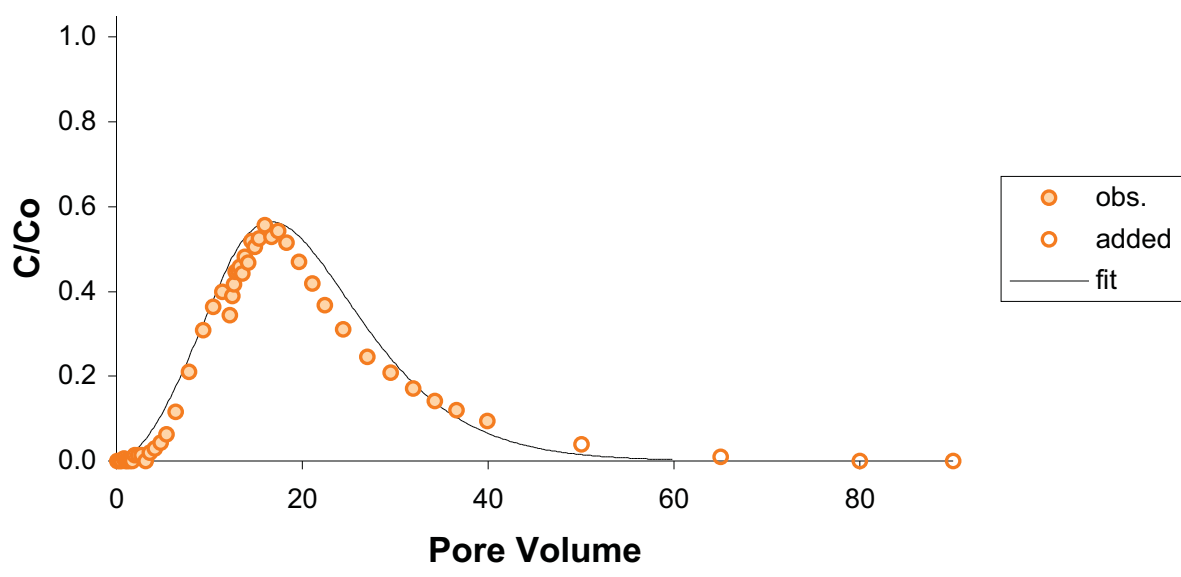

Figure J.54 Breakthrough curve and model output for CL-20 in unsaturated Burbank soil. CL-20 was modeled with a two-site sorption nonequilibrium approach. $\left(\theta=0.24 ; v=15.2 \mathrm{~cm} / \mathrm{hr} ; D=7.40 \mathrm{hr}^{-1} ; \beta=\right.$ $\left.0.0696 ; \omega=8.22 \mathrm{hr}^{-1} ; R=14 ; K_{\text {d-app }}=2.1\right)$ 


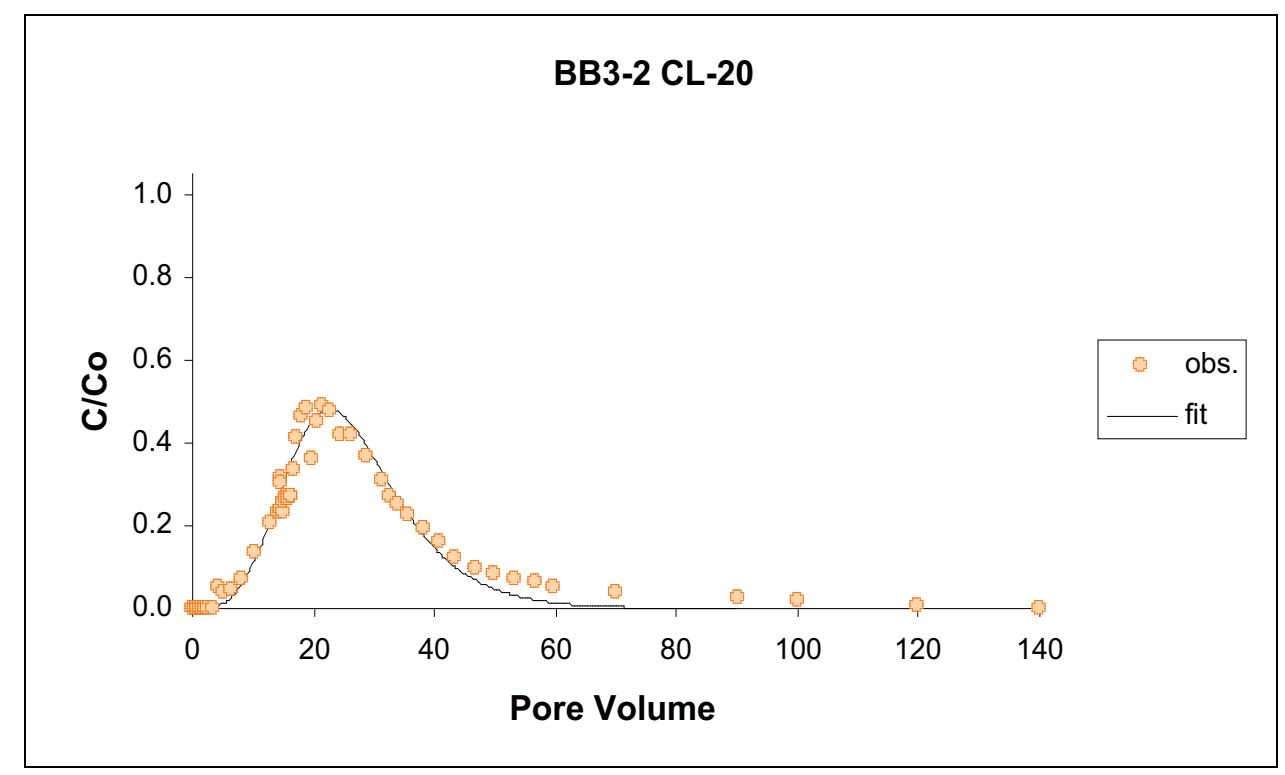

Figure J.55 Breakthrough curve and model output for CL-20 in unsaturated Burbank soil. CL-20 was modeled with a two-site sorption nonequilibrium model with the calculated first-order degradation rate coefficient $\left(\mu_{1}\right)$ fixed at $0.129 \mathrm{hr}^{-1} .\left(\theta=0.22 ; v=3.37 \mathrm{~cm} / \mathrm{hr} ; D=2.00 \mathrm{hr}^{-1} ; \beta=0.0481 ; \omega=32.4 \mathrm{hr}^{-1} ; R\right.$ $\left.=21 ; K_{d-a p p}=2.9 ; \mu_{1}=0.129 \mathrm{hr}^{-1}\right)$

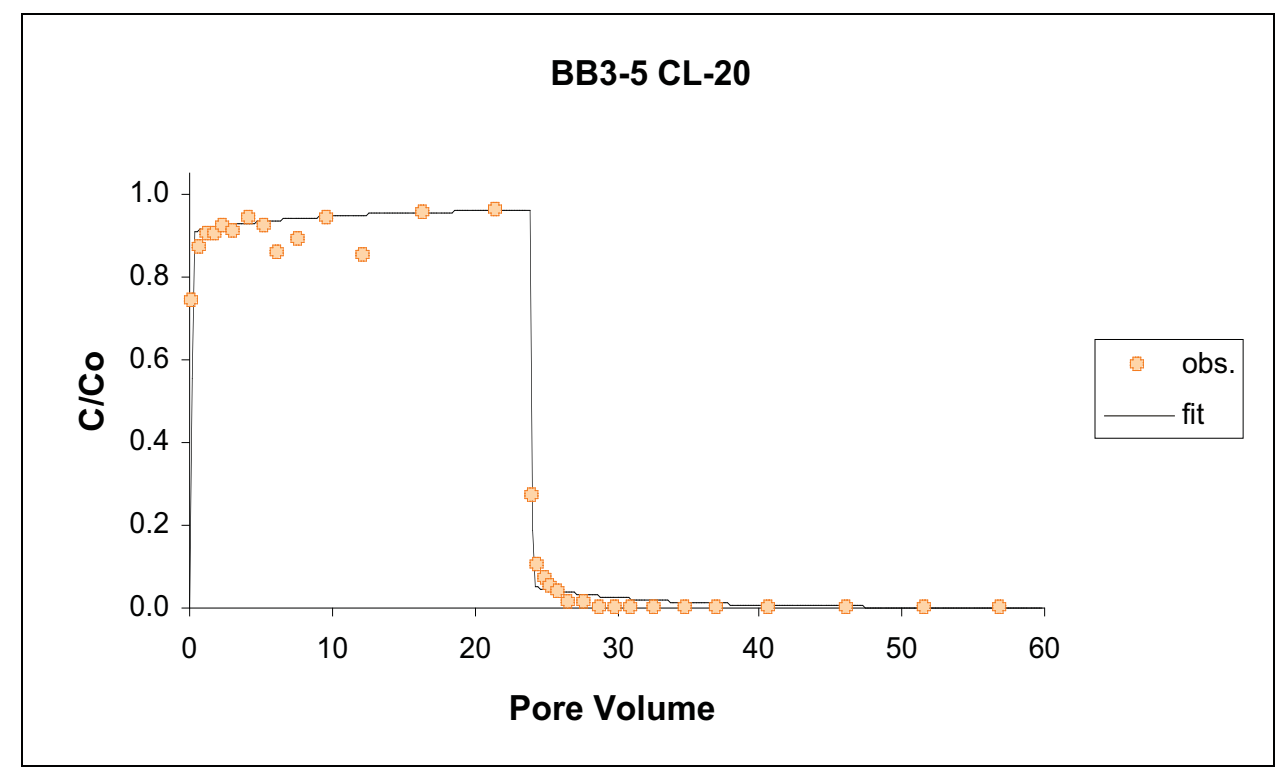

Figure J.56 Breakthrough curve and model output for CL-20 in unsaturated Burbank soil. $(\theta=0.11 ; v=$ $\left.33.3 \mathrm{~cm} / \mathrm{hr} ; D=3.43 \mathrm{hr}^{-1} ; \beta=0.290 ; \omega=0.0580 \mathrm{hr}^{-1} ; R=0.67 ; K_{d-a p p}<0 ; \mu_{1}=0.0380 \mathrm{hr}^{-1}\right)$ 


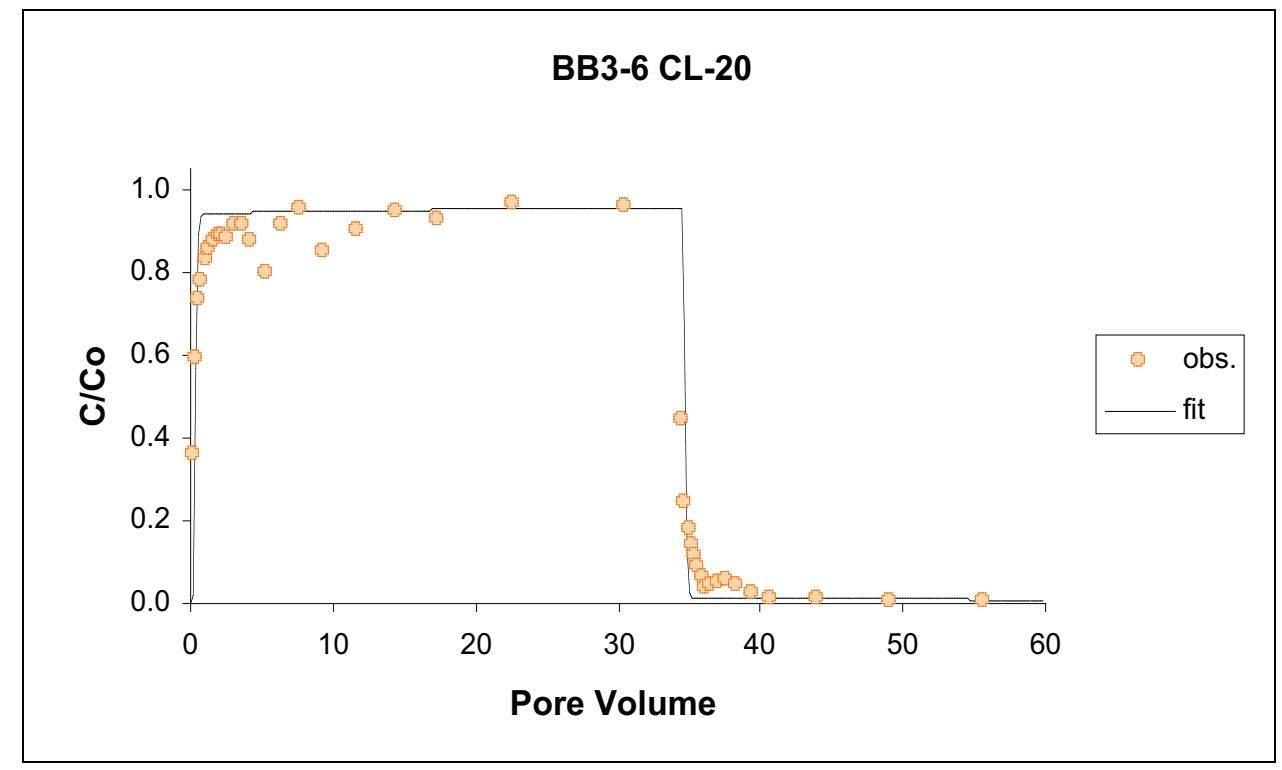

Figure J.57 Breakthrough curve and model output for CL-20 in unsaturated Burbank soil. $(\theta=0.0 .097 ; v$ $\left.=7.65 \mathrm{~cm} / \mathrm{hr} ; D=2.17 \mathrm{hr}^{-1} ; \beta=0.232 ; \omega=0.296 \mathrm{hr}^{-1} ; R=1.7 ; K_{\text {d-app }}=0.044 ; \mu_{1}=0.0310 \mathrm{hr}^{-1}\right)$

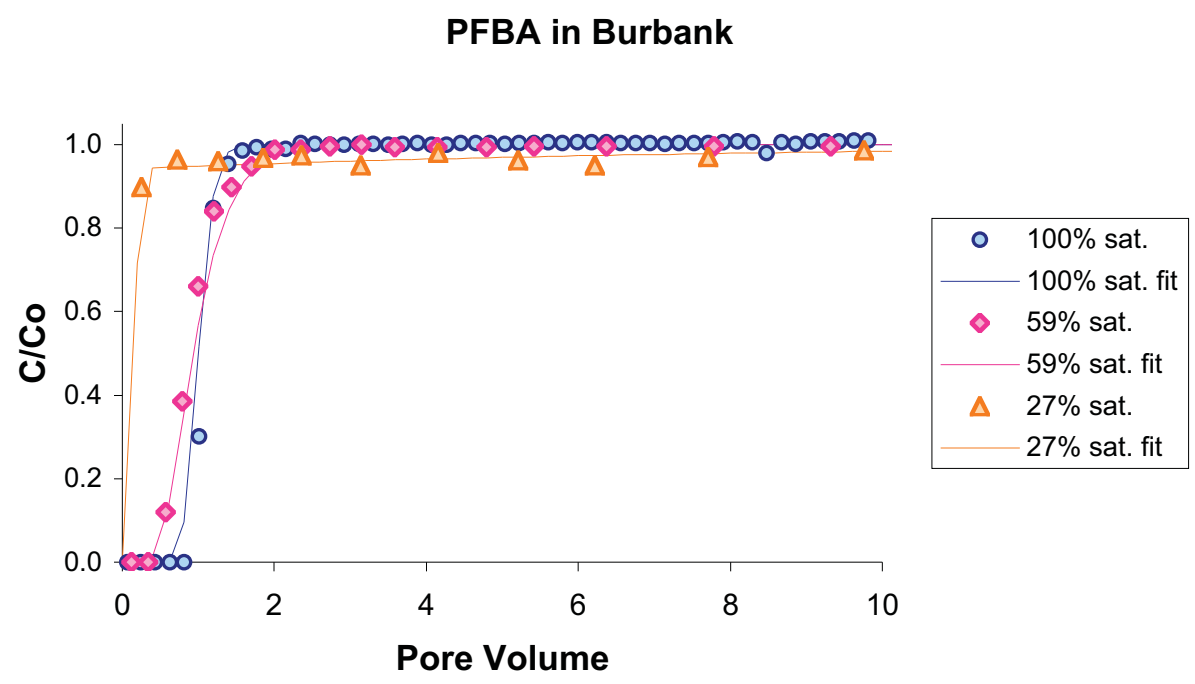

Figure J.58 Comparison of PFBA transport in Burbank soil with changing water content. Note that breakthrough accelerates with decreasing water content. (SB1-1, BB3-1, BB3-5) 


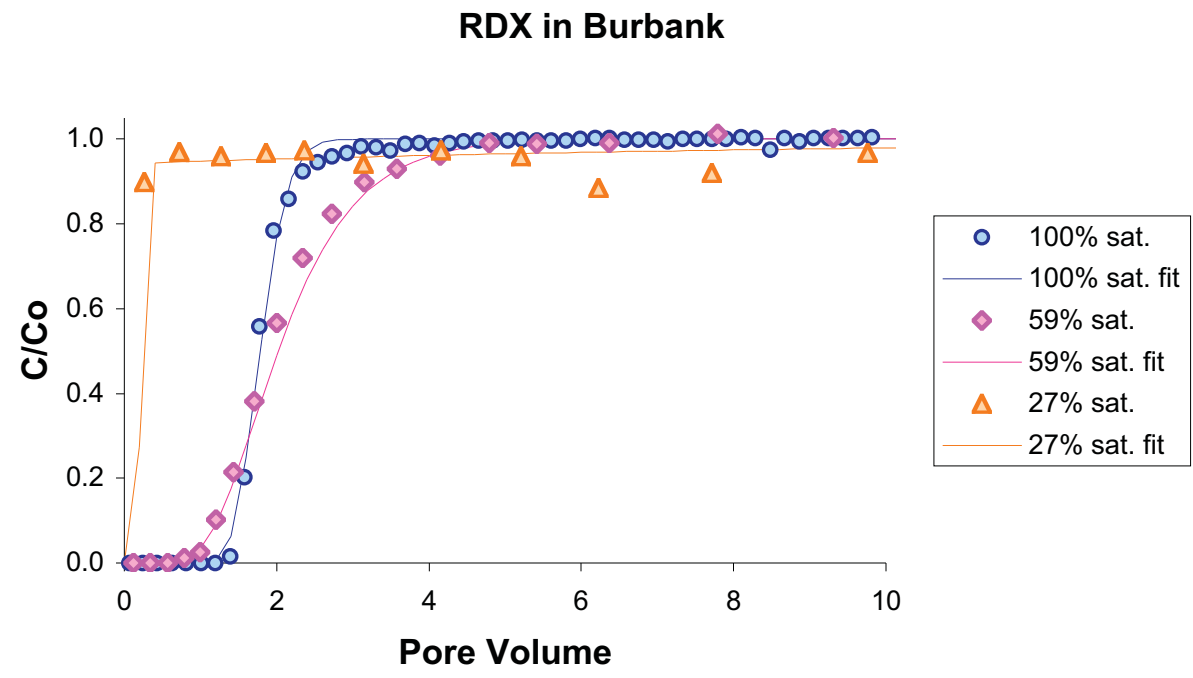

Figure J.59 Comparison of RDX transport in Burbank soil with changing water content. Note that breakthrough accelerates with decreasing water content. (SB1-1, BB3-1, BB3-5)

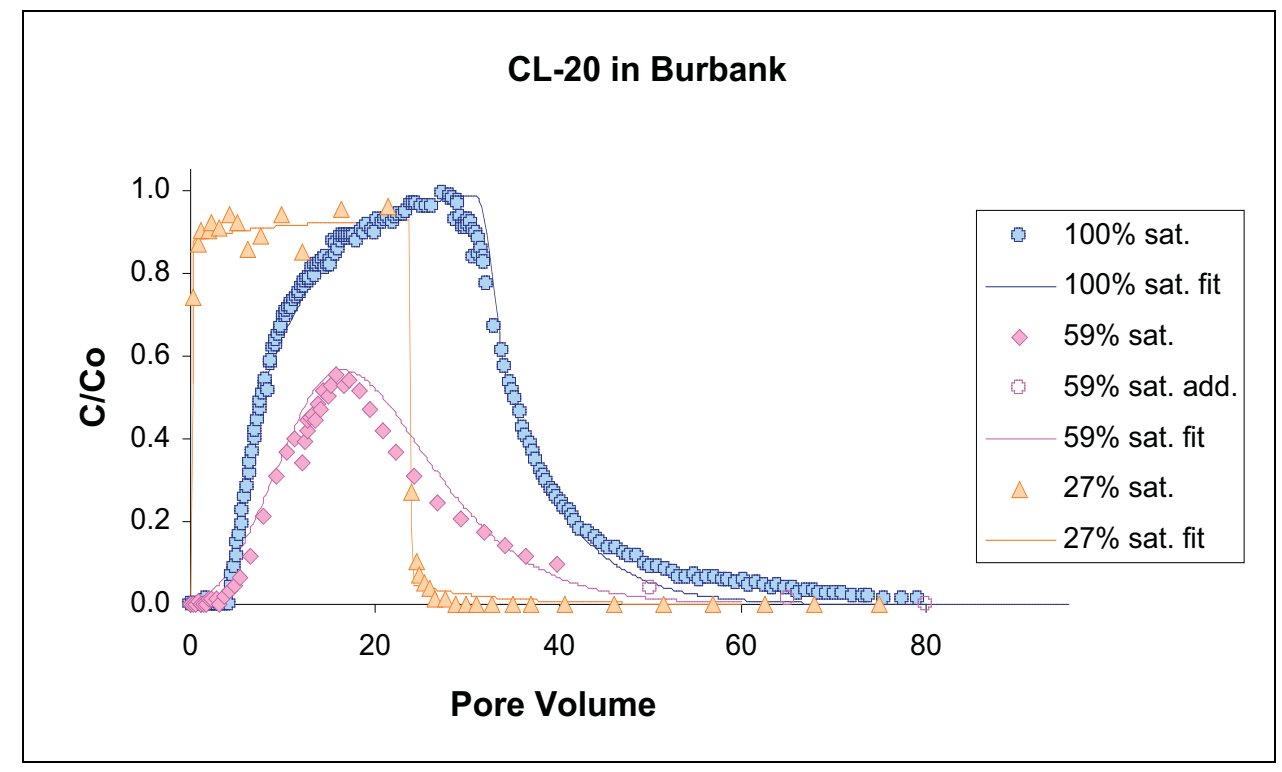

Figure J.60 Comparison of CL-20 transport in Burbank soil with changing water content. Note the extreme variation in CL-20 transport with varying water content. The apparent soil water distribution coefficient appears to increase when saturation is lowered from $100 \%$ to $59 \%$, but then decreases dramatically at the lowest water content. (SB1-1, BB3-1, BB3-5) 